\title{
WERDC
}

\section{Integration of Autonomous Electric Transport Vehicles into a Tactical Microgrid}

Final Report

Angela Rolufs, Amelia Trout, Kevin Palmer, Clark Boriack,

September 2021

Bryan Brilhart and Annette L. Stumpf

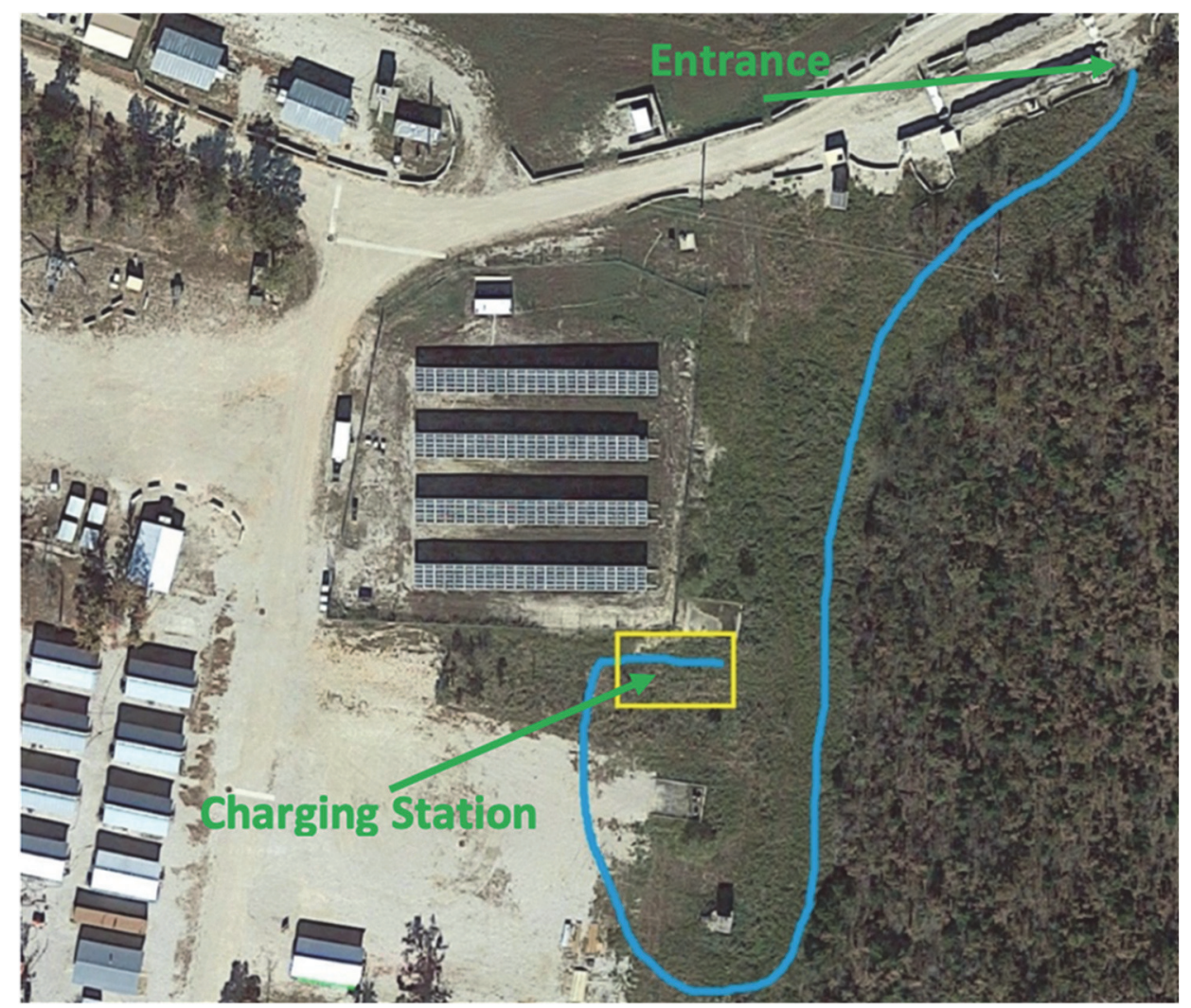


The U.S. Army Engineer Research and Development Center (ERDC) solves the nation's toughest engineering and environmental challenges. ERDC develops innovative solutions in civil and military engineering, geospatial sciences, water resources, and environmental sciences for the Army, the Department of Defense, civilian agencies, and our nation's public good. Find out more at www.erdc.usace.army.mil.

To search for other technical reports published by ERDC, visit the ERDC online library at https://erdclibrary.on.worldcat.org/discovery. 


\section{Integration of Autonomous Electric Transport Vehicles into a Tactical Microgrid}

Final Report

Annette L. Stumpf

U.S. Army Engineer Research and Development Center (ERDC)

Construction Engineering Research Laboratory (CERL)

2902 Newmark Dr.

Champaign, IL 61824

Angela Rolufs and Amelia Trout

Paragon Business Solutions, Inc.

610 N. Olive Street

Rolla, MO 65401

Kevin Palmer

CS2 (Center for Sustainable Solutions)

2700 Vinton Ct.

Charlottesville, VA 22901

Clark Boriack

TechFlow

9889 Willow Creek Road; Suite 100

San Diego, CA 92131

Bryan Brilhart

Robotic Research

555 Quince Orchard Road, Suite 300

Gaithersburg, MD 20878

Final Technical Report (TR)

Approved for public release; distribution is unlimited.

Prepared for Headquarters, U.S. Army Corps of Engineers (HQUSACE)

Washington, DC 20314-1000

Under Project 6LKD1G “FY19 Program Increase: Autonomous Transport Innovation”; and Contract Number W9132T19C0003, "Autonomous Transport Innovation: Integration of Autonomous Electric Transport Vehicles into a Tactical Microgrid." 


\section{Abstract}

The objective of the Autonomous Transport Innovation (ATI) technical research program is to investigate current gaps and challenges and develop solutions to integrate emerging electric transport vehicles, vehicle autonomy, vehicle-to-grid (V2G) charging and microgrid technologies with military legacy equipment. The ATI research area objectives are to: identify unique military requirements for autonomous transportation technologies; identify currently available technologies that can be adopted for military applications and validate the suitability of these technologies to close need gaps; identify research and operational tests for autonomous transport vehicles; investigate requirements for testing and demonstrating of bidirectional-vehicle charging within a tactical environment; develop requirements for a sensored, living laboratory that will be used to assess the performance of autonomous innovations; and integrate open standards to promote interoperability and broad-platform compatibility. This final report summarizes the team's research, which resulted in an approach to develop a sensored, living laboratory with operational testing capability to assess the safety, utility, interoperability, and resiliency of autonomous electric transport and $\mathrm{V}_{2} \mathrm{G}$ technologies in a tactical microgrid. The living laboratory will support research and assessment of emerging technologies and determine the prospect for implementation in defense transport operations and contingency base energy resilience.

DISCLAIMER: The contents of this report are not to be used for advertising, publication, or promotional purposes. Citation of trade names does not constitute an official endorsement or approval of the use of such commercial products. All product names and trademarks cited are the property of their respective owners. The findings of this report are not to be construed as an official Department of the Army position unless so designated by other authorized documents.

DESTROY THIS REPORT WHEN NO LONGER NEEDED. DO NOT RETURN IT TO THE ORIGINATOR. 


\section{Contents}

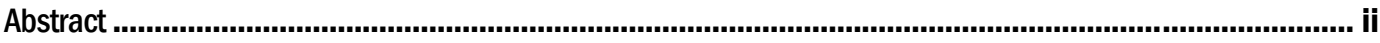

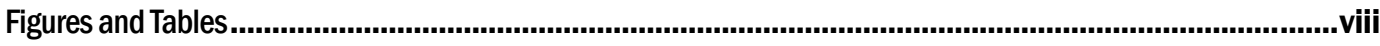

Preface................................................................................................................................... xili

1 Introduction ............................................................................................................................... 1

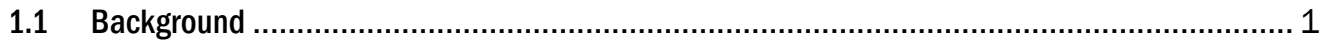

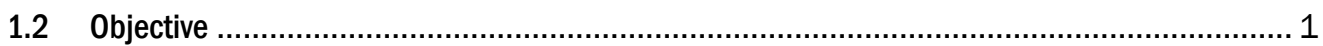

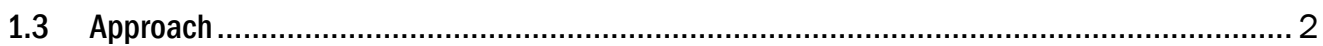

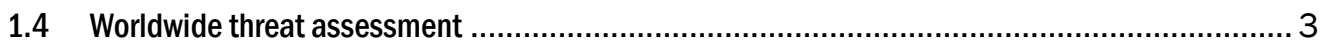

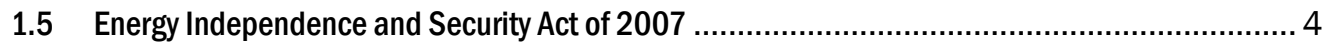

1.6 A modernized grid requires a standardized environment ................................................ 4

1.7 Electric transportation as a distributed energy resource................................................ 5

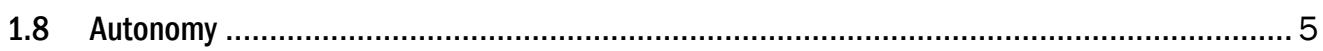

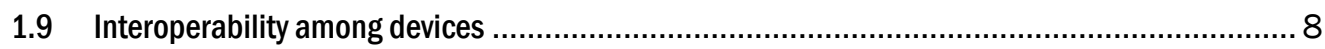

1.10 DoD 2016 operational energy strategy .................................................................... 11

1.11 U.S. Army's Energy Security and Sustainability Policy .................................................. 12

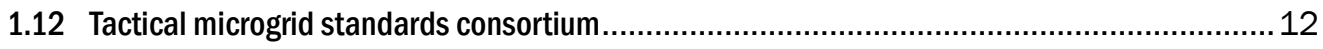

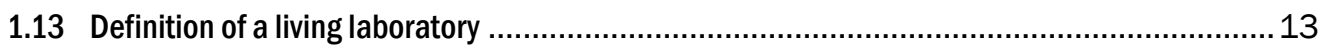

1.14 The CERL mission........................................................................................... 14

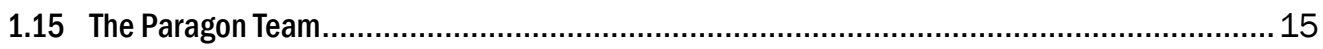

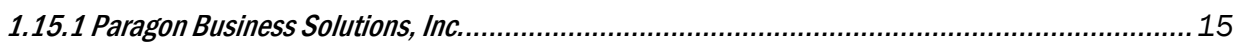

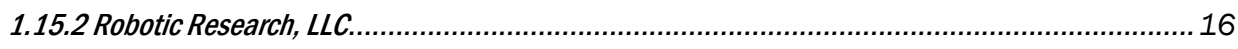

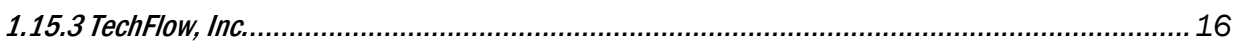

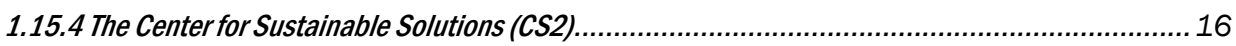

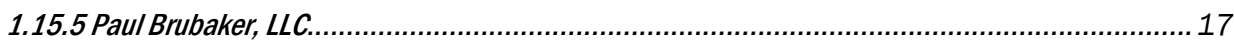

1.16 Autonomous Transport Innovation ................................................................... 17

2 Identify Military-Unique Requirements for Autonomous Transport Technologies.......................................19

2.1 Military support activities and associated transport systems ............................................. 19

2.1.1 Vehicle-dependent Military Support Activities ..................................................................... 19

2.1.2 Military Support Activity transport requirements............................................................. 22

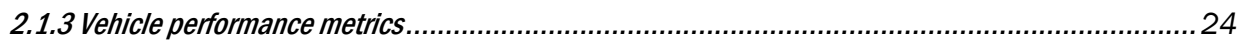

2.2 Autonomous transport vehicle military use cases...................................................... 25

2.2.1 Use case for an autonomous vehicle supply convoy................................................................. 25

2.2.2 Use case for an autonomous vehicle reconnaissance mission (command, control, communications, computing, intelligence sunveillance reconnaissance) ...............................28

2.2.3 Use case for an autonomous vehicle construction support mission..........................................30

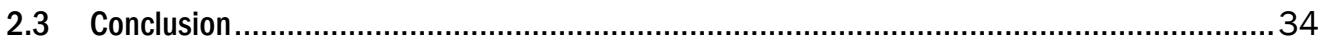

3 Research and Identify Existing Technologies That can be Adapted for Military Applications .......................35

3.1 Transport systems that can be automated to the benefit of the military .................................35

3.1.1 Candidate electric transport systems ................................................................................ 36 


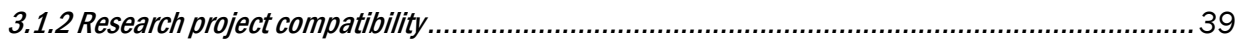

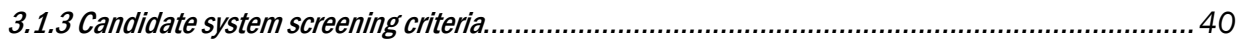

3.1.4 Prioritized categorized list of candidate systems............................................................... 43

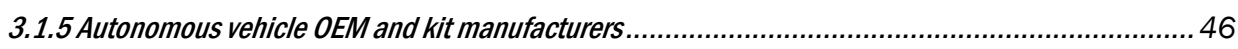

3.2 Engagement with EV transport manufacturers..........................................................46

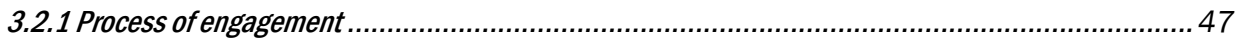

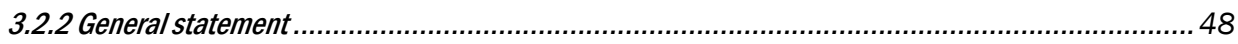

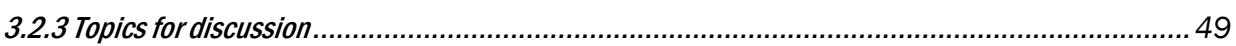

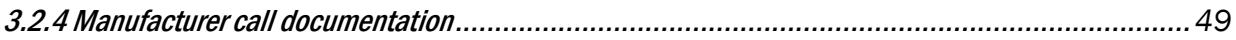

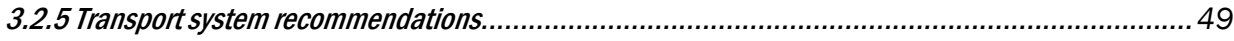

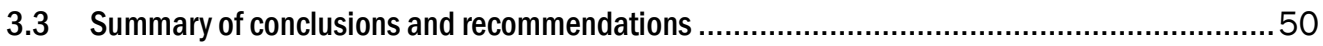

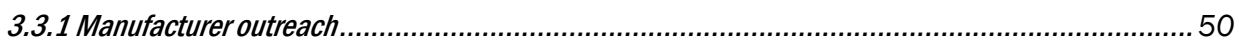

3.3.2 Manufacturer technology summaries and recommended research ............................................51

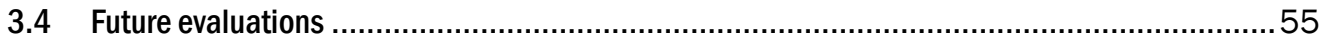

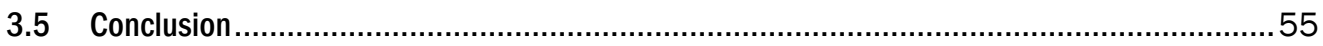

4 Research and Develop Operational Tests for Autonomous Transport Vehicles .........................................57

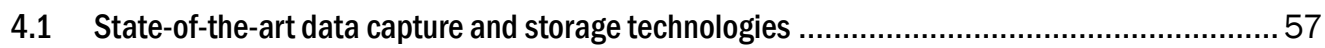

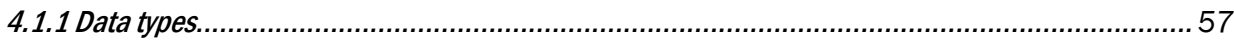

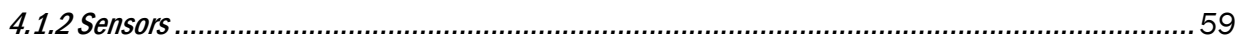

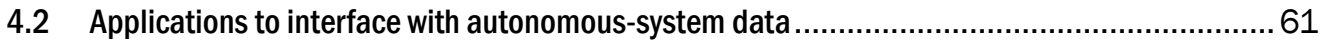

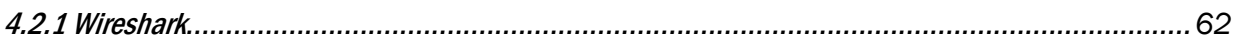

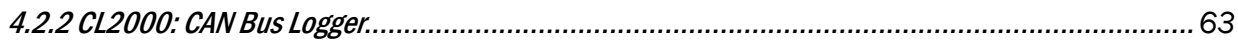

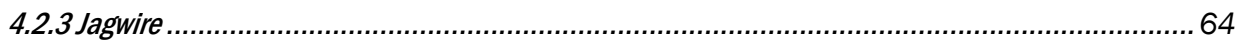

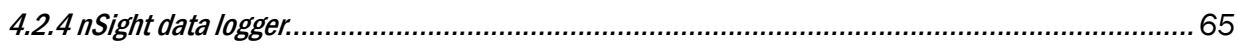

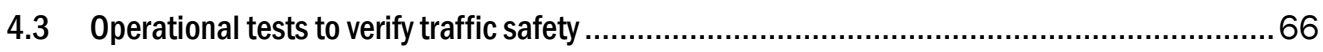

4.3.1 Compare human drive behavior to autonomous-system behavior ........................................... 67

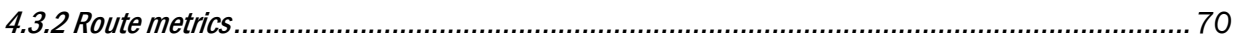

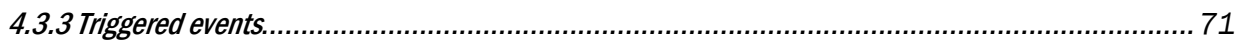

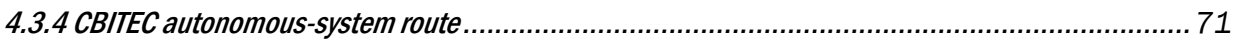

4.4 Monitoring autonomous component degradation....................................................... 72

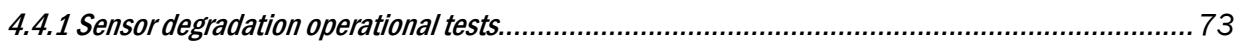

4.5 Autonomous-system terrain technologies........................................................ 74

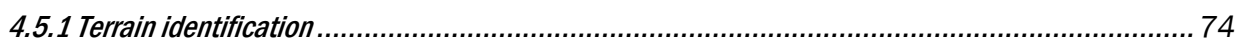

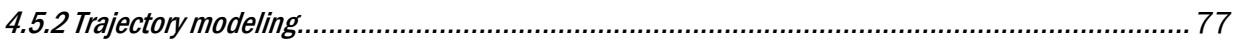

4.5.3 Autonomous docking with charging stations ....................................................................... 78

4.5.4 Optimization to reduce autonomous vehicle system power consumption.................................... 79

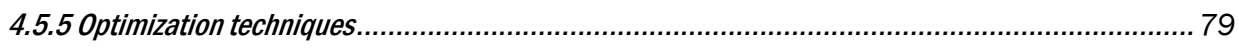

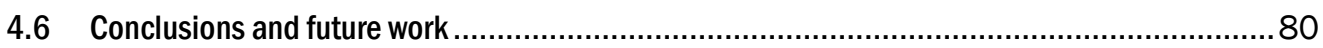

5 Research and Develop a Plan for Testing and Demonstrating Vehicle Charging within a Tactical Microgrid Environment .........................................................................................................82

5.1 Catalog CBITEC assets and identify V2G operational requirements ..................................83

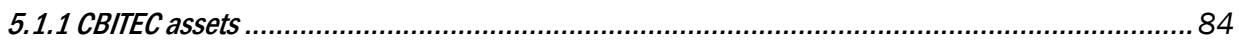

5.1.2 Current capabilities and requirements for testing and operating V2G technologies at

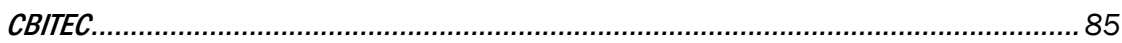

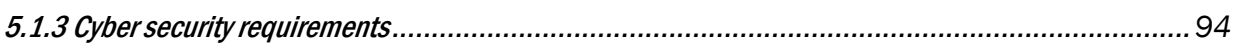


5.1.4 V2G operational requirements in a tactical environment...................................................... 94

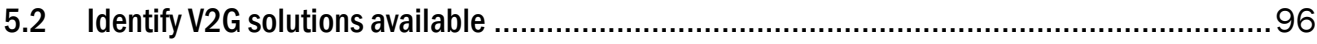

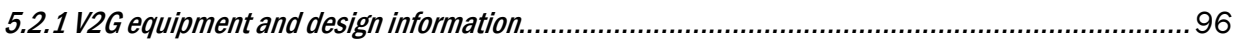

5.2.2 Design criteria for integration of a charging station into a tactical microgrid.................................100

5.2.3 Conceptual design of V2G charging station integration .......................................................100

5.2.4 Available V2G charging stations that meet performance requirements....................................101

5.2.5 Potentially suitable technologies.................................................................................. 102

5.3 Simulate V2G charging in the tactical microgrid environment.......................................103

5.3.1 Develop a computer simulation platform ..........................................................................104

5.3.2 Validation of the MATLAB microgrid computer simulation......................................................113

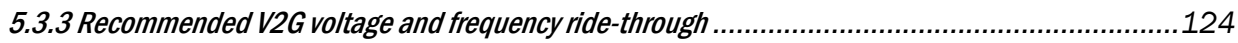

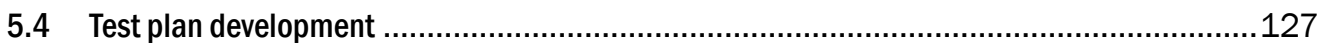

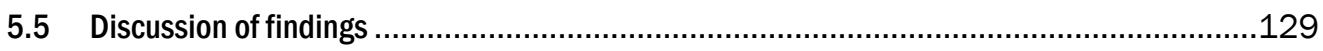

5.6 Discussions and future work ............................................................................. 131

5.7 Conclusions and recommendation......................................................................... 131

6 Identify Requirements for a Sensored, Living Laboratory to Assess Autonomous Transport

Innovations ................................................................................................................................ 133

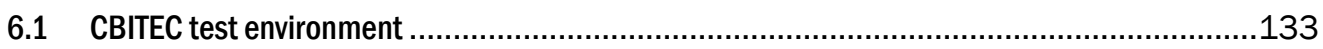

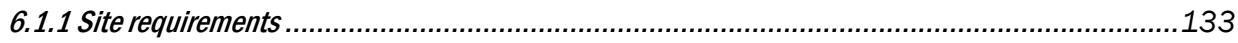

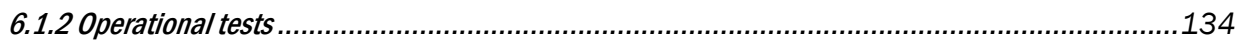

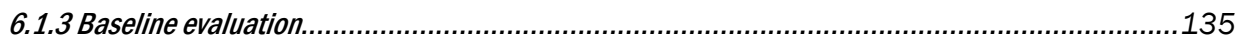

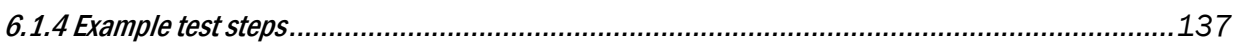

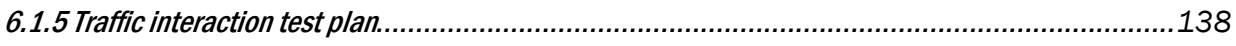

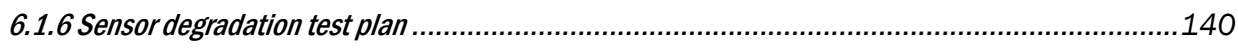

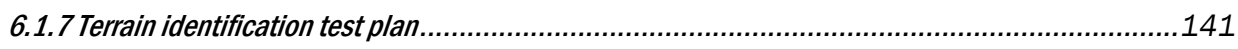

6.1.8 Charging station docking test plan.......................................................................... 142

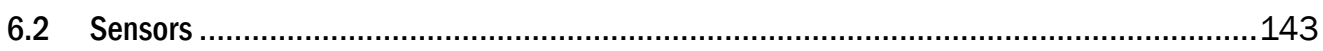

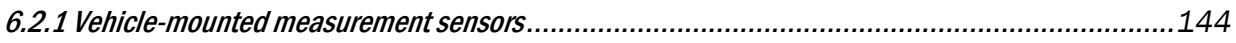

6.2.2 Site-mounted measurement sensors............................................................................. 145

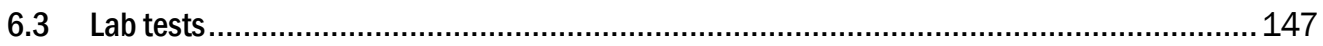

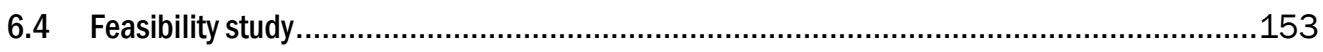

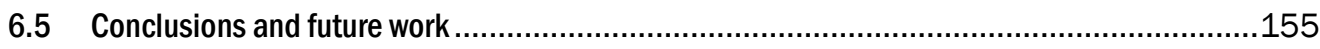

7 Identify Requirements for a Sensored, Living Laboratory to Assess the Performance of V2G

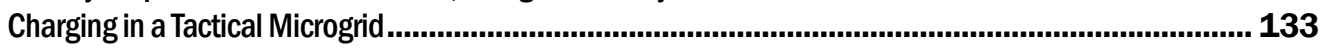

7.1 Develop requirements for a sensored, living laboratory ............................................157

7.2 Identify methods to quantify V2G system aspects......................................................158

7.3 Sensored, living laboratory requirements .............................................................159

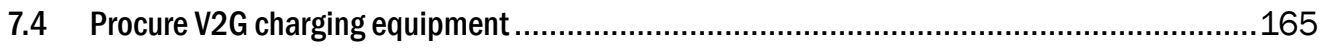

7.5 Conduct initial testing and analysis of results .........................................................172

7.6 Employ test results to model outcomes to refine recommended V2G solution set .................173

7.6.1 Protection model components................................................................................. 174

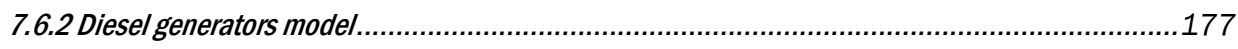

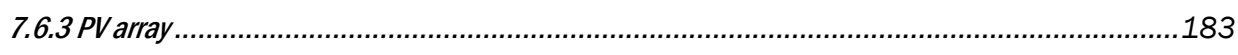

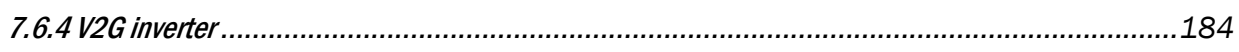

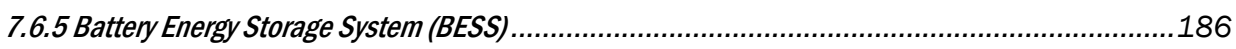




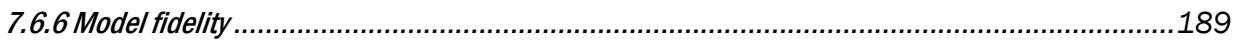

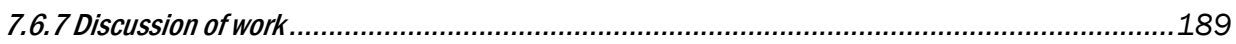

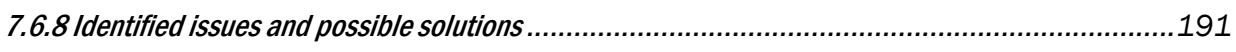

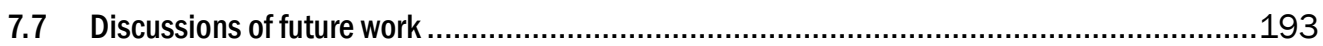

7.8 Conclusions and recommendations ........................................................................ 194

8 Integrate Open Standards to Promote Interoperability ....................................................................157

8.1 Identify and catalog currently available communication and controls technology .................195

8.2 Identify current interoperability standards ............................................................ 197

8.2.1 Interoperability standards applicable to the CBITEC tactical microgrid........................................198

8.3 Recommended communication and control products for the CBITEC tactical microgrid .........200

8.3.1 Communication and controls technology ranking methodology ...............................................200

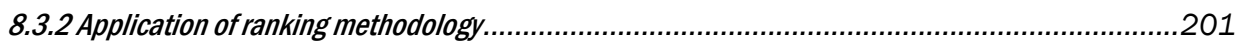

8.3.3 Recommended technologies.........................................................................................201

8.4 Approach to bridge the gap between available technologies and standards........................202

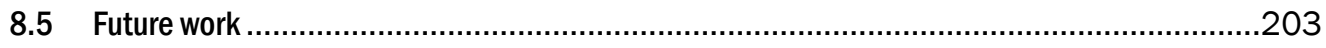

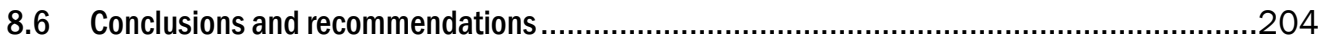

9 Summary of Findings, Recommendations, and Next Steps ......................................................... 206

9.1 Research Area 1: Identify Military-Unique Requirements for Autonomous Transport

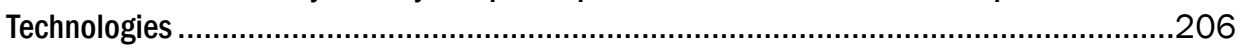

9.2 Research Area 2: Identify Existing Technologies That Can Be Adapted for Military

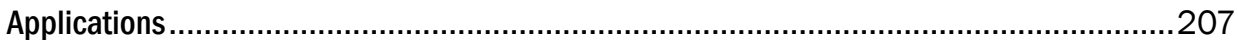

9.3 Research Area 3: Identify Research and Operational Tests for Autonomous Transport Vehicles ................................................................................................. 210

9.4 Research Area 4: Investigate Requirements for Testing and Demonstrating V2G Vehicle Charging within a Tactical Microgrid Environment .......................................................210

9.5 Research Area 5: Develop Requirements for a Sensored Living Laboratory that Could be Used to Assess the Performance of Autonomous Innovations ......................................212

9.5.1 5A - Autonomous Vehicle Living Laboratory Requirements.................................................212

9.5.2 5B - V2G Charging Living Laboratory Requirements........................................................213

9.6 Research Area 6: Integrate Open Standards to Promote Interoperability ...........................214

Appendix A : Military Strategic Planning Resource Documents........................................................... 206

Appendix B : Manufacturer Websites................................................................................................. 220

Appendix C : Electric Transport System Information Resources ..........................................................227

Appendix D : Preliminary List of Existing Electric Transportation Systems ............................................... 231

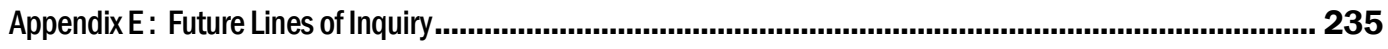

Appendix F : Research Compatibility Considerations ........................................................................237

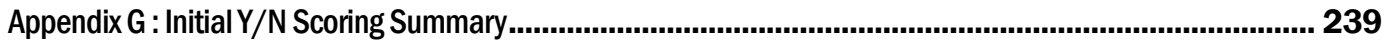

Appendix H : Candidate System Information Packets .................................................................................241

Appendix I : Autonomous Vehicle OEM and Kit Manufacturers ..........................................................277 


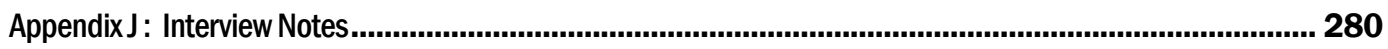

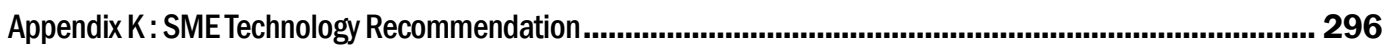

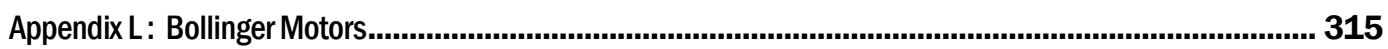

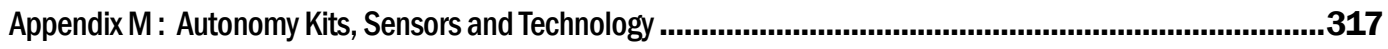

Appendix N : Bidirectional Chargers, Diesel Generators, and Controller Technologies................................... 328

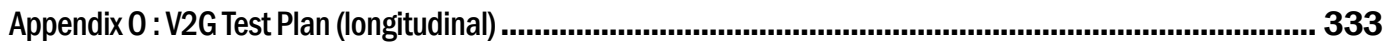

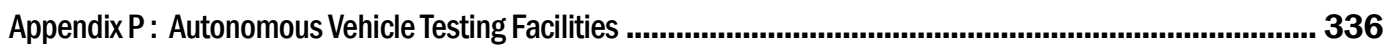

Appendix Q : 800A Intelligent Power Distribution Box Technical Manual .................................................... 339

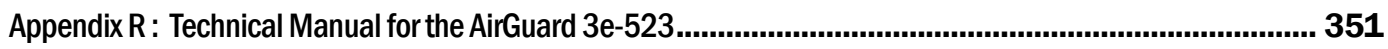

Appendix S: Tactical Microgrid Standard Data Distribution Service Implementation Guide............................. 396

Appendix T: Coritech Fast Charger Product Brochure...................................................................... 428

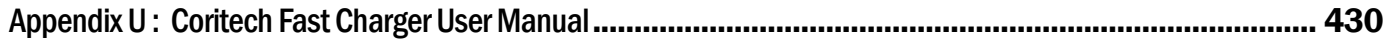

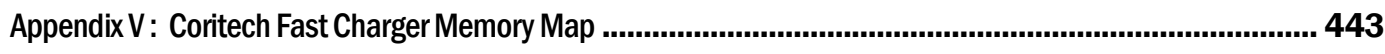

Report Documentation Page ......................................................................................................... 445 


\section{Figures and Tables}

\section{Figures}

$1 \quad$ U.S. DoD policy driver to reduce energy vulnerabilities......................................................1

$2 \quad$ U.S. Army policy driver to strengthen energy resiliency...................................................12

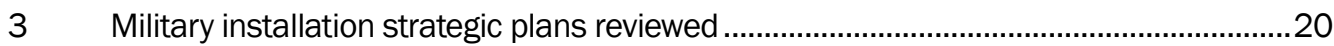

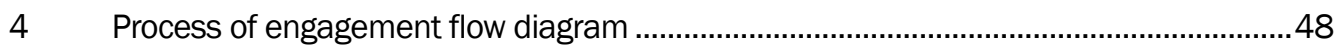

$5 \quad$ Three-dimensional LiDAR scan example ......................................................................6

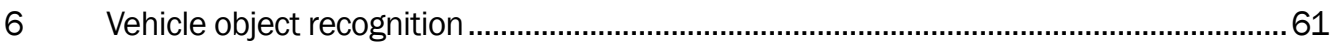

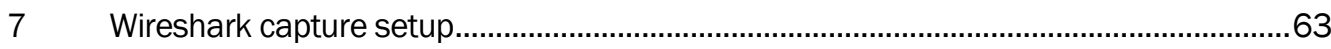

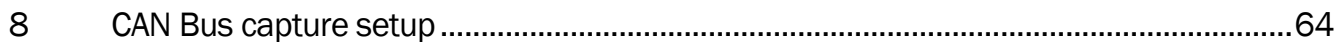

9 Proposed data management process flow using nSight tools ........................................66

$10 \quad$ Example test track .............................................................................................

11 Support surface voxel generation ......................................................................... 76

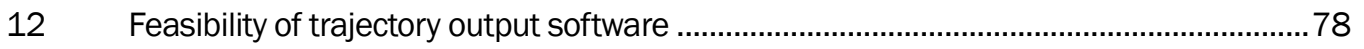

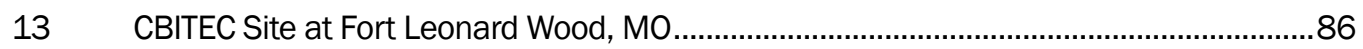

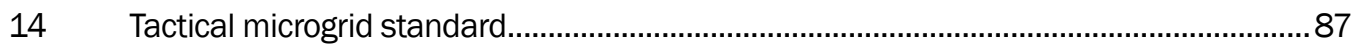

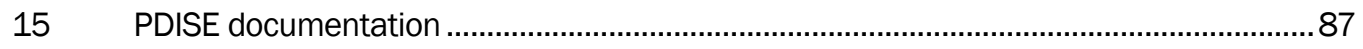

$16 \quad$ Billeting huts at CBITEC...........................................................................................90

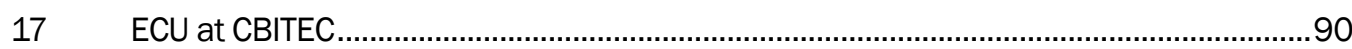

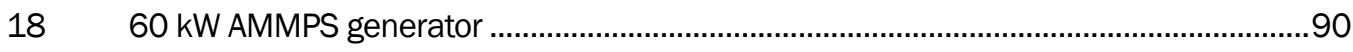

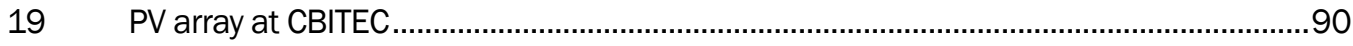

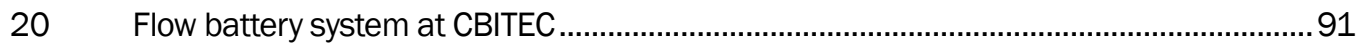

$21 \quad$ Enerdel 40 kWh lithium-ion energy storage system.....................................................91

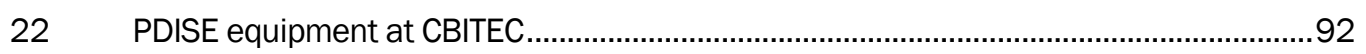

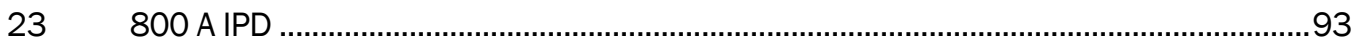

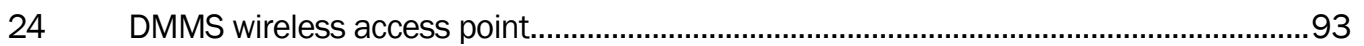

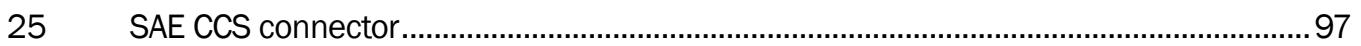

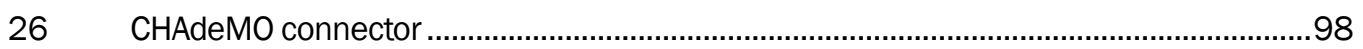

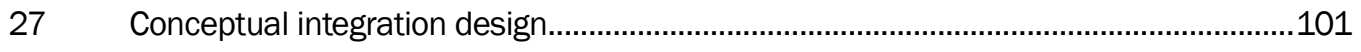

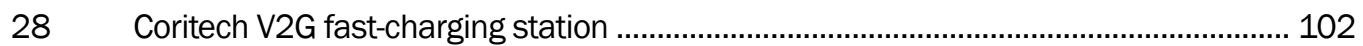

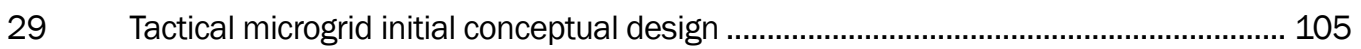

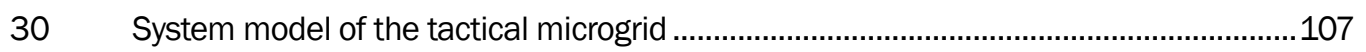

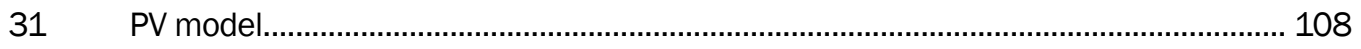

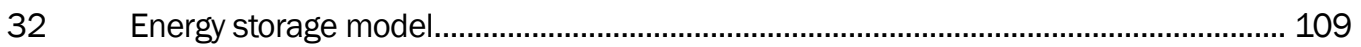

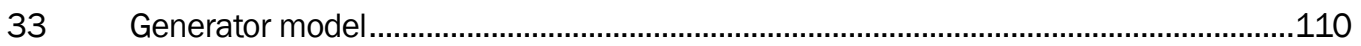

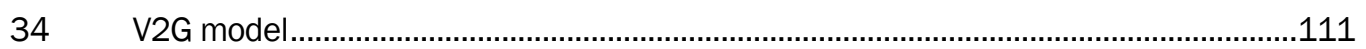

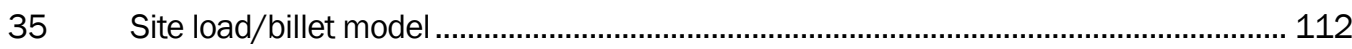

$36 \quad$ Three-phase load bank model........................................................................... 113

37 Load accept and reject for one AMMPS generator: Frequency versus seconds......... 115

38 Load accept and reject for one AMMPS generator: Power versus seconds ................ 115

39 Load accept and reject for one AMMPS generator: Voltage versus seconds .................116

40 Load accept and reject for six AMMPS generators: Frequency versus seconds ............116

$41 \quad$ Load accept and reject for six AMMPS generators: Power versus seconds ...................117

42 Load accept and reject for six AMMPS generators: Voltage versus seconds ................117 


\section{Figures}

43

44

45

46

70\% PV shading event: Power versus seconds

PV shading event: Reactive power versus seconds ................................................. 118

70\% PV shading event: Frequency versus seconds .................................................... 119

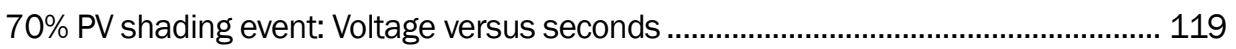

95\% PV shading event: Power versus seconds .......................................................... 120

95\% PV shading event: Reactive power versus seconds............................................. 120

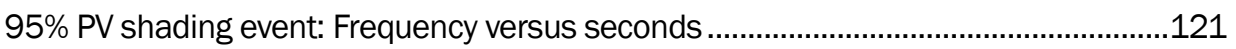

95\% PV shading event: Voltage versus seconds .........................................................121

30-kW V2G charger dispatched cyclically from 30-kW charge to 30-kW discharge-Real power versus seconds.

30-kW V2G charger dispatched cyclically from 30-kW charge to 30-kW discharge-

Reactive power versus seconds

30-kW V2G charger dispatched cyclically from 30-kW charge to 30-kW discharge-

Frequency response versus seconds ...

30-kW V2G charger dispatched cyclically from 30-kW charge to 30-kW dischargeVoltage response versus seconds.

V2G unit validation: Real power versus seconds....................................................... 125

V2G unit validation: Reactive power versus seconds................................................. 125

V2G unit validation: Frequency versus seconds ..................................................... 126

Test plan configuration .................................................................................. 129

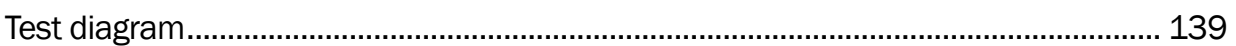

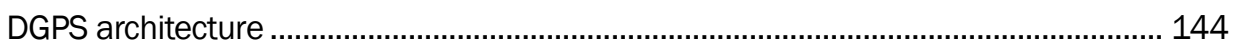

Node and distributed sensing setup........................................................................ 146

Node and distributed sensing setup....................................................................... 146

Velodyne 64 simulation (left); Velodyne 32 simulation (right) …………...................... 149

LiDAR point clouds from three different sensors........................................................ 149

Velodyne HDL-64E at 150-100 m range ................................................................. 150

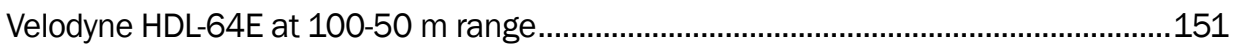

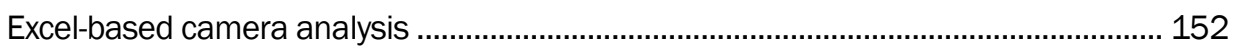

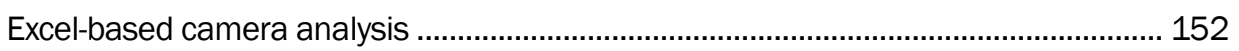

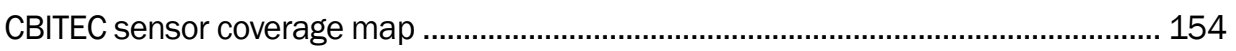

TA 231 driving course sensor coverage map .............................................................. 155

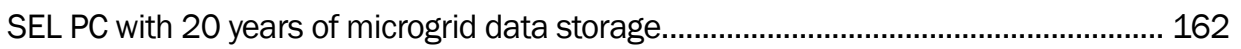

SEL's TMS demonstration project with continuous data collection ................................ 163

SEL's relays meet SOE, OSC, and CDC data collections requirements of IEEE 2030.8165

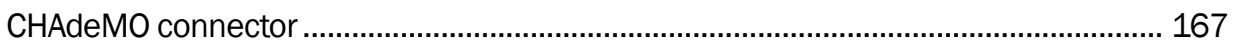

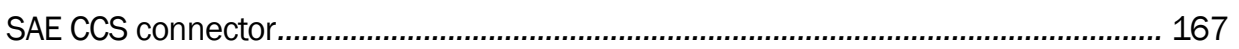

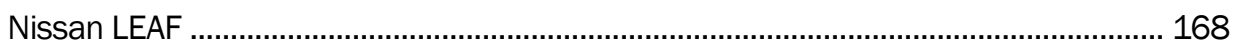

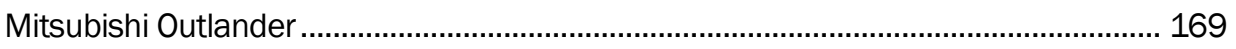

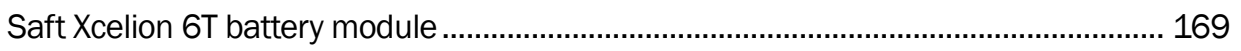

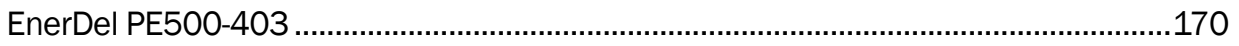

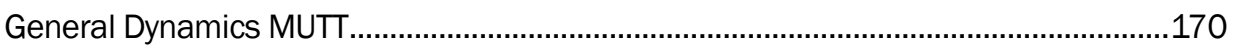

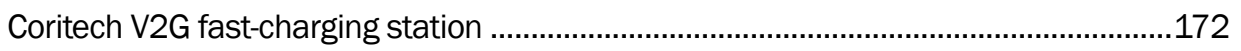

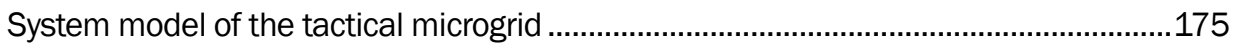

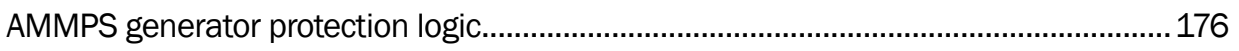




\section{Figures}

84

85

86

87

88

89

90

91

92

93

94

95

96

97

98

99

100

101

102

103

104

105

106

107

108

109

110

P-1

P-2

AMMPS generator permissive

AMMPS generator overcurrent elements ..................................................................177

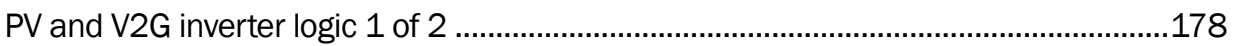

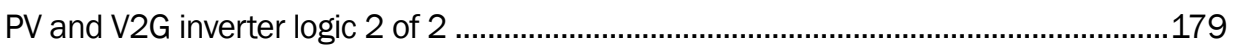

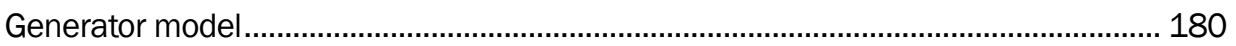

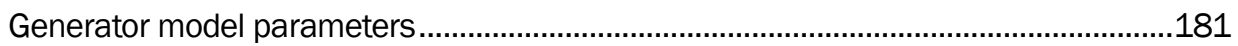

Generator governor parameters.......................................................................... 182

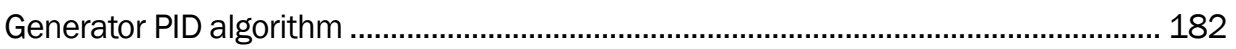

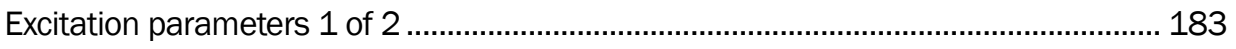

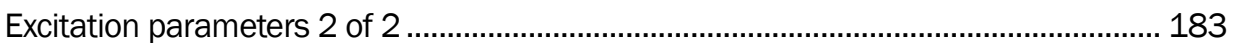

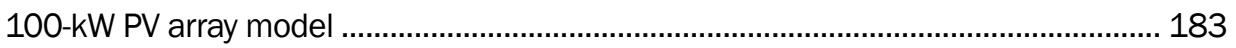

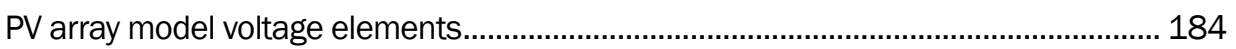

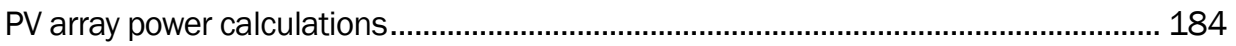

V2G inverter model....................................................................................... 185

V2G inverter charge/discharge elements 1 of 2 ...................................................... 185

V2G inverter charge/discharge elements 2 of 2 .......................................................... 185

V2G inverter charge/regulation percentage control.................................................. 186

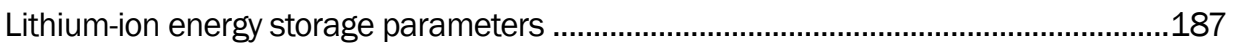

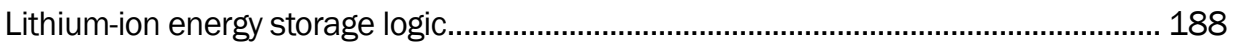

Lithium-ion energy storage inverter parameters .......................................................... 188

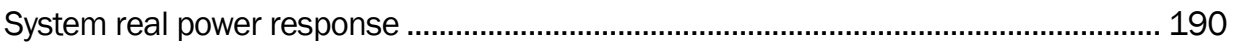

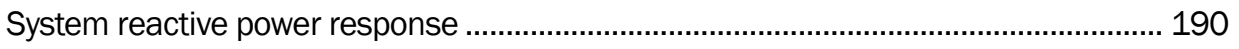

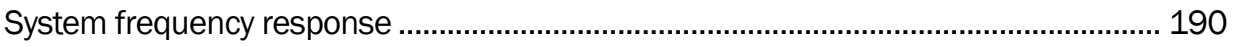

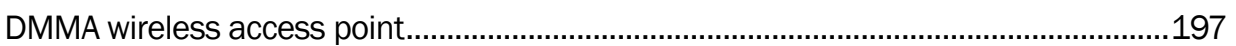

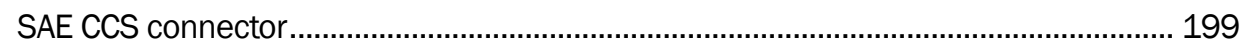

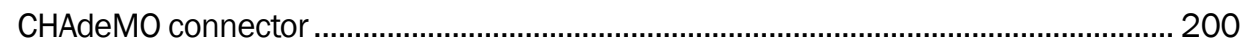

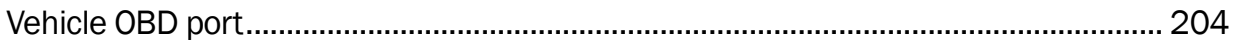

\section{Tables}

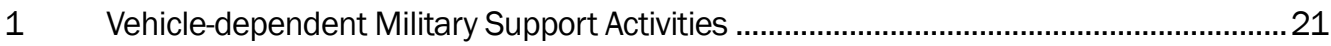

$2 \quad$ Military Support Activity 1: Personnel transport ..........................................................22

3 Military Support Activity 2: Large-scale land, training area, and range maintenance ....23

$4 \quad$ Military Support Activity 3: Materiel transport ..........................................................23

$5 \quad$ Military Support Activity 4: Off-road, transport-based security monitoring ........................24

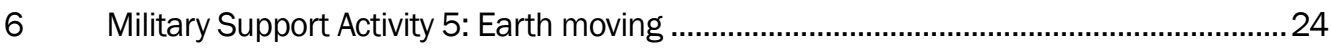

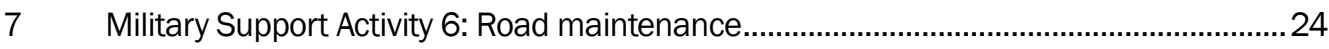

$8 \quad$ Alternative Path 1: A vehicle in the convoy fails during the trip to the FOB ......................2

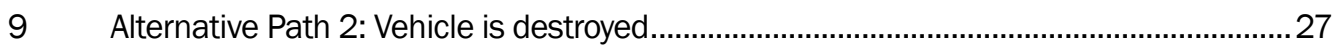

$10 \quad$ Alternative Path 3: Multiple vehicles are destroyed .....................................................28

11 Alternative Path 1: Pervasive situational awareness in the area surround the FOB.......30

12 Alternative Path 2: Platform malfunctions or is destroyed...........................................30

13 Alternative Path 1: The only AV/EV support is for site clearing and grading...................32

14 Alternative Path 2: Construction robots malfunction or are otherwise inoperable ..........33 


\section{Tables}

15 Alternative Path 3: Site is attacked as construction begins ............................................34

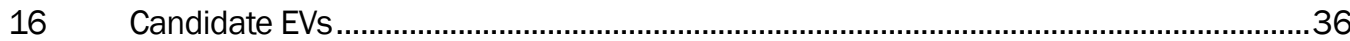

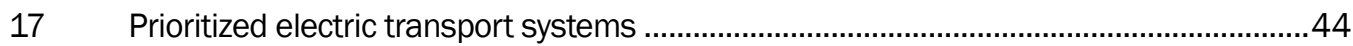

18 EV manufacturer interview correspondence..........................................................50

19 Example data collected during autonomous-system operation ....................................58

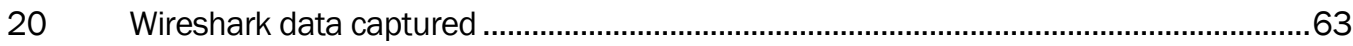

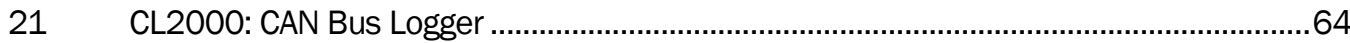

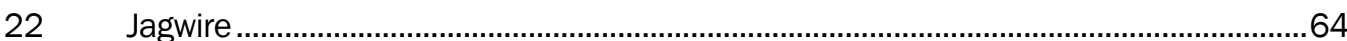

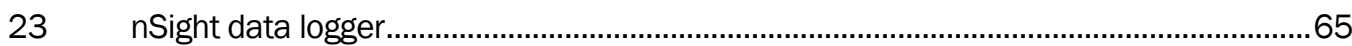

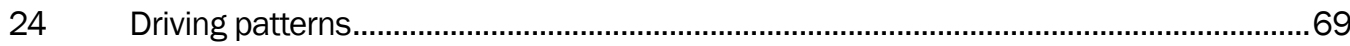

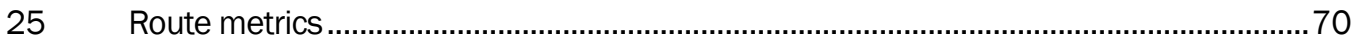

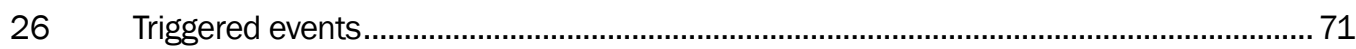

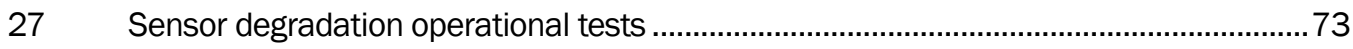

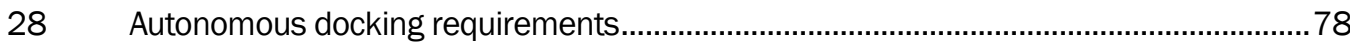

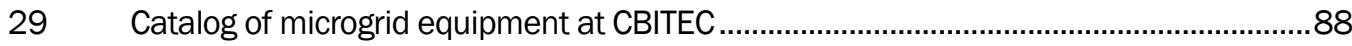

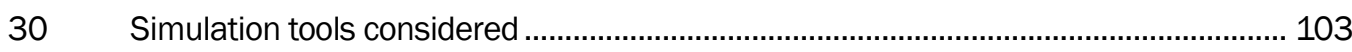

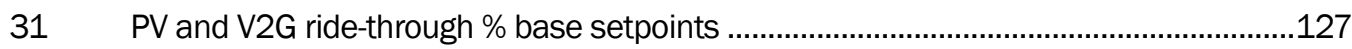

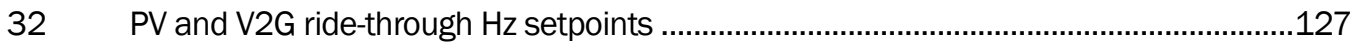

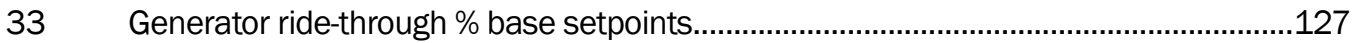

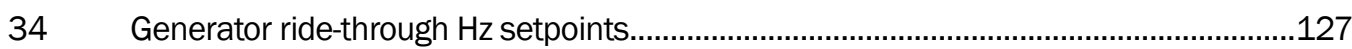

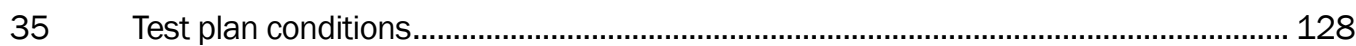

36 Requirements for infrastructure, sensors, and data collection operations ................... 134

$37 \quad$ Key personnel and test equipment during operational tests ...................................... 134

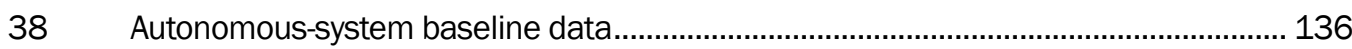

39 Sensor measurements............................................................................................ 136

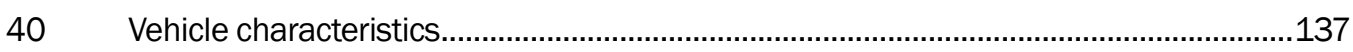

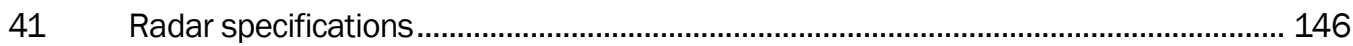

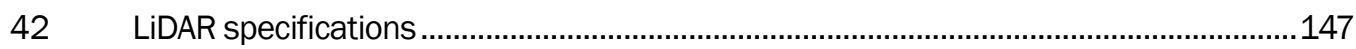

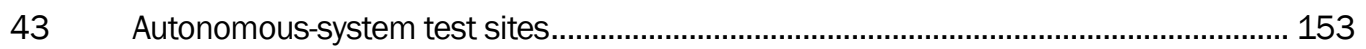

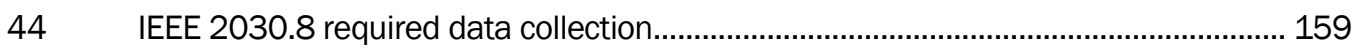

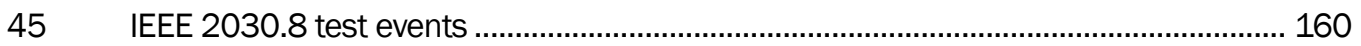

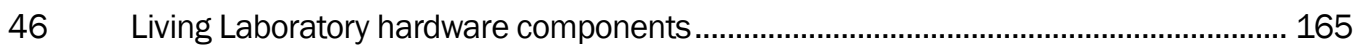

$47 \quad$ System response to abnormal voltages ................................................................... 192

48 System response to abnormal frequencies ................................................................ 192

49 Interconnection system response to abnormal voltages ............................................... 202

50 Interconnection system response to abnormal frequencies ........................................ 202

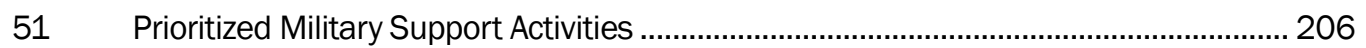

$52 \quad$ Prioritized candidate technologies ........................................................................ 208

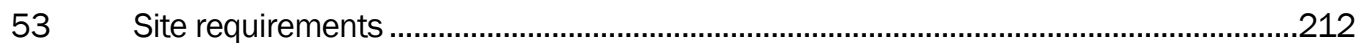

M-1 Robotic research autonomy kit for autonomous ground resupply (ARG) .......................317

M-2 Autonomy research kit (ARK) ............................................................................... 317

M-3 Perrone Robotics drop-in autonomy kit (DAK) ........................................................317

M-4 Autonomous Mobility Applique System (AMAS) .......................................................318

M-5 Unit-to-unit autonomous resupply vehicle (U2UARV) ...................................................318 


\section{Tables}

\begin{tabular}{|c|c|}
\hline M-6 & Cybernet technologies.............................. \\
\hline M-7 & Lanecruise .......................... \\
\hline M-8 & Luminar................................... \\
\hline M-9 & Shadowcam......... \\
\hline M-10 & Robosense LiDAR systems \\
\hline M-11 & Velodyne LiDAR ................................ \\
\hline $\mathrm{M}-12$ & Mobileye ..................................... \\
\hline M-13 & Innovusion LiDAR systems............ \\
\hline M-14 & Lumotive \\
\hline M-15 & Baidu Apollo Enterprise. \\
\hline M-16 & Cepton micro-motion technology (MMT) and Sensor Development Toolkits ................. 322 \\
\hline M-17 & Innoviz \\
\hline M-18 & 322 \\
\hline M-19 & 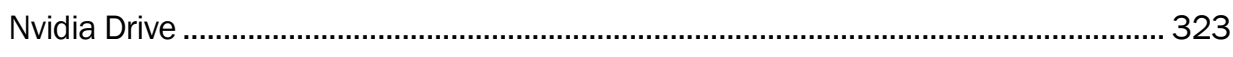 \\
\hline M-20 & R-Car \\
\hline M-21 & LiDAR USA ................................. \\
\hline M-22 & Toposens Automotive Devkit.................. \\
\hline M-23 & 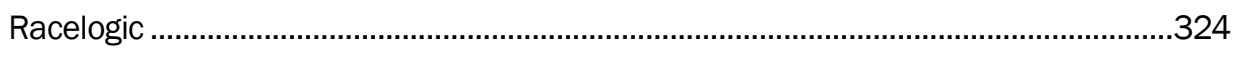 \\
\hline M-24 & Bosch Mobility Systems \\
\hline M-25 & Harman Ignite Cloud Platform \\
\hline M-26 & Certus Evo \\
\hline M-27 & Iscan modular radar demonstration and development platform ...................................... 326 \\
\hline M-28 & APPlus+ IDapt \\
\hline M-29 & Dataspeed ADAS Kit \\
\hline M-30 & Quantum R-Series. \\
\hline
\end{tabular}




\section{Preface}

This study was conducted for Headquarters, U.S. Army Corps of Engineers (HQUSACE) under Project 6LKD1G "FY19 Program Increase: Autonomous Transport Innovation," Work Unit 6LKD1G, "Autonomous Transport Innovation"; and was executed under Contract Number W9132T19Cooo3 to Paragon Business Solutions, Inc. for the Broad Agency Announcement (BAA) proposal entitled "Autonomous Transport Innovation: Integration of Autonomous Electric Transport Vehicles into a Tactical Microgrid.”

The work was performed by the Engineering Processes Branch of the Facilities Division, U.S. Army Engineer Research and Development Center, Construction Engineering Research Laboratory (ERDC-CERL). At the time of publication, Mr. James Allen was Acting Chief of the Engineering Processes Branch; Ms. Giselle Rodriguez was Chief of the Facilities Division; and Mr. Kurt Kinnevan was the Technical Director for Installations. The Acting Deputy Director of ERDC-CERL was Ms. Michelle Hanson and the Director was Dr. Andrew Nelson.

Special thanks go to the entire ERDC-CERL project team for this effort. Ms. Susan J. Bevelheimer was Contracting Officer's Technical Representative (COTR). Mr. Marcus Ferguson (CNE) was Contingency Basing Integration Technology Evaluation Center (CBITEC) Site Integration Manager. Mr. Jonathan Goebel and Mr. Thomas Decker worked on CBITEC Electrification. Mr. Thomas Bozada worked on the Charging Station Development. Ms. Heather FitzHenry, Ms. Julie L. Webster, Mr. Matthew Gross, and Ms. Emma Smith worked on the Autonomy Innovation \& Implementation Lab. Ms. Heather FitzHenry also served as co-project manager and was invaluable to envisioning the big picture for this project.

COL Teresa A. Schlosser was Commander of ERDC, and Dr. David W. Pittman was the Director. 
THIS PAGE INTENTIONALLY LEFT BLANK 


\section{Introduction}

\subsection{Background}

The Army needs a realistic testing area to test developing autonomous vehicle technology for use on Army installations and contingency operations. The military is slow to take advantage of government, academic and industry expertise to accelerate the integration of unmanned vehicle technologies into defense and civilian transport operations until the technology is tested and proven safe and reliable for transportation on post. Autonomous vehicles offer the opportunity to reduce personnel (drivers), reduce the environmental footprint and demand for individual vehicles and associated parking, and increase mobility for military and civilian personnel. Research is needed to integrate large autonomous electric vehicles into a microgrid in a way to enhance the resiliency of the installation and surrounding community.

There is a requirement to prototype the integration of unmanned vehicle technologies into defense and civilian transport operations. The ability to test and demonstrate how a variety of autonomous transportation systems could be integrated into an installation transportation network and microgrid is required to build confidence in these technologies. How can autonomous electric vehicles be integrated into contingency base operations to enhance energy security while reducing reliance on vulnerable resupply operations that put Soldiers at risk.

This effort responds to DoD research needs to accelerate the adoption and use of intelligent ground vehicle systems for military operations; and find innovative opportunities to enhance both installation and contingency basing energy resilience.

\subsection{Objective}

Develop an operational "living laboratory" on a military base to research and demonstrate the value of autonomous transportation systems in realworld, non-hostile environment. A prototype bench scale testing area will be developed to include paved and non-paved areas plus a microgrid charging capability. The goal is to be able to attract and test autonomous 
systems, monitor their performance, and identify reliability problems or safety risks to the military before the autonomous systems are exposed to civilian or military personnel at a larger scale.

Investigate how Army installations can integrate large autonomous electric vehicles into a microgrid to enhance the resiliency of the installation and surrounding community. Results will help Army installations and surrounding communities plan adoption of unmanned vehicle technologies. Research how autonomous electric vehicles can be integrated into contingency base operations to enhance energy security while reducing reliance on vulnerable resupply operations that put Soldiers at risk.

Fort Leonard Wood is a partner to the U.S. Army Tank Automotive Research, Development and Engineering Center (TARDEC) in demonstrating Autonomous Vehicle technologies. Fort Leonard Wood is the host installation for all 88M truck driver training for Army, Navy, Air Force, and Marine Corps; this provides:

- a large secure space for vehicle testing including paved, unpaved and off-road environments; and

- a realistic demonstration of technologies with Soldiers, Airmen and Marines to best assess the utility of these systems in operational environments.

\subsection{Approach}

The team members will research and demonstrate the safety and utility of autonomy-enabled vehicles in various scenarios and environmental conditions. Incorporate a demonstration microgrid that will integrate electric vehicles, charging stations, and renewable power to power adjacent facilities. The electric vehicle batteries will provide energy storage for the microgrid. The microgrid will be scalable to allow for growth as additional electric vehicles are added to the program. The center will expand to include civilian transport research applications, including autonomous commercial freight movement, underground mining equipment, and transit operations. Research results will also inform and accelerate driverless vehicle policy development. 
The purpose of the Autonomous Transport Innovation (ATI) RDTE project is to develop a roadmap for a sensored, living laboratory to integrate autonomous transport vehicles into a tactical microgrid.

The scope of the ATI technical research program is as follows:

1. Identify military-unique requirements for autonomous transport technologies

2. Identify existing technologies that can be adapted for military applications, conduct technology gap analysis and identify need gaps

3. Identify research and operational tests for autonomous transport vehicles

4. Investigate requirements for testing and demonstrating vehicle charging within tactical microgrid environment

5. Develop requirements for a sensored living laboratory to assess performance of autonomous innovations

6. Integrate open standards to promote interoperability.

\subsection{Worldwide threat assessment}

The Worldwide Threat Assessment of the U.S. Intelligence Community was presented to Congress by the Director of National Intelligence on January 29, 2019. The presentation identified threats to U.S. national security that will both expand and diversify as "development and application of new technologies introduce both risks and opportunities" to the U.S. economy. Of the global threats identified, "our adversaries and strategic competitors will increasingly use cyber capabilities-including cyber espionage, attack, and influence-to seek political, economic, and military advantage over the United States and its allies and partners." These cyber capabilities are used to attack and hold at risk the nation's critical infrastructure. " Moreover, the U.S. Department of Homeland Security (DHS) Cybersecurity and Infrastructure Security Agency (CISA), identified "16 critical infrastructure sectors whose assets, systems, and networks, whether physical or virtual, are considered so vital to the United States that their incapacitation or destruction would have a debilitating effect on security, national economic security, national public health or safety, or any

\footnotetext{
* Coats, Daniel R. "Global Threats: Cyber." Worldwide Threat Assessment of the U.S. Intelligence Community, 29 Jan. 2019, p. 5., https://www.dni.gov/files/ODNI/documents/2019-ATA-SFR---SSCI.pdf.
} 
combination thereof." The energy sector is one of the listed 16 critical infrastructure sectors. *

\subsection{Energy Independence and Security Act of 2007}

The Energy Independence and Security Act of 2007 (EISA) codified "the policy of the United States to modernize the nation's electricity transmission and distribution system to maintain a reliable and secure electricity infrastructure that can meet future demand growth and to achieve" prescribed characteristics of a modern grid. ${ }^{\dagger}$ Characteristics of a modern grid-through research, development, and demonstration of technologies-are actively deployed to advance the nation's energy critical infrastructure and to fortify against both cyber and physical attacks. The movement to a modernized grid, as outlined by the U.S. Department of Energy (DOE) Grid Modernization Initiative (GMI), is to develop a modern grid of the future that boasts "greater resilience, improved reliability, enhanced security, additional affordability, superior flexibility, and increased sustainability.”

\subsection{A modernized grid requires a standardized environment}

Progress toward a modernized grid requires a standardized environment to establish performance and operation requirements, safety considerations, commissions, and testing methodologies. Working with industry to articulate general interoperability requirements, methodology, and tools that support information and communications technology application in distributed energy resource (DER) integration is pertinent to provide guidance for device deployment without compromising grid reliability, safety, or security. $₫$ The shift to a modern grid entails distributed and dispatchable generation integration, grid storage, and microgrids-along with smart metering, time-of-use rates, networked sensors, automated controls, and the uptake of plug-in EVs. "A modernized grid enables bidirectional flows

\footnotetext{
* "Critical Infrastructure Sectors." Cyber \& Infrastructure Security Agency, U.S. Department of Homeland Security, 3 Mar. 2019, www.cisa.gov/critical-infrastructure-sectors.

† 110th Congress. "TITLE XIII-SMART GRID." Energy Independence and Security Act of 2007 [Public Law No: 110-140], 19 Dec. 2007, pp. 293-294., https://www.congress.gov/110/plaws/publ140/PLAW110publ140.pdf.

‡ "Critical Infrastructure Sectors." Cyber \& Infrastructure Security Agency, U.S. Department of Homeland Security, 3 Mar. 2019, www.cisa.gov/critical-infrastructure-sectors.

$\S$ "Grid Standards and Codes." The National Renewable Energy Laboratory.gov, U.S. Department of Energy, www.nrel.gov/grid/standards-codes.html.
} 
of energy and uses two-way communication and control capabilities that will lead to an array of new functionalities and applications."*

\subsection{Electric transportation as a distributed energy resource}

The shift from fossil fuel-reliant transportation to electric transportation presents a new paradigm in the transportation and energy sectors. The onboard energy storage electric transport vehicles provide introduces the potential to access an additional and distributed electric power supply through bidirectional power flow. V2G charging equipment, an emerging technology, is essential in enabling a bidirectional flow of electric power between electric transport and energy systems. It is this connection that allows electric transportation to provide ancillary energy and assurance to local energy generation resulting in improved reliability, flexibility, and resilience.

\subsection{Autonomy}

Electrified transport systems, coupled with $\mathrm{V} 2 \mathrm{G}$ technologies, are being evaulated for future deployment and benefit to the modernized grid. These benefits have potential to carry over to U.S. military defense transportation operations as well as contingency and enduring base energy applications. The U.S. Department of Defense (DoD) and Army have invested in research-developing technologies designed to reduce risks to the warfighter and decrease reliance on vulnderable resupply operations. This objective can be achieved through integrating autonomous technologies with electric transport.

The DoD and the Army understand the potential importance of autonomous vehicles (AVs) as illustrated by this commentary from the Army website:

The Department of Defense has been researching the use of autonomous vehicles since 2004, when the Defense Advanced Research Projects Agency (DARPA) funded specific research and technology and sponsored its first autonomous vehicle competition. While the most recent technical

\footnotetext{
* National Institute of Standards and Technology, U.S. Department of Commerce. "Purpose and Scope: Overview and Background." NIST Framework and Roadmap for Smart Grid Interoperability Standards, Release 3.0, Sept. 2014, p. 14., https://www.nist.gov/system/files/documents/smartgrid/NIST-SP1108r3.pdf.
} 
emphasis has undoubtedly been on front-line functions such as unmanned tactical vehicles or light maneuver capabilities such as bomb disarmament, those assets are not technically autonomous vehicles. Autonomous vehicles present opportunities to improve logistics, security, base operation and maintenance, transport, and eventually warfighting. As such, they represent a significant advancement in how we conduct all phases of the military mission.*

Army leadership realizes the potential of AVs. Unmanned systems will enable the Army of the future. To achieve its vision for 2028, the Army has identified equipment modernization as a key objective with efforts focused on “... experimenting with and developing autonomous systems, artificial intelligence and robotics to make our Soldiers more effective and our units less logistically dependent.” ${ }^{\prime}$

The number of applications and potential benefits for AVs are wide ranging. For use in forward areas, AV potential benefits are:

- Autonomous logistics operations could reduce the number of casualties associated with transport. According to the DoD, "Fifty-two percent of battlefield casualties occur when sustainers are delivering needed supplies to and from the battlefield." 5

- Supporting continuous (24 hours a day, 7 days a week) supply operations in theatre, efficiently creating and improving overall supply and resupply operations.

- Support security for convoys and forward-operating bases (FOB) through increased reconnaissance capacity and, eventually, warfighting capability.

Initial AV development and testing is well suited for various military installations-and, as such, is where initial testing and development will occur. In addition to supporting forward operations, there are various potential installation operations and maintenance benefits that AVs could provide to the military:

\footnotetext{
* Henry, Capt. Matthew P. “Autonomous Transportation: Combat Power in the 21st Century." U.S. Army, 1 Nov. 2018, army.mil/article/213078/autonomous_transportation_combat_power_in_the_21st_century.

† General Mark Milley, Army Chief of Staff, Honorable Mark Esper, Secretary of the Army. "Army 2028 Mission and Vision Statement."26 March 2019, pp. 7.
} 
- Reduced cost and streamlined routine or continuous, high-capacity personnel transit by reducing the number of vehicles and staff used to bus personnel-for example, a basic training Army base, such as Fort Leonard Wood, provides in excess of 1.5 million soldier trips a year* in support of basic training. Many of which are recurring transit operations that could be automated.

- Improved transit scheduling and delivery results in reduced trainingtime losses associated with current systems.

- With emerging AVs, reduced costs (personnel time, contract labor, equipment, fuel, etc.), routine mowing, and land maintenance in training areas and ranges. Installations can spend millions of dollars annually in various land management activities. ${ }^{\dagger}$

- AV land management activities in training areas and ranges that can be conducted when training is not occurring (day or night) reduces loss of areas for routine maintenance, which increases effective availability of training areas and ranges.

- $\mathrm{AV}$ point-to-point logistics systems used to establish bidirectional transportation whereby vehicles always travel hauling materials to or from storage and supply areas.

- AV systems that conduct routine road maintenance (sweeping, plowing, etc.) and unpaved trail maintenance and repair-all reducing the cost while providing improved access in support of the mission.

The development, deployment, and implementation of AVs offers a myriad of potential benefits. A report, prepared by Compass Transportation and Technology (CTT) for Securing America's Future Energy (SAFE), notes that economic benefits of AVs include: improved access to labor and jobs, access to new markets and larger markets, a reduction in public-financing capital required for traditional transportation investments, stimulated growth in shared-mobility markets, changed land use, and reduced costs for long-haul freight transportation. The report posits that, excluding economic productivity gains, the adoption of AV adoptions provides a benefit cost ratio of 8 to 1 , or about $\$ 6$ trillion $-\$ 8$ trillion. Further research from SAFE notes that "significant economic benefits from the widespread

\footnotetext{
* “ARIBO: Fort Leonard Wood." Robotic Research, LLC, roboticresearch.com/programs/aribo-fort-leonardwood/.

† Barghout, Jeff, et al. Joint Base "Lewis-McChord Feasibility Study for the Application of Automated Vehicles." pp. 1-39, Joint Base Lewis-McChord Feasibility Study for the Application of Automated Vehicles.
} 
adoption of AVs could lead to nearly $\$ 800$ billion in annual social and economic benefits by 2050."*

In a report prepared and delivered to DOE Secretary Rick Perry and U.S. Department of Transportation (DOT) Secretary Elaine Chao, that in their own previous commissioned studies, research, and analysis found the cumulative consumer and societal benefits of AVs would add $\$ 3$ trillion- $\$ 6$ trillion to the U.S. economy and, that annually, the full deployment of AVs could realize $\$ 800$ billion in economic and societal benefits. ${ }^{\dagger}$ Additionally,

In 2016, U.S. annual vehicular fatality rate was 37,$461 ; 94 \%$ of crashes are due to human error. AVs have the potential to remove/reduce human error and decrease deaths. Depending on adoption and vehicle characteristics, AVs have the potential to reduce crashes by $90 \%$, potentially saving approximately $\$ 190$ billion per year. Potential AV benefits include improvements in safety and public health; increased productivity, quality of life, mobility, accessibility and travel, especially for disabled and elderly; reduction of energy use, environmental impacts, congestion and public and private costs associated with transportation; and increased adoption of car sharing.”

\subsection{Interoperability among devices}

The rapid evolution and convergence of state-of-the-art technologies in autonomy, energy, and transportation yield uncharted technical challenges and gaps. As new and emerging technologies come online, industry standards are increasingly behind the curve. Widespread deployment of systems with onboard energy storage and cyber-based communication within the grid underscores the urgency for modernized interconnection, interoperability, and cyber security standards to achieve interoperability

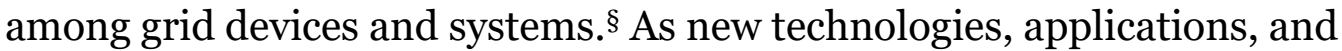
sources of value continue to emerge, the adopting of interoperability

\footnotetext{
* SAFE, Fuel Efficiency Standards Should be Modernized to Expand the Use of Advanced Fuels, Promote Driverless Technologies, and Strengthen U.S. Energy Security, Oct 2018, https://avworkforce.secureenergy.org/.

† http://secureenergy.org/wp-content/uploads/2018/10/Securing-Americas-Future-EnergyComments-on-EPA-HQOAR-2018-0283-0756.pdf.

‡ University of Michigan Center for Sustainable Systems, Autonomous Vehicles factsheet, accessed 31 Oct 2019, http://css.umich.edu/factsheets/autonomous-vehicles-factsheet.

§ “Smart Grid." NIST Engineering Laboratory, U.S. Department of Commerce, 21 Nov. 2019, www.nist.gov/el/smart-grid.
} 
standards is paramount to enable efficiency and reliability in today's increasingly complex energy sector.

As new DER technologies and capabilities emerge, the shift to a modernized grid yields new engineering challenges. The establishment of interoperability standards is fundamental for this transformation. Interoperability, as defined by the GridWise ${ }^{\mathrm{TM}}$ Architecture Council, is the

capability of two or more networks, systems, devices, applications, or components to work together, and to exchange and readily use information-securely, effectively, and with little or no inconvenience to the user. The modern grid will be a system of interoperable systems, which are different systems able to exchange meaningful, actionable information in support of the safe, secure, efficient, and reliable operations of electric systems. The systems will share a common meaning of the exchanged information, and this information will elicit agreed-upon types of response. The reliability, fidelity, and security of information exchanges between and among modernized systems must achieve requisite performance levels.*

Advances in technologies, such as smart metering, demand response, distributed generation, ESSs and EV supply equipment, are developed to offer bidirectional flows of energy and two-way communication and control capabilities. Competitive and individualized manufacturing of multilevel components drives diversity in the market and postures the need for standardize interoperability requirements. In the absence of standards, there is a risk that diverse, modern grid technologies will become prematurely obsolete or be implemented without adequate security measures. ${ }^{\dagger}$

Recognizing the necessity, federal and state agencies, utilities, stakeholders, and industry leaders are actively working to develop policies, protocols, and standards to promote system interoperability. The joint agencies of California: California Public Utilities Commission (CPUC), California Energy Commission (CEC), California Air Resources Board (CARB), and

\footnotetext{
* GridWise ${ }^{\mathrm{TM}}$ Architecture Council, "GridWise Architecture Council Interoperability Constitution Whitepaper," October 2005https://www.gridwiseac.org/pdfs/interoperability_path_whitepaper_v1_0.pdf.

† National Institute of Standards and Technology, U.S. Department of Commerce. "Purpose and Scope: Overview and Background." NIST Framework and Roadmap for Smart Grid Interoperability Standards, Release 3.0, Sept. 2014, https://www.nist.gov/system/files/documents/smartgrid/NIST-SP-1108r3.pdf.
} 
California Independent System Operator (CAISO), collaboratively make up the vehicle-grid integration working group to develop policies that support $\mathrm{V}_{2} \mathrm{G}$ integration. The initiative, to align EV charging with the needs of the electric grid, is slated to assess communication protocol necessary to enable EVs with capabilities to support bidirectional charging while economically participating in electricity markets at scale. ${ }^{*}$ The challenge: standardized connection and communication between the vehicle and the grid, optimization and price signaling, and mitigating impacts to the grid.

The Institute of Electrical and Electronics Engineers Standards Association (IEEE SA) facilitates standard development for a high-quality, market- relevant standardization environment that is respected worldwide. ${ }^{\dagger}$ Standard 1547-2018 provides standards for interconnection and interoperability of DERs with associated electrical power systems interfaces:

\begin{abstract}
The standard provides universal requirements relevant to the performance, operation, testing, safety considerations, and maintenance of the interconnection. It also includes general requirements, response to abnormal conditions, power quality, islanding, and test specifications and requirements for design, production, installation evaluation, commissioning, and periodic tests. ${ }^{*}$
\end{abstract}

Formerly, the original equipment manufacturer (OEM) designed and had complete control of EV onboard charging equipment operation, the rise in direct current (DC) fast charging with bidirectional capabilities creates a new challenge. The 2030.1.1-2015-IEEE SA help OEM engineers address the challenge of controlling an external power device in EV supply equipment. "This interoperability standard specifies the design interface of EVs and DC bidirectional chargers that utilize battery EVs as power storage devices."§

\footnotetext{
* “DRIVE OIR Vehicle-Grid Integration Working Group.” Vehicle-Grid Integration Communications Protocol Working Group, California Public Utilities Commission, www.cpuc.ca.gov/vgi/.

† “IEEE SA - Strategy." IEEE SA, https://standards.ieee.org/about/strategy.html.

‡ “1547-2018 - IEEE Standard for Interconnection and Interoperability of Distributed Energy Resources with Associated Electric Power Systems Interfaces." IEEE SA, 6 Apr. 2018, https://standards.ieee.org/standard/1547-2018.html.

§ “2030.1.1-2015 - IEEE Standard Technical Specifications of a DC Quick Charger for Use with Electric Vehicles." IEEE SA, 5 Feb. 2016, https://standards.ieee.org/standard/2030_1 1-2015.html.
} 


\subsection{DoD 2016 Operational Energy Strategy}

In alignment with U.S. grid modernization initiatives and policies, the military has concentrated efforts to improve future energy security, resiliency, and reliability through policies, including the DoD 2016 Operational Energy Strategy and the Army's Energy Security and Sustainability $\left(\mathrm{ES}^{2}\right)$ Policy. The DoD 2016 Operational Energy Strategy (Figure 1) identifies three objectives to ensure consistent delivery of energy in support of the warfighter:

1. Increase future warfighting capability by including energy throughout future force development.

2. Identify and reduce logistics and operational risks from operational energy vulnerabilities.

3. Enhance the mission effectiveness of the current force through updated equipment and improvements in training, exercises and operations. ${ }^{*}$ 


\subsection{U.S. Army's Energy Security and Sustainability Policy}

Pursuant to the DoD Operational Energy Strategy, the Army's ES ${ }^{2}$ Policy (Figure 2) defines a vision of "A ready and resilient Army, strengthened by secure access to the energy, water and land resources to preserve future choice in a rapidly changing world."* To achieve this vision, the ES 2 Policy identifies five strategic goals in an effort to provide broad direction for the integration of improved energy and water use into the Army capabilities:
1. Inform decisions
2. Optimize use
3. Assure access
4. Build resiliency
5. Drive innovation.

Figure 2. U.S. Army policy driver to strengthen energy resiliency.

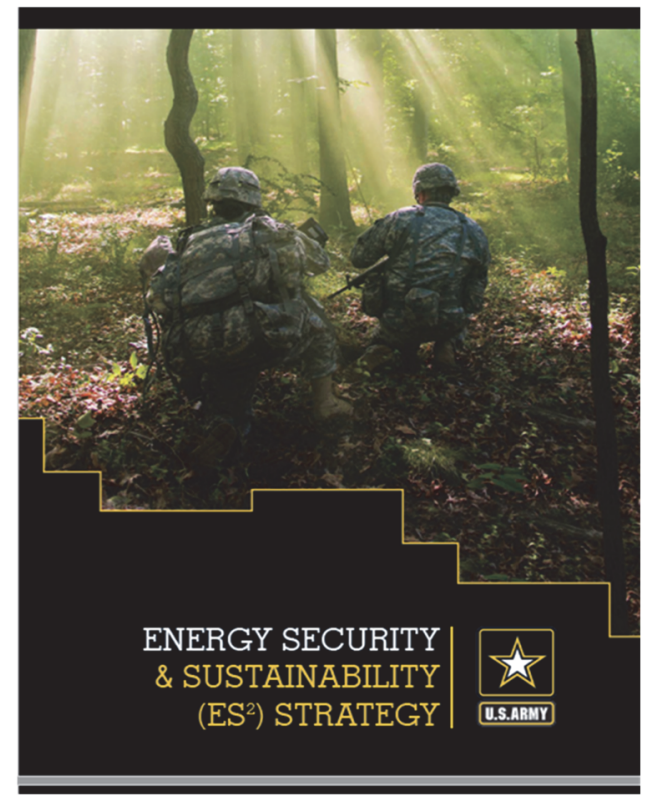

Numerous Army initiatives and programs are underway that focus on supporting this vision. They include net-zero, large-scale renewable energy, and developing energy resilient power systems for FOBs.

\subsection{Tactical microgrid standards consortium}

The Tactical Microgrid Standards Consortium (TMSC) is actively developing a standard to establish "requirements for tactical microgrids (TM), including the interoperability and interfaces of the hardware and software relevant to tactical microgrid design as well as considerations of intelligent control, stability and performance, safety of personnel, security, and protection." The standard defines an open architecture to facilitate tactical microgrid source ability to communicate, provide, and use services and information between systems, regardless of manufacturer or version, without the need for operator intervention. Progress is slated "to establish 
open standards supporting a modular, highly cohesive system structure with full design disclosure to leverage the collaborative innovation of industry, academia, and government participants and stakeholders."

USACE ERDC-CERL provided a working definition of the tactical microgrid as a:

\begin{abstract}
Warfighter operated and maintained, mobile, flexible group of interconnected sources and loads that acts as a single controllable entity, which can be organized as a system, intended to be self-contained, readily deployable that may utilize alternative-energy resources and power storage, and is capable of interfacing with other grids.*
\end{abstract}

The tactical microgrid standard supports the development of advanced power systems that can bring energy resiliency to the warfighter through a tactical microgrid that:

- Consists of interoperable devices

- Uses a resilient, open architecture that is modular and scalable

- Accommodates mission-based equipment configurations

- Enables single-component upgrades and interactions instead of forcing system upgrades

- Utilizes multilevel cybersecurity.

Policy drivers, the emergence of new technologies, and the push to develop open standards underscore the need to develop a living laboratory to conduct tests, evaluate, and demonstrate advanced technologies to assess prospect for potential incorporation into the military. A living laboratory will promote the investigation of device interoperability and allow researchers to develop an approach to bridge the gap between new technologies and standards.

\title{
1.13 Definition of a living laboratory
}

The European Network of Living Labs (ENoLL) defines a living laboratory as "user-centered, open innovation ecosystems based on a systematic user

\footnotetext{
* Tactical Microgrids Standards Consortium, Tactical Microgrid Interoperability Standardization, May 2018, U.S. Army Engineer R\&D Center (ERDC), U.S. Army Communications-Electronics RD\&E Center (CERDEC) and MIT Lincoln Laboratory
} 
co-creation approach integrating research and innovation processes in real-life communities and settings." The living laboratory is a platform for "sensing, prototyping, validating and refining complex solutions in multiple and evolving real-life contexts." ${ }^{\dagger}$ This research environment embodies "five key elements: innovation settings, operating environments, influence on innovation processes, user engagement, and expected outcomes.”

The living laboratory, an increasingly popular research and development (R\&D) concept, has gained momentum since 2006.§ While some studies suggest the concept originated from Professor William Mitchell of the Massachusetts Institute of Technology, ${ }^{* *}$ the first historical reference of the term 'living laboratory' was used by Thomas Knight in $1749 .{ }^{\dagger \dagger}$ Since then, the novel concept has modernized into a multifaceted, experimental environment in real-life context.

\subsection{The CERL mission}

The CERL mission is to "develop and infuse innovative technologies to provide excellent facilities and realistic training lands for DoD, the U.S. Army and many other customers while also supporting ERDC's R\&D mission in geospatial research and engineering, military engineering, and civil works.” " In support of this mission, the Paragon research team set out to design a roadmap for an innovative sensored, living laboratory with the capacity and capability to evaluate a nexus of emerging technologies in the fields of electric transport vehicles, vehicle autonomy, V2G, and microgrid technologies integrated with military legacy equipment. The fundamental concept of the Contingency Basing Integration Technology Evaluation Center (CBITEC) living laboratory is to serve as a testbed to facilitate realtime research and joint innovation across autonomy and microgrid

\footnotetext{
* "What Are Living Labs.” About Us, European Network of Living Labs, enoll.org/.

† Hossain, Mokter, et al. "A Systematic Review of Living Lab Literature." Journal of Cleaner Production, 1 Mar. 2019, pp. 976-988., doi:0.1016/j.jclepro.2018.12.257.

‡ “European Network of Living Labs." European Network of Living Labs, enoll.org/.

$\S$ Hossain, Mokter, et al. "A Systematic Review of Living Lab Literature.” Journal of Cleaner Production, 1 Mar. 2019, pp. 976-988., doi:0.1016/j.jclepro.2018.12.257.

** ibid.

†† Knight, Thomas. Reflections Upon Catholicons, Or Universal Medicines: With Some Remarks on the Natural Heat That Is in Animals, and the Luminous Emanations from Human Bodies. Vol. 1, T. Osborne in Gray's-Inn., 1749, books.google.com.

¥ “Mission Statement." CERL Missions, U.S. Army Corps of Engineers Engineer Research \& Development Center Construction Engineering Research Lab, www.erdc.usace.army.mil/Locations/CERL/Missions.aspx.
} 
technologies in realistic settings. An immersed, yet structured, exhibition of technologies in action will allow research and assessment of interoperability and operational performance between systems.

The CBITEC Living Laboratory roadmap facilitates an understanding of how state-of-the-art technologies can interoperate within a spectrum of possible scenarios and aid in determining the prospect for implementation to advance the military mission. This concept will support the CERL mission and posture innovation across private and public sectors where no similar applications and capabilities coexist.*

\subsection{The Paragon Team}

To develop a living laboratory roadmap, Paragon leveraged leading technical experts' ideas, knowledge, and experience in the fields of transportation, autonomy, and energy solutions. This unique collaboration led to communal information sharing and open innovation across technology borders that would have otherwise been siloed. The living laboratory is therefore a platform for the convergence of leaders across fields of interest to cocreate innovation, prompting an acceleration of technological advancement, and innovation across individualized industries. The industry experts that developed this Research, Development, Test and Evaluation (RDT\&E) project are:

\subsubsection{Paragon Business Solutions, Inc.}

Paragon Business Solutions, Inc. provides project management, quality systems, and strategic energy expertise to improve the success and resilience of our customers. Paragon's processes create a framework that is repeatable and proven to reduce risk, improve efficiency, and deliver desired results. Founded in 1997, Paragon is a U.S. Small Business Administration (SBA) Women-Owned Small Business (WOSB).

Angela Rolufs, Vice President for Strategic Energy Initiatives, and the project proponent for the ATI project, led the Paragon team by providing program management and project delivery. 


\subsubsection{Robotic Research, LLC}

Robotic Research, LLC focuses on in-ground robotics, especially in localization, sensor processing, and intelligent command and control. Robotic Research has extensive experience with developing robotic systems for various vehicles types, ranging from the man-portable Talon to small electric shuttles and large Army Palletized Load System (PLS) trucks.

Bryan Brilhart, Senior Program Manager at Robotic Research, led the R\&D efforts for improving autonomous transport technologies in a tactical environment.

\subsubsection{TechFlow, Inc.}

Founded in 1995 with deep operational roots in the bi-coastal innovation hubs of California and Washington D.C., TechFlow applies innovative engineering, technology, and integration solutions to the government's most demanding mission and business challenges, delivering impactful ingenuity on a government scale. TechFlow's Energy and Mobility Solutions business unit employs subject matter experts (SMEs) with decades of proven performance in the area of program managing, engineering, energy effectiveness, as well as $\mathrm{V} 2 \mathrm{G} /$ microgrid applications/demonstrations, energy storage, technology transition, RDT\&E, and resilience.

Clark Boriack, Director of Engineering for Energy and Mobility at TechFlow, led the research and demonstration efforts for the integration of V2G charging into a tactical microgrid.

\subsubsection{The Center for Sustainable Solutions (CS2)}

The Center for Sustainable Solutions (CS2) is a non-profit organization that works to advance sustainable practices throughout society by conducting education and outreach efforts on sustainable technologies, community systems development, long-term planning, and sustainable approaches to problem-solving within communities. CS2 works primarily with military installations and their surrounding communities to coordinate and promote sustainable technology demonstrations and validations that will advance and support state-of-the-art emerging technologies and the companies that have developed them. CS2 works to identify, 
document, and promote sustainable technology R\&D through needs identification and transition of technologies from research to use.

Kevin Palmer, Founder and Director of CS2, leveraged his knowledge of military installations and emerging technologies to lead the identification of military transport operations that can be improved through automation. $\mathrm{He}$, and the Paragon research team, engaged with military and civilian stakeholders to define requirements and identify need gaps. Palmer also contributed to the quality-assurance effort to ensure that all deliverables meet the standards set forth in the contract.

\subsubsection{Paul Brubaker, LLC}

Paul Brubaker, CEO and president of Paul Brubaker, LLC, supported the Paragon team by engaging AV SMEs to advise and provide input into the latest AV technologies that the team considered for tactical operations. He acted as a technical reviewer of the reports prepared by Paragon team members to ensure high standards are met as Paragon requires and ERDC- CERL expects.

\subsection{Autonomous Transport Innovation}

Paragon developed the ATI RDTE project in support of the Army's ERDCCERL innovative energy efficiency and energy security and contingency basecamp operational energy initiatives.

Paragon partnered with leaders in the field of electric transport vehicles, vehicle autonomy, $\mathrm{V} 2 \mathrm{G}$, and microgrid technologies to deliver the ATI RDTE project to advance the state-of-the-art of autonomous tactical vehicles and contingency-basing energy resiliency. The technical issues that have the potential to impede integrating autonomous electric transport vehicles into a microgrid were investigated and solutions documented in this report for future application. Research, results, and methodologies established can be implemented to assist ERDC-CERL in the strategic integration of new and emerging energy and resilience technologies.

The ATI technical research program investigated current gaps and challenges and develop solutions to integrate emerging electric transport vehicles, vehicle autonomy, $\mathrm{V} 2 \mathrm{G}$, and microgrid technologies alongside military legacy equipment. Research performed resulted in a roadmap to 
develop a sensored, living laboratory with operational testing capability to assess the safety, utility, interoperability, and resiliency of autonomous electric transport and $\mathrm{V}_{2} \mathrm{G}$ technologies in a tactical microgrid. The living laboratory will support ERDC-CERL research and assessment of emerging technologies and determine the prospect for implementation in defense transport operations and contingency base energy resilience.

The purpose of the ATI RDTE project is to develop a roadmap for a sensored, living laboratory to integrate autonomous transport vehicles into a tactical microgrid.

The scope of the ATI technical research program is as follows:

1. Identify military-unique requirements for autonomous transport technologies

2. Identify existing technologies that can be adapted for military applications, conduct technology gap analysis and identify need gaps

3. Identify research and operational tests for autonomous transport vehicles

4. Investigate requirements for testing and demonstrating vehicle charging within tactical microgrid environment

5. Develop requirements for a sensored living laboratory to assess performance of autonomous innovations

6. Integrate open standards to promote interoperability. 


\section{Identify Military-Unique Requirements for Autonomous Transport Technologies}

The objective of Research Area 1 is to identify military transport activities that can be automated to the benefit of the government. This research was informed through the review of government programs, standards, and practices and engagement with stakeholders.

The Paragon team identified vehicle-dependent Military Support Activities, the vehicles used to enable the activity, and the specific transport system requirements for each support activity. This information was distilled to develop specific use cases for a list of candidate transport systems that can be automated to the benefit of the military mission.

\subsection{Military support activities and associated transport systems}

The objective of this research was to identify military transport systems that have potential to benefit from automation. This was carried out by evaluating routinely conducted support activities performed by the military.

\subsubsection{Vehicle-dependent Military Support Activities}

Military Support Activities (MSA) routinely conducted in Continental United States (CONUS) installation operations were studied to document transport-dependent activities and observe transport systems used. Paragon's team engaged with stakeholders and conducted a review of literature including a thorough review of military programs, standards, and practices. CSS used a significant portion of the 46 Army Installation Strategic Plans, National Guard, Reserve, and community strategic sustainability plans that have been developed since 2000 to identify a list of common military activities that are vehicle dependent (Figure 3). These strategic plan documents included weaknesses and threats identified by a stakeholder-developed SWOT analysis. See Appendix A: Military Strategic Planning Resource Documents for detailed information on results from the planning done at Army Installations across the world used in identifying significant military activities for this study. In addition, a review of the Joint Base Lewis-McChord (JBLM) Feasibility Study for the Application of Automated Vehicles 7 identified and validated vehicle-dependent military activities that represent opportunities for autonomous 
operation. Session notes from the JBLM Scoping Session were referenced for installation stakeholder discussion around Military Support Activities that would be most useful to conduct autonomously as part of the U.S. Army Tank Automotive Research, Development and Engineering Center (TARDEC) Applied Robotics for Installation and Base Operation (ARIBO) program.6,*

Figure 3. Military installation strategic plans reviewed.

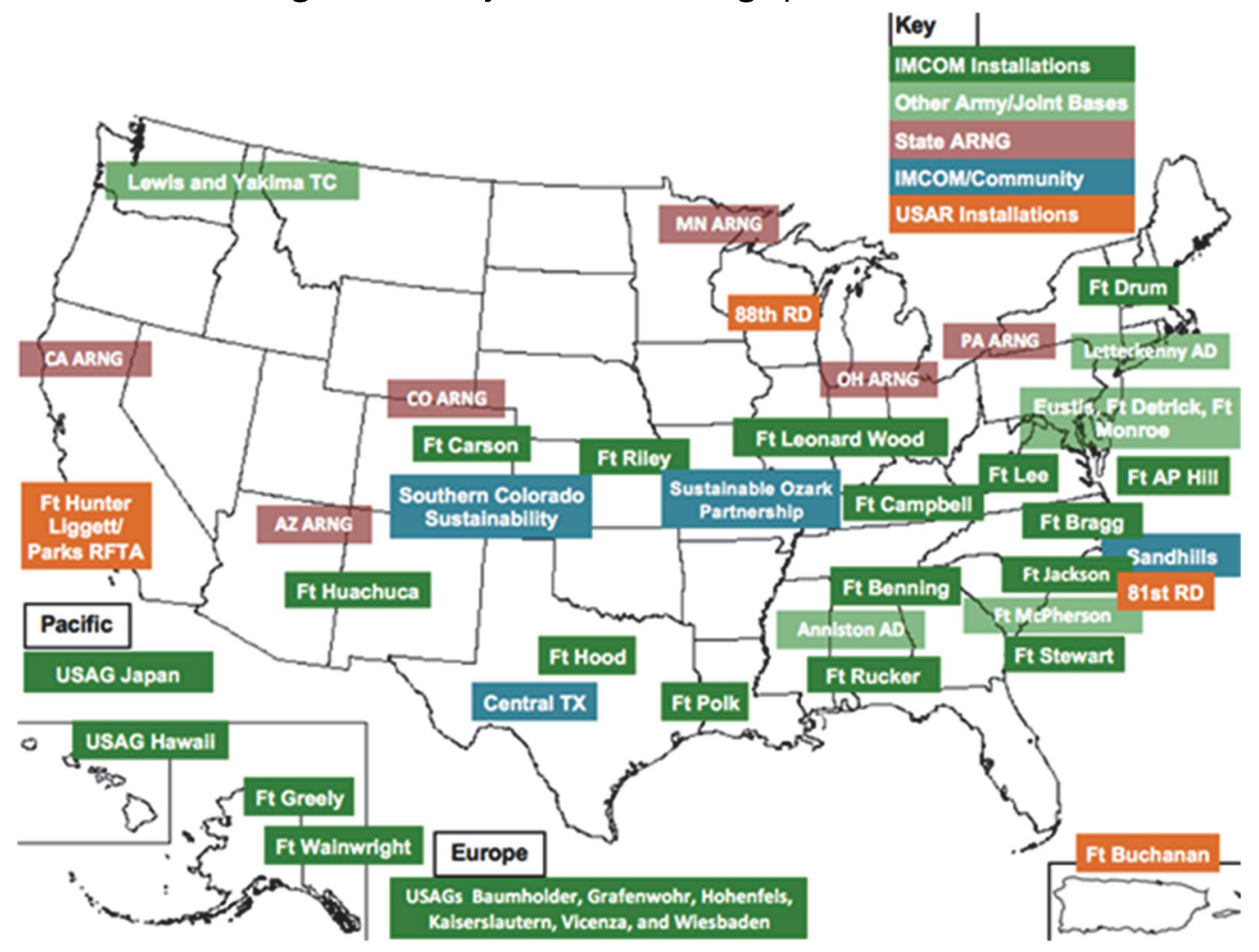

Based on reviews of strategic plans and the JBLM session, the following Military Support Activities were identified as candidates for further review and research:

- MSA 1-Personnel transport

- MSA 2-Land management

- MSA 3-Logistics

- MSA 4-Security monitoring

* Steucke, Paul. “Joint Base Lewis-McChord (JBLM) Autonomous Vehicle Scoping Session Summary.” Internal Meeting Summary with Installation Stakeholders to Identify Military Support Activities That Could Be Performed Autonomously, 31 Oct. 2014, pp. 1-4. 
- MSA 5-Land preparation

- MSA 6-Road maintenance.

Our team analyzed all of the content to document transport dependent Military Support Activities that were routinely identified in strategic plan documents as resource intensive, inefficient, costly, or poorly functioning to the point of adversely impacting or having the greatest potential to impact the mission. Transport-dependent Military Support Activities, the missions each could impact, along with the transportation system used for the Military Support Activity are provided in Table 1.

Table 1. Vehicle-dependent Military Support Activities.

\begin{tabular}{|c|c|c|c|}
\hline $\begin{array}{l}\text { MSA } \\
\text { No. }\end{array}$ & MSA Type & Mission Supported & Vehicles \\
\hline \multirow{3}{*}{1} & $\begin{array}{l}\text { Personnel Transport: } \\
\text { transport (1-4 people) }\end{array}$ & \multirow{3}{*}{$\begin{array}{l}\text { Installation Management Command (IMCOM)/Army } \\
\text { Materiel Command (AMC) missions for logistics in } \\
\text { support of installation operation-personnel transport } \\
\text { supports all CONUS housing, training and deployment } \\
\text { functions. } \\
\text { Mass transit is more common at basic training } \\
\text { installations. } \\
\text { FLW provides } 2.5 \text { million round trips annually to move } \\
\text { Soldiers to training areas, ranges, and dining facilities. }\end{array}$} & Automobile \\
\hline & $\begin{array}{l}\text { Personnel transport (5- } \\
20 \text { people) }\end{array}$ & & Shuttle \\
\hline & $\begin{array}{l}\text { Troop transport for } \\
\text { training (platoon to } \\
\text { company size--up to } \\
250 \text { Soldiers) }\end{array}$ & & $\begin{array}{l}\text { Buses }(40-80) \text { and tractor with } \\
\text { personnel trailer (125) }\end{array}$ \\
\hline \multirow[b]{2}{*}{2} & $\begin{array}{l}\text { Land Management: } \\
\text { grounds maintenance- } \\
\text { beautification and } \\
\text { security cutting }\end{array}$ & \multirow{2}{*}{$\begin{array}{l}\text { IMCOM/AMC installation operation -grounds } \\
\text { maintenance can include grass cutting, snow removal, } \\
\text { dirt removal, wood clearing (with workers), tree removal } \\
\text { (with workers), etc. } \\
\text { Two types of ground maintenance are common: grass } \\
\text { cutting in cantonment areas and less frequent grass } \\
\text { cutting in range and training areas. This effort would be } \\
\text { focused on large-scale mowers and earth movers that } \\
\text { can be used to maintain CONUS range areas (and } \\
\text { possibly cantonment areas). }\end{array}$} & Tractors and mowers \\
\hline & $\begin{array}{l}\text { Land Management: } \\
\text { range maintenance }\end{array}$ & & $\begin{array}{l}\text { Large/farm tractors with cutting } \\
\text { decks, plowing }\end{array}$ \\
\hline \multirow{3}{*}{3} & $\begin{array}{l}\text { Logistics: materiel } \\
\text { transport-food, water, } \\
\text { munitions, fuel, } \\
\text { municipal solid waste }\end{array}$ & \multirow[t]{3}{*}{$\begin{array}{l}\text { IMCOM/AMC Installation Operations directly supporting } \\
\text { training (TRADOC), Garrison (FORSCOM) missions, and } \\
\text { deployment (all installations that deploy personnel). } \\
\text { Convoys used to support all forward-operating areas. }\end{array}$} & $\begin{array}{l}\text { Delivery trucks and vans, } \\
\text { tractors with trailers, specialty } \\
\text { trucks (tankers, refrigeration } \\
\text { unit, waste hauler, bucket } \\
\text { truck), } \\
\text { secure trucks (munitions) }\end{array}$ \\
\hline & $\begin{array}{l}\text { Logistics: material } \\
\text { management and } \\
\text { warehousing-delivery, } \\
\text { storage and distribution }\end{array}$ & & $\begin{array}{l}\text { Forklifts, indoor and outdoor } \\
\text { tugs, material handling } \\
\text { equipment }\end{array}$ \\
\hline & $\begin{array}{l}\text { Logistics: airfield } \\
\text { activities-ground } \\
\text { support vehicles (GSVs) }\end{array}$ & & $\begin{array}{l}\text { Various GSVs-fuelers, pallet } \\
\text { loaders, aircraft tugs, small } \\
\text { loaders, munitions movers and } \\
\text { loaders (security), deicers, plows }\end{array}$ \\
\hline 3 & $\begin{array}{l}\text { Logistics: vehicle and } \\
\text { equipment hauling }\end{array}$ & $\begin{array}{l}\text { Vehicle and equipment recovery and delivery-supports } \\
\text { IMCOM/AMC operations. }\end{array}$ & $\begin{array}{l}\text { Tow trucks, vehicle lifts and } \\
\text { haulers, tractors with trailers }\end{array}$ \\
\hline 4 & Security Monitoring & $\begin{array}{l}\text { Reconnaissance in forward areas, as well as installation } \\
\text { security monitoring. }\end{array}$ & $\begin{array}{l}\text { Drones, reconnaissance } \\
\text { vehicles }\end{array}$ \\
\hline
\end{tabular}




\begin{tabular}{|l|l|l|l|}
\hline $\begin{array}{c}\text { MSA } \\
\text { No. }\end{array}$ & MSA Type & Mission Supported & Vehicles \\
\hline 5 & $\begin{array}{l}\text { Land Preparation: earth } \\
\text { moving, plowing, digging }\end{array}$ & $\begin{array}{l}\text { IMCOM/AMC grounds maintenance, as well as combat } \\
\text { engineering operations and training. }\end{array}$ & $\begin{array}{l}\text { Engineering equipment- } \\
\text { scraper, crane, dozer, plow, } \\
\text { hauler, dump truck, grader, } \\
\text { loader, backhoe, crawler, } \\
\text { hydraulic excavator (HYEX) }\end{array}$ \\
\hline 6 & $\begin{array}{l}\text { Road Maintenance: } \\
\text { maintenance, repair, } \\
\text { snow removal }\end{array}$ & $\begin{array}{l}\text { IMCOM/AMC installation maintenance and clearing, } \\
\text { development, and maintenance of new and existing } \\
\text { roads in forward areas. }\end{array}$ & $\begin{array}{l}\text { Grader, sweeper, dump truck, } \\
\text { asphalt paver, compactor, roller, } \\
\text { snowplow }\end{array}$ \\
\hline
\end{tabular}

\subsubsection{Military Support Activity transport requirements}

The military transports a variety of personnel, including Soldiers and civilian staff, for a variety of activities, such as training, dining, and Garrison support duties. Transport requirements for Military Support Activities identified in the previous section are summarized in Tables 2 to 7.

Table 2. Military Support Activity 1: Personnel transport.

\begin{tabular}{|c|c|}
\hline Transport System & Transport Requirements \\
\hline $\begin{array}{l}\text { Automobile, shuttle buses }(40-80) \text {, } \\
\text { tractor with personnel trailer (125) }\end{array}$ & $\begin{array}{l}\text { - On-post transit requirements can range from moving individuals to company- } \\
\text { sized units (up to } 250 \text { people) around a post to train, eat, attend medical } \\
\text { appointments, etc. } \\
\text { - Soldier transport is accomplished by bus ( } 40 \text { people with packs): hauling } \\
\text { capacity (Ib.) } \\
\text { - Distances will be a function of the demonstration: distance (burdened and } \\
\text { unburdened [mi.]) } \\
\text { - A Class 6-8 tractor (truck) pulling a trailer accommodating up to } 100 \text { people: } \\
\text { towing capacity (lb.) } \\
\text { - Personnel transport in forward areas will depend on the specific mission } \\
\text { Vehicle might need to traverse unpaved roads (gravel or dirt trails): ground } \\
\text { clearance (in.) }\end{array}$ \\
\hline \multicolumn{2}{|c|}{$\begin{array}{l}\text { Performance Attributes: } \\
\text { Vehicle capacity, seating configuration, storage area, No. of egress points, rough terrain suitability }\end{array}$} \\
\hline
\end{tabular}

The Army maintains over 9 million acres of land, ${ }^{*}$ including cantonment, training, and range areas. A majority of this acreage requires management. Cantonment area management can include grass cutting, snow removal, and grounds maintenance. Land management in training areas and ranges is required to maintain operational capacity. Grass-cutting periods depend on climate and land-use schedule. Range and training maintenance can include grass cutting, invasive species removal (bush hogging), removal of foreign materials (concertina wire), revegetation, plowing, and grading.

\footnotetext{
* Vincent, Carol Hardy, and Laurel Hanson. “Federal Land Ownership: Overview and Data." Congressional Research Service, 3 Mar. 2017, p. 6, fas.org/sgp/crs/misc/R42346.pdf.
} 
Table 3. Military Support Activity 2: Large-scale land, training area, and range maintenance.

\begin{tabular}{|c|c|}
\hline Transport System & Transport Requirements \\
\hline $\begin{array}{l}\text { Tractors and mowers: } \\
\text { large and farm tractors with cutting } \\
\text { decks, plowing }\end{array}$ & $\begin{array}{l}\text { - Maintain thousands to hundreds of thousands of acres }{ }^{*} \\
\text { - } \quad \text { Systinuous operation to mitigate invasive species and rapid growth } \\
\text { - } \text { - Systems to detect and remove foreign materials that would otherwise damage } \\
\text { - Systems to support land rehabilitation } \\
\text { - } \quad \text { Ability to work in undeveloped: ground clearance (in.) } \\
\text { - Capacity to support land-grading and earth-moving functions }\end{array}$ \\
\hline
\end{tabular}

Materiel transport has application to all military activities and is a resource-intensive activity. Logistics in forward areas represent significant risk to all personnel involved with the receipt, transport, and delivery of materiel used in operations. Transported materiel includes food, water, munitions, fuel, solid waste, warehoused materials, mail, and medical supplies.

Table 4. Military Support Activity 3: Materiel transport.

\begin{tabular}{|c|c|}
\hline Transport System & Transport Requirements \\
\hline $\begin{array}{l}\text { Delivery trucks and vans; } \\
\text { tractors with trailers; } \\
\text { specialty trucks (tankers, refrigeration unit, waste } \\
\text { hauler, bucket truck); } \\
\text { secure trucks (munitions); } \\
\text { forklifts, indoor and outdoor tugs, material handling } \\
\text { equipment } \\
\text { various GSVs }\end{array}$ & $\begin{array}{l}\text { Towing vehicles must be able to tow at least } 3 \text { tons: towing } \\
\text { capacity (lb.) } \\
\text { Delivery vehicles should have sufficient capacity to provide routine } \\
\text { materiel deliveries } \\
\text { Vehicles should be able to function at minimum required speeds } \\
\text { ( } 25-45 \mathrm{mph} \text { ) } \\
\text { Vehicle should be able to function on gravel road: ground } \\
\text { clearance (in.) } \\
\text { Specialized functions should be operable by wire }\end{array}$ \\
\hline \multicolumn{2}{|c|}{$\begin{array}{l}\text { Performance attributes: } \\
\text { Towing capacity, hauling capacity, availability of specialized functions (e.g., refrigeration) }\end{array}$} \\
\hline
\end{tabular}

\section{Security is a critical consideration at CONUS and Outside Continental} United States (OCONUS) installations. It is a critical function for FOBs and operations.

\footnotetext{
* Vincent, Carol Hardy, and Laurel Hanson. “Federal Land Ownership: Overview and Data." Congressional Research Service, 3 Mar. 2017, p. 6, fas.org/sgp/crs/misc/R42346.pdf.
} 
Table 5. Military Support Activity 4: Off-road, transport-based security monitoring.

\begin{tabular}{|l|l|}
\hline \multicolumn{1}{|c|}{ Transport System } & \multicolumn{1}{c|}{ Transport Requirements } \\
\hline $\begin{array}{l}\text { Drones, reconnaissance } \\
\text { vehicles }\end{array}$ & $\begin{array}{l}\text { - Host surveillance equipment and other platforms } \\
\text { Off-road capability required: ground clearance (in.) }\end{array}$ \\
$\begin{array}{l}\text { Duration of operation in undeveloped areas: impact of geographic features on battery } \\
\text { performance }\end{array}$ \\
$\begin{array}{ll}\text { Performance attributes: } \\
\text { Rough terrain suitability, sensors, and monitoring capability }\end{array}$ \\
\hline
\end{tabular}

Redistribution of land and materiel is a common activity that supports construction, land management, engineering operations, FOB development, and range and training area maintenance.

Table 6. Military Support Activity 5: Earth moving.

\begin{tabular}{|l|l|}
\hline Transport System & Transport Requirements \\
\hline $\begin{array}{l}\text { Engineering equipment: scraper, crane, grader, } \\
\text { dozer, plow, hauler, dump truck, etc. }\end{array}$ & $\begin{array}{l}\text { All land alteration functions associated with land maintenance and } \\
\text { management, construction, and combat engineering }\end{array}$ \\
\hline $\begin{array}{l}\text { Performance attributes: } \\
\text { Rough terrain suitability, available engineering functions (crane, dozer, dump truck, etc.) }\end{array}$ \\
\hline
\end{tabular}

Establishing and maintaining surfaced, gravel, and dirt roads is a continuous and costly activity at CONUS, OCONUS installations and FOBs.

Table 7. Military Support Activity 6: Road maintenance.

\begin{tabular}{|c|c|}
\hline Transport System & Transport Requirements \\
\hline Grader, sweeper, dump truck, asphalt paver, compactor, roller, snowplow & $\begin{array}{l}\text { - } \text { Maintain road condition } \\
\text { - } \text { Grade and repair roads } \\
\text { - } \text { Plow snow }\end{array}$ \\
\hline \multicolumn{2}{|c|}{$\begin{array}{l}\text { Performance attributes: } \\
\text { Rough terrain suitability, available engineering functions (grader, resurfacing, dozer, dump truck, etc.) }\end{array}$} \\
\hline
\end{tabular}

\subsubsection{Vehicle performance metrics}

Performance metrics can further define how well a specific vehicle can support a military activity.

Performance metrics considered include the following:

- Acceleration (burdened and unburdened)

- Battery capacity and average performance time (before batteries are empty)

- Broadcast bandwidth (Hz.) 
- Distance on a full charge (burdened and unburdened)

- Efficiency (\%)

- Ground clearance (in.)

- Handling (lb./ $\left.\mathrm{ft}^{2}\right)$

- Hauling capacity (lb.)

- Impact of geographic features on battery performance (e.g., how hills impact time between charges)

- Power (hp)

- Power to weight (hp/lb.)

- Safety ratings

- Traction (psi)

- Top speed (loaded and unloaded: lb.)

- Torque (ft-lb)

- Towing capacity (lb.)

- Traction (psi)

- Turning radius (in.).

\subsection{Autonomous transport vehicle military use cases}

Six vehicle-dependent Military Support Activities were evaluated to identify the current transport system used and the requirements necessary to maintain or improve effectiveness if automated. The following use cases demonstrate the value of AVs in logistics/supply, security/reconnaissance, and construction support activities. Each use case provides the basic flow of a support activity from receipt of request to delivery of needed support. Alternative scenarios are included to demonstrate how autonomy adds value that would not be available with a human-operated vehicle.

\subsubsection{Use case for an autonomous vehicle supply convoy}

The objective of this research is to use autonomous and EVs to move people and goods within tactical operating environments.

An FOB requires replenishing fuel and supplies to sustain military operations. The logistician receives the requisitions for supplies including fuel, food, water, munitions, ammunition, equipment, and other necessities. Supplies are loaded into autonomously operated, adequately charged electric trucks. The destination is programmed into the vehicles, which successfully navigate both paved and unpaved roads from the supply depot to the FOB. Once at the FOB, the trucks are unloaded, charged, and 
autonomously return to the supply depots with any materiel required to be returned.

Subject Area: Logistics and Supply

Actors: logisticians, supply clerks, supply specialists

Stakeholders: commanders and Soldiers

Trigger: Supply clerks request supplies from the supporting supply depot.

Precondition 1: The supply clerk orders all of the needed supplies to support the operations of the FOB.

Precondition 2: The logisticians at the depot have the materiel, fuel, and autonomous transport vehicles to supply the FOBs.

Precondition 3: Vehicles can be successfully charged.

\section{Basic Flow:}

- The supply clerk orders necessary supplies from the depot.

- The supply depot receives the order.

- Supply specialists select and load the supplies onto pallets.

- Pallets are loaded onto an autonomous and adequately charged electric truck.

- The destination is programmed into the truck(s), and the truck(s) depart(s) for the FOB.

- Drone escorts are deployed to provide additional situational awareness.

- $\mathrm{AV} / \mathrm{EV}$ trucks navigate the route from the depot to the FOB transmitting location data as well as video from the vehicle to the command center that is accessible to the FOB supply clerk.

- AV/EV trucks arrive at the FOB and then are unloaded by supply specialists.

- Vehicles are charged while being loaded with any materiel required to leave the base. 
- Depot destination is programmed into the truck(s), and the truck(s) depart(s) for the depot.

- Trucks navigate back to the depot.

- Trucks arrive at the depot.

- Supply specialists unload the trucks.

- Trucks are cleaned, inspected, maintained, and charged for next trip.
Alternative Path 1: A vehicle in the convoy fails during the trip to the FOB (Table 8).

This scenario describes the situation where a critical component, required for the safe and/or continued operation of the $\mathrm{AV} / \mathrm{EV}$, is failing or has failed.

Table 8. Alternative Path 1: A vehicle in the convoy fails during the trip to the FOB.

\begin{tabular}{|c|}
\hline Description \\
\hline $\begin{array}{l}\text { - Loaded truck begins to fail in route to the FOB. } \\
\text { - The loaded truck, its contents, and the nature of the failure are identified through onboard sensors and diagnostics. } \\
\text { - Diagnostics determine whether the truck can make it to the depot, reverse course, and return to the depot or if it } \\
\text { - should stop in route and await a repair crew. } \\
\text { - Other trucks on the supply mission continue to the FOB. } \\
\text { - If recessary, an additional drone is dispatched to observe and protect the disabled vehicle. } \\
\text { - If the truck cannot be repair crew is dispatched, necessary repairs are made, and truck resumes it supply mission. } \\
\text { the operating vehicle, and the supply mission resumes. }\end{array}$ \\
\hline $\begin{array}{l}\text { Postcondition/outcome: } \\
\text { The supplies are delivered to the FOB. }\end{array}$ \\
\hline
\end{tabular}

\section{Alternative Path 2: Vehicle is destroyed (Table 9).}

This scenario describes when a supply vehicle is attacked by the enemy and destroyed.

Table 9. Alternative Path 2: Vehicle is destroyed.

\begin{tabular}{l} 
Description \\
\hline - $\quad$ A supply vehicle and its contents are attacked by an enemy and subsequentially destroyed. \\
- The escort drone is dispatched to the supply vehicle. It provides situational awareness of the incident to the depot \\
- The level of threat is assessed by the command center. \\
- If necessary, the command center recommends a kinetic response to the threat. \\
- If necessary, and when safe, a disposal unit is deployed to remove the vehicle from the road, and any blocked vehicles \\
resume their supply mission. \\
- The supplies on board the destroyed vehicle are recorded at the depot, and an identical supply order is selected and \\
Ioaded onto a secondary AV/EV and dispatched to the FOB. \\
\hline Postcondition/outcome: \\
The supplies are delivered to the FOB.
\end{tabular}


Alternative Path 3: Multiple vehicles are destroyed (Table 10).

This scenario describes when multiple vehicles are destroyed after an enemy attack.

Table 10. Alternative Path 3: Multiple vehicles are destroyed.

\begin{tabular}{l} 
Description \\
\hline - Multiple supply vehicles attacked by an enemy and are subsequentially destroyed. \\
- depot. \\
- The level of threat is assessed. \\
- If necessary, the command center recommends a kinetic response to the threat. \\
- If ground supply cannot continue within a reasonable timeframe, designated emergency supplies are airlifted to the \\
- FOB. \\
- vehen appropriate and safe, a disposal unit is deployed to remove the vehicles from the road, and any remaining \\
- The supplies on board the destroyed vehicles are recorded at the depot, and identical supply orders are selected and, if \\
Postcondition/outcome: \\
- The ground supply mission resumes. \\
- Beeded supplies arrive at the FOB. They are unloaded and charged, allowing vehicles return to the supply depot. \\
- Supply requirements are predictable and well anticipated based on historical analysis. \\
- Depots have sufficient supplies, reserves, and vehicles to meet FOB requirements.
\end{tabular}

\subsubsection{Use case for an autonomous vehicle reconnaissance mission (command, control, communications, computing, intelligence surveillance reconnaissance)}

The objective of this research is to use autonomous and EVs as platforms for forward reconnaissance missions.

$\mathrm{AV} / \mathrm{EVs}$ can be used as reconnaissance and intelligence gathering platforms to increase military command's situational awareness. These vehicles can be operated fully autonomously, or remotely, depending on the mission situation. The platform can patrol the areas surrounding an FOB or a variety of terrain, including urban areas, to observe potential hostile or suspicious activity and/or provide desired situational awareness. The platform collects and transmits situational awareness from onboard sensors to include cameras, radar, radio signal, air quality, and/or chemical sensors. The platform is expected to calculate remaining battery life and return to the FOB for charging, as necessary. When necessary, multiple platforms can operate simultaneously to provide full-spectrum intelligence and offer resilience in observation without risk to human life. 
Subject area: Command, Control, Communications, Computing, Intelligence, Surveillance, and Reconnaissance (C4ISR)

Actors: commanders, mission planners, and platform specialists

Stakeholders: commanders, observers, intelligence analysts, mission planners

Trigger: desire for situational awareness

Precondition 1: The FOB commander requires situational awareness within AOR.

Precondition 2: The FOB has the communication capacity to receive process and monitor situational awareness data from the AV/EV C4ISR platform.

Precondition 3: The observable area is within the EV range of the FOB to allow vehicles to perform the mission and return to the FOB.

Precondition 4: There are sufficient numbers of AV/EV platforms on the FOB to perform the required $\mathrm{C}_{4}$ ISR missions.

\section{Basic Flow:}

- The commander desires situational awareness within the AOR.

- Mission planners consult with the platform specialists to plan C4ISR missions to meet commanders need.

- The platform is charged and deployed on the mission.

- The platform performs the mission.

- The platform returns to the FOB.

Alternative Path 1: Pervasive situational awareness in the area surrounding the FOB (Table 11). 
Table 11. Alternative Path 1: Pervasive situational awareness in the area surrounds the FOB.

\begin{tabular}{l} 
Description \\
\hline - The commander desires pervasive situational awareness around the FOB. \\
- Mission planners consult with platform specialists to plan platform placement, patrols, and rotation of platforms to \\
ensure the necessary charging and coverage around the FOB AOR to meet the mission requirements. \\
- Platforms perform their missions and autonomously return to base when necessary to charge. \\
Postcondition/outcome: \\
- The commander achieves the desired level of situational awareness and security objectives are achieved.
\end{tabular}

\section{Alternative Path 2: Platform malfunctions or is destroyed.}

This scenario describes when a platform becomes inoperable or is destroyed (Table 12).

Table 12. Alternative Path 2: Platform malfunctions or is destroyed.

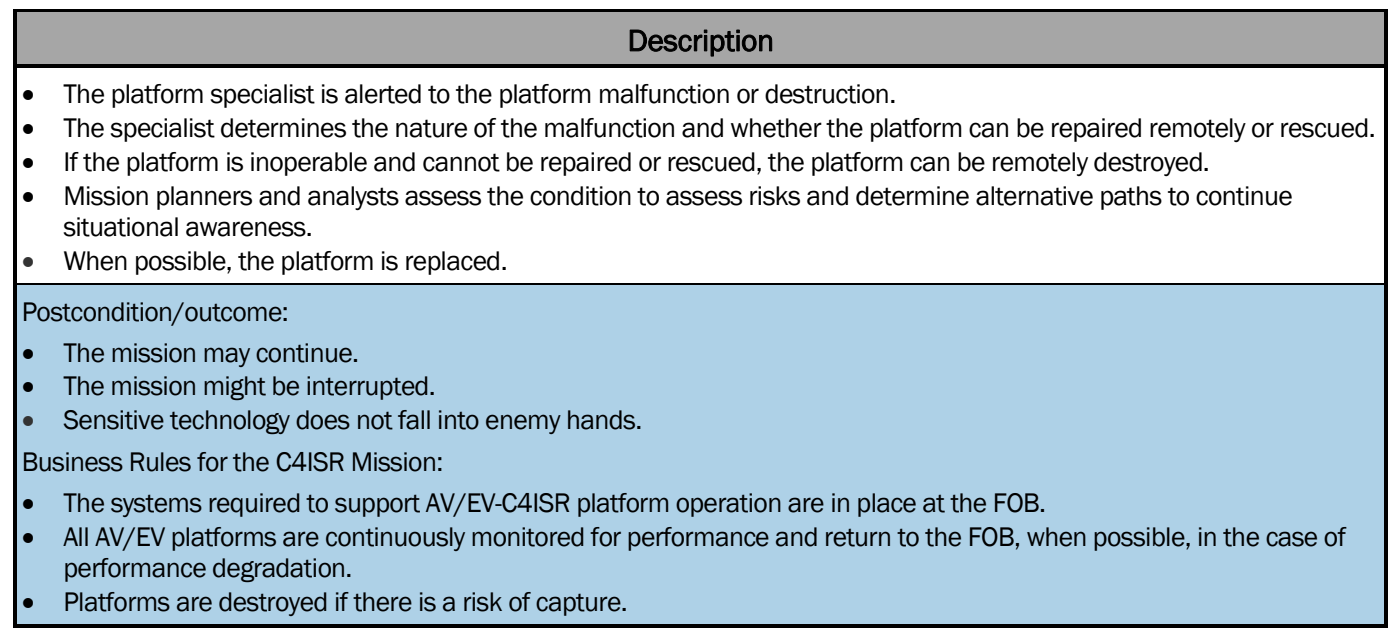

\subsubsection{Use case for an autonomous vehicle construction support mission}

The objective of this research is to use autonomous and electric platforms to support the military construction mission.

A commander decides to construct an FOB. AV/EV platforms are deployed to support land clearing, grading, site preparation, construction of perimeter fencing, security, and initial buildings.

\section{Subject Area: Military Construction}

Actors: commanders, military construction engineers, construction support 
Stakeholders: commanders and Soldiers

Trigger: commander give the order to establish an FOB at a particular location.

Precondition 1: The commander and planners have selected a site for the FOB.

Precondition 2: U.S. Army Corps of Engineers have AV/EVs capable of performing autonomous construction tasks.

Precondition 3: AV/EVs can be transported to the FOB location with generators along with a tactical microgrid for charging vehicles.

\section{Basic Flow:}

- The commander orders the construction of the FOB.

- Clearing and grading specifications are determined and programmed into the $\mathrm{AV} / \mathrm{EV}$ construction platforms.

- Computer-Aided Design (CAD) designs are developed, and construction plans and staging order requirements are programmed into the control center. Instructions are provided to the robotic construction systems and AV/EV platforms.

- USACE leverages AV/EV supply vehicles to transport AV/EV construction platforms, robots, and automated construction systems as needed to the FOB site.

- A temporary generator, fuel, and charging equipment is transported to the site to support the AV/EV clearing and grading equipment.

- $\mathrm{AV} / \mathrm{EV}$ construction equipment begins clearing and grading the site for barracks and support facilities construction.

- A tactical microgrid, EV, and robotic charging stations are transported to the FOB site and set up inside the FOB perimeter, per the CAD site design.

- $\mathrm{AV} / \mathrm{EV}$ robotic platforms drill holes to inject concrete and fence posts around the perimeter of the FOB and install fencing.

- Off-site automated prefabrication systems, such as additive manufacturing and building component manufacturing equipment, arrives. 
- Onsite automated and robotic systems, such as single-task construction robots for steel-truss assembly, welding, installation, cinder block laying, and concrete pouring, arrive on site.

- Robotic systems and AV/EV construction platforms begin construction of the FOB facilities, per the CAD specifications.

- All platforms and robotic systems are charged as needed.

- As tasks and construction is complete, specific AV/EV platforms, construction robots, and systems are loaded onto AV/EV and then returned to depots for the next assignment.

Alternative Path 1: The Only AV/EV Support is for Site Clearing and Grading (Table 13).

This scenario describes what happens when site clearing, and grading are the only available autonomous systems.

Table 13. Alternative Path 1: The only AV/EV support is for site clearing and grading.

\begin{tabular}{l} 
Description \\
\hline - The commander orders construction of the FOB. \\
- Clearing and grading specifications are determined and programmed into the AV/EV construction platforms. \\
- CAD designs and construction plans are developed. \\
- A tenstruction equipment, fuel, and supplies are transported as needed to the FOB site. \\
grading equipment as well as the traditional construction equipment. \\
- AV/EV construction equipment begins clearing and grading the site for barracks and support facilities construction. \\
- A tactical microgrid equipment and EV charging stations are transported to the FOB site and installed inside the FOB \\
perimeter, per the CAD site design to support future AV/EV missions, such as supply and C4ISR. \\
- Traditional construction equipment begins construction of the FOB facilities, per the CAD specifications. \\
equipment are loaded onto AV/EV transport vehicles. They are returned to depots for the future assignment. \\
\hline Postcondition/outcome: \\
- The FOB is built with limited autonomous support and requires more personnel support. \\
\hline
\end{tabular}




\section{Alternative Path 2: Construction Robots Malfunction or are Otherwise Inoperable (Table 14).}

Table 14. Alternative Path 2: Construction robots malfunction or are otherwise inoperable.

\begin{tabular}{l} 
Description \\
\hline - The commander orders the construction of the FOB. \\
- Clearing and grading specifications are determined and programmed into the AV/EV construction platforms. \\
- center. Instructions are provided to the robotic construction systems and AV/EV platforms. \\
- USACE leverages AV/EV supply vehicles to transport AV/EV construction platforms, robots, and automated construction \\
- $y$ Astems as needed to the FOB site. \\
grading equipment. \\
- AV/EV construction equipment begins clearing and grading the site for barracks and support facilities construction. \\
- A tactical microgrid, EV, and robotic charging stations are transported to the FOB site and set up inside the FOB \\
perimeter, per the CAD site design. \\
AV/EV robotic platforms drill holes to inject concrete and fence posts around the perimeter of the FOB and install \\
- Off-site automated prefabrication systems, such as additive manufacturing and building component manufacturing \\
equipment, arrives. \\
- Onsite automated and robotic systems, such as single-task construction robots for steel-truss assembly, welding, \\
installation, cinder block laying, and concrete pouring, arrive on site. \\
- Single-task robot(s) fail to function as performed. \\
- Robotic system software and systems are remotely examined and, if necessary, repaired if possible. \\
Other work continues if possible until the robotic system can be either repaired remotely or in person or a replacement \\
robotic system arrives. \\
- Robotic systems and AV/EV construction platforms resume construction of the FOB facilities, per the CAD \\
specifications. \\
Postcondition/outcome: \\
- All platforms and robotic systems are charged as needed. \\
AV/EV and returned to depots for the next assignment.
\end{tabular}




\section{Alternative Path 3: Site is Attacked as Construction Begins (Table 15).}

Table 15. Alternative Path 3: Site is attacked as construction begins.

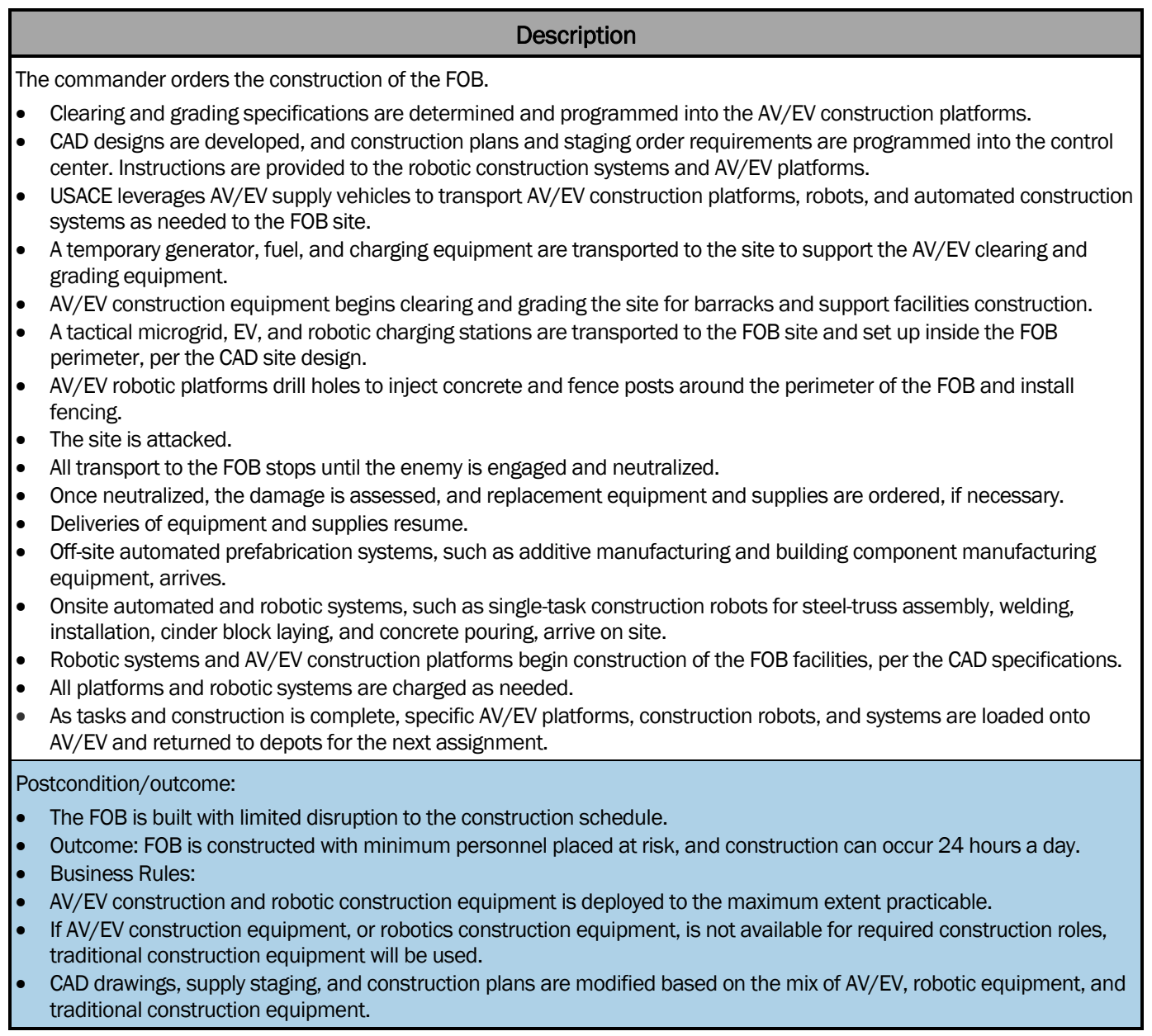

\subsection{Conclusion}

Research conducted in Research Area 1 resulted in the identification of vehicle-dependent Military Support Activities, the transport system requirements for each activity, and military transport systems that are candidates for automation. Use cases further defined the value of autonomy for specific Military Support Activity scenarios. The research conducted in this first phase of the project set the parameters for the development of a prioritized list of transport systems for automation. Research Area 2 provides an analysis of current and emerging electric vehicle technologies that can support these transport systems and Paragon's research goals of integrating the vehicles into a tactical microgrid. 


\section{Research and Identify Existing Technologies That can be Adapted for Military Applications}

The objective of this research is to identify existing transport systems that can be automated to benefit the military mission. For the purpose of this research, only existing electric transport systems were considered. These systems were cataloged in accordance with support activity application, resulting in a preliminary list of categorized candidate electric transport systems that have automation potential. Additionally, the Paragon team identified autonomous vehicle OEM and kit manufacturers that might have compatibility with identified EVs.

\subsection{Transport systems that can be automated to the benefit of the military}

Initial, broad internet searches were conducted to identify existing manufacturers and companies that develop electric transport systems. Identified electric transport system websites were recorded and analyzed for basic, available information. Reviewed websites for electric transport system manufacturers are provided in Appendix B. Appendix C summarizes addition EV and AV technology, industry, research, and DoD sources and websites reviewed for information on existing and emerging technologies. These sources (Appendices B and C) served as the foundation for this research and allowed for a more narrowed research approach going forward. Electric transport systems were then researched through review of literature-publications, journals, forums, associations, resources, journals, forums, associations, resources, and websites were-for objectivity of research and the development of a comprehensive collection of data. The validity of manufacturers or electric transport company claims were crossreferenced through review of secondary and tertiary sources. A practical limitation impacting the collection of data was the availability of information on public websites. In-depth data were gathered from priority manufacturers during the engagement process. 
The literature search did not identify an available EV for the following Military Support Activities:

- Heavy engineering equipment: scraper, crane, dozer, plow, hauler, dump truck, grader, loader, backhoe, crawler, HYEX

- Large grounds maintenance tractors-no fully EVs were identified; however, smaller EV tractors are coming onto the market as well as hybrid EV (HEV) farm tractors

- Specialty trucks, such as bucket trucks, pump trucks, etc. HEVs are available but not fully EV versions.

In general, for these cases, the activity was not considered further in the information gathering and evaluation process. In addition, many automobile manufacturers have established AV research, design, and development (RD\&D) efforts. Further, the Army Combat Capability Development Center-Ground Vehicle Systems Center (CCDCGVSC) is the lead for all research and demonstration activities conducted with automobile manufacturers. As such, automobiles were excluded from additional research, information gathering, and consideration for this effort. Future autonomous automobile demonstrations might be warranted in conjunction with the lead Army organization.

\subsubsection{Candidate electric transport systems}

Based upon the literature evaluation, Table 16 lists candidate electric transport systems that can be automated to benefit the military mission. These systems are categorized by Military Support Activity transport systems. A preliminary list of these systems identified from the initial literature search is provided in Appendix D.

Table 16. Candidate EVs.

\begin{tabular}{|l|l|}
\hline Model & Manufacturer \\
\hline \multicolumn{2}{|l|}{ Shuttles and Transit Buses (MSA 1) } \\
\hline EV22, EV27, EV3 shuttle buses & AVM \\
\hline e.Cobus shuttle & COBUS Industries \\
\hline 3000 shuttle & COBUS Industries \\
\hline K7, K95, K9, K11 shuttles & BYD \\
\hline C6, C9 \& C10 buses & BYD \\
\hline eBus & eBus \\
\hline Transit EV & Ford \\
\hline
\end{tabular}




\begin{tabular}{|c|c|}
\hline Model & Manufacturer \\
\hline Transit PHEV & Ford \\
\hline EV250, EV300, EV350, EV400 \& EV550 shuttle buses & GreenPower Bus \\
\hline Xcelsior & New Flyer \\
\hline Catalyst & Proterra \\
\hline All American RE, Microbird G5 & Blue Bird \\
\hline LEV Repower & Lightning Systems \\
\hline Ford E-450 shuttle & Lightning Systems \\
\hline Ford Transit Passenger & Lightning Systems \\
\hline Synapse bus & GreenPower \\
\hline EVStar shuttle & GreenPower \\
\hline Axess & ENC \\
\hline EZ-Rider II & ENC \\
\hline LFS HEV & Nova Bus \\
\hline \multicolumn{2}{|l|}{ Tractors and Mowers (MSA 2) } \\
\hline eDrive tractors & Autonomous Tractor Corporation (ATC) \\
\hline Class 6 \& 8 Refuse & BYD \\
\hline SESAM tractor & John Deere \\
\hline eUtility tractor & Solectrac \\
\hline eFarmer tractor & Solectrac \\
\hline Nemesis mower & Mean Green Mowers \\
\hline CXR-52/60 mower & Mean Green Mowers \\
\hline Stalker mower & Mean Green Mowers \\
\hline FL Electric \& FE Electric refuse & Volvo \\
\hline LR Electric refuse & Mack \\
\hline \multicolumn{2}{|l|}{ Trucks and Vans (MSA 3 and MSA 4) } \\
\hline Newton & Utilimaster/Smith Electric \\
\hline Newton & Utilimaster/Smith Electric \\
\hline e-100 & Workhorse \\
\hline NGEN 1000 van & Workhorse \\
\hline E100 van & Workhorse \\
\hline W-15 truck & Workhorse \\
\hline Class 6 step van & BYD \\
\hline Super Duty F Series & XL Fleet/Ford \\
\hline eVito & Mercedes-Benz \\
\hline \multicolumn{2}{|l|}{ Vocational, medium- \& heavy-duty Trucks (MSA 1 and MSA 3) } \\
\hline Class 8 Day cab & BYD \\
\hline Terminal tractor & BYD \\
\hline Class $5 \& 6$ truck & BYD \\
\hline T-Series Terminal trucks & Orange \\
\hline eM2 box truck & Freightliner \\
\hline eCascadia semi & Freightliner \\
\hline Class 8 semi & Tesla \\
\hline
\end{tabular}




\begin{tabular}{|c|c|}
\hline Model & Manufacturer \\
\hline ZeroTruck & Zero Truck \\
\hline v8011 refrigerated van & Chanje/Thermo King \\
\hline Ecantor van & Fuso \\
\hline 220EV, 520EV, 579EV & Peterbilt \\
\hline Class 6 box truck & Lightning Systems \\
\hline Ford E-450 Cutaway & Lightning Systems \\
\hline Ford Transit Chassis cab & Lightning Systems \\
\hline Ford F-59 & Lightning Systems \\
\hline NikolaTwo & Nikola \\
\hline \multicolumn{2}{|l|}{ Specialty Vehicles (MSA 3, MSA 5 and MSA 6) } \\
\hline Mobile Power System (MPS) Disaster Response Platform & Dannar \\
\hline Burden carrier hauler & Johnson Industries \\
\hline Airport carrier & Johnson Industries \\
\hline Ground support utility truck & Johnson Industries \\
\hline Large ground support truck & Johnson Industries \\
\hline Airport GSVs & Johnson Industries \\
\hline Customized hauler & Johnson Industries \\
\hline Platform carrier & Johnson Industries \\
\hline Indoor outdoor towing/lifting & Linde Material Handling \\
\hline Warehousing & Taylor-Dunn (Polaris company) \\
\hline ECB Series forklift & BYD Motors \\
\hline HyPower & Terex Utilities \\
\hline \multicolumn{2}{|l|}{ Heavy-duty Vehicles (MSA 1 and MSA 3) } \\
\hline EC15 \& EC27 compact excavators & Volvo Construction Equipment \\
\hline L20 \& L28 wheel loaders & Volvo Construction Equipment \\
\hline LX2 compact wheel loader & Volvo Construction Equipment \\
\hline EX2 compact excavator & Volvo Construction Equipment \\
\hline 988K XE wheel loader & Caterpillar \\
\hline D6 XE dozer & Caterpillar \\
\hline V8011 refrigerated van & Chanje/Thermo King \\
\hline Ecantor van & Fuso \\
\hline 220EV, 520EV, 579EV & Peterbilt \\
\hline Class 6 box truck & Lightning Systems \\
\hline Ford E-450 Cutaway & Lightning Systems \\
\hline Ford Transit Chassis cab & Lightning Systems \\
\hline Ford F-59 & Lightning Systems \\
\hline NikolaTwo & Nikola \\
\hline
\end{tabular}




\subsubsection{Research project compatibility}

Research identified several types of vehicles that could provide benefits to the military but were not further considered for one or more of the following considerations:

- Could the Military Support Activity rely on an EV of sufficient battery capacity that it can support $\mathrm{V} 2 \mathrm{G}$ and microgrid R\&D objectives? If not, are there additional considerations that could result in that activity (and its class of vehicles) being further considered as part of this initial research? Of the Military Support Activities identified in Table 1, smallscale grounds maintenance (security, grass cutting, and beautification), airfield activities, and ground service vehicles (GSV), security monitoring conducted by electric drones, warehousing activities and unmanned logistics (excluding point-to-point delivery), and vehicle towing do not offer sufficient battery storage capacity (50 kilowatt-hours [kWh]) to support tactical microgrid demonstrations envisioned for the ATI. As such, these activities were excluded from further consideration.

- To provide a charge/discharge capacity of 50 kilowatts (kW), a usable energy storage rating of $50 \mathrm{kWh}$ is needed for most battery technologies (also referred to as a 1c rating in the battery industry). The team is targeting a 50-kW charge/discharge capacity because a 50-kW charger can be tested as a reasonable replacement for the standard $60 \mathrm{~kW}$ Advanced-medium Mobile Power Source (AMMPS) generators that are to be included in the microgrid. Fifty $\mathrm{kW}$ is also the rating of the bidirectional chargers that are located at Los Angeles Air Force Base (LAAFB) that CERL would like to use in the future and enables using the charger at full capacity. A vehicle with less than a $50-\mathrm{kWh}$ rating will require multiple vehicles and chargers to be tested as a 60-kW AMMPS replacement, further increasing overall cost.

- Can testing be suitably simulated at CBITEC/FLW? The team members are familiar with the facilities and configuration of FLW and have determined that the environment would allow operation of the test vehicles and tactical microgrid and $\mathrm{V} 2 \mathrm{G}$ infrastructure in a realistic, simulated environment. Specifically, the varied terrain and diverse topography of the FLW installation allows for testing in various environments that will simulate forward-operating environments. 
Any Military Support Activities targeted for demonstration should be able to perform in this environment to provide realistic simulation scenarios that can translate to use in forward areas. No remaining Military Support Activities were precluded based upon this consideration.

- Is there ongoing R\&D in the private sector to obviate need for the program to focus on this operation? Could the Army, in the near future, purchase an $\mathrm{AV}$ system that is functional and commercially available and performs target mission support activity? Paragon's research revealed that while billions of dollars are being invested in RD\&D of electric and AVs, virtually all of this activity is aimed at simulating and testing in environments that mimic public roads and urban traffic environments. As such, the team eliminated from further consideration, personnel transport supported by passenger vehicles (commercially available electric sport utility vehicles [SUVs]/pick-up trucks were included for further research for use in off-road situations, which is not being as thoroughly researched by the automotive sector).

Several technology gaps, potential developmental areas, and project concepts were identified and/or formulated throughout this research effort. While they were not immediately germane to this specific effort, EVs that support mission activities in the context of microgrid operation, they do represent potential, future lines of effort that could be of interest to the military. Appendix E identifies and describes future lines of inquiry based upon gaps and emerging technologies/concepts related to HEVs, EVs, and AVs even though they were not directly germane to this effort.

SMEs collaboratively discussed project requirements necessary for autonomous electric transport systems to be the subject of research, testing, and demonstration for this project. This discussion resulted in a detailed set of project compatibility requirements and metrics for autonomous transport systems. Project compatibility metrics, which are provided in Appendix $\mathbf{F}$, were developed for uniformity in analysis and comparison of candidate systems considered to be integrated into our RD\&D plan.

\subsubsection{Candidate system screening criteria}

The identified Military Support Activity requirements and SMEs recommendations informed the development of candidate system screening criteria. SMEs developed screening criteria with two objectives. The first 
objective was to screen each candidate system to ensure systems maintained or exceeded identified military transport requirements. The second objective was to screen each candidate system to ensure systems were compatible with our research.

SMEs identified seven topics of interest utilized in the development of screening criteria and criteria thresholds. Screening criteria topics were technology readiness levels (TRL), target cost, location of assembly, battery capacity, complementary research, Military Support Activity requirements, and General Services Administration (GSA) availability. Screening criteria thresholds set baseline requirements to prioritize candidate systems.

SMEs collaboratively screened each candidate electric transport system utilizing the identified screening criteria. Candidate systems were prioritized by the number of thresholds met. Nomenclature used to document systems obtaining threshold requirement was labeled "Y" for "yes." Nomenclature used to document systems not obtaining a threshold requirement was labeled "N" for "no." Candidate systems were sorted by the number of Ys obtained.

The seven screening criteria and criteria threshold variables are described as follows:

\section{Technology Readiness Levels}

TRLs are utilized for estimating the maturity of technologies during the acquisition phase of a program. Using TRLs enables consistent, uniform discussions of technical maturity across different types of technology. A technology's TRL is determined during a Technology Readiness Assessment (TRA) that examines program concepts, technology requirements, and demonstrated technology capabilities. TRLs are based on a scale from 1 to 9 , with 9 being the most mature technology. The DoD has used the scale for procurement since the early 2000s. For this assessment, the team assigned a TRL value based upon the following:

\section{1-Basic principle}

2-Concept formulated 


\section{3-Proof-of-concept}

4-Valid in laboratory environment

5-Valid in relevant environment

6-Demonstration in relevant environment

7-Demonstration in operational environment

8-System completed and tested

9-System proven successful.

The threshold for these criteria was a TRL 7-9. This threshold requirement was necessary to obtain to facilitate the testing and demonstration of the candidate system. Systems meeting this criterion were assigned a value of "Y."

\section{Target Cost}

Paragon SMEs agreed to set a ceiling of $\$ 450,000$ to acquire the candidate system. The higher the cost, the less likely a system will fit within early demonstration budgets. This ceiling does not include the cost associated with retrofitting the system for autonomous operation, bidirectional charging (if modification is required) or establishing additional charging capability within the test location. A system with a published manufacturer's suggested retail price of $\$ 450,000$, or less, was identified as meeting the cost screening threshold and assigned a value of "Y."

\section{Location of Assembly}

Manufacturer's assembly systems located in the United States were used as indicators of countries of origin. At this stage in our research, available system data did inform whether Buy American Act requirements were met. A system that is manufactured or assembled in the United States were assigned a value of "Y."

\section{Battery Capacity}

Paragon's team of SMEs recommend 50-kW charge and discharge capacity for microgrid utility because: 
* A 50-kW charger can be tested as a reasonable replacement for the standard 60-kW AMMPS generators that are to be included in the microgrid.

* $50 \mathrm{~kW}$ is also the rating of the bidirectional chargers that are located at the LAAFB that CERL would like to use in the future and enables the use of the charger at full capacity.

* A vehicle with less than a 50-kWh rating will require multiple vehicles and chargers to be tested as a 60-kW AMMPS replacement, further increasing overall cost.

A system with a battery rating greater than, or equal to, $50 \mathrm{kWh}$ was assigned a value of "Y."

\section{Complements Ongoing Government Research}

SMEs' knowledge of current or planned research was identified as a useful screening criterion because of the potential to form partnerships to leverage funding. Systems that were identified as part of an ongoing research effort were assigned a value of "Y."

\section{Military Support Activity Requirements}

System data confirming the aptitude to meet at least one Military Support Activity application requirement, as outlined in Section 2.2, were identified as meeting the screening criteria and assigned a value of "Y."

\section{Available on GSA Schedule}

Manufacturer documentation is available to verify the system meets requirements for purchase by the federal government. If documentation is accessible, and stating the system is currently available on the GSA schedule, the system was assigned a value of "Y."

\subsubsection{Prioritized categorized list of candidate systems}

The $\mathrm{Y} / \mathrm{N}$ screening, employing the criteria provided above, was applied to EV technologies to prioritize the previously identified systems (Table 17) for further consideration. Screening was applied to stratify systems that resulted in a prioritized, categorized listing of candidate systems enabling the identification of optimal candidate systems to be integrated into this program. To apply the system, each of the EVs were evaluated with respect to the seven $\mathrm{Y} / \mathrm{N}$ criteria. If the system met the criteria, it was scored with 


\section{a "Y" (otherwise "N"). Each system was then assigned a score based on the number of criteria met. The initial $\mathrm{Y} / \mathrm{N}$ scoring for identified systems is provided in Appendix G.}

Table 17. Prioritized electric transport systems.

\begin{tabular}{|c|c|c|c|c|c|c|c|}
\hline $\begin{array}{c}\text { Total No. } \\
\text { of Y: }\end{array}$ & $\begin{array}{l}\text { TRL } \\
(7-9)\end{array}$ & $\begin{array}{c}\text { Cost } \leq \\
\$ 450,000\end{array}$ & $\begin{array}{l}\text { Assembled } \\
\text { In U.S. }\end{array}$ & $\begin{array}{c}\text { Battery Capacity } \\
\geq 50 \mathrm{kWh}\end{array}$ & MSA & $\begin{array}{l}\text { Related } \\
\text { Research }\end{array}$ & $\begin{array}{c}\text { Available } \\
\text { through GSA }\end{array}$ \\
\hline \multirow{3}{*}{7} & \multicolumn{7}{|c|}{ Mobile Power System (MPS) by: Dannar } \\
\hline & $\mathrm{Y}$ & Y & $\mathrm{Y}$ & $\mathrm{YY}$ & Y & $Y$ & $\mathrm{Y}$ \\
\hline & 9 & $\$ 200-400 K$ & IN & $125-630 \mathrm{kWh}$ & $\begin{array}{l}2,4,5 \\
6\end{array}$ & DHS & \\
\hline
\end{tabular}

MPS is designed to support rescue operations in emergency response and disaster mitigation operations. The system can generate power and clear debris (with attachments). This system can perform in severe conditions, in up to 4 feet of water. The drive-by-wire system should support autonomous use. This system presents a unique opportunity to support several military operations including the Maneuver Support Center of Excellence (MSCoE) Mission (Prime Power School

Engineering School) as well as routine land management functions. This robust system is designed to function in standing water and can use various attachments for recovery, debris removal, lifting, moving/towing vehicles, and structures. Power generation could also support resilience requirements in that the system can provide power to remote grids.

\begin{tabular}{|c|c|c|c|c|c|c|c|}
\hline \multirow{3}{*}{6} & \multicolumn{6}{|c|}{ Cargo Van, Chassis Cab, Cutaway Cab, Shuttle Van, Step Van by: Zenith Motors } \\
\cline { 2 - 8 } & $\mathrm{Y}$ & $\mathrm{Y}$ & $\mathrm{Y}$ & $\mathrm{Y}$ & $\mathrm{Y}$ & $\mathrm{N}$ & $\mathrm{Y}$ \\
\cline { 2 - 7 } & 9 & $\$ 100 \mathrm{~K}$ & $\mathrm{CA}$ & $\begin{array}{c}52-100 \\
\mathrm{kWh}\end{array}$ & 1,3 & & \\
\hline
\end{tabular}

Zenith Motors provides a variety of customizable EV trucks and shuttles developed on truck platforms (Dodge). The system can be ordered through DHS to meet specific function. Cost will vary depending on features. The company supports government purchase, but lag time can be long; the company cues production once order is received.

\begin{tabular}{|c|c|c|c|c|c|c|c|}
\hline \multirow{2}{*}{6} & \multicolumn{7}{|c|}{ Medium-duty Trucks, Shuttles, and Buses by: Zero Truck } \\
\cline { 2 - 8 } & $\mathrm{Y}$ & $\mathrm{Y}$ & $\mathrm{Y}$ & $\mathrm{Y}$ & $\mathrm{Y}$ & $\mathrm{N}$ & $\mathrm{N}$ \\
\cline { 2 - 8 } & 9 & $\$ 60-100 \mathrm{~K}$ & $\mathrm{CA}$ & $53-80 \mathrm{kWh}$ & 1,3 & & \\
\hline
\end{tabular}

The company offers customized vehicle platforms based on three different chassis and two power plants. It is flexible in terms of designing system to meet needs. Cost will depend on nature of specialization requested.

\begin{tabular}{|c|c|c|c|c|c|c|c|}
\hline \multirow{2}{*}{6} & \multicolumn{6}{|c|}{ Zeus Electric Shuttle bus, Utility vehicle, Flatbed truck by: Phoenix Motorcars } \\
\cline { 2 - 8 } & $\mathrm{Y}$ & $\mathrm{Y}$ & $\mathrm{Y}$ & $\mathrm{Y}$ & $\mathrm{Y}$ & $\mathrm{Y}$ & $\mathrm{N}$ \\
\cline { 2 - 8 } & 9 & $\$ 240,000+$ & $\mathrm{CA}$ & $105 \mathrm{kWh}$ & 1,3 & EPA, CA & \\
\hline
\end{tabular}

The company offers a shuttle, utility vehicle, and flatbed trucks. Utility truck and flatbed are not commonly to eTruck manufacturers and could provide a demonstration for installation maintenance functions including facility and grounds maintenance. The company is working to develop autonomous as well as microgrid (V2G) capabilities.

\begin{tabular}{|l|c|c|c|c|c|c|c|}
\hline \multirow{3}{*}{5} & \multicolumn{7}{|c|}{ T-Series Yard Hostler by: Orange Motors } \\
\cline { 2 - 7 } & $\mathrm{Y}$ & $\mathrm{Y}$ & $\mathrm{Y}$ & $\mathrm{Y}$ & $\mathrm{Y}$ & $\mathrm{N}$ & $\mathrm{N}$ \\
\cline { 2 - 7 } & 9 & \$200-300K & MO & $\begin{array}{c}80-160 \\
\mathrm{kWh}\end{array}$ & 1,3 & & \\
\hline
\end{tabular}

The T-series is manufactured by Orange Motors in Missouri. The company seems well established and provides an electric hauler that could serve low-speed purposes at an installation. Passenger transport would only be accomplished using troop trailers-Silverside design. It is common to training installations. Heavy hauling capacity increases potential for direct logistical support in terms of deployment and training material marshalling activities. Orange produces two versions of a heavy hauler. While the system can be used on roads, it has a top speed of $25 \mathrm{mph}$, and it can haul 81,000 lbs. Vehicles are Missouri-built, and the company can also retrofit older diesel frames to electric, reducing cost by approximately $\$ 50,000$.

\begin{tabular}{|l|l|}
\hline 5 & Reckless by: Nikola \\
\hline
\end{tabular} 


\begin{tabular}{|c|c|c|c|c|c|c|c|}
\hline $\begin{array}{c}\text { Total No. } \\
\text { of Y: }\end{array}$ & $\begin{array}{c}\text { TRL } \\
\mathbf{( 7 - 9 )}\end{array}$ & $\begin{array}{c}\text { Cost } \leq \\
\$ 450,000\end{array}$ & $\begin{array}{c}\text { Assembled } \\
\text { In U.S. }\end{array}$ & $\begin{array}{c}\text { Battery Capacity } \\
\mathbf{2 5 0} \mathrm{kWh}\end{array}$ & MSA & $\begin{array}{c}\text { Related } \\
\text { Research }\end{array}$ & $\begin{array}{c}\text { Available } \\
\text { through GSA }\end{array}$ \\
\hline & $\mathrm{Y}$ & $\mathrm{N}$ & $\mathrm{Y}$ & $\mathrm{Y}$ & $\mathrm{Y}$ & $\mathrm{Y}$ & $\mathrm{N}$ \\
\cline { 2 - 7 } & 9 & $\begin{array}{c}\$ 480 \mathrm{~K} / \mathrm{year} \\
\text { lease }\end{array}$ & $\mathrm{CA}$ & $52-100 \mathrm{kWh}$ & 4 & $\begin{array}{c}\text { U.S. Marine Corps } \\
\text { (USMC) }\end{array}$ & \\
\hline
\end{tabular}

The system is currently part of U.S. Marine Corps (USMC) demonstration and could provide potential for integration with tactical microgrid development portion of ATI. The system is only an EV that currently under evaluation for tactical use.

\begin{tabular}{|c|c|c|c|c|c|c|c|}
\hline \multirow{3}{*}{5} & \multicolumn{7}{|c|}{ eBus by: eBus } \\
\hline & Y & Y & Y & Y & $\mathrm{Y}$ & $\mathrm{N}$ & $\mathrm{N}$ \\
\hline & 9 & $\$ 400 K$ & CA & $130 \mathrm{kWh}$ & 1 & & \\
\hline
\end{tabular}

This commercially available system is used in mass transit. eBus is an electrification conversion service for existing buses. Cost is for electric motor, battery and charging systems. It provides a repurposing alternative and can result in vehicles configured to specific use scenarios. The company offers inductive charging system for use on routes, and it offers driveunder and drive-over charging systems. This service requires the government to provide a bus that would be converted.

\begin{tabular}{|c|c|c|c|c|c|c|c|}
\hline \multirow{3}{*}{5} & \multicolumn{7}{|c|}{ Xcelsior Charge (multiple versions) by: New Flyer } \\
\cline { 2 - 8 } & $\mathrm{Y}$ & $\mathrm{N}$ & $\mathrm{Y}$ & $\mathrm{Y}$ & $\mathrm{Y}$ & $\mathrm{Y}$ & $\mathrm{N}$ \\
\cline { 2 - 8 } & 9 & $\$ 700-1,200 \mathrm{~K}$ & $\mathrm{AL}$ & $160-466 \mathrm{kWh}$ & 1,3 & Private sector & \\
\hline
\end{tabular}

The company offers a variety of buses with varying capacity. Autonomous operation system development is under way. Partnership to demonstrate bus at an installation with integration into a microgrid could provide demonstration partnerships with the manufacturer.

\begin{tabular}{|c|c|c|c|c|c|c|c|}
\hline \multirow{3}{*}{5} & \multicolumn{7}{|c|}{ R1T-EV Pick-u by: Rivian } \\
\cline { 2 - 8 } & $\mathrm{Y}$ & $\mathrm{Y}$ & $\mathrm{Y}$ & $\mathrm{Y}$ & $\mathrm{Y}$ & $\mathrm{N}$ & $\mathrm{N}$ \\
\cline { 2 - 8 } & 8 & $\$ 70-100 \mathrm{~K}$ & $\mathrm{Y}$ & $\mathrm{IL}$ & 3,4 & & \\
\hline
\end{tabular}

The R1T all-electric pick-up truck seats five passengers and is designed for urban and off-road use. It could provide a utility truck demonstration for small transport as well as material delivery.

\begin{tabular}{|c|c|c|c|c|c|c|c|}
\hline \multirow{5}{*}{5} & \multicolumn{7}{|c|}{ Saf-T-Liner C2 Jouley Electric School Bus by: Thomas Built } \\
\cline { 2 - 8 } & $\mathrm{Y}$ & $\mathrm{Y}$ & $\mathrm{Y}$ & $\mathrm{Y}$ & $\mathrm{Y}$ & $\mathrm{N}$ & $\mathrm{N}$ \\
\cline { 2 - 9 } & 8 & $\$ 450 \mathrm{~K}$ & $\mathrm{NC}$ & $220 \mathrm{kWh}$ & 1 & & \\
\hline
\end{tabular}

This long-time bus manufacturer has gasoline vehicles traditionally used by government for student transport. Traditional units are available through GSA. Electric versions are likely to be offered, eventually, through GSA. A partnership with the company could support its effort to demonstrate vehicle as useful for government use.

\begin{tabular}{|c|c|c|c|c|c|c|c|}
\hline \multirow{3}{*}{5} & \multicolumn{7}{|c|}{ Edrive Tractor by: ATC } \\
\cline { 2 - 8 } & $\mathrm{Y}$ & $\mathrm{Y}$ & $\mathrm{Y}$ & $\mathrm{Y}$ & $\mathrm{Y}$ & $\mathrm{N}$ & $\mathrm{N}$ \\
\cline { 2 - 8 } & 9 & $\$ 250 \mathrm{~K}$ & $\mathrm{MN}$ & $250 \mathrm{~kW}$ & $2,5,6$ & & \\
\hline
\end{tabular}

ATC offers a land-maintenance system designed for agricultural use. The system can be modified to provide foreign object detection and invasive species identification and removal. Large cutting deck provides wide-area processing. The system can be purchased with digging and earth-moving attachments. It does not have batteries (no large equipment does because of weight consideration); rather it has electric motors powered by on-platform power generation. As such, it provides power generation capacity that can augment microgrids. The company can retrofit existing vehicles or new frames. The system provides cost-beneficial options for land management operations.

\begin{tabular}{|c|c|c|c|c|c|c|c|}
\hline \multirow{4}{*}{4} & \multicolumn{7}{|c|}{ eCargo, eTruck drayage-Class 8 Tractor by: US Hybrid } \\
\cline { 2 - 8 } & $\mathrm{N}$ & $\mathrm{Y}$ & $\mathrm{Y}$ & $\mathrm{Y}$ & $\mathrm{Y}$ & $\mathrm{N}$ & $\mathrm{N}$ \\
\cline { 2 - 8 } & 6 & $\$ 250 \mathrm{~K}$ & $\mathrm{CA}$ & $240 \mathrm{kWh}$ & 1,3 & & \\
\hline
\end{tabular}

Vehicles are not currently available. The system might offer better performance, as it is made available over the next 2 years.

4

\begin{tabular}{|c|c|c|c|c|c|c|}
\hline \multicolumn{7}{|c|}{ LFSe by: Nova Bus } \\
\hline$Y$ & $N$ & $Y$ & $Y$ & $Y$ & $N$ & $N$ \\
\hline 8 & $\$ 700 K+$ & $N Y$ & $152 \mathrm{kWh}$ & 1 & & \\
\hline
\end{tabular}




\begin{tabular}{|c|c|c|c|c|c|c|c|}
\hline $\begin{array}{c}\text { Total No. } \\
\text { of Y: }\end{array}$ & $\begin{array}{c}\text { TRL } \\
(7-9)\end{array}$ & $\begin{array}{c}\text { Cost } \leq \\
\$ 450,000\end{array}$ & $\begin{array}{c}\text { Assembled } \\
\text { In U.S. }\end{array}$ & $\begin{array}{c}\text { Battery Capacity } \\
\geq 50 \mathrm{kWh}\end{array}$ & MSA & $\begin{array}{c}\text { Related } \\
\text { Research }\end{array}$ & $\begin{array}{c}\text { Available } \\
\text { through GSA }\end{array}$ \\
\hline
\end{tabular}

The vehicle does not meet cost requirements, but partnership (as sought with New Flyer) might yield cost effective opportunities for a future demonstration.

In addition, Paragon's team developed information packets for each candidate system (provided in Appendix H). After reviewing the information packets, the SMEs gathered at the Robotics Research Laboratory in Clarksburg, MD. The team discussed at length each candidate system, its ranking, and the compiled information (Appendix H). The team further evaluated considered the research project compatibility metrics and screening criteria thresholds. Optimal candidate systems for continued research and engagement were collaboratively agreed upon. Candidate systems were prioritized by the total number of Ys according to the screening methodology outlined in Section 3.4. In some cases, systems were eliminated from further consideration because of significant violation of one or more of the screening criteria (i.e., assembled in China). In conducting this evaluation, the team considered all of the vehicles using the same (or very similar) platform as one system for the purposes of this evaluation.

This approach resulted in a list of 13 prioritized electric transport systems identified in Table 17.

\subsubsection{Autonomous vehicle OEM and kit manufacturers}

The Paragon team engaged AV SMEs who advised and provided input about the latest AV technologies that might have compatibility with the identified EVs. The list of AV OEM and Kit manufacturers is provided in Appendix I.

\subsection{Engagement with EV transport manufacturers}

The purpose of engagement with EV transport manufactures was to confirm identified system data from prior research and finalize research plans to collect new information for the development of SME transport system recommendation summaries. Additionally, engagement meetings allowed SMEs to assess a manufacturer's willingness to partner for future research, testing, and demonstration project. 
Paragon SMEs identified the following manufacturers for engagement: Dannar, Zenith Motors, Zero Truck, Phoenix Motorcars, Orange Motors, Nikola, Ebus, New Flyer, Rivian, Thomas Built, ATC, US Hybrid (USH) and Nova Bus.

Call leads were assigned to each manufacturer. SMEs were assigned call lead roles on the basis of previously established relationships with manufacturers. "Cold calls" were documented to state that no prior relationship with the contacted manufacturer is established.

\subsubsection{Process of engagement}

Paragon and its SMEs developed a Process of Engagement to be used when contacting manufacturers. This process ensures a consistent communication approach for scheduling and execution of manufacturer informational meetings and research plans. The Process of Engagement is outlined in the Figure 4. flow diagram. 
Figure 4. Process of engagement flow diagram.

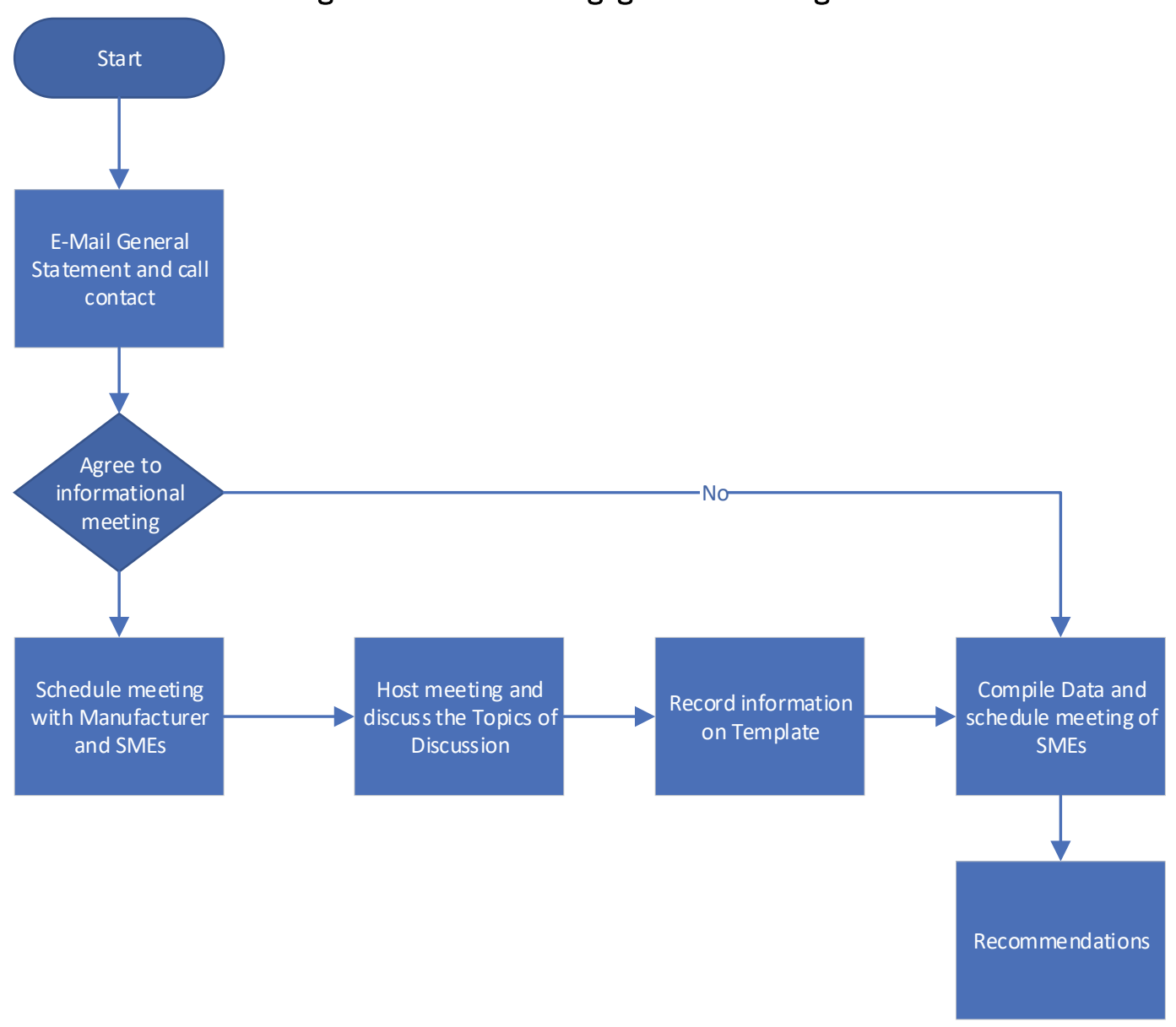

\subsubsection{General statement}

As outlined in the Process of Engagement, call leads initiated engagement with manufacturers by emailing the point of contact the following general statement:

The Paragon team is researching the integration of Autonomous Electric Transport Vehicles as part of a technical program that will enhance energy resilience for military installations and contingency bases. This research will identify and recommend specific technologies related to electric transport vehicles, vehicle autonomy, and vehicle-to-grid (V2G) charging for future integration into a tactical microgrid.

Could "insert company name" provide information about "insert vehicle(s)" to assist us with this research? Our team will follow up with a 
phone call to schedule a time that is convenient for you. We appreciate any assistance you can provide.

Call leads then followed up via phone call and email to coordinate a meeting date and time. If manufactuers agreed to an informational meeting with Paragon SMEs, call leads provided manufacturs with topics for discussion and the meeting was scheduled. If manufacters declined an informational meeting with Paragon SMEs, call leads documented manufacters response for later consideration.

\subsubsection{Topics for discussion}

Topics for discussion were collaboratively developed by Paragon SMEs. The team condensed Military Support Activity transport requirements and research compatibility considerations to create a comprehensive and concise set of topics. These topics were designed to effectively validate and fill information gaps of prior research and effectively conduct comprehensive research plans. Topics for discussion are:

1. Manufacturer and vehicle description

2. V2G charging readiness and effort implementation

3. Vehicle functions and capabilities

4. Testing and demonstrations

5. Autonomy readiness and effort to implement

6. Safety assessments

7. Pricing and warranties.

\subsubsection{Manufacturer call documentation}

For consistent documentation of manufacturer informational meetings, call leads utilized a Manufacturer Call Notes Template. Manufacturer call notes and manufacturer supplied information are listed in Appendix J.

\subsubsection{Transport system recommendations}

Documented call notes and any manufacturer supplied documents were reviewed and discussed by Paragon SMEs to develop and agree upon transport system recommendations. 
Recommendations contemplate advantages and disadvantages of each identified transport system as well as the potential for manufacturer partnerships and transport system compatibility for this research.

\subsection{Summary of conclusions and recommendations}

\subsubsection{Manufacturer outreach}

Paragon SMEs wanted to gauge the willingness of candidate technology manufacturers to form partnerships to enable future ATI demonstrations. The SMEs contacted EV technology manufacturers that closely met the aforementioned research and demonstration project criteria. They requested interviews to secure additional information critical to making a comprehensive educated recommendation. Results from initial email and phone correspondence listed in Table 18.

Table 18. EV manufacturer interview correspondence.

\begin{tabular}{|l|l|}
\hline Manufacturer & Correspondence Results \\
\hline ATC & Not interested in participating \\
\hline *Dannar & Notes in Appendix J \\
\hline eBus & No response \\
\hline First Priority Green Fleet & Not interested in participating \\
\hline *New Flyer & Notes in Appendix J \\
\hline *Nikola & Notes in Appendix J \\
\hline Nova Bus & No response \\
\hline *Orange Motors & Notes in Appendix J \\
\hline *Phoenix Motorcars & Notes in Appendix J \\
\hline Rivian & Not interested \\
\hline Thomas Built & No response \\
\hline *US Hybrid & Notes in Appendix J \\
\hline Zenith Motors & No response \\
\hline Zero Truck & No functional system to show at this time \\
\hline *Manufacturers participated in interview and provided additional information \\
\hline
\end{tabular}

The following is a list of the manufacturers that responded with interest and participated in interviews, providing additional information on technologies as they relate to the ATI testing and demonstration project:

- Dannar-MPS

- New Flyer-Xcelsior Charge (multiple versions)

- Nikola - Reckless 
- Orange Motors-T-Series Yard Hustler

- Phoenix Motorcars-Zeus Electric Shuttle bus, Utility vehicle, Flatbed truck

- US Hybrid-eCargo, eTruck drayage.

\subsubsection{Manufacturer technology summaries and recommended research}

After interview research plans were conducted, SMEs reviewed and analyzed manufacturer interview notes and held a meeting to discuss the information gathered to make recommendations for future testing and demonstration for six EV technologies in the ATI project. The EV technologies recommended for future research are listed below. Further detail of carried out research plans are provided in Appendices $\mathbf{J}$ and $\mathbf{K}$.

\subsubsection{Dannar}

The Dannar MPS is an off-road energy platform with unique versatility in application that could support a wide range of military activities with a platform base that can be converted to a multitude of transportation systems. It meets all of the key operational and programmatic ATI testing and demonstration criteria to include autonomous and $\mathrm{V} 2 \mathrm{G}$ capabilities with minimal implementation effort/cost. The system has full- remote capability, operating by onboard cab, $\mathrm{x}$-box style remote control, or wireless supervision. Dannar is currently working to develop follow-the-leader autonomous function. The system has maturity and commercial availability, which pricing is in line with project parameters and the company is interested in participating in the ATI project.

Dannar manufactures systems for the U.S. governmental market and is located in Clemente, CA; San Jose, CA; and Muncie, IN (manufactured). The MPS is a direct current (DC) energy storage asset, to whose DC power bus, one can connect various power converters for export of energy. Dannar tends to focus first on MPS power export to islanded microgrids, such as those for remote worksites where grid power is not accessible. Supply to a utility grid involves additional hardware to ensure (i) both isolation of the ungrounded MPS from the grounded grid and (ii) synchronization of exported power with grid power. DANNAR's power electronics partner has supplied both unidirectional and bidirectional power converters for both DC-DC applications, such as EV charging, and alternating current (AC)DC applications, such as either EV charging from grid or V2G transfer. 


\subsubsection{New Flyer}

New Flyer manufacturers the Xcelsior Charge electric bus and has experience in the electric transport system industry making manufactured zeroemissions buses for more than 50 years. The Xcelsior Charge is a zeroemissions bus with a battery ESS ranging from $160 \mathrm{kWh}$ to $460 \mathrm{kWh}$. While the Xcelsior Charge contains a large ESS, the New Flyer system does not have bidirectional charging capability.

New Flyer buses are interoperable with all heavy-duty vehicle charging equipment. New Flyer has partnered with Robotic Research to develop a proof-of-concept autonomous bus. Additionally, New Flyer has committed to additional autonomous bus programs with the Xcelsior Charge.

New Flyer has delivered several Xcelsior Charge buses to transit agencies across the United States and has a fleet of demonstration buses available for use. The Xcelsior Charge electric bus and the battery system is manufactured in the U.S. New Flyer warranties the ESS for 12 years and provides support and field service upon request.

The system is not a tactical vehicle but can be used on-post and even in foreign areas for transporting Soldiers and supplies. Because of its design for on-road use, testing would require paved roads and performance in conjunction with post activity. This could result in higher costs. Additionally, the entry cost of the electric bus is approximately $\$ 750,000$. This technology would be a good candidate for electric and autonomous transport research in the future when the technology is more mature in its bidirectional and autonomous capabilities.

\subsubsection{Nikola}

The Nikola Reckless is a fully electric side-by-side utility vehicle that offers flexibility with the design of its open chassis. It offers off-road performance and can reach speeds of $100 \mathrm{mph}$ in just over 3 seconds. The Reckless is estimated to have a range of 150 miles and more and a top speed of $75 \mathrm{mph}$, making it an appealing option for high-speed off-road use. The Reckless is available with powertrains ranging from $266-590$ horsepower (hp) and is powered by a 400- volts of DC (VDC) battery system with 75$125 \mathrm{kWh}$ of energy storage. 
The Nikola Reckless is interoperable with a Remote Weapon Station and military drones. A variant of the platform has steer- and throttle-by-wire capabilities, and Nikola is working to develop more autonomous capabilities. The vehicle's onboard energy storage is large for the size of the vehicle and would be beneficial for bidirectional charging. The platform does not currently support bidirectional charging; however, the company has expressed interest in working toward this capability.

Potential challenges in testing the Reckless are a lack of commercial availability, deployment risk, and cost of the system. Currently only one known system is available and additional systems are limited to production prototype, raising concern on consistency of quality and performance. Additionally, the cost of the system could be prohibitive, the system has been quoted at a leasing cost of $\$ 40,000 /$ month.

\subsubsection{Orange Motors}

Orange Motors-, based in Riverside, MO-, builds, sells, and services industrial EVs and is the leading OEM providing industrial fleets with heavy- duty electric trucks. The manufacturing location is approximately 200 miles from FLW, the recommended testing and demonstration site, FLW, which could allow for convenient operational and technical support.

The system is an electric hauler that could serve low-speed purposes at an installation. Passenger transport could be accomplished using troop trailers of a Silverside design, which is common at training installations. The trucks are purpose-built to site requirements and have heavy hauling capacity (81,00o lbs.) increases potential for direct logistical support in terms of deployment and training material marshalling activities. The system is DoD-certified for on- and off-road use, but maximum speed is currently $25 \mathrm{mph}$. The company can also retrofit older diesel frames to electric. Orange EV's trucks utilize a web-based telematics system that provides instant feedback, real-time performance statistics, and hard data, enabling fleets to understand system status, fuel efficiency, and cost savings.

The company seems well established with a mature technology that has good potential for autonomous capabilities and is priced within research project parameters. Concerns include a lack of bidirectional charging 
capabilities and potentially significant cost to develop those capabilities from scratch.

\subsubsection{Phoenix Motorcars}

Phoenix Motorcars is a Southern California based company that manufacturers electric shuttle buses, flatbeds trucks, and work trucks. While its shuttle buses are most popular, Phoenix also provides electric utility trucks, with an enclosed steel body and service and maintenance trucks. They provide box trucks, delivery, flatbed, stake bed, to anybody who uses the e450 chassis. Their next phase extends to F550 platform in 2020.

This technology has several issues that could preclude it from being a candidate for the testing and demonstration project. Most significantly, it does not have bidirectional charging capabilities and the cost and timeline for development would be prohibitive.

\subsubsection{US Hybrid}

The USH Corporation specializes in the design and manufacture of power conversion systems for medium- and heavy-duty electric, hybrid and fuel cell commercial buses and trucks. USH's products utilize power converter topologies to provide integrated system solutions for OEM applications.

USH has built a number of prototype vehicles that can accommodate bidirectional charging and the company is currently providing a vehicle to the U.S. Air Force (Air Force) that is a 230-VDC charger for sustainable power on demand. The vehicle can power most of the required AC/DC load, and the operation is monitored using a GUI-driven dashboard. Time to produce a prototype proof-of-concept vehicle can vary depending on the complexity of the design, but it typically takes 6 months to design, build, and field a single proof-of-concept vehicle.

USH has a number of other demonstration projects underway demonstrating both EV and fuel cell capabilities. They are currently testing a 480-kW wide body tug for moving $\mathrm{C}-17 \mathrm{~s}$ and a weapons loader. They have also electrified an 80,00o-lb. truck capable of moving shipping containers. USH typically builds anywhere from one to 15 vehicles for demonstration projects, often in partnership with other OEMs or third parties (e.g., Textron or Oshkosh). The prototype vehicles have a minimum TRL of 5 and are 
mostly TRL levels 6 or 7. USH is currently engaged with one such project with an R11 and R12 refuelers and is delivering jet fuel to fighter squadrons using these EVs.

USH offers all of their modified vehicles as drive-by-wire. USH has built the EV Mine Sweeper for Textron that is driven remotely through a virtual interface. USH is also supporting a number of other military related vehicles, some of which are focused on demonstrating autonomy readiness. USH does not have any autonomy capability on its own but is working on interfacing with another company's autonomous control system.

Currently all bidirectional charging has been done on prototype systems. The overall design of the technology could prove challenging with an initial modification of the base vehicle, then another modification to autotomize. For these reasons, this technology could be a good candidate for future research but currently has a high technical risk with a two- to three-year time frame for development activity.

\subsection{Future evaluations}

The EV market is constantly evolving with new and changing technologies. Even as this research was underway, transition was taking place and ongoing research reveals emerging products on a constant basis. One technology that was identified after manufacturer interviews had been conducted is Bollinger Motors. Preliminary research shows that the manufacturer offers fully EVs that support the tactical environment and are fast charging, with capabilities for transporting passengers and more (yet to be identified). Production is slated for 2 Q2020 with price starting manufacturer's suggested retail price (MSRP) is $\$ 125,000$. Appendix L provides additional information on this system.

\subsection{Conclusion}

Based on the research, there are several technologies that would be good candidates for testing and demonstration of electric transport systems with autonomous and bidirectional capabilities for Military Support Activities. The Dannar is an attractive technology for this project because it is commercially mature and has good versatility in platform design for a broad range of Military Support Activities. The technology currently has drive-by-wire operational capability and shows high probability for full 
autonomous capability in the next few years. The Nikola Reckless provides an opportunity to explore high-speed EVs that will work in a tactical environment. The Orange EV system has a large hauling capacity that makes it appealing for testing logistics support in forward warehousing as well as on-post. The other technologies identified are potential candidates for future testing as they require additional development and overall maturity. 


\section{Research and Develop Operational Tests for Autonomous Transport Vehicles}

The objective of Research Area 3 is to identify and research operational tests for autonomous transport systems. The purpose of this research is to develop autonomous-system operational test plans to be performed within the living laboratory at the CBITEC site.

Paragon SMEs identified required autonomous-system data and state-ofthe-art technologies through a review of literature and engaged with leading industry experts.

\subsection{State-of-the-art data capture and storage technologies}

The objective of this research was to identify and verify autonomous-system data and to investigate state-of-the-art technologies for capturing and storing data required to develop operational tests. The ARIBO project was referenced to identify data types required to analyze and characterize the performance of autonomous-system operation.

In order to investigate state-of-the-art technologies for capturing and storing autonomous-system data, Paragon's team of SMEs conducted a review of literature through broad internet searches. This review resulted in a list of identified autonomy kits, sensors, and other technologies available today, which is found in Appendix M.

\subsubsection{Data types}

Raw-sensor data includes LiDARs (light detection and ranging), cameras, radars (radio detection and ranging), and navigation units. The data is typically in the form of Ethernet packets, and the autonomous-system is generally on a private or isolated network. The data structure in the Ethernet packets is organized and defined by the sensor manufacturer. The data structure format is provided to the end user in an Interface Control Document (ICD) for the individual piece of hardware. In order to capture and record this data, Wireshark-the network protocol analyzer-is used. Wireshark allows the user to collect a variety of network traffic in packet capture (pcap) files. This file format is basic, and saves captured network data, and has become the "de facto" standard of network captures. The 
data is saved in a hexadecimal format and requires the user to post process, or parse, the log files to be human readable.

For the context of this report, post processing refers to the analysis and/or playback of data collected during autonomous operations after the operations have been completed.

Processed sensor data includes any of the aforementioned raw-sensor data above that has been transformed into a format usable by the autonomoussystem. An example of this is a LiDAR point cloud that has been transferred into the system's reference coordinate frame to make a $3 \mathrm{D}$-world model. This data is typically captured using Wireshark. Other data-recording tools, such as the Harris Jaqwire application, allow for LiDAR, radar, and video data to be recorded and disseminated to other users to view in real time or for post processing.

System data includes the commands, status, and control information necessary to make the system drive autonomously. Examples include obstacle detections, path-planning trajectories, velocity, steering commands, and system status information. This data can be in a variety of formats and is transferred over a Controller Area Network (CAN), serial network, or Ethernet. CAN data loggers, serial data loggers, and Wireshark are some of the common ways to collect system data. Additionally, the Robotic Operating System (ROS) command-line interface, rosbag, allows the user to create an Application Programming Interface (API) to write data into a bag file format.

Table 19 lists a set of expected generic data that would be expected to be collected during the operation of the autonomous-system. While there are many organizations designing autonomous-system software and hardware, the specific data recorded, and data formats used on platforms, are considered proprietary and unavailable without permission from the software or hardware provider. For the scope of this research area, the data types will be covered at a generic level.

Table 19. Example data collected during autonomous-system operation.

\begin{tabular}{|l|l|l|}
\hline \multicolumn{1}{|c|}{ Type } & \multicolumn{1}{|c|}{ Description } & \multicolumn{1}{c|}{ Format } \\
\hline LiDAR & 3D point clouds: raw or processed & pcap \\
\hline Radar & Radar range measurements: raw or processed & pcap \\
\hline
\end{tabular}




\begin{tabular}{|l|l|l|}
\hline \multicolumn{1}{|c|}{ Type } & \multicolumn{1}{c|}{ Description } & \multicolumn{1}{c|}{ Format } \\
\hline Camera & Camera image data: raw or processed & pcap \\
\hline Encoders & Quadrature wheel-encoder data & bag \\
\hline Estop & Recorded when estop is pressed OR an event is tagged & bag \\
\hline Localization & INS/GPS position and orientation data & pcap \\
\hline CAN & Traffic on CAN Bus & CAN \\
\hline Driving Commands & Speed and curvature commands to platform & CAN or pcap \\
\hline System Status & System diagnostic data & CAN or pcap \\
\hline
\end{tabular}

\subsubsection{Sensors}

There are typically three types of sensors used on autonomous systems to sense objects: LiDAR, camera, and radar. LiDAR and radar both emit signals and receive returns from objects. The returns are collected over time in software to create the size, shape, and distance of the objects.

Radar uses radio waves to compute the range of the object and its velocity. Radars excel at detecting metallic objects but fail to see objects without a radar signature like a pedestrian. The radar sensor usually has a narrowfocused output that detects objects at a greater distance, up to 400 meters. This ability to see metallic objects at a greater distance makes the sensor well suited for detecting vehicles. An autonomous-system will typically have a forward-facing radar mounted on the front bumper area of the platform along with radars mounted on each corner of the platform. This configuration will detect vehicle traffic in the autonomous-system's direction of travel and cross traffic.

LiDAR behaves similarly to radar but uses a laser instead of radio waves to survey the environment. LiDAR usually does not have as long of a range, typically 100 meters, as radar but has a greater field of view that does not require the object's composition to be metallic. The set of LiDAR returns is referred to as a point cloud, and contains distance and angular position components and an angular position component relative to the sensor for each point. This can be thought of as spherical coordinate representation for each LiDAR return. The point cloud is transformed into the system's reference coordinate frame to create a 3D map of the system's environment, Figure 5 shows an example of a point cloud transformed into the system's reference frame. An autonomous-system will typically have at least one LiDAR to scan the primary direction of the system's travel. Depending on the use case, speed of operations, and need for redundant 
sensing, more LiDARs may be added. For example, a system used on the road and in traffic might have LiDARs on each corner of the system whereas a system designed for lower-speed off-road use, might only have a forward-facing LiDAR.

Figure 5. Three-dimensional LiDAR scan example.

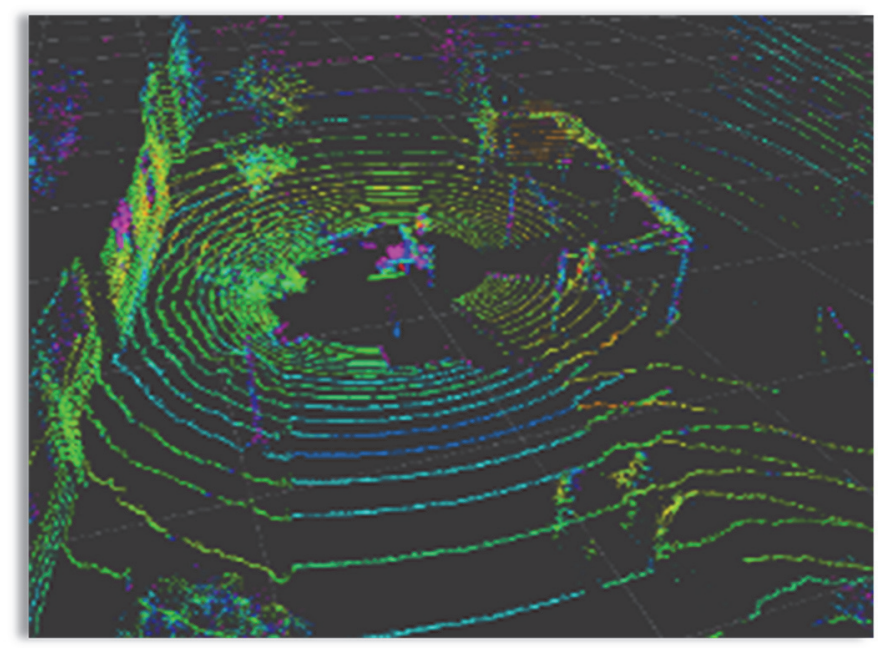

While radar and LiDAR work well for measuring an object's the range and velocity of an object, neither are not the best suited for capturing the details of an object. Because of this, cameras are a much better choice for classifying the type of object, whether it is a person, car, or the text on a road sign. Figure 6 shows of an example of a camera detecting and classifying an object as a vehicle. However, cameras have similar limitations to the human eye. For example, a camera will have a difficult time viewing the environment when looking into the sun. Sophisticated cameras can adjust to varying lighting conditions and still saturate during extreme conditions. Adding filters to the camera lens can improve camera performance but is not a viable solution for autonomous systems as lighting conditions can change rapidly and would require human interaction to change these filters. 
Figure 6. Vehicle object recognition.

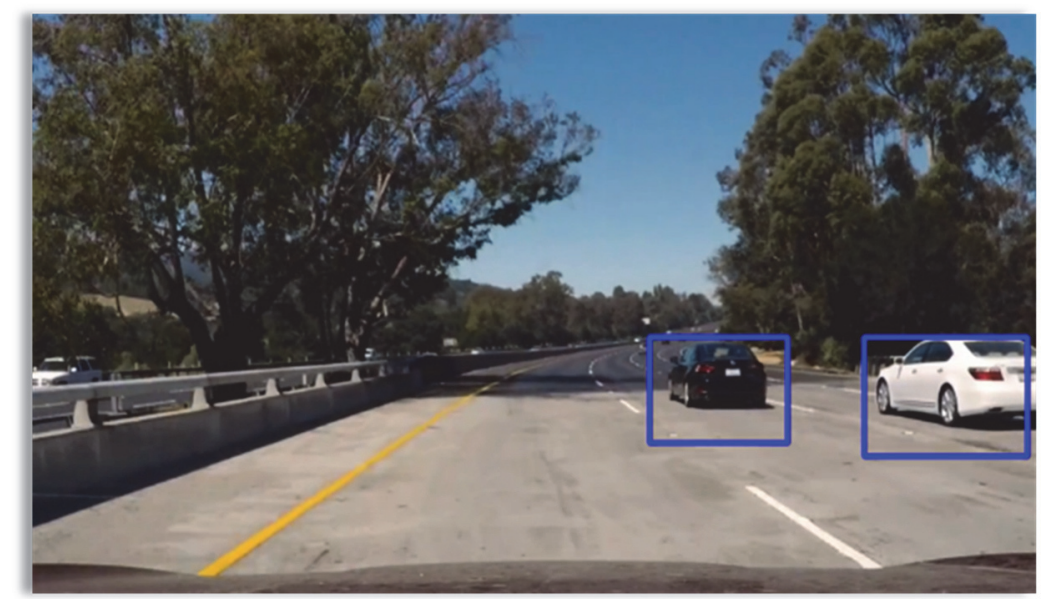

The use of stereo cameras or thermal imagers is considered beyond the scope of the report. These types of cameras are used in some robotic applications, but they generally do not perform well in the use cases described in this report.

In addition to object detection sensors, autonomous systems require a localization or navigation sensor. Most localization sensors combine a GPS sensor with an Inertial Measurement Sensor (IMU) to determine the coordinate position and the system's attitude (roll, pitch, and yaw). Localization sensors that include both GPS and IMUs are usually referred to as an Inertial Navigation System (INS).

\subsection{Applications to interface with autonomous-system data}

The tools listed in this section can capture most, if not all, the data necessary to analyze and characterize the autonomous-system's performance. The difficulty with capturing data across multiple tools is that a significant amount of post processing is needed to compare all the data against a reference timeframe. During regular operation, the autonomous-system consumes the aforementioned data, processes it, and makes decisions in real time. In order to analyze the data after it has been collected, a reference timeframe is needed to align captured data against a common timestamp. For example, it is important to compare a specific driving command to the system status message at any given position or orientation with respect to the LiDAR returns and camera images. Without a reference timeframe, it 
is not possible to correlate the multiple data streams into a single timestamp.

Most of this data is recorded locally and unavailable for viewing and post processing via web or remote applications. This data is typically proprietary or requires the manufacture's ICD to interpret. Additional steps are needed to centralize this data into a single repository.

The Robotic Research engineering team is continuously searching for new, innovative ways to capture, store, and analyze data generated by autonomous systems. Common applications to interface with autonomous-system data are Wireshark, the CL200o: CAN Bus Logger, Jagwire, and nSight Data Logger.

\subsubsection{Wireshark}

Wireshark is an open-source network packet analyzing tool. It can analyze communication protocols similar to tcpdump, but with a Graphical User Interface (GUI). Wireshark comprehends the structure of different networking protocols and can parse and display the data in the intended protocol format (Figure 7.). 
Figure 7. Wireshark capture setup.

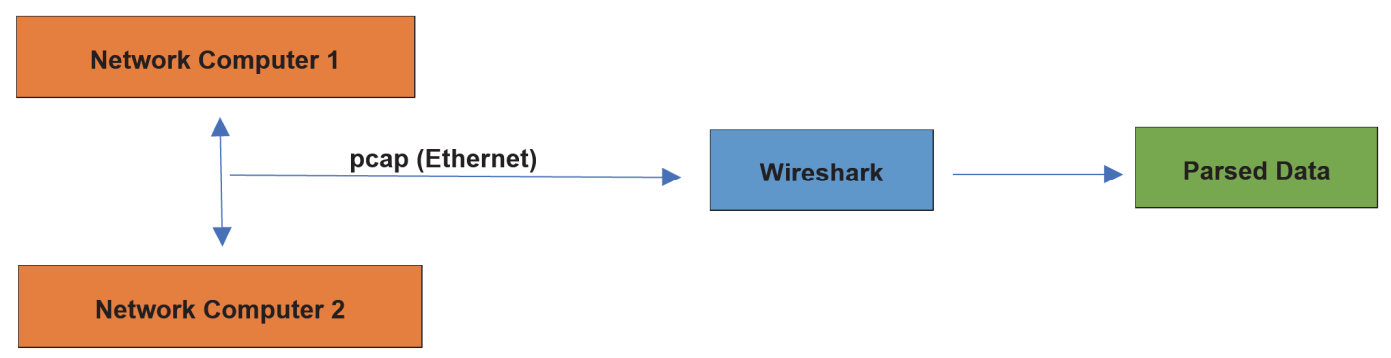

To use this tool, a computer running Wireshark must be plugged into the network being analyzed. The tool can be configured to search for user defined addresses, ports, messages, etc. It is recommended that Wireshark only be used on the private or isolated networks used by the autonomoussystem.

Wireshark data is captured in the industry standard pcap format. Additional parsing is required to put the pcap data into a human readable format (Table 20)

Table 20. Wireshark data captured.

\begin{tabular}{|c|c|}
\hline Item & Description \\
\hline Sensor & $\begin{array}{l}\text { - } \text { LiDAR } \\
\text { - } \text { Radar } \\
\text { - } \text { INS/GPS } \\
\text { - Ethernet traffic }\end{array}$ \\
\hline Data Format & - pcap \\
\hline Features & $\begin{array}{l}\text { - } \text { Capture data from live network connection or read from file } \\
\text { - } \text { Captured data can be analyzed with GUI interface } \\
\text { - } \text { Captured data can be filtered for specific inputs } \\
\text { - } \quad \text { Raw USB traffic can be captured }\end{array}$ \\
\hline Pros & $\begin{array}{l}\text { - } \quad \text { Captures all Ethernet traffic } \\
\text { - Base application is free }\end{array}$ \\
\hline Cons & $\begin{array}{l}\text { - Data requires parsing to be human readable } \\
\text { - } \quad \text { Might not be allowed on certain networks }\end{array}$ \\
\hline
\end{tabular}

\subsubsection{CL2000: CAN Bus Logger}

The CL2O0o made by CSS Electronics records live CAN Bus traffic with timestamps to a locally stored memory card. The timestamping is performed with a real-time clock to ensure accuracy. The CL2Ooo can be plugged into any CAN interface on the CAN network to record bus traffic (Figure 8). 
Figure 8. CAN Bus capture setup.

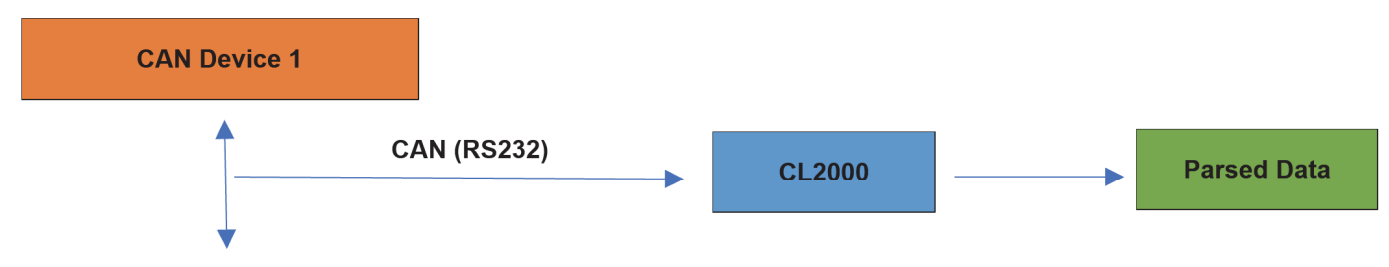

CAN Device 22

The CAN Bus traffic captures timestamped data with the CAN message header and 8-byte data field. The end user must compare the header and data field to the ICD to parse the data into a human usable format (Table 21).

Table 21. CL2000: CAN Bus Logger.

\begin{tabular}{|c|c|}
\hline Item & Description \\
\hline Sensor & - Vehicle bus (J1939, OBD2, etc.), command, and status \\
\hline Data Format & - 11-bit identifier and 8-byte data message \\
\hline Features & $\begin{array}{l}\text { - Log data to SD card-no PC needed } \\
\text { - Real-time clock timestamping } \\
\text { - Stream data in Wireshark }\end{array}$ \\
\hline Pros & $\begin{array}{l}\text { - Captures all traffic on a CAN Bus network } \\
\text { - Relatively low cost } \\
\text { - Data is time tagged }\end{array}$ \\
\hline Cons & $\begin{array}{l}\text { - Requires manufactures interface control documentation to read messages } \\
\text { - Limited to CAN/J1939/OBD2 traffic }\end{array}$ \\
\hline
\end{tabular}

\subsubsection{Jagwire}

Jagwire by Harris Geospatial Solutions is geospatial data management software that allows recording, retrieval, and playback of LiDAR, radar, and video data. The tool allows videos to be annotated or tagged for later review. Jagwire (Table 22) can save and display a variety of still imagery, such as NITF, GEOTIFF, and JPEG formats. It can also save and replay video formats including FMV and WAMI and LiDAR point clouds.

Table 22. Jagwire.

\begin{tabular}{|l|l|}
\hline Item & Description \\
\hline \multirow{3}{*}{ Sensor } & $\bullet$ LiDAR \\
& $\bullet$ Radar \\
& $\bullet$ Camera \\
\hline
\end{tabular}




\begin{tabular}{|c|c|}
\hline Item & Description \\
\hline Data Format & $\begin{array}{ll}- & \text { NITF } \\
- & \text { GEOTIFF } \\
\text { - } & \text { JPEG } \\
\text { - } & \text { FMV } \\
\text { - } & \text { WAMI } \\
& \text { LiDAR point cloud }\end{array}$ \\
\hline Features & $\begin{array}{l}\text { - } \quad \text { Manage data } \\
\text { - } \quad \text { Disseminate data at near real time } \\
\text { - }\end{array}$ \\
\hline Pros & $\begin{array}{l}\text { - Collects a wide range of sensors and data } \\
\text { - Allows for search and replay of data }\end{array}$ \\
\hline Cons & - Requires licensing agreement \\
\hline
\end{tabular}

\subsection{4 nSight data logger}

Robotic Research developed a tool, nSight (Table 23), to capture multiple streams of data with a single data-logging device that both timestamps and performs post processing operations and analytics all in one location. It is Robotic Research's understanding that there is no other non-system proprietary product available that can capture, post process, and analyze all of the data on an autonomous-system in a single location.

Table 23. nSight data logger.

\begin{tabular}{|c|c|}
\hline Item & Description \\
\hline Sensor & $\begin{array}{ll}\text { - } & \text { LiDAR } \\
\text { - } & \text { Radar } \\
\text { - } & \text { Camera } \\
\text { - Wheel encoder }\end{array}$ \\
\hline Data Format & - pcap \\
\hline Features & $\begin{array}{l}\text { - Distribute the data to the various servers } \\
\text { - Compare input command versus actual output value } \\
\text { - Measure sensor performance and predict degradation }\end{array}$ \\
\hline Pros & $\begin{array}{l}\text { - Configurable for most autonomous systems } \\
\text { - Collects raw and processed data from sensors and vehicle } \\
\text { - Automatically uploads data to cloud storage devices } \\
\text { - Hosts web application to analyze data }\end{array}$ \\
\hline Cons & $\begin{array}{l}\text { - Requires purchase of hardware to process and record data } \\
\text { - Requires configuration based on autonomous-system } \\
\text { - Might require large off-site storage dependent on amount of data collected (typically generates } 50 \\
\text { gigabytes of data in an 8-hour day) }\end{array}$ \\
\hline
\end{tabular}

The nSight platform provides an end-to-end data collection and analysis solution. The nSight suite is composed of the nSight Recorder (Onboard data recorder), nSight Upload (Data disseminator to servers), nSight after-action review (AAR) (Server and Analysis Tool). Figure 9 illustrates Robotic Research's complete data collection, storage, and management process. 
Figure 9. Proposed data management process flow using nSight tools.

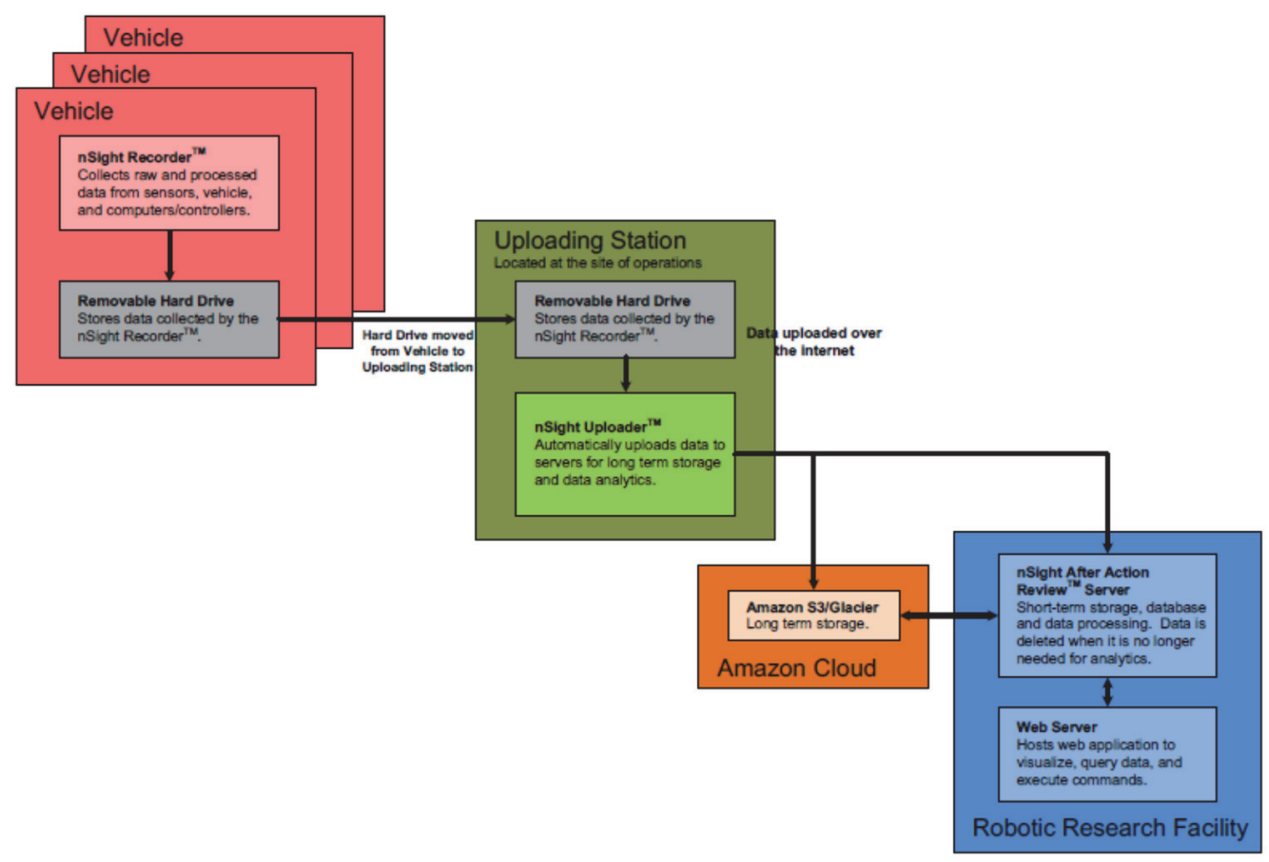

The sensor data is mostly captured in the industry standard pcap format. This is not only used for data communicated across Ethernet, but it also is used for serial data, such as CAN, USB, and RS-232. This includes sensors, such as LiDAR, radar, cameras, and wheel encoders. Processed data is outputted in ROS bag format, which was selected because it is a common format familiar to commercial and academic institutions. Robotic Research used these formats specifically to provide an open interface for data sharing.

The nSight data recorder can be installed on nearly any autonomous-system. The level of effort to install the system is based on the number of sensors, electrical signals to be recorded, and the availability of the manufacturer's ICD.

\subsection{Operational tests to verify traffic safety}

The objective of this research is to identify operational tests that can be applied to verify traffic safety of autonomous systems through analyzing and comparing human-driver behaviors to an AV system's steering-control behaviors in various traffic conditions. 


\subsubsection{Compare human drive behavior to autonomous-system behavior}

In order to complete an analysis in human-driving behavior against autonomous-driving behavior, tests can be conducted to capture human-operational inputs and decisions and compare to the AV system's data during a defined timeframe. Human-driving behavior data during operation of the system is simultaneously generated and stored with AV system data. This methodology allows for human control of vehicle steering, braking, and acceleration while the output of the robotic system is stored. The result of the human's driving actions, and the actions the robotic system would have taken, is then post processed and compared. The differences between the two then create edge cases that can be independently assessed, determining whether the autonomous-system or the human behaved more safely and in compliance with road and traffic regulations.

A database with the rules of the road pertinent to the particular locale is necessary for this data collection. In general, there are between 40 to 60 rules drivers abide by on the road network. The existence of these rules is specifically designed to minimize accidents and, to a certain degree, minimize communication between the drivers. For example, in a two-way, twolane road in the United States, the drivers will drive in the right-hand lane for each direction. In this case, both drivers, going in opposite directions, do not need to signal to one another which lane they will use. Instead, the rule of the road defines the behavior.*

For the purpose of this research, the test plan will focus on autonomoussystem operations at the CBITEC site. The road network at CBITEC is considered unimproved and without markings. In order to evaluate and verify traffic safety of the autonomous-system, rules of the road will define expected autonomous-driving behaviors, such as driving on the right side of the road, stopping at intersections, and yielding to other traffic and pedestrians. Additional infrastructure, such as stop and yield signs, might be required.

\footnotetext{
* Lacaze, Alberto. July 9, 2018. "System and Method for Verifying That a Self-Driving Vehicle Follows Traffic Ordinances."
} 
Similar work was performed at Fort Bragg on the ARIBO project from 2014-2016. ${ }^{*}+, \ddagger$ The objectives of this research are similar to the ARIBO project objectives:

- Socialize users and non-users with automated systems by creating trust and confidence

- Identify operational issues and develop mitigation strategies to offset technical risk through operational and policy adjustments

- Generate empirical data (e.g., performance, reliability, maintenance, etc.)

- Evaluate and improve reliability of AVs in a fail-safe environment

- Collaborate with industry and other agencies on autonomous systems R\&D.

This effort aims to build upon the ARIBO project by introducing the use of unimproved roads, interacting with external hardware, such as charging stations, and expanding the analysis from simple rules of the road to more complex interactions in off-road environments.

Traffic safety operational tests will occur in three phases. Autonomoussystem data will be collected using the nSight data recorder in each phase to evaluate characteristics of the human driver and the robotic vehicle. In Phase 1, a trained driver will operate the system in a manual driving mode. The autonomous-system and sensors, running in the background collecting data, will not control the system. In Phase 2, the driver becomes a safety operator. The autonomous-system will control the system, and the safety operator will be ready to take control in the event of an emergency. In Phase 3, the safety operator will be removed from the vehicle and perform safety operations from a remote location. Phase 3 will not occur until the data shows the autonomous-system is safe and reliable.

The recommended data to collect during operational tests are:

- Timestamp

\footnotetext{
* Barghout, Jeffrey. July 12, 2018. "Autonomous Shuttle Technology Safety Review Fort Bragg, NC and Greenville, SC Deployments."

† Barghout, Jeffrey. Mottern, Edward. Putney, Joseph. Patel, Rashmi. March 27, 2017. "Fort Bragg, Autonomous Warrior Transport On-Base Automated Vehicle Evaluation Plan."

¥ The University of Texas at Arlington Research Institute, September 30, 2014, "Applied Robotics for Installation and Base Operations (ARIBO) Roadmap"
} 
- Safety operator/driver

- Vehicle state (on/off, charging, park, forward, reverse)

- Autonomous-system mode (manual/autonomous)

- Vehicle location (GPS coordinates-latitude, longitude, elevation)

- Relative left path border (GPS coordinates-latitude, longitude, elevation)

- Relative right path border (GPS coordinates-latitude, longitude, elevation)

- Odometer

- Vehicle speed

- Vehicle acceleration/deceleration

- Vehicle heading (instantaneous direction the vehicle is moving)

- Moving object proximity data: captures data on all moving objects, such as cars and people that are within 100 feet or, based on their speed and heading, are projected to come within 100 feet of the vehicle on its planned route

- Distance From Vehicle

- Speed Of Objects (Including Pedestrians)

- Heading Of Objects (Including Pedestrians)

- Time Until It Intersects Vehicle Route

○ Distance From Vehicle At Route Intersect

- Environmental/ambient conditions (temperature, humidity, pressure, light).

Using the discrete data subsets listed in Table 24, driving patterns during various scenarios will be captured and analyzed to compare human- to robot-driving modes. Driving pattern observations are detailed in Table 24.

Table 24. Driving patterns.

\begin{tabular}{|l|l|}
\hline Scenario & Observation \\
\hline $\begin{array}{l}\text { Distance to } \\
\text { Stationary Objects }\end{array}$ & The average, median, maximum, and minimum distance the vehicle comes to stationary object. \\
\hline Pedestrian Reaction & The average, median, maximum, and minimum distance the vehicle initiates a response. \\
\hline Intersection Entrance & $\begin{array}{l}\text { The average, median, maximum, and minimum distance and estimated time to intersect oncoming } \\
\text { cross traffic. }\end{array}$ \\
\hline $\begin{array}{l}\text { Critical-path } \\
\text { Selection }\end{array}$ & $\begin{array}{l}\text { The autonomous-system decision to or not to enter a designated clear area if an obstacle in the } \\
\text { distance will force it to stop in an unsafe location, such as while crossing a road. }\end{array}$ \\
\hline Human Control & Frequency and circumstances that a human took control of the vehicle that was in robot-mode. \\
\hline
\end{tabular}


Additional parameters for observation will be identified during Phase 1 of the autonomous-system traffic safety evaluation. As data accumulates, patterns will emerge, warranting deeper investigation of performance.

\subsubsection{Route metrics}

Route metrics for a designed scenario will be used for comparisons. For example, data will be captured that assesses the autonomous-system's response to pedestrians crossing the street in its planned path. Where possible, route metrics (Table 25) will be identified that can be captured in multiple route segments, which will strengthen the overall performance analysis by increasing the sample size of specific situations, with a variety of influential variables. These variables can then be evaluated to determine the autonomous-system's performance in the presence of those variables and compare them to human-driver performance.

While this report covers the types of data to collect and the general testing approach, Research Area 5A expands this concept and provides a detailed test plan for performing autonomous-system testing at the CBITEC site.

Table 25. Route metrics.

\begin{tabular}{|c|c|}
\hline Scenario & Observation \\
\hline $\begin{array}{l}\text { Avoids Immediate } \\
\text { Objects }\end{array}$ & $\begin{array}{l}\text { The autonomous-system comes to a stop or alters route appropriately when an obstacle is detected } \\
\text { in its path. }\end{array}$ \\
\hline $\begin{array}{l}\text { Objects Approaching } \\
\text { Vehicle }\end{array}$ & $\begin{array}{l}\text { Objects, such as pedestrians or vehicles, move toward the autonomous-system on the pedestrian } \\
\text { roadway. }\end{array}$ \\
\hline $\begin{array}{l}\text { Approaching Objects } \\
\text { from Behind }\end{array}$ & $\begin{array}{l}\text { Objects, such as pedestrians or vehicles, move toward the autonomous-system from the crosswalk } \\
\text { or in the major roadway (from a different route segment). }\end{array}$ \\
\hline $\begin{array}{l}\text { Following Other } \\
\text { Objects }\end{array}$ & $\begin{array}{l}\text { The robotic vehicle follows another vehicle in front of it at an appropriate distance even when the } \\
\text { other vehicle is accelerating or decelerating. }\end{array}$ \\
\hline $\begin{array}{l}\text { Stopping/Stopped } \\
\text { Object }\end{array}$ & $\begin{array}{l}\text { The autonomous-system follows another vehicle and stops at an appropriate distance when the } \\
\text { vehicle stops in front of it. }\end{array}$ \\
\hline Impairs Pedestrians & $\begin{array}{l}\text { Metric that ensures that autonomous-system does not block or interfere with pedestrians from } \\
\text { crossing the street. }\end{array}$ \\
\hline Sun Blinding & $\begin{array}{l}\text { This observation evaluates conditions where the sun is positioned such that it might impair the driver } \\
\text { (robot/human). Time of day and inclination of the sun can impact the robot/human drivers at certain } \\
\text { points in the route. This metric can be layered on any route segment metric to evaluate if the sun } \\
\text { had a significant impact on driver performance (robot/human). The position of the sun can be } \\
\text { calculated based on date, time, and location of the vehicle. The References section provides links to } \\
\text { calculation resources, including an MS Excel spreadsheet developed by National Oceanic \& } \\
\text { Atmospheric Administration (NOAA). }\end{array}$ \\
\hline $\begin{array}{l}\text { Cross Traffic Turn } \\
\text { Left }\end{array}$ & The autonomous-system comes to a stop and then turns left onto a cross street. \\
\hline $\begin{array}{l}\text { Cross Traffic Turn } \\
\text { Right }\end{array}$ & The autonomous-system comes to a stop and then turns right onto a cross street. \\
\hline
\end{tabular}




\begin{tabular}{|c|l|}
\hline Scenario & \multicolumn{1}{c|}{ Observation } \\
\hline Cross Traffic Straight & $\begin{array}{l}\text { The autonomous-system comes to a stop and then proceeds across a street. When oncoming cross } \\
\text { traffic is identified, the system waits or proceeds appropriately. Distance to oncoming vehicle, speed, } \\
\text { estimated time, to intersection is collected. }\end{array}$ \\
\hline
\end{tabular}

\subsubsection{Triggered events}

Triggered events, outlined in Table 26, are scenarios where the autonomous-system operates in an unexpected or unsafe way. In the event that any of the following scenarios occur, the incident will be flagged for analysis into the mitigating circumstances. In addition to the standard data collected, audio and video will be saved for reference. For triggered events, data will be flagged for 15 seconds prior to and following the flagged event timestamp for a total of 30 seconds.

The data flagging is automatically triggered when the events listed in Table 26 occur or when the test observer requests thru a software interface. All of the data collected during the test is stored for later analysis, but the triggered or flagged events are highlighted in the dataset to locate the event more easily.

Table 26. Triggered events.

\begin{tabular}{|l|l|}
\hline \multicolumn{1}{|c|}{ Scenario } & \multicolumn{1}{c|}{ Observation } \\
\hline $\begin{array}{l}\text { Safety Operator } \\
\text { Override }\end{array}$ & $\begin{array}{l}\text { If at any time, while in robot-mode, the autonomous-system performs in an unsafe or undesirable } \\
\text { manner, the safety operator should take control and override the autonomy. The safety operator should } \\
\text { enter an explanation into the survey/log. }\end{array}$ \\
\hline $\begin{array}{l}\text { Emergency } \\
\text { Stop/Pause }\end{array}$ & $\begin{array}{l}\text { The safety operator can at any time hit the emergency stop or pause button located throughout the } \\
\text { autonomous-system. When pressed, the vehicle comes to a rapid stop. }\end{array}$ \\
\hline $\begin{array}{l}\text { System } \\
\text { Error/Failure }\end{array}$ & $\begin{array}{l}\text { In the event that the autonomous-system senses a system error or failure, it will immediately initiate } \\
\text { emergency stop procedures. }\end{array}$ \\
\hline Path Following & $\begin{array}{l}\text { Develop set of specific route boundaries that the AV is expected to use. This set should include the left- } \\
\text { and right-side boundaries for the designated route. Any events, where the robotic vehicle crosses these } \\
\text { boundaries, should be flagged automatically for closer study. }\end{array}$ \\
\hline
\end{tabular}

\subsubsection{CBITEC autonomous-system route}

Figure 10 shows a conceptual drawing of the route between the CBITEC entrance and the proposed location of the charging station. The autonomous-system will travel this segment in both directions. This is a low- to medium-vehicle traffic area with expected pedestrian traffic expected. 
Figure 10. Example test track.

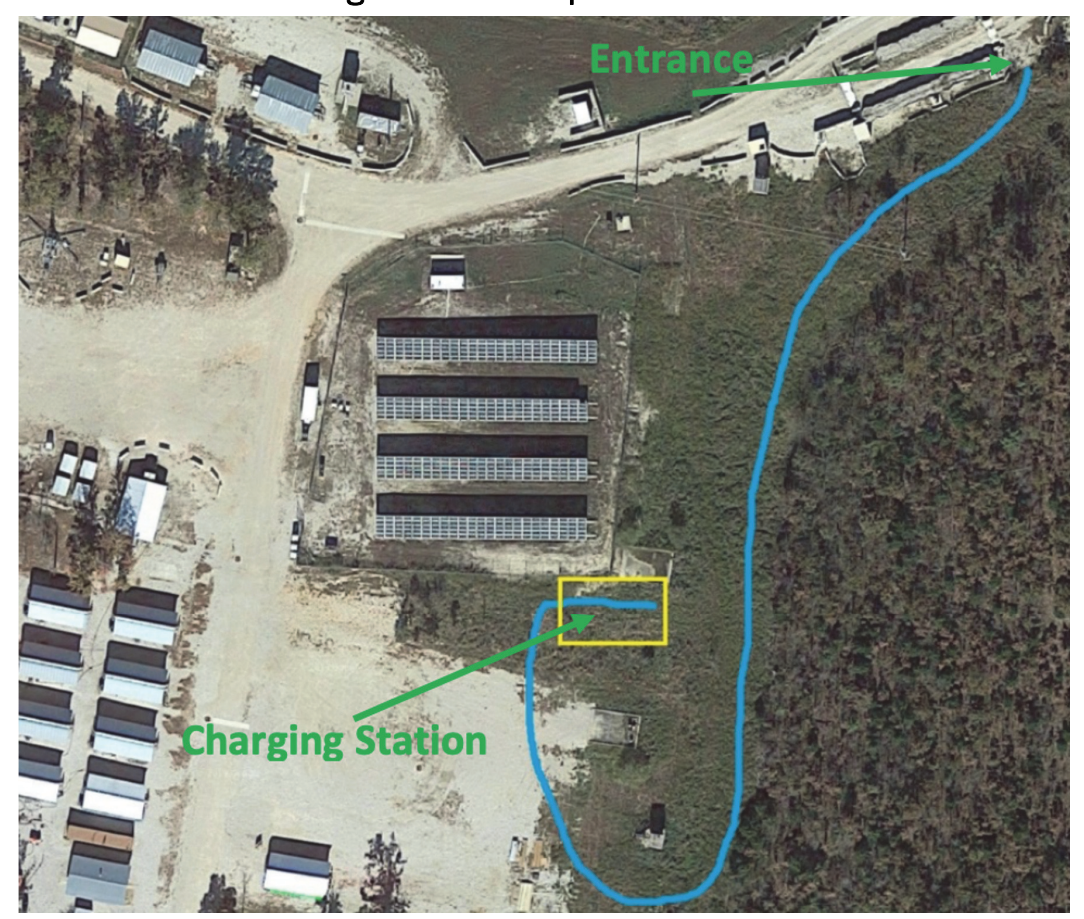

Specific observations are:

1. Vehicles turn around at the charging station area.

2. Pedestrians walk to and from the charging station area and are present in other areas of the CBITEC site.

3. There is vehicle traffic in and out of the dock area. This is the only access point to the charging station.

\subsection{Monitoring autonomous component degradation}

The objective of this research is to outline methodology for measuring autonomous component (sensor) degradation over time. For the scope of this research area, a sensor will be considered a LiDAR, radar, or camera. Just as human vision and reaction time change with age, the same is true for LiDARs, radars, and cameras, on which AVs rely.

The light intensity of a laser diode diminishing with time is an example of sensor degradation. After 3 years of operations, this intensity could be half of what it originally was. Another sensor degradation example is when the coatings of lenses and protective covers get scratched and subsequently less transparent over time, lowering the transmittivity and, therefore, 
lowering the effective range of those same sensors. Cameras and laser detectors "burn in" with time, especially after exposure to bright lights and the sun, decreasing the sensitivity and creating more noise. As with humans, these changes in performance are not usually catastrophic; however, they gradually degrade the performance of the autonomous-system. To what degree this degradation happens depends on many factors that can be tested against road rules. For example, an old LiDAR might not be able to distinguish a person wearing a black velour suit 50 meters away, while the same LiDAR could have detected that same person with the same suit when it was new. If there is a traffic safety rule that says that the autonomous systems must stay 50 meters away from pedestrians, the aged system might not be able to comply with that rule. Like with humans, aging is not predictable. Some laser manufacturers use coatings that withstand elements better than others and exposure will be vehicle dependent. Testing the performance of the system is a way of understanding the current capabilities of the system.

\subsubsection{Sensor degradation operational tests}

To measure sensor degradation, a baseline must be established when the sensor is new. Data, which will be collected and stored during operational tests, will be used as a baseline for sensor degradation analysis. It is expected that certain features of the routes or scenarios will remain constant. These features include locations of buildings or other fixed structures. Sensor data is collected and recorded every time the autonomous-system is run. When the system is run over time, the collected data can be analyzed, and the sensor degradation can be characterized. Table 27 outlines sensor degradation operational tests.

Table 27. Sensor degradation operational tests.

\begin{tabular}{|l|l|}
\hline \multicolumn{1}{|c|}{ Test } & \multicolumn{1}{c|}{ Observation } \\
\hline First Detection & $\begin{array}{l}\text { The data-recording system continuously tracks the range measurements to objects and records the } \\
\text { distance of the first detection. This data shows the overall health of the sensor and can be monitored over } \\
\text { time to compare to a baseline. This test is used for LiDARs and radars. }\end{array}$ \\
\hline $\begin{array}{l}\text { Maximum } \\
\text { Range }\end{array}$ & $\begin{array}{l}\text { The maximum range measurement compares the sensors current capability to a baseline. This can be } \\
\text { done by measuring the distance between the autonomous-system to a given feature when the feature is } \\
\text { first detected. The distance can be recorded and tracked over a given time period. Captured data will } \\
\text { show when the sensor's maximum range begins to decrease. This test is used for LiDARs and radars. }\end{array}$ \\
\hline $\begin{array}{l}\text { Range } \\
\text { Measurement }\end{array}$ & $\begin{array}{l}\text { The range measurement verifies whether the sensor is able to accurately measure distance. This can be } \\
\text { done by measuring the distance between the autonomous-system to any given feature at a set location. } \\
\text { This measurement can be recorded and tracked over time. The data will show if there is a deviation } \\
\text { between what the sensor is currently measuring and the baseline. This test is used for LiDARs and radars. }\end{array}$ \\
\hline
\end{tabular}




\begin{tabular}{|l|l|}
\hline \multicolumn{1}{|c|}{ Test } & \multicolumn{1}{c|}{ Observation } \\
\hline $\begin{array}{l}\text { Object } \\
\text { Shape/Size }\end{array}$ & $\begin{array}{l}\text { This test will characterize the sensors ability to detect the shape of objects. This can be done by } \\
\text { comparing the shape and size of a given feature over time. The data will show if the sensor has lost ability } \\
\text { to sense finer details, such as corners or edges. This test is used for LiDARs, radars, and cameras. }\end{array}$ \\
\hline $\begin{array}{l}\text { Color and } \\
\text { Focus }\end{array}$ & $\begin{array}{l}\text { This test can characterize the overall health of a camera. This can be done by comparing image data of a } \\
\text { given feature over time. The data will show if the camera has lost focus or color clarity. This test is used for } \\
\text { cameras. }\end{array}$ \\
\hline
\end{tabular}

While sensor degradation operational tests can determine if a sensor is functional or not, the intent of the tests is to establish a baseline and characterize the sensor degradation over time. Sensor performance based on observed degradation is depended upon the autonomous operations use case. For example, a degraded sensor might not be usable in a high-speed application but would still be functional for lower-speed applications.

\subsection{Autonomous-system terrain technologies}

The objective of this research is to explore how current AV system terrain identification and estimation technologies can be improved.

Autonomous systems have an advantage when traversing complex or rough terrain. Without a person onboard, the system does not need to consider the driver's or passengers' comfort. However, platform shock and vibration limits need to be accommodated. The autonomous-system can use perception sensors to predict the upcoming terrain and navigation sensors to verify the prediction. This research will evaluate techniques that can be used to predict and verify the terrain and ways to use terrain data to improve the autonomous-system's planning algorithms.

\subsubsection{Terrain identification}

Most autonomous systems use LiDAR to detect the surrounding environment. LiDAR not only looks for obstacles in the system's path but also for the ground plane or support surface on which the vehicle drives. Determining the support surface is often a trivial task for on-road environments; however, in off-road environments, with the presence of vegetation, dust, and water, the operation is much more complex.

When LiDAR scans the environment, a hit occurs as the laser-range measurement contacts a surface that reflects the beam back to the LiDAR. In contrast, if the laser does not make contact with, and is not reflected, no 
hit is recorded. The reflecting surface can be the ground, something attached to the ground, or something in the air like a bridge or an overpass. By collecting the number of hits in a given area, the autonomous-system can determine density and topography of the area surrounding the vehicle. A road surface will return several hits at a common elevation and will depict a smooth surface. A curb on the same road surface will show an abrupt elevation change. Similarly, a person or a car on the road surface will show attachment points to the ground.

Using the LiDAR information, the autonomous-system deciphers what the ground plane or support surface looks like. This becomes more challenging in areas with dense vegetation because the LiDAR might get hits off of tall grass, making the ground appear higher than it is. The autonomous-system must determine the density of those hits to better understand the surface.

Figure 11 depicts the support surface found using the detection method described above for a terrain with shoulder-height grass:

- The blue surface is the predicted support surface.

- The yellow line is the path of the vehicle.

- The green line in the middle picture is a row of hits from the grass.

- The white and red lines in bottom picture show the different density of hits returned from the grass.

- The darker red section are more dense returns where the white is less dense. The denser the vegetation, the harder it is for laser beams to penetrate, and, therefore, larger errors can be generated. ${ }^{*}$

By calculating the support surface using this methodology, the autonomous-system can predict the relative roughness of the terrain that the vehicle is about to cross. The planning system can then rate the rougher sections of terrain as less desirable to traverse or decrease the vehicle's speed to accommodate for the terrain.

\footnotetext{
* Lacaze, Alberto, Murphy, Karl, DelGiorno, Mark. July 8, 2002. “Autonomous Mobility for the Demo III Experimental Unmanned Vehicles."
} 
Figure 11. Support surface voxel generation.

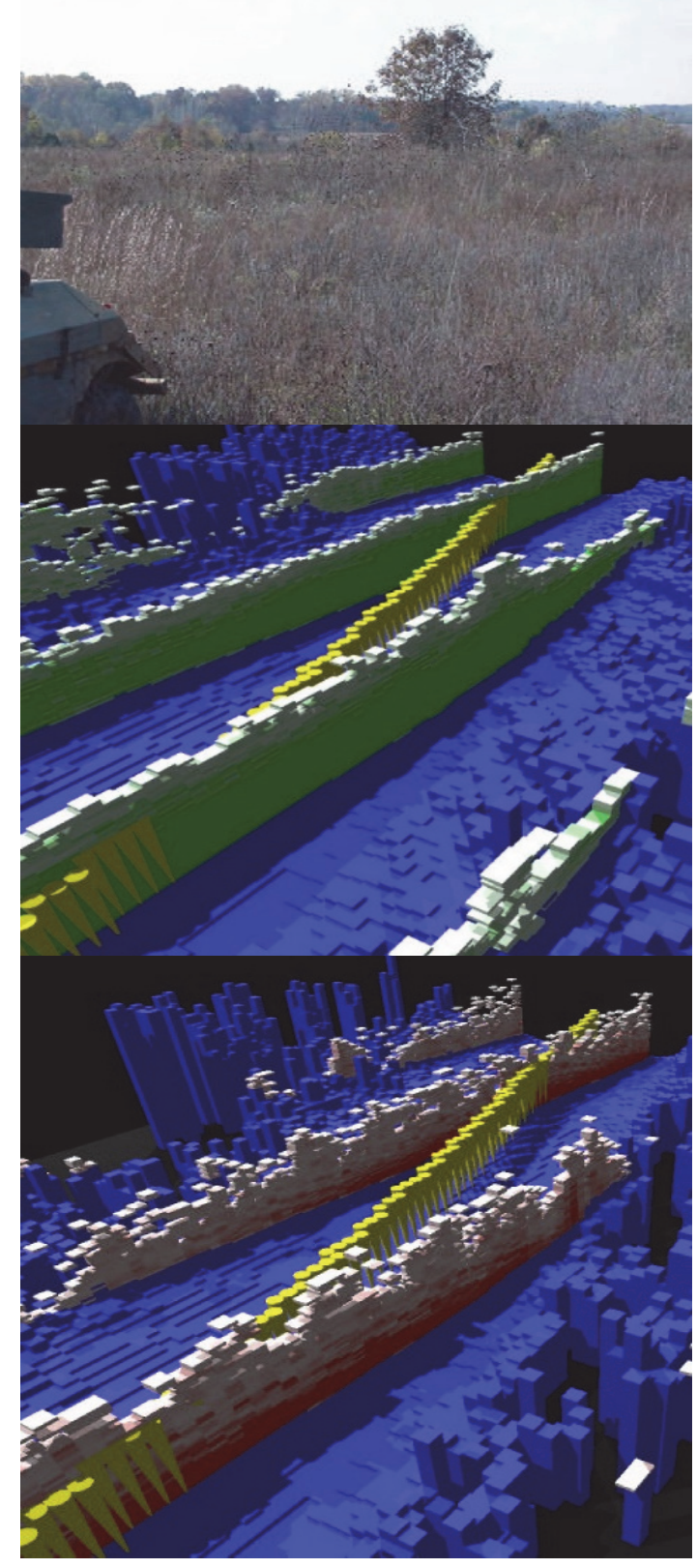

As perception sensors predict upcoming terrain, the inertial sensors can verify whether the predicted terrain equates to the platform's current attitude (roll, pitch, and yaw). This measurement can be made more accurately if the system is equipped with suspension sensors, as the tire-toground interaction will be damped by the suspension, and the GPS/INS will not sense the full roughness of the terrain. By adding the state of the suspension system to the system's current pose, terrain attributes can be better verified. 
Post processing the LiDAR and navigation data will not only verify the accuracy of the terrain prediction but also allow the terrain prediction algorithm to be tuned for better results in the future.

More fidelity can be added to the navigation sensors ability to measure the current terrain interaction by adding smaller IMUs to each wheel. The system could then sense the overall vehicle pose and the dynamics happening at each wheel in real time.

\subsubsection{Trajectory modeling}

These detailed methods can be used to create simulated models of both the terrain and the autonomous-system's interaction with the terrain. If a system's behavior can be predicted with simulation and modeling, the planning algorithms in the autonomous-system can apply feed-forward control techniques that anticipate and correct for interaction with the terrain. This is useful when navigating complex terrain or when trying to achieve higher speeds in rougher terrain.

These simulations require precisely accurate modeling of the terrain and vehicle dynamics. While the modeling will result in a smoother ride for the autonomous-system, it would not be feasible to apply such a strategy to all systems. Complex models of the both the vehicle and terrain need to be created and verified. There are times where it is not possible to model all of the terrain, or the value of the autonomous-system might not warrant the added cost of the simulation.

Considerations for the feasibility of trajectory output software include:

- Cost of vehicle

- Number of vehicles in operation

- Complexity of the operating terrain/environment.

As the complexity of the terrain increases, the difficulty and cost to model the terrain also increases. Spreading across costs of the vehicle multiplied by the number of vehicles can offset this cost. It might not be cost effective to model complex terrain for a low quantity of inexpensive vehicles could be feasible for a larger quantity of expensive vehicles. Therefore, it is recommended that terrain modeling strategy be applied per Figure 12. 
Figure 12. Feasibility of trajectory output software.

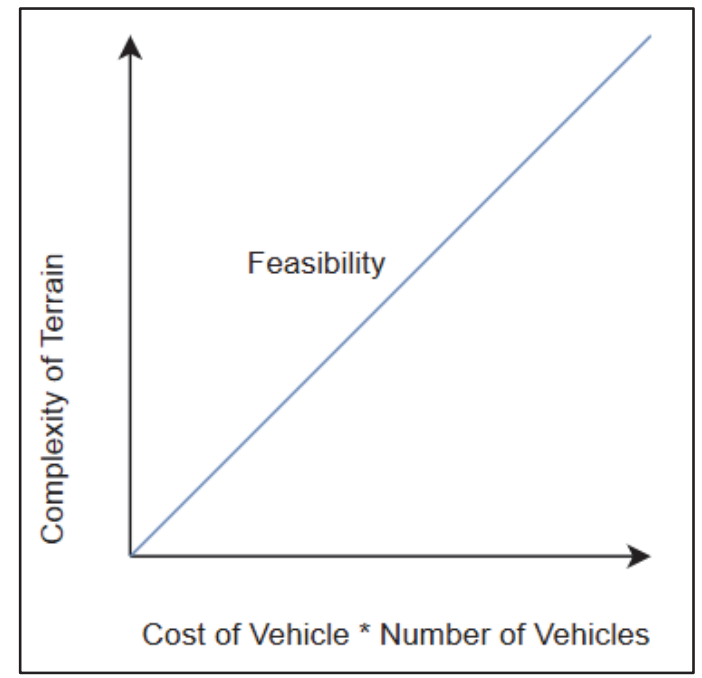

\subsubsection{Autonomous docking with charging stations}

The objective of this research is to define requirements for AV systems to autonomously dock with charging stations. Robotic Research team of engineers are SMEs in autonomous software and hardware development and integration. The following list of requirements (Table 28.) Robotic Research's engineering team developed define the high-level operations and behaviors necessary for an autonomous-system to autonomously dock with a charging station. It is expected that more specific sub-requirements will need to be defined based on the robotic vehicle selected for the operation and the final layout of the CBITEC site.

Table 28. Autonomous docking requirements.

\begin{tabular}{|c|c|}
\hline Operation & Docking Requirement \\
\hline Localization & $\begin{array}{l}\text { - Requirement 1: The autonomous-system will be able to calculate its absolute position within } 0.1 \text { meters. } \\
\text { - Requirement 2: The autonomous-system will be able calculate its relative position within } 0.1 \text { meters. } \\
\text { - Requirement 3: The autonomous-system will calculate its attitude position (roll, pitch, and yaw). } \\
\text { - Requirement 4: The autonomous-system will be able to receive differential GPS (DGPS) corrections. } \\
\text { - Requirement 5: The autonomous-system will be able to visualize odometry data for localization. } \\
\text { - Requirement 6: The autonomous-system will be able to use wheel-encoder odometry data for localization. } \\
\text { - Requirement 7: The autonomous-system will be able to operate without GPS. }\end{array}$ \\
\hline Perception & $\begin{array}{l}\text { - Requirement 8: The autonomous-system will be able to operate in varying lighting conditions, such as full } \\
\text { daylight, dusk, and night. } \\
\text { - Requirement 9: The autonomous-system will be able to detect the position of objects within the sensor's } \\
\text { field of view. } \\
\text { - Requirement 10: The autonomous-system will be able to measure the speed of objects within the } \\
\text { sensor's field of view. } \\
\text { - Requirement 11: The autonomous-system will be able to measure the trajectory of objects within the } \\
\text { sensor's field of view. }\end{array}$ \\
\hline
\end{tabular}




\begin{tabular}{|c|c|}
\hline Operation & Docking Requirement \\
\hline Planning & $\begin{array}{l}\text { - Requirement 13: The autonomous-system will be able to acquire a route via an over-the-air update, } \\
\text { through a wired connection or other means, such as a USB drive, and store it onboard. } \\
\text { - Requirement 14: The autonomous-system will be able to navigate stored routes. }\end{array}$ \\
\hline Docking & $\begin{array}{l}\text { - Requirement 15: The autonomous-system will be able to perform maintenance-yard maneuvers. } \\
\text { - Requirement 16: The autonomous-system will be able to perform point-to-point travel. } \\
\text { - Requirement 17: The autonomous-system will be able to recognize the location of the charging station. } \\
\text { - Requirement 18: The autonomous-system will be able to precision park at the charging station. } \\
\text { - Requirement 19: The autonomous-system will be able to communicate via a data interface with the } \\
\text { - Requirement 20: The autonomous-system will be able to command the charging station to begin charging. }\end{array}$ \\
\hline Vehicle & $\begin{array}{l}\text { - Requirement 22: The autonomous-system will have an emergency stop. } \\
\text { - Requirement 23: The autonomous-system will stop when the emergency stop is engaged. } \\
\text { - Requirement 24: The autonomous-system will follow the velocity commands generated by the autonomy } \\
\text { system. } \\
\text { - Requirement 25: The autonomous-system will follow the steering commands generated by the autonomy } \\
\text { system. }\end{array}$ \\
\hline
\end{tabular}

\subsubsection{Optimization to reduce autonomous vehicle system power consumption}

The objective of this research is to identify optimization techniques that the autonomous-system can exploit to reduce power consumption. Connected autonomous systems with Vehicle-to-vehicle (V2V) or Vehicle-toinfrastructure (V2I) communications and knowledge of the planned route have an advantage in reducing power consumption. Autonomous systems can intelligently exploit acceleration curves and regenerative braking.

\subsubsection{Optimization techniques}

Most, if not all, EVs not only employ regenerative braking to assist in decelerating the vehicle but also to recharge the vehicle's batteries and extend range. Human drivers practice techniques of either riding the brakes or coasting to increase brake regeneration time. This technique can be performed more efficiently by an autonomous-system. Inertial sensors, visual odometry sensors, and knowledge of the terrain allow the autonomoussystem to optimize the amount of coasting and/or braking that can be achieved to maximize regeneration time while still maintaining a given speed for a roadway. Additionally, the autonomous-system can more accurately maintain acceleration and deceleration rates during driving tasks to optimally conserve energy.

Autonomous systems with V2V or V2I communications can employ even more techniques to improve power consumption. Path planning is a major computational component of the autonomous-system. Part of the path- 
planning computation is calculating arrival time to a given location. If the autonomous-system is communicating with infrastructure, such as traffic lights, the system will know when traffic lights will turn green or red. The autonomous-system can use speed control techniques to allow it to take more time to get to a traffic light that is currently red. This technique will either allow for decreased acceleration, consuming less energy, or longer periods of deceleration, increasing regenerative braking. Similarly, an autonomous-system that is communicating with other vehicles on the road has a greater knowledge of traffic speeds and behaviors. This knowledge lets the path planner smooth the autonomous-system's speed profile to again minimize acceleration or maximize declaration times.

\subsection{Conclusions and future work}

A variety of topics and techniques regarding autonomy have been discussed in this report. Some of the techniques are established practice while others require additional development and testing. Areas where more research would be beneficial are:

- Sensor Degradation: While the tests described in this report can detect and characterize the current performance of a sensor compared to a baseline condition, the majority of this characterization is done through post processing data. More research can be done to make those comparisons in real time and report the overall sensor health to the system.

- Terrain Identification: More research can be performed in this area to better understand how the system interacts with not only the terrain but also differences in soil or road conditions. More modeling and simulation can be performed to better create feed-forward control algorithms allowing autonomous systems to traverse more complex terrain at higher speeds.

- Precision Docking: Requirements for precision docking can be derived to better constrain the platforms low-level control system. While precision docking presents challenges of localizing the autonomoussystem relative to the charging station, the more significant challenge is developing an extremely precise control system at the platform level that can accurately follow velocity and steering commands with little latency. The autonomous-system can easily overshoot the goal point by a few centimeters if the platform controls are not well tuned. 
- Improved Power Management: The techniques described in this section can be tested and improved as more V2I and V2V capabilities arise. A more connected area of operation will allow for more chances to test and verify these techniques. 


\section{Research and Develop a Plan for Testing and Demonstrating Vehicle Charging within a Tactical Microgrid Environment}

The vision to ensure energy resiliency for the warfighter in a tactical environment underscores the need for a living laboratory to test candidate technologies for potential incorporation into a tactical microgrid. The Research Area 4 collaboration with CBITEC and CERL staff identified V2G charging technology that will allow electric transport technologies to serve as an alternative-energy source, lowering fossil fuel generation dependency while supporting vehicle-mission requirements. This collaboration informed the tactical microgrid model that supports the design integration effort of the selected V2G technology along with future "what-if" scenarios. Paragon SMEs developed a test plan to validate integration and evolve the overall tactical microgrid design to support integrating future enabling technologies.

The objective of Research Area 4 is to research and develop a plan for testing and demonstrating $\mathrm{V} 2 \mathrm{G}$ charging within a tactical microgrid environment that validates EV capabilities providing energy support to critical loads, interoperating simultaneously with other generation sources, and dispatching as a load or a generation source as necessary to support the tactical microgrid's efficient operation.

In order to develop a test plan to demonstrate $\mathrm{V} 2 \mathrm{G}$ charging within a tactical microgrid environment, the Paragon research team cataloged CBITEC microgrid assets and identified $\mathrm{V} 2 \mathrm{G}$ operational requirements, developed a conceptual microgrid model, conducted "what-if" scenarios, and designed a test plan to verify performance of the V2G system.

Paragon SMEs cataloged CBITEC tactical microgrid assets through site visits and review of customer-provided documents, and engagement with CBITEC staff and technology providers to gather site requirements and current asset capabilities at CBITEC. System components and available V2G charging solutions were identified, and a conceptual design was prepared to reflect integration requirements necessary to establish a tactical microgrid. 
Because the program's intent is to build a living laboratory to support the developing and testing technologies at CBITEC, the tactical microgrid should also include additional metering and control components to help ensure safety for personnel, and equipment, along with analytical tools for equipment evaluation and data gathering. A software model of the tactical microgrid was prepared to simulate various "what-if" scenarios and aid the final tactical microgrid design and evaluation.

\subsection{Catalog CBITEC assets and identify V2G operational requirements}

The objective of this research is to identify and catalog CBITEC tactical microgrid assets and to identify $\mathrm{V} 2 \mathrm{G}$ operational requirements to integrate and use in a tactical microgrid.

To catalog the site's requirements and capabilities, Paragon SMEs collaborated with CBITEC and CERL staff, and previous equipment providers, to identify components that make up the tactical microgrid. Additionally, Paragon SMEs conducted site visits to the CBITEC facility on FLW and CERL offices in Champaign, IL to inspect existing components, location, and environment of the future living laboratory. Customer-provided documents were reviewed by SMEs, and input from vendors providing tactical microgrid technologies was documented.

During the course of this collaboration, CBITEC staff provided feedback on desired microgrid capabilities, lessons learned from previous equipment demonstrations, and practices for uniformity with current development standards as well as the Army's broader vision for tactical microgrids. Identified components were studied to ascertain capabilities and determine how to best to integrate V2G chargers with tactical microgrid components. Through engagement with CBITEC staff and equipment manufacturers, the Paragon team identified these components for:

- Specific microgrid components and equipment that must be included

- Microgrid components and equipment that must be added to meet safety and operational objectives

- Design standards that system components must adhere to. 
In addition, this research effort considered the vision of creating a living laboratory at CBITEC and necessary capabilities to test and evaluate innovative energy efficiency and energy security technologies for future integration into the tactical microgrid. Adding an evaluation capability to any energy system inherently requires the following approach: developing performance requirements for the EUT, implementing a sensored and metering system to measure performance, and a test plan that will validate the EUT to meet performance requirements by conducting applicable test use cases. This approach allows Research Area 4's test plan to evaluate integrating a $\mathrm{V}_{2} \mathrm{G}$ charger and to support the program vision of a future living laboratory bringing energy resiliency to the warfighter by:

- Providing CBITEC the capability to integrate, test, and evaluate alternate energy sources that are manufacturer agnostic and based on tactical microgrid performance requirements and not specific equipment design

- Utilizing existing tactical microgrid components readily available and familiar to the warfighter for representative results

- Identifying additional components that use open standards to ease integration, monitoring, and control of microgrid assets

- Using a modeling environment to simulate "what-if" scenarios and evolve the microgrid design and associated test plans.

By supporting the tactical microgrid short- and long-term development, this approach postures CERL to efficiently evolve test plans and evaluate technologies to ultimately develop a tactical microgrid that is resilient, flexible, and secure for the warfighter.

\subsubsection{CBITEC assets}

Work for this research effort commenced with the preparation of, and communication to CBITEC and CERL staff, a detailed list of equipment and systems that are typically present in a tactical microgrid as well as requesting available tactical microgrid documents for the Paragon SMEs to review. Critical to preparing a suitable integration design and test plan to validate performance the existing state of the tactical microgrid and site capabilities was verified. 
The following items were discussed at the project kick-off meeting held at CERL offices in Champaign, IL on April 30, 2019:

- System documentation-any available documentation to identify current and proposed design of the tactical microgrid, including equipment manuals and system drawings

- Fossil-fueled generators-power rating and quantity to be used

- ESSs-energy storage technology and power and energy ratings

- Photovoltaic (PV) systems-configuration and rating(s)

- Microgrid loads-specific load configuration, type, and size been to consume the energy produced by the microgrid's generation sources

- Distribution system-various generation sources and loads interconnection and protective devices that were included to ensure personnel and equipment safety

- Monitoring and controls system-system used to monitor and control the tactical microgrid's components and the level of control available to start and stop generation sources or loads, prioritizing available power to mission critical loads and conduct test plans

- Applicable standards-military or industry standards to incorporate

- Advanced capabilities-special capabilities and considerations that must be considered for the tactical microgrid to meet mission requirements as a living laboratory

- Site limitations-specific site limitations that could influence the tactical microgrid's design to be used as a living laboratory; how could a test plan be conducted.

\subsubsection{Current capabilities and requirements for testing and operating V2G technologies at CBITEC}

The CBITEC site at FLW (Figure 13) operates with great flexibility and authority to review and approve demonstrations. Operational requirements do not include site permitting, city inspections, state approvals, or an extensive design- review process from multiple site organizations before approval to install equipment is granted. Operational requirements mirror that of a deployed tactical microgrid outfitted with local staff with authority to review and approve installing equipment for testing and evaluation, as long as the equipment is compatible with the site's infrastructure and, as is the case with most host nation requirements, the equipment is not installed as a permanent structure. 
Figure 13. CBITEC site at Fort Leonard Wood, MO.

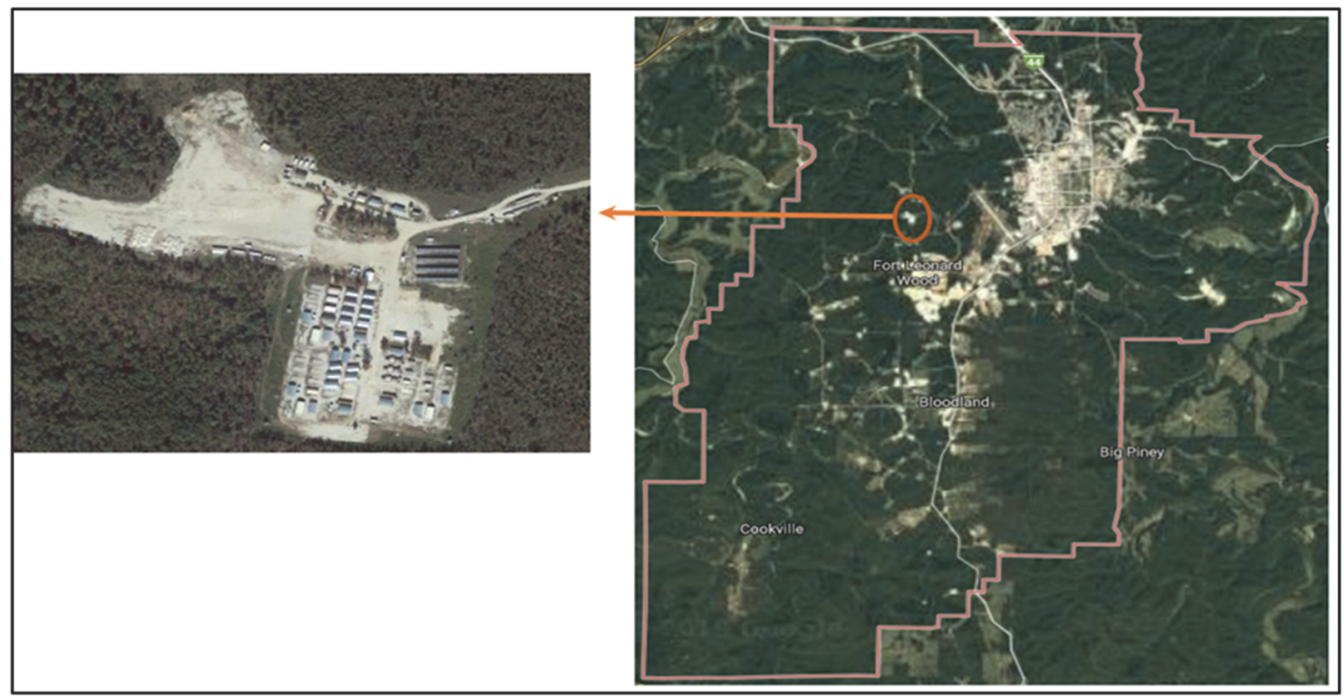

Similar to an FOB, equipment installations should be designed to accommodate a dusty environment as a byproduct from an unpaved site and the associated standing water resulting from normal rain conditions. The CBITEC site designated for the tactical microgrid contains approximately 19 acres of unpaved, substantially level ground. The dusty environment can be accommodated by selecting equipment enclosures that are properly rated for the expected operating environment by the National Electrical Manufacturers Association (NEMA).

At CBITEC, equipment installations are considered permanent if the installation includes a concrete foundation. Permanently installed equipment must acquire approvals beyond local CBITEC authority and require additional time and resources to pursue. Concrete pads placed on top of the ground, but which are portable, are not considered permanent installations and can be approved by CBITEC staff directly. Consequently, integration designs that do not use permanent installations are recommended as they might have a more efficient approval process.

Extensive engagement with staff at CBITEC; CERL; and Command, Control, Computers, Communications, Cyber, Intelligence, Surveillance and Reconnaissance (C5ISR) ultimately revealed that a formal set of microgrid design drawings is not available. While drawings of the tactical microgrid at CBITEC were also not available, information on a subset of the equipment 
to be used in the tactical microgrid was available and subsequently provided, including:

- Advanced Digital Control System (DCS) Operator's Manual; describing the parallel operation of the AMMPS generator to form a tactical microgrid

- DoD Interface Standard MIL-STD-TMS, TMS (Figure 14), version March 2018; an interface standard that is under development and has not yet been approved for release

- Army Technical Manual TM 9-6150-226-13, Operator and Field Maintenance Manual for distribution illuminations systems, electrical (DISE) and power distribution and illumination equipment (PDISE), version November 2008 (Figure 15); describing how to properly interconnect and use electrical generation sources and loads.

Figure 14. Tactical microgrid standard.

NOII: This dan, datod 23 Mrat 2018, propued by TMSC, has mot boen aproved and is subsed to modincesen DO NOT USE: PRIOR TO

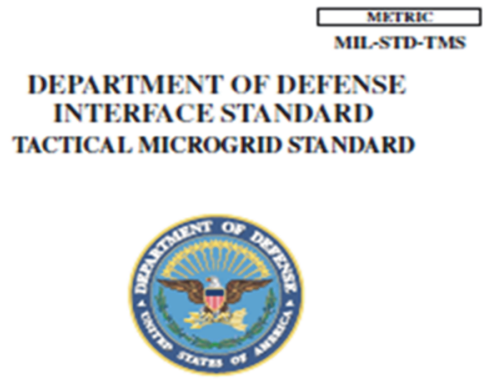

Figure 15. PDISE documentation.

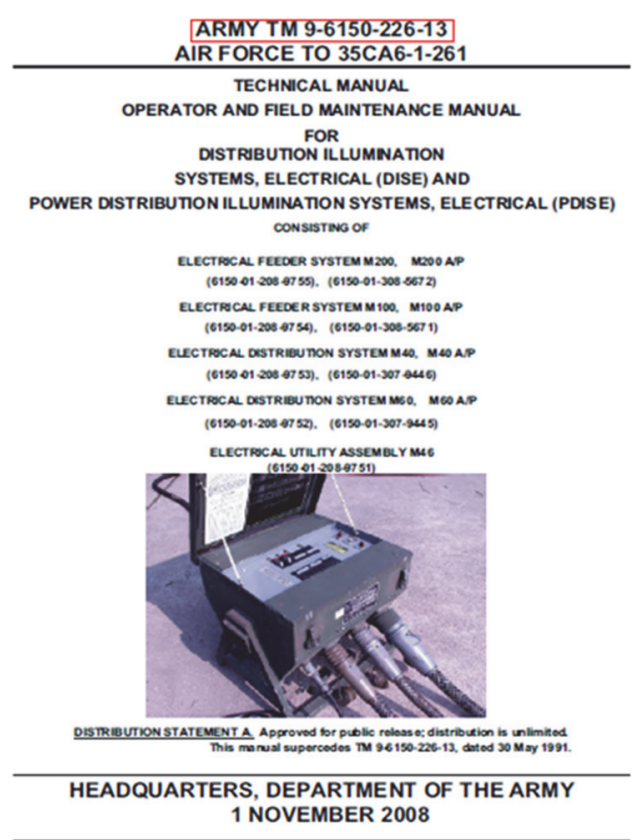


Paragon SMEs took additional steps to engage directly with vendors that have supported CERL's development and testing of legacy generators to form a tactical microgrid and acquire additional documentation and insight on the its operation, capabilities, and limitations. As a result of these engagement activities, four documents for the were developed by HumberGarrick Consulting Engineers (HG Engineers) for the government and were provided to Paragon SMEs:

- 80oA IPD Technical Manual

- IPD deployable metering and monitoring system (DMMS) User Manual

- TMS Generator Startup Procedure

- Verification of test results.

Paragon SMEs comprehensively engaged Schweitzer Engineering Laboratories (SEL) in this research given their role in the design of the controls for IPDs, test generator operation in a microgrid environment, and familiarity with the TMS and ongoing revisions. Information received from SEL included extensive discussion on existing components, the IPD design and the following documentation:

- Tactical microgrid Generator Test Procedure

- MIL-STD-TMS, TMS OMG Data Distribution Service (DDS) Implementation Guide.

On April 24, 2019, Paragon SMEs conducted a site visit to CBITEC to review available microgrid components and space to add additional equipment that would develop the living laboratory. Components of the tactical microgrid were cataloged as listed in Table 29.

Table 29. Catalog of microgrid equipment at CBITEC.

\begin{tabular}{|l|l|l|l|}
\hline No. & Component Description & \multicolumn{1}{|c|}{$\begin{array}{c}\text { Operational } \\
\text { Status }\end{array}$} & \multicolumn{1}{c|}{ Notes } \\
\hline 1 & $\begin{array}{l}\text { PDISE distribution center } \\
(100 \mathrm{~A})\end{array}$ & Available & PDISE power distribution box \\
\hline 2 & $\begin{array}{l}\text { PDISE distribution center } \\
(200 \mathrm{~A})\end{array}$ & Available & PDISE power distribution box \\
\hline 3 & $\begin{array}{l}\text { M100 distribution cables } \\
(100 \mathrm{~A})\end{array}$ & Available & PDISE power distribution cables \\
\hline 4 & $\begin{array}{l}\text { M200 distribution cables } \\
(100 \mathrm{~A})\end{array}$ & Available & PDISE power distribution cables \\
\hline
\end{tabular}




\begin{tabular}{|l|l|l|l|}
\hline No. & Component Description & \multicolumn{1}{|c|}{$\begin{array}{c}\text { Operational } \\
\text { Status }\end{array}$} & \multicolumn{1}{c|}{ Notes } \\
\hline 5 & Site electrical loads & Available & ECUs, lighting, laundry on site \\
\hline 6 & Test loads & Available & $\begin{array}{l}\text { 60-kW (minimum) three-phase resistive and reactive load banks } \\
\text { to be provided as needed }\end{array}$ \\
\hline 7 & AMMPS generation $(60 \mathrm{~W})$ & Available & Cummins generator \\
\hline 8 & Lithium-ion ESS $(40 \mathrm{kWh)}$ & Not operational & Might be removed \\
\hline 9 & $\begin{array}{l}\text { Flow battery energy storage } \\
\text { (300 kWh) }\end{array}$ & Not operational & Might be removed \\
\hline 10 & Lead acid energy storage & Not operational & Will be removed \\
\hline 11 & PV array (100 kW) & Not operational & (4) 25-kW inverters need to be upgraded \\
\hline 12 & IPD box (200A) & Available & Test units made by HG Engineers \\
\hline 13 & IPD box (800A) & Available & Test units made by HG Engineers \\
\hline 14 & Metering stations & Available & Existing deployable metering and monitoring system (DMMS) \\
\hline 15 & Microgrid controller & In development & Being developed by C5ISR \\
\hline 16 & Dashboard & Multiple & Investigating each for applicability/capability for a living laboratory \\
\hline
\end{tabular}

CBITEC staff shared that the site electrical loads consisted of numerous Billeting huts that each contain indoor lighting, convenience outlets, and an Environmental Conditioning Unit (ECU). The ECU could potentially serve as a 6.2-kW load when in air-conditioning mode or a 10.2-kW load when operating in heating mode.

In total, Billeting huts could provide approximately $200 \mathrm{~kW}$ of load for testing purposes of the tactical microgrid. Photos of a typical Billeting hut and ECU at the CBITEC site are shown in Figures 16 and 17.

Generation sources at the CBITEC site include the AMMPS 60-kW generator (Figure 18). A total of six AMMPS (6o kW) generators are expected to be available to support up to $360 \mathrm{~kW}$ of electrical load as well as the testing and interoperability evaluation of multiple generation sources on the tactical microgrid. Because the AMMPS generator is rated for three-phase, 208 volts of $\mathrm{AC}$ (VAC) at $60 \mathrm{~Hz}$, and has been selected by CERL to be the generator to serve as the backbone of the tactical microgrid, other generation sources or loads must also be rated or configured for $208 \mathrm{VAC}, 6 \mathrm{o} \mathrm{Hz}$ operation. To achieve this, appropriate power conversion devices might be required because commercially developed inverters over $10 \mathrm{~kW}$ or threephase are predominantly $480 \mathrm{~V}$.

Also located at CBITEC is a ground-mounted PV array (Figure 19) consisting of four, $25-\mathrm{kW}$ inverters with a total output capacity of $100 \mathrm{~kW}$. At the 
time of the site visit, CBITEC personnel indicated the PV array inverters were expected to be upgraded. Consequently, the array is not fully functioning. Timing of the upgrade has yet to be determined.

Figure 16. Billeting huts at CBITEC.

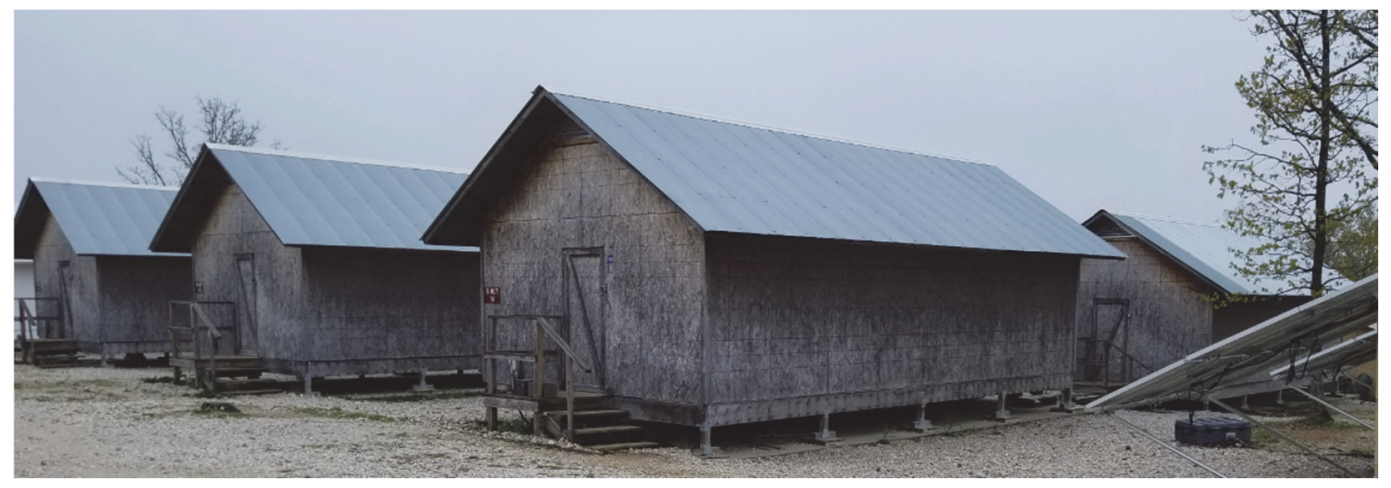

Figure 17. ECU at CBITEC.

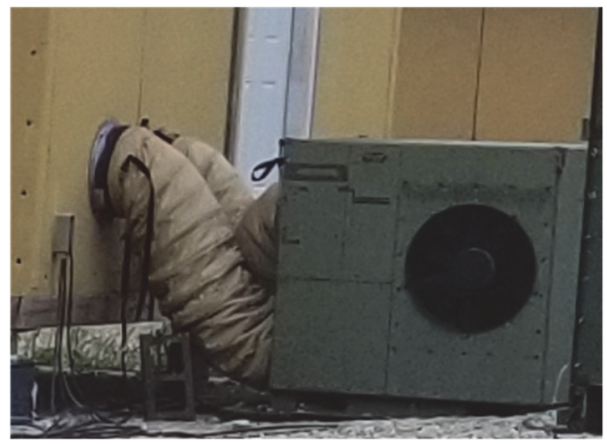

Figure 18. $60 \mathrm{~kW}$ AMMPS generator.

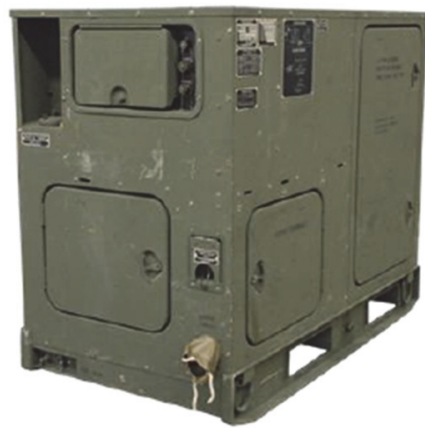

Figure 19. PV array at CBITEC.

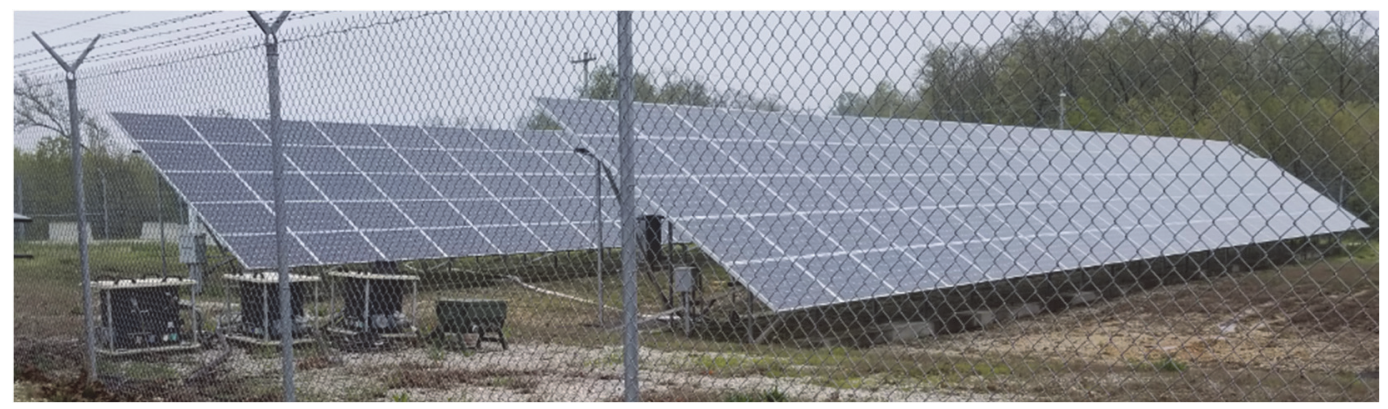

Two containerized ESSs are located at the CBITEC site. One is a $300-\mathrm{kWh}$ iron-flow battery system (Figure 20) provided by Energy Storage Systems, Inc. The other is a 40-kWh lithium-ion system (Figure 21) provided by EnerDel. Enerdel's Mobile Hybrid Power System (MHPS), which consists of two 20-kWh battery packs, is capable of charging or discharging at $30 \mathrm{~kW}$ and is rated for three-phase $208 \mathrm{VAC}, 60 \mathrm{~Hz}$. At the time of the site visit, 
the flow battery system was not operational, and repairs necessary to restore operation had not been scheduled. The MHPS was operational and available to support the tactical microgrid.

Figure 20. Flow battery system at CBITEC.

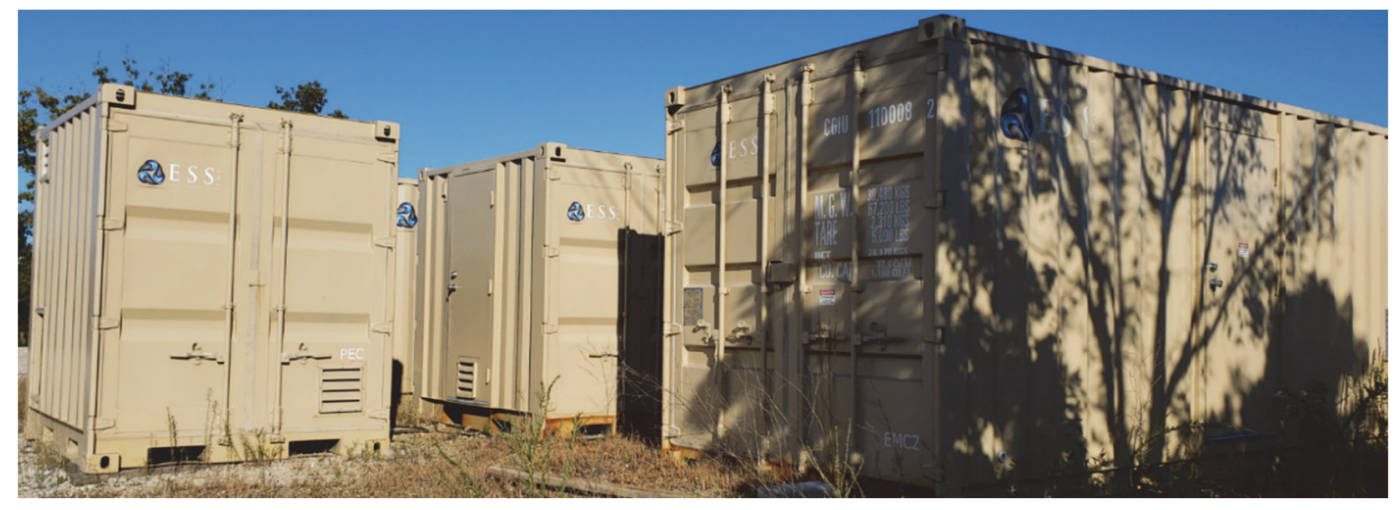

Figure 21. Enerdel $40 \mathrm{kWh}$ lithium-ion energy storage system.
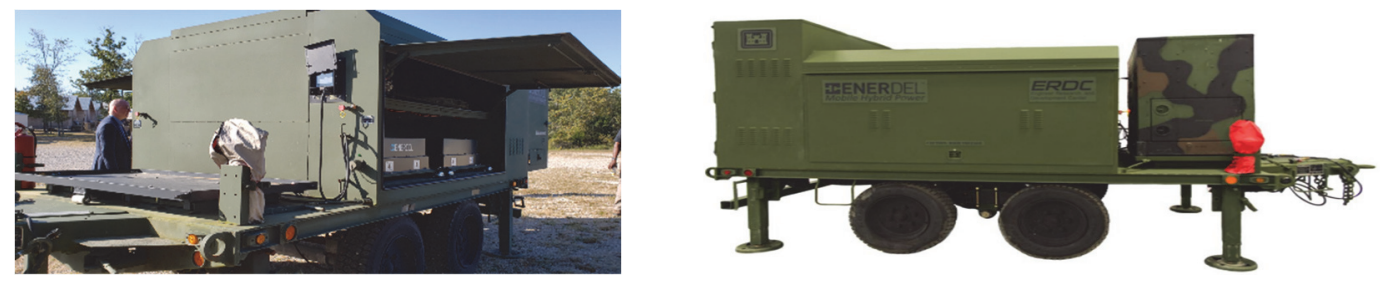

Properly interconnected generation sources and loads require properly rated conductors, power distribution boxes, and protective devices. Program of record equipment traditionally used to meet this need is the PDISE system (Figure 22), which includes conductors and power distribution boxes with protective devices designed to connect multiple loads to a generation source. The PDISE distribution boxes are not intended to connect multiple generators or equipment with bidirectional power flow nor to monitor electricity. The PDISE protective scheme solely consists of circuit breakers and does not contain any metering or sensoring to monitor power or quality, nor is there synchronization as required to establish ring connections. 
Figure 22. PDISE equipment at CBITEC.

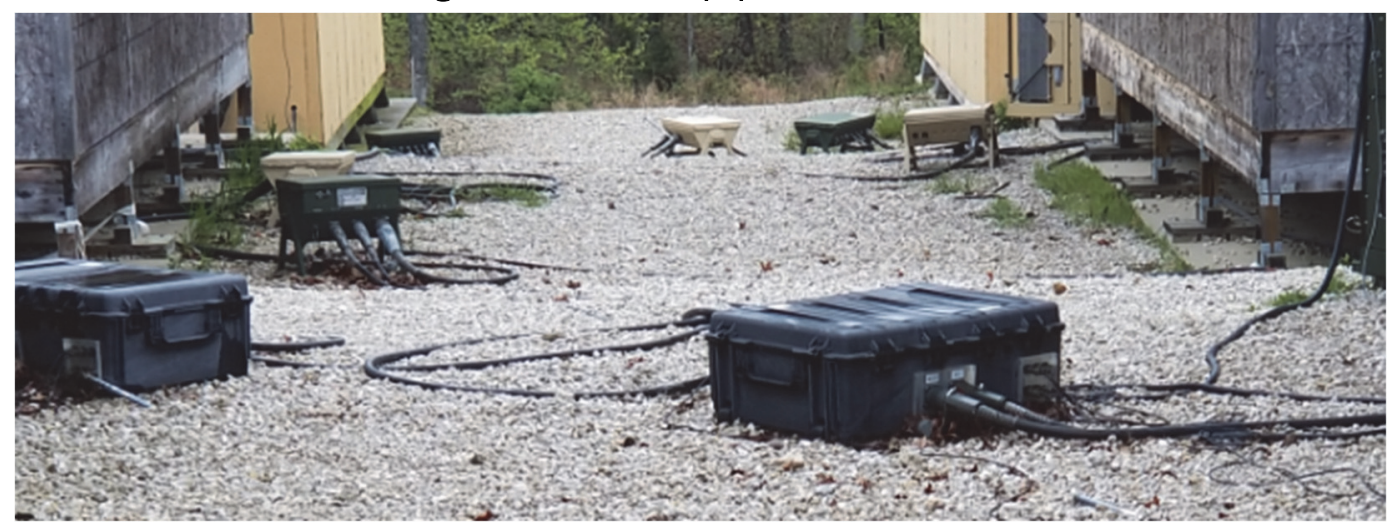

CERL, after recognizing the need to use IPD equipment for monitoring and managing power flow, contracted HG Engineers to develop the IPD (Figure 23). The IPD design specifically addresses the intent to achieve energy resiliency by incorporating:

- Multiple ports to interconnect multiple generators

- Two ports to establish a ring distribution system

- Meters on each port to monitor, on both a system and equipment level, real-time electricity flow-including voltage, frequency, current, power, and power quality

- Synchronization on the ring-bus ports for multiple sources

- Data recording for root-cause analysis of system disturbances.

The IPD included a real-time automation controller (RTAC) and controllable devices providing both intelligence and additional control of the IPD-connected device (Figure 24). The IPD includes a communications capability to interact with an principal microgrid controller via Ethernet RJ45 connections, DDS TMS protocol per TMS as well as a Modbus Transmission Control Protocol/Internet Protocol (TCP) slave connection. With these features, the IPD quantifies system power demands, remaining generator capacity, and connects or disconnects specific devices, based on commands issued by the tactical microgrid controller to help ensure resiliency of mission critical loads. 
Figure 23. $800 \mathrm{~A}$ IPD.

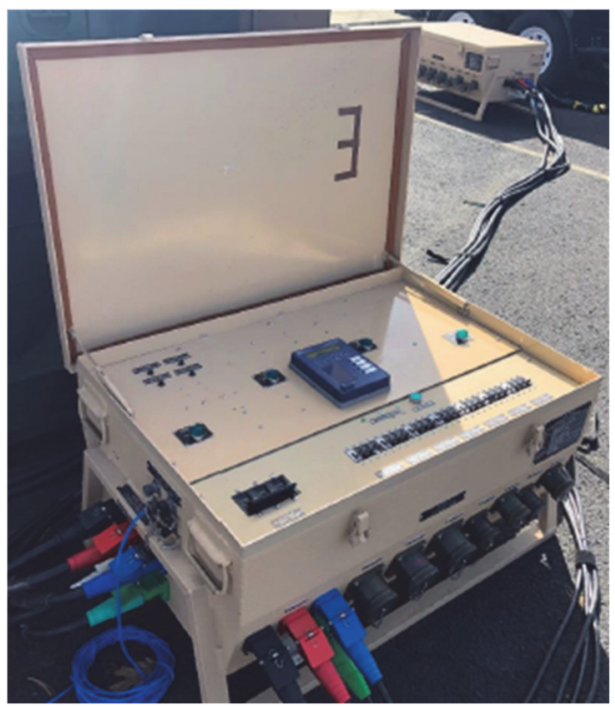

Figure 24. DMMS wireless access point.

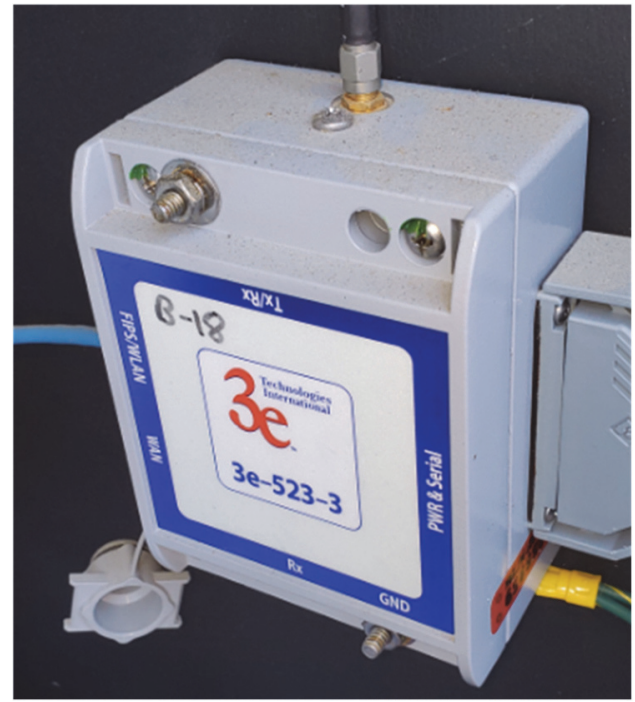

A second type of IPD is being developed now for potential use in a tactical microgrid by $\mathrm{C}_{5}$ ISR, located at Aberdeen Proving Ground in Maryland. In addtion, C5ISR is developing a microgrid controller that will provide overarching control of the tactical microgrid. Because the microgrid controller and the IPD are still in development, detailed information on these systems is not yet available to include in this report.

Currently, CBITEC's DMMS is used to wirelessly communicates with microgrid devices via a local dashboard. The DMMS only collects data is used for data collection only and does not control any of the microgrid devices. The AirGuard 3e-523 from 3e Technologies International serves provides the connection point between equipment and the CBITEC wireless local area network (WLAN) - forming a wireless mesh network at CBITEC. The DMMS allows data collection without the need for installing communications conductors.

A dashboard located in the CBITEC local office monitors equipment operations and collects data. This dashboard interface, developed by Pacific Northwest National Laboratory (PNNL), provides moderate- and high-level monitoring of system voltages and loads it collects every one to 3 seconds. A knowledgeable user can create a data collection profile on the DMMS dashboard as well as configure the operator interface to display data in different graphical forms. 
By policy, distribution equipment connected to the tactical microgrid must be manually operated when conducting tests and cannot be controlled through the dashboard. C5ISR is also developing a dashboard for monitoring a microgrid; however documentation related to it was unavailable to be included in this report.

\subsubsection{Cyber security requirements}

At the time of this research, the government did not provide the requested list of cyber security requirements beyond standard Army Risk Management Framework (RMF) procedures. However, it has stated the DMMS wireless access points (Figure 24) have been approved from a cyber security standpoint for integrating devices into CBITEC's wireless mesh communications network, which is used to monitor and collect data. The $3 \mathrm{e}-$ 523-3 wireless access points, manufactured by $3 \mathrm{e}$ TI Technology International, is the established wireless mesh network at CBITEC. This network provides sufficient cyber security and is approved for use on a Secret Internet Protocol Router Network (SIPRNet). Adding the V2G station to the tactical microgrid will not create an additional cyber security risk because it does not require specific hardware, or network architecture, to establish communications and can easily integrate within the approved DMMS network architecture.

\subsubsection{V2G operational requirements in a tactical environment}

Given CBITEC's vision to replicate the operating environment of an FOB, V2G operational requirements in a tactical microgrid mirror those identified at CBITEC. Many host nation agreements do not allow permanent structure installation, which prompts using concrete pads, or incorporating a structural frame suitable for direct earth contact as the equipment base, which is done with the AMMPS generators.

The AMMPS generator is used by the Army for providing electricity at FOBs. The AMMPS is availble in five sizes ranging from 5-60 kW. Each generator size is available in two models according to the frequency rating: $50 / 60 \mathrm{~Hz}$ or $400 \mathrm{~Hz}$. Each generator is capable of providing multiple voltages three-phase power rated at 120/208 VAC or 240/416 VAC. CBITEC and CERL staff have identified the operaing voltage and frequency for this effort to be $120 / 208 \mathrm{VAC}, 60 \mathrm{~Hz}$. Legacy generators, the 
desire to interconnect with host nation power, and specific load ratings, might require a different voltage and frequency rating.

Specific operating requirements will be documented in the TMS, which the Army is developing now, are not currently approved for release. A draft version on March 23, 2018 indicates this standard will establish both general and detailed requirements related to: interoperabilty, safety, cybersecurity, system architecture, communication interfaces, communication requirements, and electrical connections.

Drawings of the communications system at CBITEC were not available at the time of this report. Feedback from CBITEC and CERL staff indicates the tactical microgrid can use the developed TMS or Modbus protocols to communicate from the microgrid controller to all of the microgrid devices, including the system dashboard. This communication protocol will be used both over Ethernet and the wireless mesh network established by the 3e-523-3 wireless access points manufactured by 3 e TI Technology International.

The Modbus memory map for monitoring and controlling the V2G charger is provided in Appendix C. A high-level roadmap to implement $\mathrm{V}_{2} \mathrm{G}$ operations in the tactical microgrid consists of:

- Hardware installation

- Install V2G charger, step-down transformer, reference generators, loads, IPD, and interconnecting cables to form microgrid

- Commission installed equipment

- V2G charger configuration

- Configure voltage and frequency settings to avoid nuisance trips

- Establish charger communications

- Install and configure wireless access points for data collection

- Install wired communications per microgrid communications design

- Update dashboard for data collection

- IPD configuration

- Configure protective per connected equipment ratings and planned operation

- Configure communications for data collection

- Microgrid controller development 
- Identify desired control capabilities

- Develop control scheme/algorithms

- Develop communications strategy to meet monitoring and control requirements

- Install and commission microgrid controller

- System commissioning

- Verify communications

- Verify safety mechanisms/e-stops

- Develop and conduct test plans to test equipment operation/control

- Develop and conduct test plans to test system operation/control.

\subsection{Identify V2G solutions available}

The objective of this research is to identify available V2G charging solutions that can be used to apply bidirectional charging and discharging capability when integrating an EV within the tactical microgrid. Paragon SMEs researched solutions best equipped to meet the requirements outlined in Section 2. Additionally, Paragon researchers developed a list of pros. and cons. for diesel generators, and controller technologies that are potentially suitable to be used for military applications.

\subsubsection{V2G equipment and design information}

Chargers that are rated near $50 \mathrm{~kW}$ are referred to as "fast chargers" and are specifically designed to quickly charge an EV. To quickly charge an EV, fast chargers use DC to charge the vehicle's battery. By permanently installing power conversion equipment offboard of the EV, DC charging reduces both the weight and space burdens power conversation equipment place on an EV. DC fast chargers are designed to operate at much greater power levels-typically 30-100 kW compared to $1.4-19 \mathrm{~kW}$ for AC "standard" charging. In the United States, there are three fast charging-standards that specify the communications and control between an EV and a fast charger:

- Tesla

- The Combined Charging System (CCS)

- CHAdeMO.

The Tesla fast chargers, which are not bidirectional, utilize closed and proprietary protocols. Consequently, Tesla fast chargers only work with Tesla 
EVs. In Europe, Tesla recently began testing Tesla EVs that can be charged with CCS stations, as these stations are Europe's most commonly used charging standard. European systems operate at different voltages and frequencies than systems in the United States, so the operating voltage and frequency of this solution needs to be modified before being deployed with equipment designed and manufactured for use in the United States.

The CCS standard, developed by the Society of Automotive Engineers (SAE), is the most commonly used fast-charging standard in the United States. This open standard is used by multiple charging station suppliers to charge CCS-compliant EVs (Figure 25). However, the CCS standard's does not incorporate communication and command structures necessary to implement are not compatible with EV on-demand discharging of EVs. Consequently, messaging between the vehicles and chargers must be customized to include unique commands enabling a V2G-capable vehicle to use the CCS standard. This customization inherently prevents the custom charger to be readily used for multiple vehicle types. Such customization also requires extensive non-recurring engineering, development, and testing to implement the $V_{2} G$ capability, which further limits customized CCS solutions for $\mathrm{V}_{2} \mathrm{G}$ applications. By 2025, SAE plans to update the CCS standard to include communication and controls specifications necessary to enable $\mathrm{V}_{2} \mathrm{G}$ operations between CCS-compliant vehicles and charging stations.

Figure 25. SAE CCS connector.
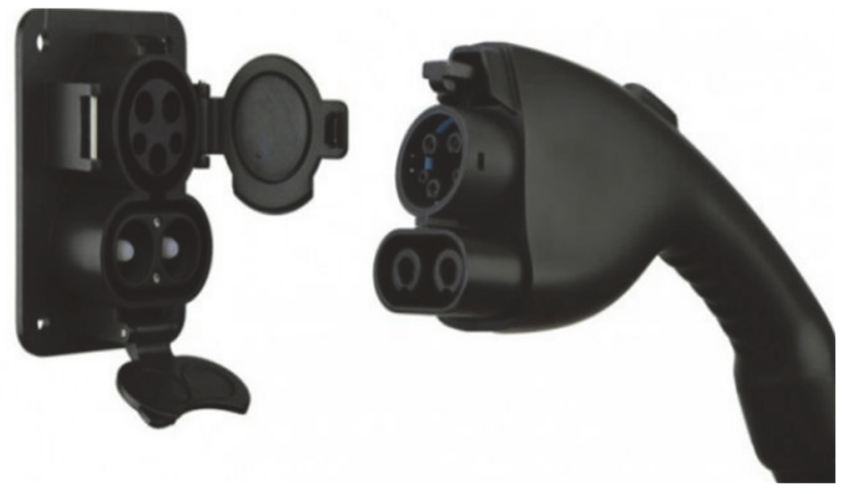

The CHAdeMO standard (Figure 26) is not only commonly used in Asia but also the only open standard that fully accommodates V2G applications. Using the CHAdeMO standard in the United States, however, is limited compared to the SAE CCS standard for fast-charging applications. 
Figure 26. CHAdeMO connector.

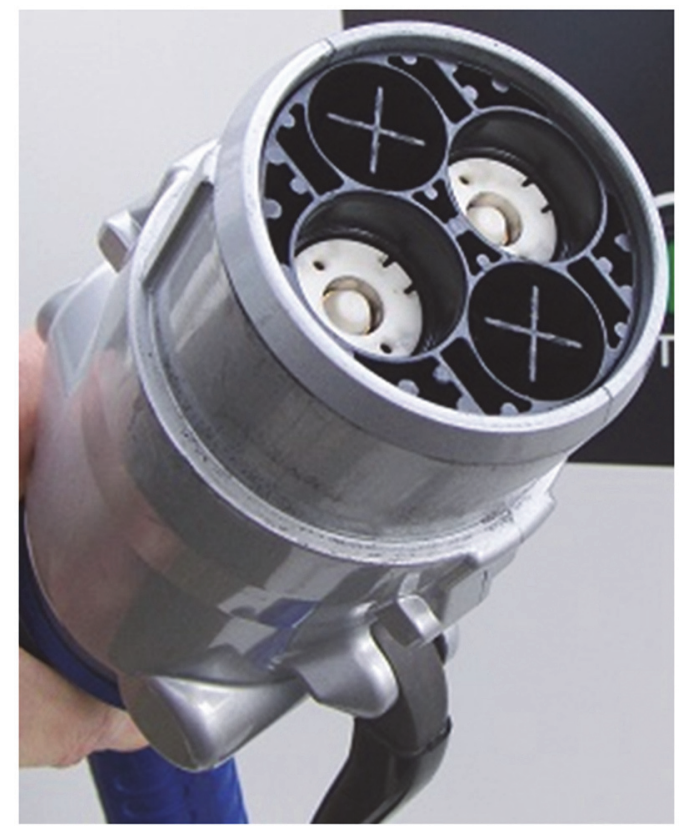

Paragon SMEs identified only two vehicles in the United States available for purchase with the CHAdeMO fast-charging option: the Mitsubishi Outlander and the Nissan LEAF. Because the Outlander is a PHEV, it comes with a battery pack that has a comparatively small energy- storage capacity-, $12 \mathrm{kWh}-$, compared to the LEAF, which is a fully EV with a much larger lithium-ion battery pack in two energy storage capacity options-40 $\mathrm{kWh}$ and $62 \mathrm{kWh}$.

In addition to ensuring compatible communications between the EV and related bidirectional charging equipment, the $\mathrm{V}_{2} \mathrm{G}$ charging equipment must also be able to communicate with an external controller. This ability enables V2G system monitoring and control. Important V2G system information to monitor includes:

- V2G system health (equipment alarms, faults, battery pack temperature)

- Operating status (available power to charge and discharge)

- Battery pack systems on chip (SOC)

- Present rates of charge and discharge, operating mode

- Capable rates of charge and discharge

- Charge and discharge setpoints. 
Through the V2G charger, EV information is accessed and does not normally require additional communications connection between the $\mathrm{EV}$ and external controller. External controllers, often capable of different communication protocols, are typically limited to those options available with the V2G charger. Protocol converters can also establish communications if the selected external controller and $\mathrm{V}_{2} \mathrm{G}$ charger cannot accommodate the same communications protocol.

Most V2G fast chargers operate with the electrical grid as opposed to stand-alone systems. Consequently, they are often certified to be compliant with electrical utilities' required specifications. A very common certification is the Underwriters Laboratories (UL) 1741, Standard for Inverters, Converters, Controllers and Interconnection System Equipment for Use with Distributed Energy Resources. This UL Standard specifies when generation sources, including V2G systems, will disconnect from the electrical grid based on system voltage and frequency deviations beyond established limits.

The standard's intent is that generation sources will disconnect when a grid disturbance is detected so that repairs can be safely made without the potential for a V2G system to back-feed the grid, which is dangerous to repair personnel. This operating requirement might pose an operational challenge for $\mathrm{V} 2 \mathrm{G}$ chargers that are UL1741 compliant as the voltage and frequency stability of a tactical microgrid could be inconsistent from that of the commercial electrical grid. Lightly loaded generators frequently deviate from voltage and frequency operating targets. Because of this, V2G chargers can disconnect from the tactical microgrid when constrained to UL1741 operating limits, even though a fluctuating voltage and frequency does not indicate a microgrid disturbance. Implementing a V2G charger in a tactical microgrid should provide voltage and frequency trip levels adjustment, accommodating normal operating conditions associated with tactical microgrid power generation sources. 


\subsubsection{Design criteria for integration of a charging station into a tactical microgrid}

CBITEC and CERL staff identified integration requirements that will:

- Integrate into the 208-VAC, 60-Hz tactical microgrid electrical distribution system

- Communicate with the DMMS

- Charge, or discharge, at a minimum of $50 \mathrm{~kW}$.

The microgrid controller, which C5ISR is developing, will control the charging station. Communication from the microgrid controller to the charging station is expected to use Modbus TCP/IP protocol.

\subsubsection{Conceptual design of V2G charging station integration}

The $\mathrm{V} 2 \mathrm{G}$ charging station should provide three-phase power to accommodate integration design criteria. This will ensure a balanced generation and consumption of electricity for the $\mathrm{V} 2 \mathrm{G}$ system that supports electrical distribution system stability. Fast chargers are designed to charge EVs between 200-600 volts of DC (VDC) and are typically supplied by threephase, 480 VAC. For 208- VAC tactical microgrid integration, a properly sized transformer needs to be installed if the selected fast charger cannot be operated at 208 VAC. Because the Coritech fast charger does not use a neutral connection, the delta side of the transformer could be connected to the charger. As this configuration is an ungrounded system that can result in excessive floating voltages, the manufacturer recommends installing a grounding transformer between the charger and the step-down transformer instead of using a floating-delta connection.

Alternatively, the manufacturer advises a delta connection on the 4-wire 208 wye distribution system and grounded 3-wire wye connection on the $48 \mathrm{o}-\mathrm{VAC}$ connection to the charger can also be used instead, avoiding the cost associated with adding the grounding transformer. Because the charger does not use a neutral, the wye neutral is not connected to the charger and only connects to the equipment ground. Figure 27 shows the charging station integration's conceptual design. 
Figure 27. Conceptual integration design.

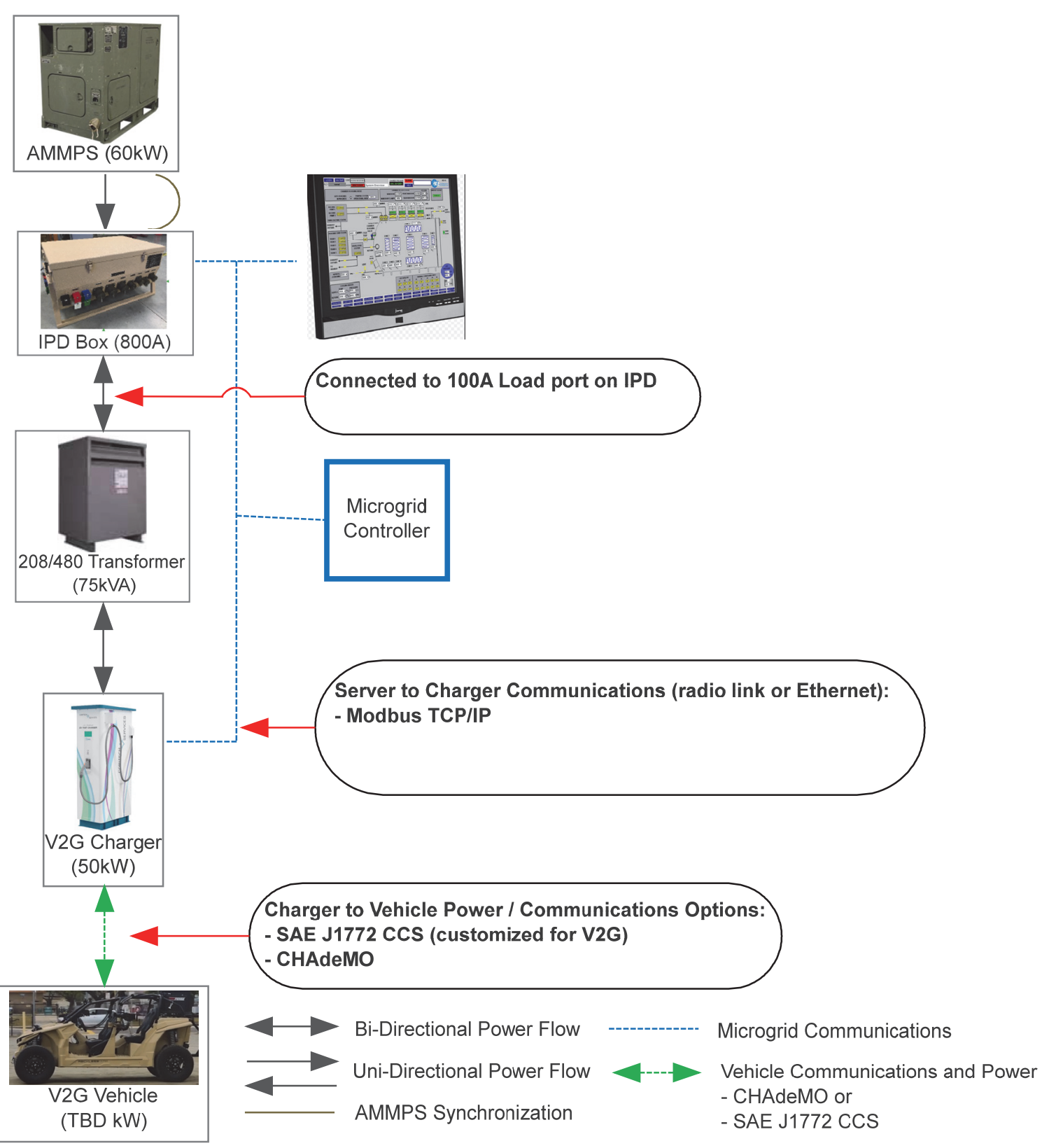

\subsubsection{Available V2G charging stations that meet performance requirements}

Paragon SMEs performed a market search for commercial-off-the-shelf (COTS) V2G chargers designed to operate in the United States. After an extensive search, the only supplier providing COTS V2G chargers is Coritech Services, Inc. from Royal Oak, MI. Coritech Services offers V2G chargers (Figure 28) in either a $30-\mathrm{kW}$ or $60-\mathrm{kW}$ power rating with the 
option of either an SAE CCS or CHAdeMO communication standard. It is noteworthy that a V2G charger ordered to operate according to the SAE CCS standard still requires additional engineering services to develop and implement additional communications messaging for the selected vehicle until SAE updates the CCS standard to include V2G operations.

Figure 28. Coritech V2G fast-charging station.

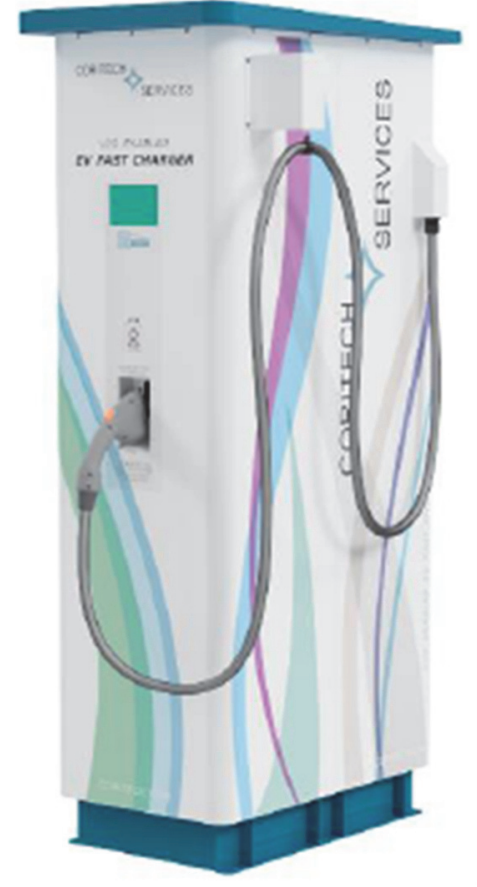

During the market search for COTS suppliers of V2G fast-charging stations, Paragon SMEs identified several power conversion equipment vendors. Each could produce a V2G fast-charging system by integrating the discreet components necessary to provide monitoring and control for charging and discharging a lithium-ion battery pack. Both Go Electric and EnerDel are experienced and can create custom systems, meeting specific fast-charging needs and operational requirements.

\subsubsection{Potentially suitable technologies}

A list of pros. and cons. for bidirectional chargers, diesel generators, and controller technologies that are considered potentially suitable for military applications is provided in Appendix N. This list catalogs both, suitable and not suitable, bidirectional chargers, diesel generators, and controller 
technologies assessed for military applicability, interoperability, cost, and plug-and-play capability.

\subsection{Simulate V2G charging in the tactical microgrid environment}

This research's objectives are to accommodate various "what-if" operational scenarios of the tactical microgrid and refine the $\mathrm{V}_{2} \mathrm{G}$ charging station integration's conceptual design, achieving performance requirements.

Numerous modeling packages were evaluated for being able to simulate power system components, conduct "what-if" scenarios, accommodate different configurations easily, and incorporate alternative and renewable energy sources. Initially, Paragon SMEs selected GINA ${ }^{\mathrm{TM}}$ as the appropriate modeling tool, given the technology can uniquely recognize system abnormalities in a real-time operating environment. After a material amount of model development using the GINA technology, the government expressed a desire for C5ISR to use the developed model for other supporting programs. To accommodate this preference, Paragon SMEs stopped developing the GINA modeling environment and began, again, using MATLAB/Simulink-because it a) met the above simulation criteria and b) is a tool DoD commonly uses, encouraging close collaboration with C5ISR, and other DoD stakeholders, to continually use the developed model. Table 30. provides a listing of the evaluated simulation tools.

Table 30. Simulation tools considered.

\begin{tabular}{|c|l|}
\hline No. & \multicolumn{1}{|c|}{ Simulation Tools Considered } \\
\hline 1 & Homer \\
\hline 2 & SimulationX \\
\hline 3 & MATLAB/Simulink \\
\hline 4 & COMSOL \\
\hline 5 & ANSYS \\
\hline 6 & Geli \\
\hline 7 & ENERGY TOOLBASE \\
\hline 8 & Energy Storage Valuation Tool (EVST) \\
\hline 9 & Paladin ${ }^{\text {Microgrid Power Management System }}$ TM \\
\hline 10 & SKM \\
\hline 11 & ETAP \\
\hline 12 & PSCAD \\
\hline 13 & PSPICE/OrCAD \\
\hline 14 & VISSIM \\
\hline 15 & PSIM \\
\hline
\end{tabular}




\begin{tabular}{|c|l|}
\hline No. & \multicolumn{1}{|c|}{ Simulation Tools Considered } \\
\hline 16 & SIMetrix/SIMPLIS \\
\hline 17 & SABER \\
\hline 18 & GINATM \\
\hline 19 & LTSpice $^{\text {TM }}$ \\
\hline
\end{tabular}

System model components are designed to enable or disable "on- the- fly" reconfiguration of the tactical microgrid simulation and "what-if" scenarios. In addition, MATLAB/Simulink can create scalable microgrid models. Each modeled component was selected to be manufacturer agnostic to evaluate a technology's energy resiliency support while avoiding the unnecessary complexity of specific manufacturer intricacies. Like the tactical microgrids it represents, the model is flexible, easy to configure, modular, and scalable.

At the time of this research, distribution boxes interconnecting multiple sources and loads have not been identified. However, given the model's simulating interoperability between sources and loads, the model does not need this detail until a full design of the tactical microgrid with specific components has commenced. At that time, specific conductors and protection schemes can be incorporated into the model.

\subsubsection{Develop a computer simulation platform}

After cataloging microgrid assets, SMEs prepared a conceptual design to construct a representative model and simulate performance. After several discussions with $\mathrm{C}_{5}$ ISR and CERL, one conceptual design of a tactical microgrid was developed, which consisted of six 60-kW AMMPS generators, three $50-k W$ V2G systems, and three critical loads connected together in a bus formation using IPD-style distribution boxes. Figure 29 shows the tactical microgrid initial conceptual design.

A tactical microgrid should be dynamic, scalable, modular, adaptable and controllable to ensure energy resiliency for mission critical loads. The tactical microgrid model was constructed to be technology focused and manufacturer agnostic. 


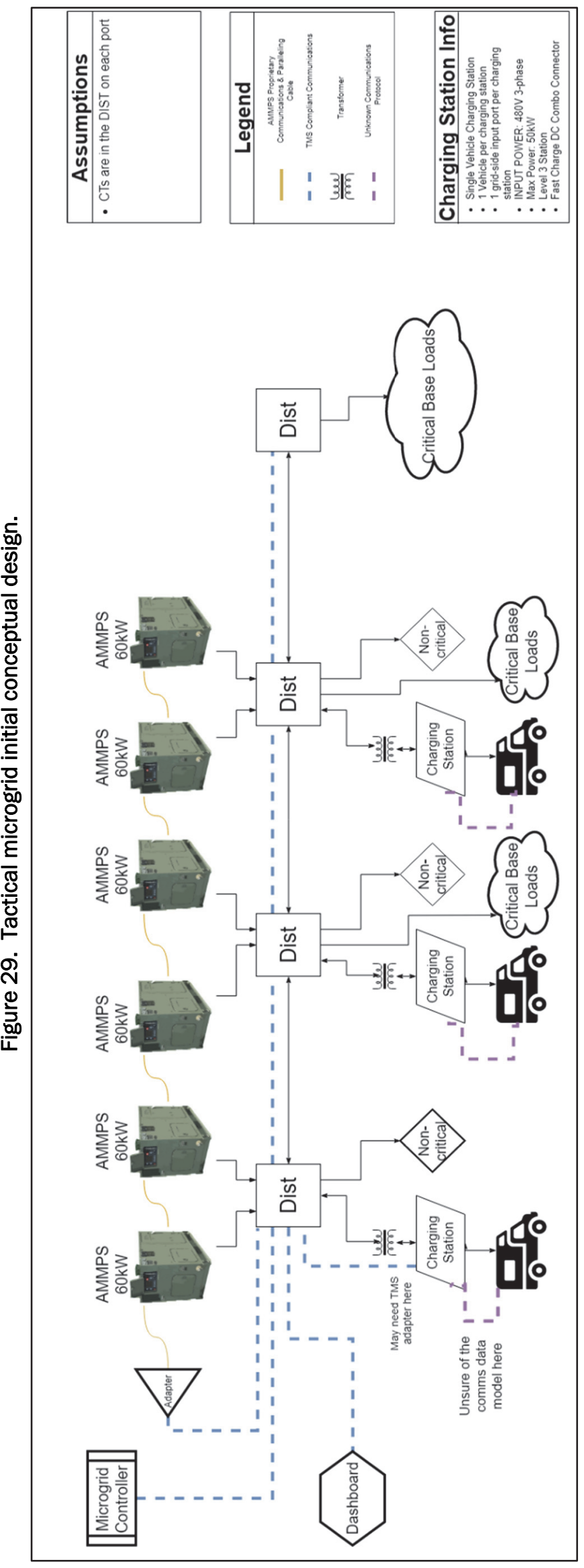


The model, which has expanded the initial conceptual design to include alternative-energy assets, is expected to be part of the living laboratory at CBITEC. The site includes a 100-kW PV array, a 40-kWh ESS, a threephase load bank, and the above referenced Billeting loads. Incorporating an ESS and a PV system into the model, with matching power and energy capabilities, enables representative model simulations to guide tactical microgrid integration and control design at CBITEC. In addition, the model can easily incorporate a utility connection to tactical microgrid simulations, which have options to connect to host nation power or local generation stations. Model components can be enabled or disabled to accommodate numerous configurations and "what-if" scenarios tactical microgrids will inevitably encounter. A system model of the tactical microgrid is shown in Figure 30.

Solar irradiance values drive the PV model, so power production for a specific location can be simulated. Both an efficiency factor, and the size of the array, were used to determine power output, ensuring a technology-focused model that reflects real-world conditions. For this model, irradiance data for FLW's closest location will be used. The PV model is shown in Figure 31 .

The energy storage model parameters reflect those of the onsite 40-kWh EnerDel MHPSs. The model has four quadrant capabilities to quantify real and reactive power. Upper- and lower-charge limits can be specified as well as recharge rates. The energy storage model can change in size easily meet different energy storage technology capacities. The ESS model is shown in Figure 32.

The system level model contains six diesel generator models simulating the six AMMPS generators to be installed at CBITEC. The generator model used the full- generator model, containing a synchronous generator, electronically fuel-injected diesel engine control unit governor, automatic voltage regulator (AVR), and excitation system to represent the dynamics of both the engine and the generator. This model (Figure 33) also allows each generator's voltage/vars and frequency/watts to be dispatched. 


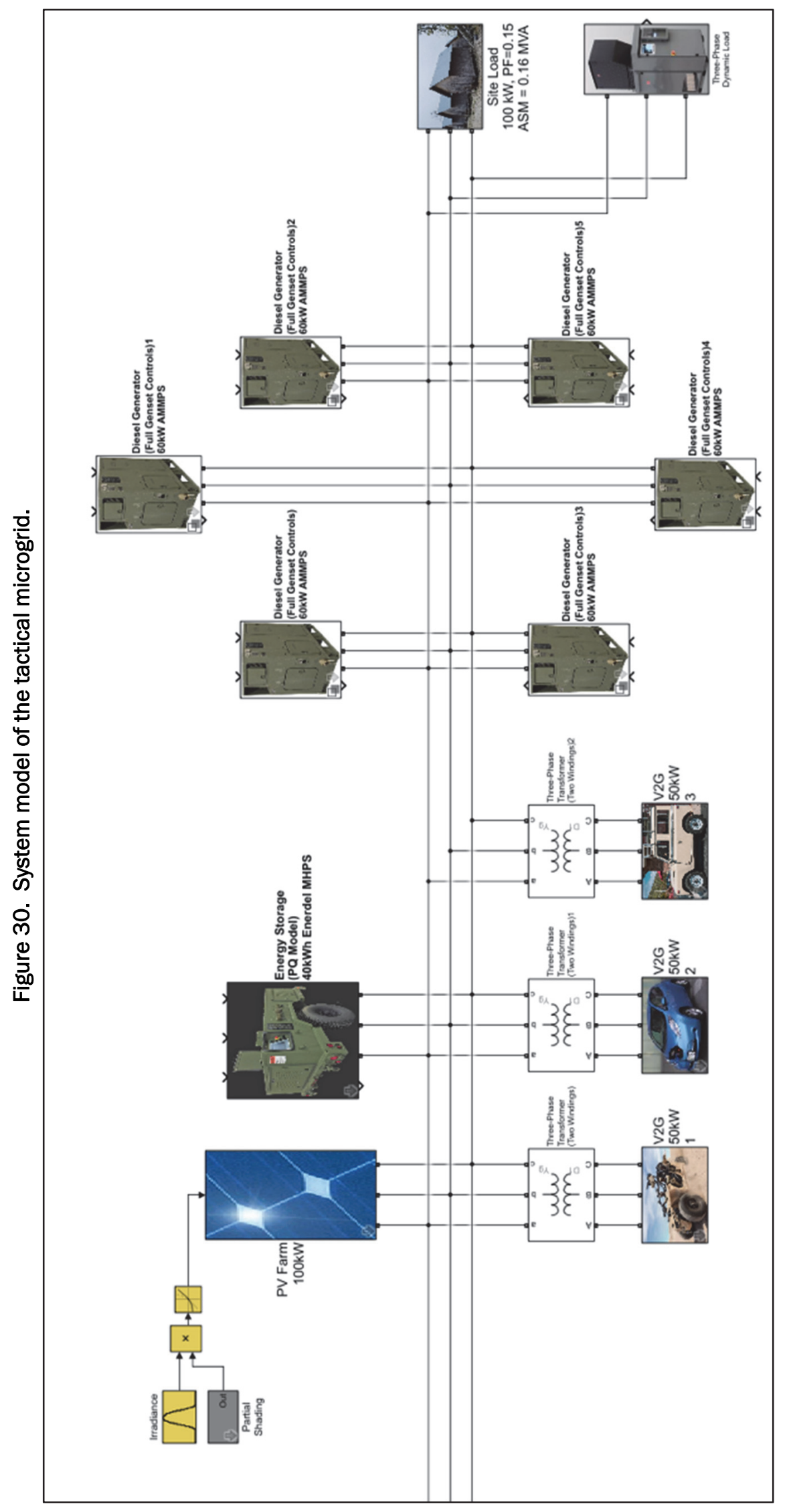




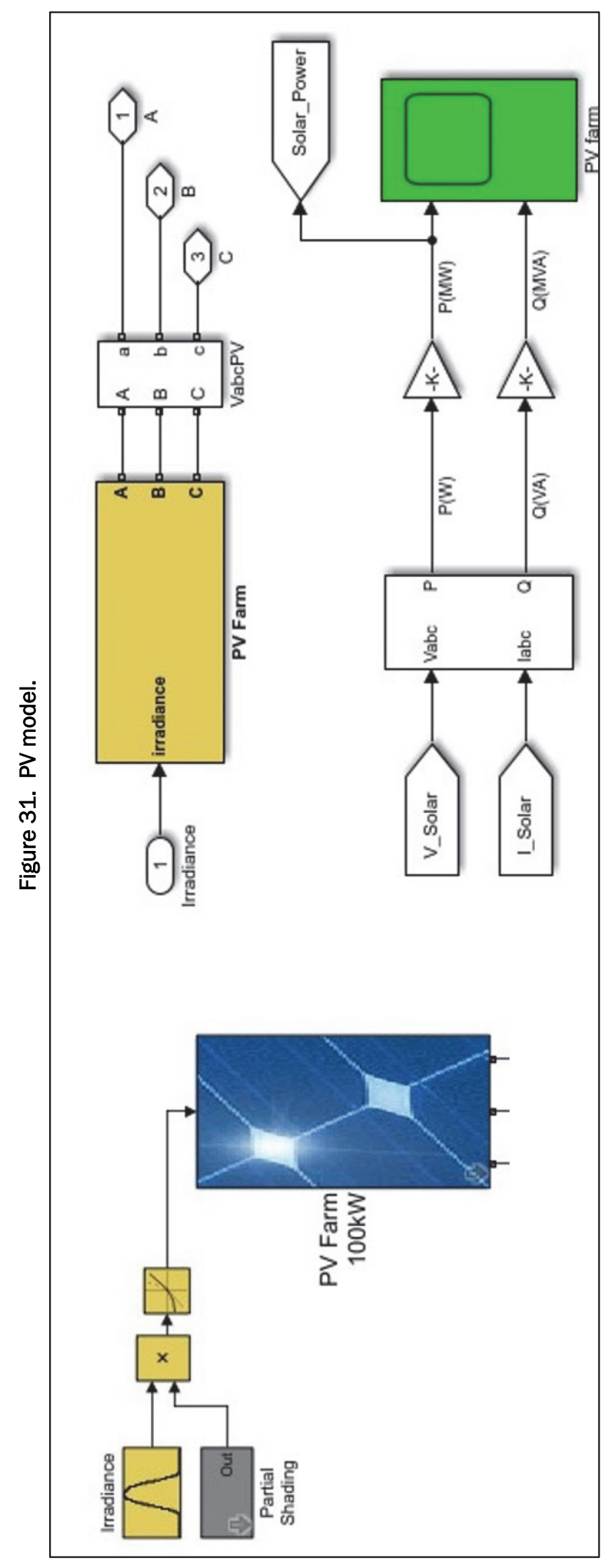




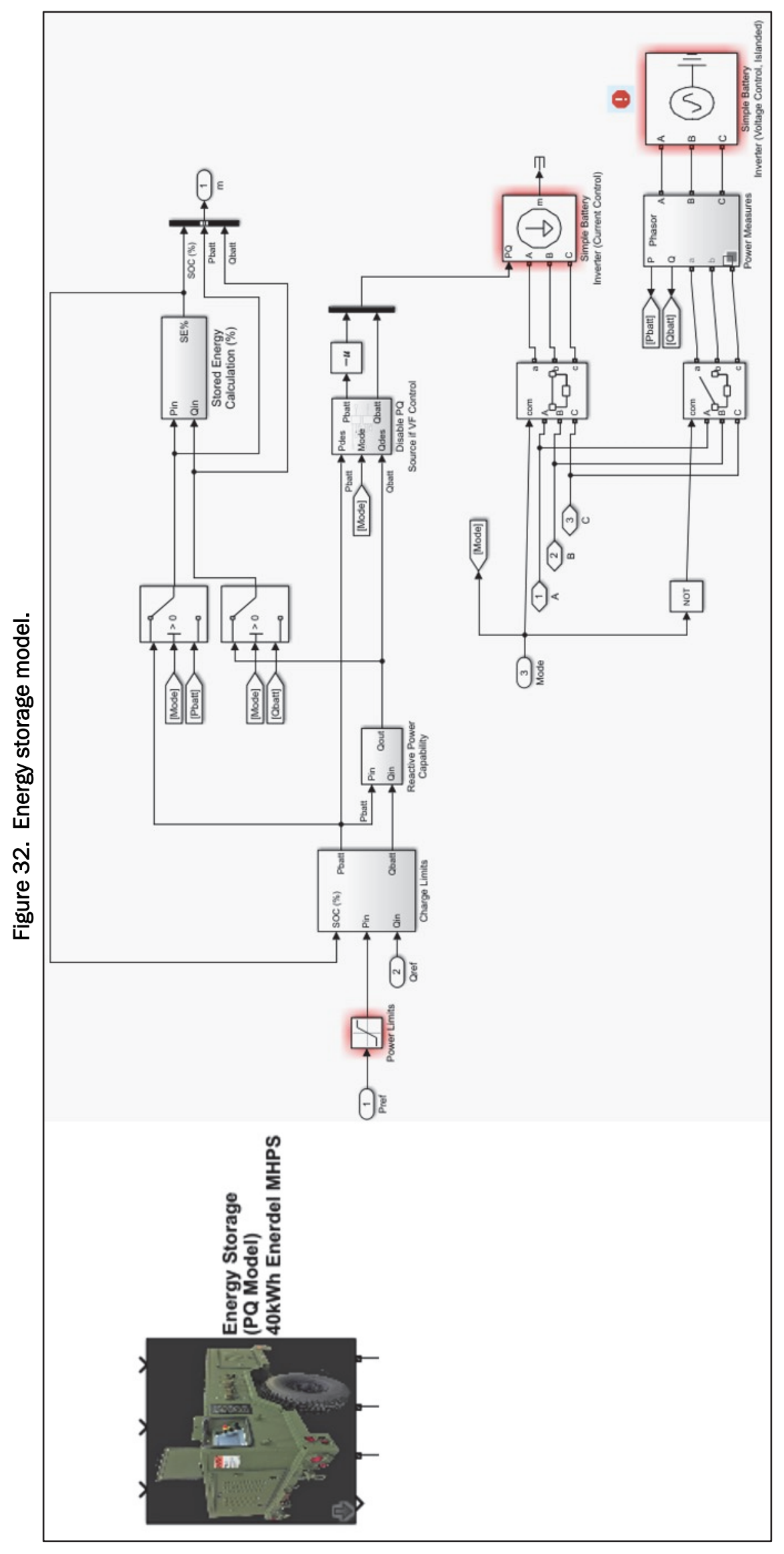




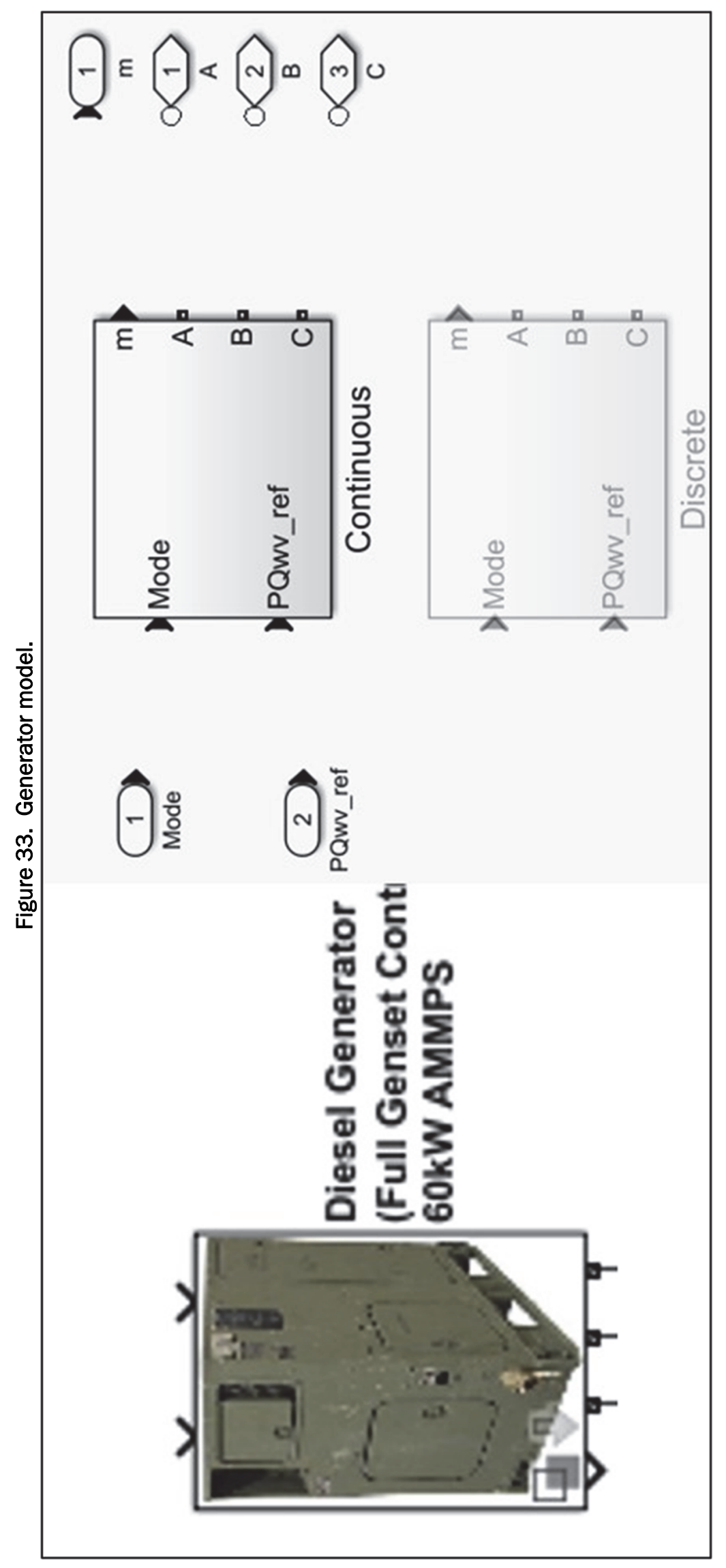


The system level model contains three $\mathrm{V} 2 \mathrm{G}$ components that can easily be reduced to one or be expanded to represent a fleet of EVs or additional lithium-ion battery storage systems. The model has a signal for control of charge or discharge for every V2G resource while monitoring power, current, and battery pack SOC. This enables total control of the resource to support generation and load requirements. Figure 34 shows the V2G component model.

Figure 34. V2G model.
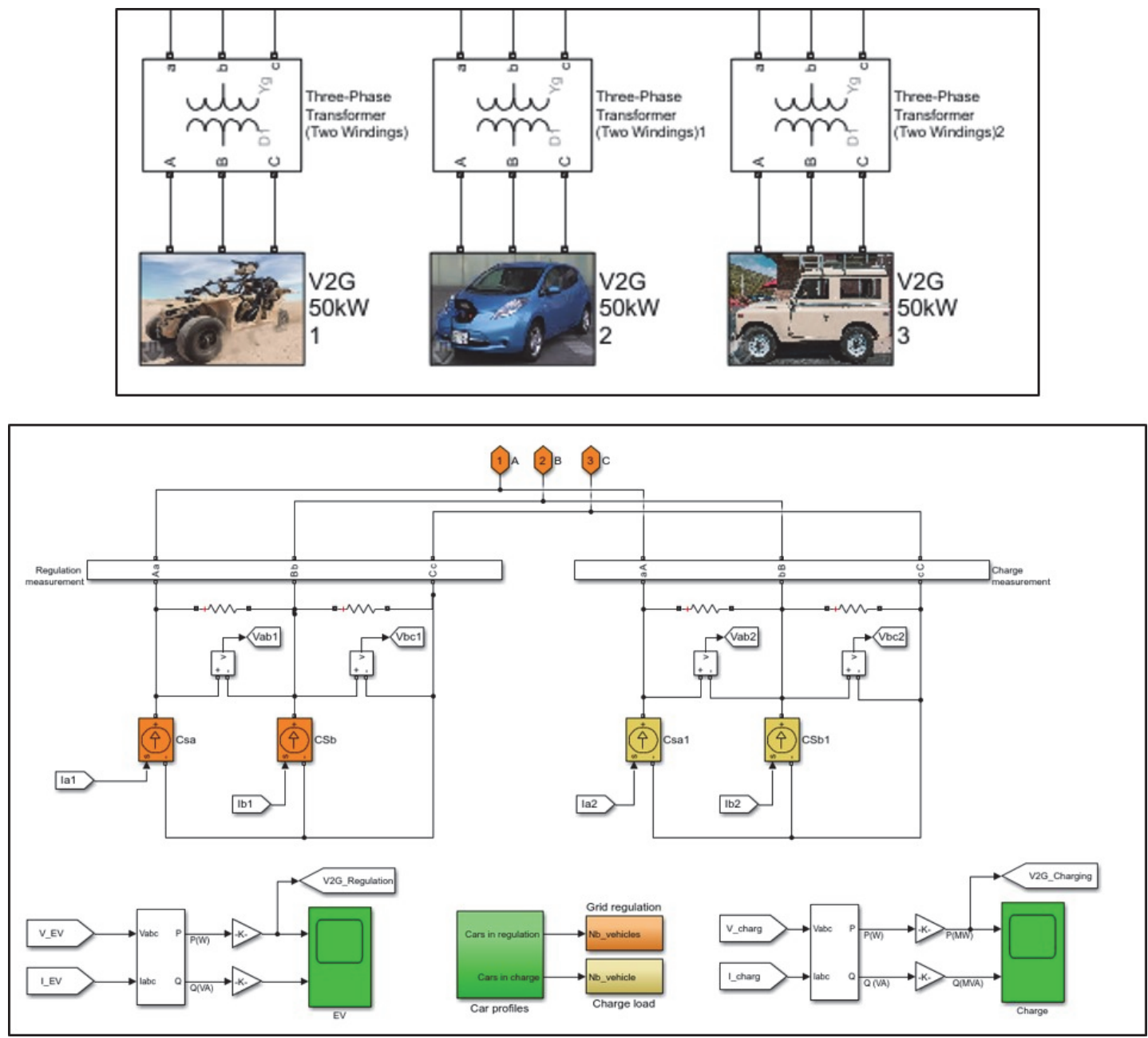

CBITEC site loads include the Billet huts and laundry facilities. The load model used contains both resistive loads as well as motor loads to represent the motors starting in-rush currents and heating operation of the ECUs feeding the Billets. The model is scalable and easily duplicated to reflect the Billet load as whole or each to disable individual huts for developing load shedding schemes. The site load model is shown in Figure 35. 


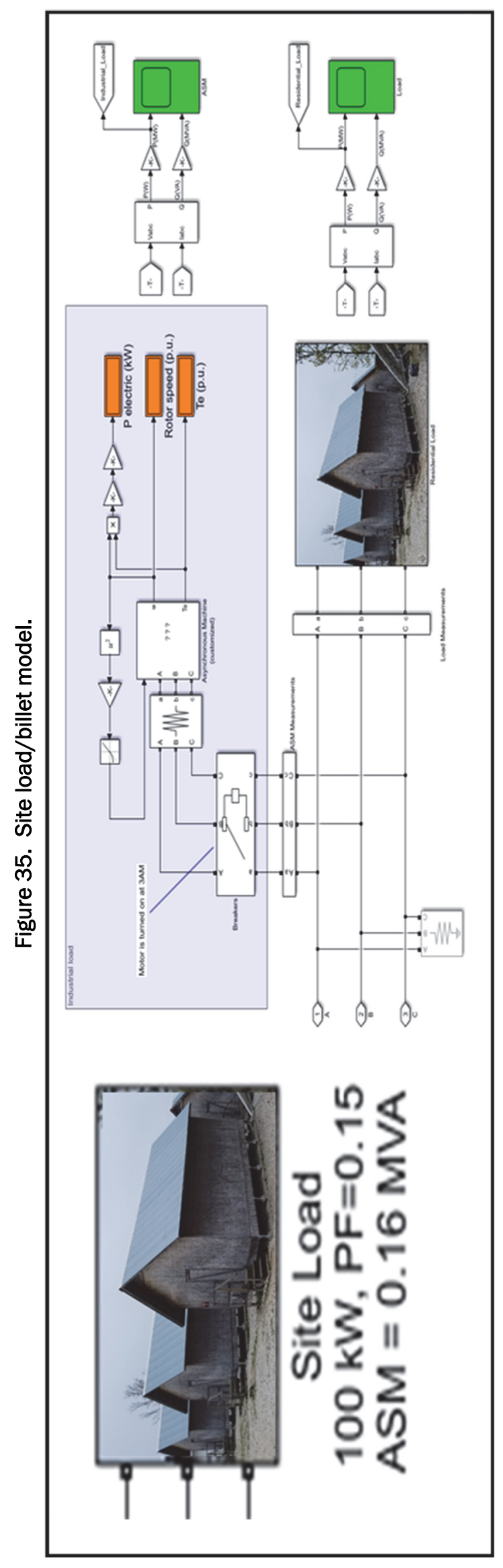


In addition to dynamic site loads, the system level model includes a threephase load bank that simulates future loads and possible configurations without needing associated equipment. The model, which can simulate real and reactive loads, is externally controlled by a reference signal. CBITEC plans on installing a load bank serving as part of the testing and evaluation capability of the living laboratory, which can be simulated by this model to support test plan development. Figure 36 shows a model of the dynamic load bank.

Figure 36. Three-phase load bank model.
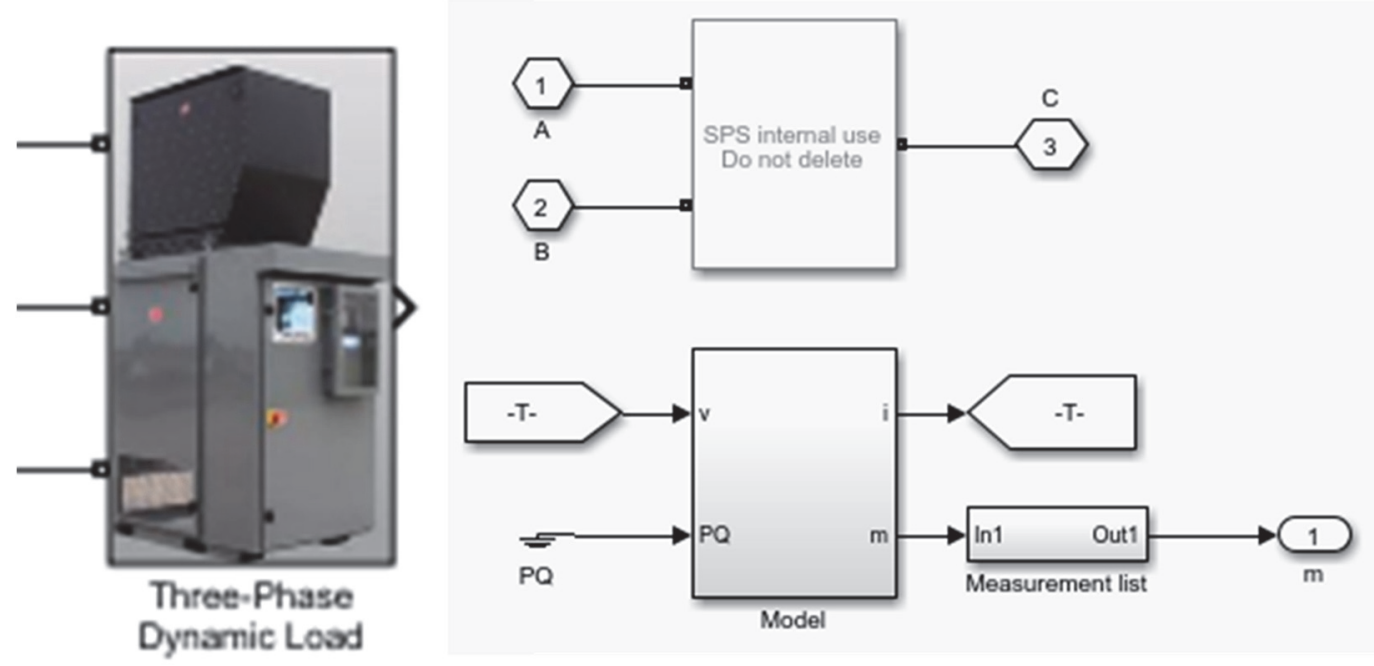

Construction of the model continues, and will be refined, through ongoing dialogue with and feedback from CERL, CBITEC, and C5ISR staff. Simulation activities will include "what-if" scenarios to establish the envisioned tactical microgrid's baseline performances and guide integration of $\mathrm{V} 2 \mathrm{G}$ components.

\subsubsection{Validation of the MATLAB microgrid computer simulation}

To establish the accuracy of any model, each component must be unit validated against known performance metrics. The second step is to establish accuracy of a model is to run the entire model with all components connected simultaneously. The following figures show this validation of the model. 
The following plots (Figures 37 to 39) show a single AMMPS 6o-kW generator increasing (at 1 second) and then decreasing (at 11 seconds) $30 \mathrm{~kW}$ of resistive and 10-kilovar (kVAr) inductive load. These ratios 10/30 indicate a power factor of 0.31 and worst-case scenario. This exercises both the ECU and AVR simultaneously on these electronically fuel-injected diesel engine generator sets. The response is within nominal operating behaviors for this type of generator.

The following plots (Figures 40 to 42) show six AMMPS 60-kW generators paralleled via their isochronous load-sharing lines. The load applied is the taking on (at 1 second) and then relieved (at 11 seconds) of $180 \mathrm{~kW}$ of resistive and 6o-kVAr inductive load. This exercises the ECU and AVR simultaneously on these electronically fuel-injected diesel- engine generator sets and the load-sharing line between the generators. The response is within nominal operating behaviors for this generator.

The following plots (Figures 43 to 46 ) show a single 10-kW-rated PV array located at the longitude and latitude of the CBITEC site experiencing a shading event (70\% drop in irradiance) while connected to the six parallel connected generators with a load of $180 \mathrm{~kW}$ and $60 \mathrm{kVAR}$. The event is simulated to occur at mid-morning when the irradiance is at approximately $80 \mathrm{~kW}$. This shows the PV operating within nominal operating behaviors for an inverter of this type. The red curve shows the generators increasing output to replace the loss of the PV generation. The yellow curve shows $V_{2} G$ generation, which is off for this unit test. The blue curve shows $\mathrm{PV}$ power production.

The following plots (Figures 47 to 50) show the PV array experiencing a more severe shading event (95\% drop in irradiance) while connected to the six parallel connected generators with the same load of $180 \mathrm{~kW}$ and 60 $\mathrm{kVAR}$. The event is simulated to occur at noon when the irradiance is at approximately $100 \mathrm{~kW}$. This shows the PV operating within nominal operating behaviors for an inverter of this type.

The following plots (Figures 51 to 54 ) show a $30-\mathrm{kW}$ V2G charger being dispatched cyclically from $30-\mathrm{kW}$ charge to $30-\mathrm{kW}$ discharge. This is done while connected to the six AMMPS generators. The response is within nominal operating behaviors for the charger. 

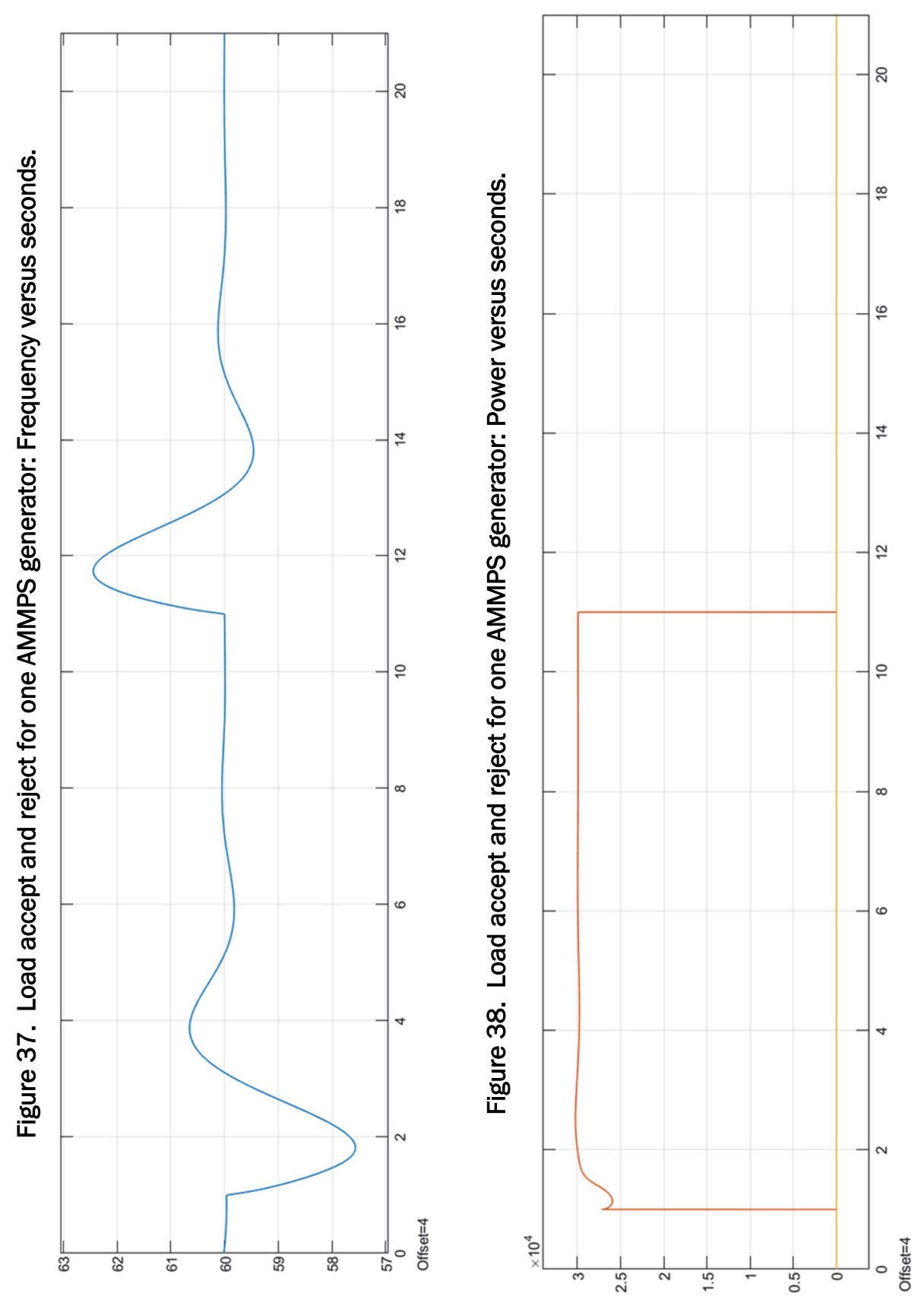

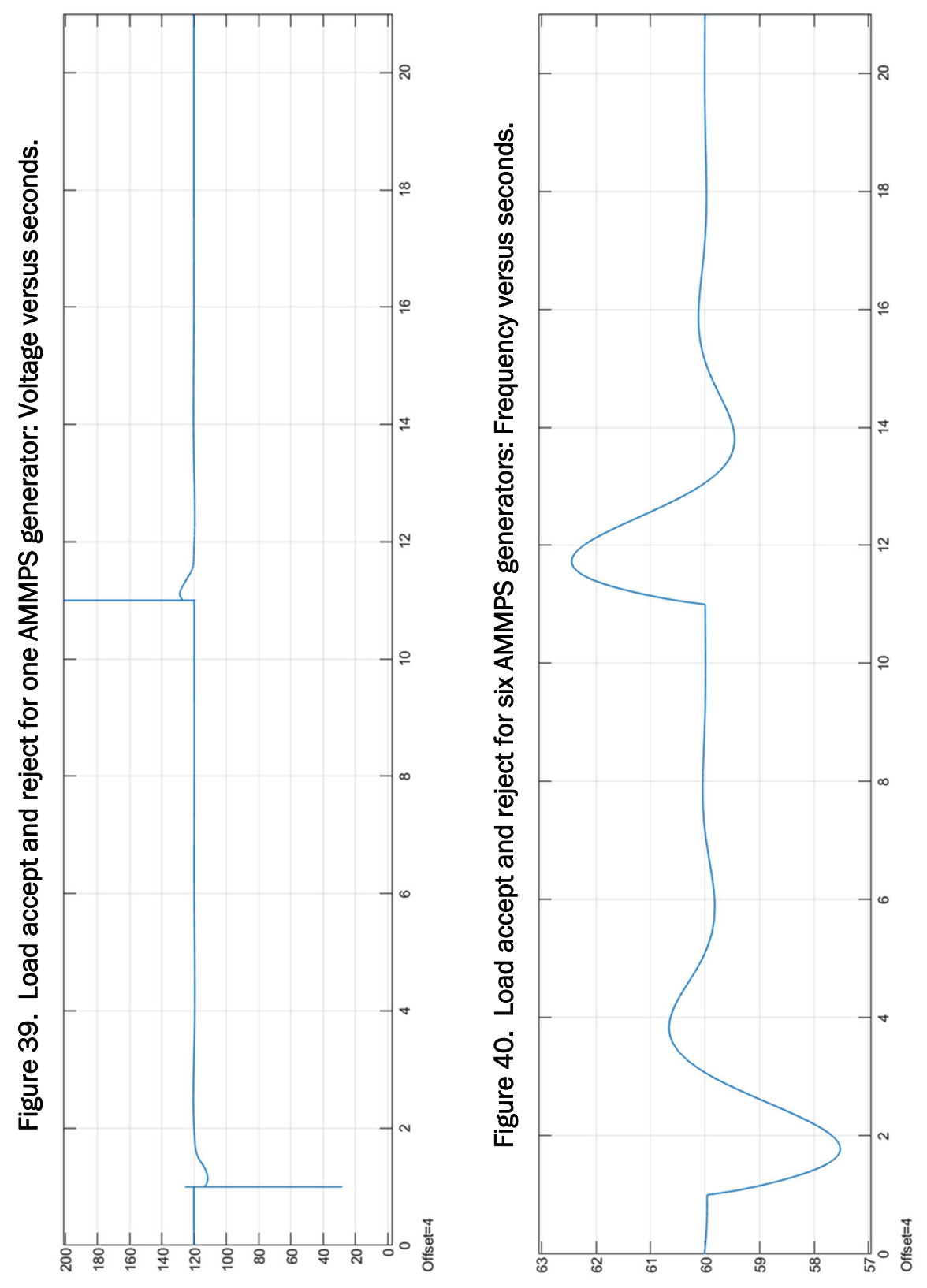

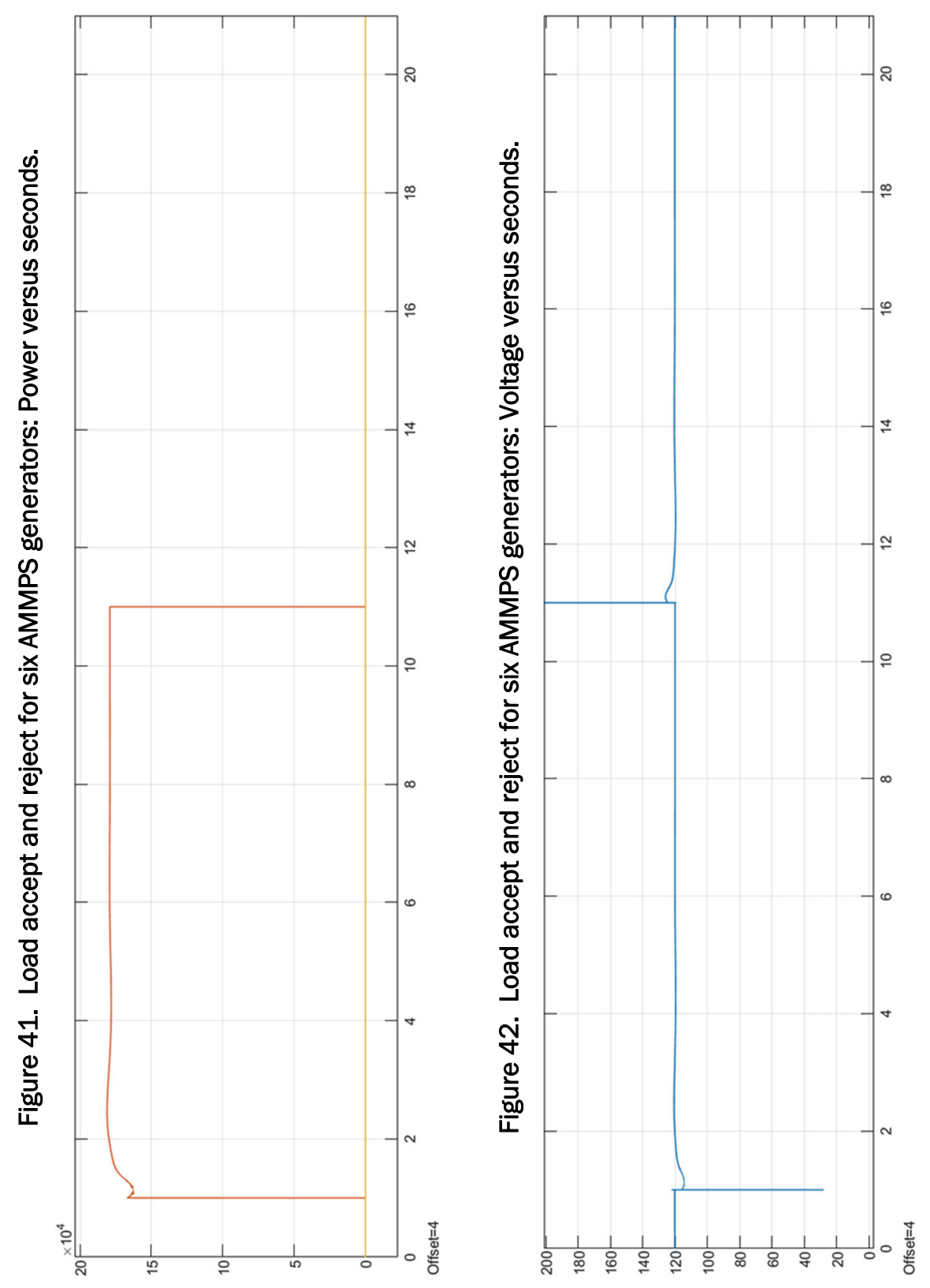

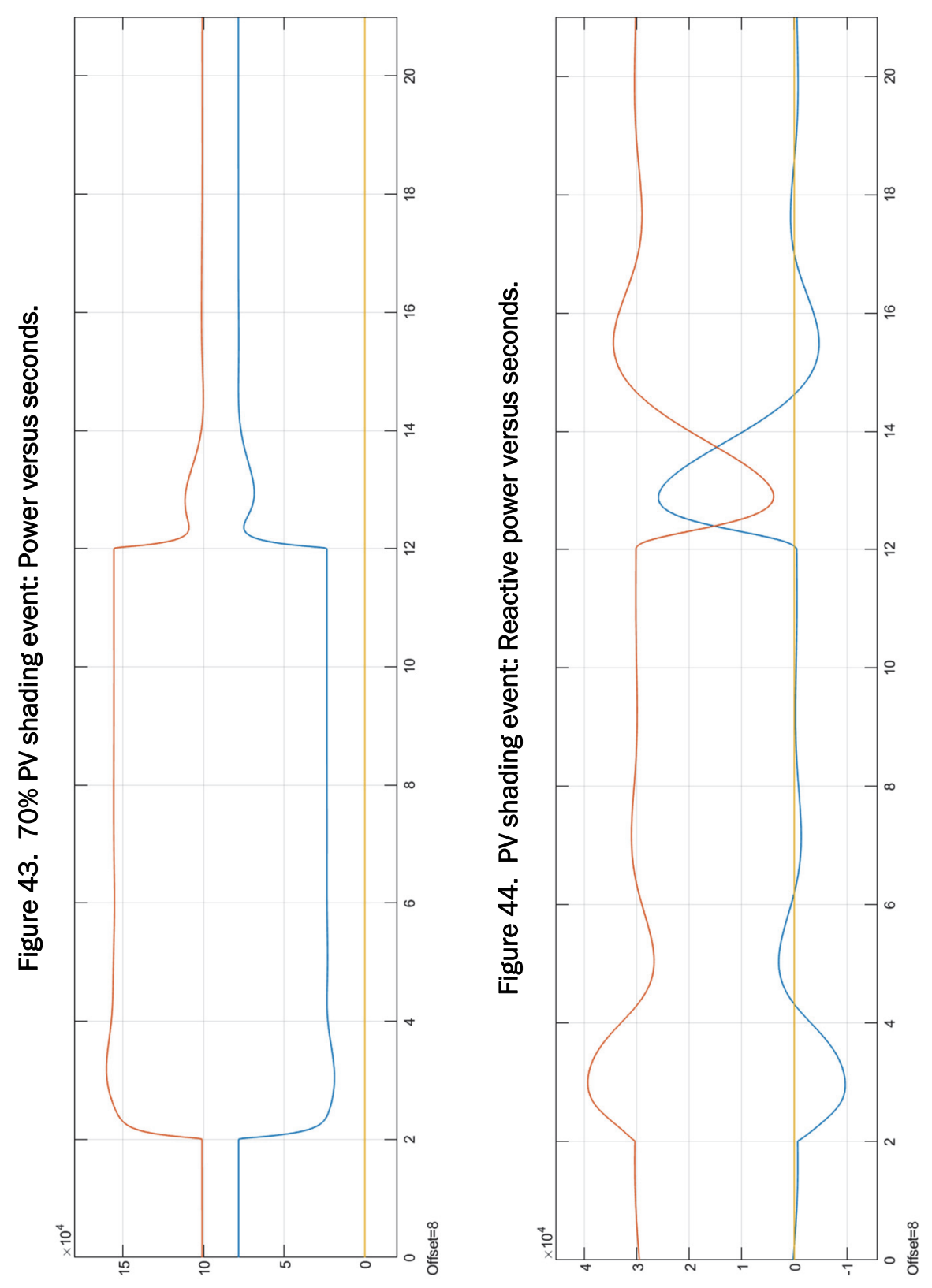

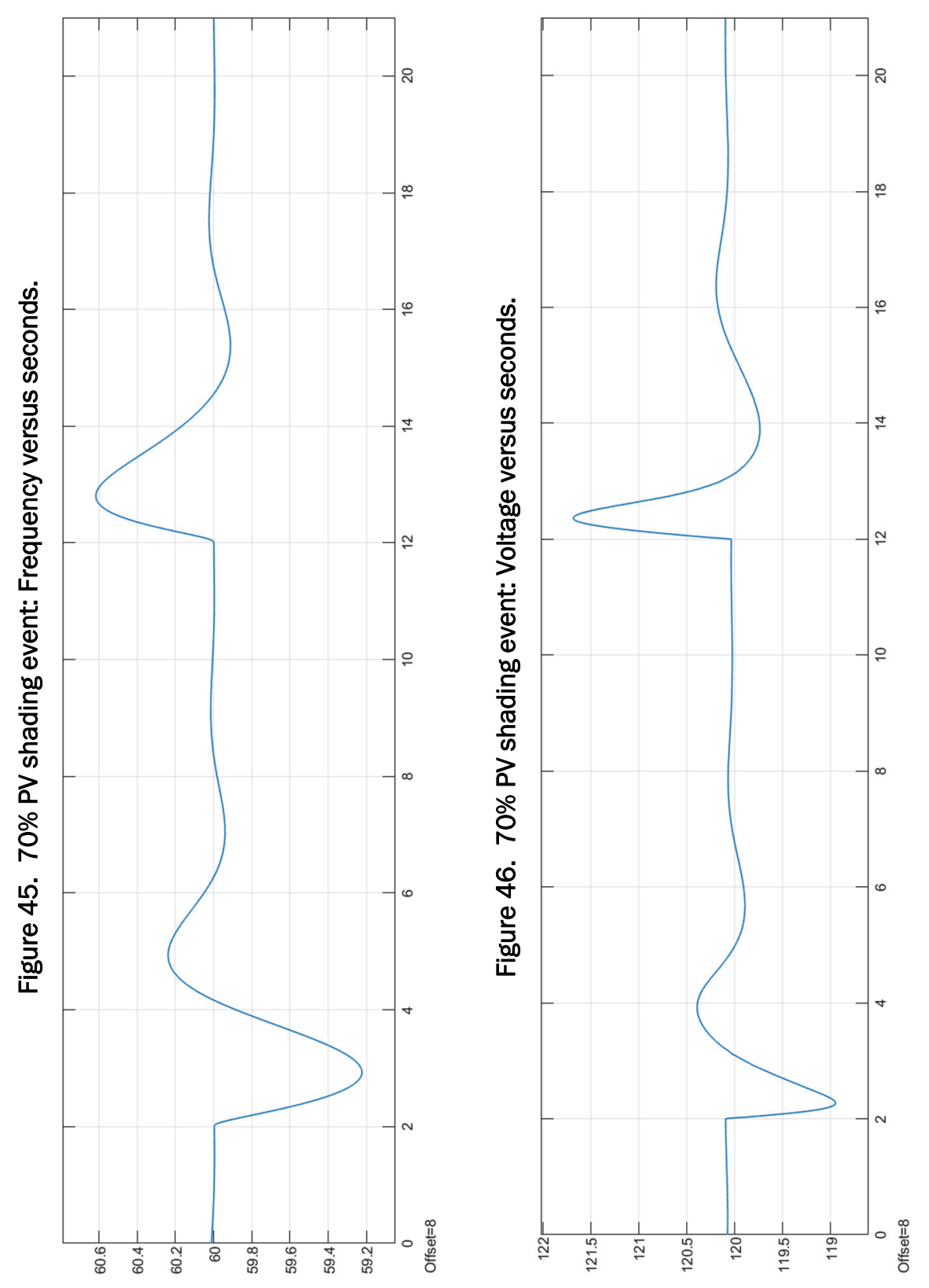

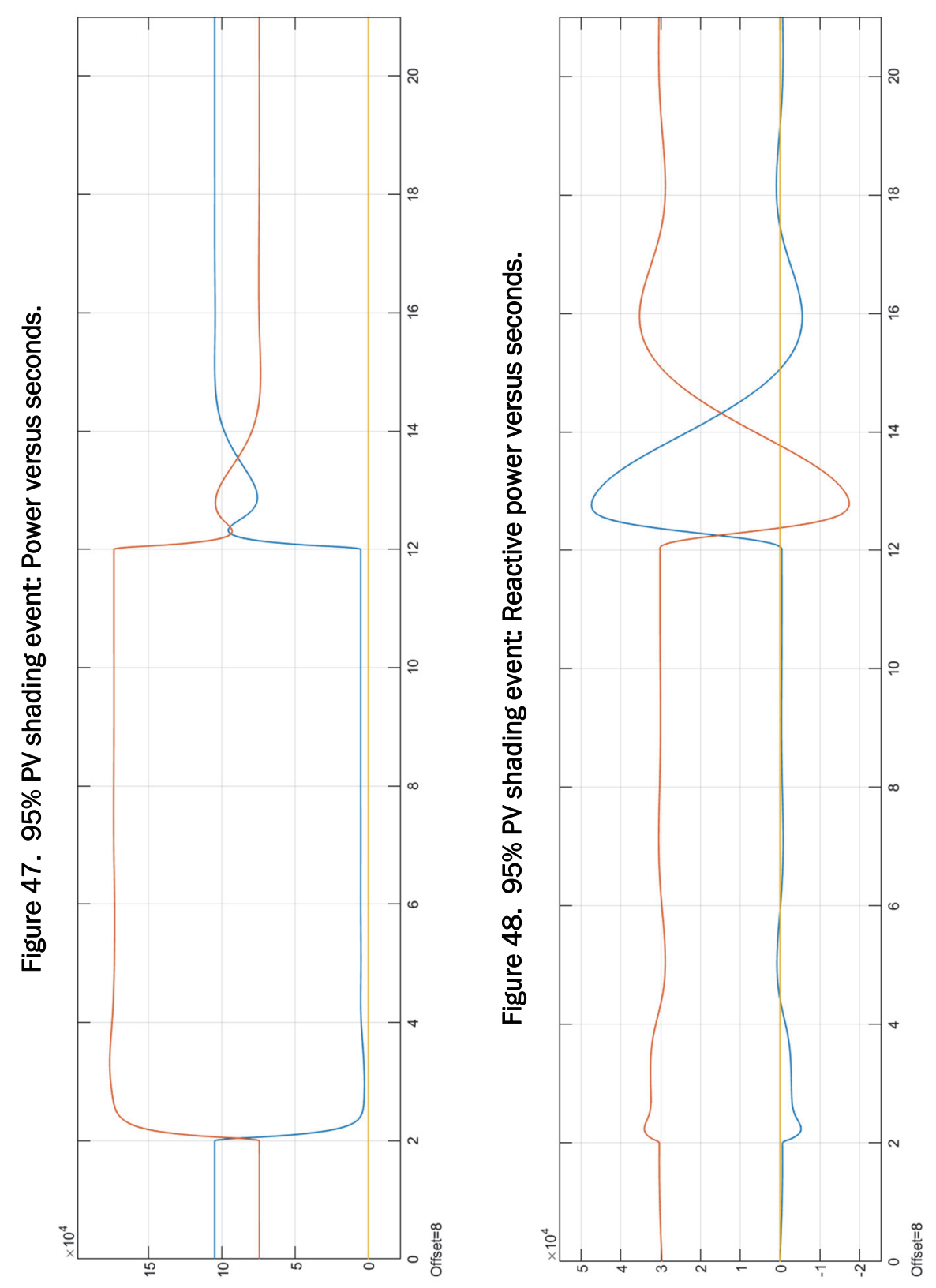

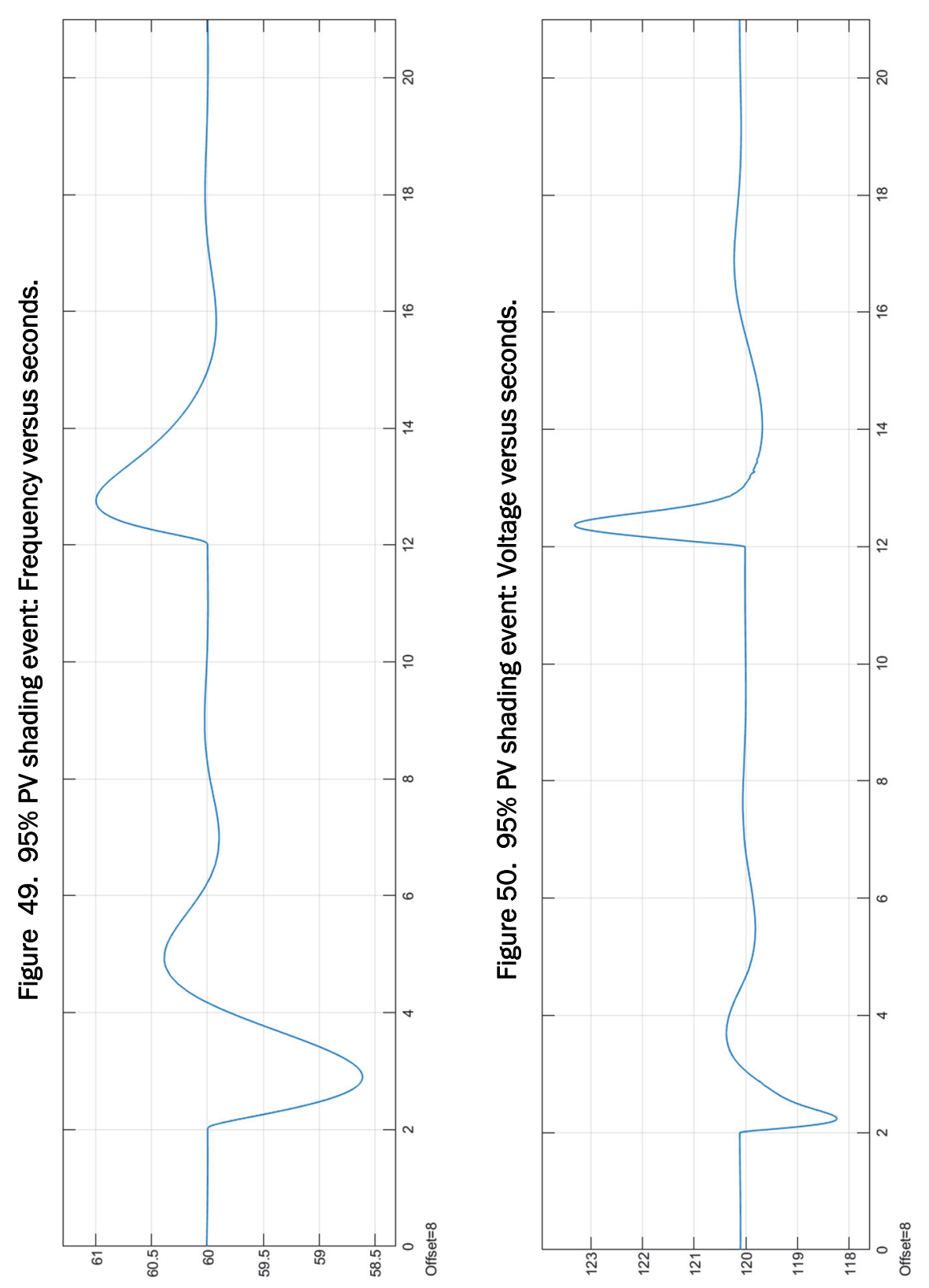

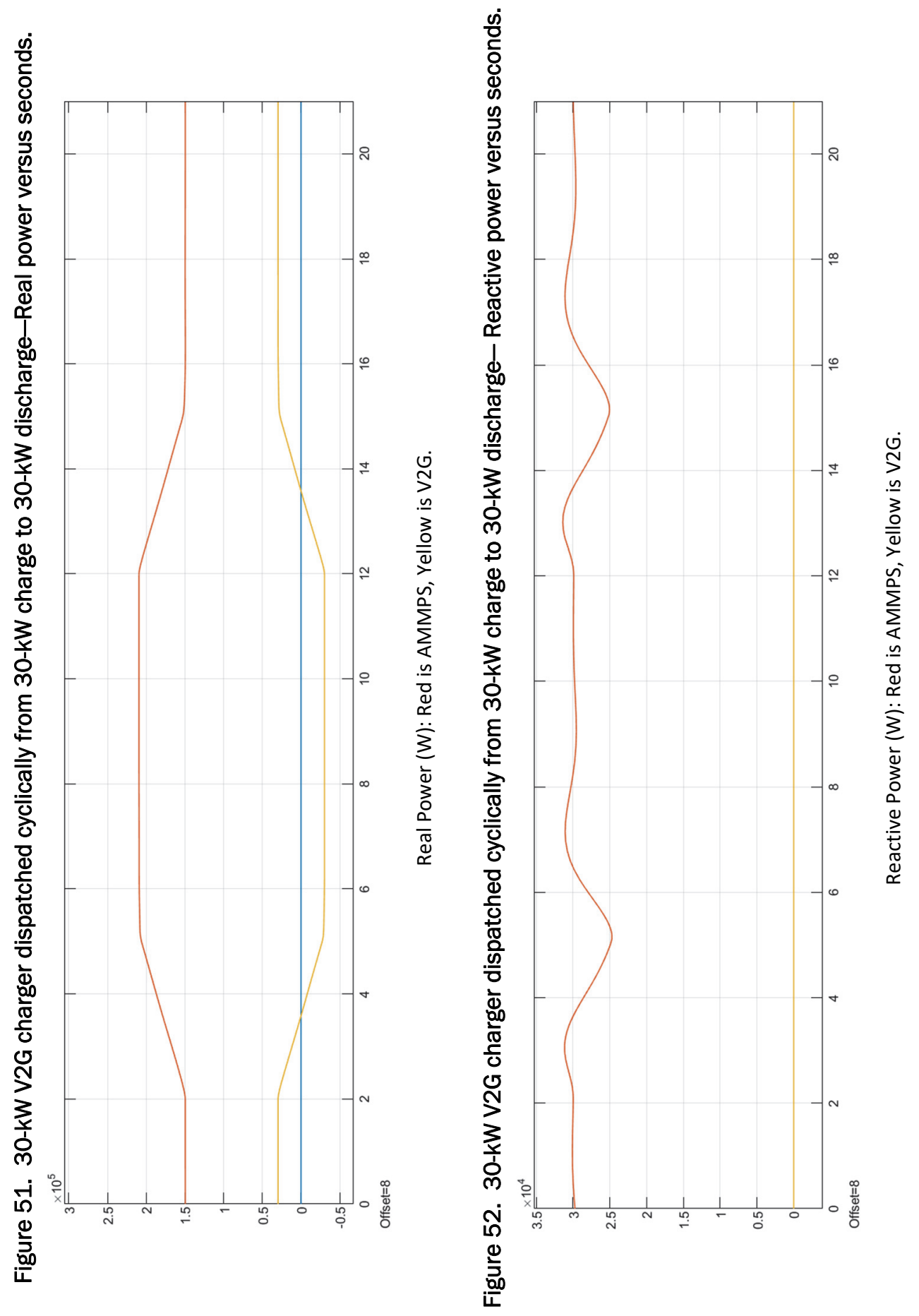

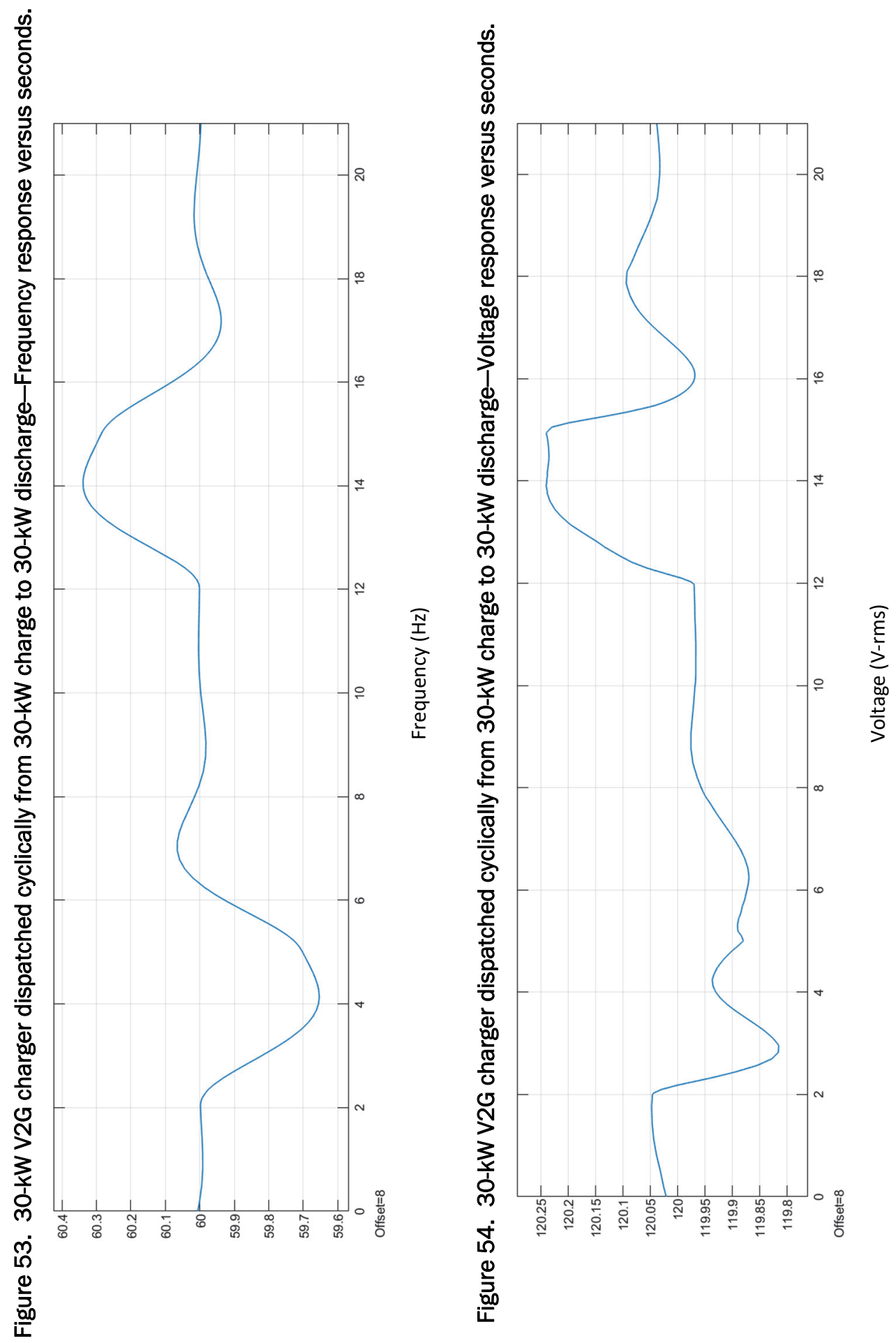
The following plots (Figures 55 to 57) show six AMMPS 60-kW generators, one $100-\mathrm{kW}$ PV, and one $30-\mathrm{kW}$ V2G paralleled and holding $50 \mathrm{~kW}$ of load. The grid then increases load (at 1 second) to $180 \mathrm{~kW}$ and $60 \mathrm{kVAr}$ of load and (at 11 seconds) loses this same load. The V2G is dispatched again from charge to discharge following the loading and returned to charging when the total load returns to $50 \mathrm{~kW}$. This proves numerical stability and a validated microgrid.

\subsubsection{Recommended V2G voltage and frequency ride-through}

The AMMPS generators, like all generators, can be programmed to stay online during larger voltage/frequency than inverters like on the PV or $\mathrm{V} 2 \mathrm{G}$ inverters. The generators will ride through severe voltage and frequency events, so the voltage and frequency tripping points of these generators should be set less restrictive than the PV or charger. As the AMMPS also dominates the grid voltage and frequency behaviors, and the PV and $\mathrm{V} 2 \mathrm{G}$ are grid following, the question that is to be answered is what ridethrough voltage and frequency the PV should and V2G be set to such as to provide reliable operation.

The most common trip limits set into inverters are UL1741. The following Tables 31 and 32. show the UL1741 disconnection points programmed into most inverters over $30 \mathrm{~kW}$ and anticipated to be enabled in most commercial inverters. The inverters in the MATLAB model have frequency and voltage trip setpoints set at these values.

Therefore, to ensure proper coordination, all the AMMPS generators should have voltage and frequency setpoints set as listed in Tables 33 and 34. 

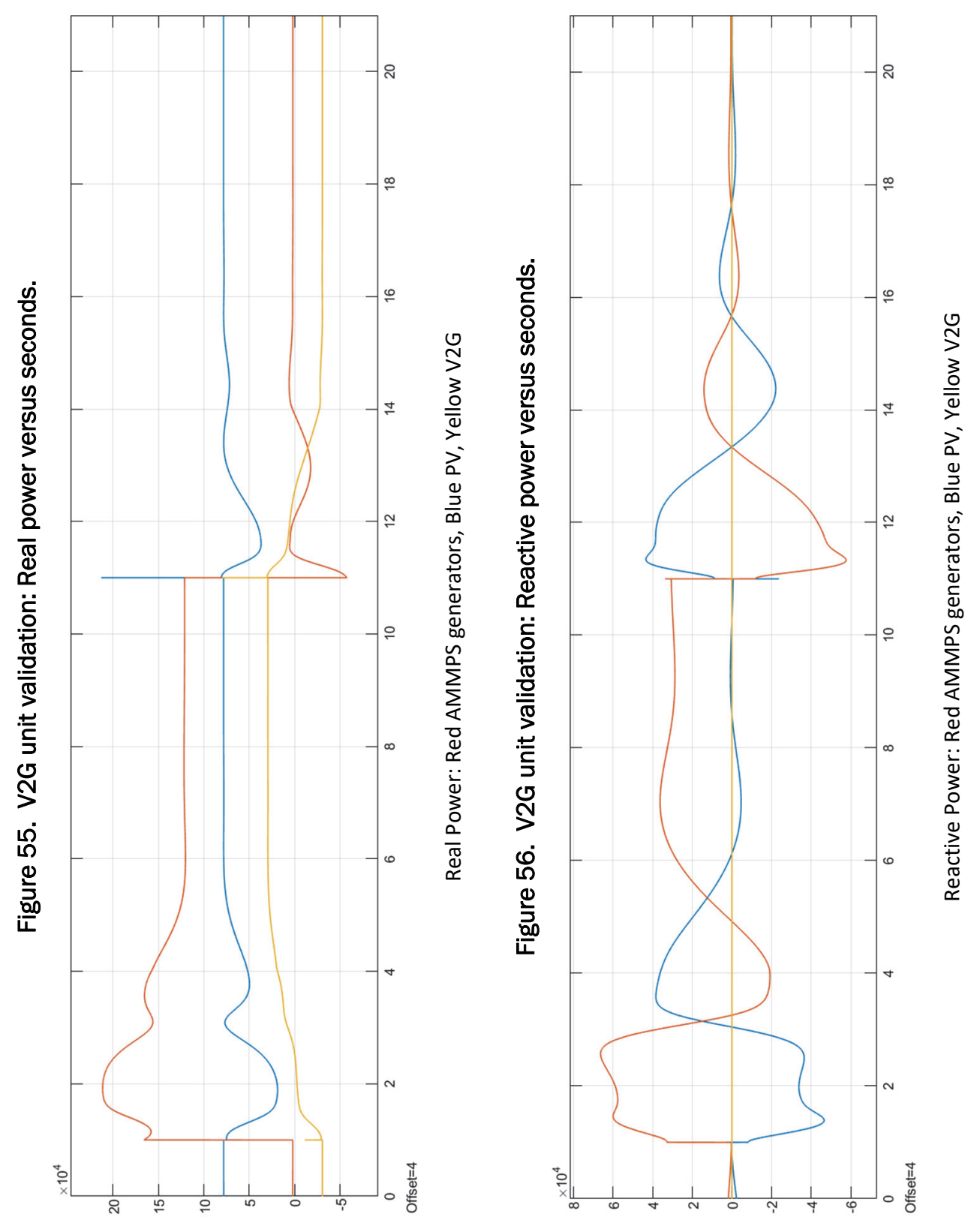


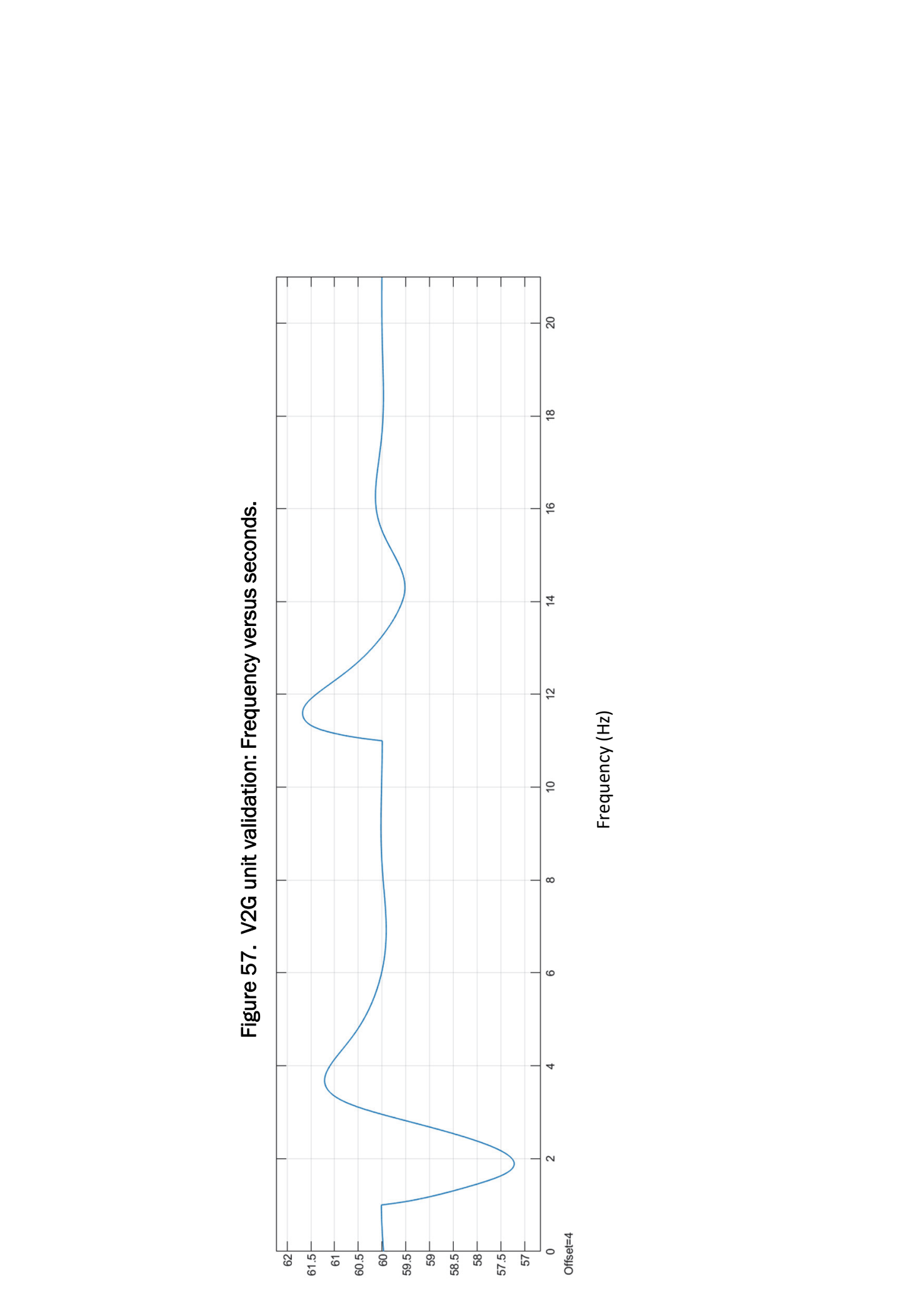


Table 31. PV and V2G ride-through \% base setpoints.

\begin{tabular}{|l|c|}
\hline Voltage range (\% base) & Clearing time (sec) \\
\hline \multicolumn{1}{|c|}{$V<50 \%$} & 0.16 \\
\hline $50 \%<V<88 \%$ & 2.00 \\
\hline $110 \%<V<120 \%$ & 1.00 \\
\hline$V>120 \%$ & 0.16 \\
\hline
\end{tabular}

Table 32. $\mathrm{PV}$ and $\mathrm{V} 2 \mathrm{G}$ ride-through $\mathrm{Hz}$ setpoints.

\begin{tabular}{|l|c|}
\hline Frequency range $(\mathrm{Hz})$ & Clearing time $(\mathrm{sec})$ \\
\hline \multicolumn{1}{|c|}{ Freq $>61.8 \mathrm{~Hz}$} & 0.16 \\
\hline Freq $>61 \mathrm{~Hz}$ & 180 \\
\hline Freq $<59 \mathrm{~Hz}$ & 180 \\
\hline Freq $<57 \mathrm{~Hz}$ & 0.16 \\
\hline
\end{tabular}

Table 33. Generator ride-through $\%$ base setpoints.

\begin{tabular}{|l|c|}
\hline \multicolumn{1}{|c|}{ Voltage range (\% base) } & Clearing time (sec) \\
\hline \multicolumn{1}{|c|}{$\mathrm{V}<50$} & 1.16 \\
\hline $50<\mathrm{V}<88$ & 3.00 \\
\hline $110<\mathrm{V}<120$ & 2.00 \\
\hline $\mathrm{V}>120$ & 1.16 \\
\hline
\end{tabular}

Table 34. Generator ride-through $\mathrm{Hz}$ setpoints.

\begin{tabular}{|l|c|}
\hline Frequency range $(\mathrm{Hz})$ & Clearing time $(\mathrm{sec})$ \\
\hline \multicolumn{1}{|c|}{ freq $>60.5$} & 1.16 \\
\hline Freq $<59.8$ & 101 \\
\hline Freq $<57$ & 1.16 \\
\hline
\end{tabular}

\subsection{Test plan development}

The objective of this task is to develop a test plan for demonstrating a V2G system within a tactical microgrid environment. It will validate a vehicle's providing energy support to critical loads and simultaneous interoperating with other generation sources while being dispatched as either a load or a generation source as needed to support efficient operation of the tactical microgrid.

The developed test plan reflects the tactical microgrid model's manufacture agnostic and technology focus. The test plan can gauge a technology's ability to support energy resilience for future use cases evaluated in the living laboratory. The test plan is designed to establish a baseline of the tactical microgrid consisting of AMMPS generators and site loading to quantify 
how those components respond to system anomalies, followed by integrating one $\mathrm{V}_{2} \mathrm{G}$ vehicle charging system, and repeating system anomalies to analyze which systems drop offline. The test plan assumes an operator dashboard previously provided to verify generator operation in a microgrid environment is still available and can be used for testing purposes.

The test plan includes establishing a microgrid operation baseline followed by connecting a $50-\mathrm{kW}$ V2G system to be operated at four power levels (25\% charge, $75 \%$ charge, $25 \%$ discharge, $75 \%$ discharge), inducing the same anomalies used for the baseline test at each power level.

The anomalies will be composed of a sudden loss of generation and load as well as a sudden increase in generation and load. In addition, the test plan includes a sudden loss of the V2G system at 100-percent charge and discharge to evaluate system impact with the loss of the $\mathrm{V} 2 \mathrm{G}$ resource. This manufacturer-agnostic approach focuses on generation source ability under test to consume or produce energy supporting the tactical microgrid. The test plan tests V2G charger response during charge and discharge modes for sudden increases and decreases for both generation and load. Table 35 lists test condition steps for reference. The current test plan is attached as Appendix $\mathbf{O}$ and is based on the configuration in Figure 58.

Table 35. Test plan conditions.

\begin{tabular}{|l|c|c|}
\hline \multicolumn{1}{|c|}{ Test Condition } & V2G Charging Steps & V2G Discharging Steps \\
\hline Sudden generation decrease & 32,35 & 26,28 \\
\hline Sudden generation increase & 33,36 & $27,29,37$ \\
\hline Sudden load decrease & 31 & 39 \\
\hline Sudden load increase & 30,34 & 38 \\
\hline
\end{tabular}


Figure 58. Test plan configuration.

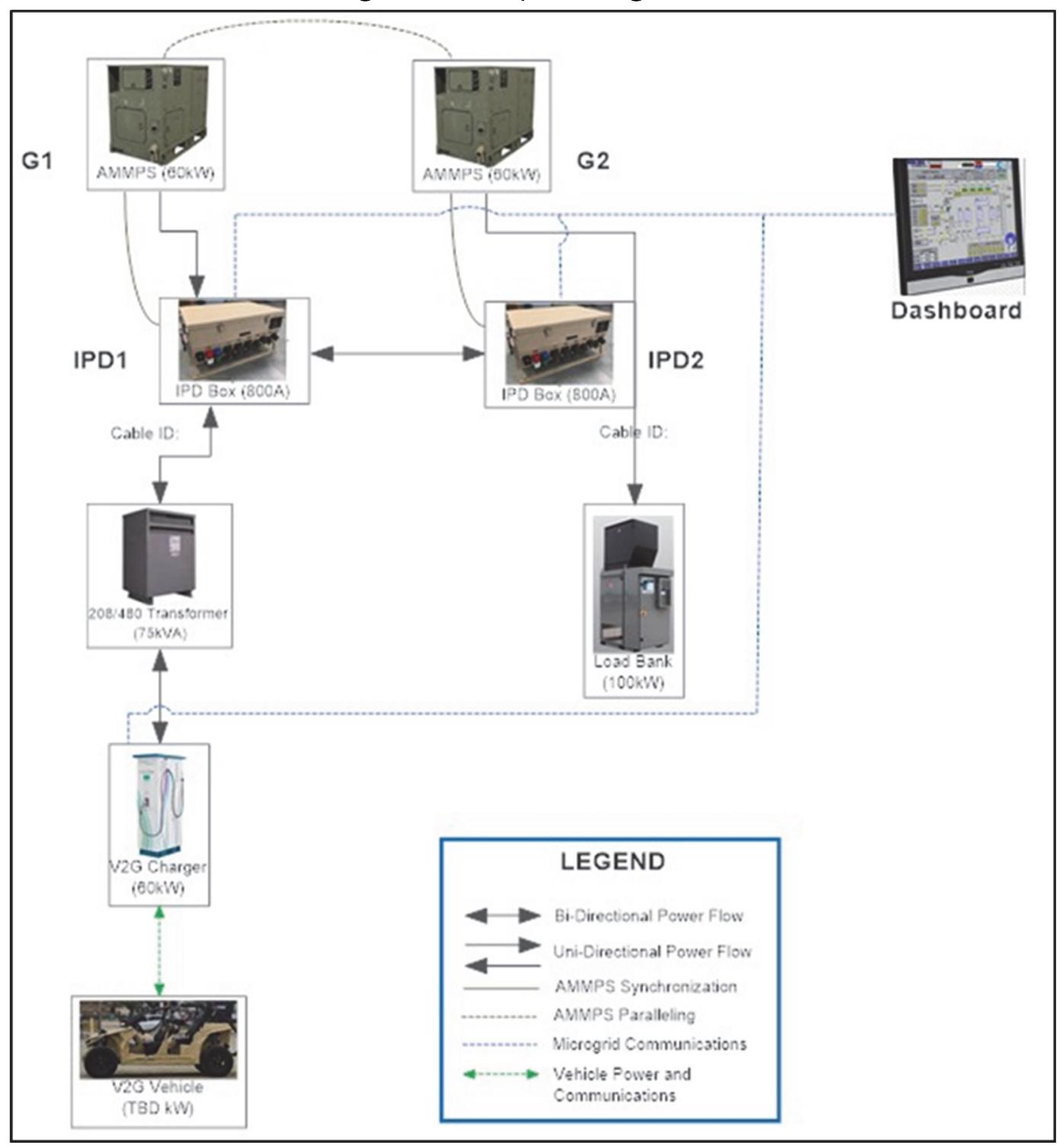

\subsection{Discussion of findings}

Paragon SMEs researched the existing assets and sought feedback from CBITEC, CERL, and C5ISR staff to prepare a viable model and test plan for the tactical microgrid. Through this effort, it is clear to SMEs that a technology focused, flexible approach is better aligned with the vision of a scalable, modular, and flexible tactical microgrid. A model that validates both equipment capabilities and performance when integrated into the tactical microgrid, while still flexible and user friendly, is preferred over a deeply complex and manufacturer- and product-specific model. Consequently, the resulting model and test plan were developed to test and evaluate $\mathrm{V}_{2} \mathrm{G}$ 
charging integration and EV charging capabilities to provide energy support to critical loads, interoperate simultaneously with other generation sources, and dispatch as a load or a generation source as necessary to support the tactical microgrid's efficient operation.

Another important finding from this research effort is the equipment used in a tactical microgrid supporting an FOB is not identical to that used in a living laboratory. The power distribution box identified in the Program of Record PDISE equipment can easily meet the distribution requirements of a tactical microgrid located at an FOB. However, testing and evaluating new equipment in a living laboratory requires additional equipment to quantify component performance and interoperability while ensuring safety for both personnel and connected equipment. The IPD box provided by HG Engineers provides metering, oscilloscope recordings, $\mathrm{C} 37.118$ data streaming, and Sequence of Events (SOE) collection for each connected device and fast-acting controls for interrupting circuits to isolate fault conditions.

The 800A IPD box provides a bidirectional load port rated at 200A, which can accommodate a $57-\mathrm{kW}$ load-per-design practice identified in the $\mathrm{Na}-$ tional Electric Code (NEC). Connecting a V2G faster charger, rated more than $50 \mathrm{~kW}$ to the 200A load port, requires the maximum allowed power flow with the V2G charger limited accordingly. The 800A IPD box also provides two ring-bus connection ports and two generator connection ports-all of which are rated at 400A. The generator ports, designed to be used with the AMMPS generators, utilize a control cable to determine with the IPD connects generator output power to the IPD main electrical bus. There is a mil spec connector on the back of the IPD80o that is connected to an AMMPS and tells the onboard IPD controls system that there is an isochronous source attached. The $\mathrm{V}_{2} \mathrm{G}$ inverter can be successfully connected to either the ring-bus, load port, or generator ports with no consequence. All of these ports support bidirectional power. The conceptual design shows the $\mathrm{V} 2 \mathrm{G}$ fast charger connected to the IPD's 200A load port to keep free the generator and ring-bus ports for other usage.

The SEL automation controller inside the IPD provides communication capability to interface with a principal TMS-compliant microgrid controller. This enables control scheme development and evaluation to be implemented, ensuring available generation sources are powering mission 
critical loads effectively. Incorporating the capabilities of the IPD into the living laboratory is optimal to limit risk and support developing future enabling technologies, even though those same capabilities might not be necessary to be deployed at an FOB.

\subsection{Discussions and future work}

The model developed for this effort included generation components that can be easily adapted as modeling needs and equipment ratings change. This enables future simulations to very quickly simulate numerous scenarios for future planning and system sizing. In particular, the V2G component has the capability to include five different EVs at any quantity to simulate the effect a fleet of $\mathrm{V}_{2} \mathrm{G}$ capable vehicles can have on the connected grid.

In addition to power distribution system sizing by scaling assets, future work should build and test control algorithms into the model to evolve the microgrid controller's control schemes. Testing control schemes in a model first is a critical step for both personnel and equipment safety.

Future could also include comparing actual field test results to the model results and refining the model for accuracy accordingly. Because the AMMPS generator will continue to be used as the standard generator, refining this model component would be very beneficial to further increase model accuracy.

\subsection{Conclusions and recommendation}

Based on the modeling results, the proposed $\mathrm{V} 2 \mathrm{G}$ charger will interoperate with the tactical microgrid as planned. The anticipated voltage and frequency variations of the AMMPS generators produced by the sudden increases or decreases in load or generation are not expected to cause nuisance trips of the $\mathrm{V} 2 \mathrm{G}$ charger.

The recommended frequency and voltage trip settings of the AMMPS generators have been provided to promote interoperability of the generation sources, even when system dynamics occur.

Paragon SMEs also recommend considering refining specific model components to increase overall simulation accuracy in cases where specific 
equipment to be used is pre-determined and commonly used. Program of Record Equipment, such as the AMMPS generator, can be very accurately modeled to further increase simulation accuracy of the overall system. The repeated benefit from accurate modeling could easily offset the cost associated with additional move development time. 


\section{Identify Requirements for a Sensored, Living Laboratory to Assess Autonomous Transport Innovations}

The objective of Research Area $5 \mathrm{~A}$ is to research and identify appropriate sensors and methods to measure autonomous-system performance within the CBITEC site. This report will detail sensors to be used and influence developing operational tests performed within the living laboratory at the CBITEC site. The report will develop a list of high-level requirements. describing the necessary site infrastructure to conduct autonomous testing, and test plans for tests that can be performed at the site. This report will not, however, define specific test procedures as the test locations and assets have yet to be selected.

For the purpose of this document, a test plan defines the scope, objective, key personnel, and approach of the test. A test procedure is a specific set of steps or test cases with measurable outcomes that result in pass/fail criteria.

Paragon's team of SMEs identified required sensors and state-of-the-art technologies through a review of literature as well as engaged with leading industry experts.

\subsection{CBITEC test environment}

The objective of this research is to define the requirements and develop a test plan for testing autonomous-system performance in the living laboratory at the CBITEC site.

\subsubsection{Site requirements}

Robotic Research's team of engineers are SMEs in autonomous software and hardware development and integration. The following list of requirements (Table 36), developed by the Robotic Research engineering team, define the high-level infrastructure, sensors, and data necessary to measure the performance of autonomous systems within the living laboratory at the CBITEC site. These requirements are meant to evolve or be further derived as more details about the test site are defined. 
Table 36. Requirements for infrastructure, sensors, and data collection operations.

\begin{tabular}{|c|c|}
\hline Operation & Site Requirement \\
\hline Infrastructure & $\begin{array}{l}\text { - Requirement 1: The living laboratory will have provisions to charge an EV. } \\
\text { - Requirement 2: The living laboratory will have electrical power connections for sensors. } \\
\text { - Requirement 3: The living laboratory will have semi-permanent mounting points for sensors. (Semi- } \\
\text { - } \text { permanent mounts can be removed from the site without causing damage or disturbing the site). }\end{array}$ \\
\hline Sensors & $\begin{array}{l}\text { - Requirement 5: The living laboratory will be able to detect autonomous systems at the site. } \\
\text { - Requirement 6: The living laboratory will be able to measure the relative position of autonomous } \\
\text { - } \text { systems at the site. } \\
\text { - } \text { within } 0.1 \text { meters. } \\
\text { - Requirement 8: The living laboratory will be able to communicate absolute position of the autonomous } \\
\text { systems at the site. } \\
\text { - Requirement 9: The living laboratory autonomous detection system will be able to operate in varying } \\
\text { lighting conditions, such as full daylight, dusk, and night. }\end{array}$ \\
\hline $\begin{array}{l}\text { Data } \\
\text { Collection }\end{array}$ & $\begin{array}{l}\text { - Requirement 10: The living laboratory will be able to collect and store time data. } \\
\text { - Requirement 11: The living laboratory will be able to collect and store weather data. } \\
\text { - Requirement 12: The living laboratory will be able to collect and store autonomous-system position } \\
\text { - Requirement 13: The living laboratory will be able to collect and store autonomous-system attitude } \\
\text { data. } \\
\text { - Requirement 14: The living laboratory will be able to collect and store autonomous-system velocity data. } \\
\text { - Requirement 15: The living laboratory will be able to collect and store autonomous-system sensor } \\
\text { health data. }\end{array}$ \\
\hline
\end{tabular}

\subsubsection{Operational tests}

This plan provides an overview of the test setup, measurements, and tests to be performed when testing autonomous-system performance at the CBITEC site in on- and off-road applications. The intent of this testing is to test at a system level. It is expected that software unit and integration testing will have already been conducted.

Table 37 lists the recommended personnel and equipment needed during the operational tests.

Table 37. Key personnel and test equipment during operational tests.

\begin{tabular}{|l|l|}
\hline Item & Description \\
\hline \multirow{3}{*}{ Key Personnel } & - Test director \\
& - Safety officer \\
& - Safety driver/operator \\
& - Autonomous-system \\
& - Flat white painted 1-meter x 1-meter poster board \\
& - $100-$ Chalk \\
Test Equipment & - Child-sized mannequin \\
& - Barricades/barrels \\
& - Compact car, utility vehicle, or terrain appropriate vehicle \\
& - DGPS base station \\
& - Non-vehicle-mounted sensor data collection system \\
& - Temporary infrastructure (stop signs, lane markers, crosswalks) \\
\hline
\end{tabular}


The start of every day will begin with setting up the course (obstacle, barriers, etc.), checking sensors and systems for proper operation, and calibrating the navigation system. Every day will end with removing obstacles from the test site, pulling data collected from the day off the autonomoussystem, and powering down the vehicle for the evening.

The maximum speed for the autonomous-system will be set by the test director and safety officer. Maximum speeds should be set for both manually and autonomously driven tests, but the speeds do not have to be the same.

Paragon SMEs do not anticipate that hardware changes will be necessary during testing, but, in the event of a hardware failure, the component will be changed as needed. Software changes and/or modifications will not occur during this event unless there is an error that prevents the completion of the test event.

Standard data files will be collected for every test performed during the test phase. Periodic data integrity checks will be performed throughout every day of testing to ensure data is accurately captured.

The raw data is captured in the industry standard pcap, CAN Bus, or serial format. This includes sensors, such as LiDAR, radar, cameras, and wheel encoders. Processed data is outputted in ROS bag format, which was selected because it is a common format familiar to commercial and academic institutions.

\subsubsection{Baseline evaluation}

The baseline evaluation is intended to include the following system capabilities:

- Vehicle physical characteristics

- Vehicle operating characteristics-includes manual and robotically operated, maximum speed, acceleration, and deceleration

- Localization-testing for navigation system

- Obstacle detection-detection distances for all sensors. 
The baseline data is common across military transportation missions. It is the basic dataset needed to measure a variety of autonomous-system performances operating within the test site.

Table 38 lists the recommended data to collect during baseline tests.

Table 38. Autonomous-system baseline data.

\begin{tabular}{|l|}
\hline Localization accuracy \\
\hline INS accuracy \\
\hline GPS satellite coverage \\
\hline LiDAR minimum/maximum range \\
\hline Vehicle dimensions \\
\hline Vehicle acceleration/deceleration \\
\hline Vehicle turning radius \\
\hline
\end{tabular}

Table 39 lists the recommended tests to be performed to verify the performance of the vehicle-mounted sensors.

Table 39. Sensor measurements.

\begin{tabular}{|l|l|}
\hline Test & Test Description \\
\hline $\begin{array}{l}\text { Localization } \\
\text { (GPS/INS) Sensor } \\
\text { Accuracy }\end{array}$ & $\begin{array}{l}\text { Manually drive a short loop and stop the vehicle in the same location as it started. Use a stopwatch } \\
\text { and record the time of data collection. (This is strictly to verify that the time and position data are } \\
\text { working correctly. The test for Absolute Accuracy will show how accurate the position estimates are.) }\end{array}$ \\
\hline INS Accuracy & $\begin{array}{l}\text { Disconnect the GPS antenna from the localization sensor, then drive a more than 1-km loop, and } \\
\text { stop the vehicle at the same location as it started. Measure the error as a percentage of distance } \\
\text { traveled. }\end{array}$ \\
\hline $\begin{array}{l}\text { GPS Satellite } \\
\text { Coverage }\end{array}$ & $\begin{array}{l}\text { Confirm the GPS antenna is connected to the localization sensor, then drive all areas of the planned } \\
\text { test route. (This can be done manually or autonomously.) Process the collected data to determine the } \\
\text { number of observed satellites along the path. }\end{array}$ \\
\hline Absolute Accuracy & $\begin{array}{l}\text { Drive the vehicle along the planned test route and stop at known surveyed locations. Process the } \\
\text { collected data to compare the reported position to the surveyed location. }\end{array}$ \\
\hline $\begin{array}{l}\text { LiDAR Minimum } \\
\text { Range }\end{array}$ & $\begin{array}{l}\text { With a tape measure, measure the minimum distance that a flat white painted 1-meter x 1-meter } \\
\text { poster board is not detected. }\end{array}$ \\
\hline $\begin{array}{l}\text { LiDAR Maximum } \\
\text { Range }\end{array}$ & $\begin{array}{l}\text { With a tape measure, measure the maximum distance that a flat white painted 1-meter x 1-meter } \\
\text { poster board is not detected. }\end{array}$ \\
\hline $\begin{array}{l}\text { LiDAR Maximum } \\
\text { Ground Distance }\end{array}$ & $\begin{array}{l}\text { Collect a single frame of LiDAR data on a flat, paved road, and measure the maximum distance } \\
\text { where the ground is returned. }\end{array}$ \\
\hline
\end{tabular}

Table 40 lists the recommended vehicle characteristics that should be measured before testing to confirm accuracy of the data collected and aide in post processing of the collected data. 
Table 40. Vehicle characteristics.

\begin{tabular}{|c|c|}
\hline Item & Test Description \\
\hline Dimensions & $\begin{array}{l}\text { The overall vehicle dimensions should be measured to analyze of data collected during testing. The } \\
\text { following measurements should be captured: } \\
\text { - Vehicle length, width, and height } \\
\text { - Vehicle wheelbase } \\
\text { - Front- and rear-track width } \\
\text { - Distance between rear axle and rear-most point of the vehicle }\end{array}$ \\
\hline $\begin{array}{l}\text { Maximum } \\
\text { Acceleration }\end{array}$ & $\begin{array}{l}\text { Manually drive the vehicle at full throttle until the maximum speed (determined by the test director and } \\
\text { safety officer) is achieved. Process the localization/inertial data to determine the maximum acceleration } \\
\text { of the run. }\end{array}$ \\
\hline $\begin{array}{l}\text { Maximum } \\
\text { Declaration }\end{array}$ & $\begin{array}{l}\text { Manually drive the vehicle at full throttle until the maximum speed (determined by the test director and } \\
\text { safety officer) is achieved, and then apply full-service brakes. Process the localization/inertial data to } \\
\text { determine the maximum deceleration of the run. }\end{array}$ \\
\hline $\begin{array}{l}\text { Minimum Left- } \\
\text { Turning Radius }\end{array}$ & $\begin{array}{l}\text { Mark the front right corner of the vehicle and front right tire with chalk. Manually drive the vehicle with a } \\
\text { full left turn until the heading has changed } 180 \text { degrees. Measure the distance between the front right } \\
\text { corner chalk mark and the front right corner of the vehicle. Repeat this measurement for the front right } \\
\text { tire chalk mark and the front right tire of the vehicle. }\end{array}$ \\
\hline $\begin{array}{l}\text { Minimum Right- } \\
\text { Turning Radius }\end{array}$ & $\begin{array}{l}\text { Mark the front left corner of the vehicle and front left tire with chalk. Manually drive the vehicle with a ful } \\
\text { right turn until the heading has changed } 180 \text { degrees. Measure the distance between the front left } \\
\text { corner chalk mark and the front left corner of the vehicle. Repeat this measurement for the front left tire } \\
\text { chalk mark and the front left tire of the vehicle. }\end{array}$ \\
\hline
\end{tabular}

\subsubsection{Example test steps}

The following procedure describes a sample set of steps for performing an autonomous-system test:

1. Record environmental conditions at the test site.

2. Follow procedure to power up autonomous-system.

3. Drive vehicle to calibrate localization sensor, and verify all subsystems are in ready to run.

4. For each test scenario:

a. Place static obstacles that will be used for tests.

b. Using the DGPS system, record the location of static obstacles.

c. Maneuver (manually drive) the vehicle to first waypoint.

d. Confirm the GPS/INS show correct position and heading for the vehicle.

e. Record start time (local and GPS time), scenario, and weather conditions.

f. Enter command for AV to follow test route.

g. Begin autonomous-system maneuvering of the returned plan.

h. Observe autonomous-system as it encounters obstacles on the test course and maneuvers to every waypoint. Make notes as applicable.

i. Restart data logs if necessary.

j. Record stop time: local and GPS time. 
5. Collect all data logs and store data on test site server or hard drive at end of the day.

\subsubsection{Traffic interaction test plan}

The purpose of this test plan is to describe operational tests that can be used to quantify an AV's performance in an on-road environment when interacting with pedestrians and other vehicles. It is expected that the autonomous systems will be bound to a single lane of travel and not allowed to drive in the oncoming traffic lane or on the shoulder.

The following data should be collected during this test:

- Time of test (local and GPS)

- Location of test

- Autonomous-system under test

- Autonomous-system telemetry data (position, attitude, velocity, acceleration)

- Autonomous-system vision sensor data (LiDAR, radar, camera)

- Environmental and ambient conditions (temperature, humidity, pressure, light)

- Living laboratory sensor data (GPS, LiDAR, radar, camera).

Entrance criteria consist of:

- Required test personnel are present

- Autonomous-system has been baselined

- Autonomous-system sensors have been verified functional

- DGPS base station and non-vehicle-mounted sensor data collection system verified functional

- Maximum speed for autonomous under-test system defined

- Temporary infrastructure (stop signs, lane markers, crosswalks) have been placed and locations have been recorded. 
The following tests should be conducted on the road surface of the TA-231 Driving Course (see Figure 59):

- Place a child-sized mannequin beyond the maximum range of the LiDAR. Drive the AV toward the mannequin at $5 \mathrm{mph}$. Process the data to determine the range of the first detection.

- Park a car beyond the maximum range of the LiDAR. Drive the AV toward the car at $5 \mathrm{mph}$. Process the data to determine the range of the first detection.

- Position a child-sized mannequin beyond the maximum detection range of the LiDAR. Autonomously drive the vehicle toward the mannequin until the vehicle autonomously stops. Measure the distance between the vehicle and mannequin with a tape measure.

- Drive a compact car at $10 \mathrm{mph}$ across the route of the autonomous-system. Autonomously drive the autonomous-system toward the compact car until the autonomous-system stops. Measure the distance between the autonomous-system and compact car with a tape measure. Conduct multiple trials increasing the compact car starting location by 10 meters until the maximum range of LiDAR is achieved.

Figure 59. Test diagram.
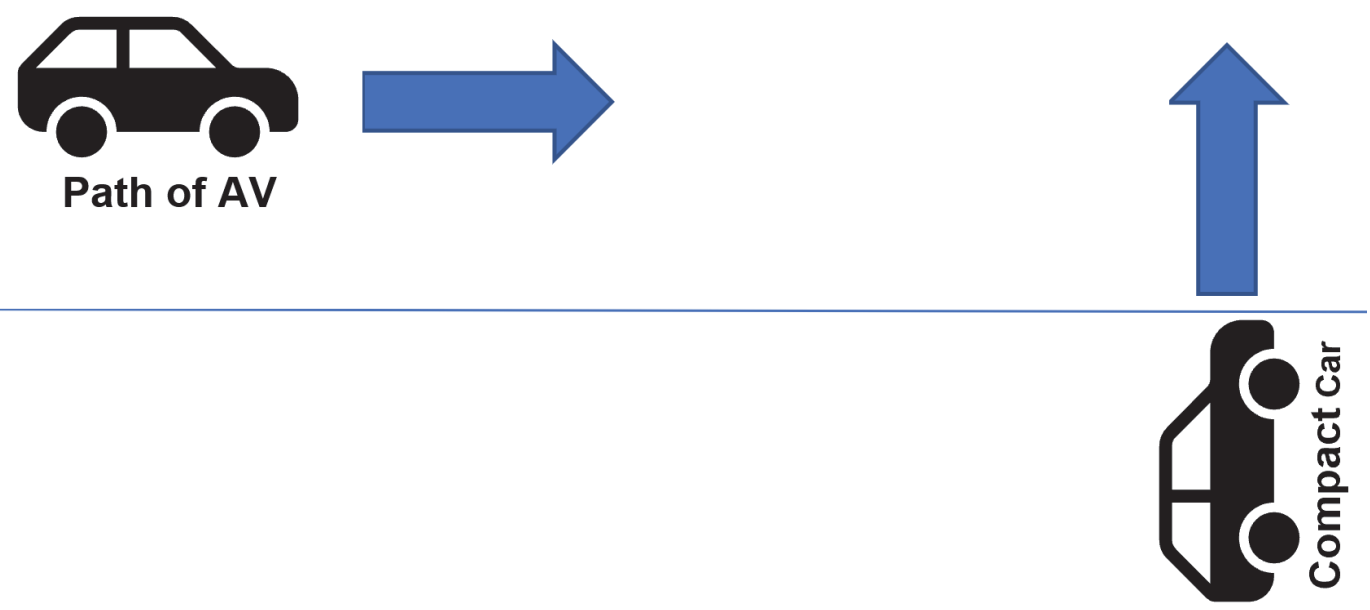

Exit criteria consist of:

- Route completed either autonomously or with safety operator's intervention

- Test data collected 
- Living laboratory sensor data collected.

\subsubsection{Sensor degradation test plan}

The purpose of this test plan is to describe operational tests that can be used to quantify the sensor performance and degradation of an autonomous-system's sensors over time.

The following data should be collected during this test:

- Time of test (local and GPS)

- Location of test

- Autonomous-system under test

- Autonomous-system telemetry data (position, attitude, velocity, acceleration)

- Autonomous-system vision sensor data (LiDAR, radar, camera)

- Environmental and ambient conditions (temperature, humidity, pressure, light)

- Living laboratory sensor data (GPS, LiDAR, radar, camera).

Entrance criteria:

- Required test personnel are present

- Autonomous-system's baseline data is available

- Autonomous-system sensors have been verified functional

- DGPS base station and non-vehicle-mounted sensor data collection system verified functional

- Test equipment (1-meter x 1-meter poster board) and measurement instruments are present.

The following tests should be conducted at the TA-231 Driving Course:

- Drive the vehicle along the planned test route and stop at known surveyed locations. Process the collected data to compare the reported position to the surveyed location.

- Disconnect the GPS antenna from the localization sensor, then drive a more than 1-km loop, and stop the vehicle at the same location as it started. Measure the error as a percentage of distance traveled. 
- With a tape measure, measure the minimum distance that a flat white painted 1-meter x 1-meter poster board is not detected.

- Collect a single frame of LiDAR data on a flat, paved road, and measure the maximum distance where the ground is seen by the LiDAR.

- Compare the results from the tests above to the initial baseline data.

Exit criteria:

- Test data collected

- Test data compared to initial baseline data and previously collected data

- Verify the sensors are still within the manufacturer specification.

\subsubsection{Terrain identification test plan}

The purpose of this test plan is to describe operational tests that can be used to measure an autonomous-system's ability to identify terrain.

The following data should be collected during this test:

- Time of test (local and GPS)

- Location of test

- Autonomous-system under test

- Autonomous-system telemetry data (position, attitude, velocity, acceleration)

- Autonomous-system vision sensor data (LiDAR, radar, camera)

- Environmental and ambient conditions (temperature, humidity, pressure, light)

- Living laboratory sensor data (GPS, LiDAR, radar, camera).

Entrance criteria:

- Required test personnel are present

- Autonomous-system has been baselined

- Autonomous-system sensors have been verified functional

- TA-231 Driving Course has been mapped by the autonomous-system

- DGPS base station and non-vehicle-mounted sensor data collection system verified functional

- Maximum speed for autonomous under test system defined. 
The following tests should be conducted at TA-231 Driving Course:

- Start with the autonomous-system on the test loop of the driving course where the foliage is high on either side of the course.

- Command the system to navigate to a point on the other side of the course. The direct line path between the autonomous-system and the end point should go through foliage and overgrowth.

- Verify that the autonomous-system plans a route that stays on the driving course surface as it navigates to the commanded endpoint.

- Return the autonomous-system to the starting point above. Place barricades, or barrels, in front of the vehicle to block the path it took above.

- Command the system to navigate to a point on the other side of the course, and verify that the system plans a route that goes the opposite direction around the course.

Exit criteria:

- Route completed either autonomously or with intervention

- Test data collected.

\subsubsection{Charging station docking test plan}

The purpose of this test plan is to describe operational tests that can be used to measure an autonomous-system's ability to dock with a charging station.

The following data should be collected during this test:

- Time of test (local and GPS)

- Location of test

- Autonomous-system under test

- Autonomous-system telemetry data (position, attitude, velocity, acceleration)

- Autonomous-system vision sensor data (LiDAR, radar, camera)

- Environmental and ambient conditions (temperature, humidity, pressure, light)

- Living laboratory sensor data (GPS, LiDAR, radar, camera). 
Entrance criteria:

- Required test personnel are present

- Autonomous-system has been baselined

- Autonomous-system sensors have been verified functional

- Charging station functional

- DGPS base station and non-vehicle-mounted sensor data collection system verified functional

- Maximum speed for autonomous under test system defined.

The following tests should be conducted at CBITEC:

- Start with the autonomous-system on the entrance to the autonomous test site, and command autonomous-system to navigate to charging station.

- Verify that the autonomous-system is able to navigate to the charging station test pad, and measure the position and attitude of the AV relative to the docking station.

- Verify and record if the autonomous-system was able to connect to the charging station.

Exit criteria:

- Route completed either autonomously or with intervention

- Test data collected.

\subsection{Sensors}

To characterize the performance of an autonomous-system, the absolute and relative position of the system must be accurately and repeatably measured. There are a variety of ways to measure the position of the systems. Some include sensors mounted on the autonomous-system, and some include sensors mounted at the test site.

The Robotic Research team of engineers are SMEs in sensor testing and integration. The following sections will describe the different types of sensors and their benefits. Additionally, data collected from a variety of sensors tested by Robotic Research will be presented. 


\subsubsection{Vehicle-mounted measurement sensors}

DGPS (Figure 60) enhances traditional GPS solutions by supplying position corrections from a fixed location. DGPS uses two GPS receivers and a communication mechanism between the two.

Figure 60. DGPS architecture.
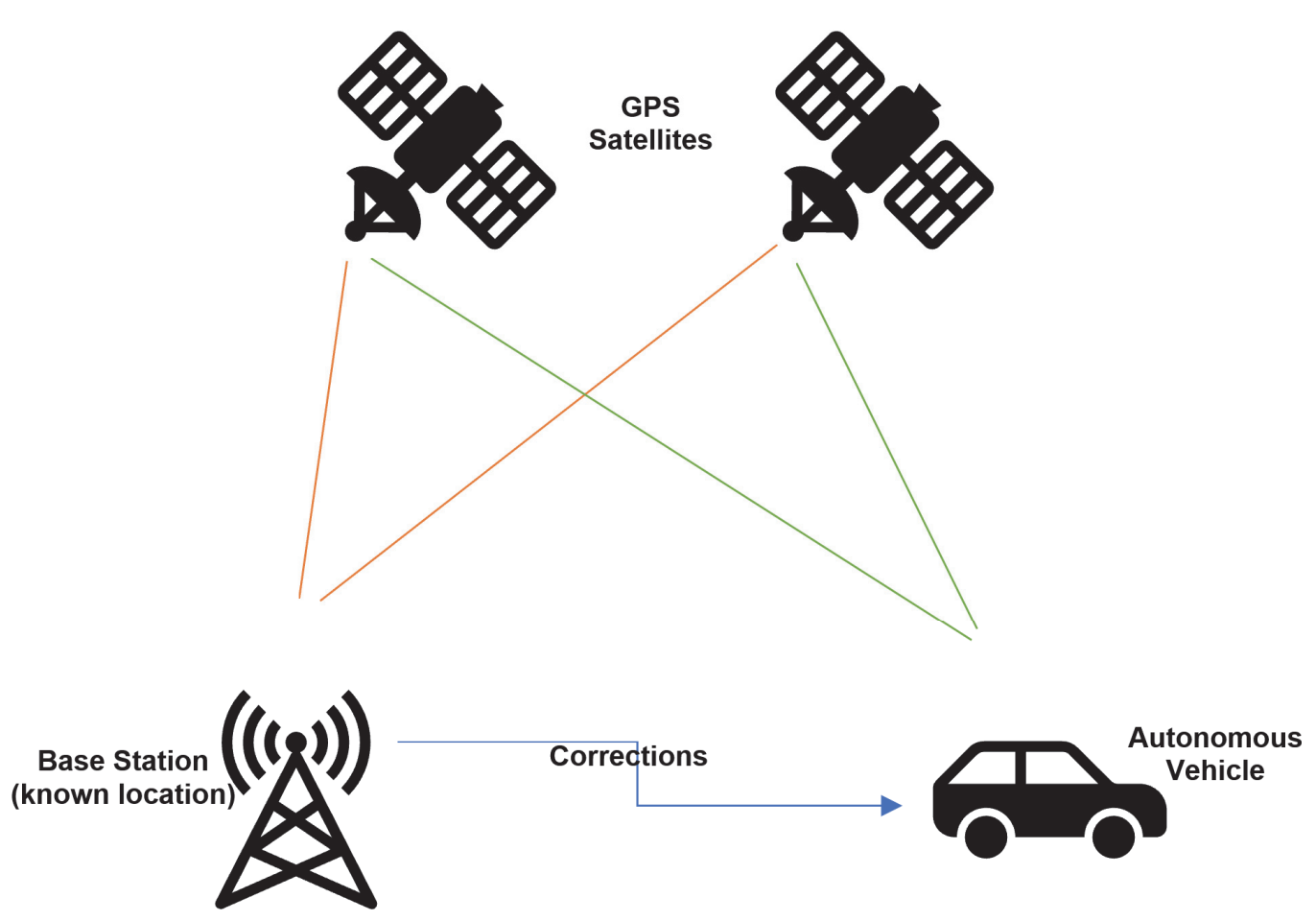

One is at a fixed location where the exact position is known, which is either by survey or measuring the GPS position for long periods of time (24-48 hours). The other receiver is mounted on the autonomous-system.

The fixed-location receiver, called a base station, computes the real-time GPS solution and compares that to its known location. The comparison is used to compute a correction factor between the real-time GPS solution and the known location. The correction factor is then sent to the GPS receiver on the autonomous-system and applied to the GPS solution of the autonomous-system.

DGPS is able to improve the position solution of the autonomous-system from over 1 meter of accuracy to less than 0.1 meter of accuracy. The typical cost of a DGPS base station is less than $\$ 20,000$. 
One drawback of the DGPS system is that the accuracy of the position solution degrades in areas with poor GPS satellite coverage, such as areas with dense tree coverage, or where GPS signals are being interfered with or jammed.

\subsubsection{Site-mounted measurement sensors}

In addition to vehicle-mounted measurement sensors, another option to track and measure autonomous-system performance is mounted sensors at fixed positions within the test site. In this scenario, vision-based sensors (LiDAR, radar, and cameras) would be mounted at locations that provide optimal viewing of the test area, and the data from every sensor would be collected in a central repository via a physical connection.

Just as the LiDARs, radars, and cameras mounted on autonomous systems classify and track different objects interacting with the vehicle, the fixedlocation sensors can classify and track the different autonomous systems operating within the test site. This type of system would be more beneficial when testing in areas with poor GPS coverage or indoors.

The Safe Autonomous Unmanned Vehicles for Installations (SAUVI) surveillance tool suite was originally developed to allow autonomous systems to have greater situational awareness by providing data to the system from sensors that were mounted along the autonomous-system's route [5A4].* The SAUVI system is composed of the sensor node and distributed sensors. An example of the node and distributed sensing setup is shown in Figure 61.

The ability to classify and track autonomous systems within the test area depends largely on the type of sensors connected to the SAUVI system. For example, a system that uses narrow field-of-view radars would be able to track autonomous systems at long ranges (up to 400 meters with line of sight) but only if the autonomous-system is within the radar's field of view (Figure 62). Conversely, a LiDAR that spins 360 degrees would be more forgiving in the field-of-view context, but the overall range is much less (approximately 100 meters).

\footnotetext{
* Lacaze, Alberto. Putney, Joseph. 1 March 2018 “SAUVI II-Safe Autonomous Unmanned Vehicles for Installations." Contractor's Final Technical Report.
} 
Figure 61. Node and distributed sensing setup.

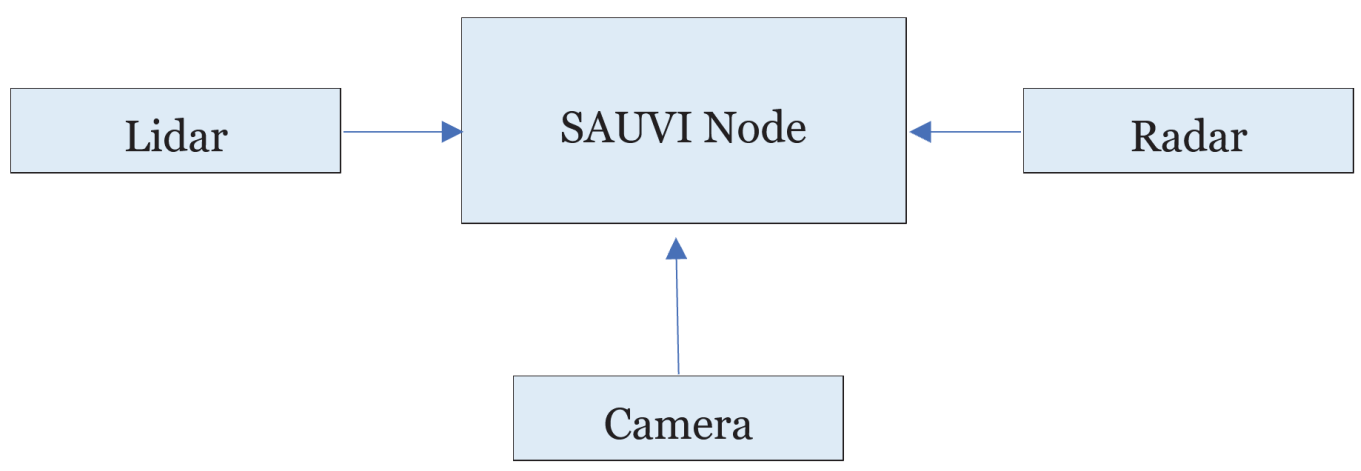

Figure 62. Node and distributed sensing setup.

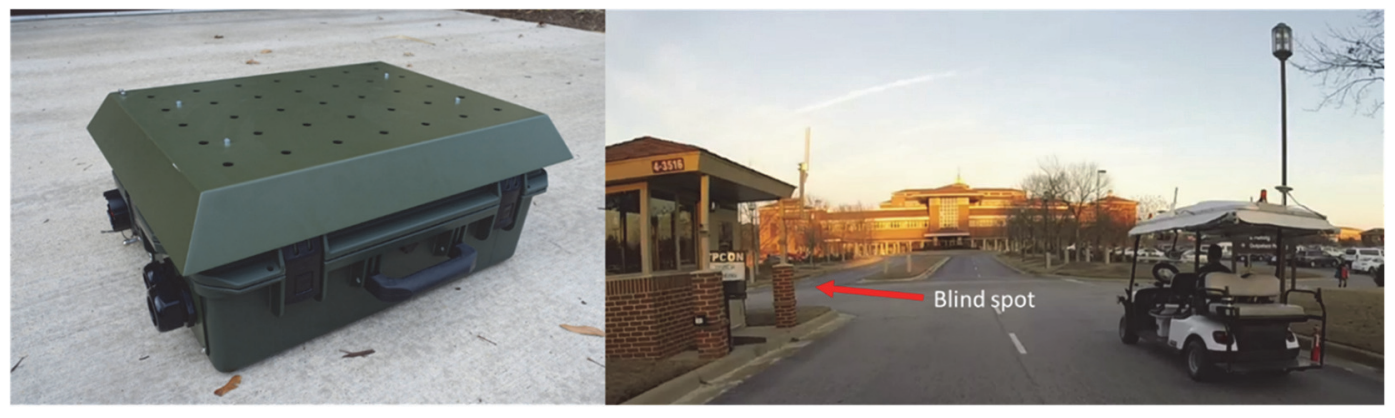

Additionally, the measurement performance of each sensor needs to be considered to achieve the desired accuracy of the collected data. The sensors listed in Table 41 can be used with the SAUVI node. A typical cost of the node is approximately $\$ 15,000$.

Table 41. Radar specifications.

\begin{tabular}{|c|c|c|c|c|c|c|c|}
\hline LiDAR & Delphi ESR & $\begin{array}{l}\text { Delphi } \\
\text { SRR2 }\end{array}$ & $\begin{array}{l}\text { SMS UMRR- } \\
\text { Oa Type } 29\end{array}$ & $\begin{array}{l}\text { SMS UMRR- } \\
\text { Oa Type } 30\end{array}$ & $\begin{array}{l}\text { SMS UMRR- } \\
\text { Oa Type } 31\end{array}$ & $\begin{array}{l}\text { Continental } \\
\text { SRR 20X }\end{array}$ & $\begin{array}{c}\text { Continental } \\
\text { ARS } 300\end{array}$ \\
\hline Frequency & $76.5 \mathrm{GHz}$ & $76.5 \mathrm{GHz}$ & $24 \mathrm{GHZ}$ & $24 \mathrm{GHZ}$ & $24 \mathrm{GHZ}$ & $24 \mathrm{GHZ}$ & $77 \mathrm{GHZ}$ \\
\hline Range & $\begin{array}{l}174 \mathrm{~m} \text { (long } \\
\text { range) } \\
60 \mathrm{~m} \\
\text { (midrange) }\end{array}$ & $80 \mathrm{~m}$ & $160 \mathrm{~m}$ & $90 \mathrm{~m}$ & $45 \mathrm{~m}$ & $50 \mathrm{~m}$ & $200 \mathrm{~m}$ \\
\hline $\begin{array}{l}\text { Max Range } \\
\text { Pedestrian }\end{array}$ & $40 \mathrm{~m}$ & & $50 \mathrm{~m}$ & $40 \mathrm{~m}$ & $20 \mathrm{~m}$ & & \\
\hline $\begin{array}{l}\text { Max Range } \\
\text { Car/Truck }\end{array}$ & $175 \mathrm{~m}$ & & $160 \mathrm{~m}$ & $90 \mathrm{~m}$ & $45 \mathrm{~m}$ & & \\
\hline $\begin{array}{l}\text { Horizontal } \\
\text { Field of View }\end{array}$ & $\begin{array}{c}+/-10^{\circ} \text { (long } \\
\text { range) } \\
+/-45^{\circ} \\
\text { (midrange) }\end{array}$ & $+/-75^{\circ}$ & $+/-18^{\circ}$ & $+/-35^{\circ}$ & $+/-50^{\circ}$ & $\begin{array}{l}+/-20^{\circ} \text { (long } \\
\quad \text { range) } \\
+/-75^{\circ} \\
\text { (midrange) }\end{array}$ & $\begin{array}{c}+/-8.5^{\circ} \text { (long } \\
\text { range) } \\
+/-28^{\circ} \\
\text { (midrange) }\end{array}$ \\
\hline
\end{tabular}




\begin{tabular}{|l|c|c|c|c|c|c|c|}
\hline \multicolumn{1}{|c|}{ LiDAR } & Delphi ESR & $\begin{array}{c}\text { Delphi } \\
\text { SRR2 }\end{array}$ & $\begin{array}{c}\text { SMS UMRR- } \\
\text { Oa Type 29 }\end{array}$ & $\begin{array}{c}\text { SMS UMRR- } \\
\text { Oa Type 30 }\end{array}$ & $\begin{array}{c}\text { SMS UMRR- } \\
\text { Oa Type 31 }\end{array}$ & $\begin{array}{c}\text { Continental } \\
\text { SRR 20X }\end{array}$ & $\begin{array}{c}\text { Continental } \\
\text { ARS } 300\end{array}$ \\
\hline $\begin{array}{l}\text { Vertical Field } \\
\text { of View }\end{array}$ & $+/-4.2-4.75^{\circ}$ & $+/-5^{\circ}$ & $+/-4^{\circ}$ & $+/-5^{\circ}$ & $+/-5^{\circ}$ & $+/-6^{\circ}$ & $+/-4.3^{\circ}$ \\
\hline Update Rate & $<=50 \mathrm{~ms}$ & $<=50 \mathrm{~ms}$ & $<=50 \mathrm{~ms}$ & $<=50 \mathrm{~ms}$ & $<=50 \mathrm{~ms}$ & $\begin{array}{c}>=33 \mathrm{~ms} \text { (typ. } \\
38 \mathrm{~ms})\end{array}$ & $66 \mathrm{~ms}$ \\
\hline $\begin{array}{l}\text { Tracking } \\
\text { Targets }\end{array}$ & 64 & 64 & 64 & 64 & 64 & $\begin{array}{c}256(126 \\
\text { reported) }\end{array}$ & 90 \\
\hline
\end{tabular}

\subsection{Lab tests}

Robotic Research has collected and analyzed data with multiple sensors. This section will describe each of the evaluated systems, discuss the pertinent specifications (and their effect on performance) for each sensor, and present some initial results. ${ }^{*}$ Table 42 lists some key specifications for each LiDAR. $^{\dagger}$

Table 42. LiDAR specifications.

\begin{tabular}{|c|c|c|c|c|c|c|c|}
\hline LiDAR & $\begin{array}{l}\text { Velodyne } \\
\text { VLP-16 }\end{array}$ & $\begin{array}{l}\text { Velodyne } \\
\text { HDL-32E }\end{array}$ & $\begin{array}{l}\text { Velodyne } \\
\text { HDL-64E }\end{array}$ & $\begin{array}{c}\text { GDRS XR } \\
\text { microLADAR }\end{array}$ & $\begin{array}{l}\text { IBEO LUX } \\
8 \mathrm{~L}\end{array}$ & $\begin{array}{l}\text { Optech } \\
\text { ILRIS-HD }\end{array}$ & $\begin{array}{c}\text { Optech } \\
\text { Lynx SG1 }\end{array}$ \\
\hline Wavelength & $903 \mathrm{~nm}$ & $905 \mathrm{~nm}$ & $905 \mathrm{~nm}$ & $905 \mathrm{~nm}$ & $905 \mathrm{~nm}$ & $1535 \mathrm{~nm}$ & $15 \mathrm{~nm}$ \\
\hline Range & $100 \mathrm{~m}$ & $100 \mathrm{~m}$ & $\begin{array}{l}120 \mathrm{~m} \text { at } \\
\sim 80 \% \\
\text { reflectivity } \\
50 \mathrm{~m} \text { at } \\
\sim 10 \% \\
\text { reflectivity }\end{array}$ & $120 \mathrm{~m}$ & $\begin{array}{l}200 \mathrm{~m} \text { for } \\
\text { "average } \\
\text { target" } \\
50 \mathrm{~m} \text { at } \\
\sim 10 \% \\
\text { reflectivity }\end{array}$ & $1250 \mathrm{~m}$ & $\begin{array}{c}250 \mathrm{~m} \text { at } \\
10 \% \\
\text { reflectivity }\end{array}$ \\
\hline Accuracy & $<3 \mathrm{~cm}$ & $<2 \mathrm{~cm}$ & $<2 \mathrm{~cm}$ & Unknown & $10 \mathrm{~cm}$ & $7 \mathrm{~cm}$ & $5 \mathrm{~cm}$ \\
\hline $\begin{array}{l}\text { Number of } \\
\text { Lasers }\end{array}$ & 16 & 32 & 64 & 1 & 8 & 1 & $\begin{array}{l}2 \text { (1 per } \\
\text { sensor) }\end{array}$ \\
\hline $\begin{array}{l}\text { Scan } \\
\text { Frequency }\end{array}$ & $5-20 \mathrm{~Hz}$ & $5-20 \mathrm{~Hz}$ & $5-15 \mathrm{~Hz}$ & $10 \mathrm{~Hz}$ & $25 \mathrm{~Hz}$ & Unknown & $\begin{array}{c}\text { Up to } 500 \\
\text { lines per } \\
\text { second }\end{array}$ \\
\hline Field of View & $\begin{array}{l}\text { HFOV: } 360^{\circ} \\
\text { VFOV: } 30^{\circ} \\
\left(\theta_{\text {up }}=15^{\circ},\right. \\
\theta_{\text {down }}= \\
\left.15^{\circ}\right)\end{array}$ & $\begin{array}{c}\text { HFOV: } 360^{\circ} \\
\text { VFOV: } 41.3^{\circ} \\
\left(\theta_{\text {up }}=\right. \\
10.67^{\circ}, \\
\theta_{\text {down }}= \\
\left.30.67^{\circ}\right)\end{array}$ & $\begin{array}{l}\text { HFOV: } 360^{\circ} \\
\text { VFOV: } 26.8^{\circ} \\
\left(\theta_{\text {up }}=2^{\circ},\right. \\
\theta_{\text {down }}= \\
\left.24.8^{\circ}\right)\end{array}$ & $\begin{array}{l}\text { HFOV: } 120^{\circ} \\
\text { VFOV: } 20^{\circ} \\
\left(\theta_{\text {up }}=10^{\circ},\right. \\
\left.\theta_{\text {down }}=10^{\circ}\right)\end{array}$ & $\begin{array}{l}\text { HFOV: } 110^{\circ} \\
\text { VFOV: } 6.4^{\circ} \\
\left(\theta_{\text {up }}=-3.2^{\circ},\right. \\
\theta_{\text {down }}= \\
\left.3.2^{\circ}\right)\end{array}$ & $\begin{array}{c}\mathrm{H}: 40^{\circ} \\
\mathrm{V}: 40^{\circ} \\
\text { (adjustable) }\end{array}$ & $360^{\circ}$ \\
\hline $\begin{array}{l}\text { Vertical } \\
\text { Angular } \\
\text { Resolution }\end{array}$ & $2^{\circ}$ & $1.33^{\circ}$ & $0.4^{\circ}$ & $\begin{array}{l}\text { Variable (non- } \\
\text { overlapping scan } \\
\text { pattern) }\end{array}$ & $0.8^{\circ}$ & $0.001146^{\circ}$ & N/A \\
\hline
\end{tabular}

\footnotetext{
* Lacaze, Alberto. Murphey, Karl, Schneider, Anne. June 2014. “Perception Sensor Study for High-Speed Autonomous Operations.

† Lacaze, Alberto. Murphey, Karl, Schneider, Anne. Del Giorno, Mark, June 2014. "Comparison and Analysis of LADAR Sensors for Object Detection in Vegetated and Obscured Environments."
} 


\begin{tabular}{|l|c|c|c|c|c|c|c|}
\hline \multicolumn{1}{|c|}{ LiDAR } & $\begin{array}{c}\text { Velodyne } \\
\text { VLP-16 }\end{array}$ & $\begin{array}{c}\text { Velodyne } \\
\text { HDL-32E }\end{array}$ & $\begin{array}{c}\text { Velodyne } \\
\text { HDL-64E }\end{array}$ & $\begin{array}{c}\text { GDRS XR } \\
\text { microLADAR }\end{array}$ & $\begin{array}{c}\text { IBEO LUX } \\
8 L\end{array}$ & $\begin{array}{c}\text { Optech } \\
\text { ILRIS-HD }\end{array}$ & $\begin{array}{c}\text { Optech } \\
\text { Lynx SG1 }\end{array}$ \\
\hline $\begin{array}{l}\text { Horizontal } \\
\text { Angular } \\
\text { Resolution }\end{array}$ & $0.1-0.4^{\circ}$ & $0.16^{\circ}$ & $0.09^{\circ}$ & $\begin{array}{c}\text { Variable (non- } \\
\text { overlapping scan } \\
\text { pattern) }\end{array}$ & $\begin{array}{c}\text { Up to } \\
0.125^{\circ}\end{array}$ & $0.001146^{\circ}$ & Unknown \\
\hline $\begin{array}{l}\text { Beam } \\
\text { Divergence }\end{array}$ & $3.0 \mathrm{mrad}$ & $2.79 \mathrm{mrad}$ & $2.0 \mathrm{mrad}$ & $3.2 \mathrm{mrad}$ & Unknown & 150 urad & Unknown \\
\hline
\end{tabular}

The range and accuracy of a LiDAR system are dependent on many factors, including the reflectivity of the object and ambient lighting conditions. For example, the Velodyne 64 specifies a 120-meter maximum range, but this figure is based off of objects with $\sim 0.8$ reflectivity (e.g., cars, foliage). The maximum range is reduced to $50 \mathrm{~m}$ for $\sim 0.1$ reflectivity (e.g., pavement).

The horizontal and vertical resolutions of a sensor are critically important when evaluating the performance of each LiDAR for the target detection application. When the desired target sizes are small (e.g., 6.5” x 6.5” x 7.75"), and the desired stand-off distances are large (e.g., 13 meters), detection is reliant on achieving enough points of the object of interest.

To illustrate this point, Figure 63 (left) shows the scan pattern of the Velodyne 64 on an object 13 meters away, which was taken from a non-moving vehicle. The red box represents the object (paint-can sized), and the blue dots represent the LiDAR scan pattern. The horizontal angular resolution is much smaller than the vertical angular resolution (0.09 vs. 0.4 degrees), producing a denser point cloud in the horizontal dimension.

In comparison, the results from the Velodyne 32 (Figure 63, right) show that the increased vertical angular resolution (1.33 degrees) produces drastically different results at the target range of 13 meters. Instead of three scan lines hitting the target of interest, only a single scan line intersects the target; at the 13 meters distance, there are close to 12 inches vertically between scan lines. 
Figure 63. Velodyne 64 simulation (left); Velodyne 32 simulation (right).
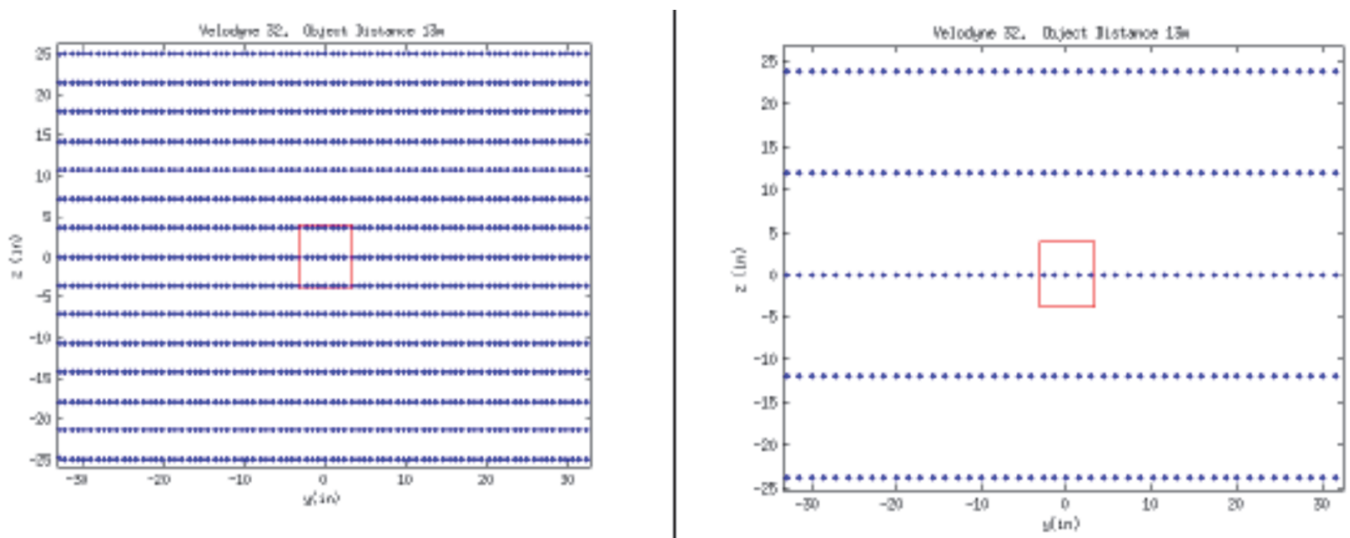

Figure 64 shows a point cloud comparison for a 30-mph collection. The top image shows the test setup, and the colored boxes represent different areas with static obstacles. The bottom images show the point clouds that are generated from driving, at speed, starting 200 meters from the first obstacles until the vehicle is approximately lined up with the first obstacles. The Velodyne HDL-64E, as expected, produces the densest point clouds; the Velodyne has 64 times as many lasers as the XR microLADAR and eight times as many lasers as the IBEO LUX 8L.

Figure 64. LiDAR point clouds from three different sensors.

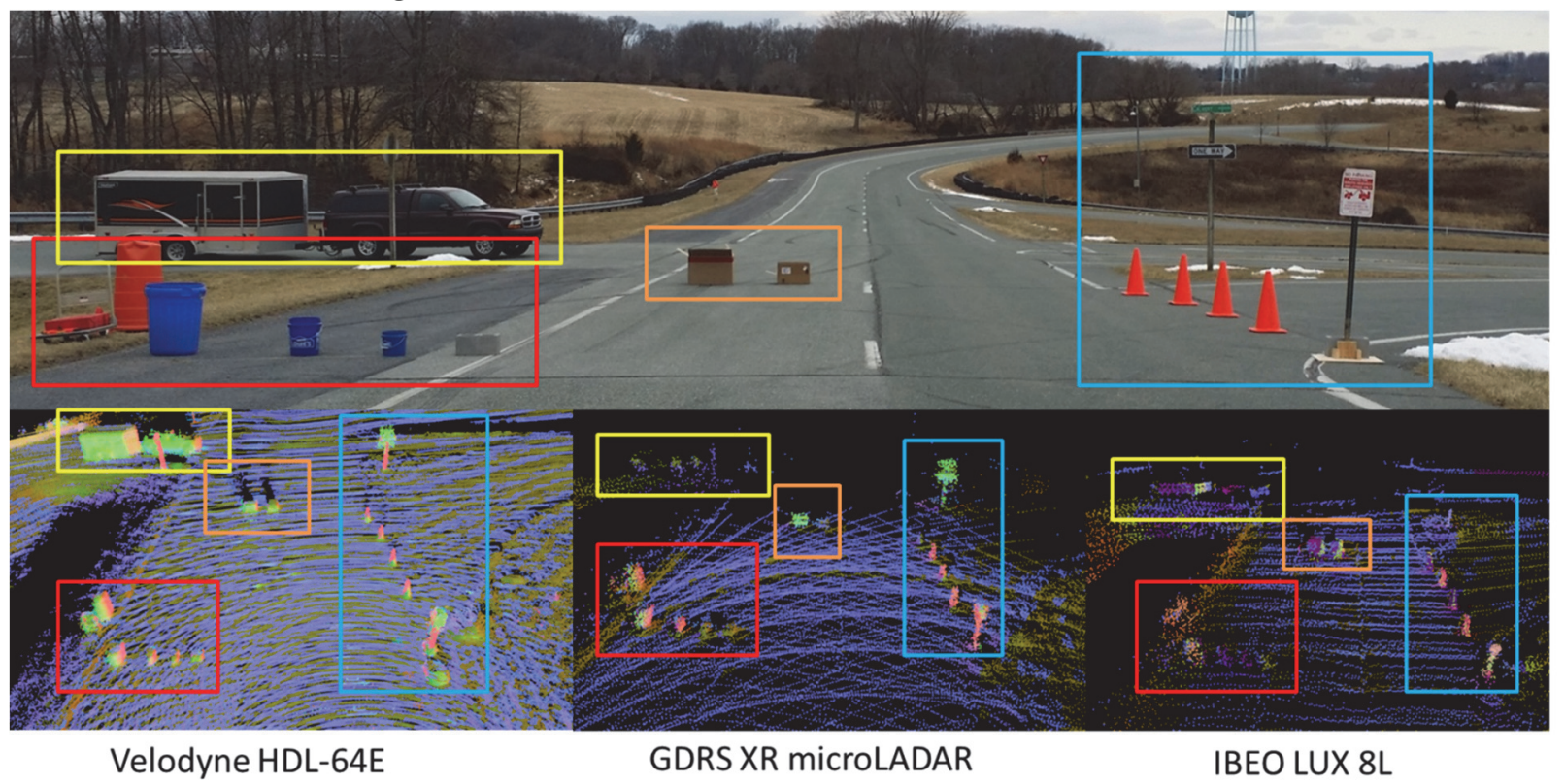

One of the objectives of the SMEs' analysis is to determine at what distances different objects can be detected. When plotting the point cloud 
collected starting at 150 meters and ending at 100 meters from the obstacles, the Velodyne HDL-64E obtained points on the barrels, cardboard boxes, and one of the street signs (Figure 65). By the 50-meter range, the point cloud was filled in with a significant number of points on each of the target objects (Figure 66).

Figure 65. Velodyne HDL-64E at 150-100 m range.

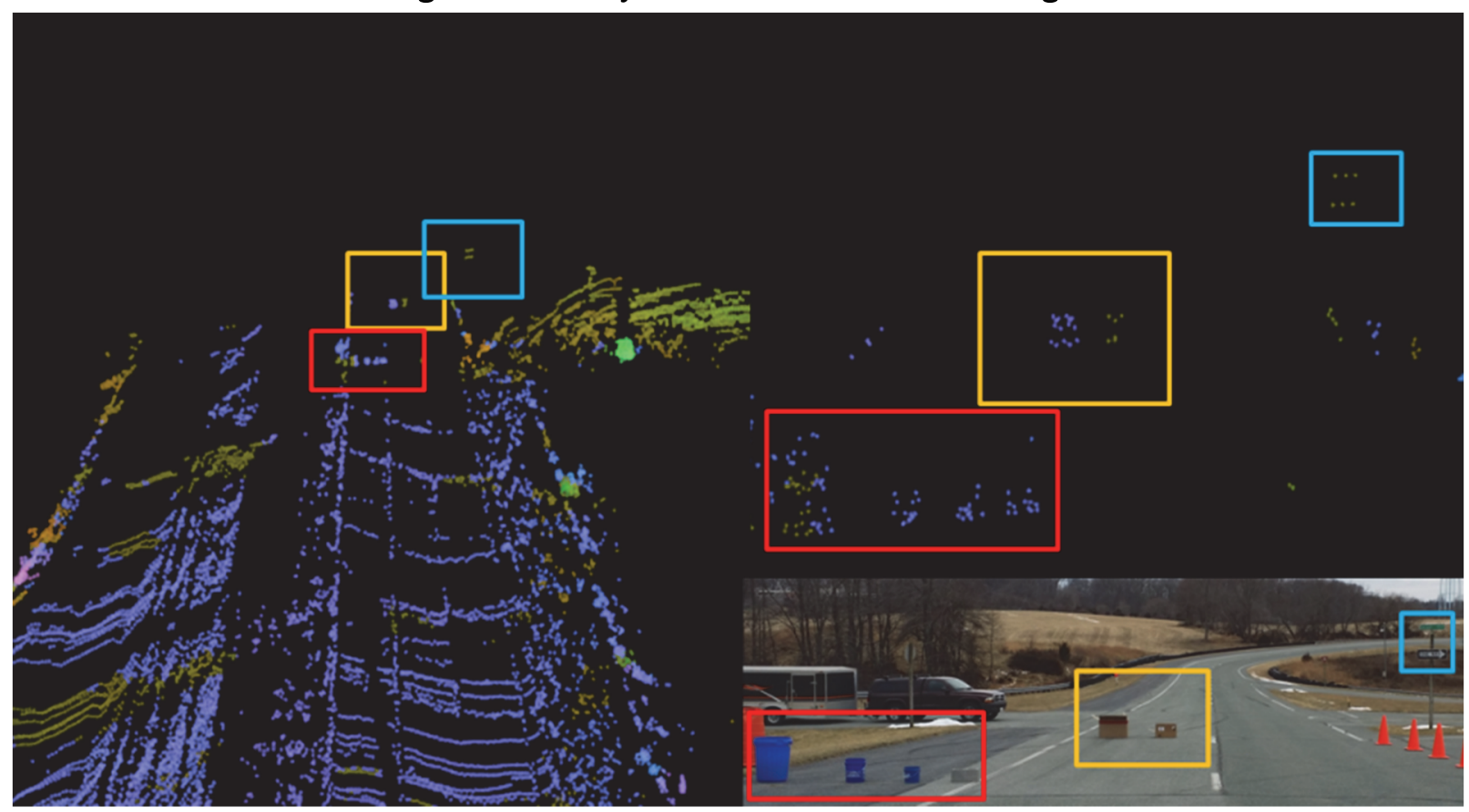


Figure 66. Velodyne HDL-64E at 100-50 m range.

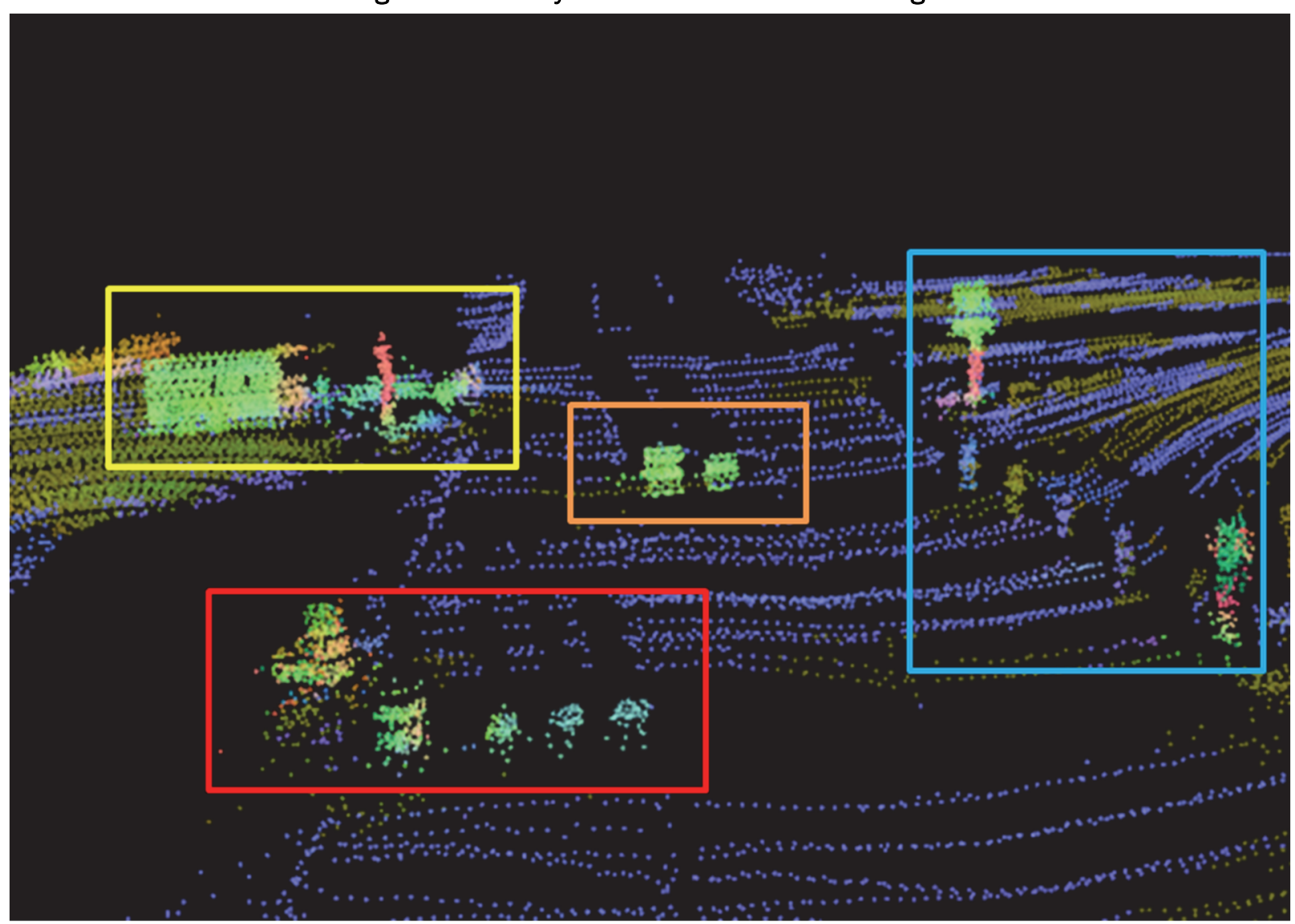

In addition to LiDARs, Electro- Optical (EO) cameras also can perceive objects from the distances required in this project.

As a starting point to determine the specifications needed for a camera system, Robotic Research is investigating the capabilities of human vision. The visual acuity of the human eye is $\approx 0.59$ arcminutes per line pair. (An arcminute is equal to $1 / 60$ th of a degree.) Because you need two pixels minimum are needed to distinguish a line pair, the necessary pixel spacing to equal human vision is $\approx 0.3$ arcminutes (0.005 degrees).

Robotic Research created an Excel tool to allow calculation of a variety of parameters (camera resolution, lens focal length, sensor size, object size, distance to the object of interest) and compute the resulting field of view, resolution (pix/m and angular), and number of pixels on target (Figure 67).

For example, using the specifications for one of the cameras, Robotic Research has in-house (Point Grey Grasshopper 3, 5MP [2448x2080], 2/3 
inched sensor size), in combination with, a 36- $\mathrm{mm}$ rectilinear lens, will generate a $15.7^{\circ} \mathrm{H} \mathrm{x} 13.8^{\circ} \mathrm{V}$ field of view and a $0.0065^{\circ}$ angular resolution (close to the human acuity values). A $10-\mathrm{mm}$ lens would increase the field of view $\left(52.8^{\circ} \mathrm{H} \mathrm{x} 47^{\circ} \mathrm{V}\right)$ but would sacrifice angular resolution (0.022 degrees). This trade-off is shown in Figure 68, which shows a plot of horizontal field of view versus focal length as well as angular resolution versus focal length for a 5-MP and 9.1-MP camera.

Figure 67. Excel-based camera analysis.

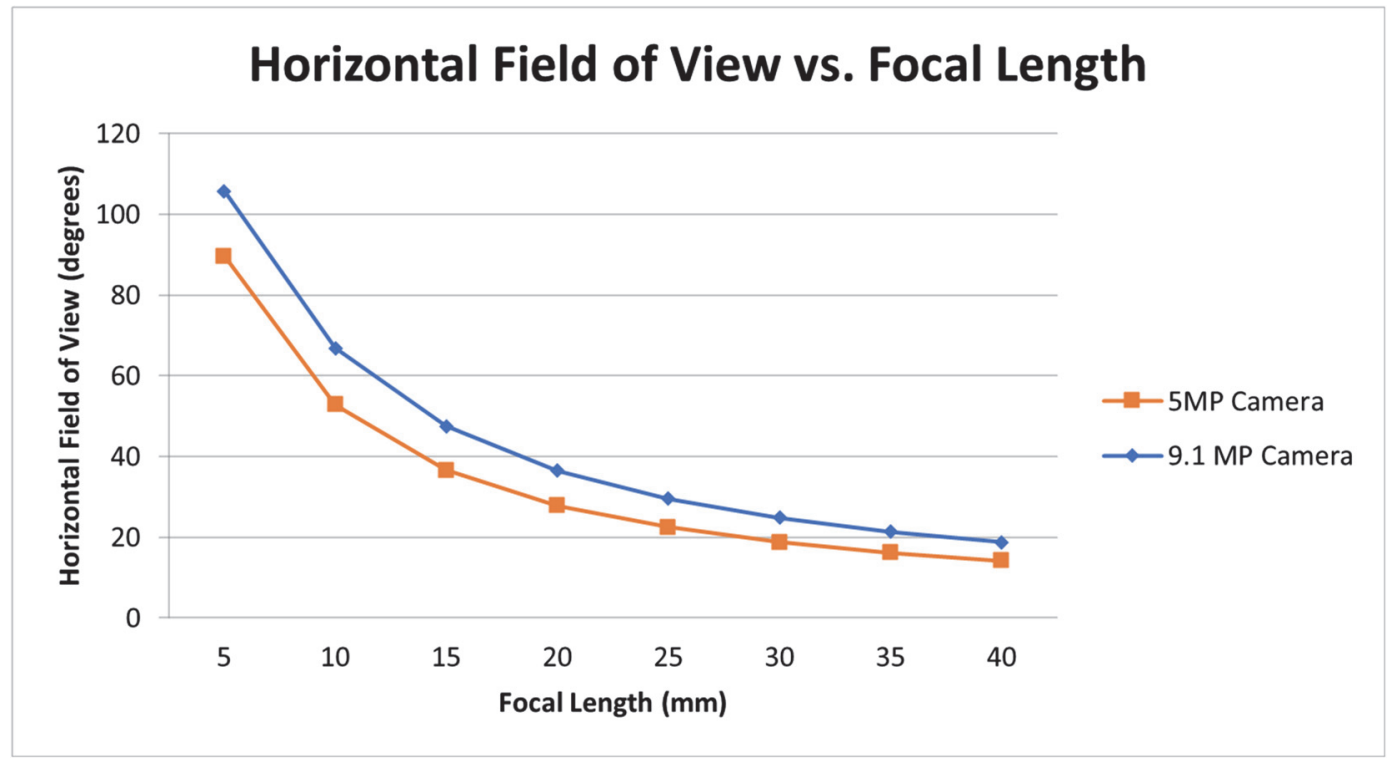

Figure 68. Excel-based camera analysis.

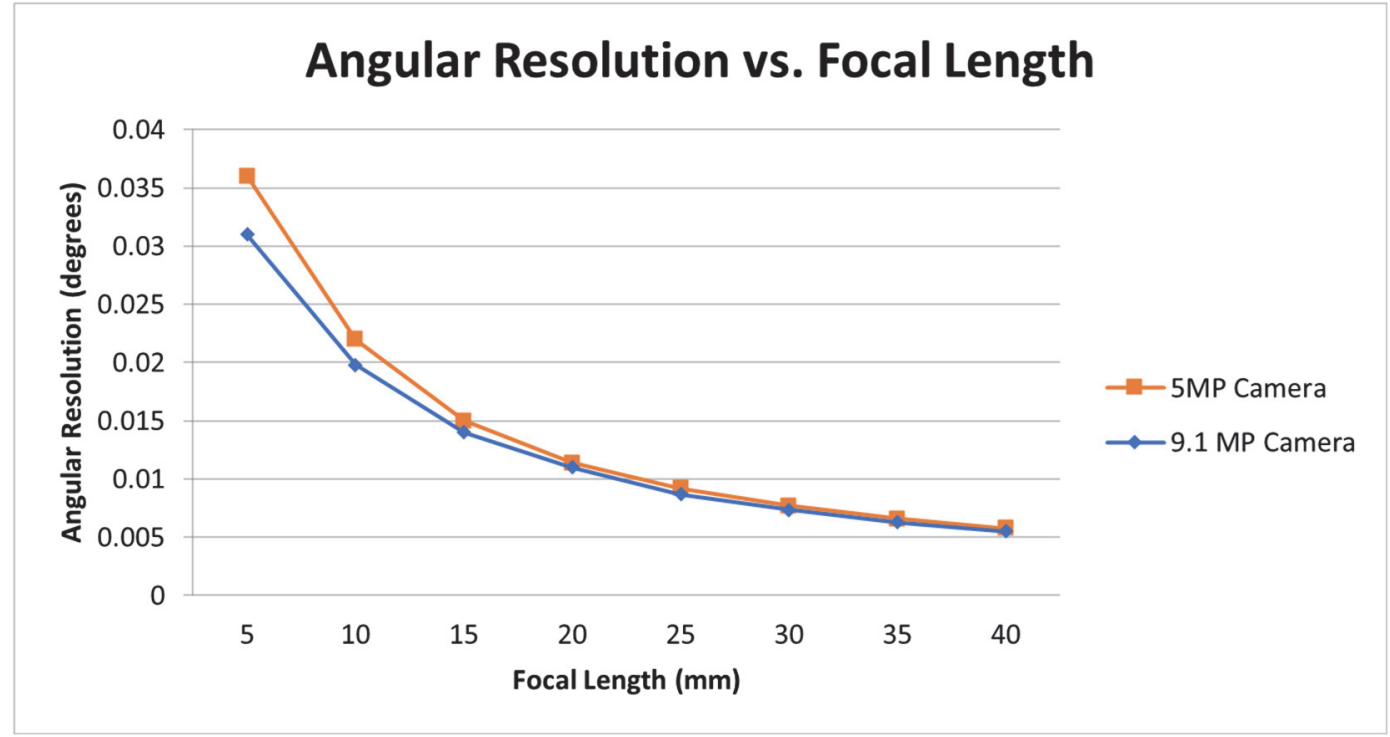


Robotic Research has also collected and analyzed data from several radar sensors. The specifications of every sensor are listed in Table 41.

\subsection{Feasibility study}

The objective of this research is to determine the feasibility of using the sensors described in the sections above in a variety of known test locations. The feasibility study will look at the number of sensors needed for a given test location and cost benefits versus the ability to collect autonomous-system data.

As described in Section 3.4 under testing, LiDAR can provide centimeter accuracy of the autonomous-system and has a range of approximately 100 meters. As the test site grows in size, more LiDARs will be needed to fully cover the site.

Table 43 lists a variety of known test locations and the number of sensors needed to measure the autonomous-system performance at each site. More details of the test locations can be found in Appendix $\mathbf{P}$.

Table 43. Autonomous-system test sites.

\begin{tabular}{|c|c|c|c|}
\hline Site & Site Details & $\begin{array}{l}\text { Number of Visual } \\
\text { Sensors }\end{array}$ & $\begin{array}{l}\text { Number of } \\
\text { Differential GPS }\end{array}$ \\
\hline $\begin{array}{l}\text { CBITEC } \\
\text { Charging Area }\end{array}$ & $\sim 100 \mathrm{~m} \times 100 \mathrm{~m}$ relatively flat parking pad area. & $\begin{array}{l}2 \text { LiDAR, } 2 \text { radar, } 2 \\
\text { cameras on opposing } \\
\text { corners of the parking pad } \\
\text { area }\end{array}$ & 1 base station \\
\hline $\begin{array}{l}\text { TA } 231 \text { Driving } \\
\text { Course }\end{array}$ & $\begin{array}{l}\sim 600 \mathrm{~m} \times 150 \mathrm{~m} \text { off-road loop with blind turns and } \\
\text { elevation changes. }\end{array}$ & $\begin{array}{l}9 \text { LiDAR, } 9 \text { radar, } 9 \\
\text { cameras spread around } \\
\text { the test track }\end{array}$ & $\begin{array}{l}1 \text { base station } \\
\text { *can use CBITEC } \\
\text { base station due to } \\
\text { proximity }\end{array}$ \\
\hline $\begin{array}{l}\text { The American } \\
\text { Center for Mobility } \\
\text { (ACM) }\end{array}$ & $\begin{array}{l}\text { ACM's facility encompasses } 500 \text { acres, multiple on- } \\
\text { road test environments, and is adjacent to the Willow } \\
\text { Run Airport. }\end{array}$ & $\begin{array}{l}4 \text { LiDAR, } 4 \text { radar, } 4 \\
\text { cameras on opposing } \\
\text { corners of the configurable } \\
\text { area } \\
\text { Visual sensor coverage is } \\
\text { not economically feasible } \\
\text { to cover road network } \\
\text { area. }\end{array}$ & 1 base station \\
\hline $\begin{array}{l}\text { Larson } \\
\text { Transportation } \\
\text { Institute }\end{array}$ & $\begin{array}{l}\text { The facility includes the Bus Research and Testing } \\
\text { Center, Center for Integrated Asset Management for } \\
\text { Multi-Modal Transportation Infrastructure Systems } \\
\text { and the Center for Dirt and Gravel Road Studies. }\end{array}$ & $\begin{array}{l}4 \text { LiDAR, } 4 \text { radar, } 4 \\
\text { cameras on opposing } \\
\text { corners of the vehicle- } \\
\text { handling area } \\
\text { Visual sensor coverage is } \\
\text { not economically feasible } \\
\text { to cover road network } \\
\text { area/ }\end{array}$ & 1 base station \\
\hline
\end{tabular}


Figures 69 and 70 show examples of how fixed-position sensors could be used at the CBITEC and the TA-231 Driving Course test locations. The sensor coverage map is a graphical representation of where sensors could be placed for optimal site coverage.

Figure 69. CBITEC sensor coverage map.

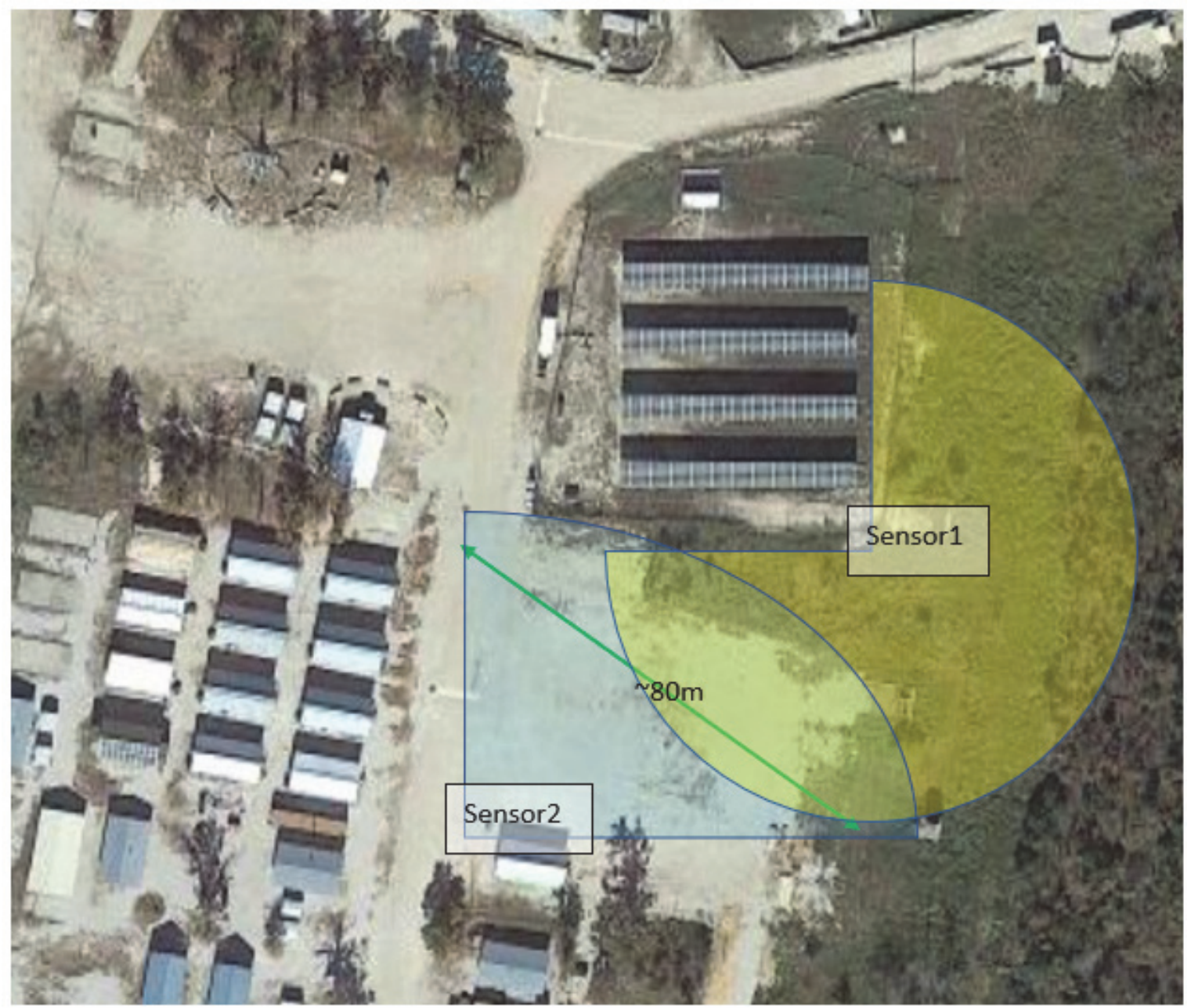


Figure 70. TA 231 driving course sensor coverage map.

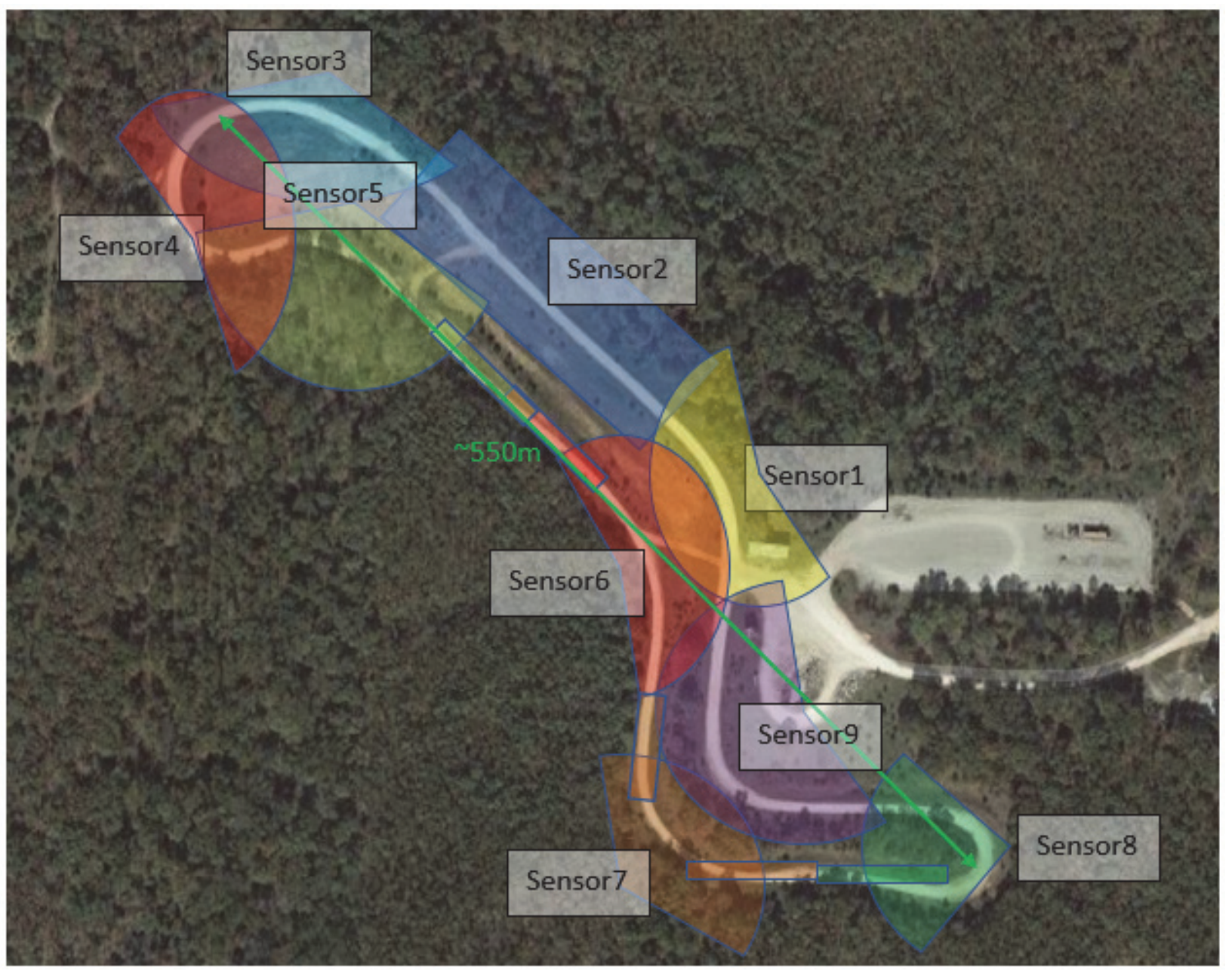

As stated in Table 43, the test areas with larger road networks become economically infeasible to outfit with vision-type sensors. The number of sensors required to cover the area and the additional infrastructure needed to power the sensors makes using a differential base station a more cost effective option. In smaller test areas, such as CBITEC, both vision-type sensors and DGPS could accurately measure the autonomous-system's position and attitude. Additionally, a hybrid approach could be used with both DGPS and a single set of vision-type sensors. The hybrid approach would allow for the vision sensors to cover areas that might have degraded GPS.

\subsection{Conclusions and future work}

Many of the testing methodologies discussed in this report are applicable to the test areas at FLW, specifically CBITEC, and the TA-231 Driving 
Course. The next step in this research is to outfit these test areas with sensor suites discussed in the report and to begin testing autonomous systems at this site. As more details about the autonomous-system and test areas are solidified, the test plans in this report can be evolved into test procedures tailored for the FLW site. 


\section{Identify Requirements for a Sensored, Living Laboratory to Assess the Performance of V2G Charging in a Tactical Microgrid}

The objective of Research Area ${ }_{5} \mathrm{~B}$ is to identify requirements for a sensored, living laboratory to assess the performance of $\mathrm{V} 2 \mathrm{G}$ charging in a tactical microgrid environment and to recommend an optimal $\mathrm{V}_{2} \mathrm{G}$ solution set for use at the CBITEC site.

To achieve this research objective, Paragon SMEs established requirements for a sensored, living laboratory, to test recommended V2G solutions, and ensure adherence to current and developing standards at CBITEC. The living laboratory must have the capacity and capability to verify the $\mathrm{V} 2 \mathrm{G}$ aspects of the system and ensure that they comply with operational requirements outlined in Research Area 4. Specific tests and measurements to determine whether measured performance meets or exceeds requirements were conducted to review procured V2G charging equipment. Paragon SMEs integrated standard test thresholds into the CBITEC tactical microgrid simulation to evaluate system performance and refine $\mathrm{V}_{2} \mathrm{G}$ solution set recommendations along with simulation results from Research Area 4.

\subsection{Develop requirements for a sensored, living laboratory}

The objective of this research is to develop requirements for the CBITEC sensored, living laboratory to have the capability to test recommended V2G solution and standards adherence.

To develop a set of operational requirements meeting this objective, Paragon SMEs conducted an extensive review of available literature. The SMEs collaborated with government personnel to capture and document requirements as they relate to recommended $\mathrm{V} 2 \mathrm{G}$ technology testing and demonstration at the CBITEC site. The following items were investigated for suitability: power distribution equipment, communications equipment, EVs, EV charging equipment, and power measurement equipment. Results 
of the investigation identified operational requirements to use for selection necessary to integrate the $\mathrm{V} 2 \mathrm{G}$ solution.

\subsection{Identify methods to quantify V2G system aspects}

Methods to quantify V2G system aspects to ensure compliance with operational requirements outlined in Research Area 4 include confirming:

- Communication capability of the V2G solution set that enable overarching control as one system, of several systems integrated together, to provide an energy resilient tactical microgrid.

- Electrical ratings are compatible with the tactical microgrid electrical ratings of voltage and frequency.

- Operating modes of the $\mathrm{V} 2 \mathrm{G}$ solution set can provide and receive power as part of the envisioned tactical microgrid.

- Power capabilities sufficiently support the tactical microgrid.

After quantifying V2G system aspects, Paragon researchers conducted an industry search to identify appropriate technology solutions to develop a V2G solution set recommendation.

Equipment safety tests must be available to conduct test plans. Current policy at CBITEC prevents using automated test plans and requires all testing be conducted manually. Consequently, conducting a test plan in a living laboratory at CBITEC requires a clear test plan documenting each test step. A well-defined test plan ensures tests are conducted correctly and repeatable to verify test results or to quantify system changes based on previous test results. When testing electrical equipment, a manual method for disconnecting equipment should be identified in case a protective device does not properly operate.

Prior to conducting any test plan on operating equipment, a "dry run" is recommended to ensure test personnel completely understand the test plan. Additionally, this action is an opportunity to update test plan instruction and verify test personnel understand proper test plan actions prior to energizing equipment. With appropriate equipment protective devices in place and adjusted for the EUT, a clear step-by-step test plan can be safely conducted by qualified personnel. Per the NEC, qualified personnel are individuals who possess the skills and knowledge related to 
construct and operate electrical equipment and installation and have also received safety training to recognize and avoid the hazards involved.

To safely test a V2G system, the existing IPD box contains SEL 849 protective relays that communicate with a SEL 3505 RTAC (HG Engineers, 2019) that can meet the protection and metering requirements for the $\mathrm{V} 2 \mathrm{G}$ system as well as communicate with the CBITEC data collection server. Each IPD will need to be configured to meet the data collection and protection requirements for the connected equipment that is connected to it.

To install a V2G solution at CBITEC, the charger should be bolted to a suitable concrete pad located on top of reasonably level ground. The equipment will be able to withstand potential winds without in-ground concrete foundations and the extensive approval process required for installation.

\subsection{Sensored, living laboratory requirements}

Testing and evaluating new equipment in a living laboratory require additional equipment to quantify component performance and interoperability while ensuring personnel and connected equipment safety. Protective devices that can be adjusted to protect and quickly disconnect EUT are critical for the safety of personnel, the EUT, and the connected test system. Protective devices, such as microprocessor relays with adjustable trips settings for current, voltage, frequency, power flow, are required. These same microprocessor relays dominate the data collection in the bulk electric power system and are likewise suggested to be employed at the laboratory.

The international ${ }^{*}$ Standard for the Testing of Microgrid Controllers, requires collecting data collection for the testing of microgrid testing as summarized in Table 44, which below: minimum sample rates in milliseconds $(\mathrm{mSec})$ for the voltage $(\mathrm{V})$ and current (I) data.

Table 44. IEEE 2030.8 required data collection.

\begin{tabular}{|c|c|l|l|}
\hline Type of Data Collection & \multicolumn{1}{|c|}{$\begin{array}{c}\text { Minimum Time-Synchronized } \\
\text { Accuracy }\end{array}$} & $\begin{array}{c}\text { Minimum Sample } \\
\text { Rate }\end{array}$ & $\begin{array}{c}\text { Required Data to } \\
\text { Collect }\end{array}$ \\
\hline Sequence of Events (SOE) & $4.16 \mathrm{mSec}$ & $4.16 \mathrm{mSec}$ & Digital data \\
\hline
\end{tabular}

* Institute of Electrical and Electronics Engineers. "2030.8-2018 - IEEE Standard for the Testing of Microgrid Controllers." IEEE Standards Association, Institute of Electrical and Electronics Engineers, 24 Aug. 2018, standards.iee.org/standard/2030_8-2018.html. 


\begin{tabular}{|l|l|l|l|}
\hline \multicolumn{1}{|c|}{ Type of Data Collection } & \multicolumn{1}{|c|}{$\begin{array}{c}\text { Minimum Time-Synchronized } \\
\text { Accuracy }\end{array}$} & $\begin{array}{c}\text { Minimum Sample } \\
\text { Rate }\end{array}$ & \multicolumn{1}{c|}{$\begin{array}{c}\text { Required Data to } \\
\text { Collect }\end{array}$} \\
\hline Event Oscillography (OSC) & $4.16 \mathrm{mSec}$ & $4.16 \mathrm{mSec}$ & V and I all phases \\
\hline $\begin{array}{l}\text { Continuous Data Collection } \\
\text { (CDC) }\end{array}$ & $4.16 \mathrm{mSec}$ & $100 \mathrm{mSec}$ & V and I all phases \\
\hline
\end{tabular}

The data listed in Table 45 is essential for root-cause analysis of events. Voltage and current are used to derive all other quantities. Frequency, root mean square (RMS) voltage, RMS current, phase angle, real and reactive power/energy, voltage sags, swells, and other characteristics, for example, are all derived from the voltage and current signals listed in Table 45 .

Considering the low cost of memory storage in 2020, and the relatively slow sampling intervals of the standard, this is a low-cost requirement that is easily accomplished with COTS equipment today. Per the standard, the data collected in the prior table must occur on the AC side of every genset, inverter, and any power-wheeling breaker or load larger than 50\% of the total load. Events to be tested are noted in Table 45.

Table 45. IEEE 2030.8 test events.

\begin{tabular}{|l|c|c|c|}
\hline Event & SOE & OSC & CDC \\
\hline Islanding & $\mathrm{X}$ & $\mathrm{X}$ & $\mathrm{X}$ \\
\hline Synchronizing & $\mathrm{X}$ & $\mathrm{X}$ & $\mathrm{X}$ \\
\hline DER trip & $\mathrm{X}$ & $\mathrm{X}$ & \\
\hline Faulted circuit & $\mathrm{X}$ & $\mathrm{X}$ & \\
\hline Load shed & $\mathrm{X}$ & $\mathrm{X}$ & $\mathrm{X}$ \\
\hline Dispatch from microgrid controller & & & $\mathrm{X}$ \\
\hline Black start & $\mathrm{X}$ & $\mathrm{X}$ & $\mathrm{X}$ \\
\hline
\end{tabular}

To measure the EUT's performance, sufficient measurement devices must be installed in the test system to capture the critical performance aspects. In the case of the V2G system, measurements include:

- Total three-phase real and reactive power

- Individual-phase real and reactive power for all three phases

- AC voltage, current, and frequency

- Accumulated energy exported-both real and reactive

- Accumulated energy imported-both real and reactive

- Total harmonic distortion

- Synchronized timestamp of each data point. 
Minimum sampling rates, called out in the 2030.8 standard, are consistent with protective relaying equipment installed throughout the bulk electric generation, transmission, and distribution power system today.

SOE reports (SERs) are recorded in most microprocessor relays manufactured today. SERs in these relays must be configured to record breaker status, inverter status, generator controller statuses, warning events, alarms, equipment health, protection events, life/safety events, and internal logic variable state changes. SERs are fundamental to debugging new systems and time-aligning events for root-cause analysis. Power systems react in fractions of a power cycle, approximately $4 \mathrm{mSec}$, which is why microprocessor relays and SERs are so essential.

Oscillographic event reports (ERs) are recorded in most microprocessor relays manufactured today. ERs in these relays must be configured to record high-speed sampled currents and voltages for periods of time intervals up to 1 second in duration. This captures the power system' oscillatory behavior, transients, sub-transients, magnetic saturation effects, X/R time constants, fault currents, and much more. ERs are commonly used to understand the root cause of events. They are also required in 2030.8 because life-safety events, such as life-threatening arc-flash events, are captured with ERs. These events are stored in the relay and are regularly retrieved with automated software to analyze system events and performance.

CDC is most commonly accomplished with synchrophasor data collection performed inside modern microprocessor relays. Modern relays include phasor measurement units (PMU), which collect time-synchronized data samples of a power system in compliance with the Institute of Electrical and Electronics Engineers*. These are proven to be an excellent conditionmonitoring system when pushed to synchrophasor data consolidation and historian software. In many applications, $\mathrm{C}_{37.118}$ data includes much more than just the data required by the 2030.8 standard. It also includes a single heads up display/historian with a single screen showing cyber-attack metrics, network traffic metrics, power system electrical parameters,

\footnotetext{
* Institute of Electrical and Electronics Engineers. "C37.118.1-2011 - IEEE Standard for Synchrophasor Measurements for Power Systems." IEEE Standards Association, Institute of Electrical and Electronics Engineers, 28 Dec. 2011, standards.ieee.org/standard/C37_118_12011.html.
} 
synchronization data, battery health, and engine mechanical health data. SEL implemented its standard off-the-shelf software in its TMS demonstration project as shown in Figure 71. This data was stored in an off-theshelf computer with over 20 years of data storage for continuous microgrid operation.

Figure 71 . SEL PC with 20 years of microgrid data storage.*

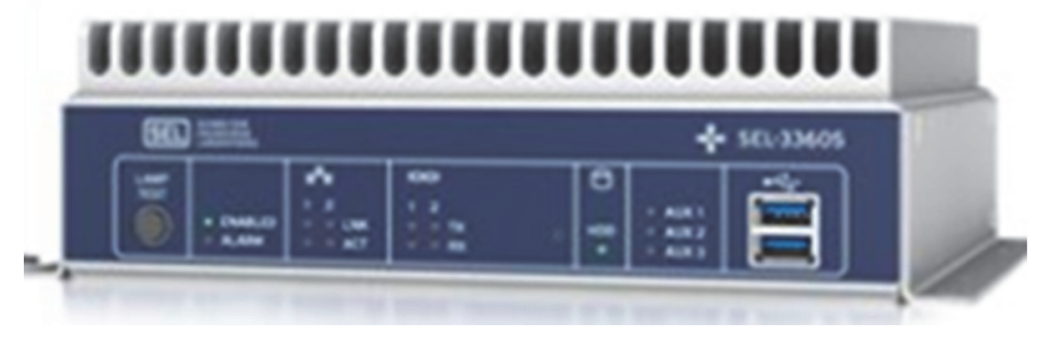

Visualization interface, such as the IEEE C37.118 synchrophasor data condition monitoring application shown above, is a user-configurable operator interface. This allows tests to be remotely monitored, with significantly slower refresh rates $(1 \mathrm{~Hz})$ to the operator display, for effective visual monitoring by zooming out to see long-term trends. When zooming, these systems scale to higher-resolution data for better precision.

Similar laboratory facilities are located at Mount Holley's in Duke Energy's ${ }^{\dagger}$ microgrid research yard. This yard contains a diverse set of generators, PV, batteries, loads, and a host of experimental equipment. This yard is instrumented with industry standard electronics primarily from SEL because of its dominance in generation, transmission, distribution, microgrid, and industrial substations throughout the United States (Figure 72).

\footnotetext{
* Schweitzer Engineering Laboratories. "SEL-3360 Compact Industrial Computer." Schweitzer Engineering Laboratories, 24 Sept. 2019, selinc.com/products/3360/.

† Duke Energy. "Duke Energy Smart Grid: Designing Ways to Operate the Grid More Simply and Cost-Effectively." Duke Energy, Duke Energy Corporation, Jan. 2016, duke-energy.com/our-company/about-us/smart-grid/coalition.
} 


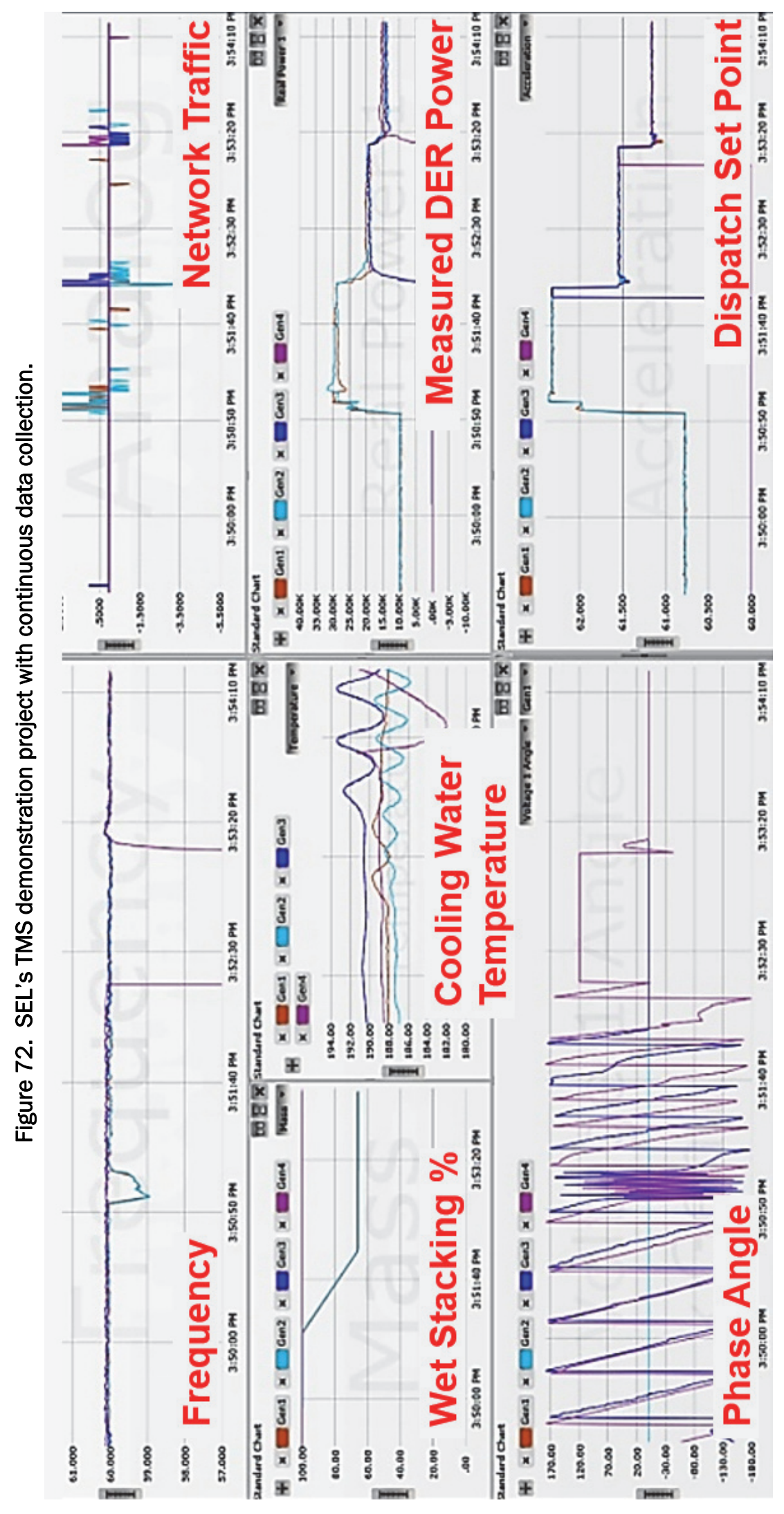


In the investigation of central versus distributed system monitoring, protective relays can provide both human safety, equipment protection, and built-in storage capabilities equal to or greater than devoted metering systems. SEL's onboard memory enables high-speed data collection that would quickly overwhelm a high-speed Ethernet network. Therefore, local storage on these relays is necessary. Off-the-shelf software is then used to download these events without undue network traffic. These data collection on the relays are first in/, last out, in that old events are eventually flushed from the relays. The protective relaying method of local (distributed) storage of data in the relay followed by a (centralized) data historian for long-term archiving appears to provide the optimal solution for power systems. This has decades of reliable operation throughout the oil and gas and utilities industries. This method avoids risks to data loss associated with noise and bandwidth limitations of communication links.

Disturbance recording systems of a non-relaying process controller-based equipment can be too slow for practical use on a microgrid. Sample rates can also be too slow, and, often, there is no remote (decentralized) storage. The systems tend to be much more expensive. One example is OSIsoft's PI System (OSISoft, n.d.), which has exorbitant license fees and complex software that requires significant coding skills to support, set up, and maintain.

Relay-based data collection methods communication requirements appear optimally designed for power systems. They are reliable with intermittent communication links; they are commonly retrieved with serial communication channels, voice channels, and other antiquated communications techniques. A restricted Ethernet system can offer sufficient bandwidth enabling connection to capture high resolution oscillography for extended tests without data loss (Figure 73). 
Figure 73. SEL's relays meet SOE, OSC, and CDC data collections requirements of IEEE 2030.8.

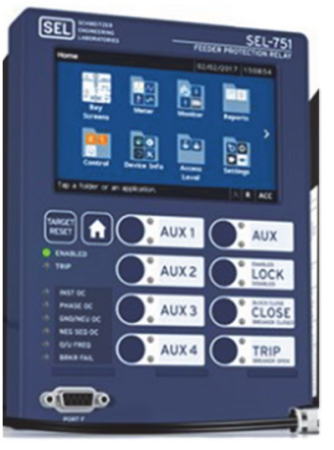

Various components needed to outfit a living laboratory will need evaluating to maintain compatibility with existing equipment and promote interoperability with open standards. SEL components listed in Table 46 are suggested as one holistic solution.

Table 46. Living Laboratory hardware components.

\begin{tabular}{|l|l|l|}
\hline \multicolumn{1}{|c|}{ Location } & \multicolumn{1}{|c|}{$\begin{array}{c}\text { Suggested SEL } \\
\text { Component }\end{array}$} & \multicolumn{1}{c|}{ Comments } \\
\hline Point of utility connection & SEL-751 + SEL-3505 & Acquire in a wall-mount outdoor enclosure \\
\hline AMMPS genset & SEL-700G + SEL-3505 & Acquire in a wall-mount outdoor enclosure \\
\hline IPD input port & SEL-700G + SEL-3505 & Exists in HG's IPD 800 \\
\hline IPD output port & SEL-849 & Exists in HG's IPD 800 \\
\hline IPD ring-bus port & SEL-849 & Exists in HG's IPD 800 \\
\hline Network Ethernet & SEL-2740S & $\begin{array}{l}\text { Used to detect and deflect outside intrusion and cyber } \\
\text { attacks }\end{array}$ \\
\hline Data concentration & SEL-3530-4 & Consolidates TMS traffic to a single C37.118 data stream \\
\hline $\begin{array}{l}\text { Human machine interface and } \\
\text { engineering workstation }\end{array}$ & SEL-3360 & $\begin{array}{l}\text { Provides two TV monitor ports and long-term data } \\
\text { archiving for all CDC, SOE, and OSC data }\end{array}$ \\
\hline Synchrophasor historian & SEL-5078 software & Historian for 20 years of microgrid run-time \\
\hline PV array & SEL-700G + SEL-3505 & Acquire in a wall-mount outdoor enclosure \\
\hline Battery & SEL-700G + SEL-3505 & Acquire in a wall-mount outdoor enclosure \\
\hline V2G & SEL-700G + SEL-3505 & Acquire in a wall-mount outdoor enclosure \\
\hline
\end{tabular}

\subsection{Procure V2G charging equipment}

The objective is to procure $\mathrm{V} 2 \mathrm{G}$ charging equipment to conduct initial testing and analyze results to confirm performance.

The approach identifying the $\mathrm{V} 2 \mathrm{G}$ charging equipment to be procured commenced with an industry search to distinguish and evaluate V2G 
charging solutions that had the potential to meet program objectives and site requirements. Program objectives included:

- Promoting interoperability of microgrid equipment through open standards

- Increasing tactical microgrid energy resiliency by using a V2G system capable of supplying, or consuming, $30 \mathrm{~kW}$ or more.

Site requirements that were considered included:

- Electrical ratings compatible with the tactical microgrid conceptual design

- Enclosure ratings suitable for an outdoor installation

- Ability for a driver to take control of the vehicle.

Paragon SMEs have extensive demonstration experience. Given the aforementioned program objectives and site requirements, they:

- Researched the EV and charging station industry for potential V2G solutions

- Contacted peers and potential suppliers to confirm latest offerings

- Participated in technical research and discussions with potential suppliers identified in Research Area 2

- Evaluated an alternative approach of mounting EV batteries and a power converter to a General Dynamics Multipurpose Unmanned Tactical Transport (MUTT).

Currently in the United States, the CHAdeMO standard (CHAdeMO, n.d.) (Figure 74) is the only open standard that is designed to fully support the bidirectional power flow of $\mathrm{V} 2 \mathrm{G}$ systems. The CHAdeMO standard is commonly used in Asia and Europe; however, use in the U.S. is limited compared to the more dominant fast-charging standard that is produced by the SAE. 
Figure 74. CHAdeMO connector.

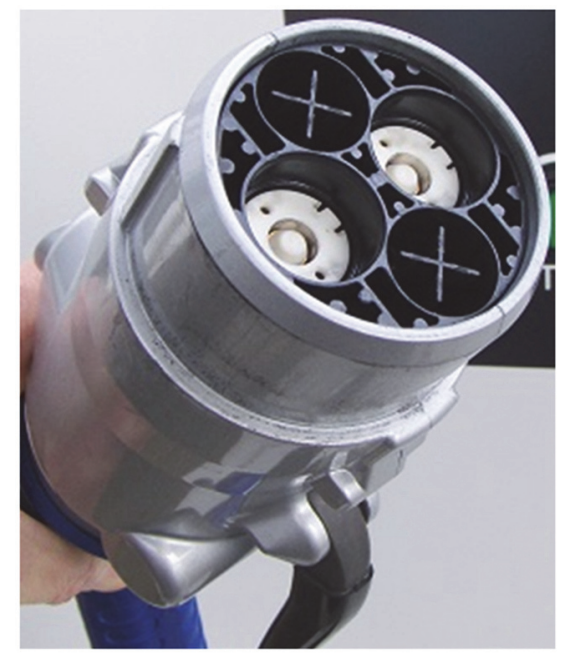

SAE's CCS (Figure 75) is the most commonly used fast-charging standard in the U.S. standard for fast-charging applications. This open standard is used by multiple charging station suppliers to charge CCS-compliant EVs. However, the CCS standard does not incorporate communication and command structures necessary to implement on-demand discharging of EVs.

Figure 75. SAE CCS connector.
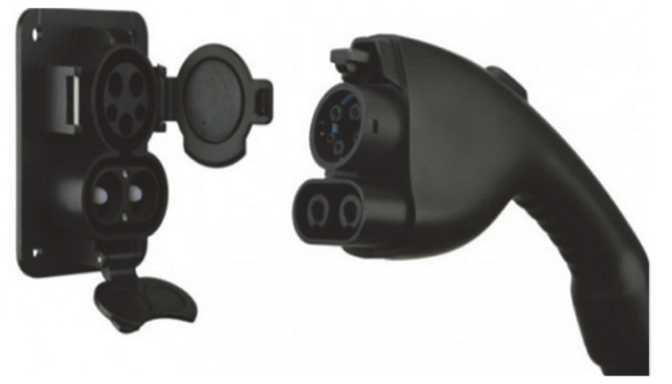

Consequently, messaging between the vehicles and chargers must be customized to include unique commands enabling a V2G-capable vehicle to use the CCS standard. This customization inherently prevents the custom charger to be readily used for multiple vehicle types. Such customization also requires extensive non-recurring engineering, development, and testing to implement the $\mathrm{V}_{2} \mathrm{G}$ capability, which further limits customized CCS solutions for V2G applications. By 2025, SAE plans to update the CCS standard to include communication and controls specifications necessary to enable $\mathrm{V} 2 \mathrm{G}$ operations between CCS-compliant vehicles and charging stations. 
While the SAE standards for vehicle charging might dominate the U.S., Paragon SMEs recommend using the CHAdeMO-compliant devices to validate $\mathrm{V}_{2} \mathrm{G}$ operation capabilities in the short term. This approach uses an open standard to promote interoperability, reduces non-recurring engineering costs, and schedules test compared to a custom solution that would be required by attempting to use SAE's present version of their CCS standard. Based on $\mathrm{V}_{2} \mathrm{G}$ validation results, purpose-built vehicles and charging solutions can be developed.

Currently in the United States, only two commercially available vehicles could be identified that are equipped with the CHAdeMO charging port: The Nissan LEAF (Figure 76) and the Mitsubishi Outlander (Figure 77). The 2019 Nissan LEAF is fully electric and can be ordered with either a 40-kWh lithium-ion battery pack or an extended range version rated at 62 $\mathrm{kWh}$. Because the LEAF is CHAdeMO-compliant, and the CHAdeMO specification fully supports V2G operation, the LEAF can export power without additional support from Nissan Motor Corporation (Nissan) or modification. Factory configuration of the Nissan LEAF limits charge and discharge rates to $30 \mathrm{~kW}$.

Figure 76. Nissan LEAF.

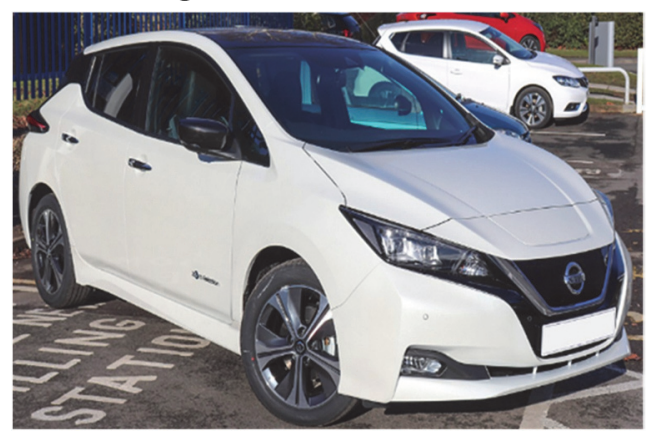

Source: (Vauxford 2018).

The 2019 Mitsubishi Outlander is a PHEV and consequently comes with a lithium-ion battery pack with a much lower energy storage capacity of only $13.8 \mathrm{kWh}$. Because a battery's power rating is directly influenced by the energy storage rating, a lower energy storage capacity also results in a lower power rating for the same battery chemistry. Factory configuration of the Mitsubishi Outlander limits charge and discharge rates to $22 \mathrm{~kW}$. Inherently, battery packs with lower energy storage ratings will typically have lower power capabilities. Further, the duration at the maximum power 
level will also be less, further reducing the vehicle's capability to support connected loads.

Figure 77. Mitsubishi Outlander.

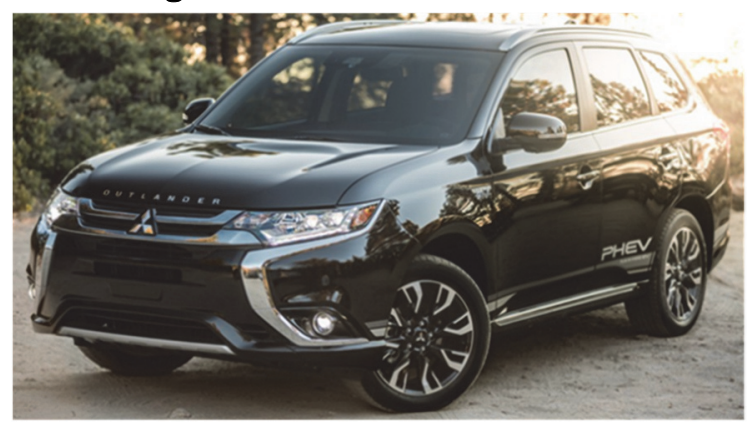

Source: (U.S. News \& World Report n.d.).

At CBITEC's request, Paragon SMEs also investigated the option of adding battery packs and a bidirectional power converter onto a modifying a mobile platform. For this approach, the mobile platform recommended was the General Dynamics MUTT by General Dynamics given the wide applicability across the Army. With the MUTT selected as the mobile platform, Go Electric and EnerDel were engaged to identify their solution for this alternative approach because both companies previously supported similar vehicle configurations that were delivered to CBITEC. Go Electric offered two battery configurations-; one of which offered was a integrating numerous individual batteries manufactured by Saft (Figures 78 and 79). The second option was to integrate three battery packs from EnerDel. EnderDel's battery solution also recommended the same EnerDel pack (Figure 79) recommended by Go Electric. Neither Go Electric nor EnerDel could offer a power converter solution to consider.

Figure 78. Saft Xcelion $6 \mathrm{~T}$ battery module.

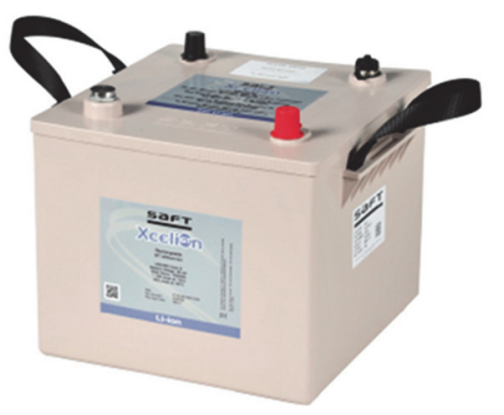

Source: (Saft 2019) 
Figure 79. EnerDel PE500-403.

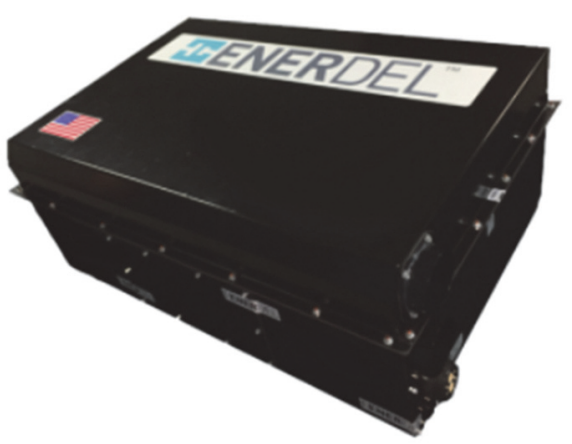

Source: EnerDel (2020).

To verify the viability of this alternate approach, Paragon SMEs compared the weight of the proposed battery solutions to the payload capacity of the MUTT (Figure 80). The weight of the battery solutions recommended by Go Electric and EnerDel ranged between 1,700 and 2,000 pounds, which exceeded the payload capacity of the largest available MUTT of 1,200 pounds by at least $40 \%$.

Figure 80. General Dynamics MUTT.

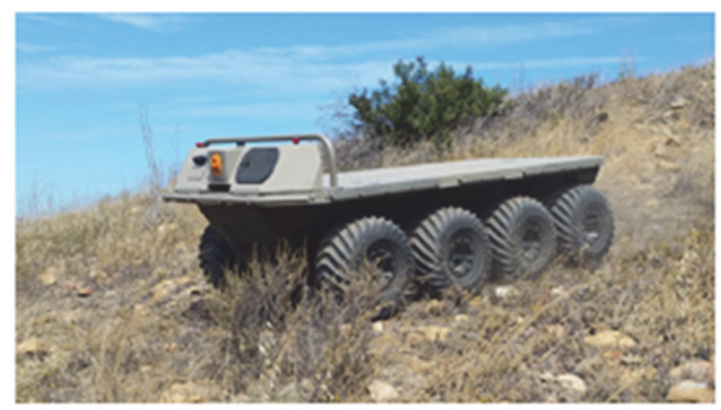

\section{X8 TRACKED/WHEELED}

- Payload (Ibs): 1200

- GVW (lbs): 3000/3500

- Length (in): 116

- Width (in): $60 / 70$

When considering the MUTT payload would increase hardware, protective devices, and an autonomy system integration, the weight of this alternate approach would overload the payload capacity of the MUTT. The payload 
would easily exceed $80 \%$ of its rating even if the required power converter were mounted offboard the vehicle rather than onboard as originally envisioned. Reducing the battery weight to one battery module still would not leave any payload capacity necessary for integration and the autonomousdriving kit. This alternate approach was deemed unviable given that MUTT does not have the payload capacity to carry an ESS rated to sufficiently interact with the envisioned microgrid.

To support energy resiliency of a microgrid, a V2G system with a power rating to significantly offset the planned 6o-kW AMMPS generator is highly recommended. While the off-road capability of the Mitsubishi Outlander might be a better fit for a tactical environment, the energy storage rating of the Outlander is only $20 \%$ that of the LEAF, which significantly limits the ability to validate impact a mobile platform might have on a microgrid. Overall, the greater power, energy, and payload capacity make the LEAF a better mobile platform for testing an EV's capability to support energy resiliency as intended by program goals.

Based on the selection of a LEAF as the mobile platform for testing purposes, Paragon SMEs searched for a V2G charger that could serve as a fast charger for the LEAF while meeting program objectives and site requirements. After searching the industry and engaging Nissan, as well as other contacts working in the $\mathrm{V}_{2} \mathrm{G}$ space, only Coritech Services, Inc. offered a suitable product, Coritech is located in Royal Oak, MI (Coritech Services, Inc.) and offers a $30-\mathrm{kW}$ fast charger that is CHAdeMO-compliant. Coritech's VGI-30-DC-CHA fast charger design (Figure 81) is suitable for integrating into the tactical microgrid as it:

- Has an AC voltage configuration that easily integrates into the planned 208-VAC microgrid distribution system with the use of a COTS stepdown transformer.

- Is specifically designed for fast charging and discharging of a CHAdeMO-compliant vehicle.

- Uses open protocols to interface with an overarching controller, monitoring and controlling an EV's charge and discharge rates.

- Uses open protocols commonly used to interface with EVs, including the Nissan LEAF.

- Is designed to U.S. standards applicable to $\mathrm{V} 2 \mathrm{G}$ charging systems and distributions systems. 
- Is designed to operate outdoors.

- Avoids the significant impacts to cost, schedule, and performance risks associated with developing a first-generation alternative.

Given the intent to use a Nissan LEAF, two complete Nissan LEAF battery packs were also procured to support initial field testing, if needed.

Figure 81. Coritech V2G fast-charging station.

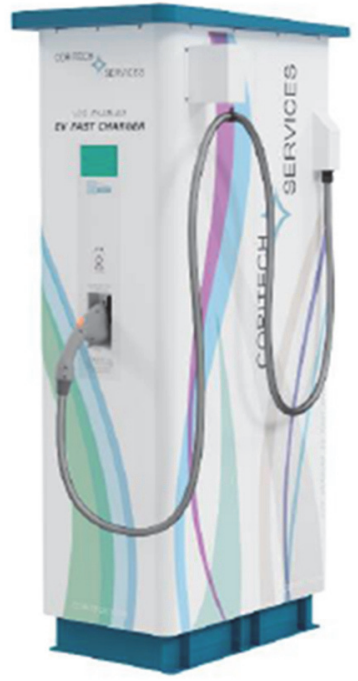

\subsection{Conduct initial testing and analysis of results}

V2G charging equipment initial testing includes Factory Acceptance Testing (FAT) the V2G charger at Coritech., where the fast charger is made. The benefit of conducting a FAT is it ensures time for the manufacturer review test results and address any identified operational issues before the product leaves the factory. This approach avoids operational down time and additional costs for removal, shipping, and reinstallation that could be incurred if an issue is discovered postinstallation.

Nissan tested the two Nissan LEAF battery packs and confirmed they were operational prior to shipping to suppliers. Paragon SMEs requested the test results but the manufacturer declined the request. Obtaining battery test results is not crucial for conducting future testing because baseline testing can be re-established. 


\subsection{Employ test results to model outcomes to refine recommended V2G solution set}

The objective of this research is to apply test results, and/or design specifications of the recommended $\mathrm{V} 2 \mathrm{G}$ solution set within the CBITEC tactical microgrid simulation, to model outcomes and develop a refined recommended solution set for review.

Paragon's research team used the MATLAB/Simulink model of the V2G solution set integrated into a conceptual microgrid developed in Research Area 4 to simulate system performance. Simulation results were used to refine the recommended $\mathrm{V} 2 \mathrm{G}$ solution set.

In Research Area 4, the tactical microgrid assets were cataloged, and a conceptual design was prepared to construct a representative model and simulate performance. The model, based on a conceptual design of the tactical microgrid, includes the $\mathrm{V} 2 \mathrm{G}$ solution set as well as alternative-energy assets anticipated to be part of the living laboratory at CBITEC-including a 100-kW PV array, a 40-kWh of lithium-ion batteries,, a three-phase load bank, and billeting loads. Incorporating an ESS and a PV system into the model, with matching power and energy capabilities, enables representative model simulations to guide integration and control design of a tactical microgrid at the CBITEC site.

In addition to including CBITEC assets, the Coritech V2G charger was added in the model to assess operation, especially during instances of voltage and frequency instability. The Coritech $\mathrm{V}_{2} \mathrm{G}$ charger, like many generation sources that are designed to discharge electricity into a local electrical utility grid, is compliant with UL Standard 1741, Standard for Inverters, Converters, Controllers and Interconnection System Equipment for Use With DERs and the IEEE 1547a Standard for Interconnecting Distributed Resources with Electric Power Systems*. The Coritech V2G charger, as a result, is designed to cease generating electricity generation if the voltage or frequency of the connected distribution system exceeds operating limits as defined in the standards. Within these V2G charger

\footnotetext{
* Institute of Electrical and Electronics Engineers. "1547a-2014 - IEEE Standard for Interconnecting Distributed Resources with Electric Power Systems - Amendment 1." IEEE Standards Association, Institute of Electrical and Electronics Engineers, 21 May 2014, standards.ieee.org/standard/1547a-2014.html.
} 
model's frequency and voltage trip settings of the $\mathrm{V} 2 \mathrm{G}$ charger model, components can be enabled or disabled to accommodate numerous configurations and subsequently conduct "what-if" scenarios that tactical microgrid will inevitably encounter.

Paragon SMEs identified four "what-if" scenarios to broadly assess performance of the recommended solutions set: sudden generation decrease, sudden generation increase, sudden load decrease, and sudden load increase. In addition, the steady state operating condition of the microgrid was simulated to serve as the baseline. Voltage and frequency stability of tactical microgrid baseline operation simulations, as well as "what-if" scenarios in the Research Area 4 modeling effort, were evaluated to determine if the inverter trip settings would need to be adjusted from the default factory settings. A system model of the tactical microgrid is shown in Figure 82.

\subsubsection{Protection model components}

Basic protection logic was constructed to emulate protective relays and inverter embedded functions. Figures 83 to 87 show these models in detail.

The following logic (Figure 83) is for under voltage, under frequency, synchronization, and one overcurrent element for the AMMPS generators. This logic monitors the key generator operational performance aspects necessary to maintain operation in the microgrid. The elements-under voltage, under frequency, synchronization, and overcurrent-were specifically created to monitor generator performance during system changes to determine if a generator would cease operation from transient conditions like an abrupt change in system loading or generation. Thresholds and associated time limits in the logic for voltage, frequency, and current monitoring apply to all operational conditions, including the what-if scenarios used to determine system stability. 


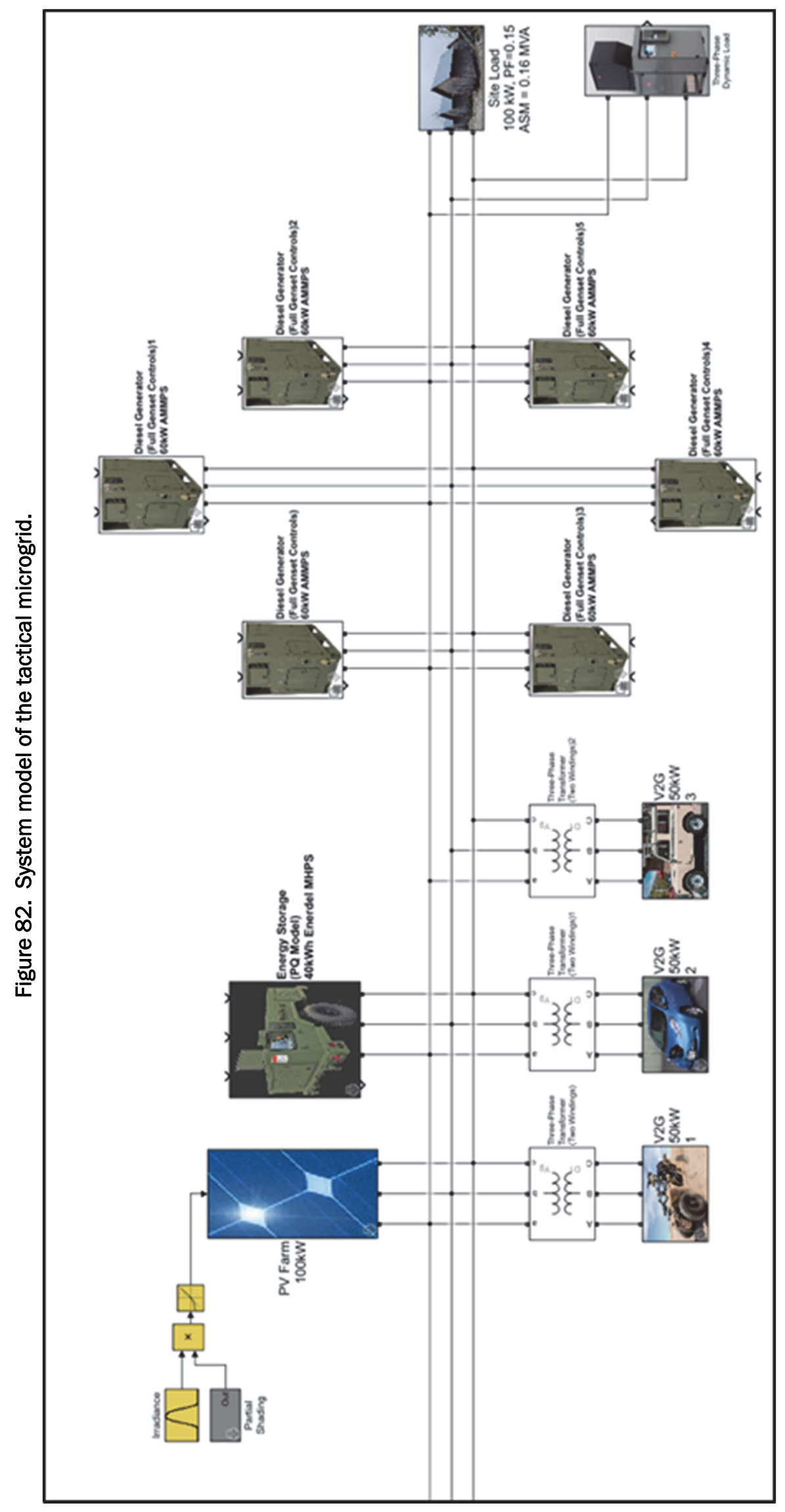




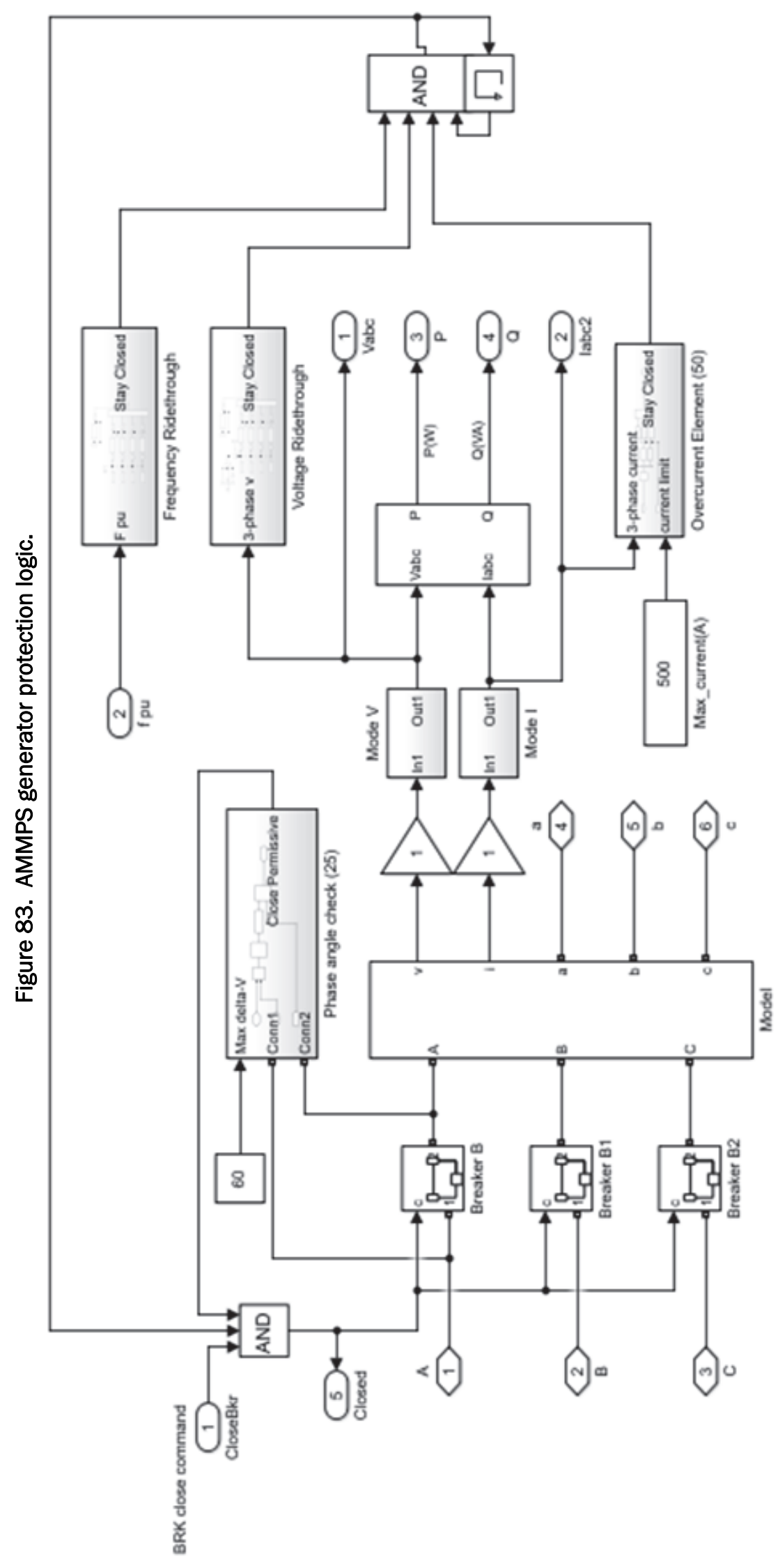


In addition to the phase angle synch check (25) shown in the figure above, the voltage permissive shown in Figure 84. was added.

Figure 84. AMMPS generator permissive.

Voltage difference for sync check

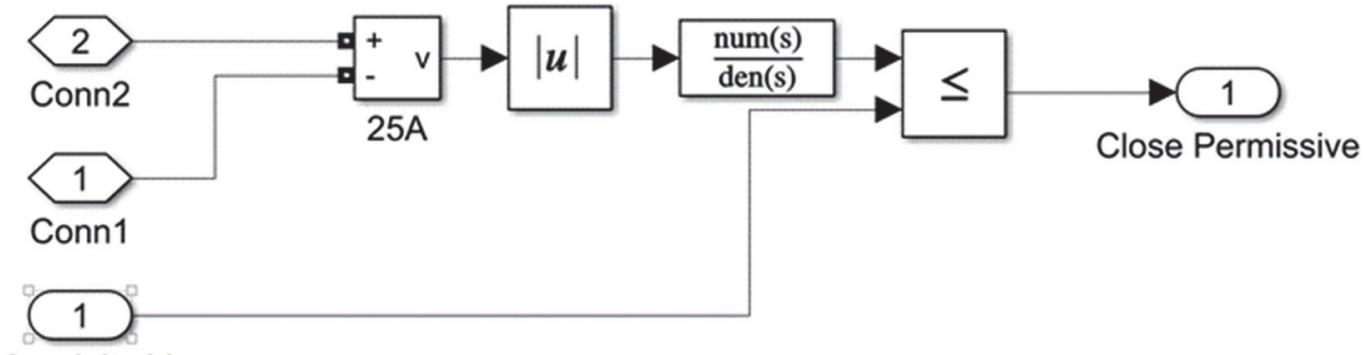

Max delta-V

Overcurrent elements were simulated with logic shown in Figure 85.

Figure 85. AMMPS generator overcurrent elements.

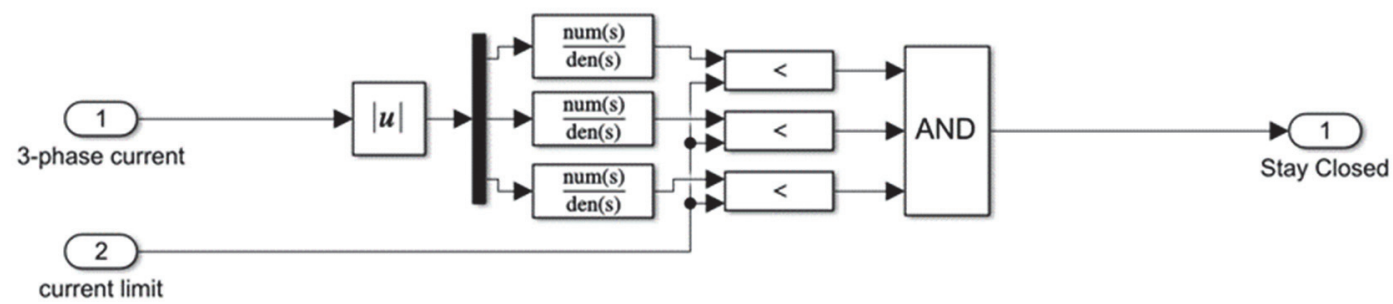

The $\mathrm{PV}$ and $\mathrm{V} 2 \mathrm{G}$ inverter voltage and frequency ride-through performance was simulated with the logic as shown in both Figures 86 and 87.

\subsubsection{Diesel generators model}

The SimScape microgrid library has two generator models, one with basic controls (frequency reference, f_ref and voltage reference, v_ref) (Figure 88 ) and one with more complex controls (f_ref, v_ref, real power (P), reactive power (Q), set to classic control, PV, or PQ). The classic portion of the more complex model was used to control the generator. However, the model still includes the ability to use the "Automatic Generator Controls" for future simulations as more complex generator control schemes are developed. 


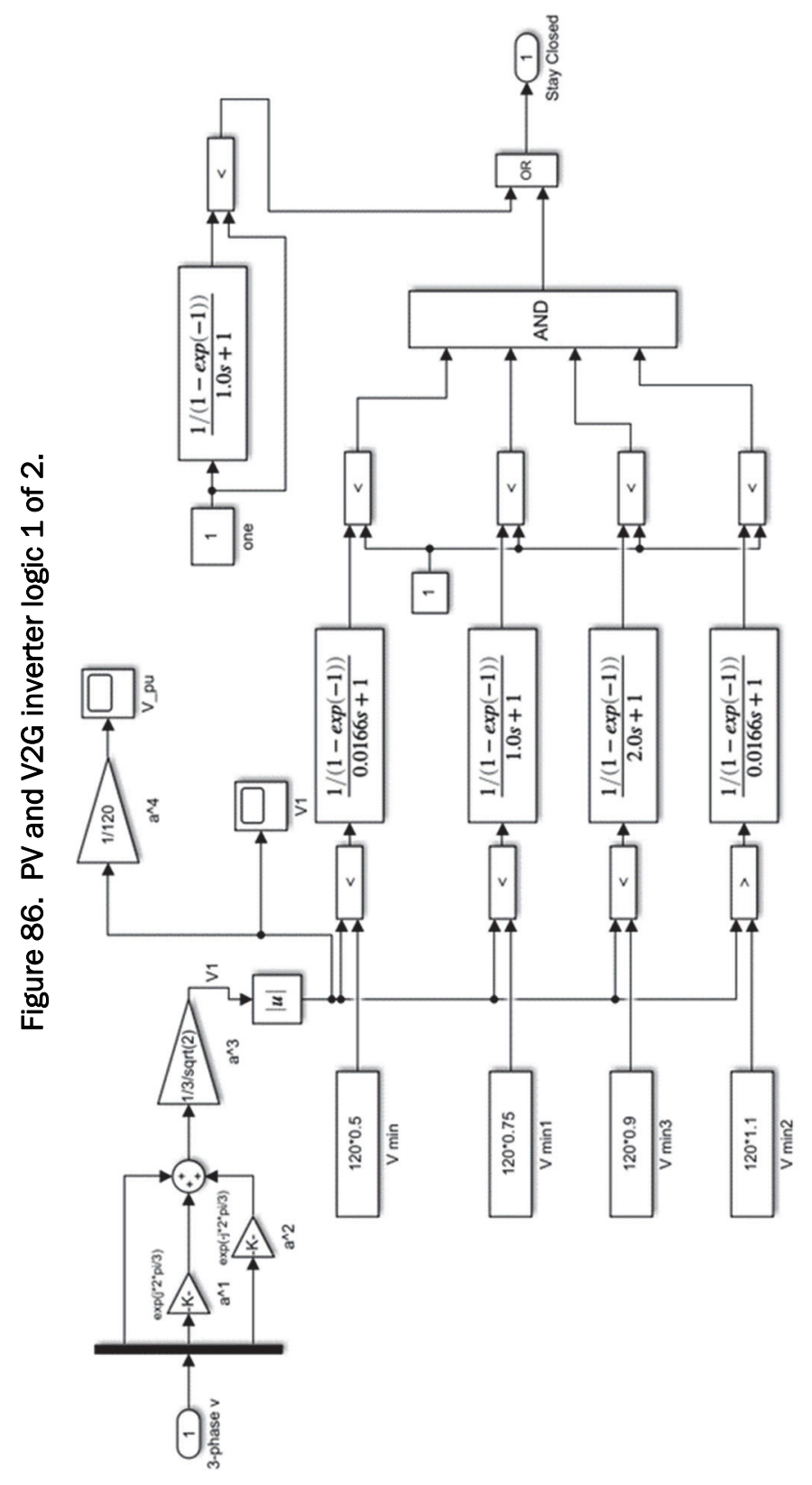




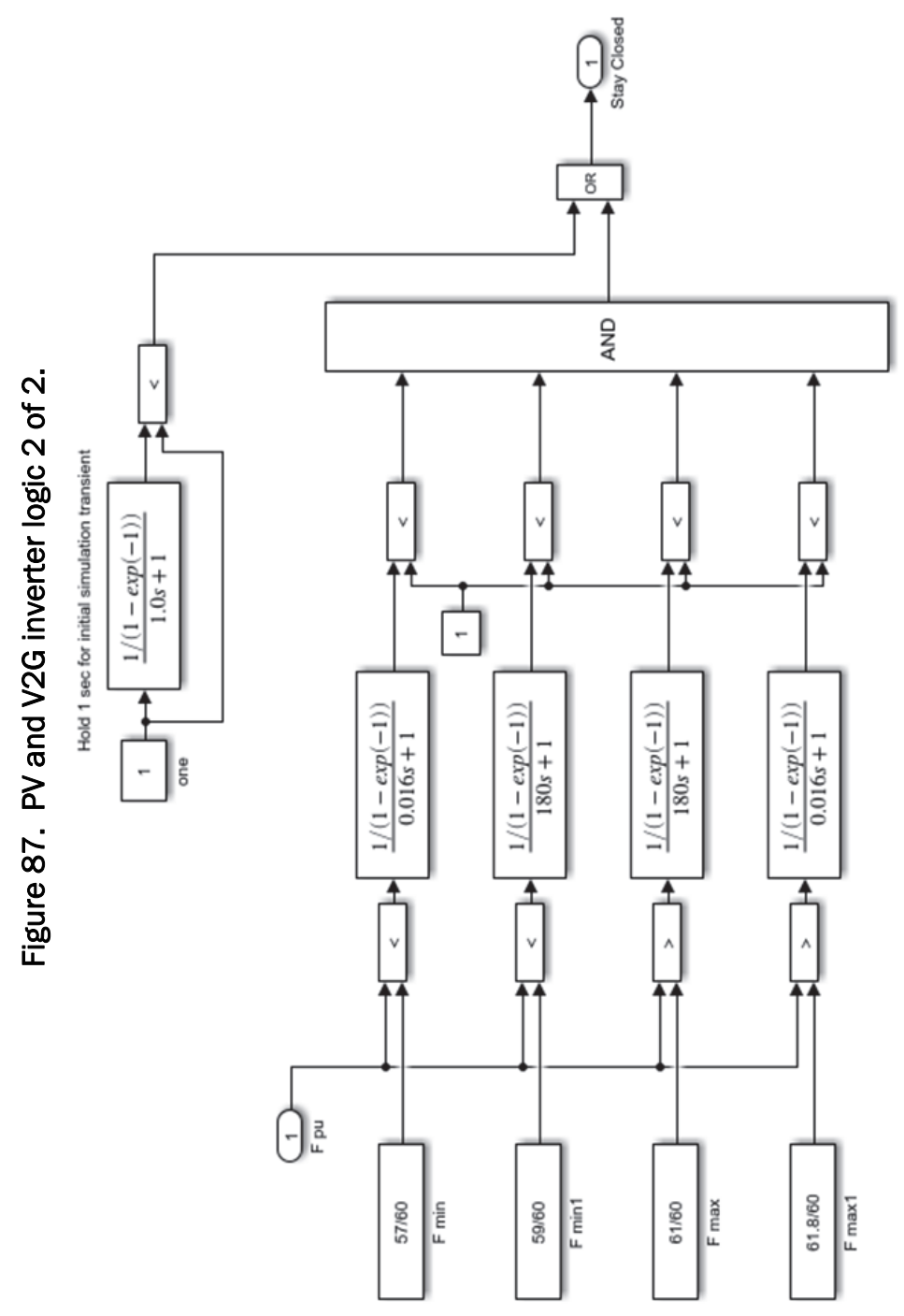




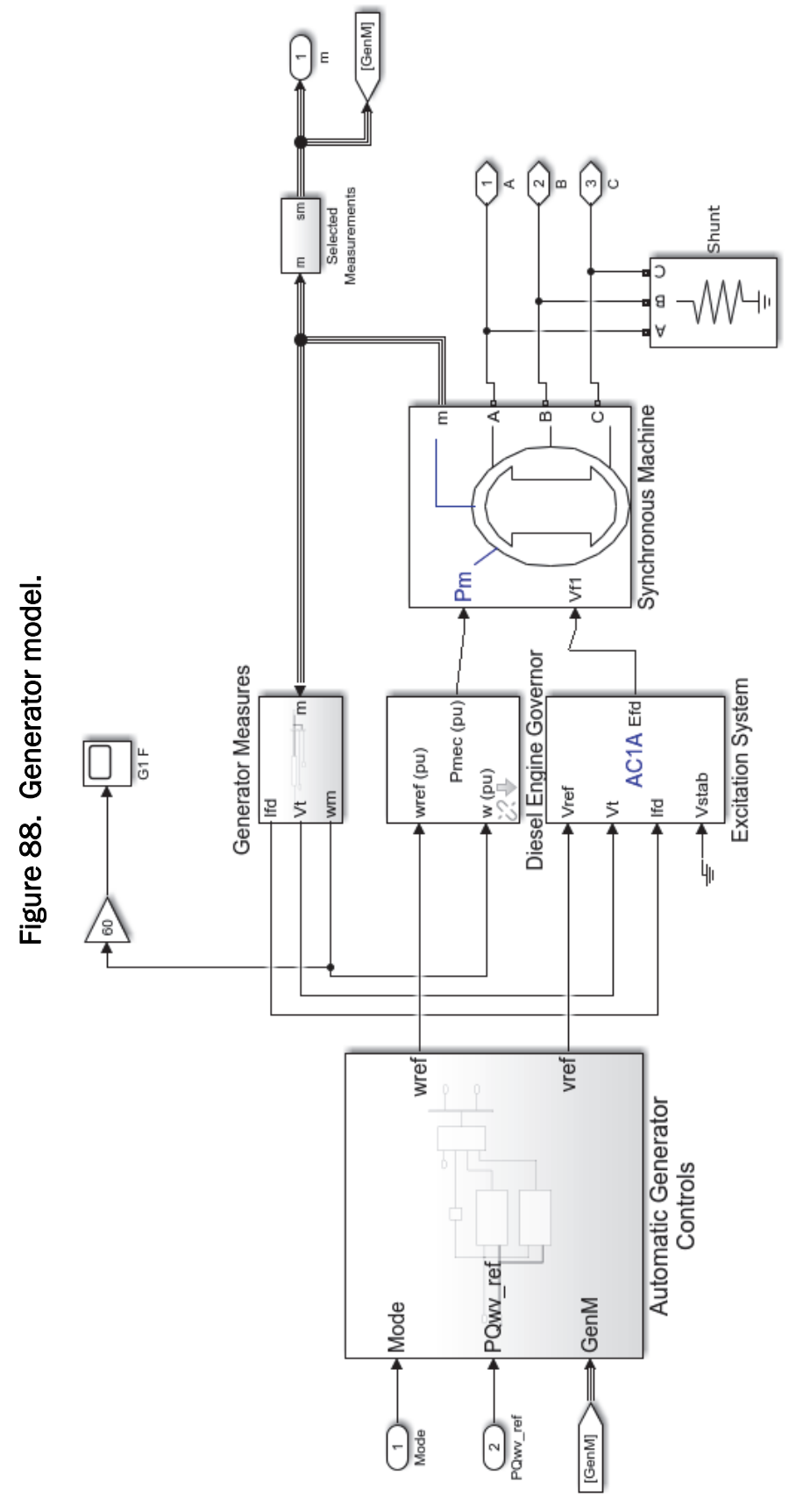


The Synchronous Machine is practically the same as the Real-time Digital Simulator (RTDS) Synchronous Machine. It allows various parameters to be set as shown in Figure 89.

Figure 89. Generator model parameters.

Block Parameters: Synchronous Machine

Synchronous Machine (mask) (link)

Implements a 3-phase synchronous machine modelled in the dq rotor reference frame. Stator windings are connected in wye to an internal neutral point.

\section{Configuration Parameters Load Flow}

Nominal power, line-to-line voltage, frequency $[\mathrm{Pn}(\mathrm{VA}) \mathrm{Vn}(\mathrm{Vrms}) \mathrm{fn}(\mathrm{Hz})]$ : $[6 \mathrm{e}+0420860]$

Reactances [ Xd Xd' Xd" Xq Xq" Xl ] (pu): [1.305, 0.296, 0.252, 0.474, 0.243, 0.18 ]

Time constants

d axis: Open-circuit

q axis: Short-circuit

[ Tdo' Tdo" Tq"] (s): $\left[\begin{array}{lll}4.49 & 0.0681 & 0.0513\end{array}\right]$

Stator resistance Rs $(\mathrm{pu}): 0.003$ :

Inertia coefficient, friction factor, pole pairs $\left[\mathrm{H}(\mathrm{s}) \mathrm{F}(\mathrm{pu}) \mathrm{p}(\mathrm{)}):\left[\begin{array}{lll}1.8 & 0 & 2\end{array}\right]\right.$ :

Initial conditions [ $\mathrm{dw}(\%)$ th(deg) ia,ib,ic(pu) pha,phb,phc(deg) vf(pu) ]: $80-1201201.30695$ ] :

Simulate saturation

Plot

[ ifd; vt] (pu): $56,1.082,1.19,1.316,1.457 ; 0.7,0.7698,0.8872,0.9466,0.9969,1.046,1.1,1.151,1.201]$

The governor is intended to be its own model (Figure 90) with only the following controls visible.

To increase accuracy, the modeling team instead built a standard Proportional Integral Derivative (PID) (Figure 91) to allow the engine genset to be tuned closer to real-life machine behavior. 
Figure 90. Generator governor parameters.

Block Parameters: Diesel Engine Governor

$\times$

Diesel Engine Governor (mask)

This block implements a diesel engine and governor system. The first input is the reference speed and the second input is the measured speed. The output is the diesel engine mechanical power. The motor inertia should be combined with the generator inertia.

The model consist of a regulator represented by the following transfer function:

$$
\mathrm{Hc}=\mathrm{K} .(1+\mathrm{T} 3 . \mathrm{s}) /\left(1+\mathrm{T} 1 . \mathrm{s}+\mathrm{T} 1 . \mathrm{T} 2 \cdot \mathrm{s}^{\wedge} 2\right)
$$

The trotte actuator is implemented as :

$$
\mathrm{Ha}=(1+\mathrm{T} 4 . \mathrm{s}) /[(\mathrm{s}(1+\mathrm{T} 5 . \mathrm{s})(1+\mathrm{T} 6 . \mathrm{s})]
$$

The motor engine is represented by a Time delay Td function.

Parameters

Regulator gain $\mathrm{K}$ :

10

Regulator time constants [T1 T2 T3 ] (s) :

$\left[\begin{array}{lll}1 & 0.25 & 0.3\end{array}\right]$

Actuator time constants [T4 T5 T6] (s) :

[0.25 0.0090 .0384 ]

Torque limits [Tmin Tmax] (pu) :

[0 1.4]

Engine time delay Td (s) :

0.024

Initial value of mechanical power $\mathrm{Pm0}(\mathrm{pu})$ :

0.27

Figure 91. Generator PID algorithm.
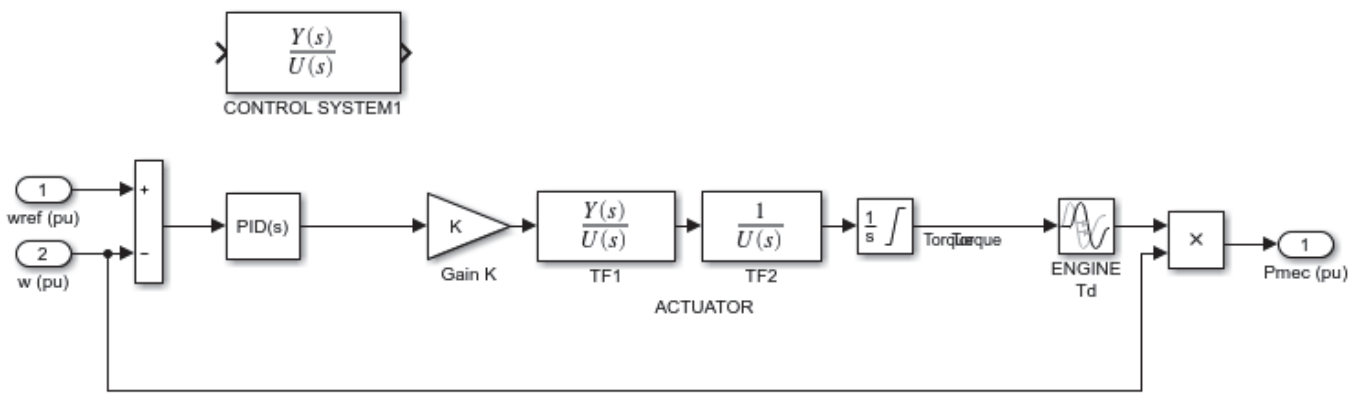
The default MATLAB generator AVR and excitation behaviors (Figures 92 and 93) were found to be reasonably accurate, so no further elaborations were made to the control other than tuning the model.

Figure 92. Excitation parameters 1 of 2.

\begin{tabular}{|c|c|c|}
\hline \multicolumn{3}{|c|}{ Block Parameters: Excitation System } \\
\hline \multicolumn{3}{|c|}{ AC1A Excitation System (mask) (link) } \\
\hline \multicolumn{3}{|c|}{$\begin{array}{l}\text { Implements a synchronous machine voltage regulator and exciter } \\
\text { based on the IEEE type ACIA excitation system model. The output is } \\
\text { the field voltage to apply to the Vf input of a Synchronous Machine } \\
\text { block. Inputs and output are in p.u. }\end{array}$} \\
\hline Controllers & Exciter and Rectifier & Initial Values \\
\hline \multicolumn{3}{|c|}{ Exciter gain and time constant $[\mathrm{Ke} \mathrm{Te}(\mathrm{s})]$ : } \\
\hline \multicolumn{3}{|l|}{$\left[\begin{array}{lll}1.0 & 0.80\end{array}\right]$} \\
\hline \multicolumn{3}{|c|}{ Exciter alternator voltage values [Ve1(pu) Ve2(pu)]: } \\
\hline \multicolumn{3}{|l|}{$\left[\begin{array}{lll}4.18 & 3.14\end{array}\right]$} \\
\hline \multicolumn{3}{|c|}{ Exciter saturation function values [SeVe1(pu) SeVe2(pu)]: } \\
\hline \multicolumn{3}{|l|}{$\left[\begin{array}{lll}0.10 & 0.03\end{array}\right]$} \\
\hline \multicolumn{3}{|c|}{ Demagnetizing factor $\mathrm{Kd}(\mathrm{pu})$ : } \\
\hline \multicolumn{3}{|l|}{0.38} \\
\hline \multicolumn{3}{|c|}{ Rectifier loading factor $\mathrm{Kc}(\mathrm{pu})$ : } \\
\hline 0.20 & & : \\
\hline
\end{tabular}

Figure 93. Excitation parameters 2 of 2.

\begin{tabular}{|c|c|c|c|}
\hline \multicolumn{3}{|c|}{ Block Parameters: Excitation System } & $x$ \\
\hline \multicolumn{4}{|c|}{ AC1A Excitation System (mask) (link) } \\
\hline \multicolumn{4}{|c|}{$\begin{array}{l}\text { Implements a synchronous machine voltage regulator and exciter } \\
\text { based on the IEEE type AC1A excitation system model. The output is } \\
\text { the field voltage to apply to the Vf input of a Synchronous Machine } \\
\text { block. Inputs and output are in p.u. }\end{array}$} \\
\hline Controllers & Exciter and Rectifier & Initial Values & \\
\hline \multicolumn{4}{|c|}{ Low-pass filter time constant $\operatorname{Tr}(\mathrm{s})$ : } \\
\hline \multicolumn{3}{|l|}{$20 \mathrm{e}-3$} & $\vdots$ \\
\hline \multicolumn{4}{|c|}{ Voltage regulator gain and time constant $[\mathrm{Ka} \mathrm{Ta}(\mathrm{s})]$ : } \\
\hline \multicolumn{3}{|l|}{$\left[\begin{array}{lll}400 & 0.02\end{array}\right]$} & $\vdots$ \\
\hline \multicolumn{4}{|c|}{ Voltage regulator internal limits [VAmin(pu) VAmax(pu)]: } \\
\hline \multicolumn{3}{|l|}{$[-14.514 .5]$} & $\vdots$ \\
\hline \multicolumn{4}{|c|}{ Voltage regulator output limits [VRmin(pu) VRmax(pu)]: } \\
\hline \multicolumn{3}{|l|}{$[-5.436 .03]$} & $\vdots$ \\
\hline \multicolumn{4}{|c|}{ Damping filter gain and time constant [Kf Tf(s)]: } \\
\hline \multicolumn{3}{|l|}{$[0.031 .0]$} & $\vdots$ \\
\hline \multicolumn{4}{|c|}{ Transient gain reduction lead and lag time constants $[\mathrm{Tb}(\mathrm{s}) \mathrm{Tc}(\mathrm{s})]$ : } \\
\hline \multicolumn{3}{|l|}{$\left[\begin{array}{ll}0 & 0\end{array}\right]$} & $\vdots$ \\
\hline
\end{tabular}

\subsubsection{PV array}

The inverter for the PV is essentially a DC power to AC current converter (having read voltage from the terminals) (Figure 94). Future improvement to this model would include interharmonic, islanding, out of step, current instability, or other problems based on a specific inverter to be used. At the time of this report, specific inverters to be used for the PV array had not been identified.

Figure 94. 100-kW PV array model.

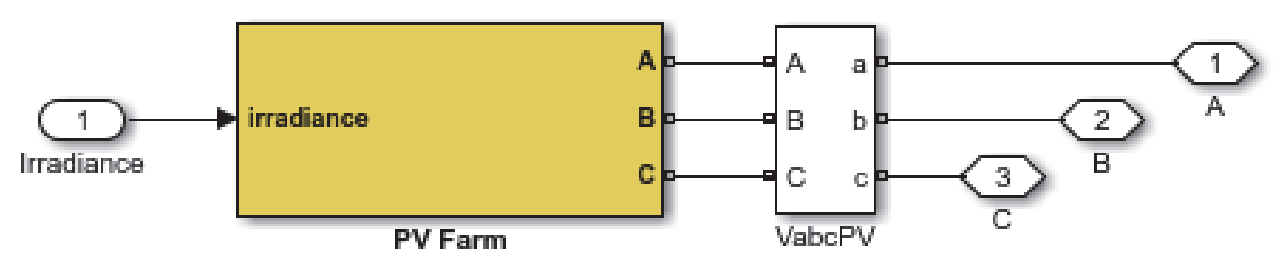


The PV array (Figure 95) is physically represented in the model solar irradiance as an input along with voltage measurements and current sources.

Figure 95. PV array model voltage elements.

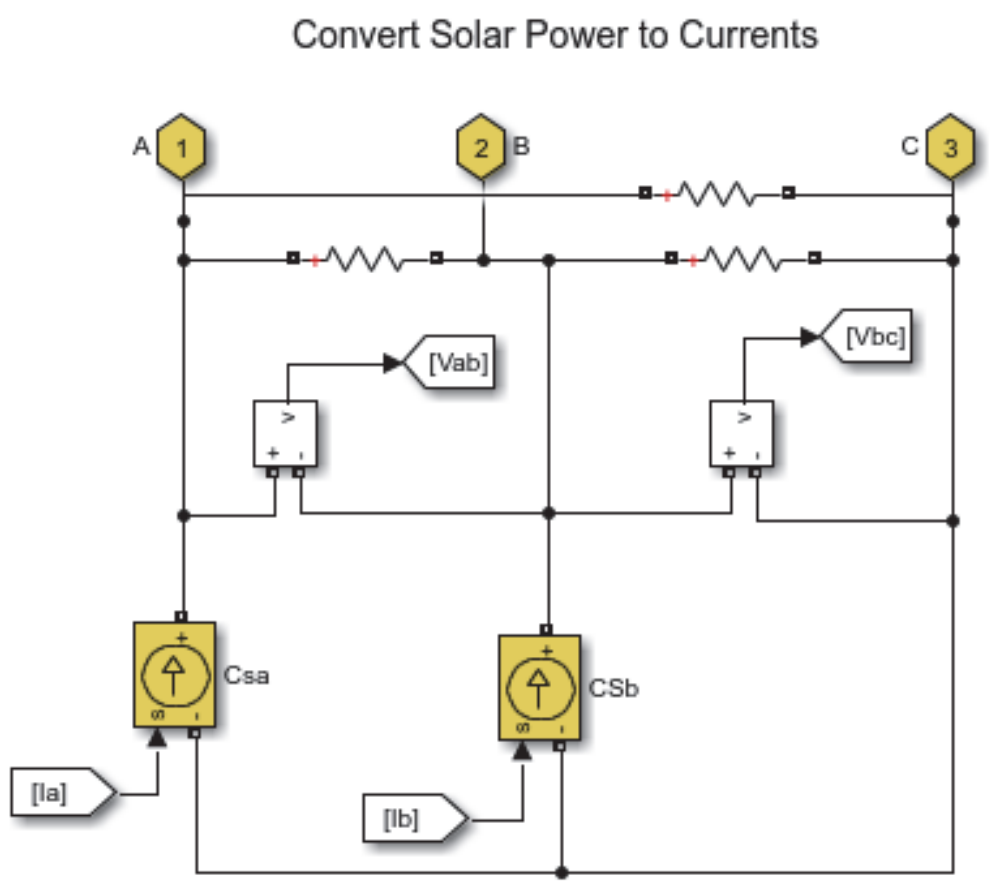

The model (Figure 96) uses voltages Vab and Vbc and power values to calculate output current.

Figure 96. PV array power calculations.

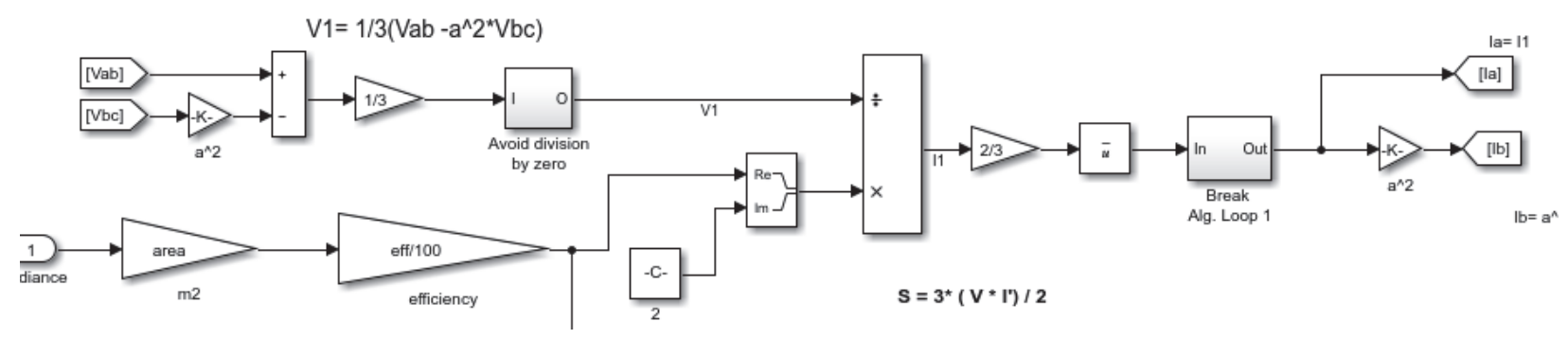

\subsubsection{V2G inverter}

The V2G inverter model (Figure 97) closely represents the PV inverter, except that it models multiple inverters, some of which are in the "Charge mode" (right), and some are in the "Discharge mode" (left). 
Figure 97. V2G inverter model.

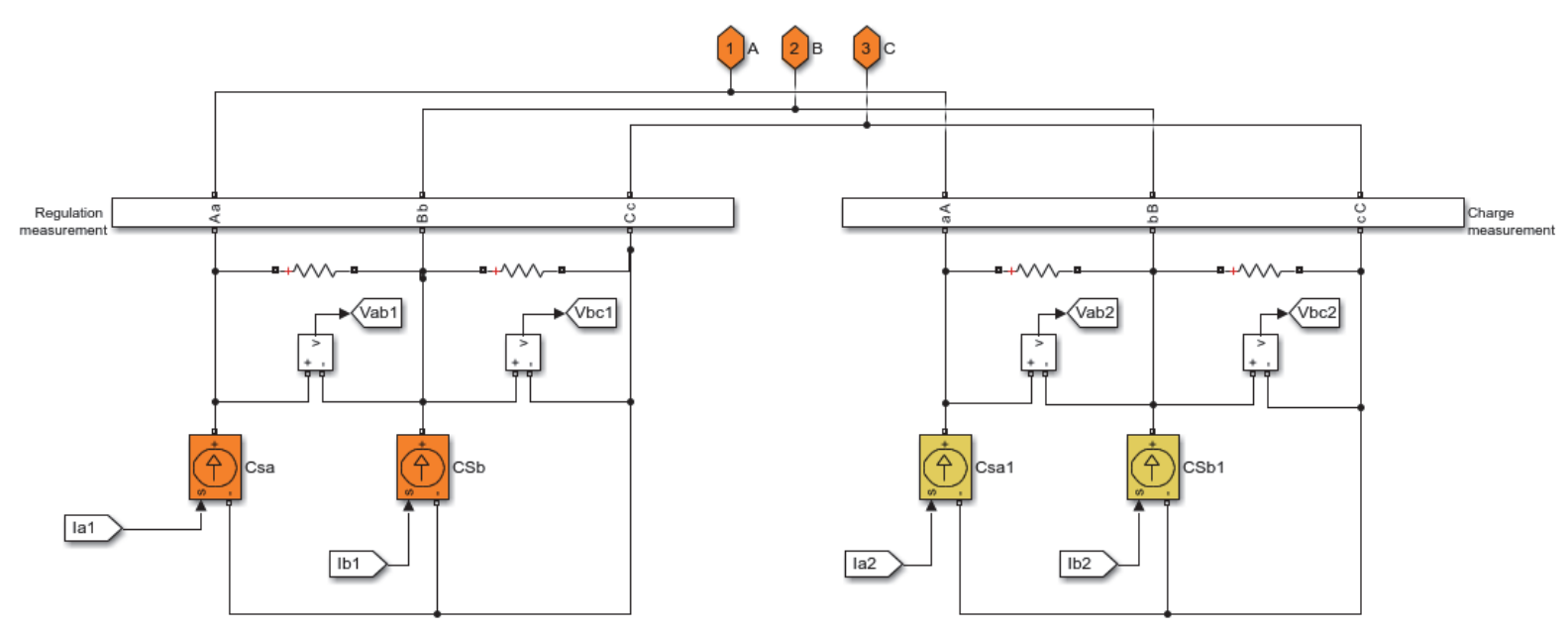

The charge/discharge fractions are determined by the blocks shown in Figures 98 and 99. The profiles specify the state of the plug (plugged in or not) and the SOC initial value to reset the internal integrator when it is newly plugged in. The block on the left contains the integrator and has the charge percentage as the output. The block on the right chooses to discharge if at above $95 \%$ charge, charge if below $85 \%$, and hold otherwise.

Figure 98. V2G inverter charge/discharge elements 1 of 2 .

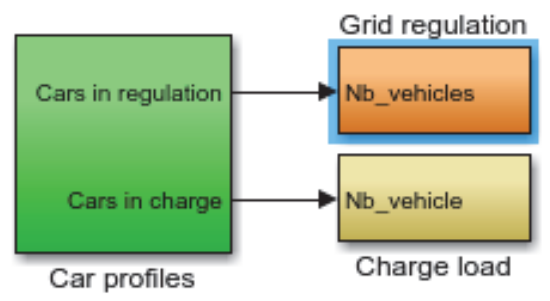

Figure 99. V2G inverter charge/discharge elements 2 of 2.

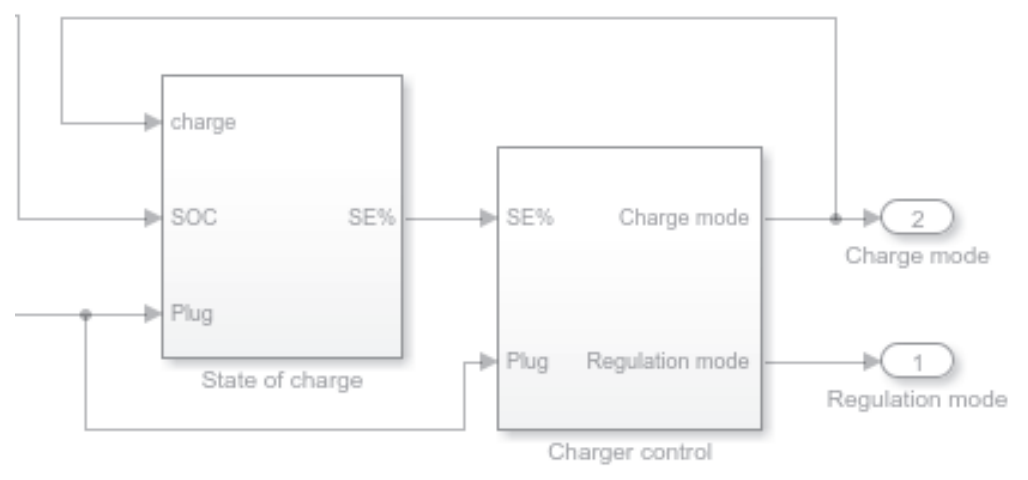


For the simulations in this report, the TechFlow team turned off (commented out) these tertiary controls (Figure 100) and directly assigned the "Charge mode" percentage and "Regulation mode" percentages as shown below in Figure 100.

Figure 100. V2G inverter charge/regulation percentage control.

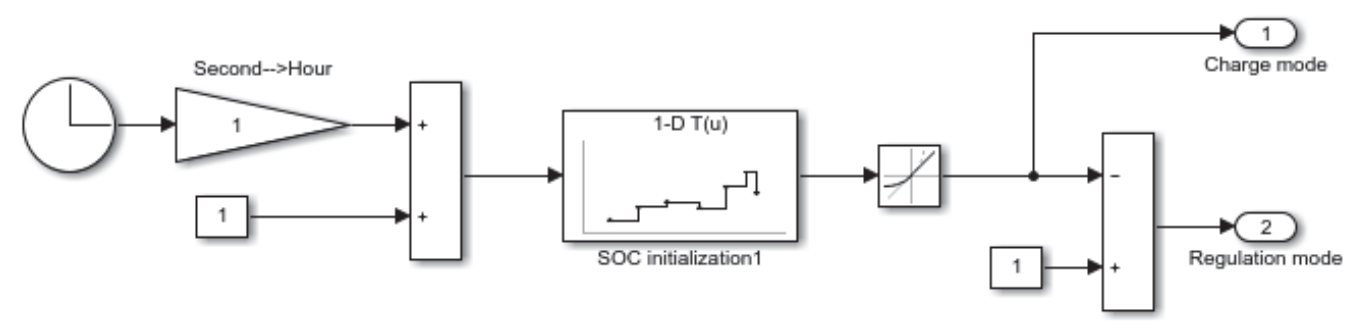

The block titled "SOC initialization1" is a look-up table that was combined with a limited-ramp rate, so the two outputs add up to 1 .

\subsubsection{Battery Energy Storage System (BESS)}

The 40-kWh battery model (Figure 101) was the standard PQ model included in SimScape. The model is also a lithium-ion technology and could use the same model as the $\mathrm{V} 2 \mathrm{G}$ lithium-ion model to provide additional capabilities, depending on how the site wants to use each ESS. The V2G model has the capability to include five vehicle profiles used for various energy storage technologies that need more extensive control. The block parameters for this model are shown in Figure 101. 
Figure 101. Lithium-ion energy storage parameters.

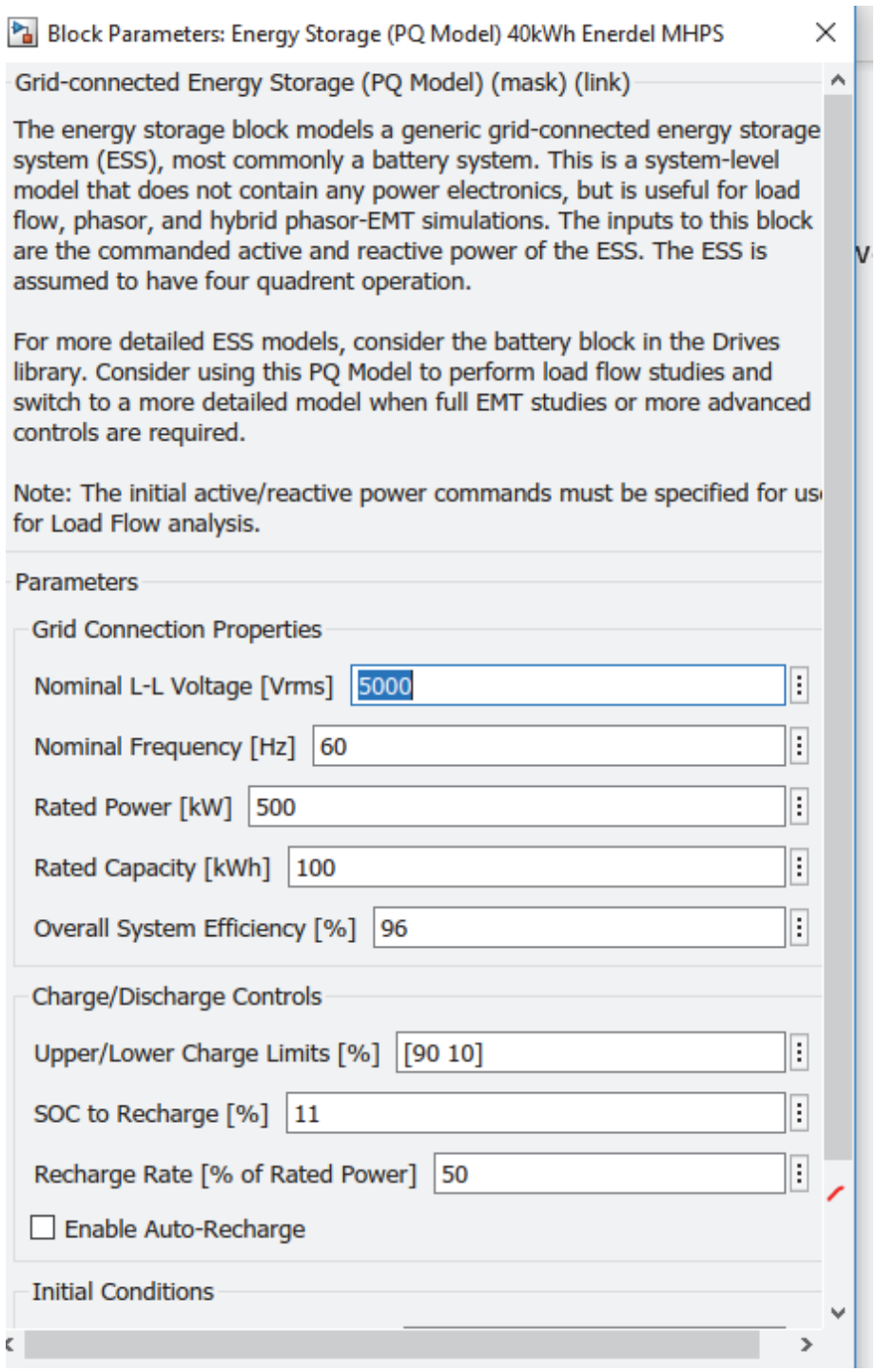

Figures 102 and 103 show the control logic for the lithium-ion ESS as well as associated parameters. 
Figure 102. Lithium-ion energy storage logic.

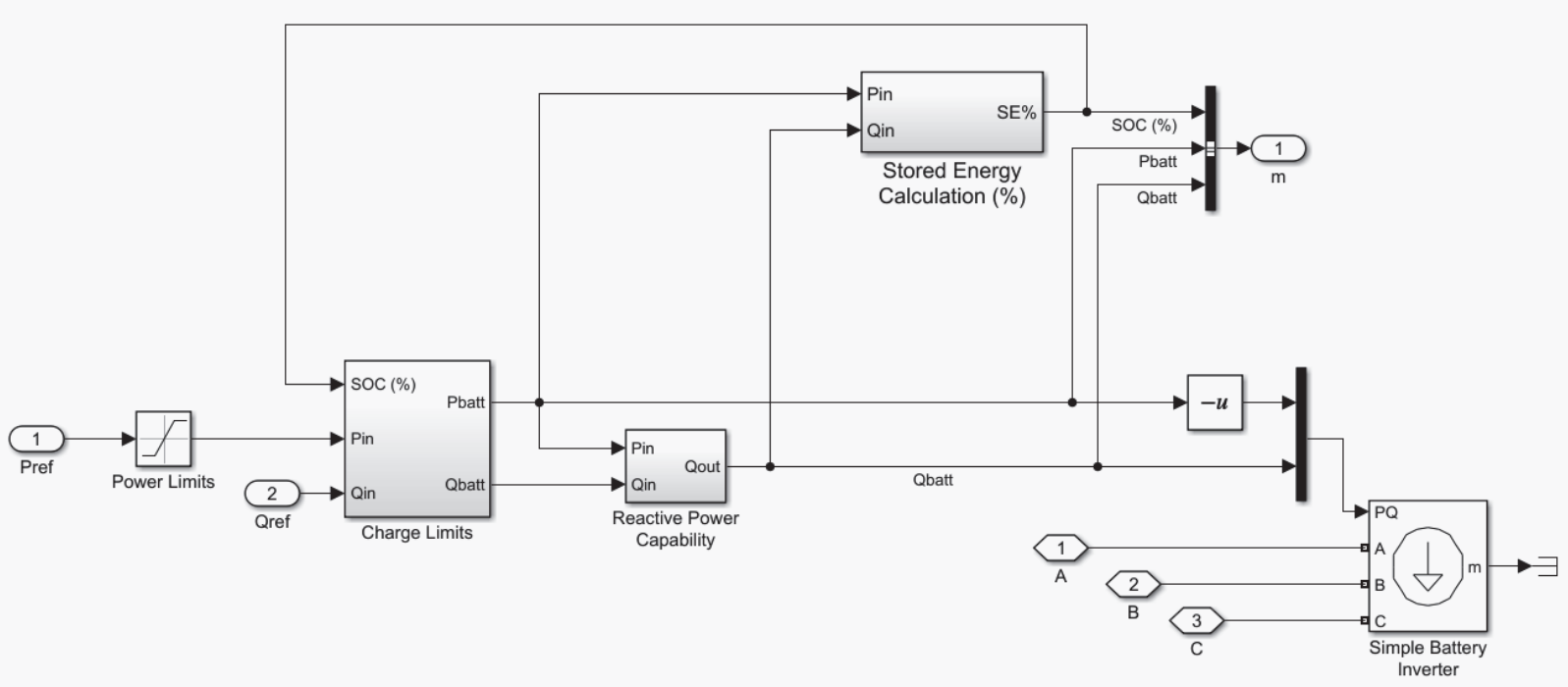

Figure 103. Lithium-ion energy storage inverter parameters.

\begin{abstract}
Block Parameters: Simple Battery Inverter
\end{abstract}
$\times$

Three-Phase Dynamic Load (mask) (link)

Implements a three-phase, three-wire dynamic load. Active power $\mathbf{P}$ and reactive power $\mathrm{Q}$ absorbed by the load vary as function of positive-sequence voltage $\mathrm{V}$.

Parameters

Nominal L-L voltage and frequency $[\mathrm{Vn}(\mathrm{Vrms}) \mathrm{fn}(\mathrm{Hz})]$ :

[Vn Fn]

Active and reactive power at initial voltage $[\mathrm{Po}(\mathrm{W}) \mathrm{Qo}(\mathrm{var})]$ :

$[-500000-3.38813 e-13]$

Initial positive-sequence voltage Vo [Mag(pu) Phase (deg.)]:

[0.999844 -0.0038635]

$\square$ External control of PQ

Filtering time constant (s):

$1 e-2$

\title{
OK
}

Cancel

Help

Apply 


\subsubsection{Model fidelity}

The MATLAB modeling tools are not an Electro Magnetic Transient Program (EMTP) equipped with differential equation solvers. Instead, the MATLAB equations developed are second-order estimates of high-level machine and electrical behavior. Even simplified, the compiling and running of one test takes several minutes. Certain attributes of model outcomes, such as frequency and voltage, power, and reactive power, are estimated to be within $10 \%$ of reality and could be tuned with actual live-system data to improve accuracy to within a three to five percentage points.

Because the MATLAB simulations are not EMTP, they also cannot accurately simulate transient or sub-transient behaviors under faulted conditions. They also will neither replicate harmonics nor sub-synchronous torsional interactions between the inverters and generators sets. To gain this level of accuracy, the modeling would require a real-time EMTP Hardware in the Loop (HIL) system similar to RTDS Simulator by RTDS Technologies, HYPERSIM by OPAL-RT Technologies, or HIL Supervisory Control and Data Acquisition (SCADA) by Typhoon.

\subsubsection{Discussion of work}

Figures 104 to 106 plot the response of six AMMPS 60-kW generators, one $100-\mathrm{kW}$ PV, and one $30-\mathrm{kW} \mathrm{V2G}$ paralleled and holding $50 \mathrm{~kW}$ of load. The grid then increases load (at 1 second) by $180 \mathrm{~kW}$ and $60-\mathrm{kVAr}$ of load and (at 11 seconds) loses this same load. The $\mathrm{V}_{2} \mathrm{G}$ is re-dispatched from charge to discharge following the loading and returned to charging when the load is lost. 
Figure 104. System real power response.

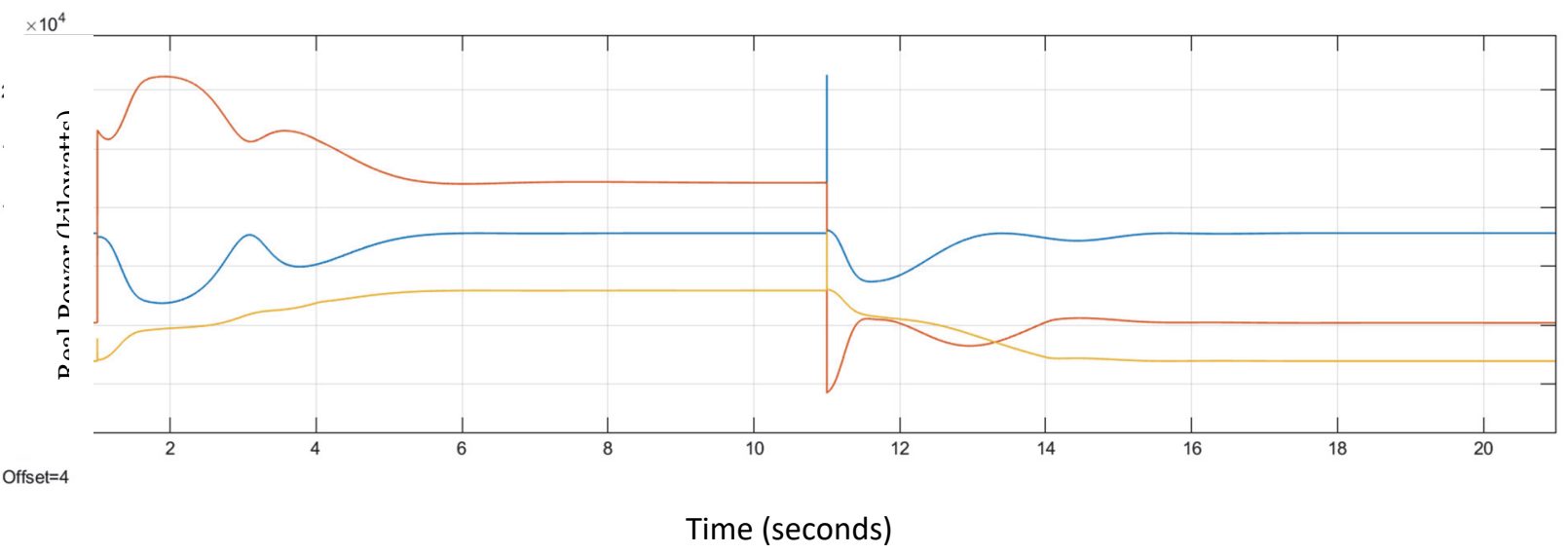

Real Power: Red AMMPS generators, Blue PV, Yellow V2G

Figure 105. System reactive power response.

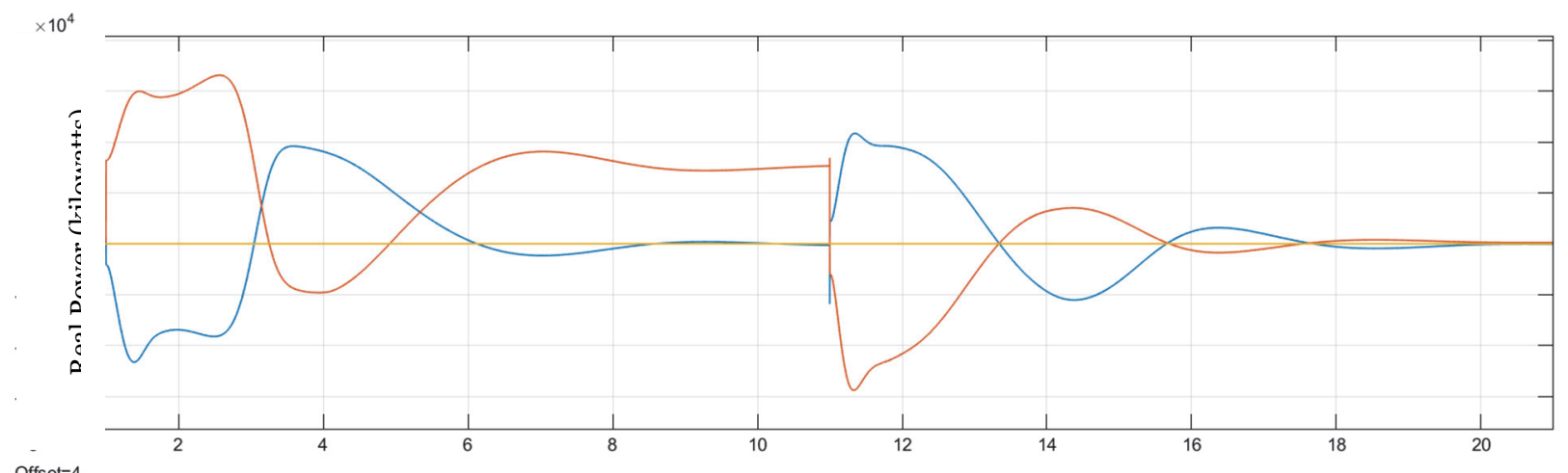

Time (seconds)

Reactive Power: Red AMMPS generators, Blue PV, Yellow V2G

Figure 106. System frequency response.

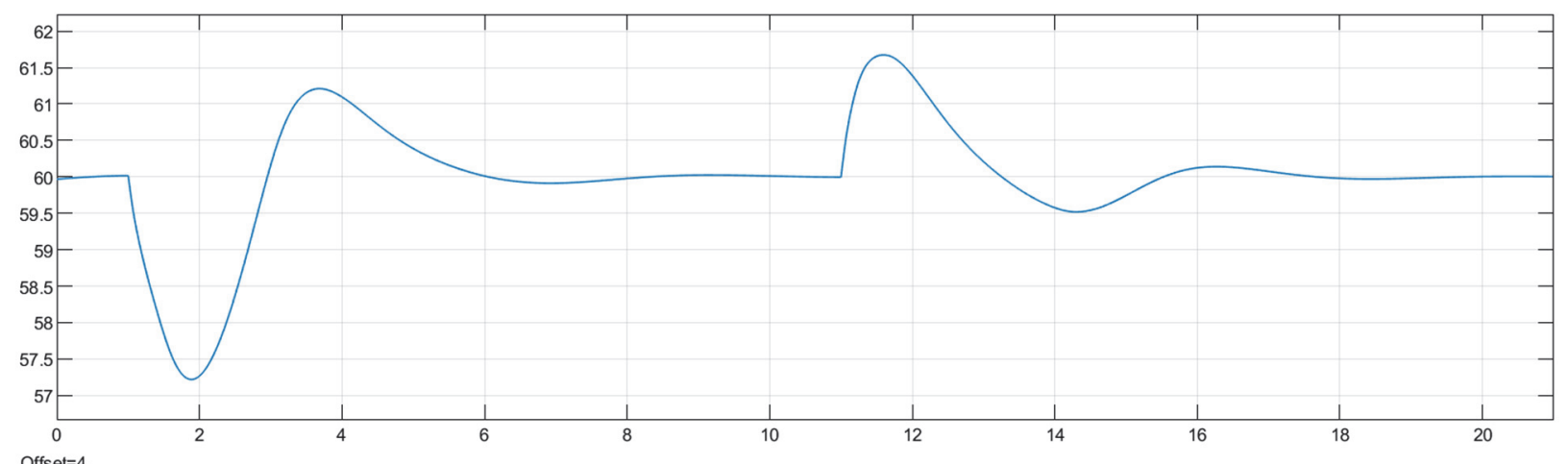

Time (seconds)

Frequency $(\mathrm{Hz})$ 
These figures represent idealized behavior, therefore the actual microgrid should be configured, tuned, and modified to inherent similar characteristics. Key behaviors that need to be replicated in the live system include:

1. Minimal oscillations among the generators, $\mathrm{PV}$, and the $\mathrm{V} 2 \mathrm{G}$ system during any form of charging or discharging. Out-of-the-box equipment will likely fight each other and require significant modification to behave in the desired manner shown.

2. PV and V2G systems do not trip offline during large load acceptances or rejections. Default PV systems will turn off from these events and settings in the PV will likely need customization to ensure this desired behavior.

3. Genset does not trip off immediately on reverse power; this is desirable for small reciprocating engines, as they work fine as compressors. This is not acceptable for turbine type engines but desired for small reciprocating engine generators.

4. Note that deviations in the Volt-Ampere Reactive (VAR) output of the PV are because of inaccuracies in the inverter's ability to track the real voltage and frequency in real time. Although these behaviors will not be identical in the field, the general behavior of PV-VAR output hunting with a genset AVR will likely happen. The only known way to eliminate this problem is to augment a PV controller with an auxiliary relay controller with energy packet controls.

5. As a result of a load increase, the $\mathrm{V} 2 \mathrm{G}$ system will ramp up to participate in a grid-support function under a high-electrical load and ramp back to a charging position once the load was removed.

6. During a load increase, the AMMPS inertia and governor response continue to dominate the frequency response characteristic of the power system.

\subsubsection{Identified issues and possible solutions}

The alternative approach of using a battery solution provided by Go Electric or EnerDel was not a viable solution because the weight of the batteries and necessary integration hardware exceeds the payload rating of the MUTT. To promote interoperability and open standards usage, the best solution is a commercial vehicle. Consequently, a bidirectional vehicle and fast charger was pursued as the solution set.

The Coritech V2G fast charger is designed to be installed on a 3-wire, electrical distribution system at $480 \mathrm{VAC}$; however, the tactical microgrid is a 
4-wire, wye connected distribution system that is rated at $208 \mathrm{VAC}$. To integrate the $\mathrm{V} 2 \mathrm{G}$ charger, a step-down transformer is required to convert the fast charger's output voltage of $480 \mathrm{VAC}$ to $208 \mathrm{VAC}$. To limit the introduction of harmonics from the fast charger's inverter to the tactical microgrid distribution system, a wye delta transformer is recommended.

Because the Coritech fast charger does not use a neutral connection, the delta side of the transformer could be connected to the charger. The manufacturer recommends installing a grounding transformer between the charger and the step-down transformer instead of using a floating-delta connection because the configuration is an ungrounded system, which can result in excessive floating voltages. The manufacturer advises a delta connection on the 4-wire 208-wye distribution system and grounded 3-wire wye connection on the $480-\mathrm{VAC}$ connection to the charger can also be used instead-avoiding the cost associated with adding the grounding transformer. The charger does not use a neutral therefore the wye neutral is not connected to the charger and only connects to the equipment ground.

By design, the Coritech fast charger is compliant with industry standard UL1741 and IEEE 1547 to cease generation if system voltage or frequency exceeds predefined limits, especially in distribution systems with multiple generation sources, voltage, and frequency stability to stay within these limits can be challenging. The speed at which the charger must cease generation, also referred to as the "Clearing Time," depends on the extent of voltage or frequency deviation as shown in the Tables 47 and 48.

Table 47. System response to abnormal voltages.

\begin{tabular}{|c|c|}
\hline Voltage Range (\% of base voltage) & Clearing Times (sec) \\
\hline$V<50$ & 0.16 \\
\hline $50 \leq V<88$ & 2.00 \\
\hline $110<\mathrm{V}<120$ & 1.00 \\
\hline$V \geq 120$ & 0.16 \\
\hline
\end{tabular}

Table 48. System response to abnormal frequencies.

\begin{tabular}{|c|c|c|}
\hline Distributed Resource Size & Frequency Range (Hz) & Clearing Times (sec) \\
\hline \multirow{2}{*}{$\leq 30 \mathrm{~kW}$} & $>60.5$ & 0.16 \\
\cline { 2 - 3 } & $<59.3$ & 0.16 \\
\hline \multirow{3}{*}{$>30 \mathrm{~kW}$} & $>60.5$ & 0.16 \\
\cline { 2 - 3 } & $<59.8-75.0$ (adjustable) & adjustable 0.16 to 300 \\
\cline { 2 - 3 } & $<57.0$ & 0.16 \\
\hline
\end{tabular}


Nuisance trips can be avoided so the charger remains online during normal operation by adjusting the trip settings and times in the fast charger. As loading conditions and system operating conditions, the fast charger trip settings can also be adjusted to better accommodate and normal voltage and frequency variations of the tactical microgrid.

\subsection{Discussions of future work}

The objective of this research area was to identify requirements for a sensored, living laboratory to assess the performance of $\mathrm{V} 2 \mathrm{G}$ charging in a tactical microgrid and to recommend an optimal V2G solution set for use at the CBITEC site.

While MATLAB is useful for simulating high-level system behavior, future efforts could include validating the model with extensive field testing and employing some of the HIL capabilities to improve upon the MATLAB model accuracy. HIL software packages are available; however, MATLAB is more commonly used in the $\mathrm{DoD}$ and tends to be considerably more affordable. Including a HIL test capability into the living laboratory will increase the accuracy of modeling and controls algorithm development.

Specific modeling actions for the future include:

1. Improve MATLAB model fidelity by including field data and revising models

2. Model the system in a true EMTP environment to improve simulated rotor angle and voltage fluctuations

3. Build more robust inverter models to replicate the complex arrangements of inverter controls-including, but not limited to, anti-islanding and overload behaviors for specific inverters

4. Build the MATLAB model to accurately simulate faulted circuit events and life-safety short-circuit and arc-flash events.

In addition to building in HIL modeling and testing capability, future work can include configuring and documenting the recommended hardware settings to record system performance for future testing. 


\subsection{Conclusions and recommendations}

Paragon SMEs determined the IPDs prepared for the site include control equipment sufficient to meet the metering and control needs for interconnecting tactical microgrid components. Additional components to complete the living laboratory and their benefits were also identified. The optimal V2G solution was recommended with parameter adjustments to avoid nuisance tripping during times of voltage and frequency instability that can be caused by sudden changes in system loading or generation.

Recommendations include:

- Completing a detailed design of the tactical microgrid

- Documenting IPD and hardware settings for interconnecting the tactical microgrid assets

- Including HIL modeling and testing to improve simulation accuracy and support control algorithm development. 


\section{Integrate Open Standards to Promote Interoperability}

To promote interoperability, the Paragon team researched and identified current interoperability standards to support the CBITEC tactical microgrid development. They also developed an approach to bridge the gap between available microgrid technologies and standards to further promote interoperability.

The objective of Research Area 6 is to identify and integrate open standards relevant to the CBITEC tactical microgrid's operational requirements. In cases where open standards did not sufficiently meet tactical microgrid operational requirements, the team worked with technology providers to build on existing open standards, minimize customization, and provide solutions that promote future demonstrated technology integration and expansion.

The Paragon team researched and identified currently available communication and control technologies as well as applicable interoperability standards for the CBITEC tactical microgrid, which are to develop an approach to bridge the gap in these standards to promote interoperability, security, and performance reliability.

\subsection{Identify and catalog currently available communication and controls technology}

The objective of this research is to identify and catalog COTS communication and controls technology.

To complete this research, SMEs reviewed material provided by $\mathrm{C}_{5} \mathrm{ISR}$, CERL, and CBITEC staff, along with previously engaged vendors, to identify communication and controls technologies with potential to provide the highest level of interoperability, security, and performance reliability. In addition, preference on design strategies and standards were also discussed for consideration.

Work related to this research effort commenced with the preparation of, and communication to CBITEC and CERL staff, a detailed list of equipment and systems that are typically present in a tactical microgrid as well 
as requesting available tactical microgrid documents for Paragon SMEs to review.

Extensive engagement with staff at CBITEC, CERL, and C5ISR revealed that a formal set of microgrid design drawings is not available; however, information on a subset of the equipment to be used in the tactical microgrid is available and was subsequently provided, including:

- Advanced DCS Operator's Manual; describing the parallel operation of the AMMPS generator to form a tactical microgrid

- DoD Interface Standard MIL-STD-TMS, TMS (Figure 14), version March 2013; an interface standard that is under development and has not yet been approved for release

- Army Technical Manual TM 9-6150-226-13, Operator and Field Maintenance Manual for DISE and PDISE.

Paragon SMEs-upon CERL's recommendation-took additional steps to engage directly with vendors that have supported CERL's development of microgrid technologies to form a tactical microgrid. They acquired additional documentation and insight on the operation, capabilities, and limitations of the existing technologies. In addition, Paragon SMEs comprehensively engaged SEL given its role in the design of the controls for IPDs, test generator operation in a microgrid environment, and familiarity with the TMS and ongoing revisions. Power quality measurements, which currently reside in IPD relays, can be collected by a future server. For future testing, the Paragon team recommends using separate metering equipment specifically designed for power quality analysis. This test equipment should be portable and easy to install/remove for testing as needed.

The communication and control technologies SMEs identified in use at the CBITEC were cataloged and reviewed to confirm operation capability and integration requirements for future equipment. Given the microgrid controller is under development, the control technologies currently in use are wholly contained in the IPD provided by Humber GEs. Within the IPD, several control devices serve as protective devices, to verify and control synchronization of sources and communicate messages for IPD monitoring and control. A detailed manual of the IPD, complete with an exhaustive parts list of all control items, is included in Appendix $\mathbf{Q}$. 
For microgrid communications, CBITEC has implemented a wireless mesh network for microgrid communications. Wireless mesh networks inherently provide redundancy while minimizing the need for extending communication conductor extensions. CBITEC's DMMS is used to wirelessly communicate with microgrid devices via a local dashboard (Figure 107). The DMMS is used for data collection only and does not control any of the microgrid devices. The AirGuard 3e-523 from 3e Technologies International serves provides the connection point between equipment and the CBITEC WLAN-forming a wireless mesh network at CBITEC. A technical manual for the AirGuard 3e-523 technical manual is provided in Appen$\operatorname{dix} \mathbf{R}$. The DMMS allows data collection without the need for communication conductors installation.

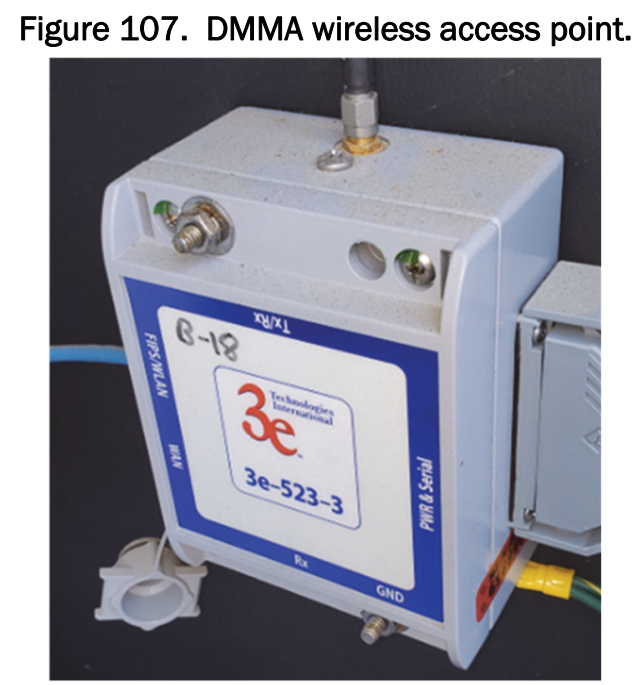

In addition to the wireless mesh network, the IPD contains Ethernet ports to support hardwired communications. By design, the Ethernet ports were intended to be used for communicating Modbus Transmission Control Protocol/Internet Protocol (TCP/IP) and DDS messages between the IPD and an overarching controller.

By policy, equipment connected to the tactical microgrid must be manually controlled.

\subsection{Identify current interoperability standards}

The objective of this research is to identify current interoperability standards applicable to the CBITEC tactical microgrid. 
Paragon SMEs identified technology providers that have developed open standards that minimize customization and provide solutions promoting future demonstrated technology integration and expansion. In order to identify and recommend an open standard, or set of standards, that promote interoperability applicable to the CBITEC tactical microgrid, SMEs reviewed the communications capabilities of the IPD, V2G fast chargers, and the wireless mesh network:

- Data Distribution Service

- Modbus

- CHAdeMO

- SAE J1772 CCS.

In addition, standards related to the installation of $\mathrm{V} 2 \mathrm{G}$ charging systems were also reviewed for applicability:

- UL 1741, Standard for Inverters, Converters, Controllers and Interconnection System Equipment for Use with DERs

- Institute of Electrical and Electronics Engineers (IEEE) 1547, Standard for Interconnecting Distributed Resources with Electric Power Systems

- UL 2202, Standard for EV Charging System Equipment

- National Fire Protection Association (NFPA) 70.

\subsubsection{Interoperability standards applicable to the CBITEC tactical microgrid}

Many open standards exist that could be reviewed for applicability and interoperability promotion. When considering the intent and desire to use the existing wire mesh communications system and IPDs developed by Humber Garick at the CBITEC site, the list of standards to review begins with those standards associated with the existing equipment as well as any new equipment to be added.

The IPD's TMS DDS Implementation Guide identifies Modbus TCP/IP and DDS by Object Management Group (OMG) as two communications protocol standards employed by the IPD. TMS DDS Implementation Guide is provided in Appendix S. As a communication protocol, Modbus is the industry standard for control communications, extremely reliable, and is built for controls communications. The Modbus protocol is also the intended 
communications protocol to be used by the microgrid controller, which is currently being developed. Moreover, the V2G fast charger also communicates Modbus for monitoring and control by an overarching controller.

The Tesla fast chargers are not bidirectional and utilize closed and proprietary protocols. Consequently, Tesla fast chargers only work with Tesla EVs. The SAE CCS standard, developed SAE, is the most commonly used fast-charging standard in the United States. This open standard is used by multiple charging station suppliers to charge CCS-compliant EVs (Figure 108). However, the CCS standard does not incorporate communication and command structures necessary to implement on-demand discharging of EVs. Consequently, messaging between the vehicles and chargers must be customized to include unique commands enabling a V2G-capable vehicle to use the CCS standard. This customization inherently prevents the custom charger to be readily used for multiple vehicle types. Such customization also requires extensive non-recurring engineering, development and testing to implement the $\mathrm{V} 2 \mathrm{G}$ capability, which further limits customized CCS solutions for $\mathrm{V} 2 \mathrm{G}$ applications. By 2025, SAE plans to update the CCS standard to include communication and controls specifications necessary to enable V2G operations between CCS-compliant vehicles and charging stations. The CHAdeMO standard (Figure 109) not only is commonly used in Asia but is also the only open standard that fully accommodates V2G applications. Using the CHAdeMO standard in the United States, however, is limited compared to the SAE CCS standard for fast-charging applications.

Figure 108. SAE CCS connector.
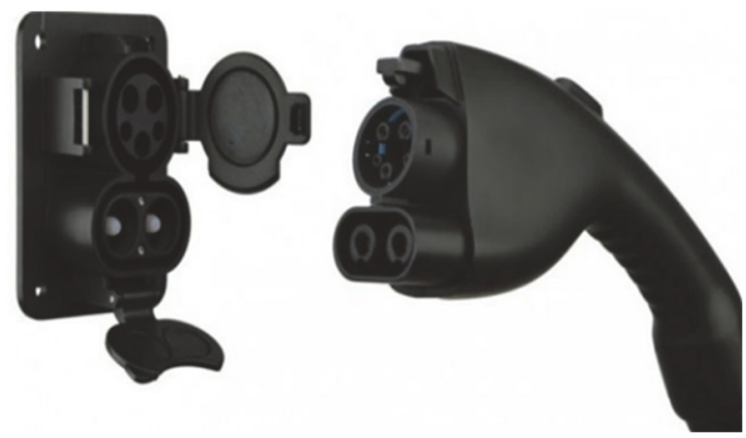


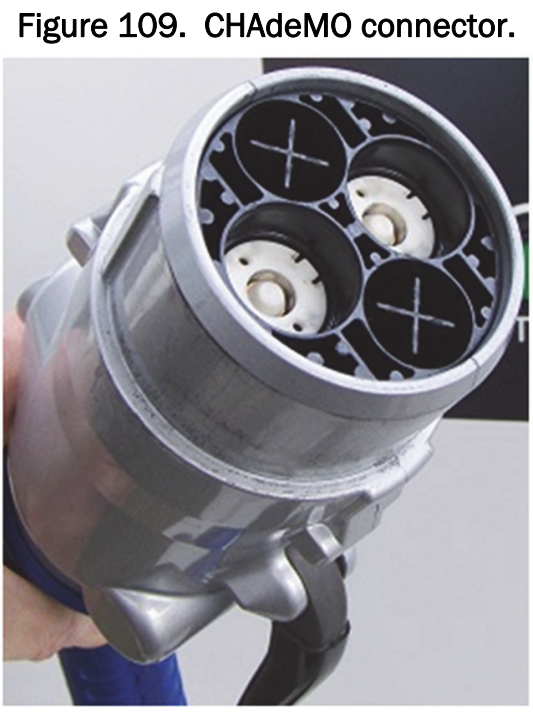

Monitoring and controlling a CHAdeMO-compliant vehicle's charge and discharge is accomplished by communicating to the V2G fast charger. The microgrid controller does not need to directly communicate to the vehicle for this purpose. The V2G fast charger communicates to the EV using the CHAdeMO communication protocol and no other communication standards are required.

Several industry standards relate to the design and installation of $\mathrm{V}_{2} \mathrm{G}$ charging equipment. From an interoperability standpoint, critical is UL1741, Standard for Inverters, Converters, Controllers and Interconnection System Equipment for Use with DERs.

\subsection{Recommended communication and control products for the CBITEC tactical microgrid}

The objective of this research is to recommend communication and control products for the CBITEC tactical microgrid. In order to recommend technologies situated to provide the highest level of interoperability, security, and performance reliability, Paragon SMEs developed a methodology to rank and stratify products.

\subsubsection{Communication and controls technology ranking methodology}

The ranking methodology utilized to select and recommend communication and control technologies was developed with the following criteria: 
- Compatibility with existing microgrid equipment to be used

- Compatibility with planned standards under development

- Use of open standards to promote interoperability

- Ability to meet performance requirements.

\subsubsection{Application of ranking methodology}

Paragon SMEs applied the ranking methodology to new equipment necessary to integrate the $\mathrm{V}_{2} \mathrm{G}$ fast-charging system. Of the fast-charging technologies available, only the CHAdeMO charging system met all items of the ranking methodology.

\subsubsection{Recommended technologies}

Given the IPD box protecting connected equipment, communicating with the planned microgrid controller and $\mathrm{V} 2 \mathrm{G}$ fast charger, and collecting operational data to verify connected equipment performance, Paragon SMEs recommend using the IPD to interconnect generation sources and loads for the tactical microgrid.

Considering the existing approvals to operate integration and cyber security features associated with the $3 \mathrm{eTi}$ 's AirGuard 3e-523 gateway, SMEs recommend the gateway be incorporated to wirelessly monitor new equipment via the system dashboard. CBITEC staff's existing operations have confirmed reliable operation that provides real-time data capture rates and resolution to enable effective monitoring.

Paragon SMEs researched industry options and found Coritech's V2G fast charger the only fast charger that fully utilizes open standards and does not require customization to implement. Integrating the fast charger will require a step-down transformer to tie the 480 -VAC output to the 208VAC power distribution system of the tactical microgrid. A Coritech fast charger product brochure is provided in Appendix T. The operation manual for the Coritech fast charger is provided in Appendix U. The communications memory map to enable monitoring and control of the charger is provided in Appendix $\mathbf{V}$. 


\subsection{Approach to bridge the gap between available technologies and standards}

The objective of this research is to develop an approach to bridge the gap between top identified technologies and standards to promote interoperability.

For generation sources to operate in parallel with the electrical grid, electrical utilities require compliance with specific design and installation standards. With compliance to specific standards, personnel safety and grid stability can be assured. Specifically, electrical utilities require UL1741 standard compliance, which requires generation sources to disconnect from the electrical grid during a grid outage. The goal of this requirement is to mitigate the risk of personnel, tasked to work on the power distribution system, electrocution and restore grid operations. Devices that are UL1741 compliant, including V2G fast chargers, are required to disconnect based on voltage and frequency deviations as identified in the standard (Tables 49 and 50).

Table 49. Interconnection system response to abnormal voltages.

\begin{tabular}{|c|c|}
\hline Voltage Range (\% of base voltage) & Clearing Times $(\mathrm{sec})$ \\
\hline $\mathrm{V}<50$ & 0.16 \\
\hline $50 \leq \mathrm{V}<88$ & 2.00 \\
\hline $110<\mathrm{V}<120$ & 1.00 \\
\hline $\mathrm{V} \geq 120$ & 0.16 \\
\hline
\end{tabular}

Table 50. Interconnection system response to abnormal frequencies.

\begin{tabular}{|c|c|c|}
\hline Distributed Resource Size & Frequency Range $(\mathrm{Hz})$ & Clearing Times (sec) \\
\hline \multirow{2}{*}{$\leq 30 \mathrm{~kW}$} & $>60.5$ & 0.16 \\
\cline { 2 - 3 } & $<59.3$ & 0.16 \\
\hline \multirow{3}{*}{$>30 \mathrm{~kW}$} & $>60.5$ & 0.16 \\
\cline { 2 - 3 } & $<59.8-75.0$ (adjustable) & adjustable 0.16 to 300 \\
\cline { 2 - 3 } & $<57.0$ & 0.16 \\
\hline
\end{tabular}

However, a tactical microgrid's voltage and frequency might not be as stable as the electrical grid. As a result, normal microgrid operation might include voltage and frequency variances in nuisance trips of the $\mathrm{V}_{2} \mathrm{G}$ fast charger-which included disconnecting during normal operation even without a "grid outage." To address this, voltage and frequency trip limits of the UL1741-compliant device might need to be adjusted. Doing this 
could void the UL1741 certification associated with the installation. However, a UL1741 certification is required when connecting to an electrical grid-not when operating the tactical microgrid off the grid.

Another prevalent industry standard used to ensure grid stability is IEEE 1547, Standard for Interconnecting Distributed Resources with Electric Power Systems. This standard includes power quality requirements for grid stability assurance. Requiring generation sources to meet standard power quality requirements will mitigate the risk of one generation device impeding other equipment connected to the microgrid by generating low quality power. While inverter technologies tend to produce more harmonics, an inverter that is IEEE1547 compliant will limit these harmonics. To further limit harmonics produced by a generation source, a delta-wye transformer, or line reactors, can be installed in between the inverter and the distribution system to limit the transmission of harmonics to other system components.

New gaps will inevitably emerge when attempting to integrate new technologies with previous technologies, in particular with communications protocols. Several potential solutions exist to help bridge gaps with communications systems, including protocol converters, media converters, and software tools. The best solution to promote interoperability completely depends on the new technology to be integrated.

\subsection{Future work}

Currently two documents that are under CERL development have potential to identify gaps in standards: the TMS and the TMS DDS Implementation Guide. These documents, as well as the need to implement new and novel technologies, will likely identify the need to adopt additional standards to promote interoperability.

Now, the CHAdeMO standard is the only open standard available to test the concept of $\mathrm{V} 2 \mathrm{G}$ technologies supporting grid stability in a tactical microgrid. The Nissan LEAF is the sole CHAdeMO-compliant vehicle currently available that also meets program power requirements. Future work could install EV battery packs on a vehicle platform considered suitable for tactical environments using the CHAdeMO open standard. 
SAE J1772 CCS standard for fast charging is more widely used in the United States. While this standard does not include V2G capability at this time, SAE is targeting by 2025 to incorporate this capability. Future work could include working group participation tasked to evolve the standard so that aspects related to military applications can also be incorporated.

Custom V2G solutions, an alternative to the two available open standards developed for vehicle charging in the United States, can be developed specifically for military applications by developing a purpose-built charger/inverter for battery packs to be mounted on a suitable vehicle platform. This approach can incorporate open standards to monitor and control the power converter and battery pack.

Sometimes, applications require additional vehicle information than what is provided through a charging standard. Battery temperature, operating status, or control of a specific vehicle's components might be required as part of the control algorithm used to determine appropriate charge or discharge rates. Most vehicle platforms use the open standard, CAN Bus, to monitor and control the various vehicle systems. By incorporating CAN Bus messaging into the microgrid controller, the ability to gather vehiclerelated operational status can be greatly extended. The CAN Bus is often accessed via the vehicle's onboard diagnostic (OBD) Port (Figure 110).

Figure 110. Vehicle OBD port.

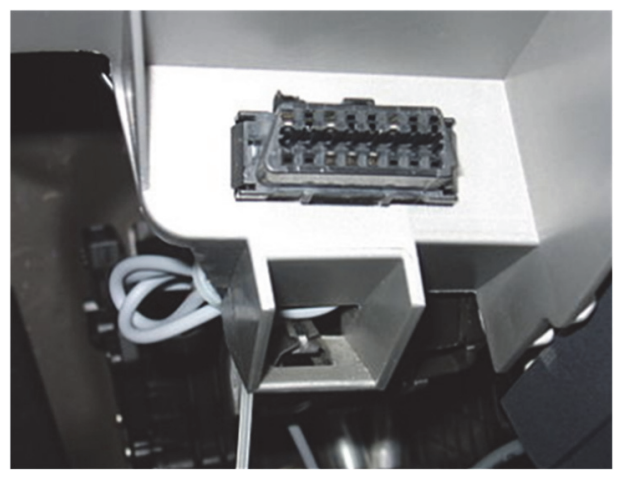

\subsection{Conclusions and recommendations}

While the tactical microgrid at CBITEC is not fully designed, the intent to use the IPD developed by HG Engineers, the wireless mesh network and the Modbus protocol are viable approaches that outline system communication requirements for future devices to interoperate. The $3 \mathrm{e}-523-3$ 
wireless access points, which were manufactured by $3 \mathrm{e}$ TI Technology International and establish the wireless mesh network at CBITEC, are cyber secure and already approved for use on a SIPRNet. Paragon SMEs recommend using the existing hardware for wireless communications as it has proven to be reliable, secure, and interoperates easily with system assets.

As testing confirms V2G systems are a viable solution that meet mission requirements and bolster energy resilience in tactical microgrids, Paragon SMEs recommend developing a V2G system that is purpose-built for military applications operating in the tactical microgrid environment. The V2G system, primarily, must have a suitable vehicle platform that meets vehicle-mission requirements. It must also have available payload capacity that can carry the weight of the lithium-ion ESS along with the necessary interconnection hardware, controls, and power converter. One vehicle platform that might be ideal is the Nikola Reckless. This off-road vehicle is built for tactical environments and is fully electric. Product information indicates the Reckless has a battery pack with an energy storage capacity of $125 \mathrm{kWh}$-enabling the possibility of charge and discharge rates equal to the 6o-kW AMMPS generator to be used at CBITEC. With an energy storage capacity nearly twice that of the proof-of-concept vehicle, the Reckless will be able to support equivalent charge/discharge rates for twice as long.

Given the program intent to serve as a living laboratory with vehicle testing capability, Paragon SMEs recommend incorporating the capability to directly communicate to vehicle CAN Bus systems for additional data gathering and the development of microgrid control algorithms. Specifically, the use of CAN Bus data loggers will provide the capability to watch the reactions of vehicle systems very closely to events in support of control algorithm development and vehicle protection. CAN Bus data loggers are directly connected to the vehicles OBD port and store time-synchronized data that is later downloaded and analyzed via software tools. 


\section{Summary of Findings, Recommendations, and Next Steps}

The Paragon team has developed the roadmap for an innovative sensored, living laboratory that can evaluate emerging electric transport vehicles, vehicle autonomy, $\mathrm{V}_{2} \mathrm{G}$ charging, and microgrid technologies. The fundamental basis of the living laboratory is to serve as a testbed to facilitate joint research and evaluation of autonomous EVs and bidirectional-vehicle charging in a real-world, non-hostile environment. The living laboratory will leverage government and industry technical expertise to accelerate technological advancement and innovation. The collaborative environment between the Paragon and CERL teams culminated in information sharing and open innovation across technology borders that might otherwise be siloed. The living laboratory is therefore also a platform for collaborative innovation by leaders in these fields.

\subsection{Research Area 1: Identify military-unique requirements for autonomous transport technologies}

The Paragon team engaged with stakeholders and conducted research to identify military-unique requirements for autonomous transport technologies. The research effort resulted in a prioritized list of transport dependent Military Support Activities that could be automated to the benefit of the government.

Table 51 provides the prioritized list of activities.

Table 51. Prioritized Military Support Activities.

\begin{tabular}{|c|c|c|c|}
\hline $\begin{array}{c}\text { MSA } \\
\#\end{array}$ & MSA Type & Mission Supported & Vehicles \\
\hline \multirow{3}{*}{1} & $\begin{array}{l}\text { Personnel Transport: } \\
\text { transport (1-4 people) }\end{array}$ & \multirow{3}{*}{$\begin{array}{l}\text { Installation Management Command (IMCOM)/AMC } \\
\text { missions for logistics in support of installation operation } \\
\text { - personnel transport supports all CONUS housing, } \\
\text { training and deployment functions. Mass transit is more } \\
\text { common at basic training installations. } \\
\text { FLW provides } 2.5 \text { million round trips annually to move } \\
\text { Soldiers to training areas, ranges, and dining facilities. }\end{array}$} & Automobile \\
\hline & $\begin{array}{l}\text { Personnel transport (5- } \\
20 \text { people) }\end{array}$ & & Shuttle \\
\hline & $\begin{array}{l}\text { Troop transport for } \\
\text { training (platoon to } \\
\text { company size-up to } \\
250 \text { Soldiers) }\end{array}$ & & $\begin{array}{l}\text { Buses (40-80) and tractor } \\
\text { with personnel trailer (125) }\end{array}$ \\
\hline 2 & $\begin{array}{l}\text { Land Management: } \\
\text { grounds maintenance- } \\
\text { beautification and } \\
\text { security cutting }\end{array}$ & $\begin{array}{l}\text { IMCOM/AMC installation operation-grounds } \\
\text { maintenance can include grass cutting, snow removal, } \\
\text { dirt removal, wood clearing (with workers), tree removal } \\
\text { (with workers), etc. }\end{array}$ & Tractors and mowers \\
\hline
\end{tabular}




\begin{tabular}{|c|c|c|c|}
\hline $\begin{array}{c}\text { MSA } \\
\#\end{array}$ & MSA Type & Mission Supported & Vehicles \\
\hline & $\begin{array}{l}\text { Land Management: } \\
\text { range maintenance }\end{array}$ & $\begin{array}{l}\text { Two types of ground maintenance are common: grass } \\
\text { cutting in cantonment areas and less frequent grass } \\
\text { cutting in range and training areas. This effort would be } \\
\text { focused on large-scale mowers and earth movers that } \\
\text { can be used to maintain CONUS range areas (and } \\
\text { possibly cantonment areas). }\end{array}$ & $\begin{array}{l}\text { Large/farm tractors with } \\
\text { cutting decks, plowing }\end{array}$ \\
\hline \multirow{3}{*}{3} & $\begin{array}{l}\text { Logistics: materiel } \\
\text { transport-food, water, } \\
\text { munitions, fuel, } \\
\text { municipal solid waste }\end{array}$ & \multirow[t]{3}{*}{$\begin{array}{l}\text { IMCOM/AMC Installation Operations directly supporting } \\
\text { training (TRADOC), Garrison (FORSCOM) missions, and } \\
\text { deployment (all installations that deploy personnel). } \\
\text { Convoys used to support all forward-operating areas. }\end{array}$} & $\begin{array}{l}\text { Delivery trucks and vans, } \\
\text { tractors with trailers, } \\
\text { specialty trucks (tankers, } \\
\text { refrigeration unit, waste } \\
\text { hauler, bucket truck), } \\
\text { secure trucks (munitions) }\end{array}$ \\
\hline & $\begin{array}{l}\text { Logistics: material } \\
\text { management and } \\
\text { warehousing-delivery, } \\
\text { storage, and distribution }\end{array}$ & & $\begin{array}{l}\text { Forklifts, indoor and outdoor } \\
\text { tugs, material handling } \\
\text { equipment }\end{array}$ \\
\hline & $\begin{array}{l}\text { Logistics: airfield } \\
\text { activities-GSVs }\end{array}$ & & $\begin{array}{l}\text { Various GSVs-fuelers, pallet } \\
\text { loaders, aircraft tugs, small } \\
\text { loaders, munitions movers and } \\
\text { loaders (security), deicers, } \\
\text { plows }\end{array}$ \\
\hline 3 & $\begin{array}{l}\text { Logistics: vehicle and } \\
\text { equipment hauling }\end{array}$ & $\begin{array}{l}\text { Vehicle and equipment recovery and delivery-supports } \\
\text { IMCOM/AMC operations. }\end{array}$ & $\begin{array}{l}\text { Tow trucks, } \\
\text { vehicle lifts and haulers, } \\
\text { tractors with trailers }\end{array}$ \\
\hline 4 & Security monitoring & $\begin{array}{l}\text { Reconnaissance in forward areas, as well as installation } \\
\text { security monitoring. }\end{array}$ & $\begin{array}{l}\text { Drones, reconnaissance } \\
\text { vehicles }\end{array}$ \\
\hline 5 & $\begin{array}{l}\text { Land Preparation: earth } \\
\text { moving, plowing, digging }\end{array}$ & $\begin{array}{l}\text { IMCOM/AMC grounds maintenance, as well as combat } \\
\text { engineering operations and training. }\end{array}$ & $\begin{array}{l}\text { Engineering equipment- } \\
\text { scraper, crane, dozer, plow, } \\
\text { hauler, dump truck, grader, } \\
\text { loader, backhoe, crawler, HYEX }\end{array}$ \\
\hline 6 & $\begin{array}{l}\text { Road Maintenance: } \\
\text { maintenance, repair, } \\
\text { snow removal }\end{array}$ & $\begin{array}{l}\text { IMCOM/AMC installation maintenance and clearing, } \\
\text { development, and maintenance of new and existing } \\
\text { roads in forward areas. }\end{array}$ & $\begin{array}{l}\text { Grader, sweeper, dump truck, } \\
\text { asphalt paver, compactor, } \\
\text { roller, snowplow }\end{array}$ \\
\hline
\end{tabular}

\subsection{Research Area 2: Identify existing technologies that can be adapted for military applications}

The focus of our research in this area was on available and emerging electric transport systems that could support the prioritized list of transport activities identified in Research Area 1. The onboard energy storage provided by electric transport vehicles introduces the potential to access an additional and distributed electric power supply to support Army energy resilience. Paragon SMEs began their research cataloging and evaluating existing and emerging electric transportation systems. This was to identify candidates for automation and to serve as a platform for future research, testing, and demonstration. The research team evaluated candidate electric transport technologies for suitability against the prioritized military transport activities and compatibility with research requirements. 
Candidate technologies were prioritized by the number of criteria thresholds met. Screening criteria thresholds were TRLs, target cost, location of assembly, battery capacity, complementary research, Military Support Activity requirements, and GSA availability. Screening criteria thresholds set baseline requirements to prioritize candidate technologies and enabled the development of the list provided in Table 52.

Table 52. Prioritized candidate technologies.

\begin{tabular}{|c|c|c|c|c|c|c|c|c|}
\hline Priority & $\begin{array}{c}\text { Total } \\
\text { No. of } Y\end{array}$ & $\begin{array}{c}\text { TRL } \\
(7-9)\end{array}$ & $\begin{array}{c}\text { Cost } \leq \\
\$ 450,000\end{array}$ & $\begin{array}{l}\text { Assembled in } \\
\text { United States }\end{array}$ & $\begin{array}{c}\text { Battery } \\
\text { Capacity } \geq \\
50 \text { kWh }\end{array}$ & MSA & $\begin{array}{l}\text { Related } \\
\text { Research }\end{array}$ & $\begin{array}{c}\text { Available } \\
\text { through GSA }\end{array}$ \\
\hline \multirow{3}{*}{1} & \multirow{3}{*}{7} & \multicolumn{7}{|c|}{ MPS by: Dannar } \\
\hline & & Y & Y & $\mathrm{Y}$ & Y & Y & Y & Y \\
\hline & & 9 & $\$ 200-400 K$ & IN & $125-630 \mathrm{kWh}$ & $\begin{array}{c}2,4,5 \\
6\end{array}$ & DHS & GSA \\
\hline \multirow{3}{*}{2} & \multirow{3}{*}{6} & \multicolumn{7}{|c|}{ Cargo Van, Chassis Cab, Cutaway Cab, Shuttle Van, Step Van by: Zenith Motors } \\
\hline & & Y & Y & Y & Y & Y & $\mathrm{N}$ & Y \\
\hline & & 9 & $\$ 100 K$ & $\mathrm{CA}$ & $52-100 \mathrm{kWh}$ & 1,3 & & GSA \\
\hline \multirow{3}{*}{3} & \multirow{3}{*}{6} & \multicolumn{7}{|c|}{ Medium-duty Trucks, Shuttles, and Buses by: Zero Truck } \\
\hline & & Y & Y & Y & Y & Y & $\mathrm{N}$ & $\mathrm{N}$ \\
\hline & & 9 & $\$ 60-100 K$ & $\mathrm{CA}$ & $53-80 \mathrm{kWh}$ & 1,3 & & \\
\hline \multirow{3}{*}{4} & \multirow{3}{*}{6} & \multicolumn{7}{|c|}{ Zeus Electric Shuttle bus, Utility vehicle, Flatbed truck by: Phoenix Motorcars } \\
\hline & & Y & Y & Y & Y & Y & Y & $\mathrm{N}$ \\
\hline & & 9 & $\$ 240,000+$ & $\mathrm{CA}$ & $105 \mathrm{kWh}$ & 1,3 & EPA, CA & \\
\hline \multirow{3}{*}{5} & \multirow{3}{*}{5} & \multicolumn{7}{|c|}{ T-Series Yard Hostler by: Orange Motors } \\
\hline & & Y & $\mathrm{Y}$ & $\mathrm{Y}$ & Y & Y & $\mathrm{N}$ & $\mathrm{N}$ \\
\hline & & 9 & $\$ 200-300 K$ & MO & $80-160 \mathrm{kWh}$ & 1,3 & & \\
\hline \multirow{3}{*}{6} & \multirow{3}{*}{5} & \multicolumn{7}{|c|}{ Reckless by: Nikola } \\
\hline & & Y & $\mathrm{N}$ & $\mathrm{Y}$ & Y & Y & Y & $\mathrm{N}$ \\
\hline & & 9 & $\begin{array}{c}\$ 480 \mathrm{~K} / \text { year } \\
\text { Lease }\end{array}$ & $\mathrm{CA}$ & $52-100 \mathrm{kWh}$ & 4 & USMC & \\
\hline \multirow{3}{*}{7} & \multirow{3}{*}{5} & \multicolumn{7}{|c|}{ eBus by: eBus } \\
\hline & & Y & Y & Y & Y & Y & $\mathrm{N}$ & $\mathrm{N}$ \\
\hline & & 9 & $\$ 400 K$ & $\mathrm{CA}$ & $130 \mathrm{kWh}$ & 1 & & \\
\hline \multirow{3}{*}{8} & \multirow{3}{*}{5} & \multicolumn{7}{|c|}{ Xcelsior Charge (multiple versions) by: New Flyer } \\
\hline & & Y & $\mathrm{N}$ & $\mathrm{Y}$ & Y & Y & $\mathrm{Y}$ & $\mathrm{N}$ \\
\hline & & 9 & $\$ 700-1,200 K$ & $\mathrm{AL}$ & $160-466 \mathrm{kWh}$ & 1,3 & Private sector & \\
\hline \multirow{3}{*}{9} & \multirow{3}{*}{5} & \multicolumn{7}{|c|}{ R1T-EV Pick-u by: Rivian } \\
\hline & & Y & $\mathrm{Y}$ & $\mathrm{Y}$ & Y & Y & $\mathrm{N}$ & $\mathrm{N}$ \\
\hline & & 8 & $\$ 70-100 K$ & IL & $105-180 \mathrm{kWh}$ & 3,4 & & \\
\hline \multirow{3}{*}{10} & \multirow{3}{*}{5} & & & -Liner C2 Jouley El & tric School Bus k & y: Thomas & Built & \\
\hline & & Y & Y & Y & Y & Y & $\mathrm{N}$ & $\mathrm{N}$ \\
\hline & & 8 & $\$ 450 K$ & NC & $220 \mathrm{kWh}$ & 1 & & \\
\hline
\end{tabular}




\begin{tabular}{|c|c|c|c|c|c|c|c|c|}
\hline Priority & $\begin{array}{c}\text { Total } \\
\text { No. of } Y\end{array}$ & $\begin{array}{c}\text { TRL } \\
(7-9)\end{array}$ & $\begin{array}{c}\text { Cost } \leq \\
\$ 450,000\end{array}$ & $\begin{array}{l}\text { Assembled in } \\
\text { United States }\end{array}$ & $\begin{array}{c}\text { Battery } \\
\text { Capacity } \geq \\
50 \mathrm{kWh}\end{array}$ & MSA & $\begin{array}{c}\text { Related } \\
\text { Research }\end{array}$ & $\begin{array}{c}\text { Available } \\
\text { through GSA }\end{array}$ \\
\hline \multirow{3}{*}{11} & \multirow{3}{*}{5} & \multicolumn{7}{|c|}{ Edrive Tractor by: ATC } \\
\hline & & Y & $\mathrm{Y}$ & Y & Y & Y & $\mathrm{N}$ & $\mathrm{N}$ \\
\hline & & 9 & $\$ 250 K$ & $\mathrm{MN}$ & $250 \mathrm{~kW}$ & $2,5,6$ & & \\
\hline \multirow{3}{*}{12} & \multirow{3}{*}{4} & \multicolumn{7}{|c|}{ eCargo, eTruck drayage-Class 8 Tractor by: US Hybrid } \\
\hline & & $\mathrm{N}$ & Y & Y & Y & Y & $\mathrm{N}$ & $\mathrm{N}$ \\
\hline & & 6 & $\$ 250 K$ & $\mathrm{CA}$ & $240 \mathrm{kWh}$ & 1,3 & & \\
\hline \multirow{3}{*}{13} & \multirow{3}{*}{4} & \multicolumn{7}{|c|}{ LFSe by: Nova Bus } \\
\hline & & Y & $\mathrm{N}$ & Y & Y & Y & $\mathrm{N}$ & $\mathrm{N}$ \\
\hline & & 8 & $\$ 700 K+$ & NY & $152 \mathrm{kWh}$ & 1 & & \\
\hline
\end{tabular}

To validate technology information and determine prospect for future research, Paragon SMEs engaged priority technology manufacturers. The manufacturers identified for engagement were: Dannar, Zenith Motors, Zero Truck, Phoenix Motorcars, Orange Motors, Nikola, Ebus, New Flyer, Rivian, Thomas Built, ATC, US Hybrid (USH), and Nova Bus. Based on research requirements and collaborative SME agreement, the following electric transport technologies are recommended for future autonomy testing and evaluation:

- Dannar MPS

- New Flyer Xcelsior Charge Bus

- Nikola Reckless

- Orange Motors Yard Hustler

- Phoenix Motorcars shuttle buses and trucks

- US Hybrid power conversion systems.

The Dannar is an attractive technology because of commercial maturity and versatility for a broad range of Military Support Activities. Additionally, the technology is drive-by-wire equipped and shows high probability for full autonomous capability in the next few years. The New Flyer Xcelsior is a full-size bus that could be utilized for soldier transport and is also a good candidate for automation. The Nikola Reckless provides the opportunity to explore high-speed EVs that will function in a tactical environment. The Orange EV system, which has a large hauling capacity, is appealing for logistic support activities. 


\subsection{Research Area 3: Identify research and operational tests for autonomous transport vehicles}

Paragon's research team researched autonomous-system data, state-ofthe-art capture and storage technologies, and recommended solutions to facilitate autonomous technology operation and performance testing. The nSight suite, developed by Robotic Research, was recommended as an end-to-end data collection and analysis solution. The nSight captures multiple streams of data with a single data-logging device, which timestamps and performs post processing operations and analytics all in one location. The nSight suite is composed of the nSight Recorder (Onboard data recorder), nSight Upload (Data disseminator to servers), nSight AAR (Server and Analysis Tool).

Paragon's research team developed test plans to verify autonomous-system safety, operation, and performance. The operational test plans are designed to assess baseline system performance, traffic safety and interaction, sensor degradation, terrain identification, and V2G station docking. These test plans are applicable to the FLW test areas, specifically CBITEC and the TA-231 Driving Course. The test plans are also scalable and can be tailored to suit technology and test cases as needed. To operationalize the test plans, the research team recommends outfitting test areas with sensor suites as detailed in Research Area 5.

\subsection{Research Area 4: Investigate requirements for testing and demonstrating V2G vehicle charging within a tactical microgrid environment}

V2G charging equipment is essential for electric power bidirectional flow between electric transport and energy systems. This connection allows electric transportation to provide ancillary energy and assurance to local energy generation resulting in improved reliability, flexibility, and resilience. Currently in the United States, the CHAdeMO standard is the only open standard that is designed to fully support $\mathrm{V}_{2} \mathrm{G}$ system bidirectional power flow. Paragon SMEs identified two electric transport technologies available for purchase in the United States with the CHAdeMO fast-charging option: the Mitsubishi Outlander and the Nissan LEAF. The Outlander is a PHEV with energy storage capacity of $12 \mathrm{kWh}$; this battery pack is comparatively small compared to the LEAF, which is solely electric and available with two energy storage capacity options $-40 \mathrm{kWh}$ and $62 \mathrm{kWh}$. 
The Coritech V2G charger was selected for this research program, based on program objectives and site requirements. The Coritech charger is a $30-\mathrm{kW}$ fast charger that is CHAdeMO-compliant. The VGI-30-DC-CHA fast charger design is suitable for integrating into a tactical microgrid because it:

- Has an $\mathrm{AC}$ voltage configuration that easily integrates into the planned 208-VAC microgrid distribution system with a COTS step-down transformer

- Is specifically designed for fast charging and discharging of a CHAdeMO-compliant vehicle

- Uses open protocols to interface with an overarching controller to monitor and control the charging and discharging rates of the EV

- Uses open protocols, commonly used to interface with EVs, including the Nissan LEAF

- Is designed to U.S. standards applicable to V2G charging systems and distributions systems rated for use in the United States

- Is designed to operate outdoors

- Avoids the significant impacts to cost, schedule and performance risks associated with developing a first-generation alternative.

Modeling results demonstrated that the Coritech $\mathrm{V}_{2} \mathrm{G}$ charger will interoperate with the military legacy equipment that make up the CBITEC tactical microgrid. Anticipated voltage and frequency variations of the AMMPS generators-produced by the sudden increases or decreases in load or generation-are not expected to cause nuisance trips for the V2G charger. Recommended frequency and voltage trip settings for the AMMPS generators are provided in this report. These settings will promote interoperability of the tactical microgrid generation sources, even when system dynamics occur.

Simulated tests also confirmed that the recommended V2G charging system is a viable solution to meet mission requirements and bolster energy resilience. Paragon SMEs recommend defining agnostic model components to increase simulation accuracy in cases where specific equipment is pre-determined and commonly used. Program of Record Equipment, such as the AMMPS generator, can be accurately modeled to further increase the overall system's simulation accuracy. The benefit from repeated and accurate modeling could easily offset the cost associated with additional development time. 


\subsection{Research Area 5: Develop requirements for a sensored living laboratory that could be used to assess the performance of autonomous innovations}

\subsubsection{A - Autonomous vehicle living laboratory requirements}

The Paragon research team, led by Robotic Research, researched and identified appropriate sensors and methods to measure performance of autonomous systems within the CBITEC site. The team provided high-level test plans that defined the scope, objective, key personnel, and approach for the following tests: traffic interaction, sensor degradation, terrain identification, and charging station docking.

Robotic Research's team of engineers are SMEs in autonomous software and hardware development and integration. The following list of requirements developed by the Robotic Research engineering team define the high-level infrastructure, sensors, and data necessary to measure autonomous-system performance within the living laboratory at CBITEC. These requirements are meant to evolve, or be further derived, as more details about the test site are defined in Table 53.

Table 53. Site requirements.

\begin{tabular}{|c|c|}
\hline Operation & Site Requirement \\
\hline Infrastructure & $\begin{array}{l}\text { - Requirement 1: The living laboratory will have provisions to charge an EV. } \\
\text { - Requirement 2: The living laboratory will have electrical power connections for sensors. } \\
\text { - Requirement 3: The living laboratory shall have semi-permanent mounting points for sensors. (Semi- } \\
\text { - } \quad \text { Rermanent mounts can be removed from the site without causing damage or disturbing the site). }\end{array}$ \\
\hline Sensors & $\begin{array}{l}\text { - Requirement 5: The living laboratory will be able to detect autonomous systems at the site. } \\
\text { - Requirement 6: The living laboratory will be able to measure the relative position of autonomous } \\
\text { systems at the site. } \\
\text { - Requirement 7: The living laboratory will be able to measure absolute position of autonomous systems } \\
\text { within 0.1 meters. } \\
\text { - Requirement 8: The living laboratory will be able to communicate absolute position of the autonomous } \\
\text { systems at the site. } \\
\text { - Requirement 9: The living laboratory autonomous detection system will be able to operate in varying } \\
\text { lighting conditions, such as full daylight, dusk, and night. }\end{array}$ \\
\hline $\begin{array}{c}\text { Data } \\
\text { Collection }\end{array}$ & $\begin{array}{l}\text { - Requirement 10: The living laboratory will be able to collect and store time data. } \\
\text { - Requirement 11: The living laboratory will be able to collect and store weather data. } \\
\text { - Requirement 12: The living laboratory will be able to collect and store autonomous-system position } \\
\text { - Requirement 13: The living laboratory will be able to collect and store autonomous-system attitude } \\
\text { data. } \\
\text { - Requirement 14: The living laboratory will be able to collect and store autonomous-system velocity } \\
\text { data. } \\
\text { - Requirement 15: The living laboratory will be able to collect and store autonomous-system sensor } \\
\text { health data. }\end{array}$ \\
\hline
\end{tabular}


Given the program's intent to create a living laboratory with autonomous vehicle testing capability, Paragon SMEs recommend incorporating the capability to directly communicate to vehicle CAN Bus systems for additional data gathering and the development of microgrid control algorithms. Specifically, using CAN Bus data loggers will provide the capability to closely watch vehicle system reactions to events in support of control algorithm development and vehicle protection. CAN Bus data loggers are directly connected to the vehicle's onboard diagnostic port and store time-synchronized data that is later downloaded and analyzed via software tools.

Autonomous capabilities extend the benefits that electrified transportation and $\mathrm{V}_{2} \mathrm{G}$ technologies offer. Autonomous technologies show great potential to benefit military transport-dependent activities by improving logistics, security, base operation, maintenance, transport, and, eventually, warfighting. Additionally, implementing these technologies has the potential to decrease risks to the warfighter and reduce casualties through autonomously conducted missions.

\subsubsection{B - V2G charging living laboratory requirements}

Paragon's research team, led by TechFlow, identified the requirements to assess $\mathrm{V} 2 \mathrm{G}$ charging performance in a tactical environment. SMEs determined that the IPD boxes prepared for the CBITEC site include control equipment that can meet the metering and control needs for interconnecting tactical microgrid components. Additional components, to complete the living laboratory, and their benefits were identified and provided in Section 6 of this report. While the tactical microgrid at CBITEC is not fully designed, the IPD developed by HG Engineers, combined with the wireless mesh network and the Modbus protocol, are determined to be a viable design approach. This approach provides a baseline for system communication requirements and future device integration and interoperability. The 3e-523-3 wireless access points manufactured by 3 e TI Technology International and the established wireless mesh network at CBITEC are cyber secure and already approved for use on a SIPRNet. Paragon SMEs recommend using the existing hardware for wireless communications, as it has proven to be reliable, secure, and interoperates easily with system assets. 
As determined through SME research and modeling conducted in Research Area 4, the Coritech V2G charger will interoperate with the military legacy equipment that make up the CBITEC tactical microgrid. The Coritech charger was purchased by Paragon, under this research project, for future integration into the CBITEC Living Laboratory. The V2G system, primarily, must have a suitable vehicle platform that meets vehiclemission requirement. It must also have available payload capacity that can carry the weight of the lithium-ion ESS along with the necessary interconnection hardware, controls, and power converter. Various options were considered for a proof-of-concept vehicle for evaluation at CBITEC, but the only EV that is currently available for $\mathrm{V}_{2} \mathrm{G}$ integration with open protocols is the Nissan LEAF. A vehicle platform that should be considered for future evaluation is the Nikola Reckless. This fully electric, off-road vehicle is built for tactical environments. Product information indicates that, when available, the Reckless will house a battery pack with an energy storage capacity of $125 \mathrm{kWh}$, enabling the possibility of charge and discharge rates equal to the 60-kW AMMPS generators at CBITEC. With an energy storage capacity nearly twice that of the Nissan LEAF proof-of-concept vehicle, the Reckless will be able to support equivalent charge/discharge rates for twice as long.

Future research recommendations regarding the development of the CBITEC tactical microgrid are as follows: complete a detailed design of the tactical microgrid, document IPD and hardware settings for interconnecting the microgrid assets and include hardware-in-the-loop modeling and testing to improve simulation accuracy and support control algorithm development.

\subsection{Research Area 6: Integrate open standards to promote interoperability}

To promote interoperability, the Paragon team identified equipment that utilizes open standards and the standards that can guide the V2G integration equipment into the tactical microgrid.

Several industry standards relate to the design and installation of V2G charging equipment. From an interoperability standpoint, UL1741, Standard for Inverters, Converters, Controllers and Interconnection System Equipment for Use with DERs is critical. Paragon SMEs researched 
industry options and found the $\mathrm{V} 2 \mathrm{G}$ fast charger by Coritech as the only fast charger that fully utilizes open standards and does not require customization to implement. Integrating the fast charger will require a stepdown transformer to tie the 480 -VAC output to the 208-VAC power distribution system of the tactical microgrid. The CHAdeMO standard is the only open standard that fully accommodates $\mathrm{V} 2 \mathrm{G}$ applications. Using the CHAdeMO standard in the United States, however, is limited compared to the SAE CCS standard for fast-charging applications.

For generation sources to be allowed to operate in parallel with the electrical grid, electrical utilities require specific design and installation standards compliance. With compliance to specific standards, personnel safety and grid stability can be assured. Specifically, electrical utilities require compliance with the UL1741 standard, which requires generation sources to disconnect from the electrical grid during a grid outage. The goal of this requirement is to mitigate the risk of personnel, tasked to work on the power distribution system, electrocution and restore grid operations. Devices that are UL1741 compliant, including V2G fast chargers, are required to disconnect based on voltage and frequency deviations as identified in the standard. Another common industry standard used to ensure grid stability is IEEE 1547, Standard for Interconnecting Distributed Resources with Electric Power Systems. This standard includes power quality requirements to help ensure grid stability.

As new technologies evolve, gaps will inevitably emerge when attempting to integrate communications protocols with previous technologies. Some potential solutions exist to help bridge gaps with communications systems, include protocol converters, media converters, and software tools. 


\section{References}

Autonomous Ground Systems, Autonomous Ground Resupply Autonomy Kit, 2018, http://gvsets.ndia-mich.org/publication.php?documentID=613.

Automotive News, Startup prepares for production of LiDAR at scale, $29 \mathrm{Jul}$ 2019, https://www.autonews.com/mobility-report/startup-prepares-production-LiDAR-scale.

Autonomous Vehicle Computing Consortium accessed 29 Oct 2019, www.avcconsortium.org/.

Business Wire, AUVSI Xponential: Robotic Research Paves the Way for Transportation Autonomy with Commercial and Defense Solutions, 29 Apr 2019, https://www.businesswire.com/news/home/20190429005851/en/AUVSI-Xponential-RoboticResearch-Paves-Transportation-Autonomy.

Clear Path Robotics, Autonomy Research Kit, accessed 19 Oct 2019, https://clearpathrobotics.com/autonomy-research-kit/.

Clear Path Robotics, Comparing accuracy of open-source vs. proprietary localization software, accessed 19 Oct 2019, https://clearpathrobotics.com/ark-whitepaper/.

Cybernet, accessed 19 Oct 2019, www.cybernet.com/.

Cybernet, Automated Tactical Ammunition Classification System, accessed 19 Oct 2019, www.cybernet.com/products/atacs.

Cybernet, Cybernet Systems Corporation Builds and Retrofits Autonomous Vehicles in Michigan, accessed 19 Oct 2019, https://www.cybernet.com/images/stories/pdf/u2uarvds13-130603-mail.pdf.

Cybernet, Inertial Measurement and Magnetometer Module, accessed 19 Oct 2019, https://www.cybernet.com/products/i3m.

Cybernet, OpenSkies, accessed 19 Oct 2019, www.cybernet.com/products/openskies.

Cybernet, Rapidly Generated Instrument Panels for Simulation and Training and Reconfigurable Tactile Panel Technology, accessed 19 Oct 2019, www.cybernet.com/products/rtpt.

Cybernet, Robotics, accessed 19 Oct 2019, www.cybernet.com/products/vsil.

Cybernet, Virtual Systems Integration Lab, accessed 19 Oct 2019, www.cybernet.com/products/vsil.

Defense Daily, Army, Lockheed Martin, Demonstrate Fully Autonomous Convoys Can Operate in Urban Areas, 31 Jan 2014, https://www.defensedaily.com/army-lockheedmartin-demonstrate-fully-autonomous-convoys-can-operate-in-urban-areas/uncategorized/.

DSpace@MIT, ShadowCam: Real-Time Detection of Moving Obstacles Behind a Corner for Autonomous Vehicles, 2018, https://dspace.mit.edu/handle/1721.1/119439. 
EurekaAlert!, Enabling autonomous vehicles to see around corners, 28 Oct 2019, https://www.eurekalert.org/pub_releases/2019-10/miot-eav102819.php.

Lockheed Martin and U.S. Army Combat Capabilities Development Command Ground Vehicle Systems Center, Integration of the Autonomous Mobility Applique System into the Robotic Technology Kernel, 13 Aug 2019, http://gvsets.ndiamich.org/publication.php?documentID=702.

Lockheed Martin, Autonomous Mobility Applique System (AMAS), accessed 19 Oct 2019, https://www.lockheedmartin.com/en-us/products/autonomous-mobility-applique-systemamas.html.

Luminar, accessed 19 Oct 2019, https://www.luminartech.com/.

Luminar, Iris System, accessed 19 Oct 2019, https://www.luminartech.com/technology.

Perrone Robotics, accessed 19 Oct 2019, https://perronerobotics.squarespace.com/.

Perrone Robotics, Drop-In Autonomy Kit (DAK), accessed 19 Oct 2019, https://perronerobotics.squarespace.com/dak.

Perrone Robotics, Turn-key Solutions for Autonomous Robots, accessed 19 Oct 2019, http://perroneautorobot.com/.

Robotic Research, Autonomous Ground Resupply, accessed 19 Oct 2019, https://www.roboticresearch.com/programs/agr.

Xmatik, LaneCruise, accessed 19 Oct 2019, https://x-matik.com/lanecruise.html. 


\section{Appendix A: Military Strategic Planning Resource Documents}

\begin{tabular}{|c|c|}
\hline Resource & Author/Reference Site \\
\hline $\begin{array}{l}\text { U.S. Army Fort } \\
\text { Leonard Wood } \\
\text { Installation } \\
\text { Strategic } \\
\text { Sustainability Plan } \\
\text { FY16-41 }\end{array}$ & $\begin{array}{l}\text { Premont, Mark, 24, Apr. 2017, Garrison Strategic Plan Annex A FY16-41 Projects, pp. 31-81, and } \\
\text { Annex B FY11-15 Progress Summary pp. 83-93., } \\
\text { https://eko.usace.army.mil/virtualteams/issp/index.cfm?id=273971\&syspage=Documents. }\end{array}$ \\
\hline $\begin{array}{l}\text { USAG Vicenza } \\
\text { Strategic Action } \\
\text { Planning Session- } \\
\text { Command Briefing }\end{array}$ & Barber, David, 15, Jul. 2010, slides 7-9, 12-13, 16, 19-20, and 25-26. \\
\hline $\begin{array}{l}\text { Central Texas } \\
\text { Sustainable } \\
\text { Communities } \\
\text { Conference, } \\
\text { Summary of } \\
\text { Goals, and } \\
\text { Objectives- } \\
\text { Command Briefing }\end{array}$ & Rawlings, Jennifer, 17 Feb. 2010, slides 7-15, 17-20, 24-30, 32-40 and 42-26. \\
\hline $\begin{array}{l}\text { USAG } \\
\text { Kaiserslautern } \\
\text { Sustainability } \\
\text { Challenges } \\
\text { Workshop } \\
\text { Summary- } \\
\text { Command Briefing }\end{array}$ & Barber, David, 6 Mar. 2009, slides 6-23. \\
\hline $\begin{array}{l}\text { Fort Drum Goal- } \\
\text { setting Workshop } \\
\text { Facilitator } \\
\text { Playbook, Internal } \\
\text { Planning } \\
\text { Document }\end{array}$ & LaDuc, Michael, 6 Jan. 2009, pp. 33-37. \\
\hline $\begin{array}{l}\text { Fort Benning } \\
2008 \\
\text { Sustainability } \\
\text { Report }\end{array}$ & $\begin{array}{l}\text { Lukken, Peter J., and Col. Thomas J. MacDonald, } 30 \text { Sep. 2008, pp. 9, 15, 21-22, } \\
\text { issuu.com/s3ops/docs/fb_2008_sustainability_performance_1c97844ec14288. }\end{array}$ \\
\hline $\begin{array}{l}\text { Fort Riley } \\
\text { Sustainability } \\
\text { Challenges }\end{array}$ & Childs, Stanley, 24 Sept. 2008, slides 3-43. \\
\hline $\begin{array}{l}\text { Fort Huachuca } \\
\text { U.S. Army } \\
\text { Installation } \\
\text { Sustainability } \\
\text { Workshop } \\
\text { Summary Briefing }\end{array}$ & Sturgeon, Col. Melissa, 26-28 Aug. 2008, slides 10-13. \\
\hline $\begin{array}{l}\text { Transportation } \\
\text { Team Significant } \\
\text { Challenges to } \\
\text { Installation } \\
\text { Sustainability }\end{array}$ & Childs, Stanley, 4 Oct. 2007, slides 2-16. \\
\hline
\end{tabular}




\begin{tabular}{|c|c|}
\hline Resource & Author/Reference Site \\
\hline $\begin{array}{l}\text { Fort Campbell } \\
\text { Strategic Plan } \\
\text { 2008-2013, } \\
\text { Garrison } \\
\text { Commander's } \\
\text { Strategic Plan }\end{array}$ & Carr, Trudy, 30 Sept. 2007, pp. 5, 8, 19, A2-A9. \\
\hline $\begin{array}{l}\text { USAG } \\
\text { Wiesbaden- } \\
\text { Sustainability } \\
\text { Goal-Setting } \\
\text { Workshop After- } \\
\text { Action Report }\end{array}$ & Barber, David, Commander Briefing on Logistics Line of Effort, 31 Aug. 2007, slides 2-7. \\
\hline $\begin{array}{l}\text { USAG Hawaii- } \\
\text { Strategic } \\
\text { Sustainability } \\
\text { Goals and } \\
\text { Objectives } \\
\text { Development } \\
\text { Session }\end{array}$ & Zuckerman, David, Transportation Team, 8 June 2007, slides 2-10. \\
\hline $\begin{array}{l}\text { Fort Jackson } \\
\text { Sustainability } \\
\text { Planning } \\
\text { Baselining } \\
\text { Workshop } \\
\text { Outcomes- } \\
\text { Command Briefing }\end{array}$ & Odom, Dr. Lynn, 14 Oct. 2005, pp. 21-34. \\
\hline $\begin{array}{l}\text { Sustaining Fort } \\
\text { Bragg through } \\
\text { Transformation: } \\
\text { Phase 1, Fort } \\
\text { Bragg } \\
\text { Environmental } \\
\text { Baseline, Internal } \\
\text { Garrison Planning } \\
\text { Documentation }\end{array}$ & Messenger, Mannette, 12 Sept. 2003, pp. 5-63. \\
\hline
\end{tabular}




\section{Appendix B: Manufacturer Websites}



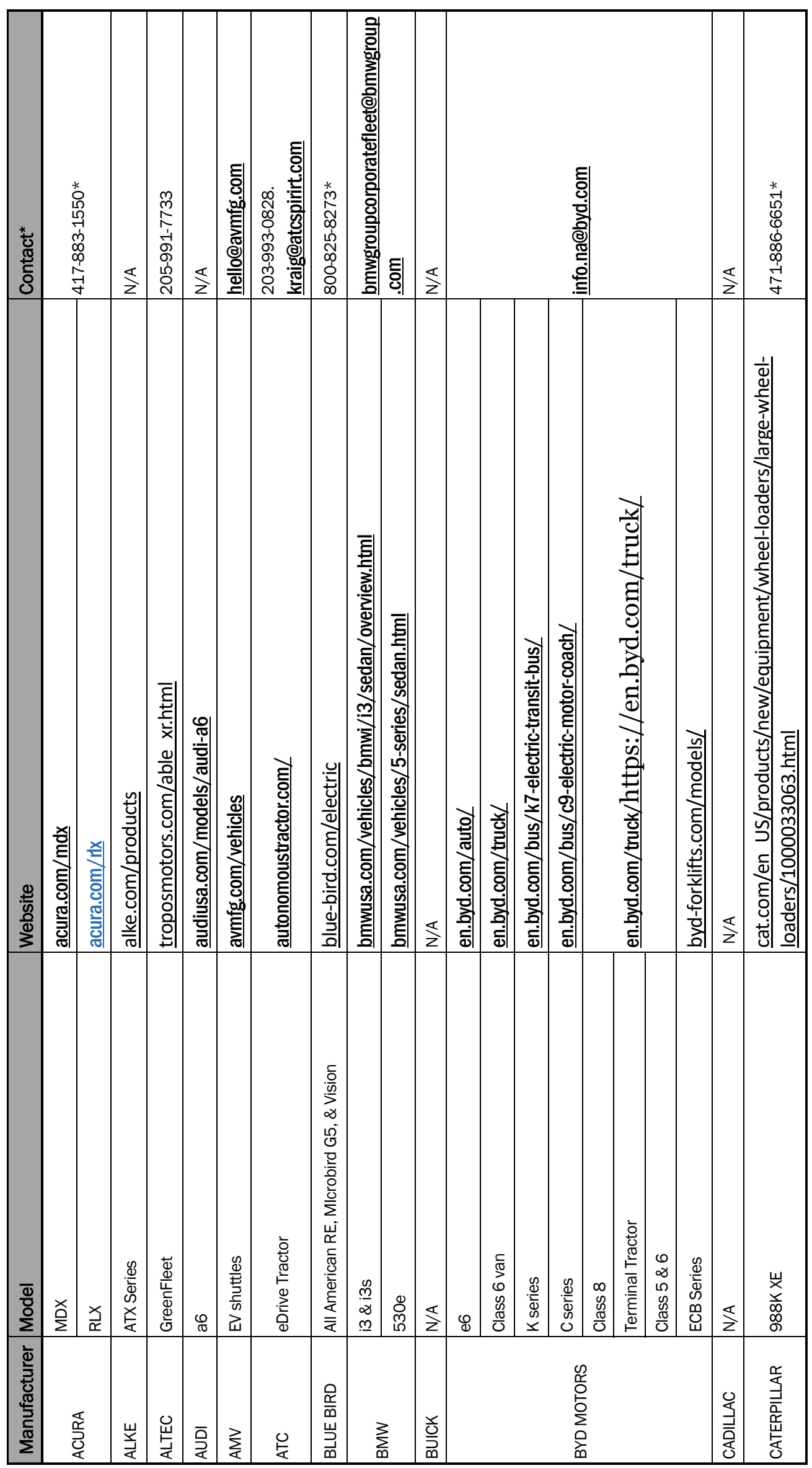

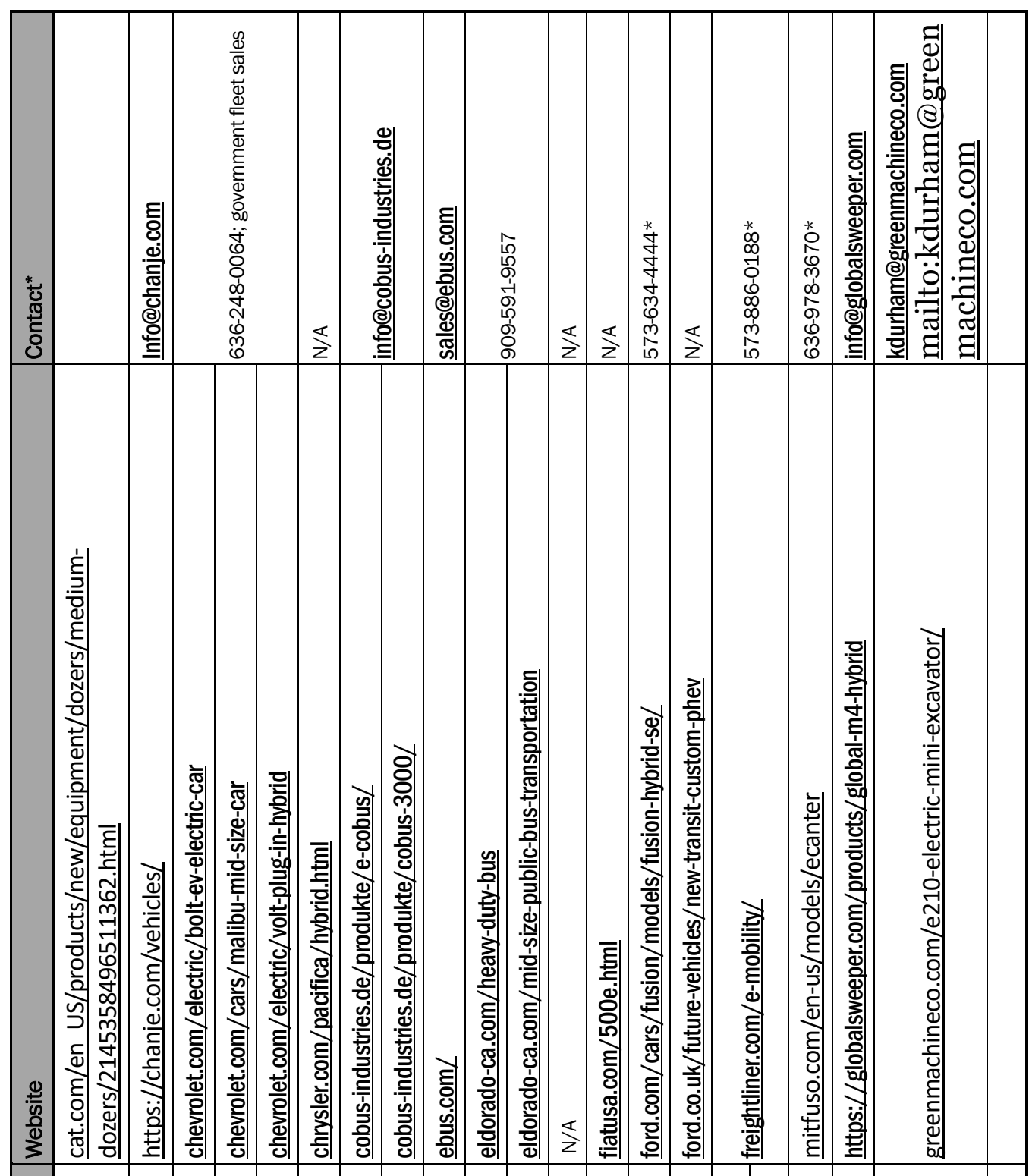

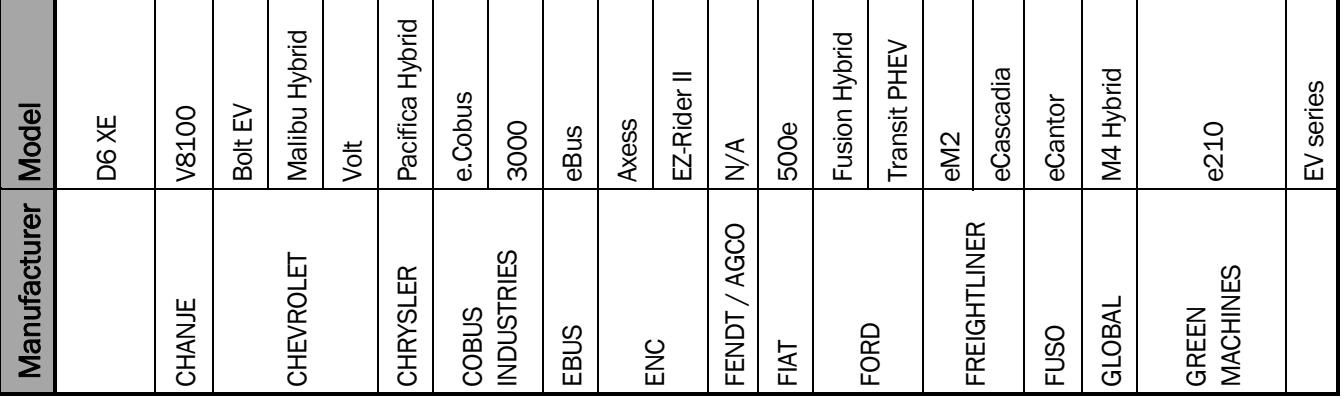




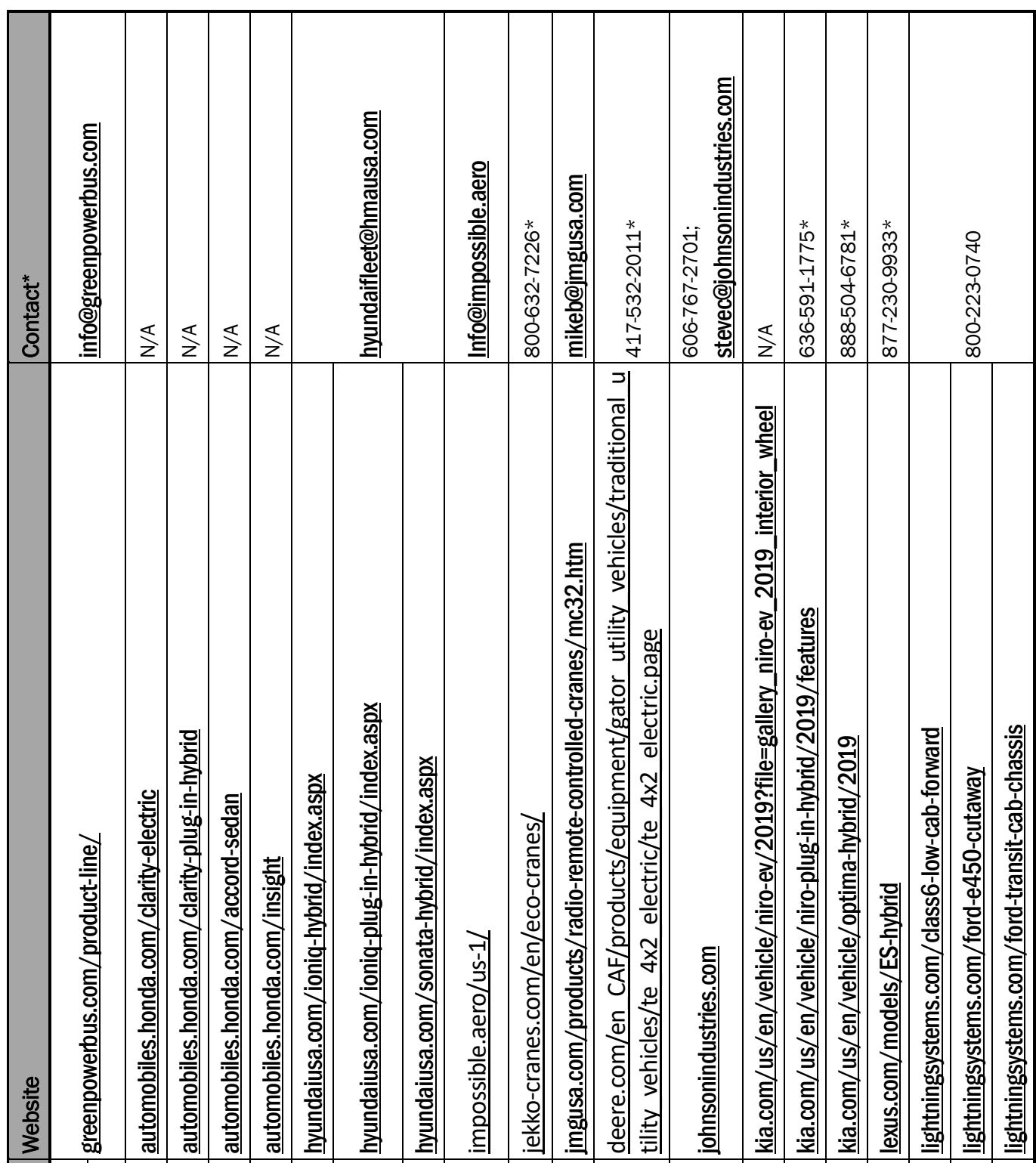

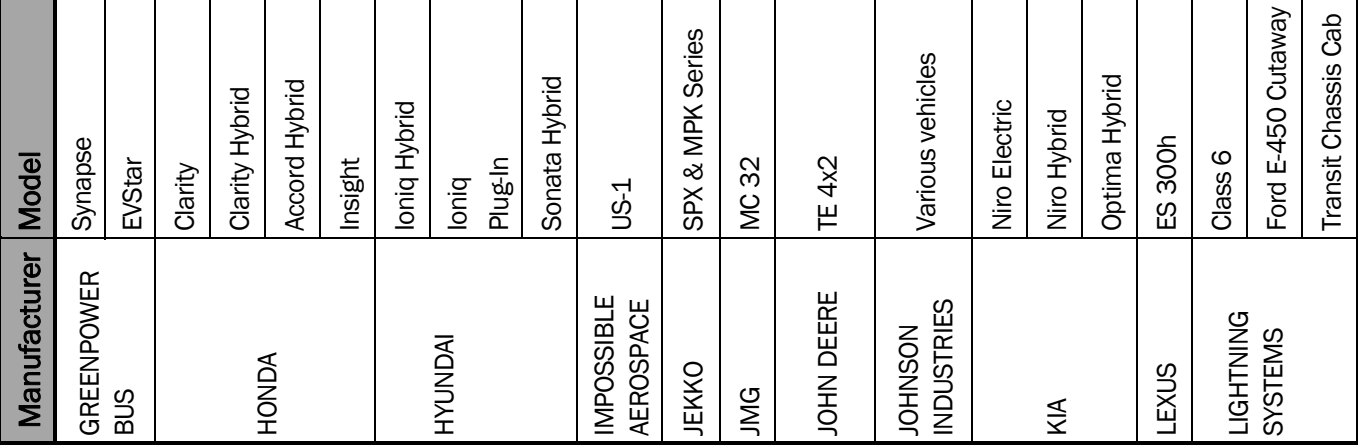



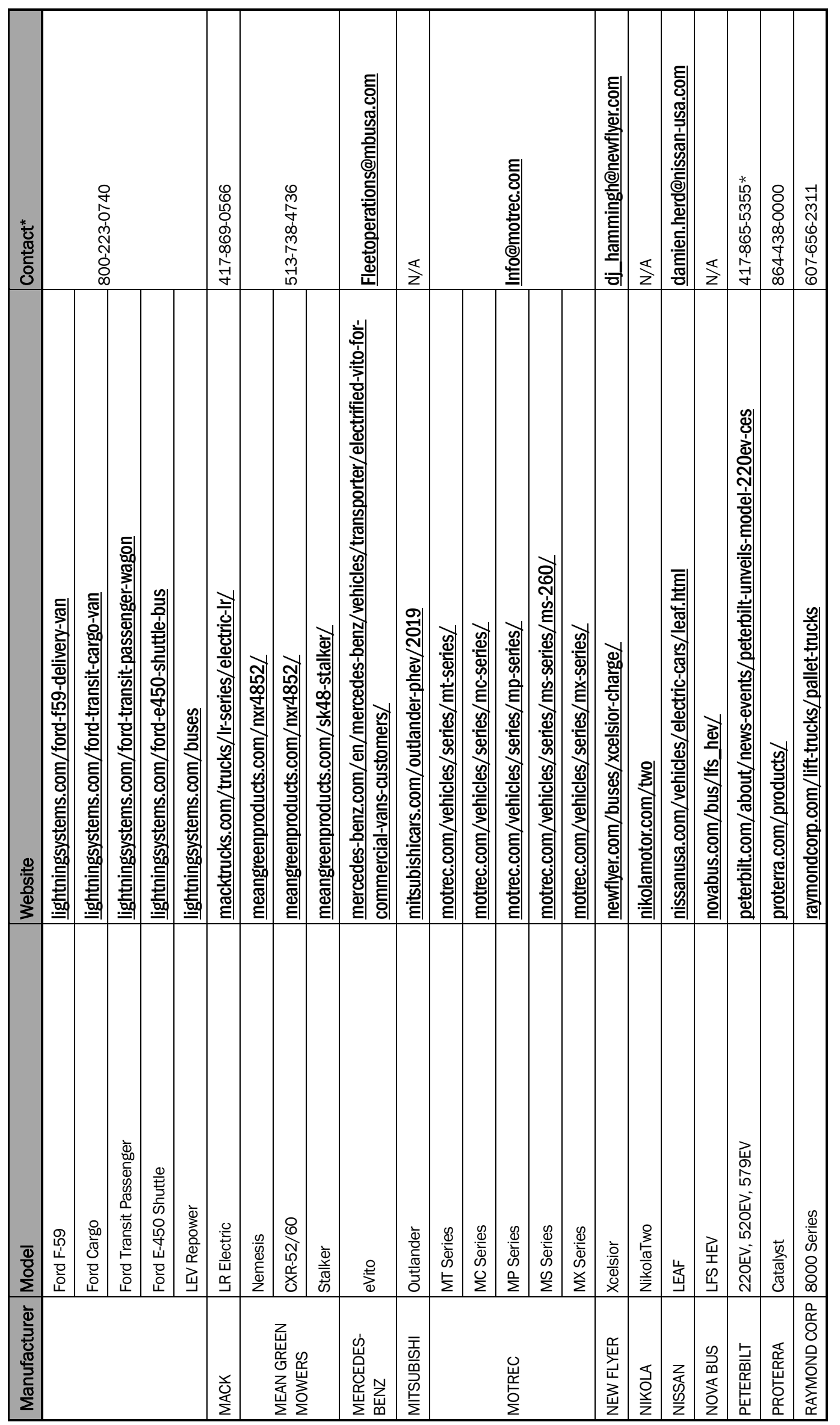

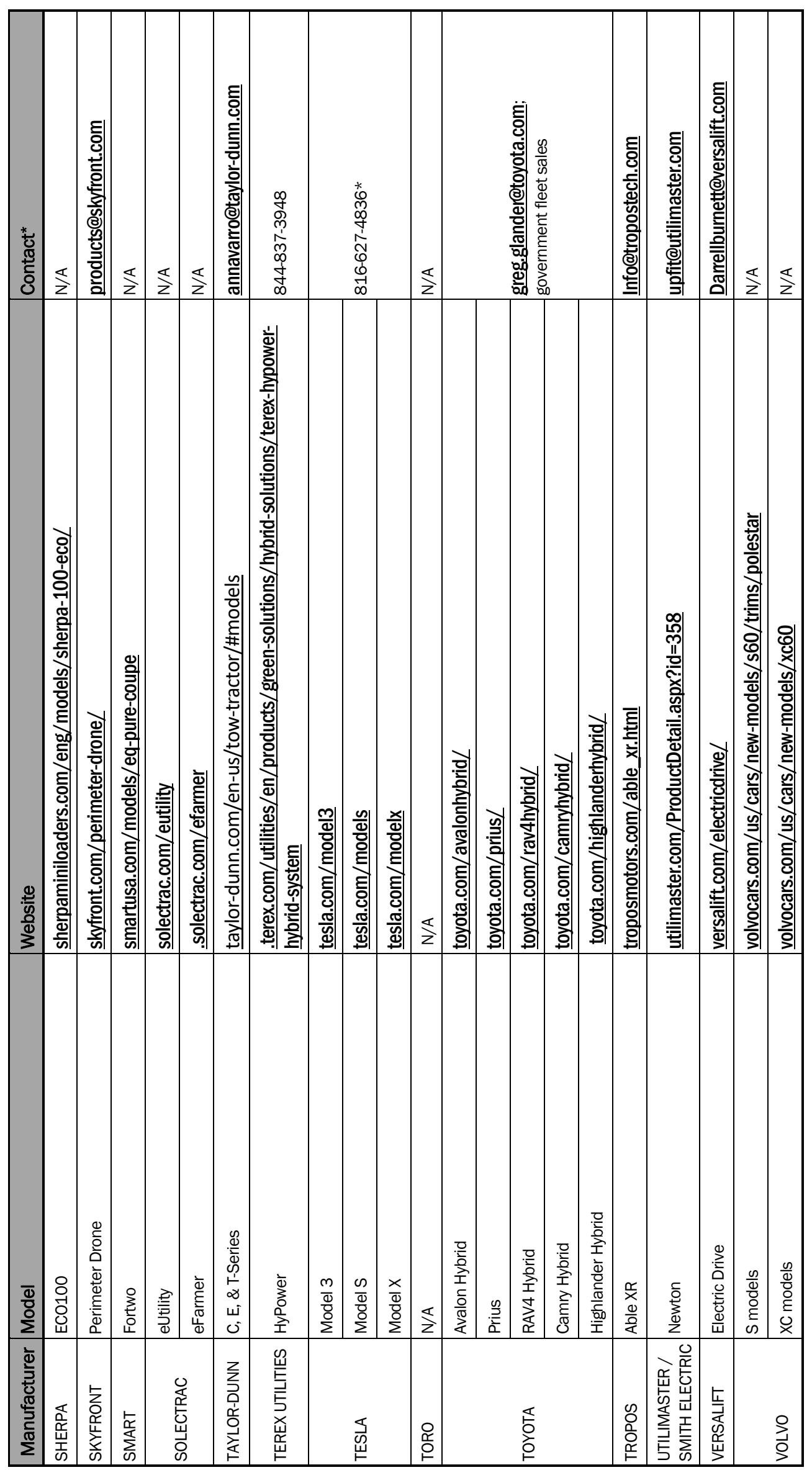

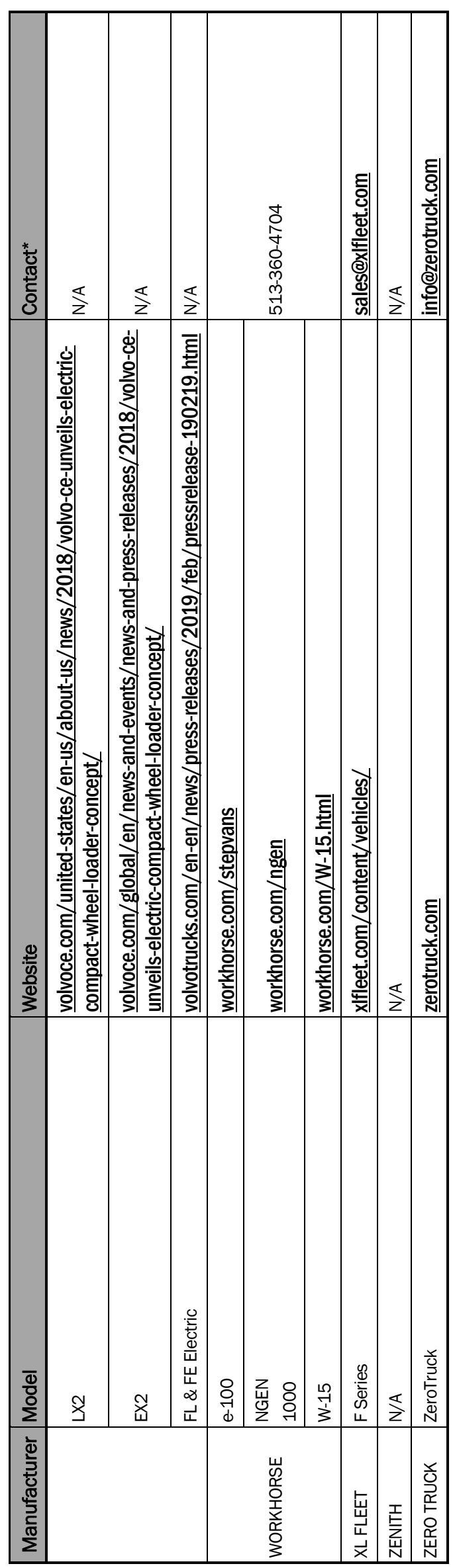


\section{Appendix C: Electric Transport System Information Resources}

\begin{tabular}{|c|c|}
\hline Resource & Author / Reference Site \\
\hline $\begin{array}{l}\text { Lewis-McChord Feasibility Study for } \\
\text { the Application of Automated } \\
\text { Vehicles; JBLM Feasibility Study for } \\
\text { the Application of Automated } \\
\text { Vehicles }\end{array}$ & Barghout, Jeff, et al. Joint Base, pp. 1-39, Joint Base Lewis-McCord. \\
\hline $\begin{array}{l}\text { JBLM Autonomous Vehicle Scoping } \\
\text { Session Summary, Internal Meeting } \\
\text { Summary with Installation } \\
\text { Stakeholders to Identify MSAs That } \\
\text { Could Be Performed Autonomously }\end{array}$ & Steucke, Paul, 31 Oct. 2014, pp. 1-4. \\
\hline ARIBO: Fort Leonard Wood & $\begin{array}{l}\text { Robotic Research, roboticresearch.com/programs/aribo-fort-leonard- } \\
\text { wood/. }\end{array}$ \\
\hline $\begin{array}{l}\text { Land Ownership: Overview and } \\
\text { Data, Congressional Research } \\
\text { Service }\end{array}$ & $\begin{array}{l}\text { Vincent, Carol Hardy, and Laurel Hanson, } 3 \text { Mar. 2017, p. 6, } \\
\text { fas.org/sgp/crs/misc/R42346.pdf. }\end{array}$ \\
\hline $\begin{array}{l}\text { Autonomous Transportation: } \\
\text { Combat Power in the 21 } 1^{\text {st }} \text { Century }\end{array}$ & $\begin{array}{l}\text { Henry, Capt. Matthew P. U.S. Army, } 1 \text { Nov. } 2018 \text {, } \\
\text { army.mil/article/213078/autonomous transportation_combat_power_in_t } \\
\text { he } 21^{\text {st }} \text { century. }\end{array}$ \\
\hline Electric Cars & Green Car Reports, greencarreports.com/news/electric-cars. \\
\hline Electric Vehicles & WIRED, Conde Nast, wired.com/tag/electric-vehicles. \\
\hline Electric Vehicle Guide & The Sierra Club, content.sierraclub.org/evguide/. \\
\hline Clean Vehicles & Union of Concerned Scientists, ucsusa.org/clean-vehicles/electric-vehicles. \\
\hline Clean Technica & Sustainable Enterprises Media, cleantechnica.com/. \\
\hline Charged EVs & Isentropic Media, chargedevs.com/. \\
\hline Electric Car Insider & electric-car-insider.com/. \\
\hline $\begin{array}{l}\text { Electric \& Hybrid: Vehicle } \\
\text { Technology International }\end{array}$ & Mark Allen Group, electrichybridvehicletechnology.com/. \\
\hline Inside EV & insideevs.com/?p=3. \\
\hline Clean Fleet Report & cleanfleetreport.com/. \\
\hline Clean Car Congress & BioAge Group, greencarcongress.com/. \\
\hline Electric Vehicle Charging News & Fleetcarma, fleetcarma.com/blog/. \\
\hline Autovolt Magazine & Autovolt Limited, autovolt-magazine.com/. \\
\hline Engineering and Technology & $\begin{array}{l}\text { The Institution of Engineering and Technology, eandt.theiet.org/tags/electric- } \\
\text { vehicles. }\end{array}$ \\
\hline Electric and Hybrid Vehicles & The New York Times, nytimes.com/topic/subject/electric-and-hybrid-vehicles. \\
\hline $\begin{array}{l}\text { TC 69: Electric Road Vehicles and } \\
\text { Electric Industrial Trucks }\end{array}$ & $\begin{array}{l}\text { International Electrotechnical Commission, } \\
\text { iec.ch/dyn/www/f?p=103:22:0:..:FSP_ORG_ID:1255. }\end{array}$ \\
\hline Electric/Hybrid & $\begin{array}{l}\text { Renewable Energy magazine, } \\
\text { renewableenergymagazine.com/electric_hybrid_vehicles. }\end{array}$ \\
\hline
\end{tabular}




\begin{tabular}{|c|c|}
\hline Resource & Author / Reference Site \\
\hline $\begin{array}{l}\text { Researchers Use Blockchain to } \\
\text { Drive Electric Vehicle Charging } \\
\text { Infrastructure }\end{array}$ & $\begin{array}{l}\text { University of Waterloo, Science News, } 14 \text { Aug 2019, } \\
\text { sciencedaily.com/releases/2019/08/190814144501.htm. }\end{array}$ \\
\hline SMERC Publications & UCLA Smart Grid Energy Research Center, smartgrid.ucla.edu/publications.htm. \\
\hline $\begin{array}{l}\text { Real-Time Bidirectional Electric } \\
\text { Vehicle Charging Control with } \\
\text { Distribution Grid Implementation }\end{array}$ & Xiong, Yingqi, et al. smartgrid.ucla.edu/publications.htm. \\
\hline $\begin{array}{l}\text { Hierarchical Distributed EV Charging } \\
\text { Scheduling in Distribution Grids }\end{array}$ & Khaki, Behnam, et al. smartgrid.ucla.edu/publications.htm. \\
\hline IEEE Electrification magazine & $\begin{array}{l}\text { IEEE Power and Energy Society, ieee-pes.org/publications/electrification- } \\
\text { magazine. }\end{array}$ \\
\hline Classic Driver magazine & $\begin{array}{l}\text { Classic Driver, classicdriver.com/en/magazine/tag/electric- } \\
\text { vehicles?page=1\&search_type=simple\&tag=8004. }\end{array}$ \\
\hline $\begin{array}{l}\text { Center for Automotive Research } \\
\text { Publications }\end{array}$ & Car Group, cargroup.org/publications/. \\
\hline Center for Automotive Research & cargroup.org/?s=electric\%20vehicle. \\
\hline Alt Energy magazine & altenergymag.com/tag/electric-vehicles. \\
\hline Green Fleet magazine & greenfleetmagazine.com/tags?tag=Battery-Electric\%20Vehicles. \\
\hline Government Fleet magazine & Green Fleet magazine, government-fleet.com/green-fleet. \\
\hline Energy Digital & BizClick Media, energydigital.com/electric-vehicles. \\
\hline Electrek & electrek.co/. \\
\hline Greentech Media & greentechmedia.com/articles/tag/electric-vehicles. \\
\hline EcoWatch & ecowatch.com/tag/electric-vehicle. \\
\hline EV News & The Driven, thedriven.io/category/ev-news/. \\
\hline Electric Vehicles & Phys.org, phys.org/tags/electric\%20vehicles/. \\
\hline $\begin{array}{l}\text { Electric and Hybrid: Vehicle } \\
\text { Technology International }\end{array}$ & Mark Allen Group Limited, electrichybridvehicletechnology.com/. \\
\hline Electric Vehicles & $\begin{array}{l}\text { American Power Association, publicpower.org/topic/electric-vehicles/public- } \\
\text { power-magazine. }\end{array}$ \\
\hline World Electric Vehicle Journal & American Public Power Association, $\underline{\text { mdpi.com/journal/wevj. }}$ \\
\hline $\begin{array}{l}\text { International Journal of Hydrogen } \\
\text { Energy }\end{array}$ & $\begin{array}{l}\text { International Association for Hydrogen Energy, } \\
\text { journals.elsevier.com/international-journal-of-hydrogen-energy. }\end{array}$ \\
\hline eTransportation & journals.elsevier.com/etransportation. \\
\hline Journal of Power Sources & $\begin{array}{l}\text { International Journal on the Science and Technology of Electrochemical Energy } \\
\text { Systems, journals.elsevier.com/journal-of-power-sources. }\end{array}$ \\
\hline $\begin{array}{l}\text { International Journal of Electrical } \\
\text { Power and Energy Systems }\end{array}$ & $\begin{array}{l}\text { journals.elsevier.com/international-journal-of-electrical-power-and-energy- } \\
\text { systems. }\end{array}$ \\
\hline $\begin{array}{l}\text { Research in Transportation } \\
\text { Economics }\end{array}$ & $\begin{array}{l}\text { ScienceDirect, sciencedirect.com/journal/research-in-transportation- } \\
\text { economics. }\end{array}$ \\
\hline Journal of Cleaner Production & ScienceDirect, sciencedirect.com/journal/journal-of-cleaner-production. \\
\hline $\begin{array}{l}\text { Synergies of Four Emerging } \\
\text { Technologies for Accelerated } \\
\text { Adoption of Electric Vehicles: } \\
\text { Shared Mobility, Wireless Charging, } \\
\text { Vehicle-to-Grid, and Vehicle } \\
\text { Automation }\end{array}$ & $\begin{array}{l}\text { Taiebat, Morteza, and Ming Xu. Journal of Cleaner Production, vol. 230, } 1 \text { Sept. } \\
\text { 2019, pp. 794-797., } \\
\text { sciencedirect.com/science/article/pii/S0959652619316683. }\end{array}$ \\
\hline Transport Policy & ScienceDirect, sciencedirect.com/journal/transport-policy. \\
\hline
\end{tabular}




\begin{tabular}{|c|c|}
\hline Resource & Author / Reference Site \\
\hline $\begin{array}{l}\text { Consumer Preferences for Public } \\
\text { Charging Infrastructure for Electric } \\
\text { Vehicles }\end{array}$ & $\begin{array}{l}\text { Globisch, Joachim, et al. Transport Policy, vol. 81, Sept. 2019, pp. 54-63., } \\
\text { sciencedirect.com/science/article/abs/pii/S0967070X1830742X. }\end{array}$ \\
\hline $\begin{array}{l}\text { International Journal of Electric and } \\
\text { Hybrid Vehicles }\end{array}$ & inderscience.com/jhome.php?jcode=ijehv. \\
\hline Peer-Reviewed Journal Publications & $\begin{array}{l}\text { Carnegie Mellon Vehicle Electrification Group, Carnegie Mellon University, } \\
\text { cmu.edu/cit/veg/publications.html. }\end{array}$ \\
\hline $\begin{array}{l}\text { Carnegie Mellon Vehicle } \\
\text { Electrification Group }\end{array}$ & Carnegie Mellon University, cmu.edu/cit/veg/index.html. \\
\hline electric://forum Blog & Electric Car Blog, electricforum.com/blog/. \\
\hline electric://forum Forum & Electric Car Blog, community.electricforum.com/. \\
\hline Speak EV & VerticalScope, speakev.com. \\
\hline Electric Vehicles & International Council on Clean Transportation, theicct.org/electric-vehicles. \\
\hline Plug-In America & Electric Auto Association, pluginamerica.org. \\
\hline Maryland EV & marylandev.org/. \\
\hline Drive Electric Northern Colorado & driveelectricnoco.org/. \\
\hline Electrification Coalition & electrificationcoalition.org. \\
\hline Midwest Evolve & midwestevolve.org/: \\
\hline Electric Vehicles Initiative & International Energy Agency, iea.org/topics/transport/evi/. \\
\hline World Electric Vehicle Association & Electric Drive Transportation Association, worldelectricvehicleassociation.info/. \\
\hline $\begin{array}{l}\text { Fuel Cell and Hydrogen Energy } \\
\text { Association }\end{array}$ & http://www.fchea.org/transportation. \\
\hline Electric Auto Association & eaa-1967.clubexpress.com/. \\
\hline $\begin{array}{l}\text { Electric Drive Transportation } \\
\text { Association }\end{array}$ & electricdrive.org/. \\
\hline $\begin{array}{l}\text { Mid-America Electric Auto } \\
\text { Association }\end{array}$ & maeaa.org. \\
\hline $\begin{array}{l}\text { Electric Vehicle Charging } \\
\text { Association }\end{array}$ & evassociation.org/. \\
\hline Hybrid and Plug-In Electric Vehicles & $\begin{array}{l}\text { Alternative Fuels Data Center, DOE's Vehicle Technologies Office, } \\
\text { afdc.energy.gov/vehicles/electric.html. }\end{array}$ \\
\hline All-Electric Vehicles & DOE, fueleconomy.gov/feg/evtech.shtml. \\
\hline $\begin{array}{l}\text { Estimating the infrastructure needs } \\
\text { and costs for the launch of zero- } \\
\text { emission trucks }\end{array}$ & $\begin{array}{l}\text { Hall, Dale, and Nic Lutsey, The International Council on Clean Transportation, } \\
\text { https://theicct.org/publications/zero-emission-truck-infrastructure. }\end{array}$ \\
\hline $\begin{array}{l}\text { Estimating electric vehicle charging } \\
\text { infrastructure costs across major } \\
\text { U.S. metropolitan areas }\end{array}$ & $\begin{array}{l}\text { Nicholas, Michael, The International Council on Clean Energy, } \\
\text { https://theicct.org/publications/charging-cost-US. }\end{array}$ \\
\hline $\begin{array}{l}\text { Early steps in the transition to } \\
\text { electric vehicles in the Midwest }\end{array}$ & $\begin{array}{l}\text { Nicholas, Mike, The International Council on Clean Energy, } \\
\text { https://theicct.org/publications/ev-transition-US-Midwest-201907. }\end{array}$ \\
\hline $\begin{array}{l}\text { Update on electric vehicle costs in } \\
\text { the United States through } 2030\end{array}$ & $\begin{array}{l}\text { Nicholas, Mike, The International Council on Clean Energy, } \\
\text { theicct.org/publications/update-US-2030-electric-vehicle-cost. }\end{array}$ \\
\hline $\begin{array}{l}\text { Quantifying the Electric Vehicle } \\
\text { Charging Infrastructure Gap across } \\
\text { U.S. Markets }\end{array}$ & $\begin{array}{l}\text { Nicholas, Mike, The International Council on Clean Energy, } \\
\text { theicct.org/publications/charging-gap-US. }\end{array}$ \\
\hline $\begin{array}{l}\text { Lessons Learned on Early Electric } \\
\text { Vehicle Fast-Charging Deployments }\end{array}$ & $\begin{array}{l}\text { Nicholas, Michael, and Dale Hall, The International Council on Clean Transportation, } \\
1 \text { Aug. 2018, theicct.org/publications/fast-charging-lessons-learned. }\end{array}$ \\
\hline
\end{tabular}




\begin{tabular}{|c|c|}
\hline Resource & Author / Reference Site \\
\hline Global EV Outlook 2019 & IEA, iea.org/publications/reports/globalevoutlook2019/. \\
\hline $\begin{array}{l}\text { Electric and Plug-In Hybrid-Electric } \\
\text { Vehicle Publications }\end{array}$ & $\begin{array}{l}\text { National Renewable Energy Laboratory, DOE, nrel.gov/transportation/fleettest- } \\
\text { publications-electric.html. }\end{array}$ \\
\hline Crash statistics & NHTSA, DOT, crashstats.nhtsa.dot.gov/\#/PublicationList/79. \\
\hline $\begin{array}{l}\text { Electric Vehicles for All: An Equity } \\
\text { Toolkit }\end{array}$ & $\begin{array}{l}\text { The Greenlining Institute, greenlining.org/publications-resources/electric- } \\
\text { vehicles-for-all/. }\end{array}$ \\
\hline DOE Vehicle Technologies Office & energy.gov/eere/vehicles/vehicle-technologies-office. \\
\hline DOE Grid Modernization Initiative & energy.gov/grid-modernization-initiative. \\
\hline Technology-to-Market & $\begin{array}{l}\text { Office of Energy Efficiency and Renewable Energy, DOE, } \\
\text { energy.gov/eere/technology-to-market/home. }\end{array}$ \\
\hline Reports and publications & $\begin{array}{l}\text { Office of Energy Efficiency and Renewable Energy: Vehicle Technologies Office, DOE, } \\
\text { energy.gov/eere/vehicles/reports-and-publications. }\end{array}$ \\
\hline $\begin{array}{l}\text { Advanced Vehicle Testing Activity } \\
\text { (AVTA) Data and Results }\end{array}$ & $\begin{array}{l}\text { Office of Energy Efficiency and Renewable Energy: Vehicle Technologies Office, DOE, } \\
\text { energy.gov/eere/vehicles/advanced-vehicle-testing-activity-avta-data-and- } \\
\text { results. }\end{array}$ \\
\hline $\begin{array}{l}\text { DARPA Electric and Hybrid-Electric } \\
\text { Vehicle Program }\end{array}$ & CTE, cte.tv/project/darpa-electric-and-hybrid-electric-vehicle-program/. \\
\hline $\begin{array}{l}\text { State Efforts to Promote Hybrid and } \\
\text { Electric Vehicles }\end{array}$ & $\begin{array}{l}\text { Hartman, Kristy, and Emily Dowd, National Conference of State Legislatures, } 26 \text { Sept } \\
\text { 2017, ncsl.org/research/energy/state-electric-vehicle-incentives-state- } \\
\text { chart.aspx. }\end{array}$ \\
\hline $\begin{array}{l}\text { Procurement Preference for Electric } \\
\text { and Hybrid-Electric Vehicles }\end{array}$ & Alternative Fuels Data Center, DOE, afdc.energy.gov/laws/431. \\
\hline $\begin{array}{l}\text { All Laws and Incentives Sorted by } \\
\text { Type }\end{array}$ & Alternative Fuels Data Center, DOE, afdc.energy.gov/laws/matrix. \\
\hline DoD Projects & $\begin{array}{l}\text { Advanced Vehicles, Idaho National Laboratory, avt.inl.gov/project-type/united- } \\
\text { states-department-defense. }\end{array}$ \\
\hline $\begin{array}{l}\text { National Transportation Research } \\
\text { Center }\end{array}$ & Oak Ridge National Laboratory, UT-Battelle, ornl.gov/facility/ntrc. \\
\hline
\end{tabular}




\section{Appendix D: Preliminary List of Existing Electric Transportation Systems}

\begin{tabular}{|c|c|c|c|c|c|c|c|}
\hline $\begin{array}{c}\text { DOT } \\
\text { Classification }\end{array}$ & Name & $\begin{array}{c}\text { MSA } \\
\text { No. }\end{array}$ & Manufacturer & Origin & TRL & Charging & GSA \\
\hline \multicolumn{8}{|l|}{ Trucks \& Vans } \\
\hline 3 & Newton & 3 & $\begin{array}{l}\text { Utilimaster/ } \\
\text { Smith Electric }\end{array}$ & $\begin{array}{l}\text { United States } \\
\text { (assembled) }\end{array}$ & 9 & L1, L2 & \\
\hline 3 & e-100 & 3 & Workhorse & $\begin{array}{l}\text { United States } \\
\text { (assembled) }\end{array}$ & $\begin{array}{l}\text { 6; in } \\
\text { production }\end{array}$ & L1, L2, DC Fast & \\
\hline 3 & NGEN 1000 van & 3 & Workhorse & $\begin{array}{l}\text { United States } \\
\text { (assembled) }\end{array}$ & $\begin{array}{l}\text { 6; in } \\
\text { production }\end{array}$ & L1, L2, DC Fast & \\
\hline 3 & E100 van & 3 & Workhorse & $\begin{array}{l}\text { United States } \\
\text { (assembled) }\end{array}$ & $\begin{array}{l}\text { 6; in } \\
\text { production }\end{array}$ & L1, L2 & \\
\hline 3 & W-15 truck & 1 & Workhorse & $\begin{array}{l}\text { United States } \\
\text { (assembled) }\end{array}$ & 7; pre-order & L1, L2, DC Fast & \\
\hline 3 & Class 6 step van & 3 & BYD & China, United States & 9 & L2, DC Fast & \\
\hline 3 & $\begin{array}{l}\text { Super Duty F } \\
\text { Series }\end{array}$ & 1 & XL Fleet/Ford & $\begin{array}{l}\text { United States } \\
\text { (assembled) }\end{array}$ & 9 & & \\
\hline 3 & eVito & 3 & Mercedes-Benz & & 9 & & \\
\hline \multicolumn{8}{|c|}{ Shuttles, Transit Buses, \& Vans } \\
\hline 4 & $\begin{array}{l}\text { EV22, EV27, EV3 } \\
\text { shuttle buses }\end{array}$ & 1 & AVM & United States & 9 & $\begin{array}{l}\text { DC Fast custom } \\
\text { system }\end{array}$ & \\
\hline 4 & e.Cobus shuttle & 1 & $\begin{array}{l}\text { COBUS } \\
\text { Industries }\end{array}$ & Germany & 9 & L2, DC Fast & \\
\hline 4 & 3000 shuttle & 1 & $\begin{array}{l}\text { COBUS } \\
\text { Industries }\end{array}$ & Germany & 9 & L2, DC Fast & \\
\hline 4 & $\begin{array}{l}\text { K7, K95, K9, K11 } \\
\text { shuttles }\end{array}$ & 1 & BYD & China, United States & 9 & $\begin{array}{l}\text { L2, DC Fast, } \\
\text { custom systems }\end{array}$ & \\
\hline 4 & $\begin{array}{l}\text { C6, C9 \& C10 } \\
\text { buses }\end{array}$ & 1 & BYD & China, United States & 9 & $\begin{array}{l}\text { L2, DC Fast, } \\
\text { custom systems }\end{array}$ & \\
\hline 4 & eBus & 1 & eBus & $\begin{array}{l}\text { United States } \\
\text { (assembled) }\end{array}$ & 9 & $\begin{array}{l}\text { In-ground } \\
450 \mathrm{~kW} 4 \mathrm{C}\end{array}$ & \\
\hline 4 & Transit EV & 3 & Ford & & $\begin{array}{l}5 ; \text { out in } \\
2021\end{array}$ & & \\
\hline 4 & Transit PHEV & 3 & Ford & $\begin{array}{l}\text { United States } \\
\text { (assembled) }\end{array}$ & $\begin{array}{l}\text { 8; delivered } \\
\text { in 2019, UK }\end{array}$ & & \\
\hline 4 & $\begin{array}{l}\text { EV250, EV300, } \\
\text { EV350, EV400 \& } \\
\text { EV550 shuttle } \\
\text { buses }\end{array}$ & 1 & GreenPower Bus & United States & 9 & L2, DC Fast & \\
\hline 4 & Xcelsior & 1 & New Flyer & United States & 9 & \begin{tabular}{|l} 
On-route, DC \\
Fast \\
\end{tabular} & \\
\hline 4 & Catalyst & 1 & Proterra & United States & 9 & \begin{tabular}{|l|} 
DC Fast, \\
overhead \& \\
custom systems
\end{tabular} & \\
\hline \multirow[t]{2}{*}{4} & $\begin{array}{l}\text { All American RE, } \\
\text { Microbird G5 }\end{array}$ & 1 & Blue Bird & United States & 9 & DC Fast & \\
\hline & LEV Repower & 1 & $\begin{array}{l}\text { Lightning } \\
\text { Systems }\end{array}$ & United States & 9 & L2, DC Fast & \\
\hline
\end{tabular}




\begin{tabular}{|c|c|c|c|c|c|c|c|}
\hline $\begin{array}{c}\text { DOT } \\
\text { Classification }\end{array}$ & Name & $\begin{array}{l}\text { MSA } \\
\text { No. }\end{array}$ & Manufacturer & Origin & TRL & Charging & GSA \\
\hline 3 & Ford E-450 shuttle & 1 & $\begin{array}{l}\text { Lightning } \\
\text { Systems }\end{array}$ & United States & 9 & L2, DC Fast & \\
\hline 3 & $\begin{array}{l}\text { Ford Transit } \\
\text { Passenger }\end{array}$ & 1 & $\begin{array}{l}\text { Lightning } \\
\text { Systems }\end{array}$ & United States & 9 & L2, DC Fast & \\
\hline 4 & Synapse bus & 1 & GreenPower & United States & 9 & L2, DC Fast & \\
\hline \multirow[t]{3}{*}{3} & EVStar shuttle & 1 & GreenPower & United States & & & \\
\hline & Axess & 1 & ENC & & 9 & DC Fast & \\
\hline & EZ-Rider II & 1 & ENC & & 9 & DC Fast & \\
\hline 4 & LFS HEV & 1 & Nova Bus & Canada & 9 & DC Fast & \\
\hline \multicolumn{8}{|c|}{ Tractors, Mowers, Street Sweepers, \& Refuse } \\
\hline 15 & eDrive tractors & 2 & ATC & United States & 9 & Hybrid-electric & Y \\
\hline 15 & Class $6 \& 8$ refuse & 3 & BYD & United States & 9 & L1, L2, DC Fast & \\
\hline 15 & SESAM tractor & 2 & John Deere & & 7 & & \\
\hline 15 & eUtility tractor & 2 & Solectrac & United States & $\begin{array}{l}\text { 8; available } \\
2020\end{array}$ & L1, L2 & \\
\hline 15 & eFarmer tractor & 2 & Solectrac & United States & $\begin{array}{l}\text { 8; available } \\
2020\end{array}$ & L1, L2 & \\
\hline 15 & Nemesis mower & 2 & $\begin{array}{l}\text { Mean Green } \\
\text { Mowers }\end{array}$ & United States & 9 & $\begin{array}{l}110 \mathrm{~V} / 22 \mathrm{VV} \\
\text { charger }\end{array}$ & \\
\hline 15 & CXR-52/60 mower & 2 & $\begin{array}{l}\text { Mean Green } \\
\text { Mowers }\end{array}$ & United States & 9 & $\begin{array}{l}110 \mathrm{~V} / 220 \mathrm{~V} \\
\text { charger }\end{array}$ & \\
\hline 15 & Stalker mower & 2 & $\begin{array}{l}\text { Mean Green } \\
\text { Mowers }\end{array}$ & United States & 9 & $\begin{array}{l}110 \mathrm{~V} / 220 \mathrm{~V} \\
\text { charger }\end{array}$ & \\
\hline 5 & $\begin{array}{l}\text { FL Electric \& FE } \\
\text { Electric refuse }\end{array}$ & 3 & Volvo & \begin{tabular}{|l|} 
Belgium, Brazil, \\
China, France, \\
Germany, India, \\
Korea, Sweden, U.K., \\
and United States \\
\end{tabular} & $\begin{array}{l}\text { Delivering in } \\
\text { Europe }\end{array}$ & & \\
\hline 6 & LR Electric refuse & 3 & Mack & United States & $\begin{array}{l}\text { Real-world } \\
\text { testing in } \\
2020\end{array}$ & & \\
\hline \multicolumn{8}{|c|}{ Vocational, Medium-, \& Heavy-duty Trucks } \\
\hline 6 & Class 8 Day cab & 3 & BYD & United States & 9 & L1, L2, DC Fast & \\
\hline 5 & Terminal tractor & 3 & BYD & United States & 9 & L1, L2, DC Fast & \\
\hline 5 & Class $5 \& 6$ truck & 3 & BYD & United States & 9 & L1, L2, DC Fast & \\
\hline 5 & $\begin{array}{l}\text { T-Series Terminal } \\
\text { trucks }\end{array}$ & 3 & Orange & United States & 9 & L1, L2, DC Fast & \\
\hline 5 & eM2 box truck & 3 & Freightliner & $\begin{array}{l}\text { Mexico, United } \\
\text { States }\end{array}$ & 9 & Custom & \\
\hline 8 & eCascadia semi & 3 & Freightliner & $\begin{array}{l}\text { Mexico, United } \\
\text { States }\end{array}$ & 9 & Custom & \\
\hline 8 & Class 8 semi & 3 & Tesla & Netherlands & $\begin{array}{l}\text { 8; available } \\
2020\end{array}$ & Megacharger & \\
\hline 8 & ZeroTruck & 3 & Zero Truck & United States & 9 & L1, L2, DC Fast & \\
\hline 3 & $\begin{array}{l}\text { V8011 } \\
\text { refrigerated van }\end{array}$ & 3 & $\begin{array}{l}\text { Chanje/Thermo } \\
\text { King }\end{array}$ & $\begin{array}{l}\text { Hong Kong, United } \\
\text { States }\end{array}$ & 9 & DC Fast & \\
\hline 3 & ECantor van & 3 & Fuso & $\begin{array}{l}\text { Egypt, the } \\
\text { Philippines, Portugal }\end{array}$ & 9 & DC Fast & \\
\hline
\end{tabular}




\begin{tabular}{|c|c|c|c|c|c|c|c|}
\hline $\begin{array}{c}\text { DOT } \\
\text { Classification }\end{array}$ & Name & $\begin{array}{l}\text { MSA } \\
\text { No. }\end{array}$ & Manufacturer & Origin & TRL & Charging & GSA \\
\hline 4 & $\begin{array}{l}\text { 220EV, 520EV, } \\
579 \mathrm{EV}\end{array}$ & 3 & Peterbilt & $\begin{array}{l}\text { Canada, United } \\
\text { States }\end{array}$ & 2020 & & \\
\hline 3 & Class 6 box truck & 3 & $\begin{array}{l}\text { Lightning } \\
\text { Systems }\end{array}$ & United States & 9 & L2, DC Fast & \\
\hline 3 & $\begin{array}{l}\text { Ford E-450 } \\
\text { Cutaway }\end{array}$ & 3 & $\begin{array}{l}\text { Lightning } \\
\text { Systems }\end{array}$ & United States & 9 & L2, DC Fast & \\
\hline 5 & $\begin{array}{l}\text { Ford Transit } \\
\text { Chassis cab }\end{array}$ & 3 & $\begin{array}{l}\text { Lightning } \\
\text { Systems }\end{array}$ & United States & 9 & L2, DC Fast & \\
\hline 5 & Ford F-59 & 3 & $\begin{array}{l}\text { Lightning } \\
\text { Systems }\end{array}$ & United States & 9 & L2, DC Fast & \\
\hline 8 & NikolaTwo & 3 & Nikola & United States & 9 & DC Fast & \\
\hline \multicolumn{8}{|l|}{ Specialty Vehicles } \\
\hline 3 & $\begin{array}{l}\text { MPS Disaster } \\
\text { Response } \\
\text { Platform }\end{array}$ & 5 & Dannar & United States & 9 & L1, L2, DC Fast & Y \\
\hline 5 & $\begin{array}{l}\text { Burden carrier } \\
\text { hauler }\end{array}$ & 3 & \begin{tabular}{|l} 
Johnson \\
Industries
\end{tabular} & United States & $\begin{array}{l}\text { 8; custom } \\
\text { build }\end{array}$ & & \\
\hline 5 & Airport carrier & 3 & \begin{tabular}{|l} 
Johnson \\
Industries
\end{tabular} & United States & 9 & & \\
\hline 3 & $\begin{array}{l}\text { Ground support } \\
\text { utility truck }\end{array}$ & 3 & \begin{tabular}{|l} 
Johnson \\
Industries
\end{tabular} & United States & 9 & & \\
\hline 3 & $\begin{array}{l}\text { Large ground } \\
\text { support truck }\end{array}$ & 3 & $\begin{array}{l}\text { Johnson } \\
\text { Industries }\end{array}$ & United States & 9 & & \\
\hline 3 & Airport GSVs & 3 & \begin{tabular}{|l} 
Johnson \\
Industries
\end{tabular} & United States & 9 & & \\
\hline 15 & Customized hauler & 3 & \begin{tabular}{|l} 
Johnson \\
Industries
\end{tabular} & United States & $\begin{array}{l}\text { 8; custom } \\
\text { build }\end{array}$ & & \\
\hline 15 & Platform carrier & 3 & $\begin{array}{l}\text { Johnson } \\
\text { Industries }\end{array}$ & United States & 9 & & \\
\hline 15 & $\begin{array}{l}\text { Indoor \& outdoor } \\
\text { towing/ } \\
\text { lifting }\end{array}$ & 3 & $\begin{array}{l}\text { Linde Material } \\
\text { Handling }\end{array}$ & United States & 9 & & \\
\hline \multirow[t]{2}{*}{15} & Warehousing & 3 & \begin{tabular}{|l} 
Taylor-Dunn \\
(Polaris \\
company)
\end{tabular} & United States & & & \\
\hline & ECB Series forklift & 3 & BYD Motors & United States & 9 & & \\
\hline 4 & HyPower & 3 & Terex Utilities & United States & 9 & & \\
\hline 5 & $\begin{array}{l}\text { Electric Drive } \\
\text { bucket truck }\end{array}$ & 3 & Versalift & United States & 9 & & \\
\hline 3 & $\begin{array}{l}\mathrm{C}, \mathrm{E}, \& \mathrm{~T} \text { Series } \\
\text { tow tractors }\end{array}$ & 3 & Taylor-Dunn & United States & 9 & & \\
\hline 15 & $\begin{array}{l}\text { MT Series tow } \\
\text { tractors }\end{array}$ & 3 & Motrec & Canada & 9 & & \\
\hline 15 & $\begin{array}{l}\text { MC Series burden } \\
\text { carriers }\end{array}$ & 3 & Motrec & Canada & 9 & & \\
\hline 15 & $\begin{array}{l}\text { MP Series } \\
\text { personnel carriers }\end{array}$ & 3 & Motrec & Canada & 9 & & \\
\hline 15 & $\begin{array}{l}\text { MS Series stock } \\
\text { chasers }\end{array}$ & 3 & Motrec & Canada & 9 & & \\
\hline
\end{tabular}




\begin{tabular}{|c|c|c|c|c|c|c|c|}
\hline $\begin{array}{c}\text { DOT } \\
\text { Classification }\end{array}$ & Name & $\begin{array}{l}\text { MSA } \\
\text { No. }\end{array}$ & Manufacturer & Origin & TRL & Charging & GSA \\
\hline 15 & $\begin{array}{l}\text { MX Series } \\
\text { (custom) }\end{array}$ & 3 & Motrec & Canada & 9 & & \\
\hline 15 & $\begin{array}{l}8000 \text { Series pallet } \\
\text { jacks }\end{array}$ & 3 & Raymond Corp & United States & 9 & & \\
\hline 15 & $\begin{array}{l}\text { ATX Series (small } \\
\text { utility, fully } \\
\text { customizable) }\end{array}$ & 3 & Alke & Italy & 9 & & \\
\hline 15 & $\begin{array}{l}\text { US-1 intelligence } \\
\text { drone }\end{array}$ & 4 & $\begin{array}{l}\text { Impossible } \\
\text { Aerospace }\end{array}$ & United States & 9 & & \\
\hline 15 & Perimeter Drone & 4 & Skyfront & United States & 9 & & \\
\hline 15 & $\begin{array}{l}\text { SPX \& MPK Series } \\
\text { mini cranes }\end{array}$ & 5 & Jekko & Italy & 9 & & \\
\hline 15 & $\begin{array}{l}\text { MC } 32 \text { portable } \\
\text { crane }\end{array}$ & 5 & $J M G$ & Italy & 9 & & \\
\hline 15 & $\begin{array}{l}\text { ECO100 mini } \\
\text { loader }\end{array}$ & 5 & Sherpa & Netherlands & 9 & & \\
\hline 15 & $\begin{array}{l}\text { e210 mini } \\
\text { excavator }\end{array}$ & 5 & Green Machines & United States & 9 & & \\
\hline 15 & TE 4X2 gator & 3 & John Deere & India, United States & 9 & & \\
\hline 3 & $\begin{array}{l}\text { Able XR } \\
\text { (customizable } \\
\text { small utility) }\end{array}$ & 3 & Tropos & United States & 9 & & \\
\hline $5 \& 6$ & $\begin{array}{l}\text { GreenFleet aerial } \\
\text { lifts }\end{array}$ & 3 & Altec & United States & 9 & & \\
\hline \multicolumn{8}{|c|}{ Heavy-duty Vehicles } \\
\hline 15 & $\begin{array}{l}\text { EC15 \& EC27 } \\
\text { compact } \\
\text { excavators }\end{array}$ & 5 & $\begin{array}{l}\text { Volvo } \\
\text { Construction } \\
\text { Equipment }\end{array}$ & EU, United States & mid-2020 & & \\
\hline 15 & $\begin{array}{l}\text { L20 \& L28 wheel } \\
\text { loaders }\end{array}$ & 5 & $\begin{array}{l}\text { Volvo } \\
\text { Construction } \\
\text { Equipment }\end{array}$ & EU, U.S & mid-2020 & & \\
\hline 15 & $\begin{array}{l}\text { LX2 compact } \\
\text { wheel loader }\end{array}$ & 5 & $\begin{array}{l}\text { Volvo } \\
\text { Construction } \\
\text { Equipment }\end{array}$ & EU, U.S & Prototype & & \\
\hline 15 & $\begin{array}{l}\text { EX2 compact } \\
\text { excavator }\end{array}$ & 5 & $\begin{array}{l}\text { Volvo } \\
\text { Construction } \\
\text { Equipment }\end{array}$ & EU, U.S & Prototype & & \\
\hline 15 & $\begin{array}{l}\text { 988K XE wheel } \\
\text { loader }\end{array}$ & 5 & Caterpillar & $\begin{array}{l}\text { Brazil, China, } \\
\text { Russian and United } \\
\text { States }\end{array}$ & 9 & & \\
\hline 15 & D6 XE dozer & 5 & Caterpillar & $\begin{array}{l}\text { Brazil, China, } \\
\text { Russian and United } \\
\text { States }\end{array}$ & 9 & & \\
\hline
\end{tabular}




\section{Appendix E: Future Lines of Inquiry}

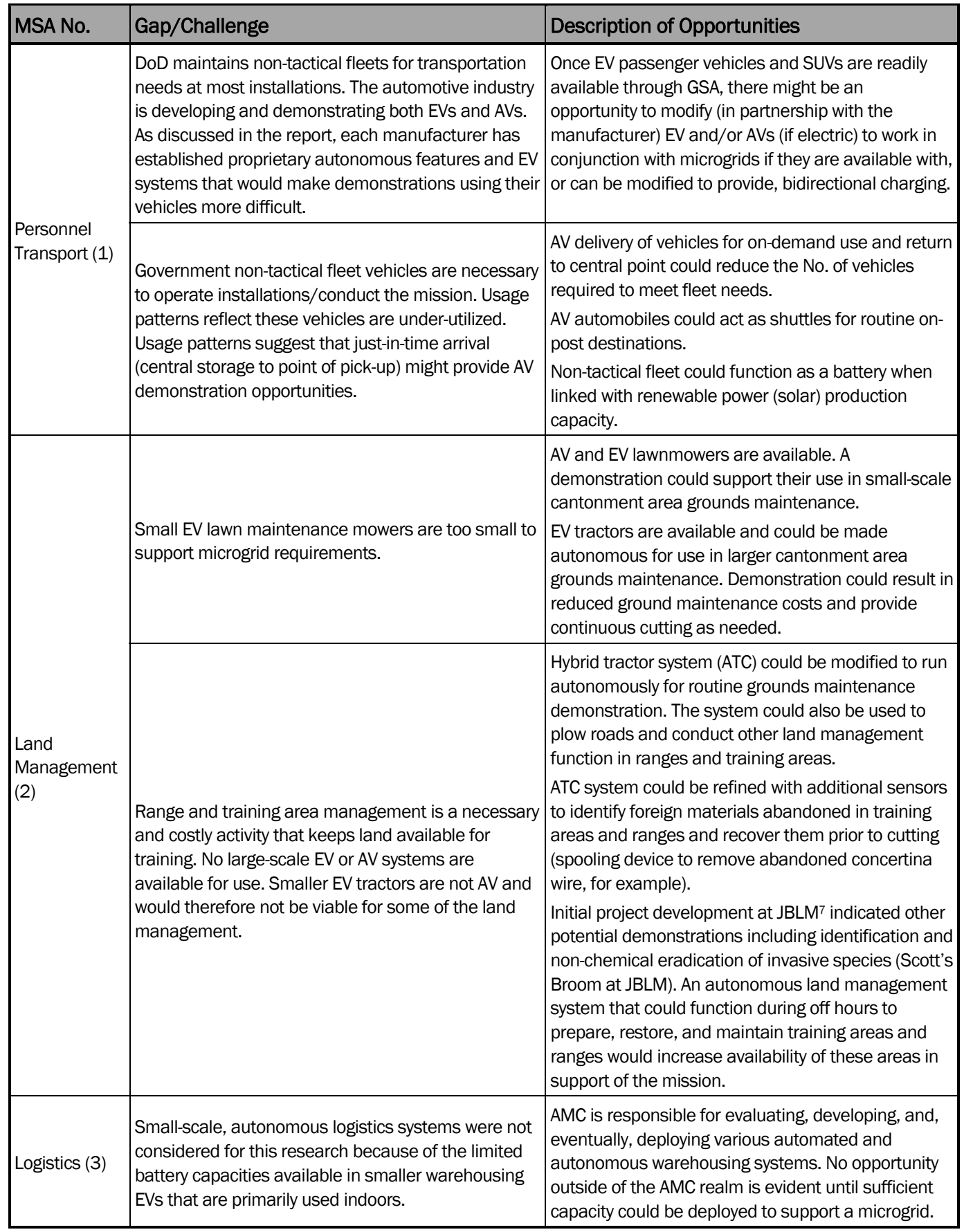




\begin{tabular}{|c|c|c|}
\hline MSA No. & Gap/Challenge & Description of Opportunities \\
\hline & $\begin{array}{l}\text { Point-to-point logistics could be supported through } \\
\text { development of small multi-use tugs with customized } \\
\text { attachment to perform routine functions including: } \\
\text { Cantonment area deliveries of material, products, } \\
\text { mail, etc. } \\
\text { Routine pick-ups for recyclables, refuse, etc. from } \\
\text { specified locations on scheduled days-these pick- } \\
\text { ups could then be used to conduct activities at night } \\
\text { or during off hours } \\
\text { Delivery of materiel to down-range locations and } \\
\text { retrieval of materiel from range areas. }\end{array}$ & $\begin{array}{l}\text { Initial tug project description developed after JBLM } \\
\text { session7 } \text {. Analysis could be updated as serve as } \\
\text { basis for EV/AV utility tug development and } \\
\text { demonstration. } \\
\text { EV tugs available from at least one manufacturer } \\
\text { who is interested in this concept-systems do not } \\
\text { meet battery capacity to support microgrid research } \\
\text { needs. }\end{array}$ \\
\hline & $\begin{array}{l}\text { Various military operations use GSVs. Operations are } \\
\text { common for logistics for aircraft (fixed and non-fixed } \\
\text { wing) loading, fueling, fitting, arming etc. Battery } \\
\text { capacity of these systems does not meet microgrid } \\
\text { research requirements. }\end{array}$ & $\begin{array}{l}\text { AV/EV GSVs could be used to directly support flying } \\
\text { missions. } \\
\text { AV loading systems would require specialized } \\
\text { detection and autonomous function to be } \\
\text { developed-mishaps could impact aircraft. }\end{array}$ \\
\hline $\begin{array}{l}\text { Land } \\
\text { Preparation } \\
\text { (5) }\end{array}$ & $\begin{array}{l}\text { HEV-heavy machinery is currently under } \\
\text { development by various manufacturers. Fully EV } \\
\text { machinery that can perform similarly to diesel- } \\
\text { powered systems is currently not possible given } \\
\text { battery technologies (weight too significant to provide } \\
\text { enough power to meet transit and functional } \\
\text { performance). }\end{array}$ & $\begin{array}{l}\text { Partnership with manufacturers will explore more } \\
\text { efficient battery technologies to be used in future } \\
\text { systems. } \\
\text { Partnership with manufacturers to test and validate } \\
\text { HEV systems and their potential autonomous use } \\
\text { could support the mission but would not support } \\
\text { microgrid research. } \\
\text { Research autonomous operation of diesel-powered } \\
\text { engineering equipment. }\end{array}$ \\
\hline \multirow{2}{*}{$\begin{array}{l}\text { Road } \\
\text { Maintenance } \\
\text { (6) }\end{array}$} & \multirow{2}{*}{$\begin{array}{l}\text { Heavy engineering equipment used in road } \\
\text { maintenance is generally not available as EV or AV } \\
\text { systems. }\end{array}$} & $\begin{array}{l}\text { Research autonomous operation of diesel-powered } \\
\text { engineering equipment } \\
\text { Evaluation of available autonomous plows used in } \\
\text { Europe for runway snow removal-not EV. } \\
\end{array}$ \\
\hline & & $\begin{array}{l}\text { Evaluate existing AV diesel-powered plow systems } \\
\text { for potential medication and use for gravel and dirt } \\
\text { trail and road development, maintenance, and } \\
\text { repair. }\end{array}$ \\
\hline
\end{tabular}




\section{Appendix F: Research Compatibility Considerations}

\begin{tabular}{|c|c|c|c|}
\hline Requirement & Metric (Quantitative) & Metric (Qualitative) & Additional Considerations \\
\hline Automation & 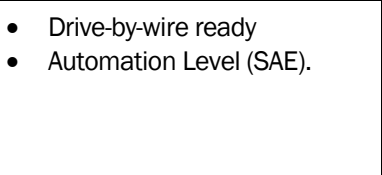 & $\begin{array}{ll}\text { - } & \text { Self-docking } \\
\text { - } & \text { Self-loading } \\
\text { - } & \text { Steering } \\
\text { - } & \text { Throttle } \\
\text { - } & \text { Braking }\end{array}$ & \\
\hline Battery & $\begin{array}{l}\text { - } \text { Maximum charge time (hour) } \\
\text { - } \text { Energy storage capacity } \\
\text { - } \text { Power capacity (kW). }\end{array}$ & $\begin{array}{ll}\text { - } & \text { Usable depth of discharge } \\
\text { - } & \text { Battery health monitoring } \\
\text { - } & \text { Battery Monitoring System } \\
& \text { (BMS) diagnostics and } \\
& \text { reporting } \\
\text { - } & \text { SOC monitoring } \\
\text { - } & \text { Battery warranty } \\
\text { - } & \text { System bidirectional } \\
& \text { capability }\end{array}$ & $\begin{array}{l}\text { Charger availability/logistics } \\
\text { Readiness for bidirectional } \\
\text { power flow } \\
\text { Industry ratings } \\
\text { Open or proprietary battery } \\
\text { management system }\end{array}$ \\
\hline Communications & $\begin{array}{l}\text { - } \text { Measurement range (V) } \\
\text { - } \text { Bandwidth (Hz) } \\
\text { - } \text { Accuracy (SNR) } \\
\text { - } \text { Central processor unit time } \\
\text { (CPU Time) } \\
\text { - } \\
\text { Space (byte) }\end{array}$ & $\begin{array}{ll}\text { - } & \text { Battery health monitoring } \\
\text { - } & \text { Telemetry (radio, cellular, } \\
\text { - } & \text { satellite) } \\
\text { - } & \text { Networface (USB/ethernet) } \\
\text { - } & \text { Network-attace } \\
\text { - } & \text { Data acquisition } \\
\text { - } & \text { Software } \\
\text { - } & \text { Cybatility/universal inputs } \\
\text { - } & \text { Communications protocol } \\
\text { - } & \text { V2G readiness of protocol, } \\
& \text { commands, data mapping }\end{array}$ & $\begin{array}{l}\text { Global communication } \\
\text { capabilities (chargers, on-road } \\
\text { vehicles) } \\
\text { Manufacturer's willingness to } \\
\text { collaborate/evolve for } \\
\text { bidirectional monitoring and } \\
\text { control } \\
\text { Level to which cybersecurity has } \\
\text { been addressed (or is planned } \\
\text { to be addressed) }\end{array}$ \\
\hline Cost Effective & $\begin{array}{ll}\text { - } & \text { Total annual maintenance } \\
& \text { Cost (USD) } \\
\text { - } & \text { Efficiency (\%) } \\
\text { - } & \text { Unit cost (USD) } \\
\text { - } & \text { Life cycle (years) }\end{array}$ & $\begin{array}{ll}\text { - } & \text { Risk reduction } \\
\text { - } & \text { Ease of acquisition } \\
\text { - } & \text { GSA availability } \\
\text { - } & \text { Warranty }\end{array}$ & $\begin{array}{l}\text { Need to include development } \\
\text { cost (NRE, materials, } \\
\text { equipment, etc.) to achieve V2G } \\
\text { readiness } \\
\text { Location of critical resources for } \\
\text { development, approvals and } \\
\text { field support/sustainment } \\
\text { Location and access to ancillary } \\
\text { equipment vendors (i.e., inverter } \\
\text { mfg.) }\end{array}$ \\
\hline Data Capture & $\begin{array}{ll}\text { - } & \text { Inputs (No.) } \\
\text { - } & \text { Input measurement range } \\
\text { - } & \text { Space (byte) } \\
\text { - } & \text { Frequency (Hz) } \\
\text { - } & \text { Environmental (temp, relative } \\
& \text { humidity, PSI). } \\
\end{array}$ & $\begin{array}{ll}\text { - } & \text { Sensors } \\
\text { - } & \text { Calibration } \\
\text { - } & \text { Maintenance } \\
\text { - } & \text { Class } \\
& \end{array}$ & \\
\hline $\begin{array}{l}\text { Environmental } \\
\text { Impacts on the } \\
\text { Vehicle }\end{array}$ & $\begin{array}{l}\text { - } \text { Temperature (F) } \\
\text { - } \text { Humidity (relative humidity, } \\
\text { dew point) } \\
\text { - } \text { Pressure (PSI) } \\
\text { - } \text { Industry and safety ratings. } \\
\end{array}$ & $\begin{array}{ll}\text { - } & \text { Battery/charger system } \\
\text { weight, footprint }\end{array}$ & $\begin{array}{l}\text { Power quality, vibration, } \\
\text { operating temperature range, } \\
\text { environmental performance } \\
\text { testing (proven; not lab level) }\end{array}$ \\
\hline
\end{tabular}




\begin{tabular}{|c|c|c|c|}
\hline Requirement & Metric (Quantitative) & Metric (Qualitative) & Additional Considerations \\
\hline $\begin{array}{l}\text { Manufacturer } \\
\text { Reliability }\end{array}$ & $\begin{array}{l}\text { - Units manufactured (No. of } \\
\text { units) } \\
\text { - } \quad \text { Total cost to manufacture } \\
\text { (USD) } \\
\text { - Utilization rate (actual } \\
\text { - } \quad \text { Cytput/optimal output) } \\
\text { - } \quad \text { Yield (No. satisfactory } \\
\text { units/total units) } \\
\text { - } \text { Recall rate (No. recalled } \\
\text { units/units produced) } \\
\text { - Inventory turnover (No. units } \\
\text { sold/units in inventory) } \\
\text { - Production downtime (No. } \\
\text { hours downtime/optimal } \\
\text { hours operation) } \\
\text { - Customer satisfaction } \\
\end{array}$ & - Customer testimonials & $\begin{array}{l}\text { Publications } \\
\text { Website/media } \\
\text { Past/present contracts } \\
\text { Financial stability }\end{array}$ \\
\hline Navigation & $\begin{array}{l}\text { - } \text { Accuracy }(\mathrm{cm}, \mathrm{ft} .) \\
\text { - } \quad \text { Frequency }(\mathrm{Hz}) \\
\text { - } \text { Accuracy (degrees, radians)) } \\
\text { - } \text { GPS Band (C, M). }\end{array}$ & $\begin{array}{ll}\text { - } & \text { GPS } \\
\text { - } & \text { Cameras } \\
\text { - } & \text { Radar } \\
\text { - } & \text { LiDAR } \\
\text { - } & \text { Inertial measurement unit } \\
& \text { systems } \\
\text { - } & \text { Receiver } \\
\text { - } & \text { Tracker }\end{array}$ & \\
\hline $\begin{array}{l}\text { Programmability } \\
\text { (Computer) }\end{array}$ & 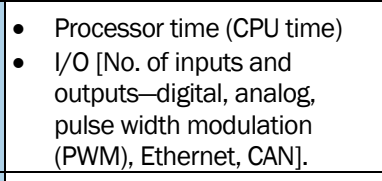 & $\begin{array}{ll}\text { - } & \text { Scheduling } \\
\text { - } & \text { Operating system } \\
\text { - } & \text { Real-time synchronization }\end{array}$ & $\begin{array}{l}\text { Willingness of manufacturer to } \\
\text { collaborate/develop V } 2 \mathrm{G} \\
\text { solution }\end{array}$ \\
\hline Range & $\begin{array}{ll}\text { - } & \text { Time (hour) } \\
\text { - } & \text { Distance (mile) } \\
\end{array}$ & $\bullet$ & Regenerative capabilities \\
\hline Safety & $\begin{array}{l}\text { - Industry ratings/certifications } \\
\text { - DOT } \\
\text { - Static stability factor } \\
\text { - }\end{array}$ & $\begin{array}{l}\text { - } \\
\text { - } \text { Armorsonnel protection } \\
\text { - Incognito/camouflage } \\
\text { - Redundant controls } \\
\text { - Weaponry } \\
\text { - Hazmat transport } \\
\end{array}$ & \\
\hline $\begin{array}{l}\text { Personnel } \\
\text { Transport }\end{array}$ & - Occupants (No.) & $\begin{array}{ll}\text { - } & \text { Accessibility } \\
\text { - } & \text { Seating } \\
\end{array}$ & \\
\hline $\begin{array}{l}\text { Vehicle } \\
\text { Performance } \\
\text { Metrics }\end{array}$ & $\begin{array}{ll}\text { - } & \text { Top speed (mph) } \\
\text { - } & \text { Acceleration range (seconds) } \\
\text { - } & \text { Traction (psi) } \\
\text { - } & \text { Torque (ft-lb) } \\
\text { - } & \text { Power (hp) } \\
\text { - } & \text { Power to weight (hp/lb.) } \\
\text { - } & \text { Handling (lb./sq. ft) } \\
\text { - } & \text { Weight distribution (\%) } \\
\text { - } & \text { Towing (max capacity) } \\
\text { - } & \text { Efficiency (\%) } \\
\text { - } & \text { Suspension (spring rate) } \\
\text { - } & \text { Turning clearance (ft) } \\
\end{array}$ & $\begin{array}{ll}- & \text { Drivetrain } \\
\text { - } & \text { Electronic stability control } \\
\text { - } & \text { Off-road } \\
& \text { durability/performance }\end{array}$ & \\
\hline Versatile & $\begin{array}{ll}\text { - } & \text { Versatility (No. of } \\
& \text { attachments) } \\
\text { - } & \text { Modularity (No. of common } \\
\text { - } & \text { variants) } \\
\text { - } & \text { Variants (No. of variants). }\end{array}$ & $\begin{array}{l}\text { - } \text { Supports multiple missions } \\
\text { - } \text { Ease of maintenance/repair } \\
\text { - } \\
\text { Function (personnel } \\
\text { transport, cargo transport, } \\
\text { refrigeration, hydraulics, } \\
\text { towing) }\end{array}$ & Supports multiple programs \\
\hline
\end{tabular}




\section{Appendix G: Initial Y/N Scoring Summary}

\begin{tabular}{|c|c|c|c|c|c|c|c|}
\hline $\begin{array}{l}\text { Total No. } \\
\text { of Y: }\end{array}$ & $\begin{array}{l}\text { TRL } \\
(7-9)\end{array}$ & $\begin{array}{c}\text { Cost } \leq \\
\$ 450,000\end{array}$ & $\begin{array}{c}\text { Assembled } \\
\text { In U.S. }\end{array}$ & $\begin{array}{c}\text { Battery Capacity } \\
\geq 50 \mathrm{kWh}\end{array}$ & MSA & $\begin{array}{c}\text { Related } \\
\text { Research }\end{array}$ & $\begin{array}{c}\text { Available } \\
\text { through GSA }\end{array}$ \\
\hline \multirow{3}{*}{7} & \multicolumn{7}{|c|}{ MPS by: Dannar } \\
\hline & Y & Y & Y & Y & Y & Y & Y \\
\hline & 9 & $\$ 200-400 K$ & IN & $125-630 \mathrm{kWh}$ & $\begin{array}{c}2,4,5 \\
6\end{array}$ & DHS & \\
\hline \multirow{3}{*}{6} & \multicolumn{7}{|c|}{ Cargo Van, Chassis Cab, Cutaway Cab, Shuttle Van, Step Van by: Zenith Motors } \\
\hline & Y & Y & Y & Y & Y & $\mathrm{N}$ & Y \\
\hline & 9 & $\$ 100 K$ & $\mathrm{CA}$ & $52-100 \mathrm{kWh}$ & 1,3 & & \\
\hline \multirow{3}{*}{6} & \multicolumn{7}{|c|}{ Zeus Electric Shuttle bus, Utility vehicle, Flatbed truck by: Phoenix Motorcars } \\
\hline & Y & Y & Y & Y & Y & $\mathrm{Y}$ & $\mathrm{N}$ \\
\hline & 9 & $\$ 240,000+$ & $\mathrm{CA}$ & $105 \mathrm{kWh}$ & 1,3 & EPA, CA & \\
\hline \multirow{3}{*}{5} & \multicolumn{7}{|c|}{ Medium-Duty Trucks, Shuttles, and Buses by: Zero Truck } \\
\hline & Y & $\mathrm{Y}$ & Y & Y & Y & $\mathrm{N}$ & $\mathrm{N}$ \\
\hline & 9 & $\$ 60-100 K$ & $\mathrm{CA}$ & $53-80 \mathrm{kWh}$ & 1,3 & & \\
\hline \multirow{3}{*}{5} & \multicolumn{7}{|c|}{ T-Series Yard Hostler by: Orange Motors } \\
\hline & $\mathrm{Y}$ & $\mathrm{Y}$ & Y & $\mathrm{Y}$ & $\mathrm{Y}$ & $\mathrm{N}$ & $\mathrm{N}$ \\
\hline & 9 & $\$ 200-300 K$ & MO & 80-160 kWh & 1,3 & & \\
\hline \multirow{3}{*}{5} & \multicolumn{7}{|c|}{ Reckless by Nikola } \\
\hline & Y & $\mathrm{N}$ & $\mathrm{Y}$ & Y & Y & $\mathrm{Y}$ & $\mathrm{N}$ \\
\hline & 9 & $\begin{array}{c}\$ 480 \mathrm{~K} / \mathrm{year} \\
\text { lease }\end{array}$ & $\mathrm{CA}$ & $52-100 \mathrm{kWh}$ & 4 & USMC & \\
\hline \multirow{3}{*}{5} & \multicolumn{7}{|c|}{ eBus by: eBus } \\
\hline & Y & Y & Y & $\mathrm{Y}$ & Y & $\mathrm{N}$ & Y \\
\hline & 9 & $\$ 400 K$ & $\mathrm{CA}$ & $130 \mathrm{kWh}$ & 1 & & \\
\hline \multirow{3}{*}{5} & \multicolumn{7}{|c|}{ Xcelsior Charge (multiple versions) by: New Flyer } \\
\hline & Y & $\mathrm{N}$ & Y & Y & Y & $\mathrm{Y}$ & $\mathrm{N}$ \\
\hline & 9 & $\begin{array}{l}\$ 700- \\
1,200 K\end{array}$ & AL & 160-466 kWh & 1,3 & Private sector & \\
\hline \multirow{3}{*}{5} & \multicolumn{7}{|c|}{ R1T - EV Pick-up by: Rivian } \\
\hline & Y & $\mathrm{Y}$ & Y & $\mathrm{Y}$ & Y & $\mathrm{N}$ & $\mathrm{N}$ \\
\hline & 8 & $\$ 70-100 K$ & $\mathrm{IL}$ & $105-180 \mathrm{kWh}$ & 3,4 & & \\
\hline \multirow{3}{*}{5} & \multicolumn{7}{|c|}{ Saf-T-Liner C2 Jouley Electric School Bus by: Thomas Built } \\
\hline & Y & Y & Y & Y & Y & $\mathrm{N}$ & $\mathrm{N}$ \\
\hline & 8 & $\$ 450 K$ & $\mathrm{NC}$ & $220 \mathrm{kWh}$ & 1 & & \\
\hline \multirow{3}{*}{5} & \multicolumn{7}{|c|}{ Shuttles, buses, Class 5-7 Tractor, Class 8 Day Cab by: BYD } \\
\hline & Y & $\mathrm{Y}$ & $\mathrm{Y}$ & $\mathrm{Y}$ & $\mathrm{Y}$ & $\mathrm{N}$ & $\mathrm{N}$ \\
\hline & 9 & $\$ 250-550 \mathrm{~K}$ & $\mathrm{CA}$ & $150-250 \mathrm{kWh}$ & 1,3 & & \\
\hline \multirow{3}{*}{5} & \multicolumn{7}{|c|}{ Edrive Tractor by: ATC } \\
\hline & $\mathrm{Y}$ & $\mathrm{Y}$ & $Y$ & $\mathrm{Y}$ & $\mathrm{Y}$ & $\mathrm{N}$ & $\mathrm{N}$ \\
\hline & 9 & $\$ 250 K$ & $\mathrm{MN}$ & $250 \mathrm{~kW}$ & $2,5,6$ & & \\
\hline 5 & \multicolumn{7}{|c|}{ EV250, EV300, EV350, EV400 \& EV550 shuttle buses by: Green Power Bus } \\
\hline
\end{tabular}




\begin{tabular}{|c|c|c|c|c|c|c|c|}
\hline $\begin{array}{c}\text { Total No. } \\
\text { of Y: }\end{array}$ & $\begin{array}{l}\text { TRL } \\
(7-9)\end{array}$ & $\begin{array}{c}\text { Cost } \leq \\
\$ 450,000\end{array}$ & $\begin{array}{l}\text { Assembled } \\
\text { In U.S. }\end{array}$ & $\begin{array}{c}\text { Battery Capacity } \\
\geq 50 \text { kWh }\end{array}$ & MSA & $\begin{array}{l}\text { Related } \\
\text { Research }\end{array}$ & $\begin{array}{c}\text { Available } \\
\text { through GSA }\end{array}$ \\
\hline & Y & Y & Y & Y & Y & N & $\mathrm{N}$ \\
\hline & 9 & $\$ 250-600 K$ & $\begin{array}{l}\text { China/United } \\
\text { States }\end{array}$ & $210-478 \mathrm{kWh}$ & 1 & & \\
\hline \multirow{3}{*}{4} & \multicolumn{7}{|c|}{ E210 and E240 (mini excavator) by: Green Machine } \\
\hline & Y & Y & Y & $\mathrm{N}$ & $\mathrm{Y}$ & $\mathrm{N}$ & $\mathrm{N}$ \\
\hline & 9 & $\$ 50 \mathrm{~K}$ & $\mathrm{NH}, \mathrm{NY}$ & $48 \mathrm{kWh}$ & $2,5,6$ & & \\
\hline \multirow{3}{*}{4} & \multicolumn{7}{|c|}{ eCargo, eTruck drayage - Class 8 Tractor by: US Hybrid } \\
\hline & $\mathrm{N}$ & Y & $\mathrm{Y}$ & Y & $\mathrm{Y}$ & $\mathrm{N}$ & $\mathrm{N}$ \\
\hline & 6 & $\$ 250 K$ & $\mathrm{CA}$ & $240 \mathrm{kWh}$ & 1,3 & & \\
\hline \multirow{3}{*}{4} & \multicolumn{7}{|c|}{ LFSe by: Nova Bus } \\
\hline & Y & $\mathrm{N}$ & Y & $\mathrm{Y}$ & $\mathrm{Y}$ & $\mathrm{N}$ & $\mathrm{N}$ \\
\hline & 8 & $\$ 700 K+$ & NY & $152 \mathrm{kWh}$ & 1 & & \\
\hline \multirow{3}{*}{4} & \multicolumn{7}{|c|}{ EV22, EV27, EV33 shuttle buses by: AVM } \\
\hline & Y & Y & $\mathrm{N}$ & Y & $\mathrm{Y}$ & $\mathrm{N}$ & $\mathrm{N}$ \\
\hline & 9 & $\$ 300 K$ & China & $250 \mathrm{kWh}$ & 1 & & \\
\hline \multirow{3}{*}{4} & \multicolumn{7}{|c|}{ Catalyst by: Proterra } \\
\hline & Y & N & $\mathrm{Y}$ & $\mathrm{Y}$ & $\mathrm{Y}$ & N & $\mathrm{N}$ \\
\hline & 9 & $\$ 800 K$ & $\mathrm{FL}$ & $650 \mathrm{kWh}$ & 1 & & \\
\hline \multirow{3}{*}{4} & \multicolumn{7}{|c|}{ eUtility, eFarmer tractors by: Soletrac } \\
\hline & Y & Y & Y & $\mathrm{N}$ & $\mathrm{Y}$ & N & $\mathrm{N}$ \\
\hline & 7 & $\$ 45-60 K$ & $\mathrm{CA}$ & $28-48 \mathrm{kWh}$ & 2,5 & & \\
\hline \multirow{3}{*}{4} & \multicolumn{7}{|c|}{ V8011 Refrigerated Truck by: Chanje Energy and Thermoking } \\
\hline & Y & Y & $\mathrm{N}$ & Y & $\mathrm{Y}$ & $\mathrm{N}$ & $\mathrm{N}$ \\
\hline & 7 & $\$ 75 \mathrm{~K}$ & China & $100 \mathrm{kWh}$ & 3 & & \\
\hline \multirow{3}{*}{4} & \multicolumn{7}{|c|}{ ECantor light-duty truck by: Mitsubishi Fuso } \\
\hline & Y & Y & $\mathrm{N}$ & Y & $\mathrm{Y}$ & $\mathrm{N}$ & $\mathrm{N}$ \\
\hline & 9 & $\$ 125 K$ & EU & $42-84 \mathrm{kWh}$ & 3 & & \\
\hline \multirow{3}{*}{4} & \multicolumn{7}{|c|}{ ECobus by: Cobus Industries } \\
\hline & $\mathrm{Y}$ & Y & $\mathrm{N}$ & Y & $\mathrm{Y}$ & N & $\mathrm{N}$ \\
\hline & 8 & $\$ 250 K$ & EU & $85 \mathrm{kWh}$ & 1 & & \\
\hline \multirow{3}{*}{3} & \multicolumn{7}{|c|}{ Class 8 Tractor by: Tesla } \\
\hline & $\mathrm{N}$ & Y & $\mathrm{N}$ & $\mathrm{Y}$ & $\mathrm{Y}$ & $\mathrm{N}$ & $\mathrm{N}$ \\
\hline & 6 & $\$ 150-200 K$ & EU & 600-1000 kWh & 1,3 & & \\
\hline
\end{tabular}




\section{Appendix H: Candidate System Information Packets}

\section{H.1 Green Fleet Manufacturer: Altec}

Altec Green Fleet offers a collection of products that encompass the most advanced technology and includes Jobsite Energy Management Systems (JEMS) HybridElectric Technology, an integrated plug-in hybrid system

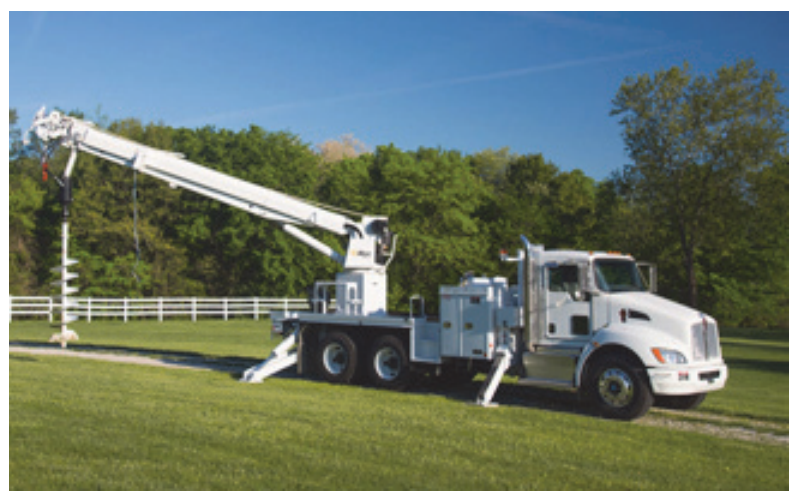
that uses stored electrical energy to power aerial devices, hydraulic tools, exportable power, and engine-off cab comfort. The company manufactures aerial devices (overcenter, non-overcenter, telescopic, and telescopic articulating) and digger derricks.

The company provides products and services to the electric utility, telecommunications, tree care, lights and signs, and contractor markets and was founded in 1929.

\begin{tabular}{|c|c|}
\hline \multicolumn{2}{|r|}{ Green Fleet by: Altec } \\
\hline Cost & $\begin{array}{l}\text { From the GSA catalog: } \\
\text { Articulating telescopic aerial device (base): } \$ 132,106 \\
\text { Overcenter articulating aerial device (base): } \$ 144,266 \\
\text { Non-overcenter aerial device with material handling (base): } \\
\$ 178,559 \\
37 \text { ' digger derrick: } \$ 133,368 \\
41^{\prime} \text { 'digger derrick: } \$ 173,266 \\
50 \text { ' digger derrick: } \$ 221,883 \\
65 \text { ' digger derrick: } \$ 272,971\end{array}$ \\
\hline Application Maturity & Commercially available \\
\hline $\begin{array}{l}\text { Maintenance } \\
\text { Requirements }\end{array}$ & $\begin{array}{l}\text { Altec provides courses to technicians to enable an understanding of the fundamental operational } \\
\text { principles on the JEMS hybrid systems, and } \\
\text { there is an Altec service center in St. Joseph, MO. }\end{array}$ \\
\hline
\end{tabular}




\section{Comments}

The Green Fleet line consists of:

28 overcenter aerial devices

24 non-overcenter aerial devices

5 telescopic aerial devices

31 telescoping articulating aerial devices

28 digger derricks

Hybrid bucket trucks/aerial devices are a combination of two power units in one vehicle. An auxiliary electric motor is located under the mid-chassis section and is connected to a hydraulic pump. Powered by high-capacity battery packs, the electric motor provides power to the hydraulic system.

More information on Altec Green Fleet government sales can be found here.

Website altec.com/products/green fleet

Contact $\quad$ For federal government sales: 205-408-2341; fedgovtsales@altec.com

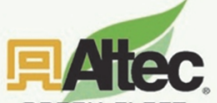

GREEN FLEET

Altec Green Fleet ...

providing innovative solutions for/today's demands

HYBRID, ELECTRIC \& ALTERNATIVE ENERGY

Integrated hybrid technology reduces fuel consumption, decreases greenhouse

gas emissions and significantly lowers noise pollution.

- Altec JEMS

- Eaton Full-Hybrid Drivetrain

- EPRI - Plug-lin Hybrid Electric Vehicle

- Smith Electric Vehicle (SEV) - All-Electric Work Trucks

RECYCLED MATERIALS

- Recycled material choices, like aluminum, increase payload and reduce fuel
consumption.

ALTERNATIVE FUELS

- Biopure hydraulic oil has the highest ASTM rating. and is non-toxic to plants
and animals

Vehicles converted to operate on compressed natural gas and green
diesel reduce operating costs, while burning cleaner and reducing taipipe

diesel reduce operating costs, while burning cleaner and reducing tailpipe
emissions.

SUSTAINABILITY

- Powder coat paint has zero VOC emissions, minimal waste and is durable and

- Vacuum infusion fiberglass process uses $30 \%$ less resin and reduces styrene

. Manufacturing

- Manufacturing processes utilize high-efficiency lasers, lighting and

- E-Coat paint prevents corrosion with low VOC emissions, low energy
consumption and minimal waste.

\section{드도.}

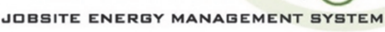

Altec JEMS ${ }^{\circ}$ is an integrated plug-in system that uses stored electrical energy to power the aerial device, tools 作

The energy storage system is recharged by plugging into shore power or by the truck's internal combustion engine.
This innovative system:

$\mathbf{D}$ Eliminates idle time at the jobsite

$\geq$ Reduces fuel consumption

$\geq$ Lessens noise pollution

$>$ Decreases carbon footprint

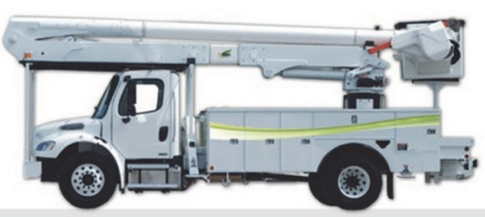




\section{Green Fleet Products}

Altec has several hybrid and/or alternative energy options for the utility industry. Whether you need an insulating or non-insulating aerial device, telescopic or telescopic articulating, Altec's Green Fleet lineup has what will work for you, with heights up to $66.0 \mathrm{ft}$. For more information, select the model that fits your application or specifications.

Aerial Devices

Overcenter

\begin{tabular}{|c|c|c|c|c|c|}
\hline A50OC & A55EOC & A550C & AM50 & AM50E & AM55 \\
\hline AM55E & AM60 & AM60E & AN $46 \mathrm{E}-\mathrm{OC}$ & AN50E-OC & AN50EOC-E80 \\
\hline AN50OC-E80 & AN55E-OC & AN55EOC-E88 & AN55OC-E88 & L36A & L37M \\
\hline L42A & L42E & L42M & L4AE & L45M & LR752 \\
\hline LR756 & LR758 & LR760 & LR760-E70 & & \\
\hline
\end{tabular}

Non-Overcenter

\begin{tabular}{|c|c|c|c|c|c|}
\hline AA50 & AA50-E80 & AA50E & AA50E-E80 & AA55 & AA55-E88 \\
\hline AA55E & AA55E-E88 & AA60 & AA60E & AA67-E100 & AA67E \\
\hline AA67E-E100 & AN32E & AN50-E80 & AN50E & AN50E-E80 & AN55-E88 \\
\hline AN55E & AN55E-E88 & AN60E & AN67-E100 & AN67E & AN67E-E100 \\
\hline
\end{tabular}

Telescopic

AT200A

AT200A Cutaway Van AT200AV

AT30G

AT40C

Telescopic Articulating

\begin{tabular}{l} 
AT235P \\
\hline AT37M/P \\
\hline AT40S \\
\hline AT48S \\
\hline TA45M-T50 \\
\hline TA60S
\end{tabular}

AT237

AT37ME/PE

AT2375

AT37S

AT $41 \mathrm{M} / \mathrm{P}$

ATA1ME/PE

TA40

TA50S

AT248F

AT40G

AT41S

TA41M

AT35G

AT4OM - JEMS

AT37G

TA50

TA55

AT $48 \mathrm{M} / \mathrm{P}$

AT 4OP - JEMS

AT48ME/PE

TA41M-T46 TA45M

\section{Digger Derricks}

\begin{tabular}{|c|c|c|c|c|c|}
\hline D2045B & D2050B & D2055B & D3050E & $\mathrm{D} 3050 \mathrm{H}$ & D3055E \\
\hline $\mathrm{D} 3055 \mathrm{H}$ & D3060E & $\mathrm{D} 3060 \mathrm{H}$ & D4050B & D4055B & D4060B \\
\hline D $4065 B$ & DC45 & DC47 & $\mathrm{DH} 45 \mathrm{~B}$ & $\mathrm{DH} 45 \mathrm{E}$ & $\mathrm{DH} 45 \mathrm{H}$ \\
\hline DH48B & DH48E & $\mathrm{DH} 48 \mathrm{H}$ & $\mathrm{DH} 50 \mathrm{~B}$ & DH50E & $\mathrm{DH} 50 \mathrm{H}$ \\
\hline DL42B & DL45B & DM45B & DM478 & & \\
\hline
\end{tabular}




\section{Altec a class} Specifications

\section{STANDARD FEATURES}

- Altec ISO-Grip, Control Systern

- Working Heights to $60 \mathrm{ft}(27.4 \mathrm{~m})$

- Side Reach to $48.3 \mathrm{ft}$ (14.7 m) - Non-Overcenter Position to $41.2 \mathrm{ft}(12.6 \mathrm{~m})$

- Unique Hydraulic Leveling Systern, Accu-Level ${ }^{T M}$ and Platform Tilt

- No Major Scheduled Maintenance Required

- Automatic Upper Boom Stow

- Material Handling Capacity - $1000 \mathrm{lb}$ Minimum in any Non-Overcenter Position

- Designed for Low Life-Cycle Cost

- Designed and Manufactured in ISO 9001 Certified Facility

- Insulated, ANSI Category C, $46 \mathrm{kV}$ and Below

- Lower Boom Insulator

- Boom Stow Protection

- Continuous Rotation

- Positive Hydraulic Leveling

- Positive Hydraulic Platform Tilt

- Continuous Autornatic Purging of Upper Controls

- Pilot Operated or Full Pressure Hydraulic System

- Dual Hydraulic Tool Circuits at the Platform with Adjustable Pressure and Flow

- Machine/Ground Control Selector Valve

- Outrigger Motion Alarm

- Outrigger Boom Interlock System

- Back-Up Alarm

\section{RECOMMENDED FEATURES}

- Fall Protection System

- Outrigger Pads

- Platform Liner

- Wheel Chocks

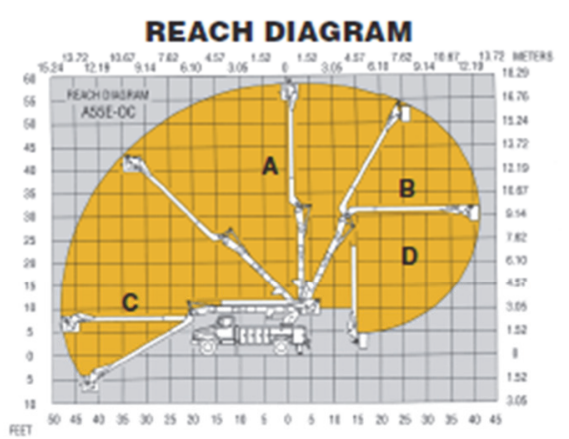

\begin{tabular}{|c|c|c|c|c|}
\hline & $A$ & $\mathrm{~B}$ & $C$ & D \\
\hline Tूode & Platiorm helght & $\begin{array}{c}\text { Slde-Reach } \\
\text { Non-Overcenter }\end{array}$ & $\begin{array}{l}\text { SIde-Reach } \\
\text { Overcenter }\end{array}$ & $\begin{array}{l}\text { Platerm to } \\
\text { Ground }\end{array}$ \\
\hline A47-OC & $47.3 \mathrm{ft}(1<2 \mathrm{~m})$ & $31.1 \mathrm{tt}(0.5 \mathrm{~m})$ & $40.7 \mathrm{ft}(12.4 \mathrm{~m})$ & $26.8 \mathrm{ft} \mathrm{( \textrm {B } 2 \mathrm { m } )}$ \\
\hline$A 50-O C$ & $49.9 \mathrm{ft}(15.2 \mathrm{~m})$ & $32.6 \mathrm{ft}(2.9 \mathrm{~m})$ & $43.3 \mathrm{ft}(13.2 \mathrm{~m})$ & $28.1 \mathrm{ft}(\mathrm{B} 6 \mathrm{~m})$ \\
\hline A55-OC & $54.9 \mathrm{ft}(16.7 \mathrm{~m})$ & $35.5 \mathrm{ft}(10.8 \mathrm{~m})$ & $48.3 \mathrm{ft}(14.7 \mathrm{~m})$ & $30.6 \mathrm{ft}(2.3 \mathrm{~m})$ \\
\hline A55E-OC & $54.9 \mathrm{ft}(16.7 \mathrm{~m})$ & $41.2 \mathrm{ft}(126 \mathrm{~m})$ & $48.3 \mathrm{ft}(14.7 \mathrm{~m})$ & $28.7 \mathrm{ft}(\mathrm{s} 7 \mathrm{~m})$ \\
\hline
\end{tabular}

\section{H.2 eDrive Tractors \& AutoDrive Autonomous Systems Manufacturer: Autonomous Tractor Company (ATC)}

ATC, specializing in system design and third-party collaboration, develops patented retrofit electric drive technology, which claims to make today's tractors two to five times more durable and $20 \%-40 \%$ more fuel efficient. ATC's technology works in diverse applications that require long duty cy-

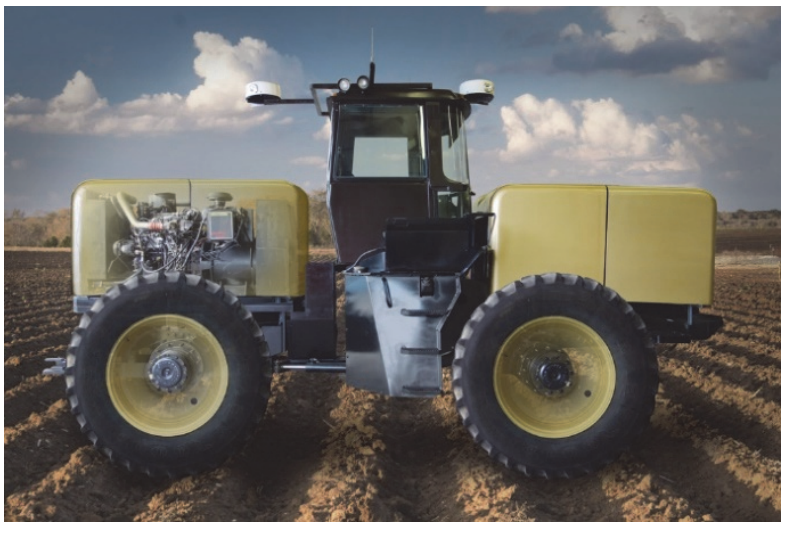
cles, and its systems run power directly from diesel generators, fuel cells, super capacitors, etc. ATC also develops AutoDrive, an autonomous navigation system for tractors. 


\section{ATC is based in Fargo, ND and St. Michaels, MN. The company uses mostly off-the-shelf components, contract manufacturers, and dealers for sales, installation, and customer support.}

\begin{tabular}{|c|c|c|}
\hline & eDrive by: ATC & AutoDrive by: ATC \\
\hline Cost & $\$ 500$ per horsepower installed & $\$ 30,000$ (in beta offering) \\
\hline $\begin{array}{l}\text { Application } \\
\text { Maturity }\end{array}$ & Commercially available & Commercially available \\
\hline Safety & & $\begin{array}{l}\text { FieldSmart artificial intelligence; associate } \\
\text { investigator (Al) systems provide system } \\
\text { management and } \\
\text { tractor tracking, and systems include fail safes. } \\
\text { Systems also } \\
\text { use ATC's patented Laser- } \\
\text { Radio Navigation System } \\
\text { (LRNS), a laser and radio } \\
\text { system used to navigate fields } \\
\text { and remain in set boundaries. }\end{array}$ \\
\hline $\begin{array}{l}\text { Extras and } \\
\text { Attachments }\end{array}$ & $\begin{array}{l}\text { Includes } 1-2 \text { diesel generators, } \\
2 \text { or } 4 \text { electric, variable speed wheel motors, and } \\
\text { joystick } \\
\text { control. }\end{array}$ & \\
\hline $\begin{array}{l}\text { Operability in } \\
\text { Adverse } \\
\text { Weather or } \\
\text { Conditions }\end{array}$ & $\begin{array}{l}\text { eDrive motors are liquid cooled and totally } \\
\text { sealed, making them ideal for use in mud, dust, } \\
\text { and } \\
\text { high-temperature field } \\
\text { conditions. }\end{array}$ & \\
\hline $\begin{array}{l}\text { Projected Life } \\
\text { Span }\end{array}$ & $\begin{array}{l}25,000 \text { hours ( } 50 \% \text { longer than typical tractor } \\
\text { lifespan) }\end{array}$ & 20 years \\
\hline \multicolumn{3}{|c|}{ Comments } \\
\hline \multicolumn{3}{|c|}{$\begin{array}{l}\text { Electric motors drive wheels but power is provided by an onboard diesel generator (high efficiency, though not electric). } \\
\text { The generator can be used to support microgrid and installation resilience. The system is implemented through existing } \\
\text { tractor conversion or installation on an } \\
\text { existing tractor frame. }\end{array}$} \\
\hline \multirow{2}{*}{\multicolumn{3}{|c|}{$\begin{array}{l}\text { While not currently commercially available, ATC is developing Spirit: a prototype tractor that is truly autonomous. } \\
\text { Also, of note, ATC's first sale of its drivetrain package was to FamilyFarms Group, a farm } \\
\text { business consulting group headquartered in St Louis, MO. }\end{array}$}} \\
\hline & & \\
\hline Website & \multicolumn{2}{|c|}{ autonomoustractor.com } \\
\hline Contact & \multicolumn{2}{|l|}{ 203-993-0828; kraig@atcspirit.com } \\
\hline
\end{tabular}




\section{H.3 C Series Buses Manufacturer: BYD}

BYD, headquartered in Los Angeles, $\mathrm{CA}$, is the largest $\mathrm{EV}$ manufacturer in the world. The company is also one of the largest battery manufacturers worldwide, and its buses are manufactured in a 450,000-square foot facility in Lancaster, CA.

BYD is the automotive subsidiary of

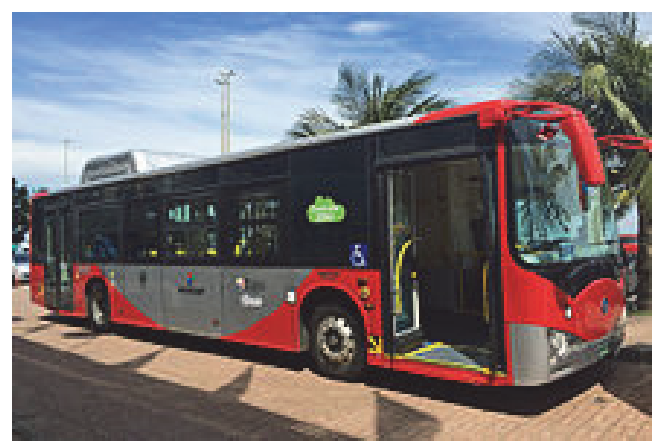
the Chinese multinational BYD Co. LTD, which was founded in 2003 and is now based in Xi'an, Shaanxi Province and was founded in 2003 .

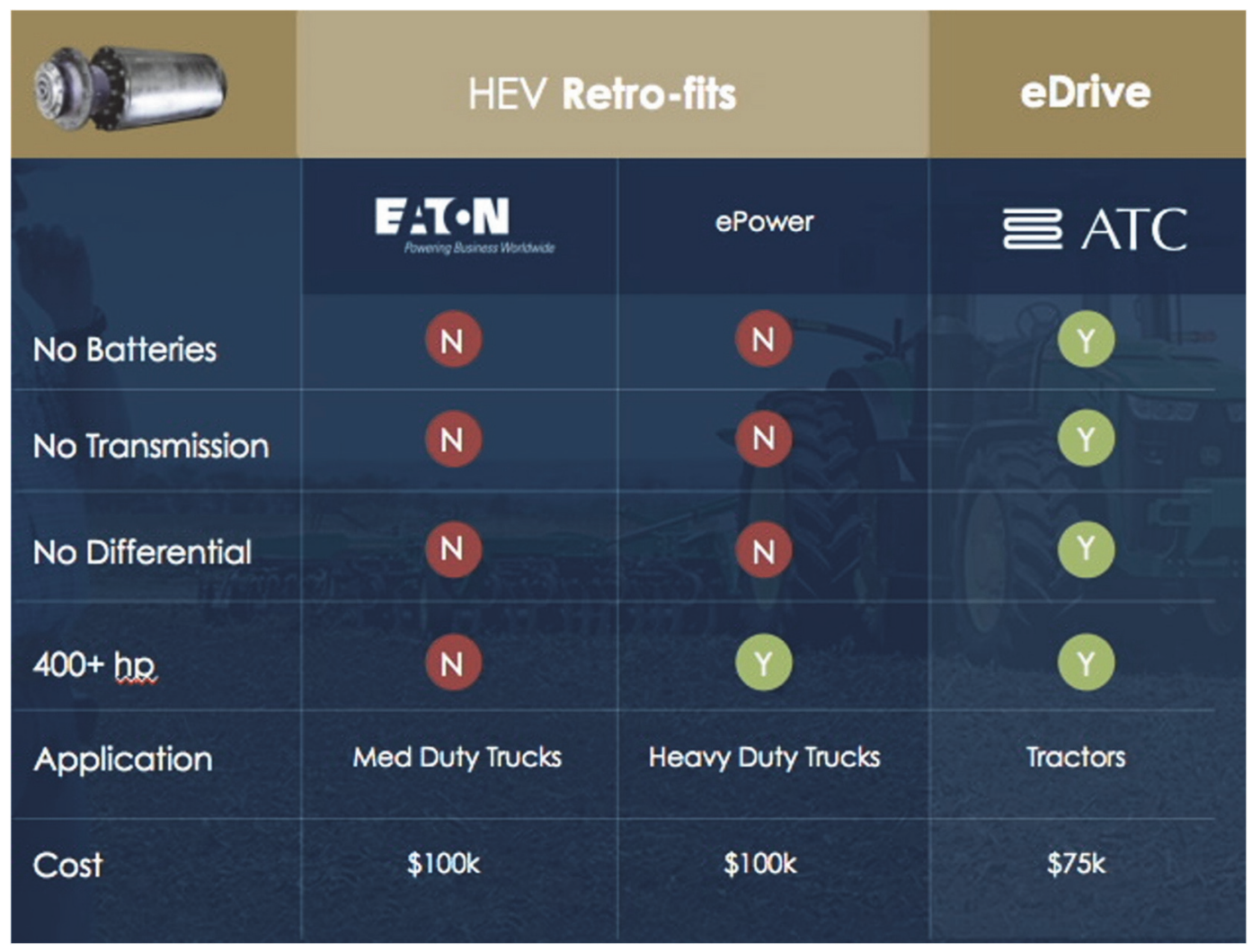

\begin{tabular}{|c|c|c|c|c|}
\hline & C6M by: BYD & C8M by: BYD & C9M by: BYD & C10M by: BYD \\
\hline Cost & \multicolumn{4}{|l|}{ Average: $\$ 297,000$} \\
\hline Application Maturity & \multicolumn{4}{|c|}{ Commercially available } \\
\hline $\begin{array}{l}\text { Charging Time } \\
\text { and Capacity }\end{array}$ & $\begin{array}{l}\text { 3-4 hours; } \\
40 \mathrm{~kW}\end{array}$ & $\begin{array}{l}4.5 \text { hours; } \\
80 \text { kW x } 2\end{array}$ & $\begin{array}{l}4-5 \text { hours; } \\
40 \mathrm{~kW} \times 2\end{array}$ & $2-2.5$ hours; $200 \mathrm{~kW}$ \\
\hline Charging Infrastructure & & & & \\
\hline
\end{tabular}




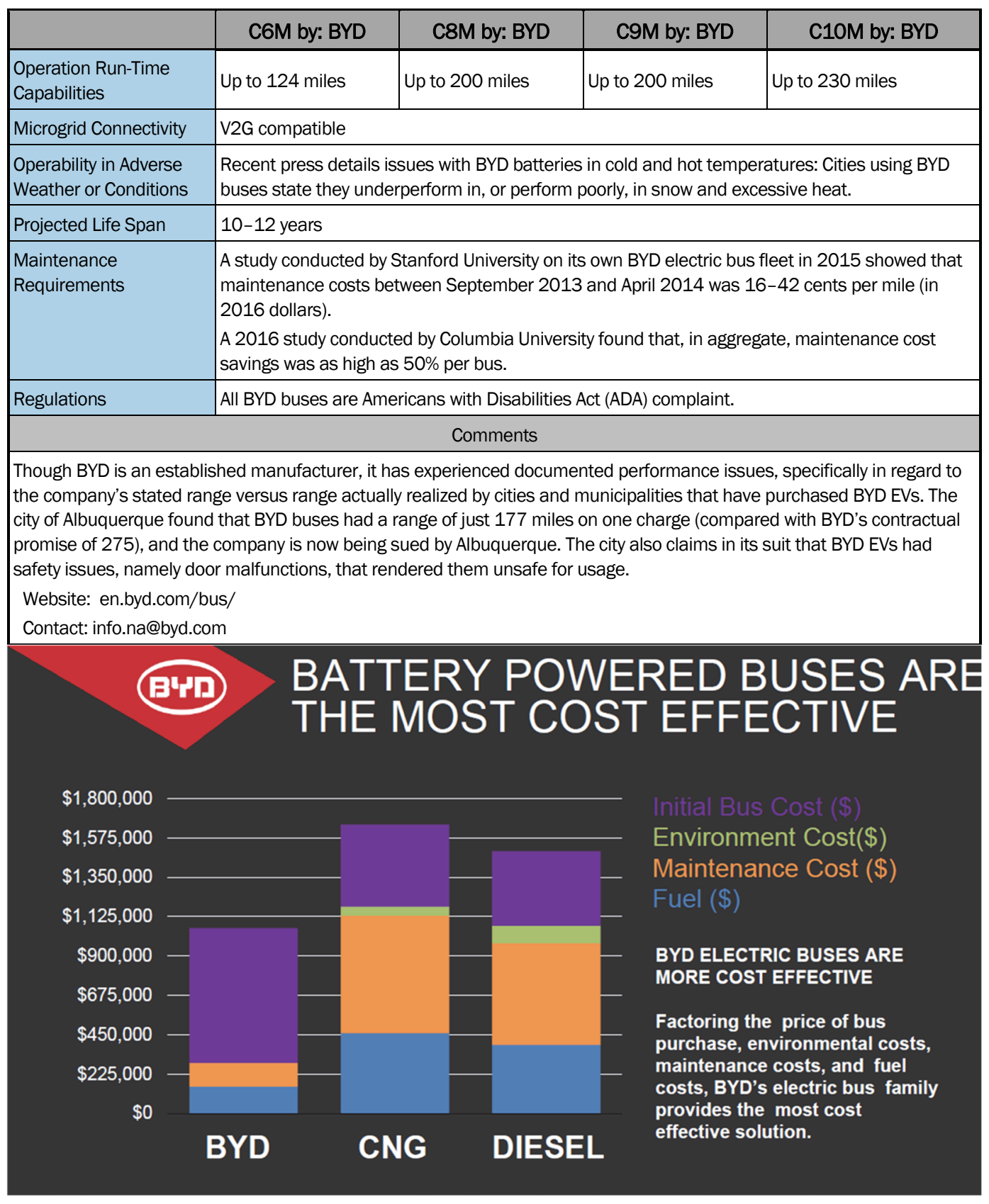




\section{Zero Emission City Bus Solution}

BYD -- the LARGEST electric bus manufacturer in the world. 1,500 units will be launched to the global market by the year end 2012.

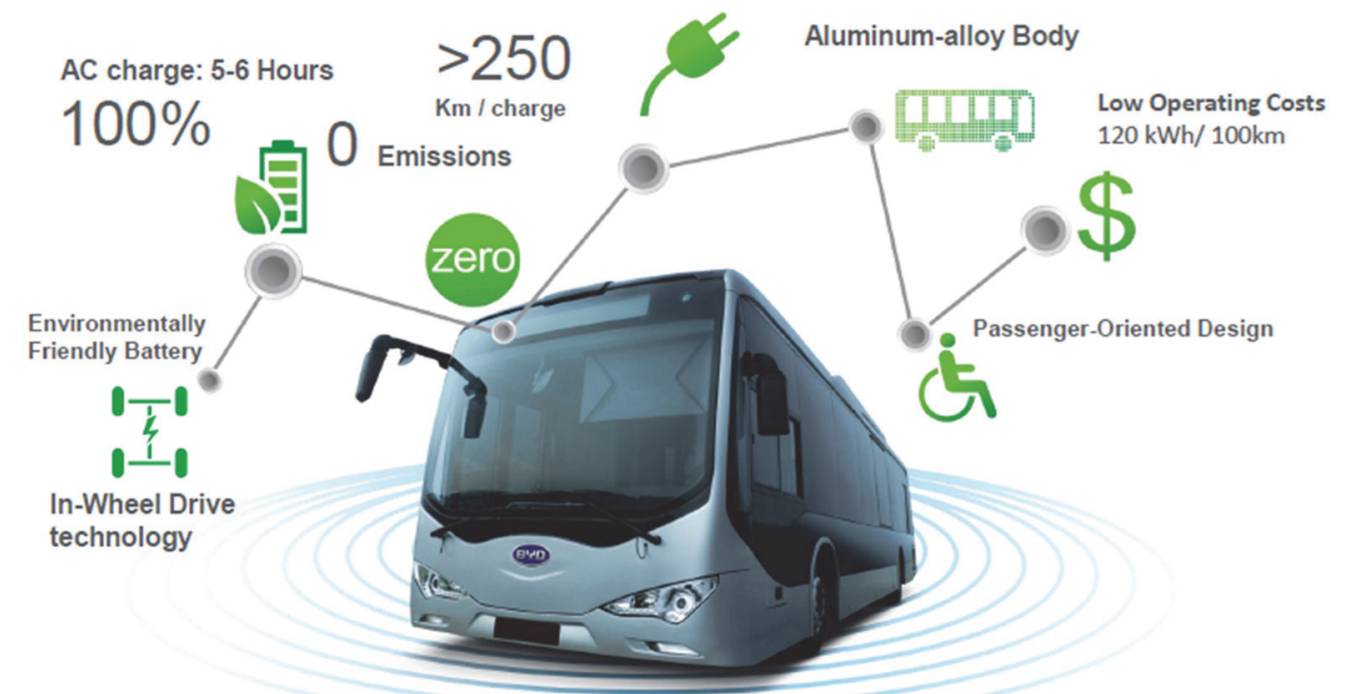

BYD I wmw.byd.com

\section{H.4 Class $5 \& 6$ Trucks Manufacturer: BYD}

BYD, headquartered in Los Angeles, CA, is the largest EV manufacturer in the world. The company is also one of the largest battery manufacturers worldwide, and its buses are manufac-

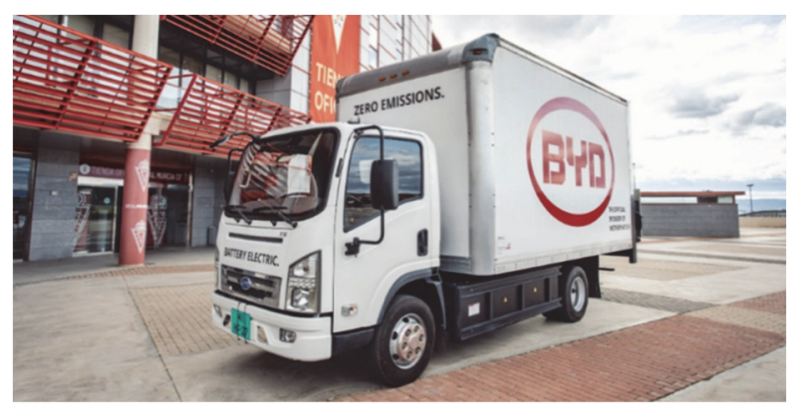
tured in a 450,000-square foot facility in Lancaster, CA.

BYD is the automotive subsidiary of the Chinese multinational BYD Co. LTD, which was founded in 2003 and is now based in Xi'an, Shaanxi Province and was founded in 2003.

\begin{tabular}{|l|l|l|}
\hline \multicolumn{1}{|c|}{ Class 5 by: BYD } & \multicolumn{1}{c|}{ Class 6 by: BYD } \\
\hline $\begin{array}{l}\text { Application } \\
\text { Maturity }\end{array}$ & Commercially available \\
\hline $\begin{array}{l}\text { Charging Time } \\
\text { and Capacity }\end{array}$ & 5 hours AC $(40 \mathrm{~kW}), 145 \mathrm{kWh}$ battery capacity & $\begin{array}{l}4.5 \text { hours AC/1.5 hours DC; } 221 \mathrm{kWh} \text { battery } \\
\text { capacity }\end{array}$ \\
\hline
\end{tabular}




\begin{tabular}{|c|c|c|}
\hline & Class 5 by: BYD & Class 6 by: BYD \\
\hline $\begin{array}{l}\text { Operation Run- } \\
\text { Time Capabilities }\end{array}$ & 155 miles (half load) & 124 miles (full load) \\
\hline $\begin{array}{l}\text { Microgrid } \\
\text { Connectivity }\end{array}$ & \multicolumn{2}{|l|}{ V2G compatible } \\
\hline Projected Life Span & \multicolumn{2}{|l|}{$10-12$ years. } \\
\hline $\begin{array}{l}\text { Maintenance } \\
\text { Requirements }\end{array}$ & \multicolumn{2}{|c|}{$\begin{array}{l}\text { BYD testing suggests average maintenance cost per mile is } 23 \text { cents per mile. While BYD provides up } \\
\text { to } 40 \text { hours of driver and preventative maintenance training to fleets purchasing their EVs, the } \\
\text { company is in CA, and accessing these services might prove problematic given the geographical } \\
\text { distance and lack of local service centers. }\end{array}$} \\
\hline $\begin{array}{l}\text { Hauling and } \\
\text { Towing Capacity }\end{array}$ & 16,141 GCWR & 26,000 lbs. GVWR \\
\hline \multicolumn{3}{|c|}{ Comments } \\
\hline \multicolumn{3}{|c|}{$\begin{array}{l}\text { Though BYD is an established manufacturer, it has experienced documented performance issues, specifically in regard to } \\
\text { the company's stated range versus range actually realized by cities and municipalities that have purchased BYD EVs. The } \\
\text { city of Albuquerque found that BYD buses had a range of just } 177 \text { miles on one charge (compared with BYD's contractual } \\
\text { promise of 275), and the company is now being sued by Albuquerque. The city also claims in its suit that BYD EVs had } \\
\text { safety issues, namely door malfunctions, that rendered them unsafe for usage. }\end{array}$} \\
\hline \multicolumn{3}{|c|}{ Website: en.byd.com/truck/\#models } \\
\hline \multicolumn{3}{|c|}{ Contact: info.na@byd.com } \\
\hline
\end{tabular}

\section{BYD Class 6 - Cab Chassis}

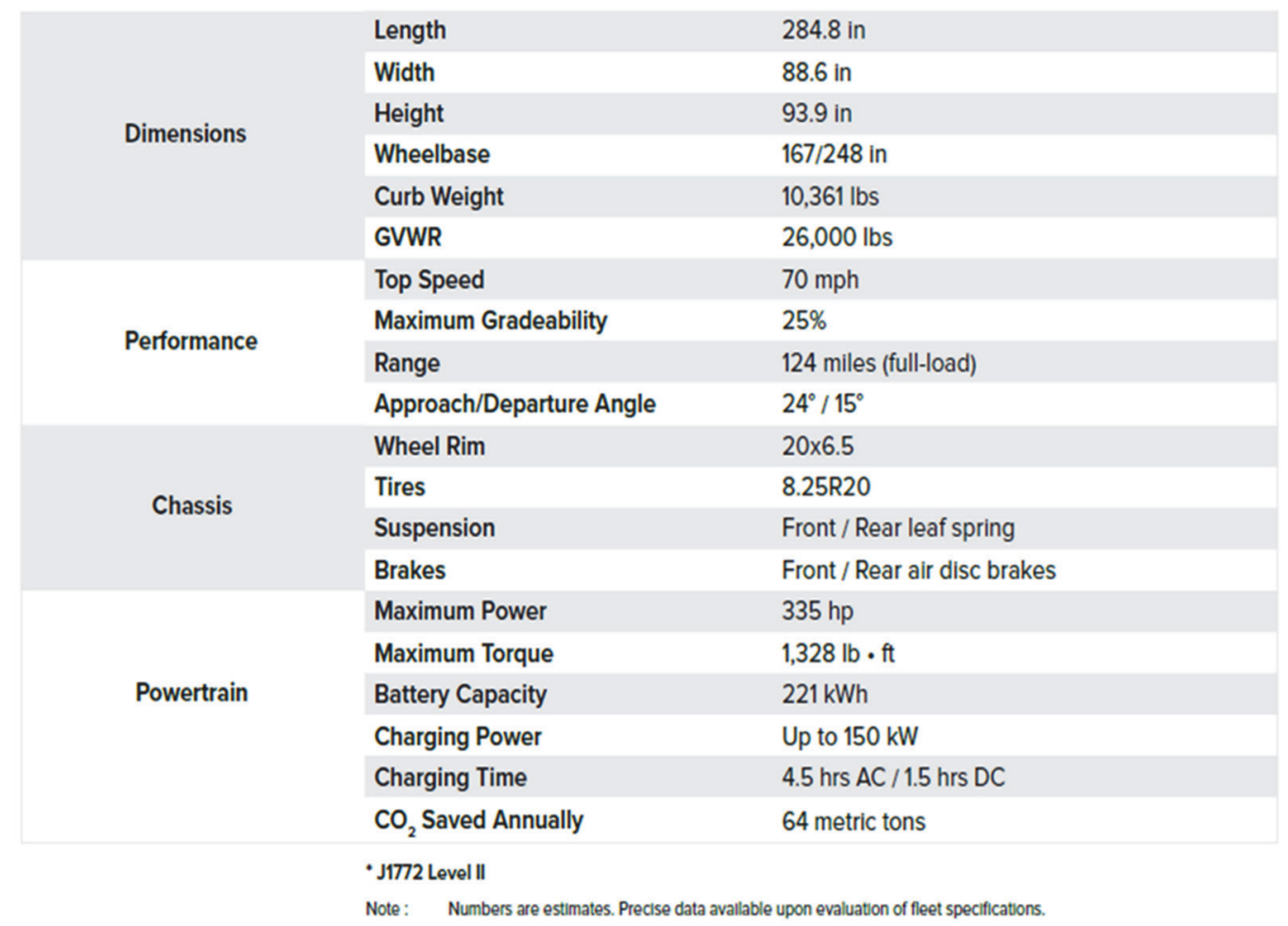




\section{BYD Class 5 - Cab Chassis}

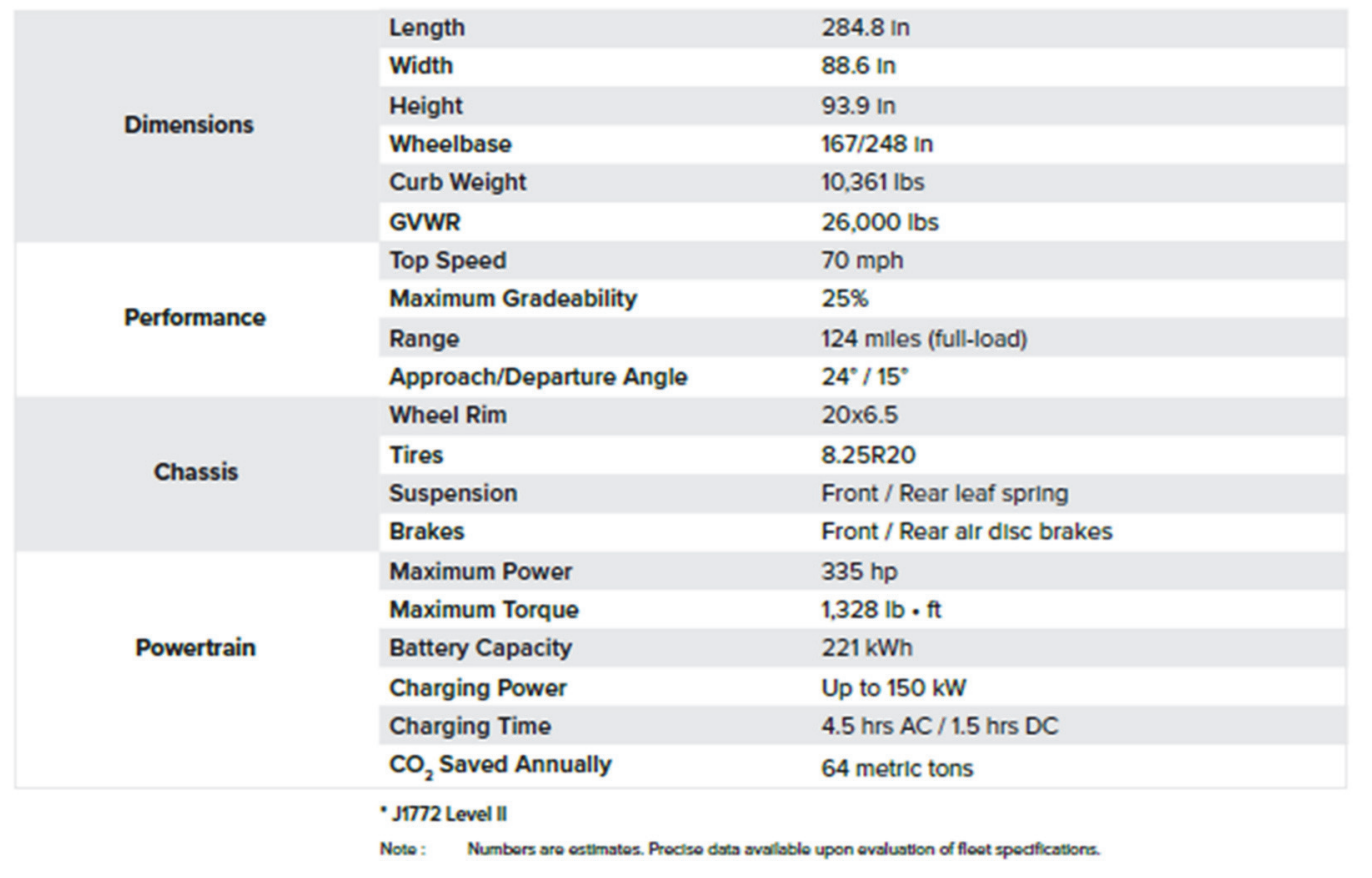

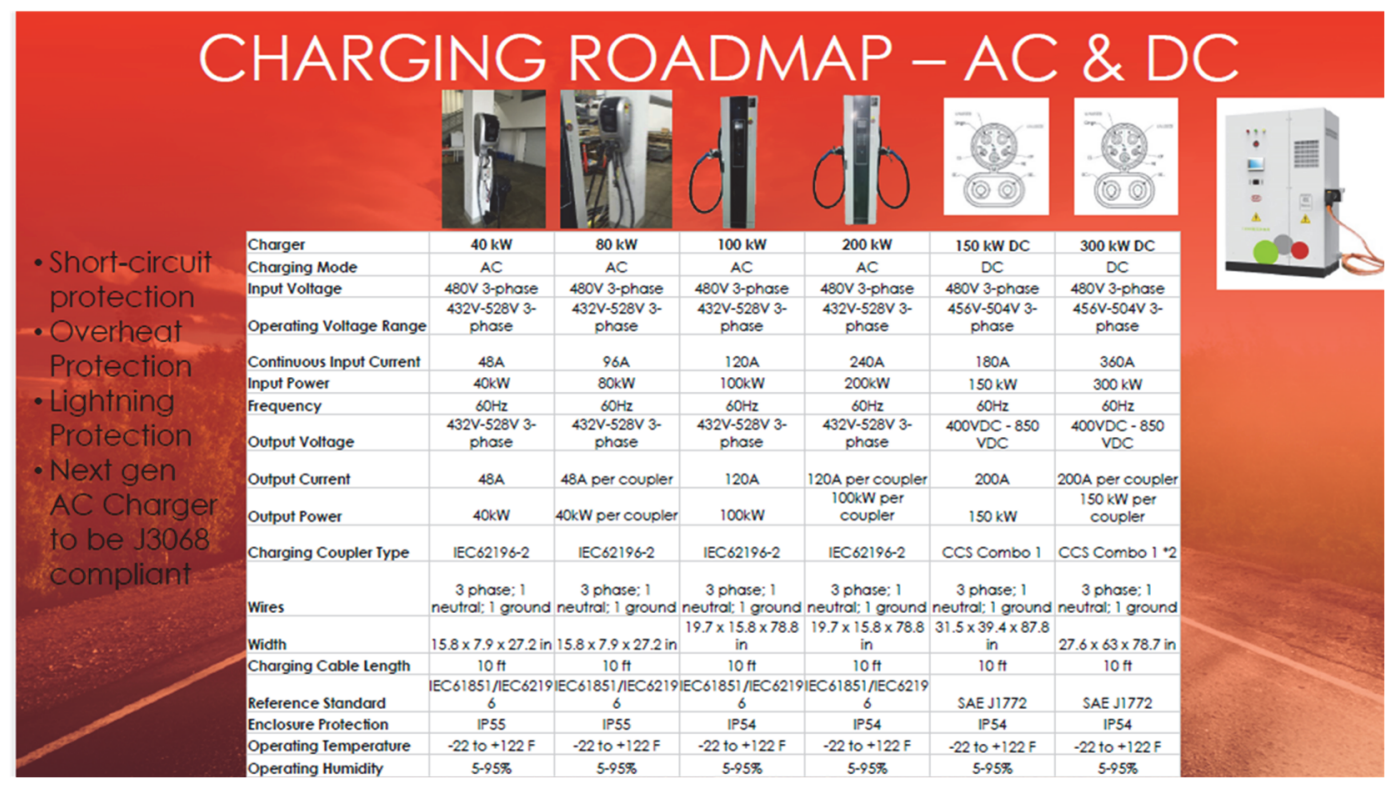




\section{H.5 BYD Class 8 Day Cab Manufacturer: BYD}

BYD, headquartered in Los Angeles, CA, is the largest EV manufacturer in the world. The company is also one of the largest battery manufacturers worldwide, and its buses are manufactured in a 450,000square foot facility in Lancaster, CA.

BYD is the automotive subsidiary of the Chinese multinational BYD Co. LTD, which was founded in 2003 and is now based in Xi'an, Shaanxi Province and was founded in 2003.

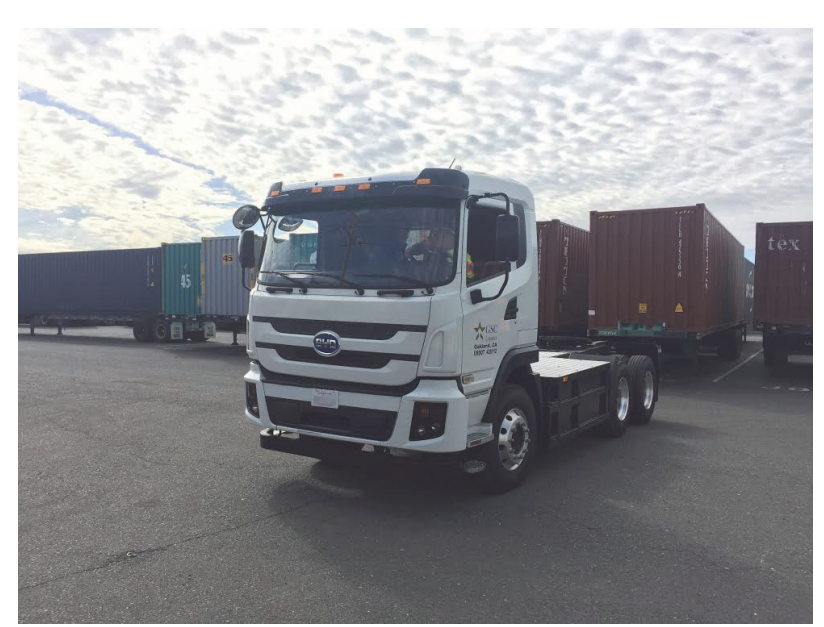

\begin{tabular}{|l|l|}
\hline \multicolumn{2}{|c|}{ BYD Class 8 Day Cab by: BYD } \\
\hline Cost & $\$ 300,000-\$ 350,000$ \\
\hline Application Maturity & Commercially available \\
\hline $\begin{array}{l}\text { Charging Time } \\
\text { and Capacity }\end{array}$ & 3 hours AC/1.5 hours DC.; $435 \mathrm{kWh}$ battery capacity \\
\hline $\begin{array}{l}\text { Charging } \\
\text { Infrastructure }\end{array}$ & Up to 300 kW \\
\hline $\begin{array}{l}\text { Operation Run-Time } \\
\text { Capabilities }\end{array}$ & 124 (full load), 167 (half load) \\
\hline $\begin{array}{l}\text { Microgrid } \\
\text { Connectivity }\end{array}$ & Compatible \\
\hline Projected Life Span & $\begin{array}{l}10-12 \text { years. A 2018 study from Carnegie Mellon states that the Class 8 trucks have a typical } \\
\text { lifetime mileage of 1 million miles. }\end{array}$ \\
\hline $\begin{array}{l}\text { Maintenance } \\
\text { Requirements }\end{array}$ & $\begin{array}{l}\text { BYD testing suggests average maintenance cost per mile is 23 cents per mile. While BYD provides up } \\
\text { to 40 hours of driver and preventative maintenance training to fleets purchasing their EVs, the } \\
\text { company is in CA, and accessing these services might prove problematic given the geographical } \\
\text { distance and lack of local service centers. }\end{array}$ \\
\hline $\begin{array}{l}\text { Hauling and } \\
\text { Towing Capacity }\end{array}$ & \begin{tabular}{l}
105,000 GCWR \\
\hline
\end{tabular} \\
\hline $\begin{array}{l}\text { Though BYD is an established manufacturer, it has experienced documented performance issues, specifically in regard to } \\
\text { the company's stated range versus range actually realized by cities and municipalities that have purchased BYD EVs. The } \\
\text { city of Albuquerque found that BYD buses had a range of just 177 miles on one charge (compared with BYD's contractual } \\
\text { promise of 275), and the company is now being sued by Albuquerque. The city also claims in its suit that BYD EVs had } \\
\text { safety issues, namely door malfunctions, that rendered them unsafe for usage. } \\
\text { Contact: info.na@byd.com }\end{array}$ \\
\hline
\end{tabular}




\begin{tabular}{|c|c|c|}
\hline \multirow{6}{*}{ Dimensions } & Length & 217.52 In \\
\hline & Width & 101.57 In \\
\hline & Height & 129.92 in \\
\hline & Wheelbase & 118 in \\
\hline & Curb Weight & 19,800 lbs \\
\hline & GCWR & $102,000 \mathrm{lbs}$ \\
\hline \multirow{4}{*}{ Performance } & Top Speed & $32^{*} \mathrm{mph}$ \\
\hline & Maximum Gradeability & $15 \%$ \\
\hline & Range & $10+$ hours \\
\hline & Approach/Departure Angle & $27^{\circ} / 33^{\circ}$ \\
\hline \multirow{4}{*}{ Chassis } & Wheel Rim & $22.5 \times 8.25$ \\
\hline & Tires & $11 R 22.5$ \\
\hline & Suspension & Front: Leaf spring, Rear: Solıd mount \\
\hline & Brakes & Front / Rear Alr drum brakes \\
\hline \multirow{6}{*}{ Powertrain } & Maximum Power & $241 \mathrm{hp}$ \\
\hline & Maximum Torque & $1,106 \mathrm{lb} \cdot \mathrm{ft}$ \\
\hline & Battery Capacity & $217 \mathrm{kWh}$ \\
\hline & Charging Power & Up to $200 \mathrm{~kW}$ \\
\hline & Charging Time & $3 \mathrm{hrs}$ AC / 1.5 hrs DC \\
\hline & $\mathrm{CO}_{2}$ Saved Annually & 105.9 metric tons \\
\hline
\end{tabular}

\section{H.6 K Series Shuttles Manufacturer: BYD}

BYD, headquartered in Los Angeles, CA, is the largest EV manufacturer in the world. The company is also one of the largest battery manufacturers worldwide, and its buses are manufactured in a 450,000-square foot facility in Lancaster, CA.

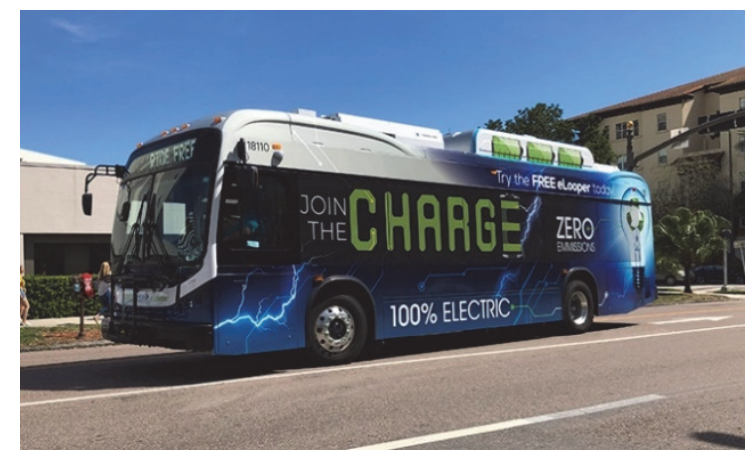

BYD is the automotive subsidiary of the Chinese multinational BYD Co. LTD, which was founded in 2003 and is now based in Xi'an, Shaanxi Province and was founded in 2003.

\begin{tabular}{|c|c|c|c|c|}
\hline & K7 by: BYD & K9S by: BYD & K9 by: BYD & K11 by: BYD \\
\hline Cost & \multicolumn{4}{|l|}{ Average: $\$ 750,000$} \\
\hline Application Maturity & \multicolumn{4}{|c|}{ Commercially available } \\
\hline $\begin{array}{l}\text { Charging Time and } \\
\text { Capacity }\end{array}$ & 3-4 hours; $80 \mathrm{~kW}$ & $\begin{array}{l}2-3 \text { hours; } \\
352 \mathrm{kWh}\end{array}$ & $\begin{array}{l}2-3 \text { hours; } \\
80 \mathrm{kWh}\end{array}$ & $\begin{array}{l}3-4 \text { hours; } \\
200 \mathrm{~kW}\end{array}$ \\
\hline $\begin{array}{l}\text { Operation } \\
\text { Run-Time Capabilities }\end{array}$ & Up to 137 miles & $\begin{array}{l}\text { Up to } 215 \text { miles (high- } \\
\text { capacity) }\end{array}$ & Up to 156 miles & Up to 220 miles \\
\hline Microgrid Connectivity & \multicolumn{4}{|c|}{$\begin{array}{l}\text { Relying on its own lithium-ion, iron-phosphate battery technology, BYD offers large-scale ESSs, } \\
\text { distributed ESSs, and microgrid systems. }\end{array}$} \\
\hline $\begin{array}{l}\text { Operability in Adverse } \\
\text { Weather or Conditions }\end{array}$ & \multicolumn{4}{|c|}{$\begin{array}{l}\text { Recent press details issues with BYD batteries in cold and hot temperatures: Cities using BYD } \\
\text { buses state they underperform in, or perform poorly in, snow and excessive heat. }\end{array}$} \\
\hline
\end{tabular}




\begin{tabular}{|c|c|c|c|c|}
\hline & K7 by: BYD & K9S by: BYD & K9 by: BYD & K11 by: BYD \\
\hline Projected Life Span & \multicolumn{4}{|c|}{ Typically, battery lifespan is $6-8$ years while bus lifespan is $10-12$ years. } \\
\hline \multirow{2}{*}{$\begin{array}{l}\text { Maintenance } \\
\text { Requirements }\end{array}$} & \multirow{2}{*}{\multicolumn{4}{|c|}{$\begin{array}{l}\text { A study conducted by Stanford University on its own BYD electric bus fleet in } 2015 \text { showed that } \\
\text { maintenance costs between September } 2013 \text { and April } 2014 \text { was } 16-42 \text { cents per mile (in } \\
2016 \text { dollars). } \\
\text { A } 2016 \text { study conducted by Columbia University found that, in aggregate, maintenance cost } \\
\text { savings was as high as } 50 \% \text { per bus. }\end{array}$}} \\
\hline & & & & \\
\hline Regulations & \multicolumn{4}{|c|}{ All BYD buses are ADA compliant. } \\
\hline \multicolumn{5}{|c|}{ Comments } \\
\hline \multicolumn{5}{|c|}{$\begin{array}{l}\text { Though BYD is an established manufacturer, it has experienced documented performance issues, specifically in regard to } \\
\text { the company's stated range versus range actually realized by cities and municipalities that have purchased BYD EVs. The } \\
\text { city of Albuquerque found that BYD buses had a range of just } 177 \text { miles on one charge (compared with BYD's contractual } \\
\text { promise of 275), and the company is now being sued by Albuquerque. The city also claims in its suit that BYD EVs had } \\
\text { safety issues, namely door malfunctions, that rendered them unsafe for usage. }\end{array}$} \\
\hline \multicolumn{5}{|c|}{ Website; en.byd.com/bus/ } \\
\hline \multicolumn{5}{|c|}{ Contact: info.na@byd.com } \\
\hline
\end{tabular}




\section{H.7 Terminal Tractor Manufacturer: BYD}

$\mathrm{BYD}$, headquartered in Los Angeles, CA, is the largest $\mathrm{EV}$ manufacturer in the world. The company is also one of the largest battery manufacturers worldwide, and its buses are manufactured in a 450,000-square foot facility in Lancaster, CA.

BYD is the automotive subsidiary of the Chinese multinational BYD Co. LTD, which was founded in 2003 and is now

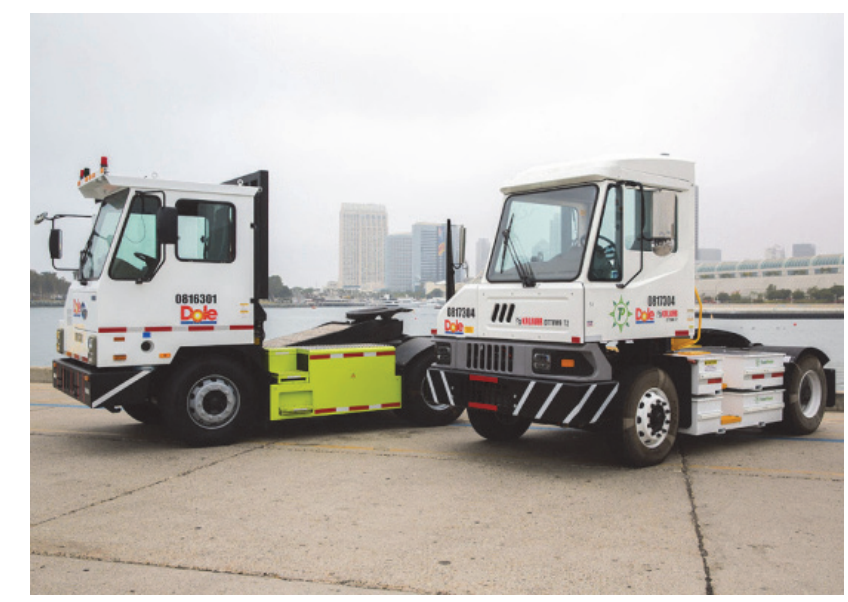
based in Xi'an, Shaanxi Province and was founded in 2003.

\begin{tabular}{|l|l|}
\hline \multicolumn{2}{|c|}{ Terminal Tractor by: BYD } \\
\hline Cost & $\$ 300,000$ \\
\hline Application Maturity & Commercially available \\
\hline $\begin{array}{l}\text { Charging Time } \\
\text { and Capacity }\end{array}$ & 3 hours AC/1.5 hours DC; 217 kWh battery capacity \\
\hline Charging Infrastructure & $66-k W$ AC or 120-kW DC \\
\hline Operation Run-Time Capabilities & $10+$ hours \\
\hline Microgrid Connectivity & Compatible \\
\hline Projected Life Span & $10-12$ years \\
\hline Maintenance Requirements & BYD testing suggests average maintenance cost per mile is 23 cents per mile. \\
\hline $\begin{array}{l}\text { Hauling and } \\
\text { Towing Capacity }\end{array}$ & 102,000 GCWR \\
\hline \multicolumn{2}{|c|}{ Comments } \\
\hline $\begin{array}{l}\text { Though BYD is an established manufacturer, it has experienced documented performance issues, specifically in regard to } \\
\text { the company's stated range versus range actually realized by cities and municipalities that have purchased BYD EVs. The } \\
\text { city of Albuquerque found that BYD buses had a range of just 177 miles on one charge (compared with BYD's contractual } \\
\text { promise of 275), and the company is now being sued by Albuquerque. The city also claims in its suit that BYD EVs had } \\
\text { safety issues, namely door malfunctions, that rendered them unsafe for usage. } \\
\text { Website: en.byd.com/truck/ } \\
\text { Contact: info.na@byd.com }\end{array}$ \\
\hline
\end{tabular}




\section{H.8 V8100 Refrigerated Van Manufacturer: Chanje}

Chanje Energy Inc., founded in 2015 and based in Los Angeles, CA, is an OEM delivering medium-duty EVs and turn-key energy infrastructure services for the last mile energy. The company is a subsidiary of FDG EVs, the international brand for FDG. In mid-2018, Chanje announced

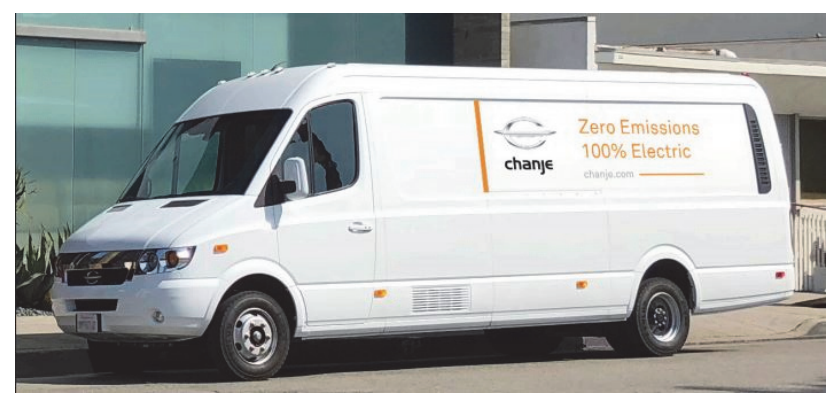
that Ryder would be the exclusive sales channel and service partner for the company and its fleet. Ryder will offer leasing and preventative maintenance solutions as part of its ChoiceLease and SelectCare fleet maintenance portfolio.

The V810o is manufactured in Hangzhou, China by FDG.

\begin{tabular}{|l|l|}
\hline \multicolumn{2}{|c|}{ V8100 Refrigerated Van by: Chanje } \\
\hline Cost & $\begin{array}{l}\text { Ryder's ChoiceLease program offers the V8100 for \$999 per month for a 3-year term or \$899 per } \\
\text { month for a 7-year lease. Pricing includes 1 EV mobile charger by eMotorWerks and four preventative } \\
\text { maintenance services annually. }\end{array}$ \\
\hline Application Maturity & $\begin{array}{l}\text { Ryder serves as the exclusive sales partner for Chanje, offering lease and rental of the V8100. The } \\
\text { company states the lease program will be available in “strategic markets" throughout 2019. That } \\
\text { said, FedEx purchased 100 V8100's from Chanje in November 2018. }\end{array}$ \\
\hline $\begin{array}{l}\text { Charging Time } \\
\text { and Capacity }\end{array}$ & 10 hours or 80\% at 1 hour for DC Fast; 100 kWh \\
\hline $\begin{array}{l}\text { Charging } \\
\text { Infrastructure }\end{array}$ & $13.2-k W$ onboard charger, Level 2 and DC Fast \\
\hline $\begin{array}{l}\text { Operation Run- } \\
\text { Time Capabilities }\end{array}$ & 150 miles \\
\hline $\begin{array}{l}\text { Hauling and Towing } \\
\text { Capacity }\end{array}$ & 6,000 lbs. payload/16,535 GVWR, 675 cu. ft. storage \\
\hline \multicolumn{2}{|c|}{ Comments } \\
\hline $\begin{array}{l}\text { The V8100 is built to meet the specific needs of the last mile industry. } \\
\text { Website chanje.com/vehicles/ } \\
\text { Contact info@chanje.com }\end{array}$ \\
\hline
\end{tabular}




\section{H.9 Mobile Power Station (MPS) Manufacturer: Dannar}

Dannar is an OEM of mobile, off-road energy platforms for the U.S. governmental market. Dannar is located in Clemente, CA; San Jose, CA; and Muncie, IN (where it is also manufactured). The company currently offers the Dannar 3.00, a base configuration that comes with standard two 33-kWh BMW i3 lithium-ion battery packs (66 kWh total), which can be

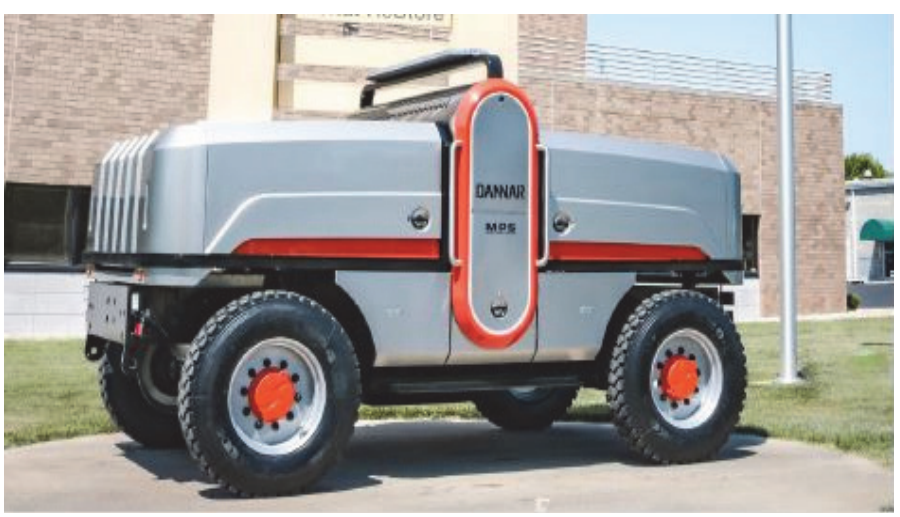
upgraded to four packs for a total of $132 \mathrm{kWh}$ onboard electricity. The Dannar 4.00 base configuration comes standard with three 42-kWh BMW i3 lithium-ion battery packs (126 kWh total); the Dannar 4.00 can be upgraded with up to nine additional packs for a total of $504 \mathrm{kWh}$ of onboard electricity. The Dannar 4.00 is built on a heavy-duty steel ladder frame and can be ordered with or without an operator cab or platform.

\begin{tabular}{|c|c|c|}
\hline & Dannar 3.00 by: Dannar & Dannar 4.00 by: Dannar \\
\hline \multicolumn{3}{|l|}{ Cost } \\
\hline $\begin{array}{l}\text { Application } \\
\text { Maturity }\end{array}$ & Commercially available & Commercially available \\
\hline Safety & Light bar comes standard & Light bar comes standard \\
\hline $\begin{array}{l}\text { Extras and } \\
\text { Attachments }\end{array}$ & $\begin{array}{l}\text { Optional open operator cab platform equipped } \\
\text { with joystick, foot pedal, and touchscreen tool } \\
\text { control. Base model is equipped with } \\
\text { universal quick mounting plates that can } \\
\text { receive CAT, Bobcat, or John Deere } \\
\text { attachments, and an optional Cummins Tier IV } \\
\text { generator ( } 60 \mathrm{~kW} \text { ) is available. Submersible } \\
\text { up to four feet and carries Type I, II, and III } \\
\text { hydraulic tool circuits. Four-wheel, multi-mode } \\
\text { steering. } \\
\text { At minimum, Dannar } 3.00 \text { and } \\
4.00 \text { microgrids can charge two Tesla EVs or } \\
\text { operate for } 8-10 \text { hours on site. At max, } \\
\text { systems offer enough energy to power nearly } \\
17 \text { houses for } 24 \text { hours. }\end{array}$ & $\begin{array}{l}\text { Export panel is configurable with multiple } 110-V A C \text { and } \\
208-V A C \text { outlets. The base model comes standard with } \\
\text { dual flat beds and can accommodate scissor lifts, } \\
\text { forklift masts, dump beds, water tanks, electric water } \\
\text { pumps, portable light units, or mobile telescoping cell } \\
\text { towers. Can accommodate all standard Caterpillar, } \\
\text { Bobcat, and John Deere attachments. Its submersible } \\
\text { up to } 4 \text { feet. Carries Type I, II, and II hydraulic tool } \\
\text { circuits, four-wheel drive, and four-wheel multi-mode } \\
\text { steering. } \\
\text { At minimum, Dannar } 3.00 \text { and } 4.00 \text { microgrids can } \\
\text { charge two Tesla EVs or operate for } 8-10 \text { hours onsite. } \\
\text { At max, systems offer enough energy to power nearly } \\
17 \text { houses for } 24 \text { hours. }\end{array}$ \\
\hline $\begin{array}{l}\text { Operability in } \\
\text { Adverse Weather } \\
\text { or Conditions }\end{array}$ & $\begin{array}{l}\text { eDrive motors are liquid cooled and totally } \\
\text { sealed, making them ideal for use in mud, } \\
\text { dust, and } \\
\text { high-temperature field } \\
\text { conditions. }\end{array}$ & \\
\hline $\begin{array}{l}\text { Projected Life } \\
\text { Span }\end{array}$ & $\begin{array}{l}25,000 \text { hours ( } 50 \% \text { longer than typical tractor } \\
\text { lifespan) }\end{array}$ & 20 years \\
\hline Communication & \multicolumn{2}{|c|}{$\begin{array}{l}\text { Operator cab, operator platform, or fully remote for distance safety. TBD wireless supervision-"follow } \\
\text { me" and autonomous operation in development. }\end{array}$} \\
\hline
\end{tabular}




\begin{tabular}{|c|c|c|}
\hline & Dannar 3.00 by: Dannar & Dannar 4.00 by: Dannar \\
\hline $\begin{array}{l}\text { Charging Time } \\
\text { and Capacity }\end{array}$ & \multicolumn{2}{|l|}{ Up to $504 \mathrm{kWh}$ of battery storage } \\
\hline $\begin{array}{l}\text { Charging } \\
\text { Infrastructure }\end{array}$ & \multicolumn{2}{|c|}{ Base unit-DC Fast charge capable (CCS2 Combo), roadmap to $500 \mathrm{~kW}$ inbound } \\
\hline $\begin{array}{l}\text { Microgrid } \\
\text { Connectivity }\end{array}$ & \multicolumn{2}{|l|}{$\begin{array}{l}\text { Up to } 504 \text { kWh of battery storage } \\
\text { Optional } 60-k W \text { hydrogen-fuel cell } \\
\text { Optional multi-fuel 60-W gen set } \\
\text { V2G compatibility } \\
\text { Mobile, off-grid power source }\end{array}$} \\
\hline $\begin{array}{l}\text { Operability in } \\
\text { Adverse Weather } \\
\text { or Conditions }\end{array}$ & \multicolumn{2}{|c|}{ Designed for disaster relief; vehicles can function in wet conditions. } \\
\hline $\begin{array}{l}\text { Climate and } \\
\text { Geographical } \\
\text { Restrictions }\end{array}$ & \multicolumn{2}{|c|}{ Climate limitations not described in terms of temperature-can function in up to 4 feet of water. } \\
\hline $\begin{array}{l}\text { Environmental } \\
\text { Risks }\end{array}$ & \multicolumn{2}{|c|}{ System is zero emission (electric) and designed for use in various disaster relief scenarios. } \\
\hline $\begin{array}{l}\text { Off-road } \\
\text { Capabilities and } \\
\text { Limitations }\end{array}$ & \multicolumn{2}{|c|}{$\begin{array}{l}\text { Rugged MPS units able to be deployed safely. Heavy-duty, water submergible to } 4 \text { feet, work tools can } \\
\text { be attached for clearing debris. }\end{array}$} \\
\hline $\begin{array}{l}\text { Current } \\
\text { Automation } \\
\text { Capabilities }\end{array}$ & \multicolumn{2}{|c|}{$\begin{array}{l}\text { Operate by onboard cab, X-box style remote control or TBD wireless supervision-"follow me" and } \\
\text { autonomous operation in development. }\end{array}$} \\
\hline $\begin{array}{l}\text { Future } \\
\text { Automation } \\
\text { Capabilities }\end{array}$ & \multicolumn{2}{|c|}{ Dannar is currently working to develop follow-the-leader autonomous function. } \\
\hline \multicolumn{3}{|c|}{ Comments } \\
\hline \multicolumn{3}{|c|}{$\begin{array}{l}\text { Features, specs, and industry examples in airports, agriculture, first responders, public works, universities, and } \\
\text { warehouses here. } \\
\text { Website: dannar.us.com } \\
\text { Contact: } 765-216-7191 \text {; Contact Don Gonneville for GSA sales at 949-248-7297 or sales@gonneville.com }\end{array}$} \\
\hline
\end{tabular}




\section{H.10 Ebus}

\section{Manufacturer: Ebus, Inc.}

Founded in 1998, Ebus is based in Downey, CA and develops electric bus technology. The company's electric propulsion system consists of a proprietary oil-cooled traction motor, a liquid-cooled traction converter, and an advanced liquid-cooled nickel-manganese-co-

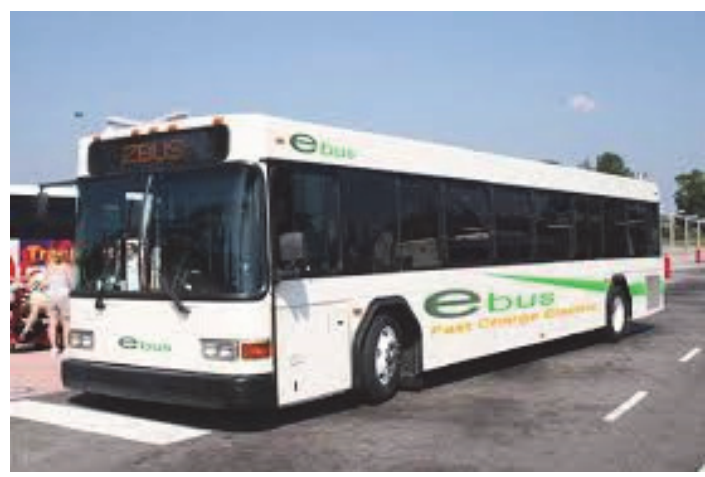
balt $89-\mathrm{kWh}$ battery system composed of 320 cells, which are manufactured in Midland, MI, by Dow Kokam LLC. Ebus's systems allow for a diesel or CNG bus to be retrofitted as a battery-electric bus via a pre-engineered conversion kit.

Ebus utilizes a fully integrated fast-charge system for its buses called Gamma. Fast charging en route allows buses to operate all day, and the Gamma-IP is based on the Gamma self-contained fast-charge station. It was redesigned for a top down Inverted Pantograph and complies with OppCharge and the SAE J-3105 Overhead Charging Standard.

\begin{tabular}{|c|c|}
\hline \multicolumn{2}{|r|}{ Ebus by: Ebus } \\
\hline Cost & $\$ 395,000$ \\
\hline $\begin{array}{l}\text { Application } \\
\text { Maturity }\end{array}$ & $\begin{array}{l}\text { The Ebus } 22 \text { is currently commercially available, and the Ebus Composite bus became available for delivery } \\
\text { in 2019-2020. }\end{array}$ \\
\hline $\begin{array}{l}\text { Charging } \\
\text { Time and } \\
\text { Capacity }\end{array}$ & 6 hours; $300 \mathrm{kWh}$ lithium-iron-phosphate battery \\
\hline $\begin{array}{l}\text { Charging } \\
\text { Infrastructure }\end{array}$ & $\begin{array}{l}\text { Ebus's utilize both fast and opportunity charging systems, known as BS\&CS and Gamma-IP, respectively. } \\
\text { The former allows for removable batteries to be swapped out via a "station" roughly every } 4 \text { hours, and the } \\
\text { latter system, with } 45-\mathrm{kW} \text { peak-charging power, is a self-contained fast-charge station, which is designed } \\
\text { for a top down IP. The company maintains that installation of the Gamma-IP system is simple and } \\
\text { inexpensive, requiring only a small concrete pad with J-bolts and a } 480-\mathrm{V} 3 \text {-phase power source located } \\
\text { under the pad. Ebus's can also be charged via a concealed conductive charger connection (4C) for in- } \\
\text { ground } 450-\mathrm{kW} \text { fast charging. }\end{array}$ \\
\hline $\begin{array}{l}\text { Operation } \\
\text { Run-Time } \\
\text { Capabilities }\end{array}$ & 125-mile range from nightly charge. \\
\hline $\begin{array}{l}\text { Projected Life } \\
\text { Span }\end{array}$ & 7 years/200,000 miles \\
\hline $\begin{array}{l}\text { Maintenance } \\
\text { Requirements }\end{array}$ & $\begin{array}{l}\text { A } 2006 \text { case study from the National Renewable Energy Laboratory (NREL) found that the average parts } \\
\text { and maintenance cost over the course of a year was } 88 \text { cents per mile. }\end{array}$ \\
\hline
\end{tabular}




\section{Comments}

The Ebus propulsion system and charging technology can be ordered from OEM bus suppliers. Retrofitted systems include electric motor and battery and charging systems. The systems provide a repurposing alternative resulting in vehicles configured to specific uses.

The Ebus automated Battery Swap and Charging Station system allows for the bus's removable 2000-pound battery to be removed and swapped out from above in less than one minute. The company states that the BS\&CS accommodates batteries for up to 24 buses, making it a more effective charging system than fast charging.

Website: ebus.com/

Contact: 562-904-3474 or 888-925-4263; sales@ebus.com 


\section{H.11 ZeroTruck Medium-duty Trucks Manufacturer: Electrorides, Inc.}

Founded in 2008, ZeroTruck manufactures all-electric medium-duty trucks with advance regenerative braking systems. The company is located in Santa Ana, CA. All vehicles are delivered to the customer via dealers. ZeroTruck's parent company is

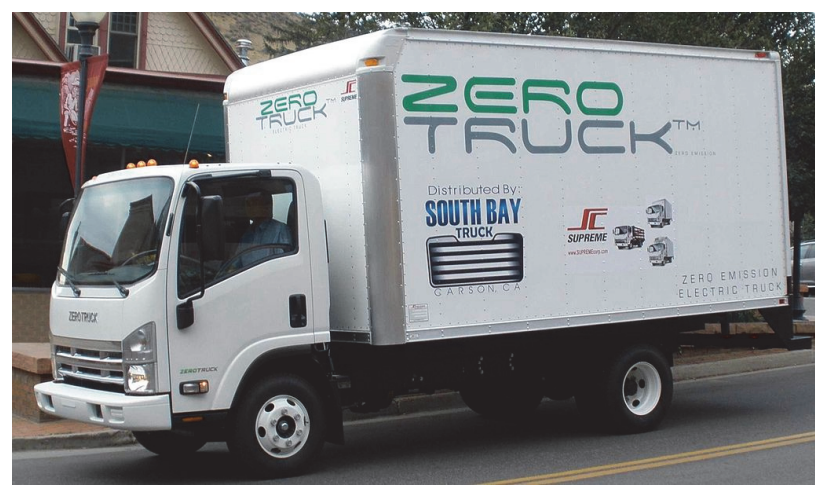
Electrorides, Inc., based in Laguna Niguel, CA.

ZeroTrucks are Class 4 or 5, based on the Isuzu NPR, feature the company's Electric Drive Integration System: proprietary battery modules using lithium-polymer cells. The battery packs are liquid cooled and single-speed, 3-speed, and 6-speed transmissions are offered.

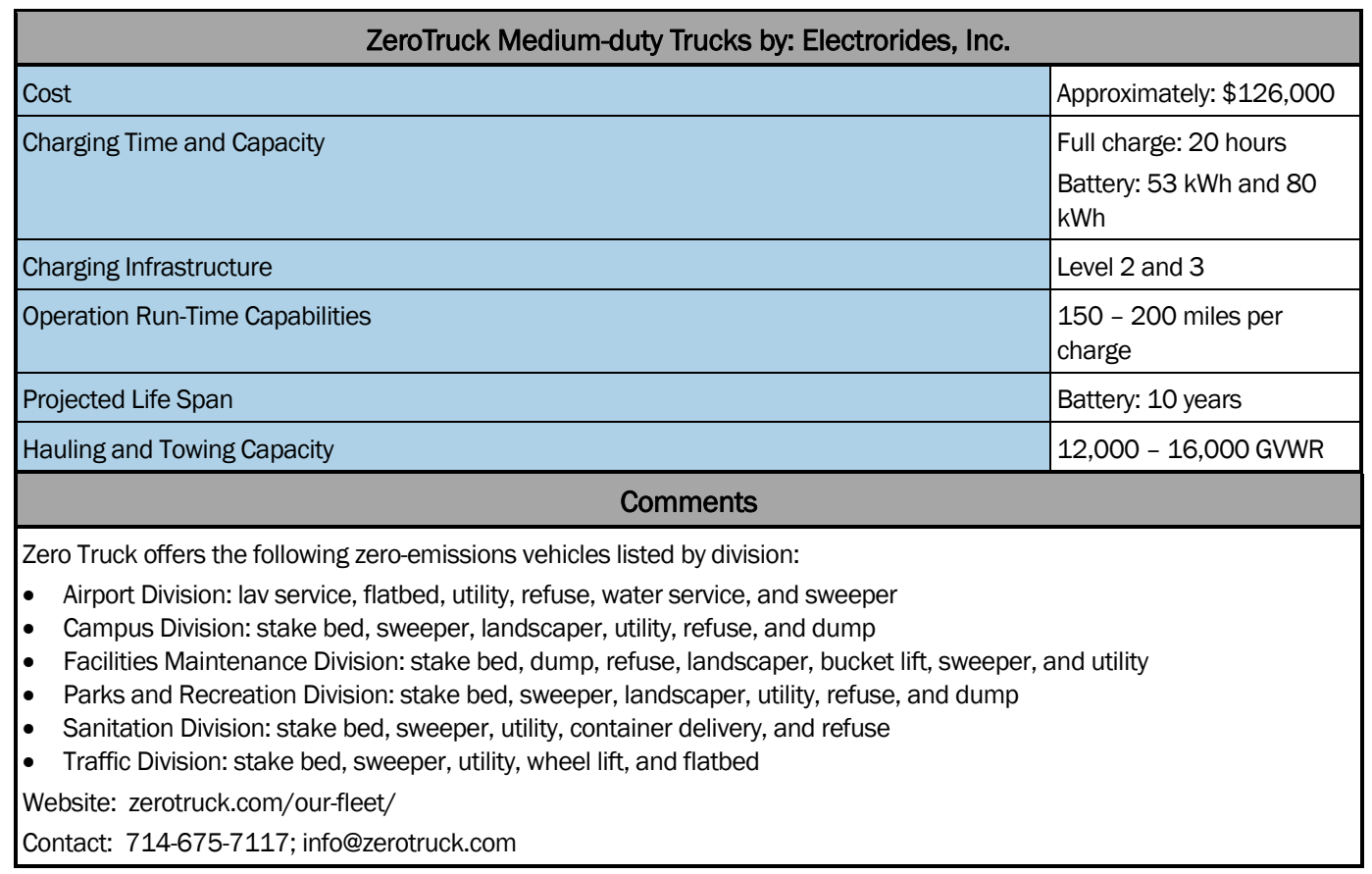




\begin{tabular}{|c|c|c|c|c|c|}
\hline \multicolumn{6}{|c|}{ SPECIFICATIONS } \\
\hline & & & ZT std & & ZT Optional Heavy Duty Chassis \\
\hline $\begin{array}{l}\text { Chassis } \\
\text { - Wheelbase } \\
\text { - Cab to Axle } \\
\text { - Cab to End of Frame } \\
\text { - Overall Length } \\
\text { Body Length (ft.) }\end{array}$ & $\begin{array}{c}109^{\prime \prime} \\
86.5^{\prime \prime} \\
129.6^{\prime \prime} \\
200.5^{\prime \prime} \\
10-12^{\prime}\end{array}$ & $\begin{array}{l}132.5^{\prime \prime} \\
110^{\prime \prime} \\
153.1^{\prime \prime} \\
224^{\prime \prime} \\
14^{\prime}\end{array}$ & $\begin{array}{c}150^{\prime \prime} \\
127.5^{\prime \prime} \\
170.6^{\prime \prime} \\
241.5^{\prime \prime} \\
16-18^{\prime}\end{array}$ & $\begin{array}{c}176^{\prime \prime} \\
153.5^{\prime \prime} \\
196.6^{\prime \prime} \\
267.5^{\prime \prime} \\
20^{\prime}\end{array}$ & $\begin{array}{c}\text { (same as standard ZT) } \\
. \\
. \\
.\end{array}$ \\
\hline $\begin{array}{l}\text { GVWR/GCWR } \\
\text { Body/Payload Allowance ( }\end{array}$ & $\begin{array}{l}12,( \\
\text { ith body) }\end{array}$ & $\begin{array}{r}000 / 18,00 \\
6000 \mathrm{lb}\end{array}$ & & & $\begin{array}{c}19,500 / 20,500 \text { lbs. } \\
7000 \text { lbs. }\end{array}$ \\
\hline $\begin{array}{ll}\text { GAWR } \\
\text { - } & \text { Front } \\
\text { - } & \text { Rear } \\
\text { - } & \text { Front Axle Capacity } \\
\text { - } & \text { Rear Axle Capacity } \\
\text { - } & \text { Rear Axle Ratio }\end{array}$ & & $\begin{array}{c}4,700 \mathrm{lbs} \\
7,950 \mathrm{lbs} \\
6,830 \mathrm{lbs} \\
11,020 \mathrm{lb} \\
4,777\end{array}$ & & & $\begin{array}{l}5,360 \text { lbs. } \\
9,880 \text { lbs. }\end{array}$ \\
\hline $\begin{array}{ll}\text { Suspension, Front \& Rea } \\
\text { - } & \text { Type Tapered/Multi-l } \\
\text { - } & \text { Front Suspension Cap } \\
\text { - } & \text { Rear Suspension Cap }\end{array}$ & & $\begin{array}{l}8,440 \mathrm{lbs} \\
9,880 \mathrm{lbs}\end{array}$ & & & \\
\hline
\end{tabular}

\begin{tabular}{|c|c|}
\hline Model Description & ZT Optional Heavy Duty \\
\hline $\begin{array}{ll}\text { Service Brakes } \\
\text { - } & \text { System Vacuum/Hydraulic } \\
\text { - } & \text { Front Disc } \\
\text { - } & \text { Rear Drum } \\
\text { Gearbox ZT VR2E (single, multi-speed) }\end{array}$ & \\
\hline $\begin{array}{l}\text { Tires } \\
\text { - Goodyearß G647 RSS 245/70R 19.5" 14-ply } \\
\text { 225/70R-19.5F (12 Ply) } \\
\text { Steering } \\
\text { - Integral Hydraulic Power Steering Column } \\
\text { - } \quad \text { Tilt \& Telescopic } \\
\text { - } \quad \text { Heat \& Air Conditioning (std) }\end{array}$ & \\
\hline $\begin{array}{l}\text { Motor } \\
\text { - Advanced liquid cooled brushless } 150 \mathrm{~kW} \quad 480 \mathrm{ft} / \mathrm{bs} \text { torque } \\
\text { Battery Pack } \\
\text { - Lithium standard } \\
\text { - Optional Charger - } 70 \mathrm{~kW}-208 \mathrm{v} \text { on-board charger } \\
\text { RANGE } \\
\text { - } \quad 70-75 \text { miles City driving (dependent on payload and driving }\end{array}$ & $\begin{array}{l}\text { Warranty } \\
\cdot 3 \text { year } \\
\text { Optional V2G } \\
\text { - Available now } \\
\text { Optional to } 125 \text { miles City) }\end{array}$ \\
\hline
\end{tabular}




\section{H.12 CXR-52 and CRX-60 Mowers Manufacturer: Mean Green Mowers}

\author{
Based in Ross, Ohio, Mean \\ Green Mowers manufactures \\ electric mowers that are quiet, \\ produce zero emissions, require \\ minimal maintenance, and pay \\ for themselves in fuel savings. \\ All mowers are manufactured in \\ the United States.
}

Mowers are manufactured from

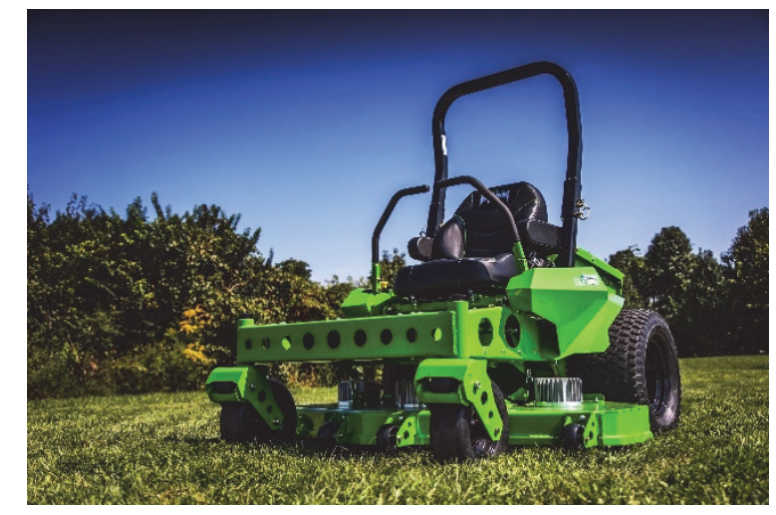
ing technologies. The company's mower designs are patented, as is its lithium-battery technology.

\begin{tabular}{|l|l|}
\hline \multicolumn{2}{|c|}{ CXR-52 and CRX-60 Mowers by: Mean Green Mowers } \\
\hline Cost & $\$ 19,530$ \\
\hline Application Maturity & Commercially available \\
\hline Safety & Roll over protection system, optional LED orange flashing light \\
\hline Extras and Attachments & $\begin{array}{l}\text { Aluminum grass catcher, rear-discharge mulching deck, debris blower, 3-kW converter, SAM } \\
\text { system, a flexible canopy that attaches to the CRX and provides solar power; 200W charge to } \\
\text { batteries. }\end{array}$ \\
\hline $\begin{array}{l}\text { Charging Time and } \\
\text { Capacity }\end{array}$ & $8-12$ to full charge \\
\hline Charging Infrastructure & 240 V \\
\hline $\begin{array}{l}\text { Operation Run-Time } \\
\text { Capabilities }\end{array}$ & $\begin{array}{l}2-3 \text { green-lithium-LEM48140 batteries, battery options enable from 2 hours to 7 hours of } \\
\text { mowing time/12 acres }\end{array}$ \\
\hline $\begin{array}{l}\text { Performance } \\
\text { Built to perform on sloping hills with cutting heights of up to } 5 \text { inches }\end{array}$ \\
\hline Projected Life Span & $6,000-9,000$ mowing hours on LEM battery \\
\hline \multicolumn{2}{|c|}{ Comments } \\
\hline $\begin{array}{l}\text { Mean Green Mowers' smaller units are in line with smaller scale mowing requirements. Through multiple units, the } \\
\text { company could provide sufficient coverage for large acres. Autonomous-system might yield cost savings benefit for } \\
\text { installation management but might not be sufficient to provide substantial power for microgrid. } \\
\text { Website: meangreenproducts.com/cxr5260/ } \\
\text { Contact: 513-738-4736 }\end{array}$ \\
\hline
\end{tabular}




\section{H.13 Nemesis NXR-48/52 Manufacturer: Mean Green Mowers}

\author{
Based in Ross, Ohio, Mean \\ Green Mowers manufactures \\ electric mowers that are quiet, \\ produce zero emissions, re- \\ quire minimal maintenance, \\ and pay for themselves in fuel \\ savings. All mowers are manu- \\ factured in the United States.
}

\section{Mowers are manufactured}

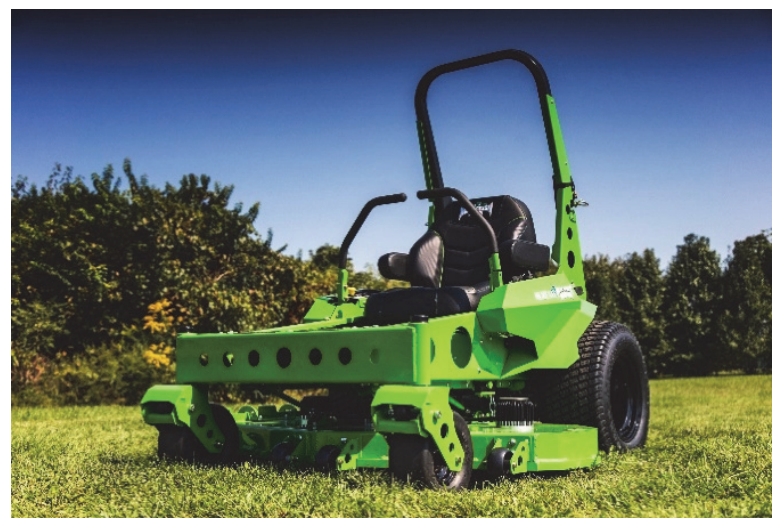
bending technologies. The company's mower designs are patented, as is its lithium-battery technology.

\begin{tabular}{|l|l|}
\hline \multicolumn{2}{|c|}{ Nemesis NXR-48/52 by: Mean Green Mowers } \\
\hline Cost & $\begin{array}{l}\text { Starts at \$11,925 for the 48" deck/LEM4880 battery model; \$12,599 for the 52" deck/LEM48140 } \\
\text { battery }\end{array}$ \\
\hline Application Maturity & Commercially available \\
\hline Safety & $\begin{array}{l}\text { Roll over protection system, operator blade speed warning system, optional LED floodlight, and } \\
\text { orange flashing light }\end{array}$ \\
\hline $\begin{array}{l}\text { Extras and } \\
\text { Attachments }\end{array}$ & $\begin{array}{l}\text { Optional Rite-Ride suspension, 48" or 52" heavy-gauge welded aluminum deck, optional 200W solar } \\
\text { flex panels, optional 3-kW converter. } \\
\text { Mean Green Mowers also offers a Solar Assisted Mower, an array of solar panels that attaches to the } \\
\text { mower. The SAM system is eligible for a 30 percent Federal Solar Tax Credit, and energy collected by } \\
\text { the system is immediately converted to power to assist in mowing operation. }\end{array}$ \\
\hline $\begin{array}{l}\text { Charging Time } \\
\text { and Capacity }\end{array}$ & 3-6 hours to complete charge \\
\hline $\begin{array}{l}\text { Charging } \\
\text { Infrastructure }\end{array}$ & 10A charger or optional 25A fast charger \\
\hline $\begin{array}{l}\text { Operation Run-Time } \\
\text { Capabilities }\end{array}$ & $\begin{array}{l}\text { LEM4880 green-lithium battery allows for 1.5 hours/3 acres mowing time and for 2.5 hours / 5 acres } \\
\text { mowing time }\end{array}$ \\
\hline Projected Life Span & green-lithium batteries last 1500-3000 mowing hours \\
\hline & \multicolumn{1}{c|}{ Comments } \\
\hline $\begin{array}{l}\text { Mean Green Mowers' smaller units are in line with smaller scale mowing requirements. Through multiple units, the } \\
\text { company could provide sufficient coverage for large acres. Autonomous-system might yield cost savings benefit for } \\
\text { installation management but might not be sufficient to provide substantial power for microgrid. More information here. } \\
\text { Website: meangreenproducts.com/nxr4852/ } \\
\text { Contact: 513-738-4736 }\end{array}$ \\
\hline
\end{tabular}




\begin{tabular}{|c|c|c|c|}
\hline Deck Size & $48^{\circ}$ or $52^{\circ}$ & Speed & up to $8.5 \mathrm{mph}$ \\
\hline Voltage & 48 Volts & Operational Savings/hour & $\$ 5.00 /$ hour \\
\hline Batteries (LEMs) & $\begin{array}{l}1 \text { each LEM4880 } \\
1 \text { each LEM48140 }\end{array}$ & Lithium Energy Module (LEM) Life & $1500-3000$ mowing hours \\
\hline Weight & $655-710 \mathrm{lbs}$. & EMISSIONS & ZERO \\
\hline Mow Time/ Charge & $\begin{array}{l}1 \text { each } 4880 \text { : } \\
-48^{\circ} 1.5 \mathrm{hrs} / 52^{\circ} 1.25 \mathrm{hrs} \\
1 \text { each } 48140 \text { : } \\
-48^{\circ} 25 \mathrm{hrs} / 52^{\circ} 2.25 \mathrm{hrs}\end{array}$ & Sound Level & $\begin{array}{l}75 \mathrm{db}-\mathrm{NXR} 48^{\circ} \\
77 \mathrm{db}-\mathrm{NXR} 52^{\circ}\end{array}$ \\
\hline Blades/ Deck & 3 each & Peak Horsepower & $24 \mathrm{HP}$ \\
\hline Recharge Time & 3-6 hrs. Complete charge & Fuel/ Oil & ZERO \\
\hline
\end{tabular}

*Varies with terrain, operator performance, and grass conditions

\section{STANDARD EQUIPMENT}

- Side Discharge Deck

- Custom Lumber Comfort Seat

- No Flat Front Wheel

- Drive Speed Control System

- Cup Holder
- Electronic Deck Lift System

High/Low Blade Speed Control

- Dual Support Anti-Scalp

Wheel Mounts

Rollover Protection System
OPTIONS \& ACCESSORIES

- 52" Side Discharge Deck Upgrade . 3KW Inverter

- Side Aluminum Grass Catcher - Monster Mode - Dual LED Floodlight Kit with USB . ORV-BLAST Package
Port

- Front Suspension Yokes • LED Orange Flashing Light

- 200W Solar Package S.A.M. Solar Canopy 


\section{H.14 SK-48 Stalker \\ Manufacturer: Mean Green Mowers}

Based in Ross, Ohio, Mean Green Mowers manufactures electric mowers that are quiet, produce zero emissions, require minimal maintenance, and pay for themselves in fuel savings. All mowers are manufactured in the United States.

Mowers are manufactured from aluminum alloys, high-strength steel, lightening holes, and modern bending technologies. The company's mower designs are patented, as is its lithium-battery technology.

\begin{tabular}{|l|l|}
\hline \multicolumn{2}{|c|}{ SK-48 Stalker by: Mean Green Mowers } \\
\hline Cost & Approximately: \$18,000 \\
\hline Application Maturity & Commercially available \\
\hline Safety & Optional LED orange flashing light \\
\hline Extras and Attachments & $\begin{array}{l}\text { LED floodlight kit with a USB port, aluminum grass catcher, rear-discharge mulching deck, debris } \\
\text { blower, 3-kW inverter }\end{array}$ \\
\hline $\begin{array}{l}\text { Charging Time and } \\
\text { Capacity }\end{array}$ & 2.5 hours for full charge (240V) \\
\hline $\begin{array}{l}\text { Charging Infrastructure } \\
\text { 25A (110V) or 35A (220V) 8-hour charger, optional LEMC-1548 overnight charger, or } \\
\text { LEMC2548 fast charger }\end{array}$ \\
\hline $\begin{array}{l}\text { Operation Run-Time } \\
\text { Capabilities }\end{array}$ & $\begin{array}{l}\text { 3.25 hours via one LEM48140 battery, } 7 \text { hours via two LEM48140; 1 LEM48112 battery, } \\
\text { additional batteries can be added to increase run-time }\end{array}$ \\
\hline Projected Life Span & Battery: 9,000 hours \\
\hline \multicolumn{2}{|c|}{ Comments } \\
\hline $\begin{array}{l}\text { Mean Green Mowers' smaller units are in line with smaller scale mowing requirements. Through multiple units, the } \\
\text { company could provide sufficient coverage for large acres. Autonomous-system might yield cost savings benefit for } \\
\text { installation management but might not be sufficient to provide substantial power for microgrid. } \\
\text { Website: meangreenproducts.com/sk48-stalker/ } \\
\text { Contact: 513-738-4736 }\end{array}$ \\
\hline
\end{tabular}




\begin{tabular}{|c|c|c|c|}
\hline Deck Size & $48^{\circ}$ & Speed & $8 \mathrm{mph}$ \\
\hline Voltage & 48 Volts & Operational Savings/hour & $\$ 6.00 /$ hour \\
\hline Batteries (LEMs) & 1-2 each LEM48140 & Lithium Energy Module (LEM) Life & $6000-9000$ mowing hours \\
\hline Weight & $\begin{array}{l}730-845 \mathrm{lbs} . \star \star \\
\star \star d e p e n d i n g \text { on the number of batteries }\end{array}$ & EMISSIONS & ZERO \\
\hline Mow Time/ Charge & $\begin{array}{l}1 \text { each LEM48140-3.25hrs* } \\
2 \text { each LEM48140- } 7 \mathrm{hrs}^{\star}\end{array}$ & Sound Level & $76 \mathrm{db}$ (gas engines tvice as loud 90-95 db) \\
\hline Blades/ Deck & 3 each & Peak Horsepower & $24 \mathrm{HP}$ (wheel dives peak torque $644 \mathrm{lb} / \mathrm{t}$ ) \\
\hline Recharge Time & $\begin{array}{l}4-10 \mathrm{hrs} \text {. (240V) Complete } \\
\text { charge }\end{array}$ & Fuel/ Oil & ZERO \\
\hline
\end{tabular}

*Varies with terrain operator performance, and grass conditions

\section{STANDARD EQUIPMENT \\ OPTIONS \& ACCESSORIES}

- Side Discharge Deck

- No Flat Front Tires

- 7 Gauge Steel Deck Chassis/Deck Design

- "Easy Adjust" Deck Height System
- Rear Discharge Mulching Deck
3KW inverter
- Aluminum Grass Catcher
- LED Orange Flashing
- LED Floodlight Kit with USB Light

High/Low Blade Speed Control Dual Support Anti-Scalp
Wheel Mounts 


\section{H.15 Reckless UTV Manufacturer: Nikola}

Nikola designs and manufactures EVs, vehicle components, ESSs, and EV drivetrains. The company was founded in 2014 and is based in Phoenix, AZ.

The Reckless UTV is a militarized version of Nikola's NZT off-road utility vehicle and is suited for combat, reconnais-

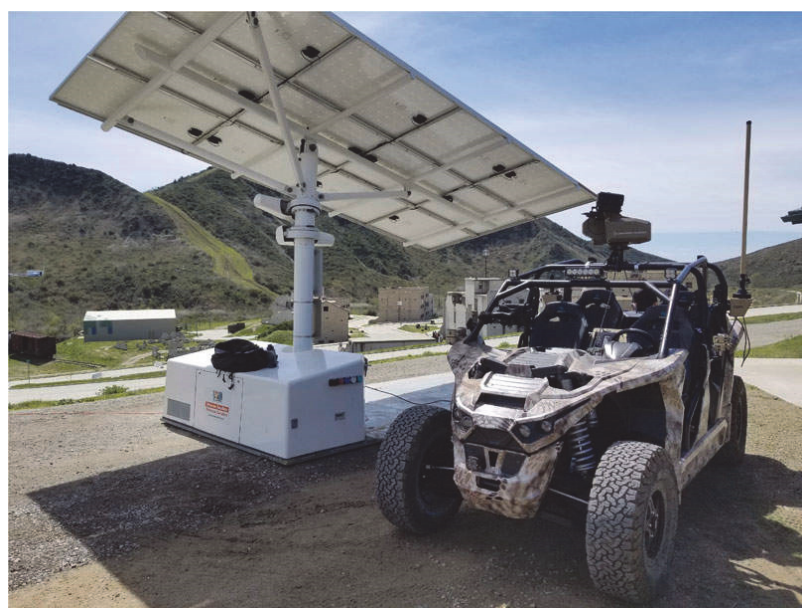
sance, and prime mover roles. The UTV is based on a $4 \times 4$ chassis and can slip into a 2x4 drive system based on mission requirement. It can accommodate four crew members and has the power capacity to support weapons systems and power command centers. The UTV, which has a low thermal signature, operates with four motors-in the event one fails, Reckless can still operate at high performance.

\begin{tabular}{|c|c|}
\hline \multicolumn{2}{|r|}{ Reckless UTV by: Nikola } \\
\hline Cost & $\$ 85,000$ \\
\hline Application Maturity & Available in 2021 \\
\hline Safety & $\begin{array}{l}\text { LED high/low, integrated signature light and SAE J588 turn signal, red LED tail, turn, and brake } \\
\text { lights }\end{array}$ \\
\hline Extras \& Attachments & $\begin{array}{l}\text { A remote weapon system with a } 12.7-\mathrm{mm} \text { machine gun is mounted atop the Reckless. The UTV } \\
\text { also features automatic grenade launchers and 0.3- and 0.17-m display monitors, which display } \\
\text { mission information and enhance the driver's situational awareness. A 4-kW DC charger powered } \\
\text { by solar energy is optional. }\end{array}$ \\
\hline $\begin{array}{l}\text { Charging Time and } \\
\text { Capacity }\end{array}$ & $\begin{array}{l}\text { Battery capacity: } 75 \mathrm{kWh}, 100 \mathrm{kWh} \text {, or } 125 \mathrm{kWh} \text { lithium-lon battery pack } \\
\text { DC Fast: } 2 \text { hours } \\
240 V \text { to } 1772: 15 \text { hours } \\
\text { EV ARC: } 19 \text { hours }\end{array}$ \\
\hline $\begin{array}{l}\text { Charging } \\
\text { Infrastructure }\end{array}$ & DC Fast, 240 V to J1772 charging, or EV ARC solar station charging \\
\hline $\begin{array}{l}\text { Operation Run-Time } \\
\text { Capabilities }\end{array}$ & 100-200 miles \\
\hline Performance & Waterproof, instant torque, direct drive, low center of gravity \\
\hline $\begin{array}{l}\text { Operability in Adverse } \\
\text { Weather or Conditions }\end{array}$ & $\begin{array}{l}\text { The Reckless has an IP67 rating-all parts can be submerged, and the UTV does not need air to } \\
\text { operate. }\end{array}$ \\
\hline Stealth Capabilities & Incredibly low noise level, low heat signature \\
\hline $\begin{array}{l}\text { Off-Road Capabilities } \\
\text { and Limitations }\end{array}$ & Off-road capable, on demand $2 \times 4$ and $4 \times 4$. Tires are 35 -inch Kevlar reinforced. \\
\hline
\end{tabular}




\section{Reckless UTV by: Nikola}

Hauling and Towing $\quad$ 1,4000-llb. payload capacity. Tow hitch enables the chassis to tow objects weighing up to 3,000 lbs. Capacity Front- and rear-mounted winches can be used to pull up to $10,000 \mathrm{lbs}$.

Weapons system is autonomous, and the Reckless features a Planck AeroSystems system that

Current Automation allows drones to take off and land autonomously on moving vehicles without GPS guidance. The

Capabilities platform can also be used for medical evacuations. The Reckless can be remotely controlled using a 3D vision system.

\section{Comments}

The Reckless has been off-road tested at Camp Pendleton and the USMC Air Ground Combat Center at Twentynine Palms, CA. Nikola plans to work with the DoD to add a hydrogen-fuel cell to the Reckless and is working with Envision Solar on portable battery banks.

The Reckless UTV was built via a contract between Sofwerx and Nikola to "develop a roadmap and demonstration of autonomous and mixed-autonomy capabilities."

Website: nikolamotor.com/reckless

Contact: 559-464-5652 


\section{H.16 T-Series Yard Truck/Hostler Manufacturer: Orange EV}

Orange EV, based in Riverside, MO, builds, sells, and services industrial EVs. The company is the leading OEM providing industrial fleets with heavy-duty EV solutions. Orange EV's terminal trucks are purpose-built to site requirements, and the company states its technology increases energy efficiency

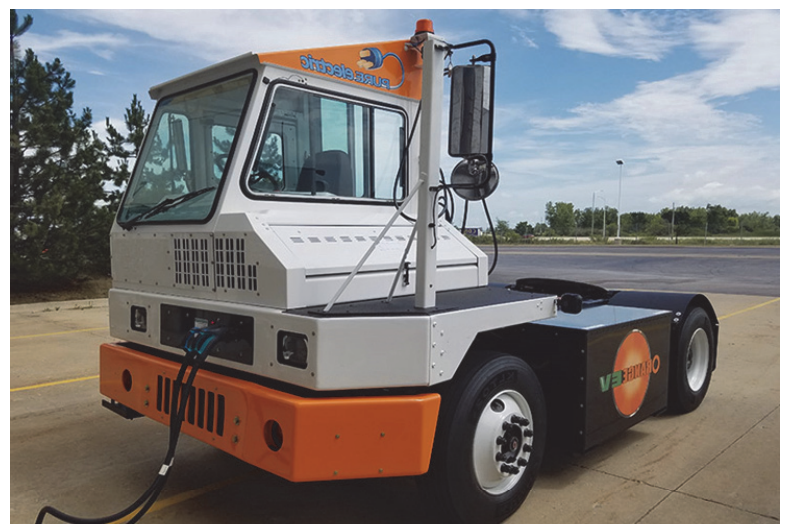
compared to traditional diesel systems by over 400 percent.

Orange EV's trucks utilize a web-based telematics system that provides instant feedback, real-time performance statistics, and hard data, which enables fleets to understand system status, fuel efficiency, and cost savings.

\begin{tabular}{|c|c|}
\hline \multicolumn{2}{|r|}{ T-Series Yard Truck/Hostler by: Orange EV } \\
\hline Cost & $\begin{array}{l}\$ 244,950-\$ 284,950 \text { (new, 2016); } \$ 199,950-\$ 239,950 \text { (re-manufactured, 2016). } \\
\text { Subsidies are available. }\end{array}$ \\
\hline Charging Time \& Capacity & $\begin{array}{l}\text { 2- or 4-hour fast charge. Orange offers two battery packs: } 80-K w \text { hour and } 160-\mathrm{kW} \text { hour. The } \\
\text { company matches charging rates to the customers' duty cycles and usage patterns. }\end{array}$ \\
\hline Charging Infrastructure & $\begin{array}{l}\text { Offboard, standard charging cabinet (440-480 VAC, up to } 22-k W \text { charging capacity) or } \\
\text { offboard, fast-charging cabinet ( } 440-480 \text { VAC, up to } 80-k W \text { charging capacity). }\end{array}$ \\
\hline $\begin{array}{l}\text { Operation Run-Time } \\
\text { Capabilities }\end{array}$ & $\begin{array}{l}\text { Commercially deployed and tested Orange EV trucks are working more than } 24 \text { hours on a } \\
\text { single charge. Trucks can run for up to } 100 \text { miles between charges. }\end{array}$ \\
\hline $\begin{array}{l}\text { Operability in Adverse } \\
\text { Weather or Conditions }\end{array}$ & $\begin{array}{l}\text { While cold weather is a concern with regard to charging, Orange builds in extra battery } \\
\text { capacity for cold climates and conditions. }\end{array}$ \\
\hline Maintenance Requirements & $\begin{array}{l}\text { All Orange EV trucks are sold with a warranty that includes onsite warranty service. The } \\
\text { company also offers additional preventative maintenance and non-warranty repair. }\end{array}$ \\
\hline $\begin{array}{l}\text { Off-Road Capabilities \& } \\
\text { Limitations }\end{array}$ & Appropriate for non-plated, in yard use. \\
\hline Hauling \& Towing Capacity & 81,000 lbs. GCWR \\
\hline Regulations & Exempt from Tier 4 emission controls. \\
\hline \multicolumn{2}{|r|}{ Comments } \\
\hline \multicolumn{2}{|c|}{$\begin{array}{l}\text { Orange EV, which has been collecting real-world operating data since } 2015 \text {, has shown that typical distribution operations } \\
\text { report savings up to } \$ 40,000 \text { per truck annually in fuel, maintenance, and emission control. Orange has also demonstrated } \\
\text { increased safety through its data collection. Further, Orange trucks reduce emissions by } 1.7 \text { tons of nitrogen oxides, } 1.6 \\
\text { tons of carbon monoxide, } 180 \text { pounds of particulate matter, and } 166 \text { tons of carbon dioxide (per } 6,000 \text { operating hours). }\end{array}$} \\
\hline \multicolumn{2}{|c|}{$\begin{array}{l}\text { Orange EV produces two versions of a heavy hauler. While systems can be used on roads, top speed maxes out at } 25 \mathrm{mph} \text {. } \\
\text { Vehicles are Missouri-built, and the company can retrofit older diesel frames to electric, resulting in a potential cost savings } \\
\text { of approximately } \$ 50,000 \text {. }\end{array}$} \\
\hline \multicolumn{2}{|c|}{ Website: orangeev.com/ } \\
\hline \multicolumn{2}{|c|}{ Contact: 866-688-5223 x702; mikes@orangeev.com } \\
\hline
\end{tabular}




\section{Deployed Industry Segments}

- Rail Intermodal

- Seaport

- Parcel

- Global Retail Distribution

- LTL Freight

- 3PL firms

- Manufacturing

- Spotting

- Waste Mgt

- Warehouse/DCs
In use with top ramp operators and railroads

Supporting California Ports of LA, Long Beach and Oakland

The sorting operations of global brand leaders

The big boxes

The largest, busiest break-bulk sites

Global leaders and regional firms alike

Global and regional consumer brand leaders and commercial brands

Innovative firms electrifying and optimizing yard management

Municipal waste transfer sites

McDonald's distribution network (NALC) as well as

national and regional logistics firms

\section{ORANGE EV TRUCKS: OWNED AND OPERATED ACROSS SITES AND FLEET TYPES}

\section{Built to Site Specifications}

Single axle (4x2) with GCWR of $81 \mathrm{k} \mathrm{LB}$ up to $25 \mathrm{MPH}$. Major configurations include:

Truck:

- New

- Remanufactured

Battery:

- Standard $80 \mathrm{kWh}$

- Extended $160 \mathrm{kWh}$

Charging:

- Standard 15-22 kW per hour

- Fast $80 \mathrm{~kW}$ per hour

DOT:

- Off-road

- On-road (DOT compliant)

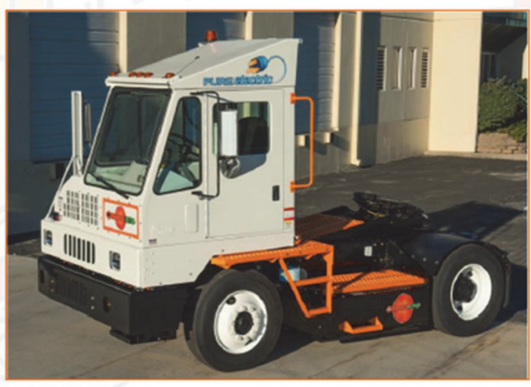




\section{H.17 Zeus}

\section{Manufacturer: Phoenix Motors}

\section{Based in Ontario, Phoenix Motors} was founded in 2003. The company develops light- and medium-duty EVs for the service and government fleet markets. Phoenix is a wholly owned subsidiary of AYL, a privately held company based in Dubai, UAE.

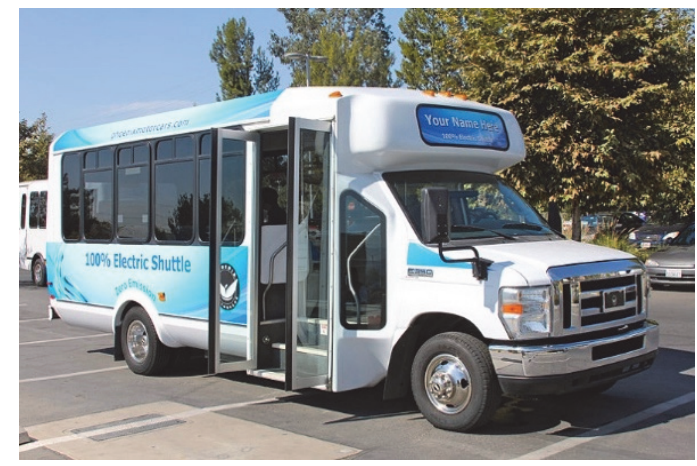

Phoenix's Zeus models are built out from Ford chassis' and the company maintains a scalable second-stage production line in the United States for their initial production activities, including the development and manufacturing of the Phoenix Drive System and its components. The company's $\mathrm{R} \& \mathrm{D}$ facility is located in Chino, CA.

\begin{tabular}{|c|c|c|c|}
\hline & $\begin{array}{l}\text { Electric Shuttle by: Phoenix } \\
\text { Motors }\end{array}$ & $\begin{array}{l}\text { Electric Flatbed by: Phoenix } \\
\text { Motors }\end{array}$ & $\begin{array}{l}\text { Utility Vehicle by: Phoenix } \\
\text { Motors }\end{array}$ \\
\hline Cost & \multicolumn{3}{|l|}{ Approximately: $\$ 290,000$} \\
\hline Application Maturity & \multicolumn{3}{|l|}{ Commercially available } \\
\hline Safety & \multicolumn{3}{|c|}{ All vehicles have an ADA-compliant option. } \\
\hline $\begin{array}{l}\text { Charging Time and } \\
\text { Capacity }\end{array}$ & \multicolumn{3}{|c|}{ J1772 Lvl II: 8-9 hours; DC fast: 3-4 hours } \\
\hline Run-Time Capabilities & Up to 110 miles & Up to 100 miles & Up to 100 miles \\
\hline Microgrid Connectivity & \multicolumn{3}{|l|}{ V2G capable } \\
\hline Regulations & \multicolumn{3}{|c|}{$\begin{array}{l}\text { Phoenix's Zeus models are currently undergoing testing at Altoona to receive its FTA certification, } \\
\text { which is expected to happen no later than May } 31,2020 .\end{array}$} \\
\hline \multicolumn{4}{|c|}{ Comments } \\
\hline \multicolumn{4}{|c|}{$\begin{array}{l}\text { In 2005, Phoenix delivered a Zeus flatbed to the Naval Base Ventura County Port Hueneme, CA, and a large number of } \\
\text { Phoenix buses was scheduled for delivery in } 2019 \text {, including } 29 \text { for the EPA-funded South Coast Air Quality Management } \\
\text { District project to electrify shuttles across key airports in Southern California. The Zeus shuttle was among GSA's FY19 } \\
\text { Electric Offerings. }\end{array}$} \\
\hline
\end{tabular}




\section{MANUFACTURING}
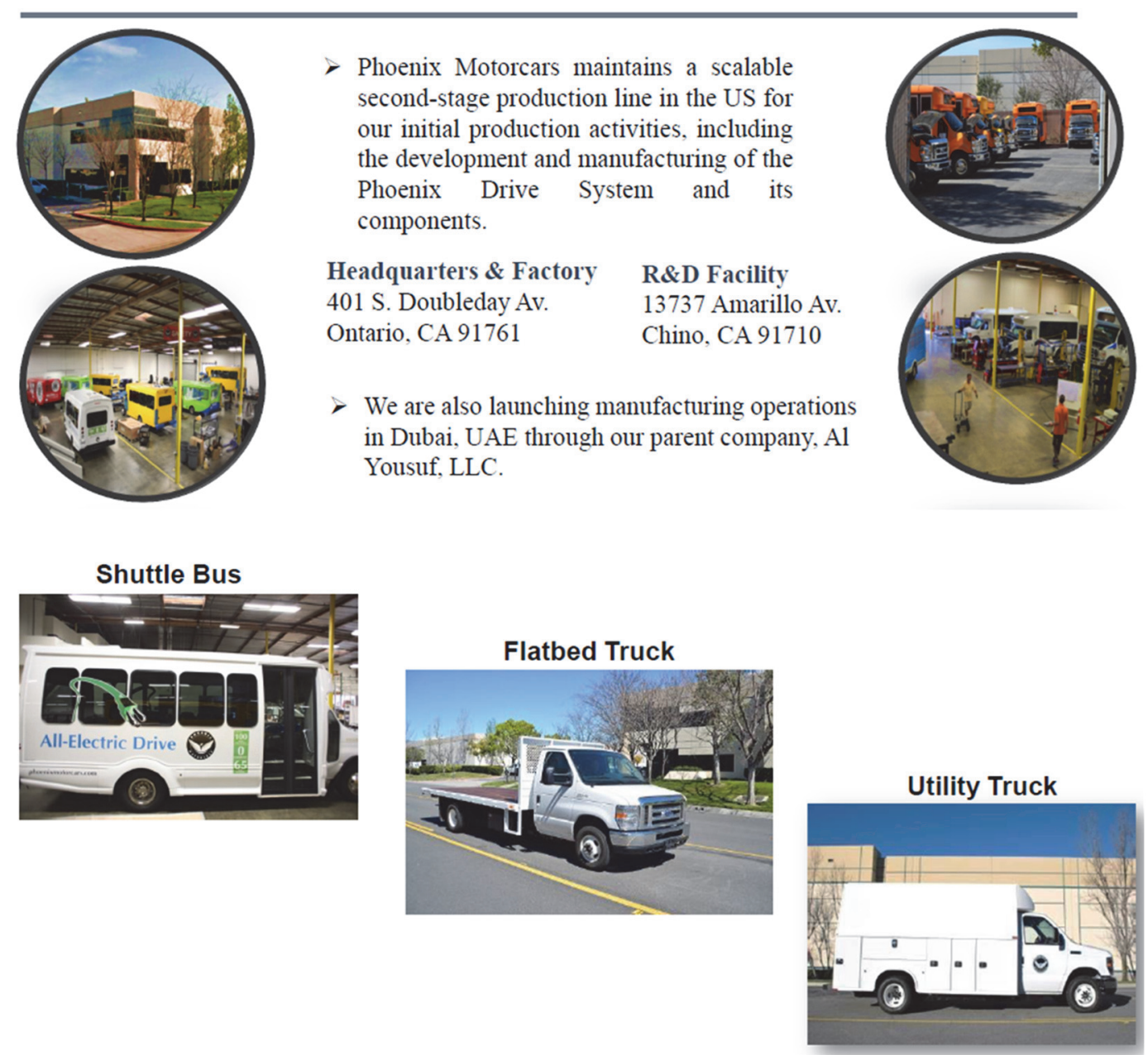

CUSTOMIZABLE CONFIGURATIONS

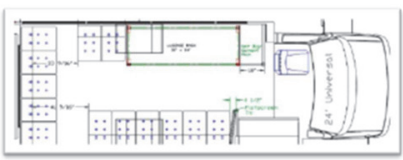

> 13 Passengers, Perimeter, 80" Luggage Rack

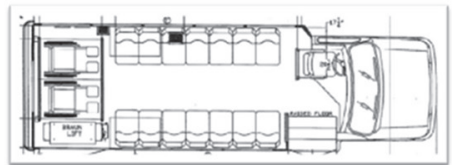

- 14 Passengers, Perimeter Rear Lift, 2 Wheelchair Positions

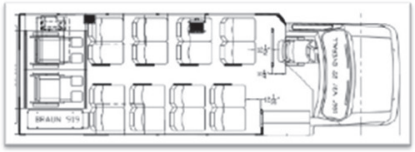

$>16$ Passengers, Fwd Facing Rear Lift, 2 Wheelchair Positions 


\section{H.18 Sherpa 100ECO Mini Skid-Steer Loader Manufacturer: Hanenberg Materiel B.V.}

Hanenberg Materiel B.V. is based in the Netherlands.

The ECO10o is a $76-\mathrm{cm}$ wide mini skid-steer powered by a 2-HP front-mounted electric motor. A 360-Ampere battery powers the mini skid-steer.

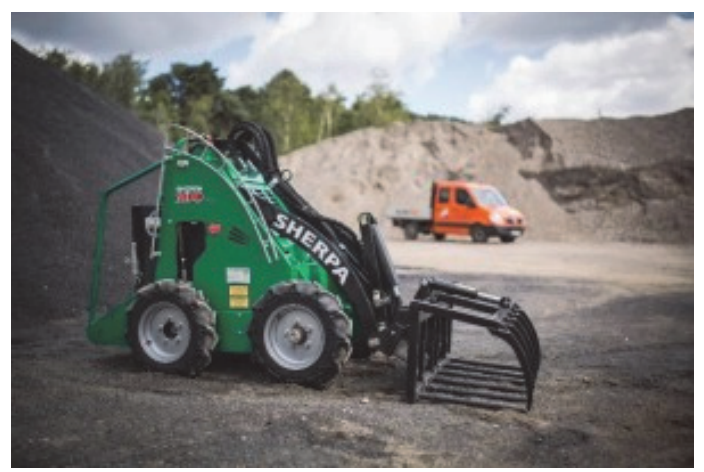

\begin{tabular}{|c|c|}
\hline Parameter & Value \\
\hline Cost & $\$ 32,995$ \\
\hline $\begin{array}{l}\text { Application } \\
\text { Maturity }\end{array}$ & Commercially available \\
\hline $\begin{array}{l}\text { Extras and } \\
\text { Attachments }\end{array}$ & $\begin{array}{l}\text { Optional bucket, volume bucket, 4-1 bucket, bucket with grab, hydraulic manure forks, weed brush, slab } \\
\text { bucket, pin bucket, pallet forks, snow push/feeding scraper, hydraulic snow push, trailer coupling, lifting } \\
\text { arm, mast with electric wench, levelling framework, broom, roller broom, hydraulic earth drill, demolition } \\
\text { hammer, hydraulic trencher, hydraulic tile/curb clamp, hydraulic stone clamp, hydraulic tree grapple, } \\
\text { hydraulic curbstone clamp, dumper, and hydraulic concrete mixer attachments available. }\end{array}$ \\
\hline $\begin{array}{l}\text { Charging Time } \\
\text { and Capacity }\end{array}$ & $\begin{array}{l}\text { Overnight charging } \\
\text { Battery capacity: } 360 \mathrm{Ah}\end{array}$ \\
\hline $\begin{array}{l}\text { Charging } \\
\text { Infrastructure }\end{array}$ & Standard 110-volt outlet \\
\hline $\begin{array}{l}\text { Operation } \\
\text { Run-Time } \\
\text { Capabilities }\end{array}$ & Up to 8 hours \\
\hline Regulations & OSHA-approved for indoor and outdoor use \\
\hline $\begin{array}{l}\text { Current } \\
\text { Automation } \\
\text { Capabilities }\end{array}$ & The Sherpa $100 E H D$ is fully remote-control capable. \\
\hline & Comments \\
\hline \multicolumn{2}{|c|}{$\begin{array}{l}\text { There is only one authorized Sherpa dealer in the United States: Triple E Equipment, located in Pompano Beach, FL. } \\
\text { Website sherpaminiloaders.com/eng/models/sherpa-100-eco/ } \\
\text { U.S. dealer: e3equipment.com/ } \\
\text { Contact: Dealer: } 954-978-3440\end{array}$} \\
\hline
\end{tabular}




\section{Technical data}

\begin{tabular}{lr} 
Engine & \\
\hline Type: DC Motor (brushless) & $1,5 / 2$ \\
Capacity (kW/HP): & 1800 \\
Revolutions per minute (rpm): & \\
Drive system & \\
\hline Hydraulic through 2 hydraulic wheel motors & $\mathbf{0 - 4}$ \\
Speed (mph): & 1851 \\
\hline Capacity data & 1230 \\
\hline Breakout force (lbs): & 2756 \\
Tractive force (lbs): & 11 \\
Hydraulic drive system (psi): & \\
Hydraulic tank capacity (gal): &
\end{tabular}

Tipping load in straight position on LCD point ( )

Pallet forks with weight low: $\quad 771$

Pallet forks with weight high: $\quad 486$

Bucket with weight low : $\quad 770$

Bucket with weight high: $\quad 484$

$\begin{array}{ll}\text { Tires: } & 4.00 \times 10\end{array}$

0-4 Brakes

Service brake: Hydrostatic on 2 rear wheels / 2 front wheels through chain transmission

Steering

Type: Skid steering

Electrical system

Battery type: Traction battery

Voltage (V):

Battery capacity (Ah):

Kerb weight (Ibs): 


\section{H.19 eUtility \& eFarmer Tractors Manufacturer: Solectrac}

Solectrac, based in Albion, CA., manufactures tractors that provide portable power, home power, water pumping, and other mechanical functions including hauling and transportation needs. Solectrac batteries can be charged

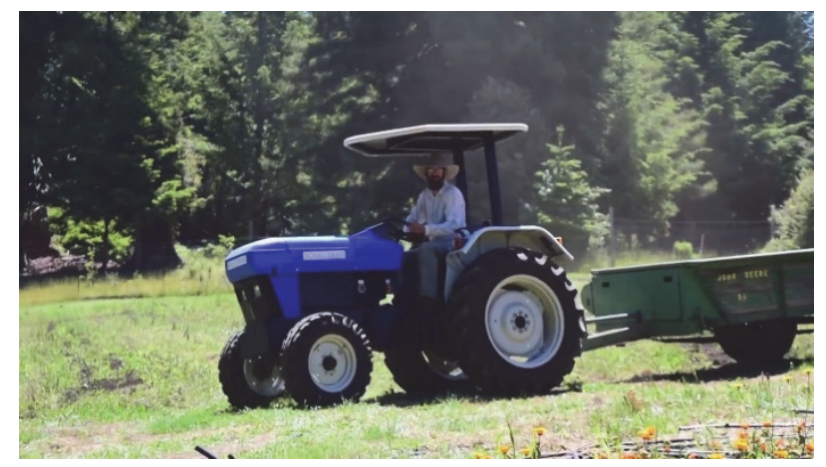
directly by clean renewable energy or at a low cost from the electric utility grid at off-peak rates. Quick change battery packs extend operational periods.

\begin{tabular}{|c|c|c|}
\hline & eUtility & eFarmer \\
\hline Cost & $\$ 45,000$ & $\$ 45,000$ \\
\hline Application Maturity & $\begin{array}{l}\text { Available on a first-deposit basis, } \\
\text { commercially available } 2020\end{array}$ & Available in 2020 \\
\hline $\begin{array}{l}\text { Extras and } \\
\text { Attachments }\end{array}$ & $\begin{array}{l}\text { Accepts all Category } 1-540 \mathrm{rpm} \text { PTO } \\
\text { implements on rear hitch. Front loader } \\
\text { option available for } \$ 10,000 \text {. }\end{array}$ & $\begin{array}{l}\text { Can accommodate all Category } 1 \text { implements, with the } \\
\text { exception of those requiring hydraulics. A hydraulic pump } \\
\text { for legacy implements can be added for an additional cost } \\
\text { Can also accommodate front-mounted implements, such } \\
\text { as a reaper or low lift loader bucket. }\end{array}$ \\
\hline $\begin{array}{l}\text { Charging Time and } \\
\text { Capacity }\end{array}$ & $\begin{array}{l}\text { Level } 2 \text { fast for } 80 \text { percent charge in } 3 \\
\text { hours/full charge overnight; } 26-k W h \\
\text { battery pack }\end{array}$ & $\begin{array}{l}\text { 3-hour quick charge for one pack, overnight slow charge } \\
\text { for two packs }\end{array}$ \\
\hline $\begin{array}{l}\text { Charging } \\
\text { Infrastructure }\end{array}$ & 240 VAC-30A & \\
\hline $\begin{array}{l}\text { Operation Run-Time } \\
\text { Capabilities }\end{array}$ & 5-8 hours, depending on load & $\begin{array}{l}4-8 \text { hours with onboard battery pack. Additional battery } \\
\text { packs may be added to extend run-time. }\end{array}$ \\
\hline Projected Life Span & $\begin{array}{l}\text { Battery: } 10 \text { years }(3000 \text { cycles at } 80 \\
\text { percent DoD) } \\
\text { Tractor: } 80,000 \text { hours }\end{array}$ & $\begin{array}{l}\text { Battery: } 10 \text { years ( } 3000 \text { at } 80 \text { percent DoD) } \\
\text { Tractor: } 80,000 \text { hours }\end{array}$ \\
\hline \multicolumn{3}{|c|}{ Comments } \\
\hline eUtility & & eFarmer \\
\hline \multicolumn{2}{|c|}{$\begin{array}{l}\text { Exchangeable } 26-k \text { Wh battery packs for the eUtility are } \\
\text { available. Can be ordered with either } 12 \text { or } 18 \text { inches of } \\
\text { ground clearance, the former of which is suitable for steep } \\
\text { slopes. Can accommodate snow blower attachment. }\end{array}$} & $\begin{array}{l}\text { Exchangeable } 26-k W h \text { battery packs will be available. } \\
2020 \text { model is } 2 \text { WD, but future models will be } 4 W D \\
\text { compact. Standard } 18 \text { inches of ground clearance, } \\
\text { suitable for gentle slopes. Can accommodate snow blower } \\
\text { attachment. }\end{array}$ \\
\hline $\begin{array}{l}\text { Website: eFarmer: } \underline{\mathrm{n}} \\
\text { // eUtility: } \underline{\mathrm{https}} / / / \mathrm{w}\end{array}$ & $\begin{array}{l}\text { ttps://www.solectrac.com/efarmer } \\
\text { vw.solectrac.com/eutility }\end{array}$ & \\
\hline
\end{tabular}




\section{eUtility}

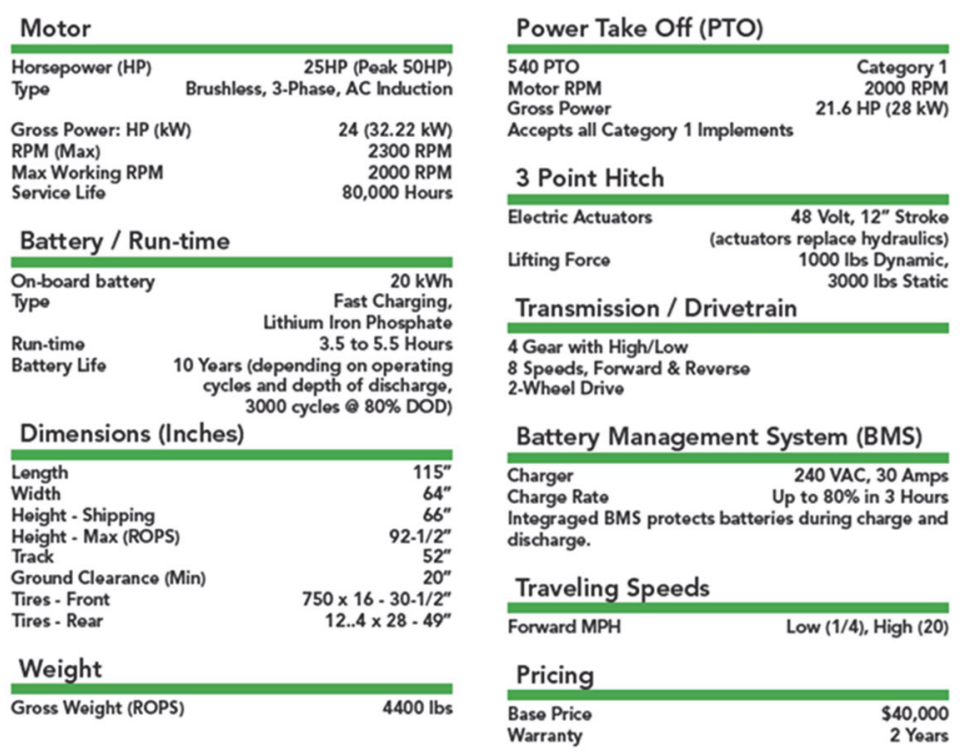




\section{Appendix I: Autonomous Vehicle OEM and Kit Manufacturers}

\begin{tabular}{|c|c|c|c|c|c|c|}
\hline & AV FIRM & U.S. Firm & $\begin{array}{l}\text { State/ } \\
\text { Country }\end{array}$ & OEM or Kit & $\begin{array}{c}\text { Cars, } \\
\text { Trucks, or } \\
\text { Other }\end{array}$ & Website \\
\hline 1 & Almotive Inc. & N & Hungary & KIT & Cars/Vans & https://aimotive.com \\
\hline 2 & Amazon Web Services & $\mathrm{Y}$ & WA & $\mathrm{Al} / \mathrm{ML}$ & Yes & https://amzn.to/2GtDH2a \\
\hline 3 & Ambarella Corporation & N & $\begin{array}{l}\text { China, } \\
\text { Japan, } \\
\text { US, EU }\end{array}$ & Computer Vision & Yes & https://www.ambarella.com \\
\hline 4 & Apex.Al & & & & & https://www.apex.ai \\
\hline 5 & Apple Inc. & Y & $\mathrm{CA}$ & TBD & TBD & https://www.drive.ai \\
\hline 6 & Argo Al, LLC & $\mathrm{Y}$ & PA & KIT & Cars & https://www.argo.ai \\
\hline 7 & Aurora Innovation & Y & PA & KIT & Yes & https://aurora.tech \\
\hline 8 & AutoX Technologies Inc. & Y & CA & KIT & Yes & https://autox.ai \\
\hline 9 & Baidu USA, LLC & N & China & KIT & Yes & http://usa.baidu.com/adu/ \\
\hline 10 & BMW & $\mathrm{N}$ & Germany & OEM & Yes & https://bit.ly/2BdM6EN \\
\hline 11 & Bosch & N & Germany & KIT & Yes & https://bit.ly/2JsIDrD \\
\hline 12 & Boxbot Inc. & $\mathrm{Y}$ & CA & KIT & $\begin{array}{l}\text { Vans and } \\
\text { delivery } \\
\text { robots }\end{array}$ & https://www.boxbot.io \\
\hline 13 & CarOne, LLC & Y & CA & KIT & Custom & https://www.udelv.com \\
\hline 14 & Changan Automobile & N & China & OEM & Yes & http://www.globalchangan.com/index.html \\
\hline 15 & Continental Automotive Systems Inc. & N & Germany & KIT & TBD & https://bit.ly/2Hej4ad \\
\hline 16 & CYNGN Inc. & Y & CA & KIT & Custom & https://www.cyngn.com \\
\hline 18 & Deka Labs & Y & $\mathrm{NH}$ & KIT & Specialized & http://www.dekaresearch.com/ \\
\hline 19 & Delphi Automotive (Now Aptiv) & N & Ireland & KIT & Yes & https://bit.ly/2POJOI3 \\
\hline 20 & DiDi Research America, LLC & N & China & KIT & TBD & www.didi-labs.com \\
\hline 21 & EasyMile & N & France & Shuttle & Shuttle & https://easymile.com \\
\hline 22 & Embark & Y & CA & KIT & Trucks & https://embarktrucks.com \\
\hline 22 & Faraday \& Future Inc. & N & China & OEM & $\begin{array}{l}\text { Car and } \\
\text { SUV }\end{array}$ & https://www.ff.com \\
\hline 23 & Ford & Y & $\mathrm{MI}$ & OEM & All & Ford Self-Driving \\
\hline 24 & Gatik Al. Inc. & $\mathrm{Y}$ & CA & KIT & $\begin{array}{l}\text { Trucks and } \\
\text { vans }\end{array}$ & http://www.gatik.ai \\
\hline 25 & GM Cruise, LLC & Y & CA & KIT & Cars & https://getcruise.com \\
\hline 26 & Helm.Al Inc. & & & & & \\
\hline 27 & Honda & $\mathrm{N}$ & Japan & Other & Specialized & Honda Autonomous Work Vehicle \\
\hline 28 & IKE & Y & CA & KIT & Trucks & https://www.ikerobotics.com/ \\
\hline 29 & Imagry Inc. & $\mathrm{N}$ & Israel & KIT & YES & https://imagry.co \\
\hline 30 & Intel Corp & $Y$ & CA & Chips & See Notes & https://intel.ly/2s8x6nU \\
\hline 31 & Jingchi Corp & $N$ & China & KIT & Yes & https://s.nikkei.com/33KCVHK \\
\hline
\end{tabular}




\begin{tabular}{|c|c|c|c|c|c|c|}
\hline & AV FIRM & U.S. Firm & $\begin{array}{c}\text { State/ } \\
\text { Country }\end{array}$ & OEM or Kit & $\begin{array}{c}\text { Cars, } \\
\text { Trucks, or } \\
\text { Other } \\
\end{array}$ & Website \\
\hline 32 & Kodiak Robotics & $\mathrm{Y}$ & TX & KIT & Trucks & http://kodiak.ai \\
\hline 33 & Lyft, Inc. & Y & CA & KIT & Yes & https://self-driving.lyft.com \\
\hline 34 & Mando America Corporation & N & Korea & KIT & Cars & https://www.mando.com/eng/ \\
\hline 35 & May Mobility & $\mathrm{Y}$ & MI & OEM & Shuttles & https://maymobility.com \\
\hline 36 & Mercedes-Benz & N & Germany & Possibly & Yes & https://bit.ly/30c0yat \\
\hline 37 & Navya Inc. & N & France & OEM & Shuttle & https://bit.ly/2P4Xqvr \\
\hline 38 & NIO USA, Inc. & $\mathrm{N}$ & China & OEM & Cars & https://www.nio.com \\
\hline 39 & Nissan & N & Japan & OEM & $\begin{array}{l}\text { Cars and } \\
\text { trucks }\end{array}$ & https://bit.ly/2TJlvXn \\
\hline 40 & Nullmax & $N$ & China & Kit/Supplier & Yes & https://bit.ly/2zag8aM \\
\hline 41 & Nuro, Inc. & Y & CA & KIT & \begin{tabular}{|l|} 
Prius and \\
customized \\
vehicle
\end{tabular} & https://nuro.ai \\
\hline 42 & NVIDIA Corporation & Y & CA & $\begin{array}{l}\text { Chips, software } \\
\text { deep learning, } \\
\text { Al }\end{array}$ & Yes & https://bit.ly/2KJhxeB \\
\hline 42 & Perrone Robotics & $\mathrm{Y}$ & VA & KIT & Yes & https://www.perronerobotics.com/ \\
\hline 43 & Phantom Al & $\mathrm{Y}$ & CA & KIT & \begin{tabular}{|l|} 
Specialized \\
yard \\
trucks, \\
forklifts, \\
etc.
\end{tabular} & https://phantom.ai \\
\hline 44 & PlusAi Inc & Y & CA & KIT & Trucks & https://plus.ai \\
\hline 45 & Pony.Al & N & China & KIT & & https://www.pony.ai \\
\hline 46 & Qcraft.ai & Y & CA & Possibly KIT & TBD & http://qcraft.ai \\
\hline 47 & Qualcomm Technologies, Inc. & Y & CA & Chips & TBD & https://ubm.io/2Mrcrp8 \\
\hline 48 & Renovo.auto & & & & & https://www.renovo.auto \\
\hline 49 & Ridecell Inc. & Y & CA & KIT & TBD & https://bit.ly/20ZFVwu \\
\hline 50 & Rivian & Y & MI & OEM & Yes & https://rivian.com \\
\hline 51 & Roadstar.Ai & & & & & http://roadstar.ai \\
\hline 52 & Robomart & Y & CA & Other & \begin{tabular}{|l} 
Mobile \\
Mini-Marts \\
\end{tabular} & https://robomart.co/ \\
\hline 53 & Robotics Research & Y & MD & KIT & Yes & https://www.roboticresearch.com \\
\hline 54 & SAIC Innovation Center, LLC & N & China & & & https://bit.ly/31QkKia \\
\hline 55 & Samsung Electronics & N & Korea & KIT & TBD & https://bit.ly/3101zp6 \\
\hline 56 & SF Motors Inc. & & & & & https://bit.ly/31NmHM7 \\
\hline 57 & Starship Technologies & Y & CA & Other & $\begin{array}{l}\text { Delivery } \\
\text { robots }\end{array}$ & https://www.starship.xyz/ \\
\hline 58 & Starsky Robotics & Y & CA & KIT & Trucks & https://www.starsky.io \\
\hline 59 & Subaru & N & Japan & & & https://bit.ly/30l2HzT \\
\hline 60 & Telenav, Inc. & & & & & https://bit.ly/30mfdiU \\
\hline 61 & Tesla Motors & Y & CA & OEM & $\begin{array}{l}\text { Cars and } \\
\text { trucks }\end{array}$ & https://bit.ly/2elBzhj \\
\hline
\end{tabular}




\begin{tabular}{|c|c|c|c|c|c|c|}
\hline & AV FIRM & U.S. Firm & $\begin{array}{l}\text { State/ } \\
\text { Country }\end{array}$ & OEM or Kit & $\begin{array}{c}\text { Cars, } \\
\text { Trucks, or } \\
\text { Other }\end{array}$ & Website \\
\hline 62 & ThorDrive Inc. & N & Korea & KIT & TBD & https://www.thordrive.ai \\
\hline 63 & TORC Robotics Inc. & $\mathrm{Y}$ & VA & KIT & Yes & https://torc.ai \\
\hline 64 & Toyota Research Institute & Y & MA & KIT & Yes & https://bit.ly/2KKdcrr \\
\hline 65 & TuSimple & N & China & KIT & Yes & https://www.tusimple.com \\
\hline 66 & Udacity, Inc & & & & & https://bit.ly/2aEAnki \\
\hline 67 & Valeo North America, Inc. & N & France & $\begin{array}{l}\text { Supplier/ } \\
\text { KIT }\end{array}$ & Yes & https://bit.ly/2TNJqVF \\
\hline 68 & Volkswagen Group of America & N & Germany & OEM & Cars & https://bit.ly/33JcHFn \\
\hline 69 & Voyage & & & & & https://voyage.auto \\
\hline 70 & Waymo LLC & Y & $\mathrm{CA}$ & KIT & $\begin{array}{l}\text { Cars and } \\
\text { vans }\end{array}$ & https://waymo.com \\
\hline 71 & WeRide & N & China & KIT & Specialized & https://www.weride.ai \\
\hline 72 & Xmotors.ai, Inc. & N & China & KIT & Yes & https://bit.ly/30kRAap \\
\hline 73 & Zoox, Inc. & $Y$ & $\mathrm{CA}$ & KIT & Specialized & https://zoox.com \\
\hline
\end{tabular}




\title{
Appendix J: Interview Notes
}

\author{
Call with Dannar
}

9/27/2019 | 1 p.m.

\author{
Location: Telecon
}

\section{Participants:}

Birgitte Dodd, CS2

John Conger, Dannar

Scott Crepeau, Dannar

Bob Sudah, Paul Brubaker, LLC

Paul Brubaker, Paul Brubaker, LLC

Bryan Brilhart, Robotic Research

Clark Boriack, TechFlow

Michael Genseal, TechFlow

\section{Notes:}

1. Manufacturer and Vehicle Description

Dannar is an OEM of mobile, off-road energy platform for the U.S. governmental market. Dannar is located in Clemente, CA, San Jose, CA, and Muncie, IN (manufactured). The company currently offers the Dannar 3.00, a base configuration that comes with standard two 33-kWh BMW i3 lithiumion battery packs ( $66 \mathrm{kWh}$ total) that can be upgraded to four packs for a total of 132-kWh onboard electricity. The Dannar 4.00 base configuration comes standard with three 42-kWh BMW i3 lithium-ion battery packs (126 $\mathrm{kWh}$ total) and can be upgraded with up to nine additional packs for a total of $504 \mathrm{kWh}$ of onboard electricity. The Dannar 4.00 is built on a heavy-duty steel ladder frame and can be ordered with or without an operator cab or platform.

2. Vehicle-to-Grid Charging Readiness/Effort to Implement

Vehicle charging, battery, plugs, and range and V2G capabilities - It is easiest to envision an MPS as a DC-energy storage asset, to whose DC power bus one can connect various power converters for export of energy.

Dannar tends to focus first on MPS power export to islanded microgrids, such as those for remote worksites where grid power is not accessible. 
Implementations for these applications are simpler because onboard power converters do not need to synchronize with a/the larger utility grid.

Supply to a utility grid involves additional hardware to ensure (i) isolation of the ungrounded MPS from the grounded grid and (ii) synchronization of exported power with grid power. Dannar's power electronics partner has supplied both unidirectional and bidirectional power converters for both DC-DC applications (such as EV charging) and AC-DC applications (such as either EV charging from grid or V2G transfer), so Dannar definitely can make arrangements for this nature of export.

- Implementing V2G on an MPS amounts to installing a V2G-capable EVSE (EV charger) on the MPS, and then connecting the AC/infrastructure end of that converter to grid. The EVSE would include the isolation means. Dannar's power electronic partner has in the past implemented for DoD such electronics in more vehicle-compatible packaging.

- Higher-power V2G transfer amounts to scaling the V2G-capable EVSE on an MPS or on multiple, interconnected MPSs.

- For most EV's packaging an EVSE onboard would present a challenge. The MPS, however, was designed to provide mechanical support and mobility for such electrical/electronic attachments.

\section{Vehicle Functions and Capabilities}

Dannar 3.00: Optional open operator cab platform equipped with joystick, foot pedal, and touchscreen tool control. Base model is equipped with universal quick mounting plates that can receive CAT, Bobcat, or John Deere attachments and an optional Cummins Tier IV generator (60 kW) is available. Submersible up to 4 feet and carries Type I, II, and III hydraulic tool circuits. Four-wheel, multi-mode steering.

Dannar 4.00: Export panel, which is configurable with multiple 110-VAC and 208-VAC outlets, base model, which comes standard with dual flat beds and can accommodate scissor lifts, forklift masts, dump beds, water tanks, electric water pumps, portable light units, or mobile telescoping cell towers. Can accommodate all standard Caterpillar, Bobcat, and John Deere attachments, submersible up to 4 feet. Carries Type I, II, and II hydraulic tool circuits, fourwheel drive, and four-wheel multi- mode steering.

At minimum, Dannar 3.00 and 4.00 microgrids can charge two Tesla EVs or operate for 8-10 hours onsite. At max, systems offer enough energy to power nearly 17 houses for 24 hours. Designed for disaster relief, can function in wet conditions. System is zero emission (electric) and designed for use in various disaster relief scenarios. Rugged MPS units able to be 
deployed safely-heavy-duty, water submergible to 4 feet, work tools can be attached for clearing debris.

4. Testing and Demonstrations

Seven vehicles have been built to date: One has been in the field for 3 years; two have been in demonstration for 1.5 years. The first two deliveries to Portes Stockten, CA are being used as fork lifts. No official testing has been done to date, but many system users can offer information/act as references regarding their experience using the system.

5. Autonomy Readiness/Effort to Implement System has full remote capability, operating by onboard cab, $x$-box style remote control, or TBD wireless supervision. Dannar is currently working to develop follow-the-leader autonomous function.

\section{Safety Assessments}

The system uses high volume off-shelf parts, so assessments would be specific to the parts and their manufactures. As such, Dannar has not conducted technology specific safety assessments, safety standards apply to individual parts.

7. Pricing, Warranties, and Support

Axel and battery pack are under a 10-year warranty; no parts obsolescence battery guarantee for early adopters; stand by unit for 10 years; Portes Stockten, CA.

\section{Additional Information:}

\section{Environmental Impacts}

No battery disposal issues because Dannar will lease the battery, taking it back for repurposing.

\section{Communications Systems}

Onboard communication systems; sensors and data collection-data dumps every 10 seconds; augmented reality glasses (see what you see, see what I see- in field diagnostics) 
Dannar has multiple CAN: ISO 11898 busses for user interface access, communication with attachments (in response to user interface communications), power management, and energy management.

1. We use multiple software platforms for various systems of the MPS, but Parker Hannifin's IQAN mobile control platform is what we use for user interface communication, and that is how we tend to let outside entities communicate with an MPS for movement and to control other functions. We favor Parker IQAN at this point for user interface communication because of industry familiarity with the platform for mobile industrial control and for the platform's reputation for ease of use. Ease of use is important to Dannar, as we expect some customers to use the MPS as a multipurpose platform, upon which the customer can develop their own end-functions.

2. If a customer wishes to integrate an external communication system to an MPS, the entry point for communication is via the user interface CAN Bus. If a customer has a special communication for offboard communication (e.g., secure wireless link), which might or might not be based on IQAN, they may access the user interface CAN Bus through an interface between their special communication system and the MPS's user interface CAN Bus physical media (twisted wire pair). The CAN format on the MPS user interface bus can be either Parker IQAN (Parker proprietary message format) or SAE J1939 (published and maintained by SAE).

3. Dannar usually does not allow direct access to low-level movement, power management, and energy management control systems. This arrangement inherently shields MPS lowlevel systems from external communication access. The general philosophy is to "ask the MPS at high-level; let the MPS handle functions at low-level."

Technology Information Analysis: New Flyer

\section{Technology}

New Flyer has been providing innovative transit solutions for more than 85 years, with over 50 years of experience manufacturing zero-emissions buses. New Flyer has developed the Xcelsior Charge electric bus available in $35, ' 40$,' and 60' lengths. The zero emissions bus contains a battery ESS ranging in size from $160 \mathrm{kWh}$ to $460 \mathrm{kWh}$.

The Xcelsior Charge is commercially available and is currently being used in revenue service by several transit agencies across the United States. 
ATI Research Pros

New Flyer buses are interoperable with all heavy-duty vehicle charging equipment. New Flyer has partnered with Robotic Research, LLC to develop a proof-of-concept autonomous bus. Additionally, New Flyer has committed to additional autonomous bus programs with the Xcelsior Charge.

The Xcelsior Charge electric bus and the battery system is manufactured in the United States. New Flyer has delivered several Xcelsior Charge buses to transit agencies across the United States and has a fleet of demonstration buses available for use.

New Flyer warranties the ESS for 12 years and provides support and field service upon request.

Safety standards should be acceptable for ATI research conditions. All electrical components are UL-rated and qualified. In addition to the UL-rated electrical components, the buses comply with Federal Motor Vehicle Safety Standards and have passes Altoona Testing.

ATI Research Cons

While the Xcelsior Charge contains a large ESS, the bus is designed for onroad transit application and might not be suitable for off-road use. Additionally, the entry cost of the electric bus is approximately $\$ 750 \mathrm{k}$.

\section{Technology Information Analysis: Nikola}

\section{Technology}

Nikola Powersports is a division of Nikola Motor Company that is dedicated to the powersports industry, which includes UTVs for military and commercial markets as well as watercraft. Nikola Motor Company designs and manufactures EVs, vehicle components, ESSs, and EV drivetrains. The Reckless vehicle is a fully electric side-by-side UTV that can reach speeds of $100 \mathrm{kph}$ in just over 3 seconds.

The Reckless is available with powertrains ranging from 266-590 horsepower and is powered by a 400-VDC battery system with 75-125 kWh of 
energy storage. The Reckless is estimated to have a range of $150+$ miles and a top speed of $75 \mathrm{mph}$.

The Reckless has been demonstrated for the USMC. The availability is currently limited to production prototype models.

\section{ATI Research Pros}

The Nikola Reckless is interoperable with a Remote Weapon Station (RWS) and military drones. A variant of the platform has steer and throttle-by-wire capabilities, and Nikola is working to develop more autonomous capabilities. The vehicle's onboard energy storage is large for the size of the vehicle and would be beneficial for bidirectional charging.

The Reckless boasts performance numbers that are ahead of any other sideby-side UTV currently on the market. The vehicle's capabilities make it an appealing option for high-speed off-road use.

\section{ATI Research Cons}

While the Reckless contains a large ESS, the platform does not currently support bidirectional charging. A separate effort is being made to integrate a hydrogen-fuel cell onto the vehicle. The vehicle's availability is limited. There are a few production prototypes available for lease at a cost of $\$ 40,000$ per month.

\section{Call with Orange EV}

$11 / 21 / 2019$ I noon

Location: Conference Call

\section{Participants:}

Kurt Neutgens, Orange EV

Angie Rolufs, Paragon

Bryan Brilhart, Robotic Research 
Clark Boriack, TechFlow

Michael Genseal, TechFlow

\section{Notes:}

\section{Manufacturer and Vehicle Description}

- From Kansas City, MO; Orange EV's website: orangeev.com/

- Electric Terminal Truck - called a "Yard Dog"-moves the trailers that attach to semi-trucks around a shipping yard, used by commercial warehouses and distribution centers.

- Critical component of a distribution center-yard dog cannot go down, so no fewer than two trucks at any location; most do not have more than 20 trucks; one customer has 160 trucks.

- Also used in railyards to load and offload shipping containers from trains.

- Maximum speed is $25 \mathrm{mph}$.

- Includes a hydraulic fifth wheel that allows the truck to lift the trailer-Orange uses CAN Bus to manage this communication.

- Trucks get the job done but at a lower cost than diesel and with no diesel fumes; also quieter and smoother ride.

- Orange EV is looking toward faster vehicles with more capabilities.

- Orange EV is the only commercial player in this space.

- 350,000 hours, 1.1 million miles on their fleet-can track all of the usage remotely.

- Started company in 2012; profitable last year with substantial increase in profit this year.

- Now in a 52,000-square-foot facility with a team of 55 people with trucks in 14 states.

- Orange EV performs their own service on their trucks.

2. Vehicle-to-Grid Charging Readiness/Effort to Implement

- Charging equipment: fast charge at 80-kW rate and level II

- Battery pack sizes: $80 \mathrm{kWh}$ and $160 \mathrm{kWh}$

- Chargers: cabinet, harnesses, software, heating/cooling designed in-house.

- The charging component, an AC/DC power converter, is purchased separately from two different suppliers. Three different $A C$ voltage options are available depending upon site availability: 240 VAC, 208 VAC three-phase and 480 VAC three-phase.

- Charger time depends on battery pack size $(80 \mathrm{kWh}$ or $160 \mathrm{kWh})$ and ranges from 8 hours to 2 hours ( 3 in parallel at $80 \mathrm{~kW}$ ).

- V2G: Have you considered changes to system and how ready your system is for a bidirectional power flow?

- Clark-If you have the capability to customize your charger, then it is definitely viable as compared to starting from scratch. 
- Kurt-Not beyond Orange EV capabilities, other than actual power conversion; not their expertise.

- Clark-Sounds like you are already to set up to communicate from vehicle to charger, so to adapt to a bidirectional power converter would not be hard to develop the communication (handshake) expertise.

- Kurt-Building to different voltage than the rest of the world is doing. Maximum voltage is 120 VDC-doing this for multiple reasons, but main reason is safety. They deliver these to small operations where there are one or two people moving groceries around, wanted to make sure that people who were not qualified to work on the trucks will not get hurt. Proud of being able to do this without having to be at the higher voltage. Positive for company that they are doing this at the lower voltage. The inverters are at a lower voltage than we may have worked with in the past. Voltage of battery pack is only 120 VDC.

- The output of the charger is 120 VDC.

- Capacity to handle the higher current resulting from charging at lower voltages has been a successful approach for them.

- Vehicle designed for specific use-can upsize cables without having a detrimental effect on the vehicle; heavy vehicle.

- 120 VDC is a safety approach?

- Yes, and also for reliability, per Kurt

- Kurt familiar with how Ford did this. Large vehicle with small niche market-hard to make custom systems

- Clark, interested in V2G? Any thoughts on how soon? 1-2 years forward, or is there a timeline?

- Clark-No real work yet, non-military customers not really interested in V2G. If power is down for the site, they would go home. Hard to keep a warehouse running without grid power.

- See themselves doing stationary power capability so they can do load-level cost management-charge over 8 hours and discharge over 2 or less hours. Could there be a tie-in with this to shift energy from one truck to another? Not as important for current customer but understands the value for a military customer.

3. Vehicle Functions and Capabilities

- Electric Terminal Truck-called a "Yard Dog"-moves the trailers that attach to semi-trucks around a shipping yard, used by commercial warehouses and distribution centers

- Critical component of a distribution center-yard dog cannot go down so no fewer than two trucks at any location; most do not have more than 20 trucks; one customer has 160 trucks

- Also used in railyards to load and offload shipping containers from trains

- Maximum speed is $25 \mathrm{mph}$

- Includes a hydraulic fifth wheel which allows the truck to lift the trailer-Orange usesCAN Bus to manage this communication 
- Trucks get the job done but at a lower cost than diesel and with no diesel fumes, also quieter and smoother ride

4. Testing and Demonstrations

- Public Demonstration Ride and Drive on December 6 in Kansas City, but would prefer that we visit on our own after the first of the year, so Kurt can show us everything.

5. Autonomy Readiness/Effort to Implement

- Bryan-What capabilities aiming for, who working with?

a. Kurt cannot share but not an exclusive agreement. Can slow truck with regenerative breaking. Driver is currently main source of braking. Acceleration and deceleration can be controlled autonomously. One of the values of vehicles is hydraulic fifth wheel that allows the truck to lift trailer; use CAN Bus to manage this communication

- All auxiliary functions will be on CAN Bus by third quarter next year-turn headlights on or windshield wipers from a CAN Bus message

- Steering is in the roadmap-working with a partner to do this-would be interested in working with a partner to do braking and other things

- Working with suppliers to develop, but they are all a little slow

- More driver assistance functions, or automate backing into loading dock?

a. Kurt wants to start with crash avoidance, then go to things like backing trailer up. Current partner is working toward full autonomy.

- Kurt asked Bryan if Robotic Research has systems that are fully autonomous. Bryan identified the Army's Oshkosh tractor trailer truck and RR's work on making it a fully AV.

6. Safety Assessments

- Truck is DOT compliant for on- and off-road use.

- Self-certified: no certification required if within compliance.

- Stopping distance is half that of diesel Yard Dog, with a much lower accident rate.

7. Pricing, Warranties, and Support

- Base unit with $80-k W h$ battery pack is $\$ 240,000$

- Cost of unit with 160-kWh battery pack is $\$ 289,950$

- Level II charger (8 hours to charge) is $\$ 6,000$, and DC fast charger is $\$ 60,000$

- 3-year warranty

Next Steps: 
- Paragon team member call lead will provide email correspondence PDF.

- Load all call information captured on CERL deliverables Research Area 2 EV Company Informational Meeting file.

Call with Phoenix Motorcars

9/17/2019, 2 p.m.

Location: Conference Call

\section{Participants:}

Sharad Agarwal, VP, Easy Mile

Angie Rolufs, Paragon

Thomas Allen, Sales, Phoenix Motorcars

Jose Paul Plackal, Business Director, Phoenix Motorcars

Bryan Brilhart, Robotics Research

Clark Boriack, TechFlow

Michael Genseal, TechFlow

Tarek Helou,

\section{Notes:}

\section{Manufacturer and Vehicle Description (Thomas)}

- Southern California based company. Currently on the second-generation drive system. To date, 1.8 million miles driven. Current platform is Ford E450; partnered with Ford. Will be able to take all Phoenix vehicles to any Ford dealer for service soon.

- GVWR is 14,500\#; has a reasonable payload.

- Shuttle buses are most popular. Can provide electric utility trucks with enclosed steel body or service truck body/maintenance trucks. Can provide box trucks, delivery, flatbed, stake bed, and anybody that uses the e450 chassis. Next phase extends to F550 platform with a GVWR of $19,000 \#$ in 2020. 
2. Vehicle-to-Grid Charging Readiness/Effort to implement

(Jose)

- Already deployed a flatbed to the Navy that was V2G; used

CPS fast charger standard.

- V2G is not a standard offering.

- Typical level 2 or 3 charging capability.

- Will work with partners to enable.

- CHAdeMO standard level 3 charging.

- V2G should be easy to implement.

- Can use j1772 combo connector also for V2G.

- 4-6 months to implement V2G. 120-day manufacturing. Capabilities are in-house. Has partners for bidirectional EVSE.

3. Vehicle Functions and Capabilities

- Most shuttle bus options are available.

- 6500\# payload for shuttles.

- Can mount cranes, racks, inverters up to $125 \mathrm{~W}$ for offboard power, LED lighting, worklights, toolboxes.

- Flatbed truck has lift gates (aluminum 1300-lb. lift capacity) stake bed, animal control.

- Refrigeration.

- Open to customization per client needs.

- All vehicles come with telematics for GPS tracking, emission savings; great tool for fleets monitoring.

- Can disable GPS tracking within vehicle but keep data.

- Telematics can help acquire incentives, up to $\$ 8,000 /$ year per vehicle.

- $105 \mathrm{kWh}$ is standard, 25 miles in range, can drop to 87 or increase to $129 \mathrm{kWh}$. 1 mile of range per kWh. 50-kW charge rate or discharge max;

- Production models will have a top speed of $58 \mathrm{mph}$

4. Testing and Demonstrations

- Had all-electric shuttle bus at LAX, first electric fleet at LAX.

- Demonstration with U.S. Air Force (USAF) at LAAFB.

- 4 vehicle with NASA at Caltec in Pasadena, CA, and they bought another 4.

- Multitude of municipal contracts.

- Port of Oakland; Austin, TX airport, 15-20 for Sacremento, CA.

- Grant with federal transit for six at rural transit agencies in Wisconsin.

- Grant to replace 29 ICE shuttles in SoCal area airports.

5. Autonomy Readiness/Effort to Implement (see table below for timeline)

- Easymile is the autonomy supplier for Phoenix

- Level IV autonomous for Phoenix vehicle platform

- No drive-by-wire kit on e450 but has done on a car and will expand to 450 
- 3 years out for autonomy package

6. Safety Assessments

- Buy-American certified

- CARB approved

- NIST compliant

- Approved for on-road and highway use throughout U.S.

7. Pricing, Warranties, and Support

- Have baseline pricing for all vehicles.

- All come with warranties.

- 3-year, 36-month bumper-to-bumper with Ford; 5-year, 60 of drive system, 5 -year 150,000 -mile warranty on batteries with option to extend to 8 years and 300,00 miles.

- All customers go straight to Phoenix, who handles it all. Three types of service packages, include all-inclusive maintenance.

- Services on site, provides training.

- Also partnered with creative bus sales where they can also be serviced for warranty or maintenance.

Phoenix Motorcars Shuttle Bus Pricing:

MSRP \$234,950

Includes listed vehicle specifications and a 105-kWh battery system.

Excluding tax, title, shipping and registration

Lead time: 5-6 months

Phoenix Motorcars Cargo Truck Pricing:

MSRP $\$ 214,950$

Includes listed vehicle specifications and a 105-kWh battery system.

Excluding tax, title, shipping and registration

Lead time: 5-6 months

V2G Solutions Pricing:

CHAdeMO V2G Development $\$ 99,950$

SAE CCS V2G Development \$249,950 
V2G Option for Series Production $\$ 11,950 /$ vehicle

Lead time: 9-12 months

Level 4 Autonomous Prototype Pricing:

Phase 1 Development: $\$ 4,448,000$

Phase 2 Development: $\$ 6,230,000$

Phase 3 Series Production: Level 4 Autonomous Shuttle or Cargo

Truck for $\$ 399,950 /$ vehicle

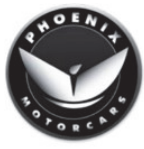

Autonomous Vehicle Development Timeline
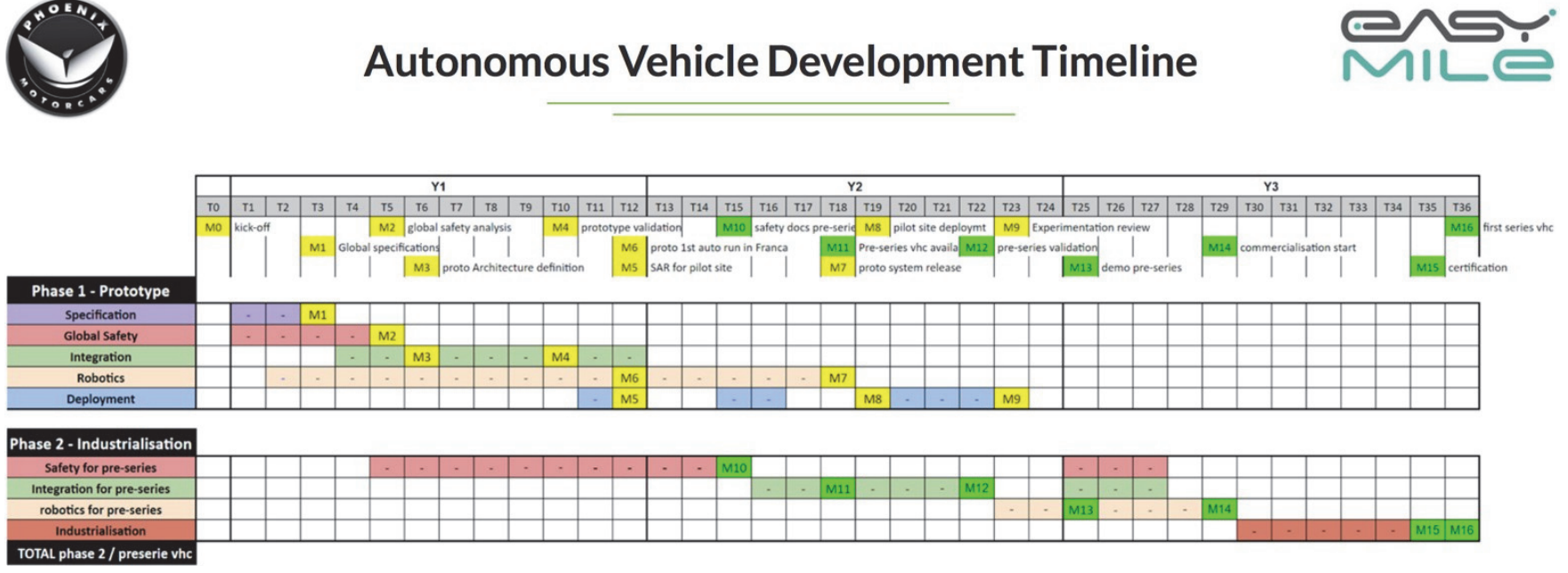

Call with US Hybrid Corporation

9/20/2019, 2 p.m.

Location: Telecon

\section{Participants:}

Bob Suda, Brubaker LLC (Birdsong Partners)

Paul Brubaker, Brubaker LLC

Alice Reeves, Paragon Business Solutions 
Angie Rolufs, Paragon Business Solutions

Amelia Trout, Paragon Business Solutions

Bruce Brilhart, Robotics Research

Eddie Mottern, Robotics Research

Clark Boriack, Techflow

Abas Goodarzi, President and Chief Executive Officer, US Hybrid Corporation

Don Kang, Chief Engineer, US Hybrid Corporation

Daniel Orlowski, VP Business Development, US Hybrid Corporation

\section{Notes:}

1. Software Manufacturer and Vehicle Description

US Hybrid Corporation (USH) specializes in the design and manufacture of power conversion systems for medium and heavy-duty electric, hybrid, and fuel cell commercial buses and trucks to enable them to become more reliable and fuel efficient, with lower emissions and better responsiveness.

USH offers comprehensive engineering expertise in electric and hybrid vehicle systems and components, including Integrated Motor Drives DC-DC Power Conversions and High- Performance AC Motors and Controllers. USH's products utilize Integrated Digital Signal Processor power converter topologies to provide reliable and cost effective integrated system solutions for OEM applications.

2. Vehicle-to-Grid Readiness/Effort to Implement USH has built a number of prototype vehicles that can accommodate $\mathrm{V} 2 \mathrm{G}$ applications, and they are currently providing a vehicle to the USAF that is a 230-VDC charger for sustainable power on demand. The vehicle can power most of the required $\mathrm{AC} / \mathrm{DC}$ load, and the operation is monitored using a GUI-driven dashboard. 
3. Implement Vehicle Functions and Capabilities

Time to produce a prototype proof-of-concept vehicle that can vary depending on the complexity of the design, but it typically take 6 months to design, build, and field a single proof-ofconcept vehicle.

\section{Testing and Demonstrations}

USH EV vehicles are currently being used to support USAF flight line operations, and they have an EV step van currently being tested. USH has built an EV Special Operations Vehicle that is being tested by U.S. Special Operations Command (SOCOM), but USH could not offer specifics without more information and suggested that background information would need to be provided in a classified enviornment.

USH has a number of other demonstration projects underway demonstrating both EV and fuel cell capabilities. They are currently testing a 480-Kw wide body tug for moving $\mathrm{C}-17 \mathrm{~s}$ and a weapons loader. They have also electrified a $80,000-\mathrm{lb}$ truck capable of moving shipping containers.

USH typically builds anywhere from one to 15 vehicles for demonstration projects often in partnership with other OEMs or third parties (e.g., Textron or Oshkosh). The prototype vehicles have a minimum (TRL of 5 and are mostly TRL levels 6 or 7). USH is currently engaged with one such project with an R11 and R12 refuelers and is delivering jet fuel to fighter squadrons using these EVs.

\section{Autonomy Readiness/Effort to Implement}

USH offer all of their modified vehicles as drive-by-wire. USH has built the EV Mine Sweeper for Textron that is driven remotely through a virtual interface. USH is also supporting a number of other military related vehicles some of which are focused on demonstrating autonomy readiness. USH does not have any autonomy capability on its own but is working on interfacing with another company's autonomous control system and is aware that that company is interfacing with the braking and steering controls through the vehicle's CAN Bus.

6. Safety Assessments 
All electrical components are UL-rated and qualified. All vehicles are prototypes, do not operate on public roadways, and, consequently, do not assess against the Federal Motor Vehicle Safety Standards (FMVSS). In addition to the UL-rated electrical components, USH vehicles are built and modified in compliance with SAE standards.

7. Pricing, Warranties, and Support

The USH POC said that he needs more information about the project requirements before he could offer specifics on pricing, warranties, and support.

\section{Additional Notes:}

USH would like to offer additional detail but cannot until they know more about the requirements of a potential pilot project. The Paragon team said it would review the information gathered from all of the interviews and be in contact if additional information is needed. 


\section{Appendix K: SME Technology \\ Recommendation}

Dannar Review by: Brubaker LLC

Do you recommend this technology for ATI demon-

stration? $\mathrm{Y} / \mathrm{N}$ : Yes

If No, please explain: N/A

If Yes, please explain below based on the following demonstration considerations:

\begin{tabular}{|c|c|}
\hline $\begin{array}{l}\text { Military Activity: What makes this } \\
\text { technology particularly useful to } \\
\text { supporting one or more military } \\
\text { transportation support activities? }\end{array}$ & $\begin{array}{l}\text { Demonstration concepts: } \\
\text { - } \quad \text { Flexible platform that can be adopted } \\
\text { for a variety of military applications } \\
\text { - Rugged platform }\end{array}$ \\
\hline $\begin{array}{l}\text { Autonomous Vehicle: What makes } \\
\text { this system a good candidate for } \\
\text { AV research, demonstration? Why } \\
\text { is it unique and potentially useful } \\
\text { to CERL research objectives? }\end{array}$ & $\begin{array}{l}\text { Demonstration concepts: } \\
\text { - Can be outfitted with AV capabilities } \\
\text { - Drive-by-wire capable } \\
\text { - Unique platform that can be easily } \\
\text { adapted for a number of uses - proba- } \\
\text { bly low-speed operation that will re- } \\
\text { duce complexity and risk for AV outfit- } \\
\text { ting }\end{array}$ \\
\hline $\begin{array}{l}\text { Microgrid: How can this system } \\
\text { support microgrid research and } \\
\text { demonstration objectives con- } \\
\text { sistent with CERL objectives and } \\
\text { CBITEC capabilities? }\end{array}$ & $\begin{array}{l}\text { Demonstration concepts: } \\
\text { - Great storage } \\
\text { - Bidirectional charging } \\
\text { - } \quad \text { Flexibility for microgrid }\end{array}$ \\
\hline \multicolumn{2}{|c|}{$\begin{array}{l}\text { Partnering in Research: Describe the potential for and interest in establishing a part } \\
\text { nership for demonstration and research application between this manufacturer and } \\
\text { CERL. }\end{array}$} \\
\hline $\begin{array}{l}\text { Based on our interview I believe } D \\
\text { opportunity with CERL. }\end{array}$ & very interested in pursuing a RD\& \\
\hline
\end{tabular}


Other Comments and Considerations:

Danner appears to already be working with an autonomy provider. This may provide a challenge for Robotic Research.

Dannar Review by: TechFlow, Inc.

Do you recommend this technology for ATI demonstration? Y/N: Yes, if there is not a more viable option.

If No, please explain: N/A

If Yes, please explain below based on the following demonstration considerations:

\begin{tabular}{|l|l|}
\hline $\begin{array}{l}\text { Military Activity: What makes this } \\
\text { technology particularly useful to } \\
\text { supporting one or more military } \\
\text { transportation support activities? }\end{array}$ & Demonstration concepts: \\
$\begin{array}{l}\text { Payload capacity to move equipment } \\
\text { fits for FOBs. }\end{array}$ & \\
\hline $\begin{array}{l}\text { Autonomous Vehicle: What makes } \\
\text { this system a good candidate for } \\
\text { AV research, demonstration? Why } \\
\text { is it unique and potentially useful } \\
\text { to CERL research objectives? }\end{array}$ & Demonstration concepts: \\
\hline $\begin{array}{l}\text { Microgrid: How can this system } \\
\text { support microgrid research and } \\
\text { demonstration objectives con- } \\
\text { sistent with CERL objectives and } \\
\text { CBITEC capabilities? }\end{array}$ & Demonstration concepts: \\
$\begin{array}{l}\text { Can share power be- } \\
\text { tween charging sta- } \\
\text { tions. Provides fast- } \\
\text { charging capabilities. }\end{array}$ & \\
Can export power from vehicles.
\end{tabular}


Partnering in Research: Describe the potential for and interest in establishing a partnership for demonstration and research application between this manufacturer and CERL.

Dannar was not able to articulate their vehicles could synchronize with other power sources. This may be a feasible development for Dannar given their product already has other flexible power delivery capabilities.

Other Comments and Considerations:

New Flyer Review by: Brubaker LLC

Do you recommend this technology for ATI demonstration? Y/N: No

If No, please explain: The New Flyer bus platform is of limited utility from a military perspective. It does not support bidirectional charging and is very expensive. For these reasons, New Flyer is not a suitable candidate for inclusion in this RD\&D project.

If Yes, please explain below based on the following demonstration considerations:

\begin{tabular}{|l|l|}
\hline $\begin{array}{l}\text { Military Activity: What makes this } \\
\text { technology particularly useful to } \\
\text { supporting one or more military } \\
\text { transportation support activities? }\end{array}$ & Demonstration concepts: \\
\hline $\begin{array}{l}\text { Autonomous Vehicle: What makes } \\
\text { this system a good candidate for AV } \\
\text { research, demonstration? Why is it } \\
\text { unique and potentially useful to } \\
\text { CERL research objectives? }\end{array}$ & Demonstration concepts: \\
\hline $\begin{array}{l}\text { Microgrid: How can this system } \\
\text { support microgrid research and } \\
\text { demonstration objectives con- } \\
\text { sistent with CERL objectives and } \\
\text { CBITEC capabilities? }\end{array}$ & Demonstration concepts: \\
\hline
\end{tabular}


Partnering in Research: Describe the potential for and interest in establishing a partnership for demonstration and research application between this manufacturer and CERL.

Other Comments and Considerations:

New Flyer Review by: Robotic Research

Do you recommend this technology for ATI demonstration? Y/N: No

If No, please explain: The New Flyer bus platform is of limited utility from a military/off-road perspective and is very expensive. Additionally, NF is not looking at bidirectional charging until more standards are defined.

If Yes, please explain below based on the following demonstration considerations:

\begin{tabular}{|l|l|}
\hline $\begin{array}{l}\text { Military Activity: What makes this } \\
\text { technology particularly useful to } \\
\text { supporting one or more military } \\
\text { transportation support activities? }\end{array}$ & Demonstration concepts: \\
\hline $\begin{array}{l}\text { Autonomous Vehicle: What makes } \\
\text { this system a good candidate for AV } \\
\text { research, demonstration? Why is it } \\
\text { unique and potentially useful to } \\
\text { CERL research objectives? }\end{array}$ & Demonstration concepts: \\
\hline $\begin{array}{l}\text { Microgrid: How can this system sup- } \\
\text { port microgrid research and demon- } \\
\text { stration objectives consistent with } \\
\text { CERL objectives and CBITEC capabili- } \\
\text { ties? }\end{array}$ & Demonstration concepts: \\
\hline $\begin{array}{l}\text { Partnering in Research: Describe the potential for and interest in establishing a } \\
\text { partnership for demonstration and research application between this manufac- } \\
\text { turer and CERL. }\end{array}$ \\
\hline
\end{tabular}


Other Comments and Considerations:

New Flyer Review by: TechFlow, Inc.

Do you recommend this technology for ATI demonstration? Y/N: No

If No, please explain: Does not appear to be align with bidirectional power or tactical applications.

If Yes, please explain below based on the following demonstration considerations:

\begin{tabular}{|l|l|}
\hline $\begin{array}{l}\text { Military Activity: What makes } \\
\text { this technology particularly } \\
\text { useful to supporting one or } \\
\text { more military transportation } \\
\text { support activities? }\end{array}$ & Demonstration concepts: \\
\hline $\begin{array}{l}\text { Autonomous Vehicle: What } \\
\text { makes this system a good can- } \\
\text { didate for AV research, } \\
\text { demonstration? Why is it } \\
\text { unique and potentially useful } \\
\text { to CERL research objectives? }\end{array}$ & Demonstration concepts: \\
\hline $\begin{array}{l}\text { Microgrid: How can this system } \\
\text { support microgrid research and } \\
\text { demonstration objectives con- } \\
\text { sistent with CERL objectives } \\
\text { and CBITEC capabilities? }\end{array}$ & Demonstration concepts: \\
\hline $\begin{array}{l}\text { Partnering in Research: Describe the potential for and interest in establishing a part- } \\
\text { nership for demonstration and research application between this manufacturer and } \\
\text { CERL. }\end{array}$ & - \\
\hline \begin{tabular}{l} 
Other Comments and Considerations: \\
\hline
\end{tabular} & \\
\hline
\end{tabular}


budget or schedule constraints associated with the demonstration.

Nikola Review by: Brubaker LLC

Do you recommend this technology for ATI demonstration?

$\mathrm{Y} / \mathrm{N}$ : Yes (top pick)

If No, please explain: N/A

If Yes, please explain below based on the following demonstration considerations:

\begin{tabular}{|c|c|}
\hline $\begin{array}{l}\text { Military Activity: What makes } \\
\text { this technology particularly } \\
\text { useful to supporting one or } \\
\text { more military transportation } \\
\text { support activities? }\end{array}$ & $\begin{array}{l}\text { Demonstration concepts: } \\
\text { - Reconnaissance capabilities } \\
\text { - } \text { Autonomous Weapons Platform } \\
\text { - Ruggedized transport } \\
\text { - } \quad \text { Flexible modular capabilities }\end{array}$ \\
\hline $\begin{array}{l}\text { Autonomous Vehicle: What } \\
\text { makes this system a good can- } \\
\text { didate for AV research, } \\
\text { demonstration? Why is it } \\
\text { unique and potentially useful } \\
\text { to CERL research objectives? }\end{array}$ & $\begin{array}{l}\text { Demonstration concepts: } \\
\text { - Great EV platform for an autonomous } \\
\text { - demonstration } \\
\text { - } \quad \text { Rrive-by-wire modern control system } \\
\text { - Reatform for outfitting }\end{array}$ \\
\hline $\begin{array}{l}\text { Microgrid: How can this system } \\
\text { support microgrid research and } \\
\text { demonstration objectives con- } \\
\text { sistent with CERL objectives } \\
\text { and CBITEC capabilities? }\end{array}$ & $\begin{array}{l}\text { Demonstration concepts: } \\
\text { - Great power } \\
\text { - Bidirectional } \\
\text { - Uses J1772 charger } \\
\text { - DC fast-charging capable }\end{array}$ \\
\hline
\end{tabular}

Partnering in Research: Describe the potential for and interest in establishing a partnership for demonstration and research application between this manufacturer and CERL.

High potential for partnership. This would be an exceptional platform to test-Nikola is willing to partner, but the cost of the vehicle is high. 


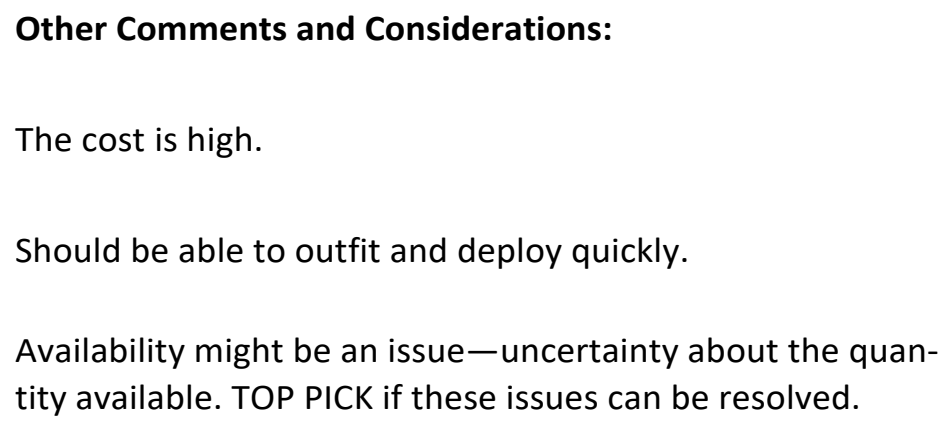

Nikola Review by: Robotic Research

Do you recommend this technology for ATI demonstration? Y/N: Yes

If No, please explain: N/A

If Yes, please explain below based on the following demonstration considerations:

\begin{tabular}{|c|c|}
\hline $\begin{array}{l}\text { Military Activity: What makes } \\
\text { this technology particularly use- } \\
\text { ful to supporting one or more } \\
\text { military transportation support } \\
\text { activities? }\end{array}$ & $\begin{array}{l}\text { Demonstration concepts: } \\
\text { - High power/weight capability } \\
\text { - Long range } \\
\text { - Quite operation }\end{array}$ \\
\hline $\begin{array}{l}\text { Autonomous Vehicle: What } \\
\text { makes this system a good can- } \\
\text { didate for AV research, demon- } \\
\text { stration? Why is it unique and } \\
\text { potentially useful to CERL re- } \\
\text { search objectives? }\end{array}$ & $\begin{array}{l}\text { Demonstration concepts: } \\
\text { - Steer and throttle-by-wire ready } \\
\text { - High energy storage system }\end{array}$ \\
\hline $\begin{array}{l}\text { Microgrid: How can this system } \\
\text { support microgrid research and } \\
\text { demonstration objectives con- } \\
\text { sistent with CERL objectives and } \\
\text { CBITEC capabilities? }\end{array}$ & $\begin{array}{l}\text { Demonstration concepts: } \\
\text { - Bidirectional capabilities } \\
\text { - High-capacity energy storage system }\end{array}$ \\
\hline \multicolumn{2}{|c|}{$\begin{array}{l}\text { Partnering in Research: Describe the potential for and interest in establishing a part- } \\
\text { nership for demonstration and research application between this manufacturer and } \\
\text { CERL. }\end{array}$} \\
\hline
\end{tabular}


Nikola appears to want to lease vehicles, might not be ready to enter partnership.

Other Comments and Considerations:

Very high cost (lease/month) and limited availability.

Nikola Review by: TechFlow, Inc.

Do you recommend this technology for ATI demonstration? Y/N: Yes, pending cost resolution.

If No, please explain: N/Q

If Yes, please explain below based on the following demonstration considerations:

\begin{tabular}{|l|l|}
\hline $\begin{array}{l}\text { Military Activity: What makes } \\
\text { this technology particularly } \\
\text { useful to supporting one or } \\
\text { more military transportation } \\
\text { support activities? }\end{array}$ & Demonstration concepts: \\
$\begin{array}{l}\text { Off-road capability fits very } \\
\text { well within a tactical environ- } \\
\text { ment and would support all } \\
\text { program goals. }\end{array}$ & \\
\hline $\begin{array}{l}\text { Autonomous Vehicle: What } \\
\text { makes this system a good } \\
\text { candidate for AV research, } \\
\text { demonstration? Why is it } \\
\text { unique and potentially useful } \\
\text { to CERL research objectives? }\end{array}$ & Demonstration concepts: \\
\hline
\end{tabular}




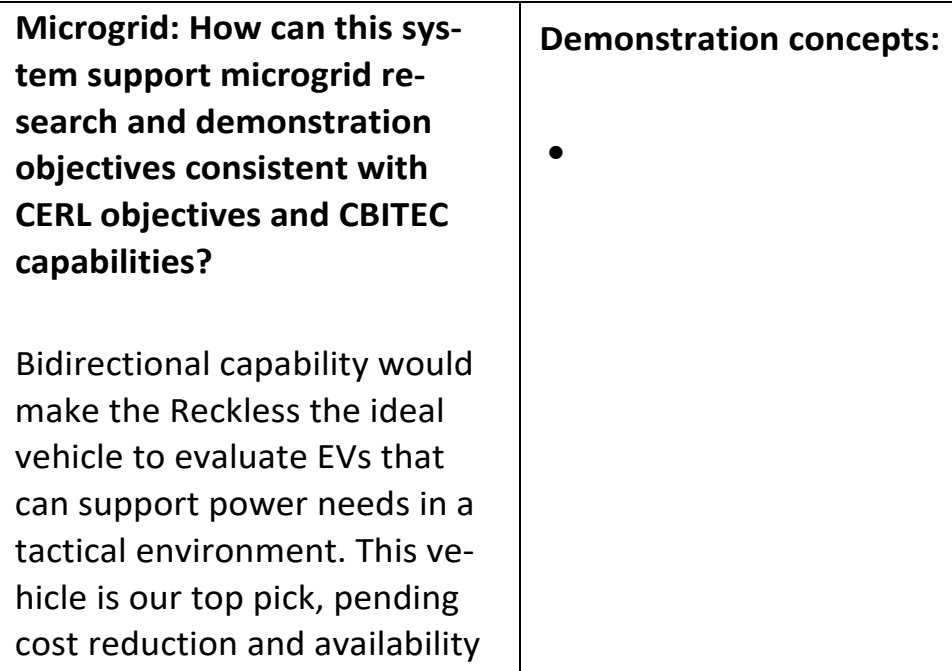

\section{Demonstration concepts:}

$\bullet$

Partnering in Research: Describe the potential for and interest in establishing a partnership for demonstration and research application between this manufacturer and CERL.

Other Comments and Considerations:

We believe the open chassis of the Reckless would greatly facilitate the integration of components to implement autonomy and bidirectional power flow. Since the DoD tactical space is a target market for Nokia, their products and capabilities might be a very good fit for program objectives.

Orange EV Review by: Brubaker LLC

Do you recommend this technology for ATI demonstration? Y/N: No

If No, please explain: No demonstrated bidirectional charging capability. Limited platform. Not a good candidate for this RD\&D project.

If Yes, please explain below based on the following demonstration considerations:

\begin{tabular}{|l|l|}
\hline Military Activity: What makes & Demonstration concepts: \\
this technology particularly use- & \\
ful to supporting one or more & \\
military transportation support & \\
activities? & \\
\hline
\end{tabular}




\begin{tabular}{|l|l|}
\hline $\begin{array}{l}\text { Autonomous Vehicle: What } \\
\text { makes this system a good candi- } \\
\text { date for AV research, demon- } \\
\text { stration? Why is it unique and } \\
\text { potentially useful to CERL re- } \\
\text { search objectives? }\end{array}$ & Demonstration concepts: \\
\hline $\begin{array}{l}\text { Microgrid: How can this system } \\
\text { support microgrid research and } \\
\text { demonstration objectives con- } \\
\text { sistent with CERL objectives and } \\
\text { CBITEC capabilities? }\end{array}$ & Demonstration concepts: \\
\hline $\begin{array}{l}\text { Partnering in Research: Describe the potential for and interest in establishing a part- } \\
\text { nership for demonstration and research application between this manufacturer and } \\
\text { CERL. }\end{array}$ \\
\hline
\end{tabular}

Orange EV Review by: Robotic Research

Do you recommend this technology for ATI demonstration? Y/N: No

If No, please explain: Platform is limited to on-road use, does not support bidirectional charging, and does not have drive-by-wire capabilities on their roadmap.

If Yes, please explain below based on the following demonstration considerations:

\begin{tabular}{|l|l|}
\hline $\begin{array}{l}\text { Military Activity: What makes } \\
\text { this technology particularly use- } \\
\text { ful to supporting one or more } \\
\text { military transportation support } \\
\text { activities? }\end{array}$ & Demonstration concepts: \\
\hline $\begin{array}{l}\text { Autonomous Vehicle: What } \\
\text { makes this system a good can- } \\
\text { didate for AV research, demon- } \\
\text { stration? Why is it unique and } \\
\text { potentially useful to CERL }\end{array}$ & Demonstration concepts: \\
\hline
\end{tabular}




\begin{tabular}{|l|l|}
\hline research objectives? & \\
\hline $\begin{array}{l}\text { Microgrid: How can this system } \\
\text { support microgrid research and } \\
\text { demonstration objectives con- } \\
\text { sistent with CERL objectives and } \\
\text { CBITEC capabilities? }\end{array}$ & Demonstration concepts: \\
\hline $\begin{array}{l}\text { Partnering in Research: Describe the potential for and interest in establishing a part- } \\
\text { nership for demonstration and research application between this manufacturer and } \\
\text { CERL. }\end{array}$ \\
\hline
\end{tabular}

Orange Review by: TechFlow, Inc.

Do you recommend this technology for ATI demonstration? Y/N: No

If No, please explain: Effort to develop bidirectional power and autonomy requirements will likely not meet budget, or schedule constraints, and present more risk compared to other options.

If Yes, please explain below based on the following demonstration considerations:

\begin{tabular}{|l|l|}
\hline $\begin{array}{l}\text { Military Activity: What makes } \\
\text { this technology particularly } \\
\text { useful to supporting one or } \\
\text { more military transportation } \\
\text { support activities? }\end{array}$ & Demonstration concepts: \\
\hline $\begin{array}{l}\text { Autonomous Vehicle: What } \\
\text { makes this system a good } \\
\text { candidate for AV research, } \\
\text { demonstration? Why is it } \\
\text { unique and potentially useful } \\
\text { to CERL research objectives? }\end{array}$ & Demonstration concepts: \\
\hline
\end{tabular}




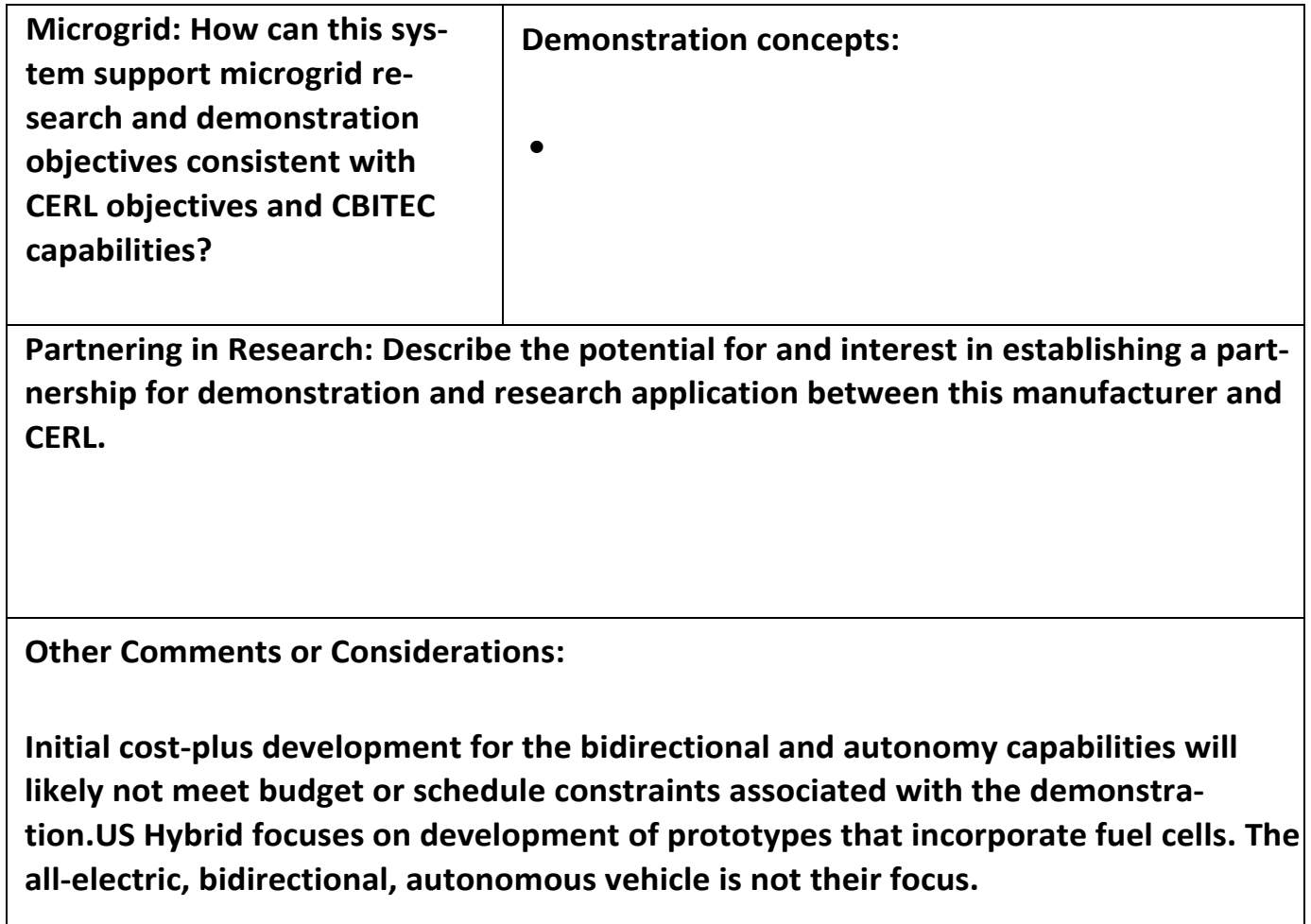

Phoenix Motorcars Review by: Brubaker LLC

Do you recommend this technology for ATI demonstration? Y/N: Yes

If No, please explain: N/A

If Yes, please explain below based on the following demonstration considerations:

\begin{tabular}{|c|c|}
\hline $\begin{array}{l}\text { Military Activity: What makes } \\
\text { this technology particularly } \\
\text { useful to supporting one or } \\
\text { more military transportation } \\
\text { support activities? }\end{array}$ & $\begin{array}{l}\text { Demonstration concepts: } \\
\text { - Shuttle bus } \\
\text { - Flatbeds }\end{array}$ \\
\hline $\begin{array}{l}\text { Autonomous Vehicle: What } \\
\text { makes this system a good } \\
\text { candidate for AV research, } \\
\text { demonstration? Why is it } \\
\text { unique and potentially useful } \\
\text { to CERL research objectives? }\end{array}$ & $\begin{array}{l}\text { Demonstration concepts: } \\
\text { - Good EV platform for an autonomous } \\
\text { - } \text { demonstration } \\
\text { Partners have experience in outfitting simi- } \\
\text { - Data collection }\end{array}$ \\
\hline
\end{tabular}




\begin{tabular}{|l|l|}
\hline $\begin{array}{l}\text { Microgrid: How can this sys- } \\
\text { tem support microgrid re- } \\
\text { search and demonstration ob- }\end{array}$ & Demonstration concepts: \\
$\begin{array}{l}\text { jectives consistent with CERL } \\
\text { objectives and CBITEC capabil- } \\
\text { ities? }\end{array}$ & $\begin{array}{l}\text { - } \\
\text { - Storage capacity }\end{array}$ \\
\hline
\end{tabular}

Partnering in Research: Describe the potential for and interest in establishing a partnership for demonstration and research application between this manufacturer and CERL.

Good potential for partnership. These are fairly simple platforms to outfit and test.

Other Comments and Considerations:

Unsure about off-road capacity of the flatbed platform in an operational environment. Should be able to outfit and deploy quickly.

Some risk in that they are mostly familiar with fuel cell systems.

Phoenix Motorcars Review by: Robotic Research

Do you recommend this technology for ATI demonstration? Y/N: No

If No, please explain: The base platform does not seem well suited for off-road/military use. I would be more interested in a smaller truck variant as opposed to the shuttle vans.

If Yes, please explain below based on the following demonstration considerations:

\begin{tabular}{|l|l|}
\hline $\begin{array}{l}\text { Military Activity: What makes } \\
\text { this technology particularly } \\
\text { useful to supporting one or } \\
\text { more military transportation } \\
\text { support activities? }\end{array}$ & Demonstration concepts: \\
\hline $\begin{array}{l}\text { Autonomous Vehicle: What } \\
\text { makes this system a good } \\
\text { candidate for AV research, } \\
\text { demonstration? Why is it } \\
\text { unique and potentially useful } \\
\text { to CERL research objectives? }\end{array}$ & Demonstration concepts: \\
\hline
\end{tabular}




\begin{tabular}{|l|l|}
\hline $\begin{array}{l}\text { Microgrid: How can this sys- } \\
\text { tem support microgrid re- } \\
\text { search and demonstration ob- } \\
\text { jectives consistent with CERL } \\
\text { objectives and CBITEC capabil- } \\
\text { ities? }\end{array}$ & Demonstration concepts: \\
\hline $\begin{array}{l}\text { Partnering in Research: Describe the potential for and interest in establishing a part- } \\
\text { nership for demonstration and research application between this manufacturer and } \\
\text { CERL. }\end{array}$ \\
\hline Other Comments or Considerations: \\
\hline
\end{tabular}

Phoenix Motorcars Review by: TechFlow, Inc.

Do you recommend this technology for ATI demonstration? Y/N: No

If No, please explain: Effort to develop bidirectional power and autonomy requirements will likely not meet budget, or schedule constraints, and are more risk compared to other options.

If Yes, please explain below based on the following demonstration considerations:

\begin{tabular}{|l|l|}
\hline $\begin{array}{l}\text { Military Activity: What makes } \\
\text { this technology particularly } \\
\text { useful to supporting one or } \\
\text { more military transportation } \\
\text { support activities? }\end{array}$ & Demonstration concepts: \\
\hline $\begin{array}{l}\text { Autonomous Vehicle: What } \\
\text { makes this system a good } \\
\text { candidate for AV research, } \\
\text { demonstration? Why is it } \\
\text { unique and potentially useful } \\
\text { to CERL research objectives? }\end{array}$ & Demonstration concepts: \\
\hline $\begin{array}{l}\text { Microgrid: How can this sys- } \\
\text { tem support microgrid re- } \\
\text { search and demonstration ob- } \\
\text { jectives consistent with CERL }\end{array}$ & Demonstration concepts: \\
\hline
\end{tabular}




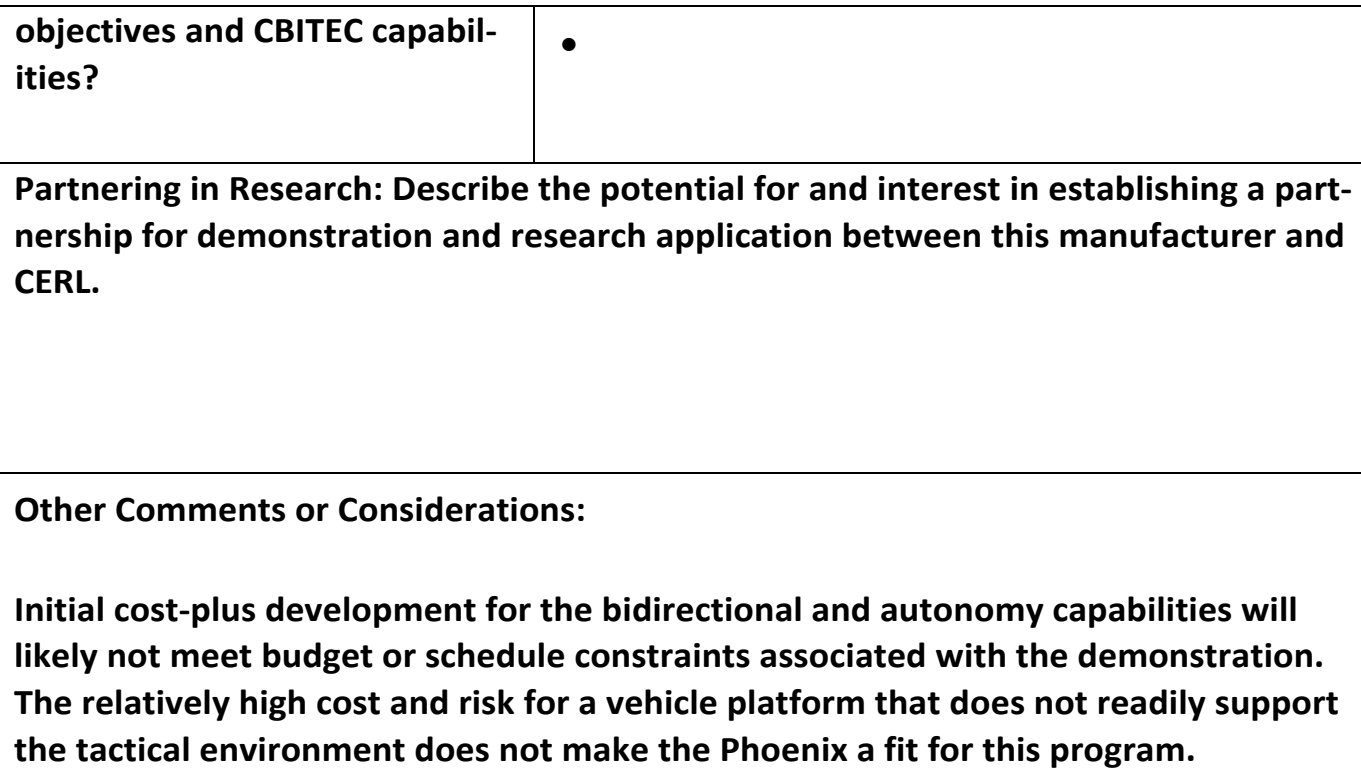

Other Comments or Considerations:

Initial cost-plus development for the bidirectional and autonomy capabilities will likely not meet budget or schedule constraints associated with the demonstration. The relatively high cost and risk for a vehicle platform that does not readily support the tactical environment does not make the Phoenix a fit for this program.

US Hybrid Review by: Brubaker LLC

Do you recommend this technology for ATI demonstration? Y/N: No

If No, please explain: Company builds one-off prototypes. Too much execution and timing risk to spec out and build a custom platform.

If Yes, please explain below based on the following demonstration considerations:

\begin{tabular}{|l|l|}
\hline $\begin{array}{l}\text { Military Activity: What makes } \\
\text { this technology particularly } \\
\text { useful to supporting one or } \\
\text { more military transportation } \\
\text { support activities? }\end{array}$ & Demonstration concepts: \\
\hline $\begin{array}{l}\text { Autonomous Vehicle: What } \\
\text { makes this system a good can- } \\
\text { didate for AV research, } \\
\text { demonstration? Why is it } \\
\text { unique and potentially useful } \\
\text { to CERL research objectives? }\end{array}$ & Demonstration concepts: \\
\hline $\begin{array}{l}\text { Microgrid: How can this system } \\
\text { support microgrid research and } \\
\text { demonstration objectives con- } \\
\text { sistent with CERL objectives }\end{array}$ & Demonstration concepts: \\
\hline
\end{tabular}




\begin{tabular}{|l|}
\hline and CBITEC capabilities? \\
\hline $\begin{array}{l}\text { Partnering in Research: Describe the potential for and interest in establishing a part- } \\
\text { nership for demonstration and research application between this manufacturer and } \\
\text { CERL. }\end{array}$ \\
\hline Other Comments or Considerations: \\
\hline
\end{tabular}

US Hybrid Review by: Robotic Research

Do you recommend this technology for ATI demonstration? Y/N: No

If No, please explain: I am concerned about the producibility of this vehicle. It seems to be second-order modified from the OEM.

If Yes, please explain below based on the following demonstration considerations:

\begin{tabular}{|l|l|}
\hline $\begin{array}{l}\text { Military Activity: What makes } \\
\text { this technology particularly } \\
\text { useful to supporting one or } \\
\text { more military transportation } \\
\text { support activities? }\end{array}$ & Demonstration concepts: \\
\hline $\begin{array}{l}\text { Autonomous Vehicle: What } \\
\text { makes this system a good } \\
\text { candidate for AV research, } \\
\text { demonstration? Why is it } \\
\text { unique and potentially useful } \\
\text { to CERL research objectives? }\end{array}$ & Demonstration concepts: \\
\hline $\begin{array}{l}\text { Microgrid: How can this sys- } \\
\text { tem support microgrid re- } \\
\text { search and demonstration ob- } \\
\text { jectives consistent with CERL } \\
\text { objectives and CBITEC capabil- } \\
\text { ities? }\end{array}$ & Demonstration concepts: \\
\hline
\end{tabular}


Partnering in Research: Describe the potential for and interest in establishing a partnership for demonstration and research application between this manufacturer and CERL.

Other Comments or Considerations:

US Hybrid Review by: TechFlow, Inc.

Do you recommend this technology for ATI demonstration? Y/N: No

If No, please explain: Effort to develop bidirectional power and autonomy requirements will likely not meet budget, or schedule constraints, and present more risk compared to other options.

If Yes, please explain below based on the following demonstration considerations:

\begin{tabular}{|l|l|}
\hline $\begin{array}{l}\text { Military Activity: What makes } \\
\text { this technology particularly } \\
\text { useful to supporting one or } \\
\text { more military transportation } \\
\text { support activities? }\end{array}$ & Demonstration concepts: \\
\hline $\begin{array}{l}\text { Autonomous Vehicle: What } \\
\text { makes this system a good } \\
\text { candidate for AV research, } \\
\text { demonstration? Why is it } \\
\text { unique and potentially useful } \\
\text { to CERL research objectives? }\end{array}$ & Demonstration concepts: \\
\hline $\begin{array}{l}\text { Microgrid: How can this sys- } \\
\text { tem support microgrid re- } \\
\text { search and demonstration } \\
\text { objectives consistent with } \\
\text { CERL objectives and CBITEC } \\
\text { capabilities? }\end{array}$ & Demonstration concepts: \\
\hline
\end{tabular}


Partnering in Research: Describe the potential for and interest in establishing a partnership for demonstration and research application between this manufacturer and CERL.

Other Comments and Considerations:

Initial cost-plus development for the bidirectional and autonomy capabilities will likely not meet budget or schedule constraints associated with the demonstration. US Hybrid focuses on development of prototypes that incorporate fuel cells. The allelectric, bidirectional, autonomous vehicle is not their focus. 


\title{
Appendix L: Bollinger Motors
}

\author{
Bollinger B1 and Bollinger B2 \\ Manufacturer: Bollinger Motors
}
Bollinger manufactures all-electric, all-aluminum Class 3 trucks in Detroit, $\mathrm{MI}$ and was founded in 2015.

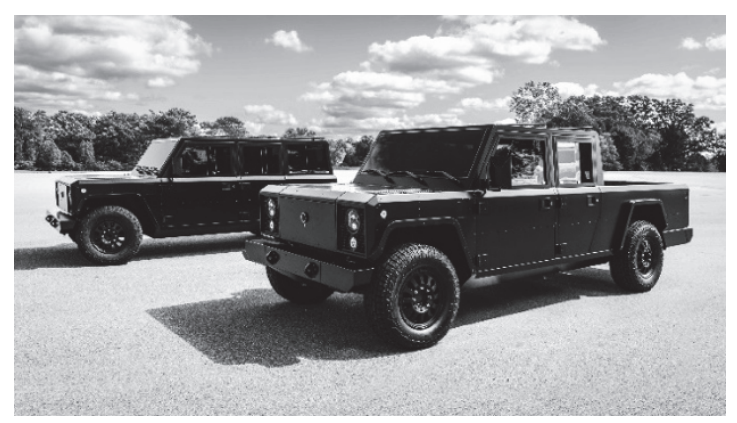

\begin{tabular}{|c|c|c|}
\hline & Bollinger B1 & Bollinger B2 \\
\hline Cost & \multicolumn{2}{|l|}{$\$ 125,000$ MSRP } \\
\hline Application Maturity & \multicolumn{2}{|c|}{$\begin{array}{l}\text { Production is set to start in the second half of } 2020 \text { with customer deliveries beginning in } 2021 . \\
\text { Bollinger's trucks are only available for reservation though the company states that process is "NOT a } \\
\text { vehicle purchase." Buyers will be "required to configure vehicle and options at a later date subject to } \\
\text { availability and production results at that time" after which buyers will finalize pricing with their } \\
\text { independent dealers, not Bollinger. The company is targeting low-volume production. }\end{array}$} \\
\hline Safety & \multicolumn{2}{|c|}{$\begin{array}{l}\text { The B1 and B2 are to be built to exceed federal regulations for seat belt safety standards, but air } \\
\text { bags are not available. }\end{array}$} \\
\hline Communication & \multicolumn{2}{|l|}{ Bluetooth } \\
\hline $\begin{array}{l}\text { Charging Time and } \\
\text { Capacity }\end{array}$ & \multicolumn{2}{|l|}{10 hours for $220 \mathrm{~V}$ and 75 minutes for $\mathrm{DC}$ fast } \\
\hline $\begin{array}{l}\text { Charging } \\
\text { Infrastructure }\end{array}$ & \multicolumn{2}{|c|}{ Level 1 and 2: J1772 Port (110 V and 220 V), Level 3: DC fast } \\
\hline $\begin{array}{l}\text { Run-Time } \\
\text { Capabilities }\end{array}$ & \multicolumn{2}{|l|}{ 200-mile range } \\
\hline Performance & \multicolumn{2}{|l|}{$614 \mathrm{HP}, 668 \mathrm{ft}-\mathrm{lb}$ torque } \\
\hline $\begin{array}{l}\text { Operability in } \\
\text { Adverse Weather or } \\
\text { Conditions }\end{array}$ & \multicolumn{2}{|l|}{ Removable glass, doors, and windshields } \\
\hline Projected Life Span & \multicolumn{2}{|c|}{$\begin{array}{l}\text { The company says the trucks are built to last "a lifetime," and it hopes to eventually offer updated, } \\
\text { lighter batteries for its current trucks as they become available }\end{array}$} \\
\hline Stealth Capabilities & \multicolumn{2}{|l|}{ None } \\
\hline $\begin{array}{l}\text { Off-Road } \\
\text { Capabilities and } \\
\text { Limitations }\end{array}$ & $\begin{array}{l}\text { 15-inch ground clearance with } 10 \text {-inch } \\
\text { wheel travel }\end{array}$ & 16-inch ground clearance and 10 -inch wheel travel \\
\hline $\begin{array}{l}\text { Hauling and Towing } \\
\text { Capacity }\end{array}$ & $\begin{array}{l}\text { 5,000-Ib. payload, } 14 \text { cubic feet of "frunk" } \\
\text { storage capacity, } 7,500-\text { Ib. towing capacity }\end{array}$ & $\begin{array}{l}\text { 5,000-lb. payload, } 72 \text {-inch bed length, } 96 \text {-inch } \\
\text { expandable bed, } 14 \text { cubic feet of "frunk," and 7,500-lb. } \\
\text { towing capacity }\end{array}$ \\
\hline $\begin{array}{l}\text { Current Automation } \\
\text { Capabilities }\end{array}$ & \multicolumn{2}{|l|}{ None } \\
\hline $\begin{array}{l}\text { Future Automation } \\
\text { Capabilities }\end{array}$ & \multicolumn{2}{|c|}{ The CEO of the company has stated that Bollinger has no plans to pursue autonomy } \\
\hline
\end{tabular}


Comments

Bollinger bills the B2 as the "big brother of the B1," which is essentially the same vehicle with the rea seats removed, enabling a trunk bed and a wider wheelbase. Both trucks are Class 3.

Website: bollingermotors.com/

Contact: Chet Parsons, Sales Director: 313-486-0500; chet@bollingermotors.com 


\section{Appendix M: Autonomy Kits, Sensors and Technology}

Table M-1. Robotic research autonomy kit for autonomous ground resupply (ARG).

\begin{tabular}{|l|l|}
\hline Element & Summary \\
\hline Description & $\begin{array}{l}\text { Robotic Research was engaged by both the National Advanced Mobility Consortium (NAMC) and Army's } \\
\text { TARDEC to develop the autonomy kit for the AGR Sustainment Operations program. Under the six-year } \\
\text { contract, Robotic Research will develop a fault-tolerant vehicle-agnostic applique kit to enable higher-level } \\
\text { autonomous-driving and planning functions for integration onto existing military ground vehicles. }\end{array}$ \\
\hline $\begin{array}{l}\text { The kit implements an interoperability profile (IOP) and higher-level robotic modes, including-but not limited } \\
\text { to-teleoperation, waypoint navigation, and leader/follower technology. The kit hardware consists of sensing } \\
\text { and computing resources necessary for perception, world modeling, planning, and the execution of } \\
\text { Leader/Follower and teleoperation and it contains all power conditioning, signal switching, and routing. } \\
\text { Sensor suite includes: } \\
\text { COTS LiDAR sensors } \\
\text { Automotive radar } \\
\text { Color cameras } \\
\text { RR-N-140 Navigation System and Ultra-wideband (UWB) Ranging Nodes }\end{array}$ \\
\hline $\begin{array}{l}\text { Website: roboticresearch.com/programs/agr } \\
\text { Robotic Research and TARDEC presentation at the 2018 NDIA Ground Vehicle Systems Engineering and }\end{array}$ \\
$\begin{array}{l}\text { Technology Symposium } \\
\text { AUVSI Xponential: Robotic Research Paves the Way for Transportation Autonomy with Commercial } \\
\text { and Defense Solutions, 29 Apr 2019 }\end{array}$ \\
\hline
\end{tabular}

Table M-2. Autonomy research kit (ARK).

\begin{tabular}{|l|l|}
\hline Description & $\begin{array}{l}\text { ARK is an all-in-one autonomy kit, enabling point-to-point autonomous navigation of mobile robots. Kit provides } \\
\text { advanced software with a powerful processor and sensor suite. ARK is ROS ready and fully extensible with a } \\
\text { well document API. ARK is ideal for indoor, single-level, infrastructure free-navigation. }\end{array}$ \\
\hline Features & $\begin{array}{l}\text { ARK provides point-to-point navigation with dynamic path planning, simultaneous mapping and localization, } \\
\text { and obstacle avoidance. Its web-based interface, ARK Mapper, allows for manual controls of robots and the } \\
\text { creation of 2D maps of indoor environments. Supported LiDARs include Hokuyo UST-10LX and SICK LMS1xx. }\end{array}$ \\
\hline $\begin{array}{l}\text { Supported } \\
\text { Technology }\end{array}$ & $\begin{array}{l}\text { Kit integrates with Clearpath mobile robotic development platforms, including the Husky UGV and Ridgeback } \\
\text { mobile manipulation platform. ARK also integrates with the Jackal UGV. }\end{array}$ \\
\hline References & $\begin{array}{l}\text { Website: clearpathrobotics.com/autonomy-research-kit/ } \\
\text { White paper: Comparing accuracy of open source vs. proprietary localization software }\end{array}$ \\
\hline
\end{tabular}

Table M-3. Perrone Robotics drop-in autonomy kit (DAK).

\begin{tabular}{|l|l|}
\hline Element & \multicolumn{1}{|c|}{ Summary } \\
\hline Description & $\begin{array}{l}\text { DAK is an autonomous kit system for outfitting existing vehicles with autonomous and remote-control driving } \\
\text { capabilities. }\end{array}$ \\
\hline Features & $\begin{array}{l}\text { DAK was built using the MAX general purpose robotics and automated vehicle operating system. } \\
\text { The DAK kit includes all necessary actuators, sensors, electronics, and software needed for operation. Key } \\
\text { features include: } \\
\text { Steering, brake, and throttle control } \\
\text { 30-minute or less install } \\
\text { Programmatic fully autonomous navigation } \\
\text { Route and trajectory planning } \\
\text { Maneuver planning } \\
\text { Remote teleoperation available }\end{array}$ \\
\hline
\end{tabular}




\begin{tabular}{|l|l|}
\hline Element & \multicolumn{1}{|c|}{ Summary } \\
\hline \multirow{3}{*}{$\begin{array}{l}\text { Supported } \\
\text { Technology }\end{array}$} & $\begin{array}{l}\text { Compatible with Locata, an alternative local position system that uses a constellation of LocataLite. Locata } \\
\text { allows DAK to deliver tunable accuracy indoors or outdoors and in adverse weather, 24/7. Embedded } \\
\text { software in DAK allows for defining and controlling testing and transferring and reviewing data. DAK complies } \\
\text { with requirements from the Insurance Institute for Highway Safety and features software for performing } \\
\text { NHTSA Crash-Imminent Braking, NHTSA Dynamic Brake Support, Euro NCAP Autonomous Emergency Braking, } \\
\text { and others. }\end{array}$ \\
\hline References & $\begin{array}{l}\text { Website: perronerobotics.squarespace.com/dak } \\
\text { Other Perrone turn-key products for autonomous robotics is found here. }\end{array}$ \\
\hline
\end{tabular}

Table M-4. Autonomous Mobility Applique System (AMAS).

\begin{tabular}{|l|l|}
\hline Element & \multicolumn{1}{|c|}{ Summary } \\
\hline Description & $\begin{array}{l}\text { Developed by Lockheed Martin, the AMAS autonomy kit provides a low-cost, low-risk, kit-based solution to } \\
\text { retrofit active safety, semi-autonomy, and autonomy capability onto any vehicle in the military's logistics fleet. } \\
\text { The AMAS Operational Demonstration and Military User Assessment (MUA) was conducted in 2014 and the } \\
\text { system was tested more recently at the Aberdeen Proving Ground in 2015. }\end{array}$ \\
\hline Features & $\begin{array}{l}\text { AMAS features Driver Warning/Driver Assist functionality, Leader-Follower convoy operations, Waypoint } \\
\text { following capabilities, and provides growth to fully autonomous operations. }\end{array}$ \\
\hline References & $\begin{array}{l}\text { Website: lockheedmartin.com } \\
\text { Army, Lockheed Martin, demonstrate fully autonomous convoys can operate in urban areas, }\end{array}$ \\
$\begin{array}{l}\text { 31 Jan 2014 } \\
\text { Integration of the Autonomous Mobility Applique System into the Robotic Technology Kernel } \\
\text { presentation from U.S. Army Combat Capabilities Development Command Ground Vehicle Systems Center and } \\
\text { Lockheed Martin at the 2019 NDIA Ground Vehicle Systems Engineering and Technology Symposium. }\end{array}$ \\
\hline
\end{tabular}

Table M-5. Unit-to-unit autonomous resupply vehicle (U2UARV).

\begin{tabular}{|c|c|}
\hline Element & Summary \\
\hline Description & $\begin{array}{l}\text { Cybernet's U2UARV bills itself as a general purpose "Swiss Army knife" vehicle automation kit that can } \\
\text { autonomously deliver and return ordnance, food, water, ammunition, and general logistics and resupply. }\end{array}$ \\
\hline $\begin{array}{l}\text { Supported } \\
\text { Technology }\end{array}$ & Compatible with the Gladiator UGV, Polaris, and the Hyster fork truck. \\
\hline References & $\begin{array}{l}\text { Website: cybernet.com } \\
\text { More information here. }\end{array}$ \\
\hline
\end{tabular}

Table M-6. Cybernet technologies.

\begin{tabular}{|l|l|}
\hline Element & \multicolumn{1}{|c|}{ Summary } \\
\hline \multirow{3}{*}{ Features } & $\begin{array}{l}\text { Automated Tactical Ammunition Classification (ATACS) } \\
\text { Inertial Measurements and Magnetometer Module (I3M) }\end{array}$ \\
& $\begin{array}{l}\text { OpenSkies } \\
\text { Rapidly Generated Instrument Panels for Simulation and Training: developed specifically for the Navy } \\
\text { Virtual Systems Integration Lab (VSIL): an integrated simulation platform for the development, modification, } \\
\text { and prototyping of vehicle systems and components. }\end{array}$ \\
\hline References & Website: cybernet.com/defense-and-security-technologies/robotics More information here. \\
\hline
\end{tabular}

Table M-7. Lanecruise.

\begin{tabular}{|c|l|}
\hline Element & \multicolumn{1}{|c|}{ Summary } \\
\hline Description & $\begin{array}{l}\text { Developed by X-Matix, LaneCruise autonomy kit is a complete system of hardware components and software } \\
\text { elements that enables semi-autonomy, active safety, and fleet management. }\end{array}$ \\
\hline
\end{tabular}




\begin{tabular}{|c|c|}
\hline Element & Summary \\
\hline \multirow{5}{*}{ Features } & The kit includes four major hardware components: \\
\hline & $\begin{array}{l}\text { Linx: a fully integrated vision and location sensor and computation system capable of SAE Level } 3 \text { autonomy } \\
\text { and HD telematics data gathering. }\end{array}$ \\
\hline & Brake actuators \\
\hline & $\begin{array}{l}\text { Gas control module: an electronic by-pass device that utilizes onboard vehicle drive-by-wire technology to } \\
\text { sense and control throttle position. }\end{array}$ \\
\hline & Wheel actuator \\
\hline References & Website: $\underline{x-m a t i k . c o m / l a n e c r u i s e ~}$ \\
\hline
\end{tabular}

Table M-8. Luminar.

\begin{tabular}{|l|l|}
\hline Element & Summary \\
\hline Description & $\begin{array}{l}\text { Luminar bills its sensor as the “first and only LiDAR sensing system to meet the stringent performance and } \\
\text { safety requirements needed to achieve safer-than-human level perception.” }\end{array}$ \\
\hline Features & $\begin{array}{l}\text { The sensor offers a true } 250-\text {-meter range with }<10 \% \text { reflectively, } 200 \mathrm{px} / \mathrm{Deg}^{2} \text { camera-like resolution, }<1 \mathrm{~cm} \\
\text { range precision with high-precision reflectance, and a } 120^{\circ} \text { field of view with } 30^{\circ} \text { dynamic vertical resolution. } \\
\text { Rated IP67 for dust and water ingress. } \\
\text { Vibration: SAE J1211 } \\
\text { Shock: IEC } 60068-2-27 \text { to } 20 \text { g. }\end{array}$ \\
\hline References & $\begin{array}{l}\text { Website: luminartech.com } \\
\text { Luminar prepares for production of LiDAR at scale, } 29 \text { Jul } 2019 . \\
\begin{array}{l}\text { Luminar also offers Iris, a sensor for production vehicles. Iris offers the same core Luminar technology and } \\
\text { performance in an efficient auto-grade, low-cost package for series production programs beginning SOP } 2022 .\end{array}\end{array}$ \\
\hline
\end{tabular}

\section{Table M-9. Shadowcam.}

\begin{tabular}{|c|l|}
\hline Element & \multicolumn{1}{|c|}{ Summary } \\
\hline Description & $\begin{array}{l}\text { On 28 Oct 2019, MIT announced the development of its Shadowcam, a system that can sense tiny changes in } \\
\text { shadows on the ground to determine if there's a moving object coming around a corner. In a paper being } \\
\text { presented at International Conference on Intelligent Robots and Systems, MIT researchers will describe } \\
\text { successful experiments with an autonomous car driving around a parking garage and an autonomous } \\
\text { wheelchair navigating hallways. When sensing and stopping for an approaching vehicle, the car-based system } \\
\text { Shadowcam beats traditional LiDAR-which can only detect visible objects-by more than half a second. }\end{array}$ \\
\hline References & $\begin{array}{l}\text { ShadowCam: Real-Time Detection of Moving Obstacles Behind a Corner for Autonomous Vehicles, } \\
\text { 2018 } \\
\text { Enabling autonomous vehicles to see around corners, 28 Oct 2019 }\end{array}$ \\
\hline
\end{tabular}

Table M-10. Robosense LiDAR systems.

\begin{tabular}{|c|l|}
\hline Element & \multicolumn{1}{|c|}{ Summary } \\
\hline Description. & $\begin{array}{l}\text { RoboSense manufactures multiple LiDAR systems (mechanical, solid-state, and static 3D laser scanner), } \\
\text { LiDAR Perception Algorithms, and Perception solutions. }\end{array}$ \\
\hline
\end{tabular}




\begin{tabular}{|c|c|}
\hline Element & Summary \\
\hline \multirow{13}{*}{ Features } & Multi-Beam LiDAR Series \\
\hline & Ruby \\
\hline & BPearl \\
\hline & LiDAR-32 \\
\hline & LiDAR-16 \\
\hline & LiDAR-M1 \\
\hline & Medium-low Speed AD LiDAR Perception Solution \\
\hline & RoboTaxi LiDAR Perception Solution \\
\hline & V2R LiDAR Perception Solution \\
\hline & LiDAR Software \\
\hline & RSView 3.1.5 for Windows $7 / 8 / 10$ \\
\hline & RSView 3.1.5 for Ubuntu 16.04 \\
\hline & ROS Driver for RS-LiDAR-16 and RS-LiDAR-32 \\
\hline \multirow{2}{*}{ References } & Website: robosense.ai \\
\hline & More information on RoboSense's available technologies is here. \\
\hline
\end{tabular}

Table M-11. Velodyne LiDAR.

\begin{tabular}{|c|c|}
\hline Element & Summary \\
\hline Description & $\begin{array}{l}\text { Velodyne manufactures multiple LiDAR solutions, detailed below. } \\
\text { Velodyne's solutions allow customers to unlock advanced capabilities for ADA features including pedestrian } \\
\text { and bicycle avoidance, Lane Keep Assistance, Automatic Emergency Braking, Adaptive Cruise Control, Traffic } \\
\text { Jam Assist, and more. }\end{array}$ \\
\hline Features & 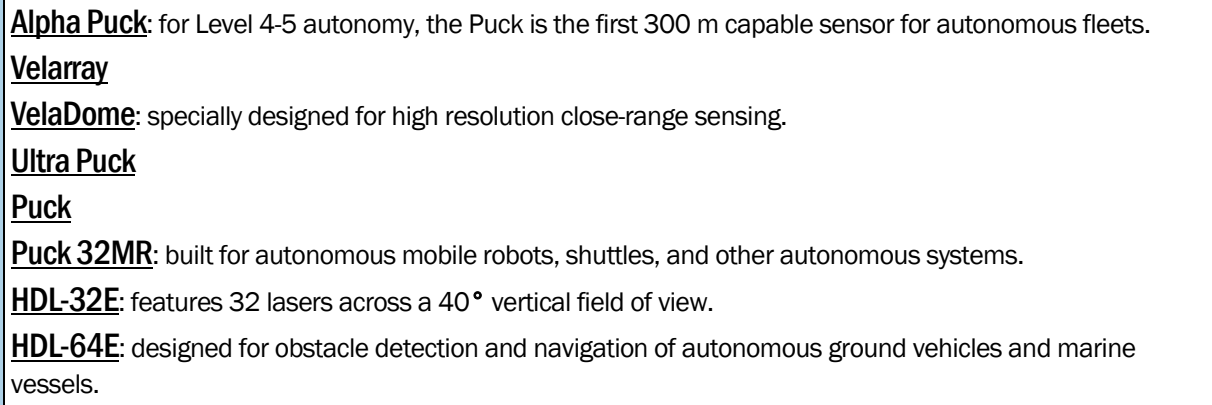 \\
\hline References & $\begin{array}{l}\text { Website: velodyneLiDAR.com } \\
\text { Product guides, data sheets, white papers, and more here. } \\
\text { A breakdown of Velodyne models and capabilities is found here. }\end{array}$ \\
\hline
\end{tabular}

\section{Table M-12. Mobileye.}

\begin{tabular}{|c|c|}
\hline Element & Summary \\
\hline Description & $\begin{array}{l}\text { Mobileye, a subsidiary of Intel, develops vision-based advanced driver assistance systems providing warnings } \\
\text { for collision prevention and mitigation. The company is a Tier } 2 \text { automotive supplier working with all major Tier } \\
1 \text { suppliers and their hardware and software is developed in-house. }\end{array}$ \\
\hline \multirow{3}{*}{ Features } & $\begin{array}{l}\text { EyeQ: Mobileye's system-on-chip technology family provides processing power to support ADAS functions } \\
\text { based on a single camera sensor. }\end{array}$ \\
\hline & $\begin{array}{l}\text { Road Experience Management (REM): end-to-end mapping and localization engine for full autonomy. REM } \\
\text { comprises three layers: harvesting agents, map aggregating server, and map-consuming agents. }\end{array}$ \\
\hline & Responsibility-Sensitive Safety (RSS): a mathematical model for AV safety. \\
\hline \multirow{2}{*}{ References } & Website: mobileye.com \\
\hline & Mobileye solutions specifically for fleets can be found $\underline{\text { here. }}$. \\
\hline
\end{tabular}


Table M-13. Innovusion LiDAR systems.

\begin{tabular}{|l|l|}
\hline Element & Summary \\
\hline Description & $\begin{array}{l}\text { Innovusion develops image-grade LiDAR systems for Level } 4 \text { and } 5 \text { AVs and ADAS markets. } \\
\text { Applications for Innovusion systems include vehicle safety systems, AV systems, and vehicle-to-everything } \\
\text { (V2X) for autos, trucks, buses, trains, and off-road vehicles. }\end{array}$ \\
\hline Features & $\begin{array}{l}\text { Cheetah: Cheetah utilizes the industry's first proprietary dual-rotating polygon design and is billed as } \\
\text { "compact, flexible, and easy for vehicle integration." The system draws under 40W, making it the most energy } \\
\text { efficient system of high-performance LiDAR currently on the market. } \\
\text { Jaguar: utilizes dual-rotating polygons to achieve detail out to } 280 \mathrm{~m} \text { and boasts a.13 } 13^{\circ} \text { resolution coupled with } \\
\text { a frame rate of } 10 \text { fps and selectable field of vision (FOV) of } 110^{\circ} \text { or } 65^{\circ} .\end{array}$ \\
\hline References & $\begin{array}{l}\text { Website: innovusion.com } \\
\text { Innovusion announces high-resolution image-grade LiDAR system Cheetah, } 17 \text { Jun } 2019\end{array}$ \\
\hline
\end{tabular}

Table M-14. Lumotive.

\begin{tabular}{|l|l|}
\hline Element & \multicolumn{1}{|c|}{ Summary } \\
\hline Description & $\begin{array}{l}\text { Lumotive develops high-performance, solid-state LiDAR powered by liquid crystal metasurface technology } \\
\text { based on beam-steering technology. The latter leverages semiconductor chips designed using metamaterials } \\
\text { principles and enables Lumotive's systems to perform at a high level while remaining cheaper than other } \\
\text { systems. } \\
\text { Initial production units will be available to select customers for beta testing in the 3rd quarter of } 2019 .\end{array}$ \\
\hline Features & $\begin{array}{l}\text { Lumotive's system offers: } \\
\text { Large optical aperture }(25 \times 25 \mathrm{~mm}) \text { which delivers long range } \\
120^{\circ} \text { field-of-view with high angular resolution } \\
\text { Fast random-access beam steering }\end{array}$ \\
\hline References & $\begin{array}{l}\text { Website: lumotive.com } \\
\text { Lumotive says it's got a solid-state LiDAR that really works, 21 Mar 2019 } \\
\text { Lumotive Unveils High-Performance LiDAR to Enable Autonomous Vehicles, } 20 \text { Mar } 2019\end{array}$ \\
\hline
\end{tabular}

Table M-15. Baidu Apollo Enterprise.

\begin{tabular}{|l|l|}
\hline \multicolumn{1}{|c|}{ Element } & \multicolumn{1}{c|}{ Summary } \\
\hline Description & $\begin{array}{l}\text { Baidu's Apollo Enterprise is a suite of intelligent driving products and solutions for mass production } \\
\text { vehicles that is scalable and highly customizable based on enterprise customer needs. Apollo solutions } \\
\text { support vehicle and information safety and can be upgraded via over-the-air programming. }\end{array}$ \\
\hline Features & $\begin{array}{l}\text { IOV 0S: a mass-produced and complete operation system solution to internet of vehicles (loV). } \\
\text { DuerOS: a set of Al-based loV solutions with voice assistant, augmented reality, and motion detected } \\
\text { capabilities. } \\
\text { Apollo 3.5: the first open-source autonomous-driving platform able to perform in complex urban and } \\
\text { suburban driving scenarios. The system utilizes the Apollo Cyber RT framework. } \\
\text { Apollo V2X: an intelligent vehicle infrastructure cooperation solution. }\end{array}$ \\
\hline $\begin{array}{l}\text { Supported } \\
\text { Technology }\end{array}$ & $\begin{array}{l}\text { The Apollo 3.5 system is compatible with most advanced sensor suites, including those from Velodyne and } \\
\text { LeddarTech. }\end{array}$ \\
\hline References & $\begin{array}{l}\text { Website: apollo.auto } \\
\text { More information on Apollo's architecture (HD map, localization, perception, prediction, planning, and } \\
\text { control) is found here. }\end{array}$ \\
\hline
\end{tabular}


Table M-16. Cepton micro-motion technology (MMT) and Sensor Development Toolkits.

\begin{tabular}{|c|c|}
\hline Element & Summary \\
\hline Description & $\begin{array}{l}\text { Cepton develops and manufactures 3D sensing solutions for automotive, industrial, and mapping } \\
\text { applications. } \\
\text { Cepton built and uses the MMT platform, Vista, and Vista LiDAR samples are available today for Cepton's } \\
\text { automotive partners to evaluate in their self-driving fleets. }\end{array}$ \\
\hline Features & $\begin{array}{l}\text { Vista-P LiDAR sensor: } 120-\text {-line equivalent scanner delivering } 200 \text { meters of range and. } 2^{\circ} \text { of spatial } \\
\text { resolution using less than } 10 \mathrm{~W} \text { of power. } \\
\text { Vista-X: } 120^{\circ} \text { FOV enables object perception and localization in an area wider than the standard Vista } \\
\text { sensor. } \\
\text { Sora-P60: mobile object classification and volumetric measurement device that provides } 1,200 \text { scan lines } \\
\text { per second and can send data over Ethernet, Wi-Fi, or LTE to a central processing server. } \\
\text { Sora-P60Lite: lightest high-performance LiDAR for Unmanned Aerial Vehicle (UAV). Lightweight payload } \\
\text { enables longer trips. } \\
\text { Vista-Edge: LiDAR evaluation kit using Nvidia's Jetson TX2 based microcomputer, providing immediate 3D } \\
\text { visualization of point cloud data. } \\
\text { Helius Smart LiDAR System: system connects multiple LiDAR sensors enabling comprehensive, high } \\
\text { resolution imaging, and seamless sensor-to-sensor tracking. Helius is a browser-based, PoE-powered and } \\
\text { processes data at the edge for low-bandwidth data output. }\end{array}$ \\
\hline References & Website: cepton.com \\
\hline
\end{tabular}

Table M-17. Innoviz.

\begin{tabular}{|c|c|}
\hline Element & Summary \\
\hline Description & Innoviz is a manufacturer of solid-state LiDAR sensors and perception software. \\
\hline Features & $\begin{array}{l}\text { InnovizPro: commercially available solid-state sensor. Applications include warehouse and delivery robotics, } \\
\text { heavy machinery, smart cities, security, HD mapping, and AVs. } \\
\text { InnovizOne: designed specifically for automakers and robo-taxi and delivery industries requiring an } \\
\text { automotive-grade, mass producible solution to achieve autonomy. Offers angular resolution }(\mathrm{HxV}) \text { of } \\
0.1^{\circ} \times 0.1^{\circ} \text {; a configurable frame rate of } 25 \mathrm{FPS} \text {; detection range of } 250 \mathrm{~m} \text {; and a field of view (HxV) of } \\
120^{\circ} \times 25^{\circ} \text { and will be available in } 2020 \text {. } \\
\text { Systems are accompanied by the supporting perception software. }\end{array}$ \\
\hline References & $\begin{array}{l}\text { Website: } \underline{\text { innoviz.tech }} \\
\text { InnovizPro datasheet } \\
\text { Innoviz's Solid-State LiDAR Wins CES } 2019 \text { “Best of Innovation" Award, } 8 \text { Nov } 2018 .\end{array}$ \\
\hline
\end{tabular}

Table M-18. Leddartech.

\begin{tabular}{|l|l|}
\hline Element & \multicolumn{1}{|c|}{ Summary } \\
\hline Description & $\begin{array}{l}\text { LedderTech provides versatile, scalable auto and mobility LiDAR platforms based on the LeddarEngine, a suite } \\
\text { of automotive-grade and functional safety certified SOCs that work in tandem with the LeddarSP signal } \\
\text { processing software. } \\
\text { LedderTech is based in Quebec City, Canada, but has offices in Silicon Valley, Detroit, and Austin. }\end{array}$ \\
\hline
\end{tabular}




\begin{tabular}{|c|c|}
\hline Element & Summary \\
\hline Features & 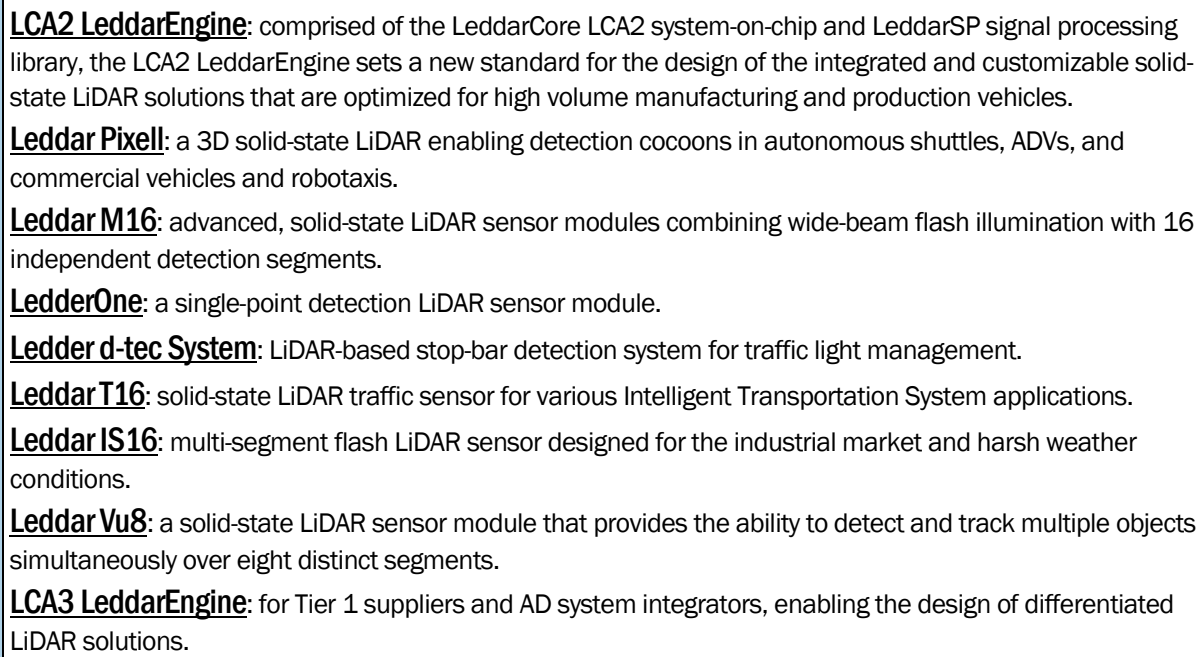 \\
\hline References & $\begin{array}{l}\text { Website: leddartech.com } \\
\text { An overview of LedderTech's auto and mobility LiDAR platform is available here. } \\
\text { LedderTech distributors are found here. }\end{array}$ \\
\hline
\end{tabular}

Table M-19. Nvidia Drive.

\begin{tabular}{|l|l|}
\hline Element & \multicolumn{1}{|c|}{ Summary } \\
\hline Features & $\begin{array}{l}\text { Drive AGX Developer Kit: provides hardware, software, and sample applications needed for the development } \\
\text { of production level AVs. } \\
\text { Drive Hyperion: a reference architecture for Nvidia's Level 2+ autonomy solution consisting of a complete } \\
\text { sensor suite, an Al computing platform, and a full software stack for autonomous-driving, driver monitoring, } \\
\text { and visualization. } \\
\text { Drive Constellation: a cloud-based virtual reality simulation platform. }\end{array}$ \\
$\begin{array}{l}\text { DGX System: an integrated system of deep learning software and simplified management tools for data } \\
\text { scientists. } \\
\text { Safety Force Field (SFF): computational framework supporting frame-by-frame, physics-based SFF } \\
\text { computations using vehicle sensor data. } \\
\text { Information on Nvidia Drive's software is here, information on Drive's safety is here, and information on } \\
\text { partners and innovation is here. }\end{array}$ \\
\hline References
\end{tabular} \begin{tabular}{l} 
Website: $\underline{\text { nvidia.com }}$ \\
\hline
\end{tabular}

Table M-20. R-Car.

\begin{tabular}{|l|l|}
\hline Element & \multicolumn{1}{|c|}{ Summary } \\
\hline Description & $\begin{array}{l}\text { Developed by Renesas, R-Car is the company's SoC family for car information systems designed for } \\
\text { automotive computing in AVs. R-Car is a scalable and flexible hardware platform and the company offers plug- } \\
\text { ins for multiple open-source software tools. } \\
\text { Renesas is based in Japan though it does have Design Centers, distributors, manufacturers, sales outlets, and } \\
\text { value-added resellers in the United States. }\end{array}$ \\
\hline Features & $\begin{array}{l}\text { Links for the below listed technologies details the features, specifications, and block diagrams for each: } \\
\text { Information on all available technologies from Renesas is here. } \\
\text { V3H: platform supporting ADAS and Level 3+ autonomy. }\end{array}$ \\
$\begin{array}{l}\text { SH-Navi3: a dual-core SOC built around a pair of SH-4A CPUs for processing performance of up to } 1920 \\
\text { MIPS. } \\
\text { H3: automotive computing platform that is compliant with ISO 26262 (ASIL-B) functionality safety standard } \\
\text { and can be applied to driving safety support systems. }\end{array}$ \\
\hline
\end{tabular}




\begin{tabular}{|c|l|}
\hline Element & \multicolumn{1}{|c|}{ Summary } \\
\hline References & $\begin{array}{l}\text { Website: } \underline{\text { renesas.com }} \\
\text { ADAS and Automated Driving Applications from Renesas can be found here. }\end{array}$ \\
\hline
\end{tabular}

Table M-21. LiDAR USA.

\begin{tabular}{|c|c|}
\hline Element & Summary \\
\hline Description & LiDAR USA developed and manufactures the Snoopy and ScanLook LiDAR systems \\
\hline Features & $\begin{array}{l}\text { ScanLook mapping systems } \\
\text { Cameras } \\
\text { UAV and drones } \\
\text { GNSS equipment } \\
\text { Commercially available LiDAR sensors } \\
\text { Snoopy Highway Mapper Z+F } \\
\text { PhaseOne Industrial IXU cameras }\end{array}$ \\
\hline References & $\begin{array}{l}\text { Website: LiDARusa.com } \\
\text { Sample data can be found here and includes examples of city, power line, and railroad mapping. } \\
\text { Case studies can be found here. } \\
\text { CAGE Code: } 6 J 1 G 4\end{array}$ \\
\hline
\end{tabular}

Table M-22. Toposens Automotive Devkit.

\begin{tabular}{|l|l|}
\hline Element & \multicolumn{1}{|c|}{ Summary } \\
\hline Description & $\begin{array}{l}\text { Based in Germany and California, Toposens develops and manufactures 3D ultrasonic sensors, enabling } \\
\text { users to implement applications like autonomous parking. The company's sensors can be integrated on the } \\
\text { outside and inside, providing the vehicle with information about objects, obstacles, and movements in the } \\
\text { vehicle's environment and the sensors can be adjusted, in hardware and software, to suit specific user needs. }\end{array}$ \\
\hline Features & $\begin{array}{l}\text { Toposens “typical work packages" includes: } \\
\text { Joint planning of proof-of-concept } \\
\text { Preparation and implementation of test scenarios } \\
\text { Preparation and installation of sensor systems and auxiliary devices } \\
\text { Setup of sensor network via UART or CAN } \\
\text { Setup of ROS environment for evaluation and demonstration purposes } \\
\text { Optimization of sensor parameters and installation positions } \\
\text { Conception and presentation of a final demonstration } \\
\text { Documentation for respective work packages }\end{array}$ \\
\hline References & \begin{tabular}{l} 
Website: toposens.com/automotive-poc \\
\hline
\end{tabular}
\end{tabular}

Table M-23. Racelogic.

\begin{tabular}{|l|l|}
\hline Element & \multicolumn{1}{|c|}{ Summary } \\
\hline Description & $\begin{array}{l}\text { RaceLogic designs and manufactures electronic systems to measure, record, display, analyze, and simulate } \\
\text { data from moving vehicles }\end{array}$ \\
\hline Features & $\begin{array}{l}\text { Data loggers } \\
\text { Speed sensors } \\
\text { Video data loggers }\end{array}$ \\
& $\begin{array}{l}\frac{\text { Indoor positioning }}{\text { Telemetry systems }} \\
\text { Modules } \\
\text { Software } \\
\frac{\text { AD and ADAS systems }}{\text { Handling and dynamics }} \\
\text { Performance testing }\end{array}$ \\
\hline
\end{tabular}




\begin{tabular}{|c|lc|}
\hline Element & \\
\hline References & Website: vboxautomotive.co.uk & Summary \\
\hline
\end{tabular}

Table M-24. Bosch Mobility Systems.

\begin{tabular}{|l|l|}
\hline Element & \multicolumn{1}{|c|}{ Summary } \\
\hline Description. & $\begin{array}{l}\text { Bosch Mobility, a division of Bosch Global, utilizes its extensive technical expertise to develop and } \\
\text { manufacture connected and AV smart mobility solutions. }\end{array}$ \\
\hline Features & $\begin{array}{l}\text { Bosch Mobility solutions include: } \\
\text { Traffic Jam Assist } \\
\text { Highway Assist }\end{array}$ \\
\hline $\begin{array}{l}\text { Driver assistance system domain controllers } \\
\text { Localization for automated driving }\end{array}$ \\
\hline $\begin{array}{l}\underline{\text { V2X connectivity solutions }} \\
\text { Multi-camera driver assistance solutions }\end{array}$ \\
\hline References & Website: $\underline{\text { bosch-mobility-solutions.us }}$ \\
\hline
\end{tabular}

Table M-25. Harman Ignite Cloud Platform.

\begin{tabular}{|l|l|}
\hline Element & \multicolumn{1}{|c|}{ Summary } \\
\hline Description & $\begin{array}{l}\text { The Harman Ignite is an automotive digital ecosystem that enables connectivity, device management, } \\
\text { application enablement, analytics, and managed services capabilities. }\end{array}$ \\
\hline Features & $\begin{array}{l}\text { Ignite and Harman's solutions offer multiple capabilities, including (but not limited to): } \\
\text { Remote Vehicle Updating Service } \\
\text { Intelligent Reasoning Assistant } \\
\text { Device virtualization for connected vehicles } \\
\text { Vehicle analytics dashboard } \\
\text { V2X solutions }\end{array}$ \\
\hline References & Weud-based driver profiles \\
\hline
\end{tabular}

Table M-26. Certus Evo.

\begin{tabular}{|l|l|}
\hline Element & \multicolumn{1}{|c|}{ Summary } \\
\hline Description & $\begin{array}{l}\text { Manufactured by Advanced Navigation, the Certus Evo is a miniature GPS aided inertial navigation system and } \\
\text { AHRS that provide positioning, velocity, acceleration, and orientation under adverse conditions. }\end{array}$ \\
\hline Features & $\begin{array}{l}\text { Certus Evo offers: } \\
\text { Dual frequency RTK GNSS receiver providing 8 mm accuracy positioning } \\
\text { Multiple interfaces including Ethernet, CAN, RS232, RS422, and GPIOs. } \\
\text { An advanced sensor fusion filter } \\
\text { MEM intertial sensors } \\
\text { Dual antennae moving baseline RTK } \\
\text { IP67 standard casing }\end{array}$ \\
\hline Supported \\
Technology
\end{tabular} \begin{tabular}{l}
$\begin{array}{l}\text { All current and future satellite navigation systems, including GPS, GLONASS, GALILEO, BeiDou, and QZSS. The } \\
\text { Certus Evo also supports all standard protocols, including NMEA 0183, NMEA 2000, TSS, PASHR, Simrad, } \\
\text { and others. }\end{array}$ \\
\hline $\begin{array}{l}\text { Website: advancednavigation.com } \\
\text { Rertus Evo specifications can be found here. } \\
\text { Other Advanced Navigation systems and solutions, including other MEMS GNSS/INS, speed sensors, and } \\
\text { web-based GNSS/INS post processing software, can be found here. }\end{array}$ \\
\hline
\end{tabular}


Table M-27. Iscan modular radar demonstration and development platform.

\begin{tabular}{|c|c|}
\hline Element & Summary \\
\hline Description & $\begin{array}{l}\text { Developed by Colorado Engineering, the iScan radar Series enables rapid prototyping of high-performance } \\
\text { radar. CEl's iScan processor, expansion and antenna modules can be mixed and matched to create a } \\
\text { customized development platform. }\end{array}$ \\
\hline Features & 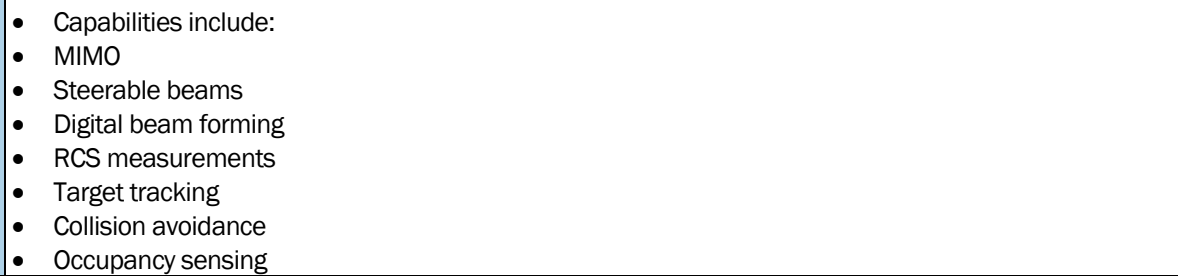 \\
\hline References & $\begin{array}{l}\text { Website: coloradoengineering.com } \\
\text { iScan Architecture brochure }\end{array}$ \\
\hline
\end{tabular}

Table M-28. APPlus+ IDapt.

\begin{tabular}{|c|c|}
\hline Element & Summary \\
\hline Description & $\begin{array}{l}\text { The IDAPT, developed by Applus+, is a vehicle onboard unit that allows for flexible and multipurpose } \\
\text { prototyping and a development tool for CAV activities. The unit can be used independently or integrated into } \\
\text { a vehicle's electronic architecture and can be retrofit into non-connected vehicles for R\&D purposes, such } \\
\text { as V2X, 4G, and GPS. }\end{array}$ \\
\hline Features & $\begin{array}{l}\text { IDAPT is a development tool built for: } \\
\text { - } \quad \text { ADAS and CAV Field Operational Tests (FOT) } \\
\text { - Automotive Internet of Things (loT) prototyping } \\
\text { - } \quad \text { AV control } \\
\text { - Camera imaging and object detection } \\
\text { - } \quad \text { Machine learning algorithm development } \\
\text { - LIDAR and radar sensor development } \\
\text { - V2X application development }\end{array}$ \\
\hline $\begin{array}{l}\text { Supported } \\
\text { Technology }\end{array}$ & $\begin{array}{l}\text { The system is currently being used in } \underline{\text { SecureloT, a platform that receives, validates, and stores information }} \\
\text { from AVs and } \underline{\text { RAIN }} \text {, a system based on cooperative communications that will enable enhanced safety } \\
\text { and advanced management services within automated proving grounds. }\end{array}$ \\
\hline References & $\begin{array}{l}\text { Website: applusidiada.com } \\
\text { Pricing, data sheet, and case studies can be found here. }\end{array}$ \\
\hline
\end{tabular}

Table M-29. Dataspeed ADAS Kit.

\begin{tabular}{|c|c|}
\hline Element & Summary \\
\hline Description & $\begin{array}{l}\text { Dataspeed's By-Wire Kit is a complete hardware and software system, allowing for seamless electronic control } \\
\text { of braking, throttle, steering, and shifting to enable testing for AV applications. }\end{array}$ \\
\hline Features & $\begin{array}{l}\text { - CAN interface module with four networks up to } 1 \text { Mbps each and configurable bit rates } \\
\text { - } \text { CAN breakout module enabling up to five additional nodes with additional available for further expansion } \\
\text { - Inverter module } \\
\text { - Brake, throttle, steer, and shift by-wire controller modules } \\
\text { - } \text { Switchboard Kit or Touchscreen Display Kit } \\
\text { - } 12 \text { channel power distribution system } \\
\text { DataSpeed also manufactures and/or distributes: } \\
\text { - } \frac{\text { Power Distribution System }}{\text { - }} \text { Cepton and Ouster LiDAR } \\
\text { - } \frac{\text { Magnetic LiDAR mounts }}{\text { CAN Breakout Board }}\end{array}$ \\
\hline References & $\begin{array}{l}\text { Website: } \underline{\text { dataspeedinc.com }} \\
\text { Other AV integrations from DataSpeed, Inc., can be found here. }\end{array}$ \\
\hline
\end{tabular}


Table M-30. Quantum R-Series.

\begin{tabular}{|c|l|}
\hline Element & \multicolumn{1}{|c|}{ Summary } \\
\hline Description & $\begin{array}{l}\text { Quantum's R-Series was designed for high-speed data capture in moving vehicles and allows for removable } \\
\text { data storage and “in the field” data capture to a Quantum StorNext shared storage environment. }\end{array}$ \\
\hline Features & $\begin{array}{l}\text { The R-Series is an in-vehicle storage system with a small form factor well suited for military vehicles, trucks, } \\
\text { and cars. Available in the following capacities and storage technologies: 60 TB HDD, 23 TB SSD, and 46 TB } \\
\text { SSD. }\end{array}$ \\
\hline References & $\begin{array}{l}\text { Website: quantum.com } \\
\text { Other Quantum products and services can be found here. } \\
\text { R-Series datasheet } \\
\text { Mass Storage for AV Development } \\
\text { Accelerate Automotive Development with Intelligent Management of Machine-Generated Data datasheet }\end{array}$ \\
\hline
\end{tabular}




\section{Appendix N: Bidirectional Chargers, Diesel Generators, and Controller Technologies}

\begin{tabular}{|c|c|c|c|c|c|}
\hline & Applicability & Interoperability & Cost & Plug-and-play & Resources \\
\hline \multicolumn{6}{|c|}{ Bidirectional Battery Chargers } \\
\hline CORITECH VGI-30 & $\begin{array}{l}\text { Battery Voltage Range: } 200- \\
600 \text { Vdc } \\
\text { Battery Charging Power: } \\
30 \text { kW 60A Max } \\
\text { Reverse Power Flow V2G: } \\
30 \text { kW 60A Max }\end{array}$ & $\begin{array}{l}\text { Aggregator } \\
\text { Connection: } \\
\text { Ethernet, Modbus } \\
\text { TCP/IP, SEP } 2.0\end{array}$ & $\$ 45 K$ & $\begin{array}{l}\text { Vehicle Connection: } \\
\text { SAE J1772-Combo } \\
\text { CCS or CHAdeMO }\end{array}$ & https://coritech.com/ev-chargers \\
\hline \multicolumn{6}{|c|}{ Diesel Generators } \\
\hline CAT XQP100 & $\begin{array}{l}\text { Generator successfully used } \\
\text { in the Smart and Green } \\
\text { Energy (SAGE) for Base } \\
\text { Camps Final Report (Jan } \\
\text { 2014) which utilized COTS } \\
\text { equipment within a } \\
\text { smartgrid }\end{array}$ & $\begin{array}{l} \\
\text { Uses a proprietary } \\
\text { control system } \\
\text { which required } \\
\text { changing the } \\
\text { Tactical Quiet } \\
\text { Gensets (TQG) } \\
\text { control units for CAT } \\
\text { control units to } \\
\text { allow for system } \\
\text { communication. }\end{array}$ & \begin{tabular}{|l} 
|l \\
No response to \\
multiple \\
request for \\
information
\end{tabular} & $\begin{array}{l}\text { Literature indicates } \\
\text { only within the } \\
\text { Caterpillar control } \\
\text { system } \\
\text { architecture. } \\
\text { However as noted } \\
\text { in the SAGE for } \\
\text { Base Camps Final } \\
\text { Report these } \\
\text { generators were } \\
\text { successfully } \\
\text { integrated into the } \\
\text { Decision Support } \\
\text { for Operations and } \\
\text { Maintenance } \\
\text { (DSOM) control } \\
\text { system (see below } \\
\text { in Controller } \\
\text { Technologies) } \\
\end{array}$ & $\begin{array}{l}\text { https://www.cat.com/en_US/products/new } \\
\text { /power-systems/electric-power/mobile- } \\
\text { generator-sets/1000031338.html }\end{array}$ \\
\hline $\begin{array}{l}\text { Triton 1103D- } \\
\text { E44TG1 }\end{array}$ & $\begin{array}{l}\text { Meets general output } \\
\text { specifications of the } 60 \mathrm{~kW} \\
\text { AMMPS generator at } 60 \mathrm{~Hz}\end{array}$ & $\begin{array}{l}\text { Connection allows } \\
\text { for Modbus } \\
\text { communication, } \\
\text { Remote-control } \\
\text { sources (10) can be } \\
\text { accessed via } \\
\text { SCADA, RS232 \& } \\
\text { RS485 } \\
\text { communications, } \\
\text { DSENet }{ }^{\circledR} \\
\text { compatible }\end{array}$ & $\$ 25 K$ & $\begin{array}{l}\text { Remote-control } \\
\text { sources (10) can be } \\
\text { accessed via } \\
\text { SCADA and allows } \\
\text { for Ethernet } \\
\text { communications }\end{array}$ & $\begin{array}{l}\text { https://www.americasgenerators.com/60 k } \\
\text { W-Perkins-Diesel-Generator.aspx }\end{array}$ \\
\hline Kohler 90REOZT4 & $\begin{array}{l}\text { Meets general output } \\
\text { specifications of the } 60 \mathrm{~kW} \\
\text { AMMPS generator at } 60 \mathrm{~Hz}\end{array}$ & \begin{tabular}{|l|} 
Communication \\
with RS-485 and \\
Ethernet (RJ-45), \\
Supports Modbus \\
RTU and Modbus \\
TCP protocols, NFPA \\
110, Level 1 \\
monitoring capable
\end{tabular} & $\begin{array}{l}\text { No response to } \\
\text { multiple } \\
\text { request for } \\
\text { information }\end{array}$ & $\begin{array}{l}\text { Only within the } \\
\text { Kokler control } \\
\text { system } \\
\text { architecture. } \\
\text { Requires G6-148 } \\
\text { Mobile Generator } \\
\text { Set Paralleling Box } \\
\text { to connect } \\
\text { generators together } \\
\text { (one G6-148 per } \\
\text { two generators; } \\
\text { maximum four G6- } \\
148=\text { eight } \\
\text { generators) }\end{array}$ & $\begin{array}{l}\text { https://kohlerpower.com/en/generators/in } \\
\text { dustrial/product/90reozt4 }\end{array}$ \\
\hline \multicolumn{6}{|c|}{ Controller Technologies } \\
\hline \multirow{2}{*}{$\begin{array}{l}\text { Schweitzer } \\
\text { Engineering }\end{array}$} & \multirow{2}{*}{$\begin{array}{l}\text { Compliance to MIL-STD-TMS } \\
\text { (DoD Tactical Microgrid }\end{array}$} & \multirow{2}{*}{$\begin{array}{l}\text { Data Distribution } \\
\text { Service (DDS) }\end{array}$} & \multirow{2}{*}{$\begin{array}{l}\text { Varies } \\
\text { depending on }\end{array}$} & \multirow{2}{*}{$\begin{array}{l}\text { "Plug-and-play } \\
\text { solutions for military }\end{array}$} & https://selinc.com/solutions/microgrids/ \\
\hline & & & & & https://selinc.com/api/download/98236/ \\
\hline
\end{tabular}




\begin{tabular}{|c|c|c|c|c|c|}
\hline & Applicability & Interoperability & Cost & Plug-and-play & Resources \\
\hline $\begin{array}{l}\text { Laboratories } \\
\text { (SEL) PowerMax }\end{array}$ & Standard) & \begin{tabular}{|l|} 
communications, \\
Interoperability with \\
Advanced-Medium \\
Mobile Power \\
Sources (AMMPSs), \\
Conversion kits for \\
TQGs, customized \\
kits developed for \\
various \\
manufactures.
\end{tabular} & $\begin{array}{l}\text { system size } \\
\text { and } \\
\text { components. } \\
\text { Initial kit could } \\
\text { start } \$ 10 \mathrm{~K}- \\
15 \mathrm{~K} \text {. Initial cost } \\
\text { of } \\
\text { customization } \\
\text { for unique } \\
\text { equipment is } \\
\text { approx } \$ 40 \mathrm{~K}\end{array}$ & \begin{tabular}{|l} 
bases. \\
powerMAX for \\
Mobile Microgrids is \\
a solution made \\
specifically for \\
military bases, \\
including fixed, \\
remote, or forward \\
operations. It is \\
perfect for first \\
responders, \\
disaster relief, or \\
anyone requiring a \\
mobile microgrid. \\
Additionally, power \\
MAX is the only \\
microgrid control \\
system that meets \\
the standards for \\
tactical microgrids."
\end{tabular} & $\begin{array}{l}\text { https://selinc.com/solutions/microgrids- } \\
\text { fob/ }\end{array}$ \\
\hline $\begin{array}{l}\text { Spirae Wave } \\
\text { Microgrid and } \\
\text { energy } \\
\text { distribution } \\
\text { solutions }\end{array}$ & $\begin{array}{l}\text { "Can be configured for three } \\
\text { different types of operations: } \\
\text { i) grid-connected economic- } \\
\text { microgrid, where Wave } \\
\text { manages generation, } \\
\text { demand, and storage to } \\
\text { achieve energy cost } \\
\text { optimization, ii) resilience- } \\
\text { microgrid, where Wave } \\
\text { manages local assets to } \\
\text { maximize the resiliency of } \\
\text { the site to grid outages and } \\
\text { switches to island mode if } \\
\text { the grid fails, and iii) island } \\
\text { (off-grid) microgrid mode, } \\
\text { where Wave continuously } \\
\text { runs in island mode to } \\
\text { reliably serve loads using } \\
\text { local resources." }\end{array}$ & \begin{tabular}{|l|} 
"Wave Microgrid \\
controls are \\
agnostic to the \\
types and brands of \\
DER (distributed \\
energy resource) in \\
a microgrid. \\
Choosing the Spirae \\
solution provides \\
the ultimate \\
flexibility to mix and \\
match system \\
components \\
including legacy \\
equipment such as \\
backup generators."
\end{tabular} & $\begin{array}{l}\text { No response to } \\
\text { multiple } \\
\text { request for } \\
\text { information }\end{array}$ & $\begin{array}{l}\text { No response to } \\
\text { multiple request for } \\
\text { information }\end{array}$ & https://www.spirae.com/about-microgrid/ \\
\hline $\begin{array}{l}\text { OMNETRIC Group } \\
\text { and Siemens }\end{array}$ & $\begin{array}{l}\text { No response to multiple } \\
\text { request for information }\end{array}$ & \begin{tabular}{|l|} 
"Verified and \\
implemented a new \\
interoperability \\
reference \\
architecture, called \\
the Open Field \\
Message Bus \\
(OpenFMB) \\
framework. This \\
solution, integrated \\
with Siemens \\
Microgrid \\
Management \\
Software (MGMS), \\
allows CPS Energy \\
to overcome the \\
lack of \\
standardization and \\
interoperability \\
between the vast \\
array of equipment \\
in operation to \\
better manage load \\
behavior on the \\
power grid."
\end{tabular} & $\begin{array}{l}\text { No response to } \\
\text { multiple } \\
\text { request for } \\
\text { information }\end{array}$ & $\begin{array}{l}\text { No response to } \\
\text { multiple request for } \\
\text { information }\end{array}$ & $\begin{array}{l}\text { https://www.omnetric.com/data/files/news } \\
\text { room/omnetric_siemens_cps_milestone_re } \\
\text { lease_final_O.pdf?cat1=demonstrating- } \\
\text { microgrid-technology-cps-energy-and-utsa }\end{array}$ \\
\hline $\begin{array}{l}\text { Universal } \\
\text { Microgrid } \\
\text { Controller }\end{array}$ & $\begin{array}{l}\text { Not aimed at very small } \\
\text { systems. Most cost effective } \\
\text { option in the } 100 \mathrm{~kW} \text { to }\end{array}$ & \begin{tabular}{|l} 
Component \\
Communication: \\
Protocols: Modbus
\end{tabular} & $\$ 25-50$ per kw & $\begin{array}{l}\text { No response to } \\
\text { multiple request for } \\
\text { information }\end{array}$ & $\begin{array}{l}\text { http://www.sustainablepowersystems.com/ } \\
\text { products-services/universal-microgrid- } \\
\text { controllertm/ }\end{array}$ \\
\hline
\end{tabular}




\begin{tabular}{|c|c|c|c|c|c|}
\hline & Applicability & Interoperability & Cost & Plug-and-play & Resources \\
\hline & $20 \mathrm{MW}$ size range & \begin{tabular}{|l|} 
TCP Utility \\
Communication: \\
Protocols: IEEE \\
2030.5 Modbus \\
RTU, CAN Bus
\end{tabular} & & & $\begin{array}{l}\text { http://www.sustainablepowersystems.com/ } \\
\text { wp-content/uploads/2019/06/UMC- } \\
\text { Frequently-Asked-Questions-Rev-4.pdf }\end{array}$ \\
\hline DSOM system & $\begin{array}{l}\text { "Open, flexible configuration } \\
\text { and capability that could be } \\
\text { easily adapted for energy } \\
\text { management at base } \\
\text { camps. With documented } \\
\text { use in the SAGE for Base } \\
\text { Camps" }\end{array}$ & \begin{tabular}{|l|} 
"Talks to most \\
monitoring and \\
control systems, \\
mitigating the need \\
for expensive \\
upgrades of \\
instrumentation for \\
compatibility"
\end{tabular} & $\begin{array}{l}\text { Only prototyped } \\
\text { for the SAGE } \\
\text { experiment }\end{array}$ & \begin{tabular}{|l|} 
"Provides a flexible \\
plug-and-play \\
system to \\
communicate with \\
almost all \\
commercial data \\
acquisition systems \\
and facility control \\
systems"
\end{tabular} & https://www.pnnl.gov/dsom/ \\
\hline \multicolumn{6}{|c|}{ Bidirectional Battery Chargers } \\
\hline \multirow{2}{*}{$\begin{array}{l}\text { KI platform } \\
\text { VCharge by OVO }\end{array}$} & \multirow{2}{*}{$\begin{array}{l}\text { not available on open } \\
\text { market (Great Britain only), } \\
50 \mathrm{~Hz} \text { only }\end{array}$} & \multirow{2}{*}{$\begin{array}{l}\text { Currently only works } \\
\text { within the Kaluza } \\
\text { network } \\
\text { architecture. }\end{array}$} & \multirow{2}{*}{ NA } & \multirow{2}{*}{ CHAdeMO protocol } & $\begin{array}{l}\text { https://www.ovoenergy.com/electric- } \\
\text { cars/vehicle-to-grid-charger }\end{array}$ \\
\hline & & & & & https://www.indra.co.uk/v2g \\
\hline Fermata Energy & & & & & https://www.fermataenergy.com/ \\
\hline $\begin{array}{l}\text { NICHICON } \\
\text { CORPORATION }\end{array}$ & Single phase voltage only & NA & $\begin{array}{l}\text { Not available in } \\
\text { the United } \\
\text { States }\end{array}$ & CHAdeMO protocol & $\begin{array}{l}\text { https://www.nichicon.co.jp/english/product } \\
\text { _news/new124.html }\end{array}$ \\
\hline Nuvve PowerPort & $\begin{array}{l}\text { Vehicle must have } \\
\text { bidirectional inverter to be } \\
\text { V2G capable delivers up to } \\
100 \mathrm{~kW} \text { in three-phase } \\
\text { configuration and } 19 \mathrm{~kW} \text { in } \\
\text { single phase configuration }\end{array}$ & $\begin{array}{l}\text { Proprietary } \\
\text { communication } \\
\text { system }\end{array}$ & $\begin{array}{l}\$ 4500 \text { (does } \\
\text { not include cost } \\
\text { of compatible } \\
\text { OEM DC } \\
\text { charger for fast } \\
\text { DC charging ) }\end{array}$ & \begin{tabular}{|l|}
$J 1772$ Type 1 , \\
J3068 Type 2 and \\
compatible with \\
several charger \\
manufacturers both \\
in DC (such as \\
CHAdeMO) and AC \\
mode (vehicle must \\
have bidirectional \\
inverter)
\end{tabular} & https://nuvve.com/v2g-chargers/ \\
\hline \multicolumn{6}{|l|}{ Diesel Generators } \\
\hline $\begin{array}{l}\text { Generac } \\
\text { MDG75DF4 }\end{array}$ & $\begin{array}{l}\text { This system is designed to } \\
\text { work with generators } \\
\text { connected on the mobile } \\
\text { switching center (MSC) } \\
\text { network only. All other power } \\
\text { sources must be isolated } \\
\text { from the MSC network }\end{array}$ & $\begin{array}{l}\text { Supports Modbus } \\
\text { RTU and Modbus } \\
\text { TCP protocols }\end{array}$ & $\begin{array}{l}\text { No response to } \\
\text { multiple } \\
\text { request for } \\
\text { information }\end{array}$ & $\begin{array}{l}\text { Only within the } \\
\text { Generac control } \\
\text { system } \\
\text { architecture. This } \\
\text { system is designed } \\
\text { to work with } \\
\text { generators } \\
\text { connected on the } \\
\text { MSC network only }\end{array}$ & $\begin{array}{l}\text { https://www.generacmobileproducts.com/p } \\
\text { roducts/generators/products/mdg75df4- } \\
\text { diesel generator }\end{array}$ \\
\hline
\end{tabular}




\begin{tabular}{|c|c|c|c|c|c|}
\hline & Applicability & Interoperability & Cost & Plug-and-play & Resources \\
\hline $\begin{array}{l}\text { Spirae Wave } \\
\text { Microgrid and } \\
\text { energy } \\
\text { distribution } \\
\text { solutions }\end{array}$ & $\begin{array}{l}\text { Can be configured for three } \\
\text { different types of operations: } \\
\text { i) grid-connected economic- } \\
\text { microgrid, where Wave } \\
\text { manages generation, } \\
\text { demand, and storage to } \\
\text { achieve energy cost } \\
\text { optimization, ii) resilience- } \\
\text { microgrid, where Wave } \\
\text { manages local assets to } \\
\text { maximize the resiliency of } \\
\text { the site to grid outages and } \\
\text { switches to island mode if } \\
\text { the grid fails, and iii) island } \\
\text { (off-grid) microgrid mode, } \\
\text { where Wave continuously } \\
\text { runs in island mode to } \\
\text { reliably serve loads using } \\
\text { local resources. }\end{array}$ & \begin{tabular}{|l|} 
"Wave Microgrid \\
controls are \\
agnostic to the \\
types and brands of \\
DER in a microgrid. \\
Choosing the Spirae \\
solution provides \\
the ultimate \\
flexibility to mix and \\
match system \\
components \\
including legacy \\
equipment such as \\
backup generators."
\end{tabular} & \begin{tabular}{|l|} 
\\
No response to \\
multiple \\
request for \\
information
\end{tabular} & $\begin{array}{l}\text { No response to } \\
\text { multiple request for } \\
\text { information }\end{array}$ & https://www.spirae.com/about-microgrid/ \\
\hline \multirow{2}{*}{$\begin{array}{l}\text { GE microgrid } \\
\text { controller }\end{array}$} & & & & & $\begin{array}{l}\text { https://www.gegridsolutions.com/app/Dow } \\
\text { nloadFile.aspx?prod=microgrid\&type=1\&file } \\
=1\end{array}$ \\
\hline & & & & & $\begin{array}{l}\text { https://www.gegridsolutions.com/multilin/c } \\
\text { atalog/mcs.htm }\end{array}$ \\
\hline $\begin{array}{l}\text { OMNETRIC Group } \\
\text { and Siemens }\end{array}$ & $\begin{array}{l}\text { No response to multiple } \\
\text { request for information }\end{array}$ & \begin{tabular}{|l|} 
"Verified and \\
implemented a new \\
interoperability \\
reference \\
architecture, called \\
the OpenFMB \\
framework. This \\
solution, integrated \\
with Siemens \\
Microgrid \\
Management \\
Software (MGMS), \\
allows CPS Energy \\
to overcome the \\
lack of \\
standardization and \\
interoperability \\
between the vast \\
array of equipment \\
in operation to \\
better manage load \\
behavior on the \\
power grid."
\end{tabular} & \begin{tabular}{|l|} 
No response to \\
multiple \\
request for \\
information
\end{tabular} & $\begin{array}{l}\text { No response to } \\
\text { multiple request for } \\
\text { information }\end{array}$ & $\begin{array}{l}\text { https://www.omnetric.com/data/files/news } \\
\text { room/omnetric_siemens_cps_milestone_re } \\
\text { lease_final_0.pdf?cat1=demonstrating- } \\
\text { microgrid-technology-cps-energy-and-utsa }\end{array}$ \\
\hline $\begin{array}{l}\text { Ovation }^{\mathrm{TM}} \\
\text { Microgrid Control }\end{array}$ & $\begin{array}{l}\text { Designed specifically for the } \\
\text { power industry }\end{array}$ & \begin{tabular}{|l|} 
Ovation \\
communicates with \\
intelligent electronic \\
devices (IEDs) using \\
various protocols \\
including: \\
Modbus over \\
RS485 serial link, \\
Modbus over \\
TCP/IP, DNP, \\
Profibus, IEC \\
61850, IEC, 60870- \\
$5-104$
\end{tabular} & $\begin{array}{l}\text { No response to } \\
\text { multiple } \\
\text { request for } \\
\text { information }\end{array}$ & $\begin{array}{l}\text { No response to } \\
\text { multiple request for } \\
\text { information }\end{array}$ & $\begin{array}{l}\text { https://www.emerson.com/documents/aut } \\
\text { omation/microgrid-control-system-en- } \\
\text { 1263362.pdf }\end{array}$ \\
\hline $\begin{array}{l}\text { Eaton Power } \\
\text { Xpert Energy } \\
\text { Optimizer }\end{array}$ & $\begin{array}{l}\text { "Configured instead of } \\
\text { custom programmed, } \\
\text { allowing for maximum }\end{array}$ & $\begin{array}{l}\text { Based on open } \\
\text { standards, including } \\
\text { IEC } 61850\end{array}$ & $\begin{array}{l}\text { No response to } \\
\text { multiple } \\
\text { request for }\end{array}$ & $\begin{array}{l}\text { No response to } \\
\text { multiple request for } \\
\text { information }\end{array}$ & $\begin{array}{l}\text { https://www.eaton.com/us/en- } \\
\text { us/catalog/services/microgrid-and- } \\
\text { distributed-energy-resources.html }\end{array}$ \\
\hline
\end{tabular}




\begin{tabular}{|l|l|l|l|l|l|}
\hline & Applicability & Interoperability & Cost & Plug-and-play & Resources \\
\hline & $\begin{array}{l}\text { flexibility and scalability for } \\
\text { both microgrid and stand- } \\
\text { alone energy storage } \\
\text { applications." }\end{array}$ & communications. & information & & $\begin{array}{l}\text { https://www.eaton.com/content/dam/eato } \\
\text { n/services/eess/eess-documents/power- } \\
\text { xpert-energy-optimizer-controller- } \\
\text { pa027032en.pdf }\end{array}$ \\
\hline
\end{tabular}




\section{Appendix 0: V2G Test Plan (longitudinal)}

\begin{tabular}{|c|c|c|c|c|c|c|}
\hline Step No. & Step Description & Use Case & $\begin{array}{c}\text { V2G } \\
\text { Setting }\end{array}$ & $\begin{array}{l}\text { Load } \\
\text { Bank } \\
\text { Setting }\end{array}$ & Check & Notes \\
\hline PRE & $\begin{array}{l}\text { Visual Inspection to confirm: fluids, } \\
\text { warm engines }\end{array}$ & Baseline & $\mathrm{N} / \mathrm{A}$ & N/A & & \\
\hline PRE & $\begin{array}{l}\text { Verify all generators are off and } \\
\text { ESTOPPED }\end{array}$ & Baseline & $\mathrm{N} / \mathrm{A}$ & N/A & & \\
\hline PRE & $\begin{array}{l}\text { Confirm IPD load bank, generator } \\
\text { and bus ports are all open }\end{array}$ & Baseline & N/A & N/A & & \\
\hline PRE & $\begin{array}{l}\text { Confirm site loads and IPD load } \\
\text { ports are open }\end{array}$ & Baseline & $\mathrm{N} / \mathrm{A}$ & N/A & & \\
\hline PRE & $\begin{array}{l}\text { Confirm all breakers on IPDs are } \\
\text { open }\end{array}$ & Baseline & $\mathrm{N} / \mathrm{A}$ & N/A & & \\
\hline PRE & $\begin{array}{l}\text { Confirm all generators are } \\
\text { ESTOPPED and stopped }\end{array}$ & Baseline & N/A & N/A & & \\
\hline PRE & $\begin{array}{l}\text { Confirm all IPD bus and generator } \\
\text { breakers/contactors are open }\end{array}$ & Baseline & N/A & N/A & & \\
\hline PRE & $\begin{array}{l}\text { Confirm all generators are } \\
\text { connected to IPDs generator ports } \\
\text { per the configuration to be tested }\end{array}$ & Baseline & N/A & N/A & & \\
\hline PRE & $\begin{array}{l}\text { Confirm V2G is connected to an IPD } \\
\text { No. } 1 \text { load port }\end{array}$ & Baseline & $\mathrm{N} / \mathrm{A}$ & N/A & & \\
\hline PRE & $\begin{array}{l}\text { Confirm the load bank is connected } \\
\text { to an IPD No. } 2 \text { load port }\end{array}$ & Baseline & N/A & N/A & & \\
\hline 1 & $\begin{array}{l}\text { Hearing and eye protection on all } \\
\text { participants }\end{array}$ & Baseline & $\mathrm{N} / \mathrm{A}$ & N/A & & \\
\hline 2 & $\begin{array}{l}\text { Start and close CB on generator No. } \\
1 \text { (G1) }\end{array}$ & Baseline & $\mathrm{N} / \mathrm{A}$ & N/A & & \\
\hline 3 & $\begin{array}{l}\text { Confirm G1 MCCB closed on IPD } \\
\text { No. } 1\end{array}$ & Baseline & $\mathrm{N} / \mathrm{A}$ & $\mathrm{N} / \mathrm{A}$ & & \\
\hline 4 & $\begin{array}{l}\text { Close 200A bus breaker for IPD No. } \\
1\end{array}$ & Baseline & $\mathrm{N} / \mathrm{A}$ & N/A & & \\
\hline 5 & $\begin{array}{l}\text { Energize all bus ring and generator } \\
\text { ports }\end{array}$ & Baseline & N/A & N/A & & \\
\hline 6 & Start and synchronize G1 & Baseline & N/A & N/A & & \\
\hline 7 & $\begin{array}{l}\text { Start and close } \mathrm{CB} \text { on generator No. } \\
2 \text { (G2) }\end{array}$ & Baseline & N/A & N/A & & \\
\hline 8 & $\begin{array}{l}\text { Confirm G2 MCCB closed on IPD } \\
\text { No. } 2\end{array}$ & Baseline & $\mathrm{N} / \mathrm{A}$ & N/A & & \\
\hline 9 & $\begin{array}{l}\text { Close 200A bus breaker for IPD No. } \\
2\end{array}$ & Baseline & $\mathrm{N} / \mathrm{A}$ & N/A & & \\
\hline 10 & $\begin{array}{l}\text { Energize all bus ring and generator } \\
\text { ports }\end{array}$ & Baseline & N/A & N/A & & \\
\hline 11 & Start and synchronize G2 & Baseline & N/A & N/A & & \\
\hline 12 & Set load bank to $0 \mathrm{~kW}$ & Baseline & N/A & $0 \mathrm{~kW}$ & & \\
\hline 13 & Close load port on IPD No. 2 & Baseline & N/A & $0 \mathrm{~kW}$ & & \\
\hline 14 & Set load bank to $60 \mathrm{~kW}$ & Baseline & $\mathrm{N} / \mathrm{A}$ & $60 \mathrm{~kW}$ & & $\begin{array}{l}\text { Observe frequency } \\
\text { depression }\end{array}$ \\
\hline
\end{tabular}




\begin{tabular}{|c|c|c|c|c|c|c|}
\hline Step No. & Step Description & Use Case & $\begin{array}{c}\text { V2G } \\
\text { Setting }\end{array}$ & $\begin{array}{l}\text { Load } \\
\text { Bank } \\
\text { Setting }\end{array}$ & Check & Notes \\
\hline 15 & $\begin{array}{l}\text { Simultaneously adjust sourcing } \\
\text { machines to achieve } 60 \mathrm{~Hz} \text { and } \\
\text { equal load balance (achieve both } \\
\text { control objectives simultaneously) }\end{array}$ & Baseline & N/A & $60 \mathrm{~kW}$ & & $\begin{array}{l}\text { Note inter-dependencies } \\
\text { between generator speed } \\
\text { setpoints, power outputs, } \\
\text { and system frequency }\end{array}$ \\
\hline 16 & Open G1 port on IPD No. 1 & Baseline & N/A & $60 \mathrm{~kW}$ & & $\begin{array}{l}\text { Islanding G1. Observe no } \\
\text { instabilities and load } \\
\text { sharing continues }\end{array}$ \\
\hline 17 & Close G1 port on IPD No. 1 & Baseline & N/A & $60 \mathrm{~kW}$ & & $\begin{array}{l}\text { Reconnecting G1, G2 via } \\
\text { IPD No. } 1 \text { and } 2\end{array}$ \\
\hline 18 & Open G2 port on IPD No. 2 & Baseline & N/A & $60 \mathrm{~kW}$ & & $\begin{array}{l}\text { Islanding G2. Observe no } \\
\text { instabilities and load } \\
\text { sharing continues }\end{array}$ \\
\hline 19 & Close G2 port on IPD No. 2 & Baseline & N/A & $60 \mathrm{~kW}$ & & $\begin{array}{l}\text { Reconnecting G1, G2 via } \\
\text { IPD No. } 1 \text { and } 2\end{array}$ \\
\hline 20 & Close V2G port on IPD No. 1 & Baseline & N/A & $60 \mathrm{~kW}$ & & $\begin{array}{l}\text { Observe system voltage } \\
\text { and frequency stability }\end{array}$ \\
\hline 21 & $\begin{array}{l}\text { Set V2G charging at } 12.5 \mathrm{~kW} \\
\text { (25-percent load) }\end{array}$ & Baseline & $\begin{array}{l}\text { Charging } \\
12.5 \mathrm{~kW}\end{array}$ & $60 \mathrm{~kW}$ & & $\begin{array}{l}\text { Observe system voltage } \\
\text { and frequency stability }\end{array}$ \\
\hline 22 & $\begin{array}{l}\text { Set V2G charging at } 37.5 \mathrm{~kW} \\
\text { (75-percent load) }\end{array}$ & Baseline & $\begin{array}{l}\text { Charging } \\
37.5 \mathrm{~kW}\end{array}$ & $60 \mathrm{~kW}$ & & $\begin{array}{l}\text { Observe system voltage } \\
\text { and frequency stability }\end{array}$ \\
\hline 23 & Set V2G charging at $0 \mathrm{~kW}$ & Baseline & $\begin{array}{l}\text { Charging } 0 \\
\mathrm{~kW}\end{array}$ & $60 \mathrm{~kW}$ & & $\begin{array}{l}\text { Observe system voltage } \\
\text { and frequency stability }\end{array}$ \\
\hline 24 & $\begin{array}{l}\text { Set V2G discharging at } 12.5 \mathrm{~kW} \\
\text { (25-percent load) }\end{array}$ & Baseline & $\begin{array}{l}\text { Discharging } \\
12.5 \mathrm{~kW}\end{array}$ & $60 \mathrm{~kW}$ & & $\begin{array}{l}\text { Observe system voltage } \\
\text { and frequency stability }\end{array}$ \\
\hline 25 & $\begin{array}{l}\text { Set V2G discharging at } 37.5 \mathrm{~kW} \\
\text { (75-percent load) }\end{array}$ & Baseline & $\begin{array}{l}\text { Discharging } \\
37.5 \mathrm{~kW}\end{array}$ & $60 \mathrm{~kW}$ & & $\begin{array}{l}\text { Observe system voltage } \\
\text { and frequency stability }\end{array}$ \\
\hline 26 & Open G1 port on IPD No. 1 & $\begin{array}{l}\text { Sudden } \\
\text { generation } \\
\text { decrease }\end{array}$ & $\begin{array}{l}\text { Discharging } \\
37.5 \mathrm{~kW}\end{array}$ & $60 \mathrm{~kW}$ & & $\begin{array}{l}\text { Observe system voltage } \\
\text { and frequency stability }\end{array}$ \\
\hline 27 & Close G1 port on IPD No. 1 & $\begin{array}{l}\text { Sudden } \\
\text { generation } \\
\text { increase }\end{array}$ & $\begin{array}{l}\text { Discharging } \\
37.5 \mathrm{~kW}\end{array}$ & $60 \mathrm{~kW}$ & & $\begin{array}{l}\text { Observe system voltage } \\
\text { and frequency stability } \\
\text { and V2G operational } \\
\text { status }\end{array}$ \\
\hline 28 & Open G2 port on IPD No. 2 & $\begin{array}{l}\text { Sudden } \\
\text { generation } \\
\text { decrease }\end{array}$ & $\begin{array}{l}\text { Discharging } \\
37.5 \mathrm{~kW}\end{array}$ & $60 \mathrm{~kW}$ & & $\begin{array}{l}\text { Observe system voltage } \\
\text { and frequency stability } \\
\text { and V2G operational } \\
\text { status }\end{array}$ \\
\hline 29 & Close G2 port on IPD No. 2 & $\begin{array}{l}\text { Sudden } \\
\text { generation } \\
\text { increase }\end{array}$ & $\begin{array}{l}\text { Discharging } \\
37.5 \mathrm{~kW}\end{array}$ & $60 \mathrm{~kW}$ & & $\begin{array}{l}\text { Observe system voltage } \\
\text { and frequency stability } \\
\text { and V2G operational } \\
\text { status }\end{array}$ \\
\hline 30 & $\begin{array}{l}\text { Set V2G charging to } 50 \mathrm{~kW} \\
\text { (100 percent) }\end{array}$ & $\begin{array}{l}\text { Sudden load } \\
\text { increase }\end{array}$ & $\begin{array}{l}\text { Charging } \\
50 \mathrm{~kW}\end{array}$ & $60 \mathrm{~kW}$ & & $\begin{array}{l}\text { Observe system voltage } \\
\text { and frequency stability } \\
\text { and V2G operational } \\
\text { status }\end{array}$ \\
\hline 31 & $\begin{array}{l}\text { Decrease load bank from } 50 \mathrm{~kW} \text { to } \\
0 \mathrm{~kW}\end{array}$ & $\begin{array}{l}\text { Sudden load } \\
\text { decrease }\end{array}$ & $\begin{array}{l}\text { Charging } \\
50 \mathrm{~kW}\end{array}$ & $0 \mathrm{~kW}$ & & $\begin{array}{l}\text { Observe system voltage } \\
\text { and frequency stability } \\
\text { and V2G operational } \\
\text { status }\end{array}$ \\
\hline 32 & Decrease $\mathrm{G} 1$ to $0 \mathrm{~kW}$ & $\begin{array}{l}\text { Sudden } \\
\text { generation } \\
\text { decrease }\end{array}$ & $\begin{array}{l}\text { Charging } \\
50 \mathrm{~kW}\end{array}$ & $0 \mathrm{~kW}$ & & $\begin{array}{l}\text { Observe system voltage } \\
\text { and frequency stability } \\
\text { and V2G operational } \\
\text { status }\end{array}$ \\
\hline
\end{tabular}




\begin{tabular}{|c|c|c|c|c|c|c|}
\hline Step No. & Step Description & Use Case & $\begin{array}{l}\text { V2G } \\
\text { Setting }\end{array}$ & $\begin{array}{l}\text { Load } \\
\text { Bank } \\
\text { Setting }\end{array}$ & Check & Notes \\
\hline 33 & Increase $\mathrm{G} 1$ to $50 \mathrm{~kW}$ & $\begin{array}{l}\text { Sudden } \\
\text { generation } \\
\text { increase }\end{array}$ & $\begin{array}{l}\text { Charging } \\
50 \mathrm{~kW}\end{array}$ & $0 \mathrm{~kW}$ & & $\begin{array}{l}\text { Observe system voltage } \\
\text { and frequency stability } \\
\text { and V2G operational } \\
\text { status }\end{array}$ \\
\hline 34 & $\begin{array}{l}\text { Increase load bank from } 0 \mathrm{~kW} \text { to } \\
50 \mathrm{~kW}\end{array}$ & $\begin{array}{l}\text { Sudden load } \\
\text { increase }\end{array}$ & $\begin{array}{l}\text { Charging } \\
50 \mathrm{~kW}\end{array}$ & $50 \mathrm{~kW}$ & & $\begin{array}{l}\text { Observe system voltage } \\
\text { and frequency stability } \\
\text { and V2G operational } \\
\text { status }\end{array}$ \\
\hline 35 & Decrease $\mathrm{G} 1$ to $0 \mathrm{~kW}$ & $\begin{array}{l}\text { Sudden } \\
\text { generation } \\
\text { decrease }\end{array}$ & $\begin{array}{l}\text { Charging } \\
50 \mathrm{~kW}\end{array}$ & $50 \mathrm{~kW}$ & & $\begin{array}{l}\text { Observe system voltage } \\
\text { and frequency stability } \\
\text { and V2G operational } \\
\text { status }\end{array}$ \\
\hline 36 & Increase $\mathrm{G} 1$ to $50 \mathrm{~kW}$ & $\begin{array}{l}\text { Sudden } \\
\text { generation } \\
\text { increase }\end{array}$ & $\begin{array}{l}\text { Charging } \\
50 \mathrm{~kW}\end{array}$ & $50 \mathrm{~kW}$ & & $\begin{array}{l}\text { Observe system voltage } \\
\text { and frequency stability } \\
\text { and V2G operational } \\
\text { status }\end{array}$ \\
\hline 37 & Set V2G for discharging at $40 \mathrm{~kW}$ & $\begin{array}{l}\text { Sudden } \\
\text { generation } \\
\text { increase }\end{array}$ & $\begin{array}{l}\text { Discharging } \\
40 \mathrm{~kW}\end{array}$ & $50 \mathrm{~kW}$ & & $\begin{array}{l}\text { Observe system voltage } \\
\text { and frequency stability } \\
\text { and V2G operational } \\
\text { status }\end{array}$ \\
\hline 38 & $\begin{array}{l}\text { Increase load bank from } 50 \mathrm{~kW} \text { to } \\
100 \mathrm{~kW}\end{array}$ & $\begin{array}{l}\text { Sudden load } \\
\text { increase }\end{array}$ & $\begin{array}{l}\text { Discharging } \\
40 \mathrm{~kW}\end{array}$ & $100 \mathrm{~kW}$ & & $\begin{array}{l}\text { Observe system voltage } \\
\text { and frequency stability } \\
\text { and V2G operational } \\
\text { status }\end{array}$ \\
\hline 39 & $\begin{array}{l}\text { Decrease load bank from } 100 \mathrm{~kW} \\
\text { to } 50 \mathrm{~kW}\end{array}$ & $\begin{array}{l}\text { Sudden load } \\
\text { decrease }\end{array}$ & $\begin{array}{l}\text { Discharging } \\
40 \mathrm{~kW}\end{array}$ & $50 \mathrm{~kW}$ & & $\begin{array}{l}\text { Observe system voltage } \\
\text { and frequency stability } \\
\text { and V2G operational } \\
\text { status }\end{array}$ \\
\hline 40 & Set V2G to charge at $0 \mathrm{~kW}$ & Test end & $0 \mathrm{~kW}$ & $50 \mathrm{~kW}$ & & \\
\hline 41 & Set load bank to $0 \mathrm{~kW}$ & Test end & $0 \mathrm{~kW}$ & $0 \mathrm{~kW}$ & & \\
\hline 42 & $\begin{array}{l}\text { Open both bus ports A\&B on IPD } \\
\text { No. } 1\end{array}$ & Test end & $0 \mathrm{~kW}$ & $0 \mathrm{~kW}$ & & \\
\hline 43 & $\begin{array}{l}\text { Open both bus ports A\&B on IPD } \\
\text { No. } 2\end{array}$ & Test end & $0 \mathrm{~kW}$ & $0 \mathrm{~kW}$ & & \\
\hline 44 & $\begin{array}{l}\text { Reset (open) all IPD breakers; } \\
\text { all load banks offline; } \\
\text { estop all generators }\end{array}$ & Test end & $0 \mathrm{~kW}$ & $0 \mathrm{~kW}$ & & \\
\hline
\end{tabular}




\section{Appendix P: Autonomous Vehicle Testing Facilities}

\section{The American Center for Mobility}

The ACM at Willow Run was originally built in 1940-it was one of the first assembly line production facilities for planes, designed on a napkin by Ford's chief engineer, despite both the U.S. government and established aircraft manufacturers of the time believing assembly line plane production was impossible. At its peak, ACM produced one B-52 Liberator bomber every 55 minutes.

ACM's facility (Figure P-1) encompasses 500 acres, multiple test environments, and is adjacent to the Willow Run Airport. The test area contains highways, exit ramps, single, and multi-lane roads, intersections, and a configurable area. The configurable area is flat, approximately $400 \mathrm{~m} \mathrm{x}$ $400 \mathrm{~m}$ asphalt pad that can be made into complex intersections, round abouts, or other user desired functions.

ACM is open to private industry, government, standard bodies, and academia and is a global center for testing and validation, education, and product and standards development related to connected and autonomous systems.

ACM is currently 13 months into a study, involving the center, defense, academia, and the public sector, to further research and test automated convoy platooning featuring both military and commercial grade trucks. The study is the first of its kind in the United States. 
Figure P-1. American Center for Mobility site map.

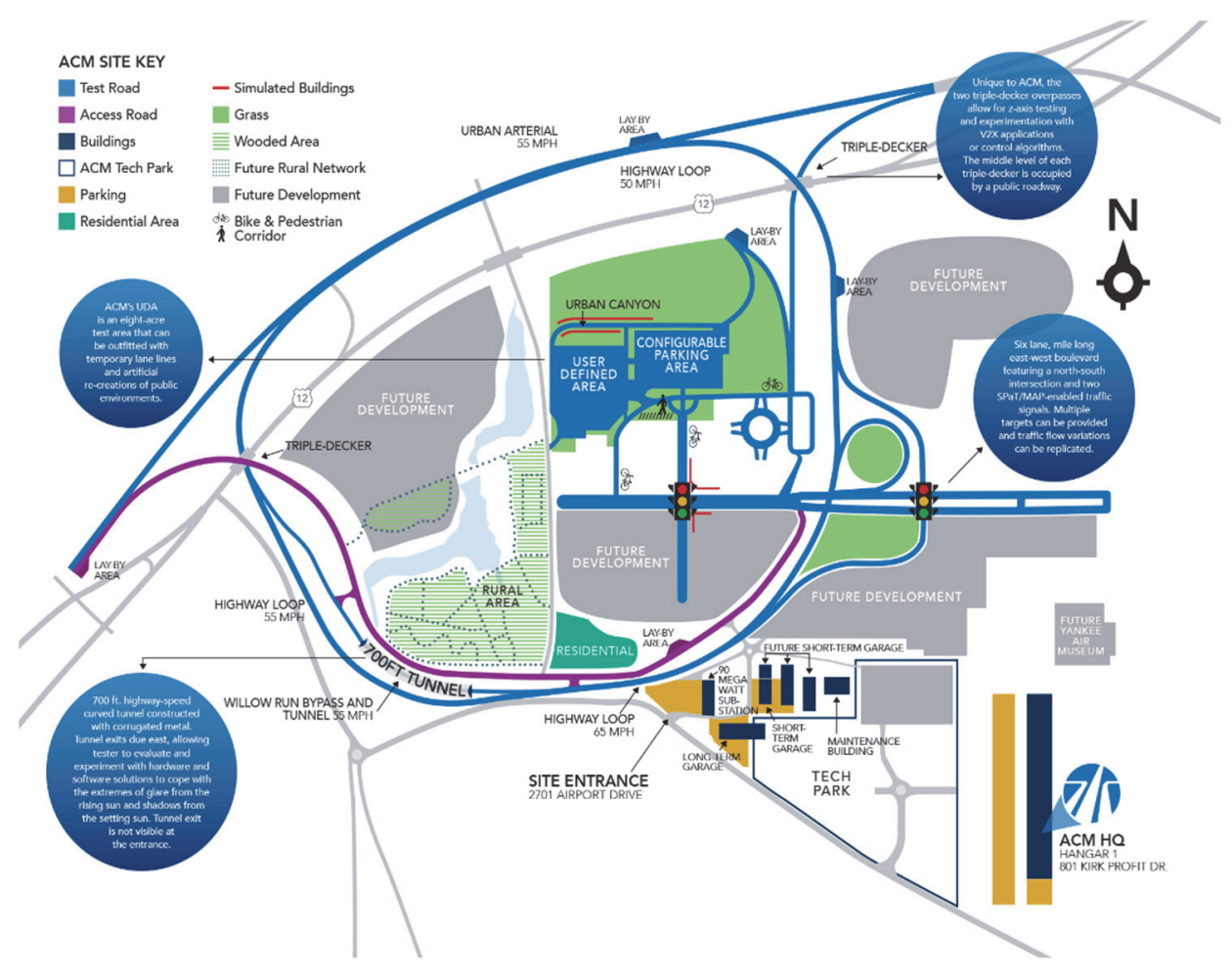

Larson Transportation Institute

The Thomas D. Larson Pennsylvania Transportation Institute at Pennsylvania State University is a transportation research center and a group of public and private stakeholders formed to address critical transportationrelated problems.

The Institute "maintains a broad range of activities that support corporate and governmental needs and goals for research and collaboration."

The facility (Figure P-2) includes the Bus Research and Testing Center, Center for Integrated Asset Management for Multi-Modal Transportation Infrastructure Systems and Center for Dirt and Gravel Road Studies. Other facilities include the Battery Application Technology and Energy Research Laboratory, a hydrogen fueling station, Hybrid and Hydrogen Vehicle Research Lab, test track, and a vehicle-handling area test pad 
Figure P-2. Larson Transportation Institute facility map

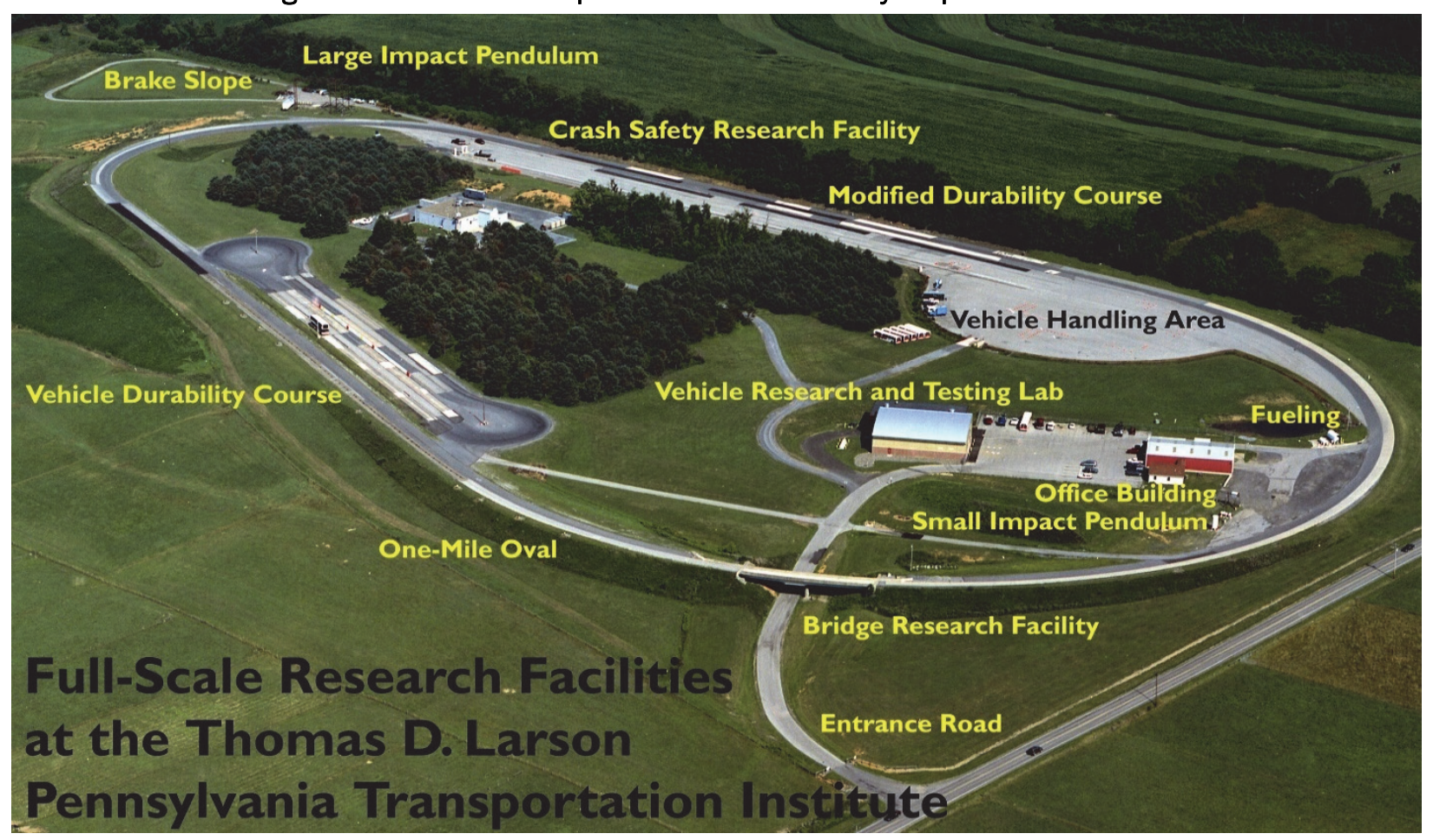




\section{Appendix Q: 800A Intelligent Power Distribution Box Technical Manual}

SEL $\begin{aligned} & \text { SCHWEITZER } \\ & \text { ENGINEERING } \\ & \text { LABORATORIES }\end{aligned}$
SEL Engineering Services, Inc.

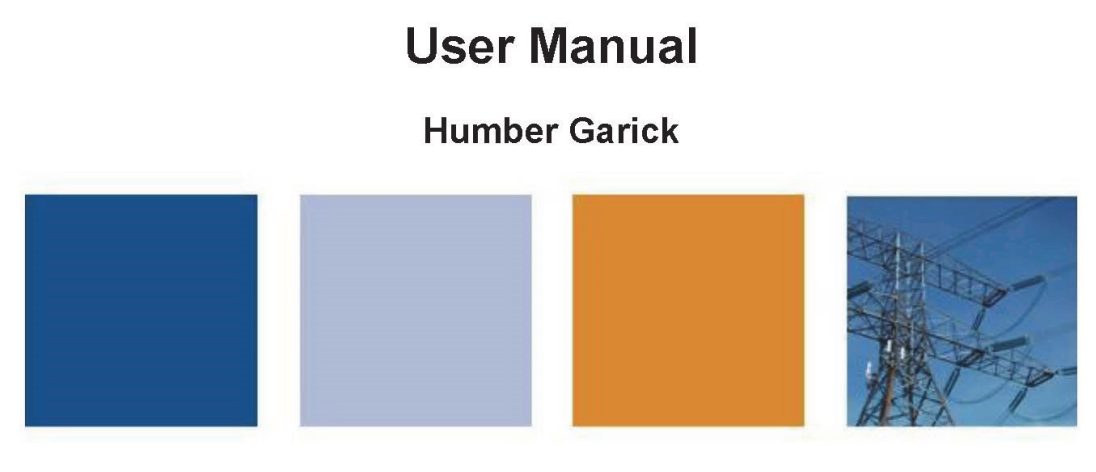

20190320

For information regarding this document, contact:

Author...

Brandon Marcum

$+1.509 .715 .0918$

brandon_marcum@selinc.com

Project Manager..

Rena McCulley

SEL ES Project Number

020124.000 .00

2350 NE Hopkins Court Pullman, WA 99163-5603 USA Tel: +1.509.332.1890 www. selinc.com

This document, and all information contained herein, is proprietary to SEL ES. Any unauthorized use, distribution, or reproduction of this document (in whole or in part) or of any information contained herein is specifically prohibited. This legend must appear on any authorized reproduction (in whole or in part).

SEL ES PROPRIETARY INFORMATION

일 2019 by SEL ES (All rights reserved)

DISTRIBUTION A. Approved for public release: distribution unlimited 
Table of Revisions

\begin{tabular}{|c|c|c|c|c|c|}
\hline $\begin{array}{c}\text { Revision } \\
\text { Number }\end{array}$ & Date & Description & Author & Reviewer & Approved \\
\hline 0 & & Initial issue & BM & SM & SM \\
\hline & & & & & \\
\hline & & & & & \\
\hline
\end{tabular}

All brand or product names appearing in this document are the trademark or registered trademark of their respective holders. No SEL trademarks may be used A

SEL products referred to in this report are manufactured by Schweitzer Engineering Laboratories, Inc. (SEL).

\begin{tabular}{lcr}
\hline Date Code 20190320 & Humber Garick & Page ii \\
SEL ES PROPRIETARY INFORMATION & User Manual & Project 020124.000.00 \\
๑ 2019 by SEL ES (All rights reserved) & & whw selinc.com
\end{tabular}

DISTRIBUTION A. Approved for public release: distribution unlimited 


\section{Table of Contents}

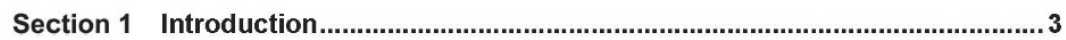

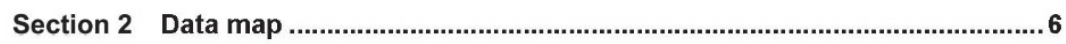

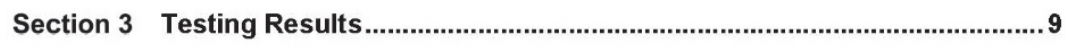

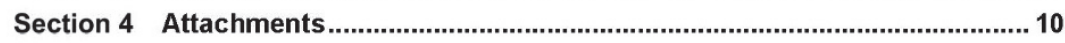

\begin{tabular}{lcr}
\hline Date Code 20190320 & Humber Garick & Page 1 \\
SEL ES PROPRIETARY INFORMATION & User Manual & Project 020124.000.00 \\
๑ 2019 by SEL. ES (All rights reselinc.com
\end{tabular}

DISTRIBUTION A. Approved for public release: distribution unlimited 


\section{Figures}

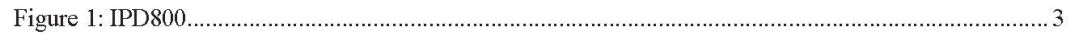

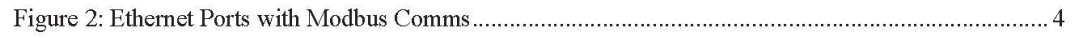

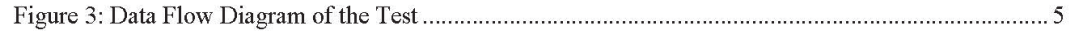

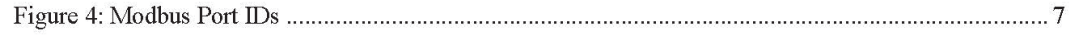

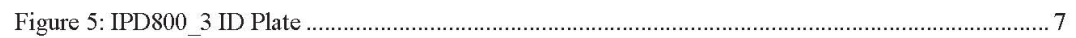

\begin{tabular}{lcr}
\hline Date Code 20190320 & Humber Garick & Page 2 \\
SEL ES PROPRIETARY INFORMATION & User Manual & Project 020124.000.00 \\
$\odot$ wrown. selinc. com
\end{tabular}

DISTRIBUTION A. Approved for public release: distribution unlimited 


\section{SECTION 1 INTRODUCTION}

This document is produced to describe the Modbus communication functionality of the $800 \mathrm{~A}$ Intelligent Power Distribution (IPD800) box. Figure 1 below shows the IPD800 control panel.

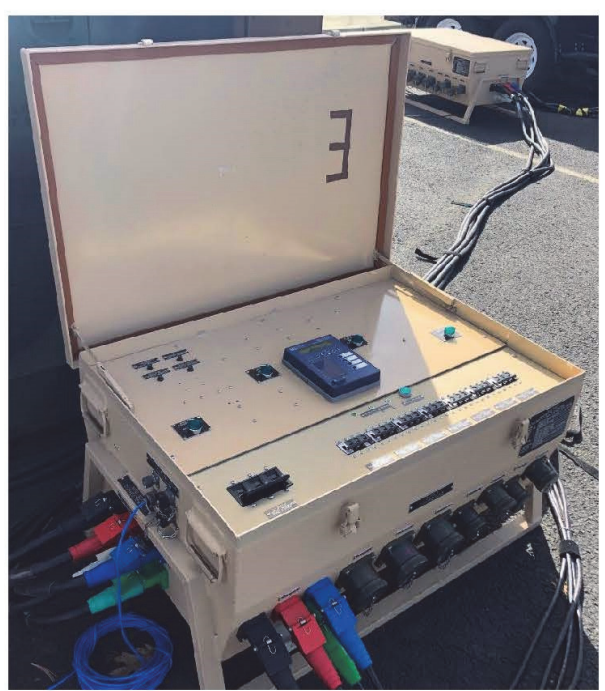

Figure 1: IPD800

Figure 2 below shows the ethernet ports that can be used for Modbus communication. They are the bottom two ports on the Bus A (left) side of the IPD. The top right port will not communicate via Modbus and is for internal programming and diagnosties only.

\begin{tabular}{|c|c|c|}
\hline $\begin{array}{l}\text { Date Code } 20190320 \\
\text { SEL ES PROPRIETARY INFORMATION } \\
\text { @ } 2019 \text { by SEL ES (All rights reserved) }\end{array}$ & $\begin{array}{l}\text { Humber Garick } \\
\text { User Manual }\end{array}$ & $\begin{array}{r}\text { Page 3 } \\
\text { Project 020124.000.00 } \\
\text { wrow selinc.com }\end{array}$ \\
\hline
\end{tabular}




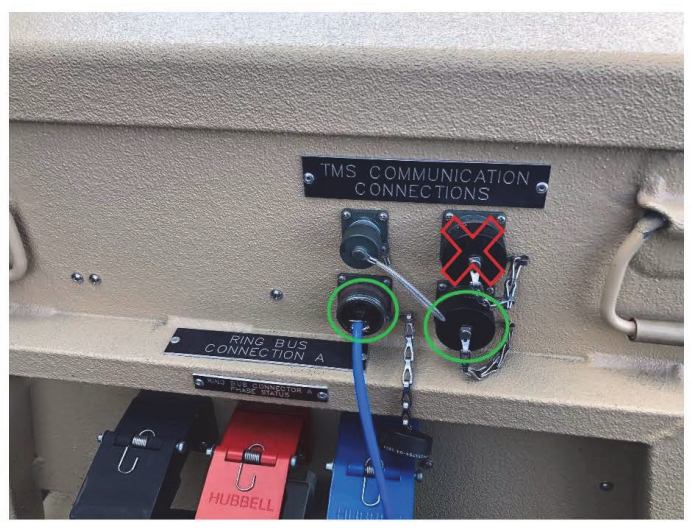

Figure 2: Ethernet Ports with Modbus Comms

Figure 3 below shows the Data Flow Diagram of the IPD and the testing PC. Both the Real-Time Automation Controller (RTAC) and relays are installed inside the IPD800. The relays talk IEC-61850 GOOSE to the RTAC, which acts as a protocol translator (gateway) and converts the GOOSE messages to Modbus and DDS messages. DDS and Modbus are both available simultaneously on the same ethernet ports.

In order to translate the Modbus data coming from the IPD800, you will need to have a PC that acts as the Modbus master. SEL used Kepware ServerEX software to act as the Modbus master, as well as Kepware OPC Quick Client to interpret the Modbus data.

\begin{tabular}{|c|c|c|}
\hline $\begin{array}{l}\text { Date Code } 20190320 \\
\text { SEL ES PROPRIETARY INFORMATION } \\
\text { (C) } 2019 \text { by SEL ES (All rights reserved) }\end{array}$ & $\begin{array}{l}\text { Humber Garick } \\
\text { User Manual }\end{array}$ & $\begin{array}{r}\text { Page } 4 \\
\text { Project 020124.000.00 } \\
\text { www.selinc.com }\end{array}$ \\
\hline
\end{tabular}




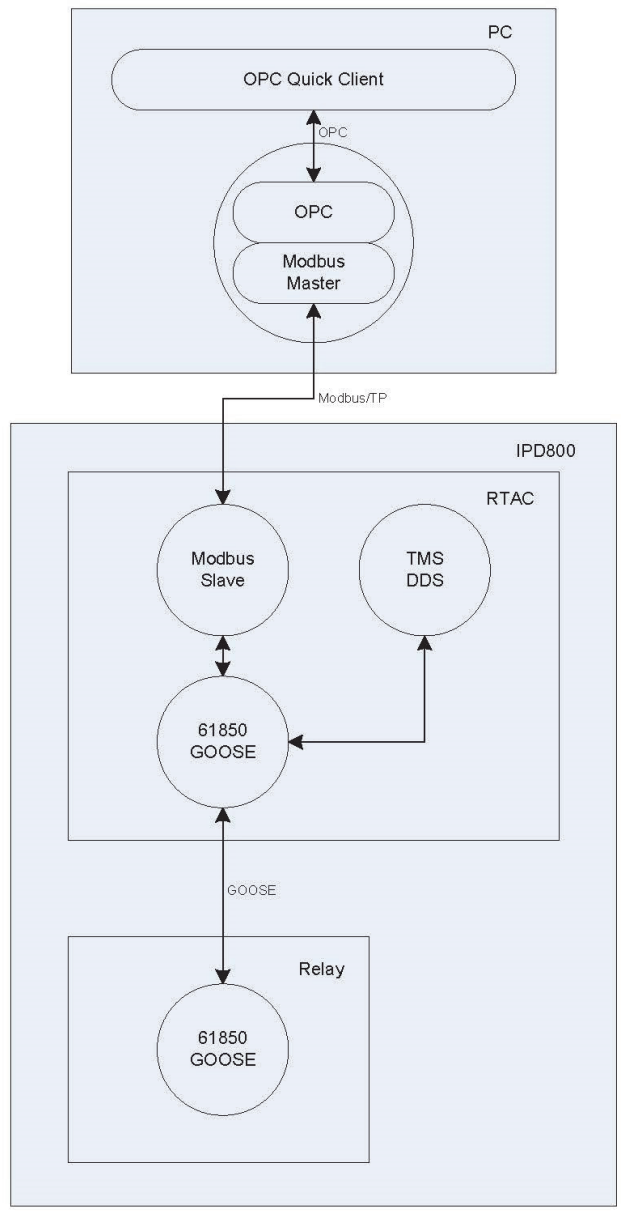

Figure 3: Data Flow Diagram of the Test

\begin{tabular}{|c|c|c|}
\hline $\begin{array}{l}\text { Date Code } 20190320 \\
\text { SEL ES PROPRIETARY INFORMATION } \\
\text { @ } 2019 \text { by SEL ES (All rights reserved) }\end{array}$ & $\begin{array}{l}\text { Humber Garick } \\
\text { User Manual }\end{array}$ & $\begin{array}{r}\text { Page } 5 \\
\text { Project } 020124.000 .00 \\
\text { wrow selinc. com }\end{array}$ \\
\hline
\end{tabular}




\section{SECTION 2 DATA MAP}

Table 1 below shows the data map of the Modbus registers used by the IPD 800 .

\begin{tabular}{|c|c|c|c|c|c|c|c|c|}
\hline $\begin{array}{l}\text { Tag } \\
\text { Name }\end{array}$ & Address & $\begin{array}{l}\text { Data } \\
\text { Type }\end{array}$ & $\begin{array}{l}\text { Tag } \\
\text { Name }\end{array}$ & Address & $\begin{array}{l}\text { Data } \\
\text { Type }\end{array}$ & $\begin{array}{l}\text { Tag } \\
\text { Name }\end{array}$ & Address & $\begin{array}{l}\text { Data } \\
\text { Type }\end{array}$ \\
\hline Breakers & 400001 & Word & P05_VA & 400034 & Word & P09_VA & 400066 & Word \\
\hline P01_VA & 400002 & Word & P05_VB & 400035 & Word & P09_VB & 400067 & Word \\
\hline P01_VB & 400003 & Word & P05_VC & 400036 & Word & P09_VC & 400068 & Word \\
\hline P01_VC & 400004 & Word & P05_IA & 400037 & Word & P09_IA & 400069 & Word \\
\hline P01_IA & 400005 & Word & P05_IB & 400038 & Word & P09_IB & 400070 & Word \\
\hline P01_IB & 400006 & Word & P05_IC & 400039 & Word & P09_IC & 400071 & Word \\
\hline P01_IC & 400007 & Word & P05_P & 400040 & Short & P09_P & 400072 & Short \\
\hline P01_P & 400008 & Short & P05_Q & 400041 & Short & P09_Q & 400073 & Short \\
\hline P01_Q & 400009 & Short & P06_VA & 400042 & Word & P10_VA & 400074 & Word \\
\hline P02_VA & 400010 & Word & P06_VB & 400043 & Word & P10_VB & 400075 & Word \\
\hline P02_VB & 400011 & Word & P06_VC & 400044 & Word & P10_VC & 400076 & Word \\
\hline P02_VC & 400012 & Word & P06_IA & 400045 & Word & P10_IA & 400077 & Word \\
\hline P02_IA & 400013 & Word & P06_IB & 400046 & Word & P10_IB & 400078 & Word \\
\hline P02_IB & 400014 & Word & P06_IC & 400047 & Word & P10_IC & 400079 & Word \\
\hline P02_IC & 400015 & Word & P06_P & 400048 & Short & P10_P & 400080 & Short \\
\hline P02_P & 400016 & Short & P06_Q & 400049 & Short & P10_Q & 400081 & Short \\
\hline P02_Q & 400017 & Short & P07_VA & 400050 & Word & P11_VA & 400082 & Word \\
\hline P03_VA & 400018 & Word & P07_VB & 400051 & Word & P11_VB & 400083 & Word \\
\hline P03_VB & 400019 & Word & P07_VC & 400052 & Word & P11_VC & 400084 & Word \\
\hline P03_VC & 400020 & Word & P07_IA & 400053 & Word & P11_IA & 400085 & Word \\
\hline P03_IA & 400021 & Word & P07_IB & 400054 & Word & P11_IB & 400086 & Word \\
\hline P03_IB & 400022 & Word & P07_IC & 400055 & Word & P11_IC & 400087 & Word \\
\hline P03_IC & 400023 & Word & P07_P & 400056 & Short & P11_P & 400088 & Short \\
\hline P03_P & 400024 & Short & P07_Q & 400057 & Short & P11_Q & 400089 & Short \\
\hline P03_Q & 400025 & Short & P08_VA & 400058 & Word & P12_VA & 400090 & Word \\
\hline P04_VA & 400026 & Word & P08_VB & 400059 & Word & P12_VB & 400091 & Word \\
\hline P04_VB & 400027 & Word & P08_VC & 400060 & Word & P12_VC & 400092 & Word \\
\hline P04_VC & 400028 & Word & P08_IA & 400061 & Word & P12_|A & 400093 & Word \\
\hline P04_IA & 400029 & Word & P08_IB & 400062 & Word & P12_IB & 400094 & Word \\
\hline P04_IB & 400030 & Word & P08_IC & 400063 & Word & P12_IC & 400095 & Word \\
\hline P04_IC & 400031 & Word & P08_P & 400064 & Short & P12_P & 400096 & Short \\
\hline P04_P & 400032 & Short & P08_Q & 400065 & Short & P12_Q & 400097 & Short \\
\hline P04_Q & 400033 & Short & & & & FREQ & 400098 & Word \\
\hline
\end{tabular}

Table 1: Modbus Data Map

\begin{tabular}{|c|c|c|}
\hline $\begin{array}{l}\text { Date Code } 20190320 \\
\text { SEL ES PROPRIETARY INFORMATION } \\
\text { @ } 2019 \text { by SEL ES (All rights reserved) }\end{array}$ & $\begin{array}{l}\text { Humber Garick } \\
\text { User Manual }\end{array}$ & $\begin{array}{r}\text { Page 6 } \\
\text { Project 020124.000.00 } \\
\text { wrow selinc.com }\end{array}$ \\
\hline
\end{tabular}


Figure 4 below maps out the port numbers assigned to the Modbus registers with the physical switches on the control panel. P01-P05 are all rated at 200A, P06-P08 are 100A rated, and P09-P12 are 60A rated. The IPD800 is rated at 400A total input per generator port, and $800 \mathrm{~A}$ total output on each bus port.

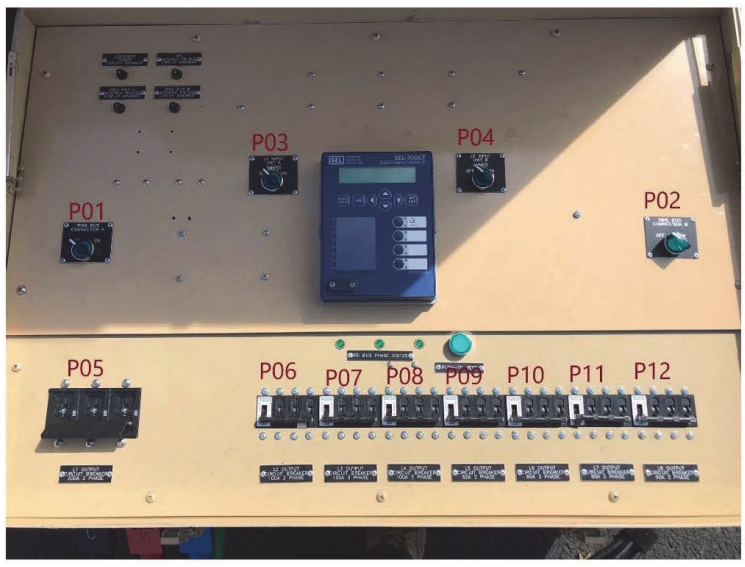

Figure 4: Modbus Port IDs

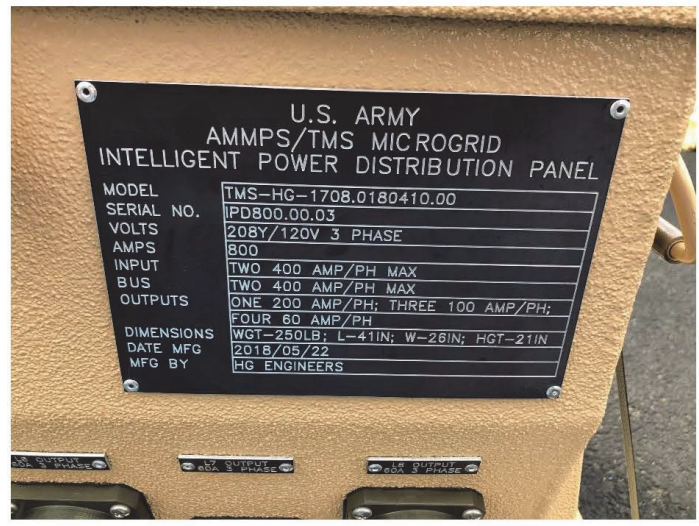

Figure 5: IPD800_3 ID Plate

\begin{tabular}{lcr}
\hline Date Code 20190320 & Humber Garick & Page 7 \\
SEL ES PROPRIETARY INFORMATION & User Manual & Project 020124.000 .00 \\
@ 2019 by SEL ES (All rights reserved) & & whww.selinc.com
\end{tabular}

DISTRIBUTION A. Approved for public release: distribution unlimited 
SEL added a scaling factor of $10 \mathrm{X}$ to each of the data points reporting over Modbus. The data sent over Modbus are integers, which means that only whole numbers can be transmitted. By adding the scaling factor, we get better resolution in the data and ean show decimal values out to one decimal place. This means that when register 40098 shows a frequency of "604", the actual frequency is $60.4 \mathrm{~Hz}$. Likewise, when PO3_VA shows a value of "1202", the actual voltage on phase A of port 3 is $120.2 \mathrm{VAC}$.

As mentioned, in the Modbus registers each data point is represented by an integer. Most of those integers are unsigned, meaning that no negative values can be represented. These values are listed as Word types in the datamap, which is a 16-bit unsigned integer type. However, a couple of the data points on each port require polarity, so they are represented as Short types in the datamap, which is a 16-bit signed integer type.

Only one frequency is measured and transmitted despite having 12 ports. The frequency data transmitted is the frequency as measured on the bus bars internal to the IPD800. Because all the ports connect to the same internal bus bars, if one port is live, all 12 ports have the same frequency internal to the IPD800. Therefore, only one frequency measurement is transmitted.

Lastly, it should be noted that address 40001 is a bit-packed register, where each bit represents the breaker status of one port. The ports are stacked in order, so the breaker status of port 1 , which is the Bus A port on the IPD, is indicated by the state of the least significant bit in the register. When port 1 (Bus A) is closed, register 40001 shows a value of " 1 ", the decimal representation of the binary value "0000 0000 0001." When ports 4 (IPD generator port J2), 6 (IPD load port 2) and 10 (IPD load port 6) are closed, the register will display the value " 552 ", the decimal representation of the binary value "0010 00101000 ".

The attached video file "02_DigitalTest.mp4" provides further explanation.

\begin{tabular}{lcr}
\hline Date Code 20190320 & Humber Garick & Page 8 \\
SEL ES PROPRIETARY INFORMATION & User Manual & Project 020124.000.00 \\
@ 2019 by SEL ES (All rights reserved) & & wow selinc. com
\end{tabular}

DISTRIBUTION A. Approved for public release: distribution unlimited 


\section{SECTION 3 TESTING RESULTS}

For test results and proof of functionality, we refer you to the attached video files.

01_TestSetup.mp4 explains the communication data flow as explained in section 1 .

02 DigitalTest.mp4 explains and demonstrates the bit-packing of the Breakers register 40001 .

03 VoltTest.mp4 shows the change in $\mathrm{AC}$ voltage on each port as each port is opened in sequence, beginning with $\mathrm{P} 12$ and ending with $\mathrm{P} 04$.

04_AnalogTests.mp4 explains the analog tests we perform in videos 05_AnalogTest1.mp4 through 08 AnalogTest4.mp4.

Note that port P06 should not be used because the breaker status does not report properly. The wire termination at the MCCB is faulty.

\begin{tabular}{|c|c|c|}
\hline $\begin{array}{l}\text { Date Code } 20190320 \\
\text { SEL ES PROPRIETARY INFORMATION } \\
\text { @ } 2019 \text { by SEL ES (All rights reserved) }\end{array}$ & $\begin{array}{l}\text { Humber Garick } \\
\text { User Manual }\end{array}$ & $\begin{array}{r}\text { Page 9 } \\
\text { Project 020124.000.00 } \\
\text { wrow selinc.com }\end{array}$ \\
\hline
\end{tabular}




\section{SECTION 4 ATTACHMENTS}

01 TestSetup.mp4

02_DigitalTest.mp4

03_VoltTest.mp4

04_AnalogTests.mp4

05_Analog Test1.mp4

06_AnalogTest2.mp4

07_AnalogTest3.mp4

08_AnalogTest4.mp4

\begin{tabular}{lcr}
\hline Date Code 20190320 & Humber Garick & Page 10 \\
SEL ES PROPRIETARY INFORMATION & User Manual & Project 020124.000 .00 \\
๑ 2019 by SEL ES (All rights reselinc.com
\end{tabular}

DISTRIBUTION A. Approved for public release: distribution unlimited 


\section{Appendix R: Technical Manual for the AirGuard 3e-523}

3eTI Technology International

Airguard 3e-525/523 Series Wireless

Access Points

(NDcPP20E/WLANAScEP10) Security

Target

3e Technologies International

9715 Key West Avenue

5 th Floor

Rockville, MD 20850 USA

Ultra

ELECTRONICS 


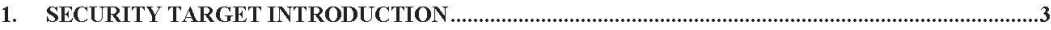

1.1 SECURITY TARGET REFERENCE

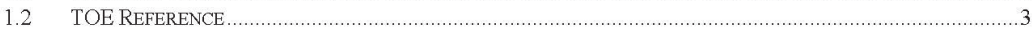

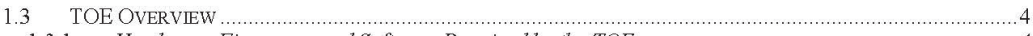

1.3.1 Hardware, Firmware, and Software Required by the TOE …....................................................... 4

1.4 TOE DESCRTPTION

1.4.1 TOE Architecture.

1.42 TOE Documentation .

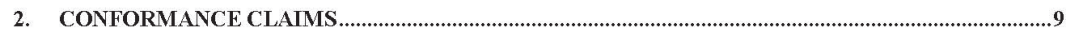

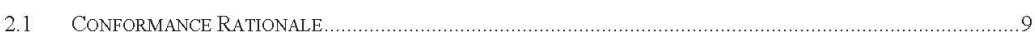

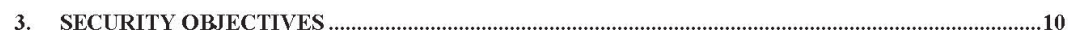

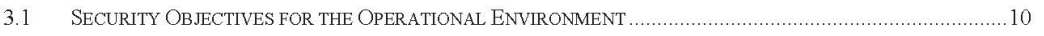

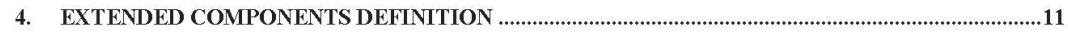

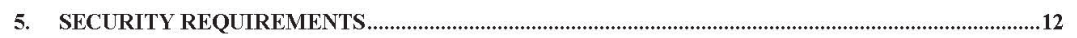

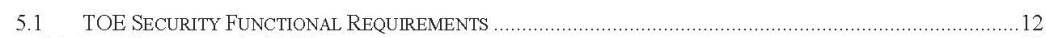

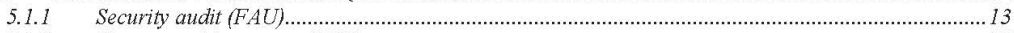

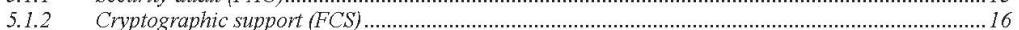

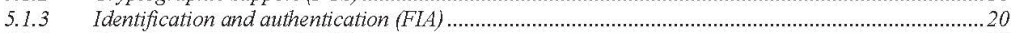

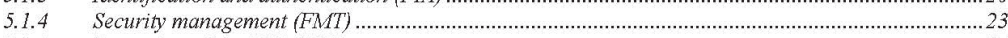

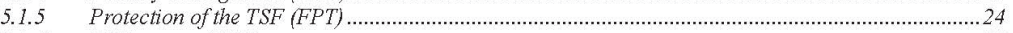

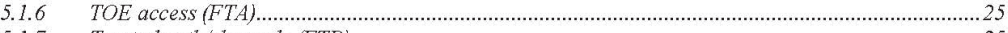

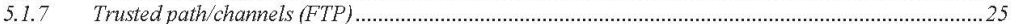

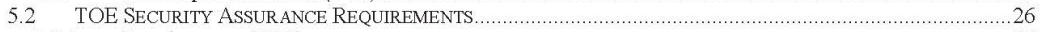

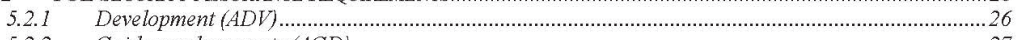

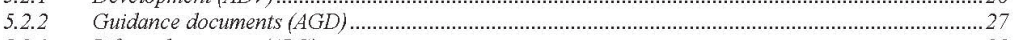

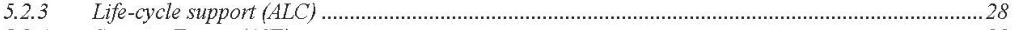

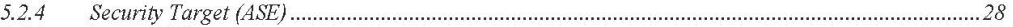

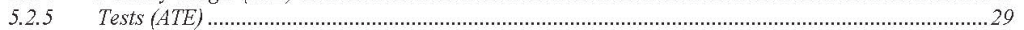

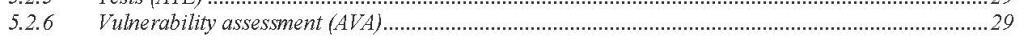

6. TOE SUMMARY SPECIFICATION ………….....................................................................................

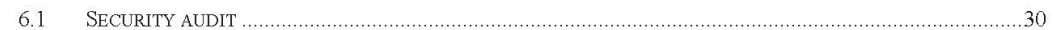

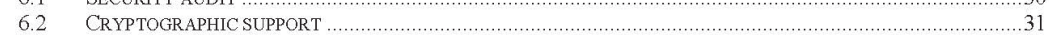

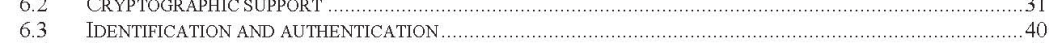

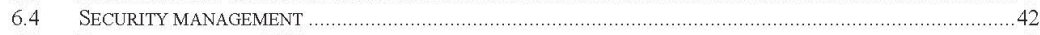

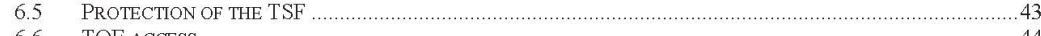

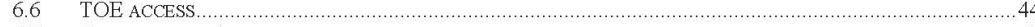

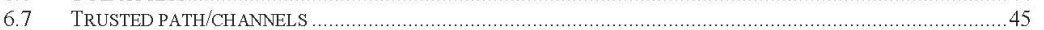

\section{LIST OF TABLES}

Table 1 TOE Security Functional Components

Table 2 Auditable Events

Table 3 Assurance Components

Table 6-1: TOE FIPS-140 Tested Algorithms 


\section{Security Target Introduction}

This section identifies the Security Target (ST) and Target of Evaluation (TOE) identification, ST conventions, ST conformance claims, and the ST organization. The TOE is 3eTI AirGuard 3e-525/523 Wireless Access Points provided by $3 \mathrm{e}$ Technologies International. The TOE is being evaluated as a Network Device and Wireless Access Point.

The Security Target contains the following additional sections:

- Conformance Claims (Section 2)

- Security Objectives (Section 3)

- Extended Components Definition (Section 4)

- Security Requirements (Section 5)

- TOE Summary Specification (Section 6)

\section{Conventions}

The following conventions have been applied in this document

- Security Functional Requirements - Part 2 of the CC defines the approved set of operations that may be applied to functional requirements: iteration, assignment, selection, and refinement.

- Iteration: allows a component to be used more than once with varying operations. In the ST, iteration is indicated by a parenthetical number placed at the end of the component. For example FDP_ACC.1(1) and FDP_ACC.1(2) indicate that the ST includes two iterations of the FDP_ACC.1 requirement.

- Assignment: allows the specification of an identified parameter. Assignments are indicated using bold and are surrounded by brackets (e.g., [assignment]). Note that an assignment within a selection would be identified in italics and with embedded bold brackets (e.g., [Iselected-assignment]]).

- Selection: allows the specification of one or more elements from a list. Selections are indicated using bold italics and are surrounded by brackets (e.g., [selection]).

- Refinement: allows the addition of details. Refinements are indicated using bold, for additions, and strike-through, for deletions (e.g., "... all objects ..." or "... big things ...")

- Other sections of the ST - Other sections of the ST use bolding to highlight text of special interest, such as captions.

\subsection{Security Target Reference}

ST Title - 3eTI AirGuard 3e-525/523 Wireless Access Points (NDcPP20E/WLANAScEP10) Security Target

ST Version - Version 0.5

ST Date $-05 / 08 / 18$

\subsection{TOE Reference}

TOE Identification - 3e Technologies International AirGuard 3e-525/523 Wireless Access Points

TOE Developer - 3e Technologies International

TOE Models - 3e-525N, 3e-525N-MP, 3e-525NV, 3e-523N, 3e-523NF and 3e-523NR Wireless Access Points, firmware version 5.1 .0 
Evaluation Sponsor - 3e Technologies International

Keywords - Encryption, Wireless Access Points, IPsec, access control, traffic filter

\subsection{TOE Overview}

The Target of Evaluation (TOE) includes the following 3eTI AirGuard ${ }^{\mathrm{TM}}$ Wireless Access Points models: 3e-525N, $3 \mathrm{e}-525 \mathrm{~N}$ MP, 3e-525NV, 3e-523N, 3e-523NF and 3e-523NR. The models share the identical hardware platform and firmware. Differences between models are limited to enclosure, power options, the number of Wi-Fi radio interfaces and extra video component.

The table below shows the differences among the 3eTI Wireless Access Points (APs)

Table 1-1 Access Point Products Comparison

\begin{tabular}{|c|c|c|c|c|}
\hline Model & Number of Radio & Radio Mode & Mechanical & Comments \\
\hline $3 e-525 N$ & 2 & Access Point & $\begin{array}{l}\text { Ruggedized for } \\
\text { industrial and } \\
\text { outdoor }\end{array}$ & \\
\hline 3e-525N MP & 2 & Access Point & $\begin{array}{l}\text { Ruggedized for } \\
\text { industrial and } \\
\text { outdoor }\end{array}$ & $\begin{array}{l}\text { Same as } 3 e-525 N \\
\text { except mobile } \\
\text { power input }\end{array}$ \\
\hline $3 e-525 N V$ & 2 & Access Point & $\begin{array}{l}\text { Ruggedized for } \\
\text { industrial and } \\
\text { outdoor }\end{array}$ & $\begin{array}{l}\text { Same as } 3 e-525 \mathrm{~N} \\
\text { with extra video } \\
\text { capture card }\end{array}$ \\
\hline $3 e-523 N$ & 1 & Access Point & Indoor Enclosure & $\begin{array}{l}\text { Operate in } \\
\text { Industrial } \\
\text { temperature range } \\
-40 \mathrm{C} \text { to } 75 \mathrm{C}\end{array}$ \\
\hline $3 e-523 N F$ & 1 & Access Point & Indoor Enclosure & $\begin{array}{l}\text { Same as } 3 e-523 N \\
\text { Operate in }-10- \\
60 C\end{array}$ \\
\hline $3 e-523 N R$ & 1 & Access Point & $\begin{array}{l}\text { Ruggedized for } \\
\text { industrial and } \\
\text { outcloor }\end{array}$ & $\begin{array}{l}\text { Same as 3e-523N } \\
\text { except enclosure } \\
\text { for outdoor } \\
\text { deployment }\end{array}$ \\
\hline
\end{tabular}

1.3.1 Hardware, Firmware, and Software Required by the TOE

The TOE consists of hardware and firmware residing on the Access Point appliances as listed in Section 1.2 above. The evaluated configuration of the TOE requires the following Operational Environment support which is not included in the TOE's physical boundary.

- RADIUS Server: The TOE requires a RADIUS Server in the Operational Environment for wireless client authentication.

- Wireless Clients: All wireless client hosts connecting to the wired network from the wireless network

- Administrator Workstations: Trusted administrators access the TOE through the HTTPS protocol. 
- Audit Servers: The TOE relies upon the audit server for storage of audit records.

- NTP Servers: The TOE relies upon an NTP server to provide reliable time.

\subsection{TOE Description}

The TOE is classified as a Wireless Local Area Network (WLAN) Access Device. The TOE employs mesh networking, which allows multiple TOEs to network within the operational environment ( $802.11 \mathrm{~s}$ is not validated).

The TOE sits between wired and wireless portions of an enterprise network and provides integrity and confidentiality of wireless traffic and restricts access of wireless endpoints to wired network systems. The TOE provides a secure, yet flexible, WLAN environment as an Access Point that mediates authenticated wireless client's data through encryption/decryption and integrity protection between the wireless link and the wired LAN.

\subsubsection{TOE Architecture}

The 3eTI 3e-525N, 3e-525N MP, 3e-525NV, 3e-523N, 3e-523NF and 3e-523NR Access Points (hereafter referred to as Access Points or APs) provide the connection point between wireless client hosts and the wired network. Once installed as trusted nodes on the wired infrastructure, the APs provide the encryption service on the wireless network between themselves and the wireless clients. The APs can also communicate among themselves through the secured channel via $3 \mathrm{eTI}$ mesh forming or 802.11s mesh network. However, this AP to AP secured communication service is not evaluated here.

The Access Points are appliances consisting of hardware and firmware. Wireless communications between clients and $\mathrm{APs}$ are carried out using the IEEE 80211 protocol standard. The 802.11 standard governs communication transmission for wireless devices. For this evaluation, the APs use $802.1 \mathrm{la}, 802.1 \mathrm{lg}$ and $802.1 \mathrm{ln}$ for wireless communication. The wireless security protocol that is to be used with the APs is WPA2, which is the Wi-Fi Alliance interoperable specification based on IEEE 802.11i security standard

The APs have one or more RF interfaces and one or more Ethernet interfaces. All these interfaces are controlled by the software executing on the APs. The Access Points included in the TOE vary by the number of RF and Ethernet interfaces and antenna support; however, the differences do not affect the security functionality claimed by the TOE. The APs maintain a security domain containing all hardware and software of the appliance for its own execution. The APs maintain this security domain by controlling the actions that can occur at the interfaces described above and providing the hardware resources that carry out the execution of tasks on the APs. The APs provide for isolation of different wireless clients that have sessions with the WLAN, which includes maintaining the keys necessary to support encrypted sessions with wireless devices.

The APs control the actions and the manner in which external users may interact with its external interfaces. Thus, the APs ensure that the TOE's enforcement functions are invoked and succeed be fore allowing the external user to carry out any other security function with or through the APs. The figure below shows the TOE and its operational environment. The trusted path between the TOE and Administration Station is TLS/HTTPS and the trusted path between the TOE and NTP, Log Server and RADIUS server is IPsec.

The figure below illustrates the typical deployment use case and operational environment setup for TOE devices. 


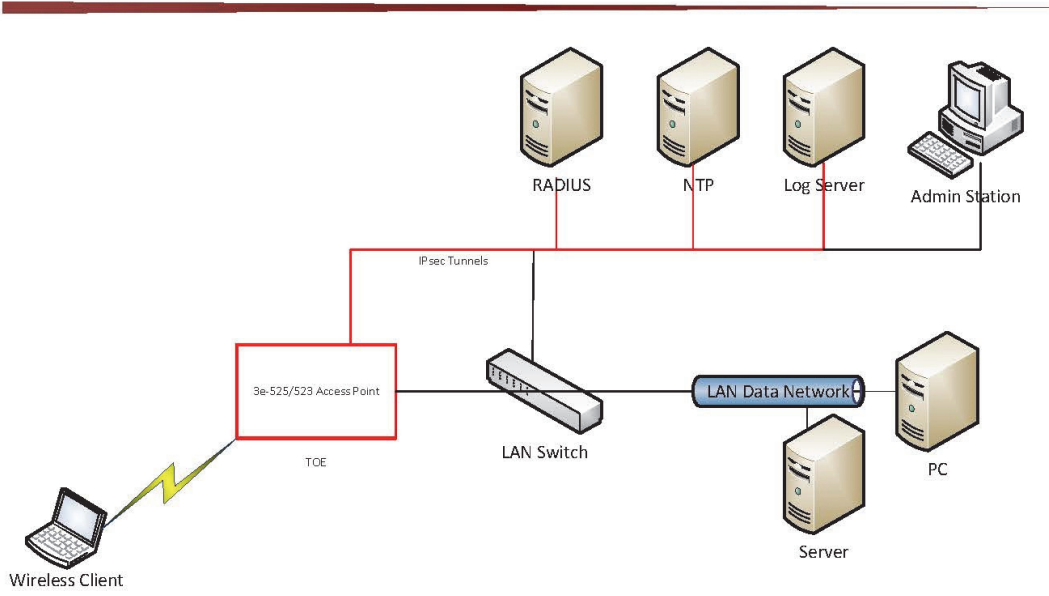

1.4.1.1 Physical Boundaries

The TOE physical boundary defines all hardware and firmware that is required to support the TOE's logical boundary and the TOE's security functions. The TOE hardware platform uses Freescale MPC8378E CPU and the TOE's firmware contains an embedded Linux Kernel customized by $3 \mathrm{eTI}$ based on kernel version 3.6. In short, the TOE's physical boundary is the physical device/appliance for all models. The APs have the following physical interfaces.

- AP antenna ports - The AP antenna ports are connected to one $802.1 \mathrm{la} / \mathrm{b} / \mathrm{g} / \mathrm{n}$ radio for wireless connectivity to secure WLAN clients.

- LAN local port - The LAN local port is used exclusively for management of the access point. It supports Ethernet 10/100/1000 Mbps wired traffic, full duplex for fast configuration and management. The LAN port is locally terminated - no data entering here goes out to the WLAN, only management data is accepted.

- WAN uplink port - The WAN uplink port is intended to connect the 3eTI access points to the wired LAN It also supports Ethernet $10 / 100 / 1000$ Mbps wired traffic in a full duplex configuration. The WAN port bridges all data between the wireless domain and the wired network

\subsubsection{Logical Boundaries}

This section summarizes the security functions provided by the TOE:

- Security audit

- Cryptographic support

- Identification and authentication

- Security management

- Protection of the TSF

- TOE access

- Trusted path/channels 


\subsection{Security audit}

The TOE generates auditable events for actions on the TOE with the capability of selective audit record generation The records of these events can be viewed within the Web User Interface (UI) or they can be exported to audit log servers in the Operational Environment. The TOE generates records for its own actions, containing information about the user/process associated with the event, the success or failure of the event, and the time that the event occurred. Additionally, all administrator actions relating to the management of TSF data and configuration data are logged by the TOE's audit generation functionality.

1.4.1.2.2 Cryptographic support

The TOE uses NIST SP 800-90 DRBG random bits generator and the following cryptographic algorithms: AES, RSA, ECDSA, SHA, HMAC to secure the wireless client data to the LAN, trusted channel and trusted path communication. The TOE zeroizes Critical Security Parameters (CSPs) to mitigate the possibility of disclosure or modification.

1.4.1.2.3 Identification and authentication

The TOE provides Identification and Authentication security functionality to ensure that all users are properly identified and authenticated before accessing TOE functionality. The TOE displays a configurable access banner and enforces a local password-based authentication mechanism to perform administrative user authentication. Passwords are obscured when being displayed during any attempted login.

The wireless users are authenticated by the RADIUS server in the Operational Environment. EAP-TLS is used for WPA2 wireless authentication via $x .509$ certificates. The TOE sets up an IPsec tunnel with a RADIUS server and supports IKEv 2 with x. 509 certificates for IPsec endpoints mutual authentication with its IPsec peer.

1.4.1.2.4 Security management

The Web User Interface (UI) of the TOE provides the capabilities for configuration and administration. The Web UI can be accessed via the dedicated local Ethernet port configured for "out-of-band" management. There is no local access such as a serial console port. Therefore, the local and remote management is considered the same for this evaluation.

An authorized administrator has the ability to modify, edit, and delete security parameters such as audit data, configuration data, and user authentication data. The Web UT also offers an authorized administrator the capability to manage how security functions behave. For example, an administrator can enable/disable certain audit functions query and set encryption/decryption algorithms used for network packets.

\subsection{Protection of the TSF}

Internal testing of the TOE hardware, software, and software updates against tampering ensures that all security functions are running and available before the TOE accepts any communications. The TSF prevents reading of preshared keys, symmetric keys, private keys, and passwords. The TOE uses electronic signature verification before any firmware/software updates are installed.

1.4.1.2.6 TOE acces

The TOE provides the following TOE Access functionality:

- Configurable MAC address and/or IP address filtering with remote management session establishment

- TSF-initiated session termination when a connection is idle for a configurable time period

- Administrative termination of own session 


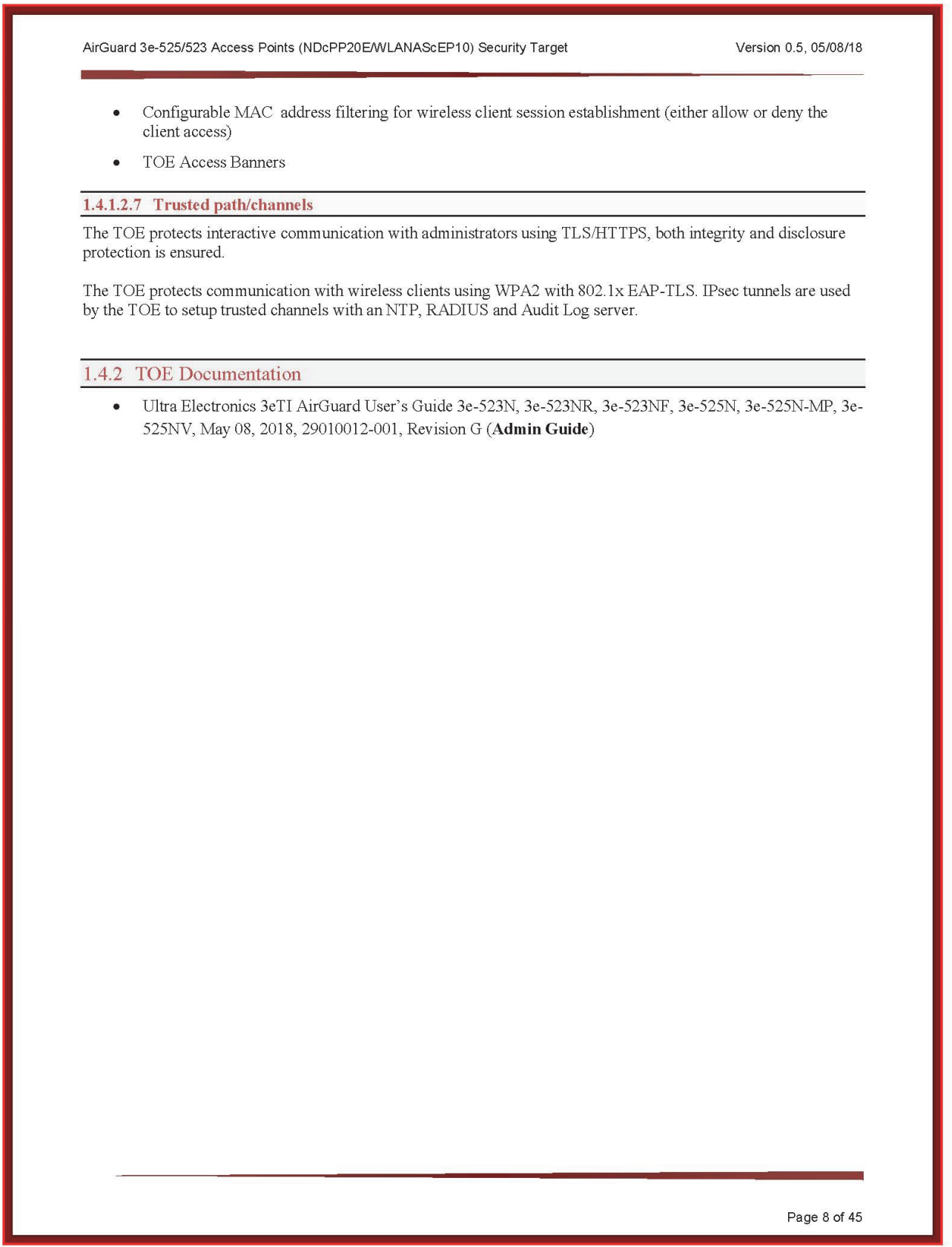




\section{Conformance Claims}

This TOE is conformant to the following CC specifications:

- Common Criteria for Information Technology Security Evaluation Part 2: Security functional components, Version 3.1, Revision 4, September 2012.

- Part 2 Extended

- Common Criteria for Information Technology Security Evaluation Part 3: Security assurance components, Version 3.1 Revision 4, September 2012.

- Part 3 Conformant

- Package Claims:

- collaborative Protection Profile for Network Devices Version 2.0 + Errata 20180314, Version 2.0, 14 March 2018 (NDcPP20E) with technical decisions TD0290 and TD0228.

- Extended Package Wireless Local Area Network (WLAN) Access Systems, Version 1.0, 29 May 2015 (NDcPP20E/WLANAScEP10) with technical decisions TD0282, TD0271, TD0277 and TD0315

\subsection{Conformance Rationale}

The ST conforms to the NDCPP20E/WLANAScEP10. As explained previously, the security problem definition, security objectives, and security requirements have been drawn from the PPs. 


\section{Security Objectives}

The Security Problem Definition may be found in the NDcPP20E/WLANAScEP10 and this section reproduces only the corresponding Security Objectives for operational environment for reader convenience. The NDcPP20E/WLANAScEP10 offers additional information about the identified security objectives, but that has not been reproduced here and the NDCPP20E/WLANAScEP10 should be consulted if there is interest in that material.

In general, the NDcPP20E/WLANAScEP10 has defined Security Objectives appropriate for Network Device and Wireless Access Point and as such are applicable to the $3 \mathrm{e}-525 / 523 \mathrm{TOE}$

3.1 Security Objectives for the Operational Environment

OE.ADMIN_CREDENTIALS_SECURE The administrator's credentials (private key) used to access the TOE must be protected on any other platform on which they reside.

OE.COMPONENTS_RUNNING (applies to distributed TOEs only)

OE.CONNECTIONS TOE administrators will ensure that the TOE is installed in a manner that will allow the TOE to effectively enforce its policies on network traffic flowing among attached networks.

OE.NO GENERAL PURPOSE There are no general-purpose computing capabilities (e.g., compilers or user applications) available on the TOE, other than those services necessary for the operation, administration and support of the TOE

OE.NO_THRU_TRAFFIC_PROTECTION The TOE does not provide any protection of traffic that traverses it. It is assume $\bar{d}$ that protection of this traffic will be covered by other security and assurance measures in the operational environment.

OE.PHYSICAL Physical security, commensurate with the value of the TOE and the data it contains, is provided by the environment.

OE.RESIDUAL INFORMATION The Security Administrator ensures that there is no unauthorized access possible for sensitive residual information (e.g. cryptographic keys, keying material, PINs, passwords etc.) on networking equipment when the equipment is discarded or removed from its operational environment.

OE.TRUSTED_ADMIN TOE Administrators are trusted to follow and apply all guidance documentation in a trusted manner

OE.UPDATES The TOE firmware and software is updated by an administrator on a regular basis in response to the release of product updates due to known vulnerabilities.

T.SECURITY FUNCTIONALITY FAILURE An external, unauthorized entity could make use of failed or compromised security functionality and might therefore subsequently use or abuse security functions without prior authentication to access, change or modify device data, critical network traffic or security functionality of the device. 


\section{Extended Components Definition}

All of the extended requirements in this ST have been drawn from the NDcPP20E/WLANAScEP10. The NDCPP20E/WLANASCEP10 defines the following extended requirements and since they are not redefined in this ST the NDcPP20E/WLANAScEP10 should be consulted for more information in regard to those CC extensions.

\section{Extended SFRs:}

- NDcPP20E:FAU_STG_EXT.1: Protected Audit Event Storage

- NDePP20E:FCS_HTTPS_EXT.1: HTTPS Protocol

- NDcPP20E:FCS_IPSEC_EXT.1: IPsec Protocol

- NDcPP20E:FCS_RBG_EXT.1: Random Bit Generation

- NDcPP20E:FCS_TLSS_EXT.1: TLS Server Protocol

- WLANAScEP10:FIA_8021X_EXT.1: Extended: 802.1X Port Access Entity (Authenticator) Authentication

- NDcPP20E:FIA_PMG_EXT.1: Password Management

- WLANAScEP10:FIA_PSK_EXT.1: Extended: Pre-Shared Key Composition

- NDcPP2OE:FIA_UAU_EXT. 2: Password-based Authentication Mechanism

- NDcPP20E:FIA_UIA_EXT.1: User Identification and Authentication

-NDcPP20E:FIA_X509_EXT.1(2):X.509 Certificate Validation (Rev)

- NDcPP20E:FIA_X509_EXT.2: X.509 Certificate Authentication

-NDcPP20E:FIA_X509_EXT.3: X.509 Certificate Requests

- NDcPP20E:FPT_APW_EXT.1: Prolection of Administralor Passwords

-NDcPP20E:FPT_SKP_EXT.1: Protection of TSF Data (for reading of all pre-shared, symmetric and private keys)

-NDcPP20E:FPT_STM_EXT.1: Reliable Time Stamps

- NDcPP20E:FPT_TST_EXT.1: TSF testing

- WLANAScEP10:FPT_TST_EXT.1: Extended: TSF Testing

- NDcPP20E:FPT_TUD_EXT.1: Trusted update

- NDcPP20E:FTA_SSL_EXT.1: TSF-initiated Session Locking 


\section{Security Requirements}

This section defines the Security Functional Requirements (SFRs) and Security Assurance Requirements (SARs) that serve to represent the security functional claims for the Target of Evaluation (TOE) and to scope the evaluation effort The SFRs have all been drawn from the NDcPP20E/WLANAScEP10. The refinements and operations already performed in the NDcPP20E/WLANAScEP10 are not identified (e.g., highlighted) here, rather the requirements have been copied from the NDcPP20E/WLANAScEP10 and any residual operations have been completed herein. Of particular note, the NDcPP20E/WLANAScEP10 made a number of refinements and completed some of the SFR operations defined in the Common Criteria (CC) and that PP should be consulted to identify those changes if necessary.

The SARs are also drawn from the NDcPP20E/WLANAScEP10 which includes all the SARs for EAL 1. However, the SARs are effectively refined since requirement-specific 'Assurance Activities' are defined in the NDcPP20E/WLANAScEP10 that serve to ensure corresponding evaluations will yield more practical and consistent assurance than the EAL 1 assurance requirements alone. The NDcPP20E/WLANAScEP10 should be consulted for the assurance activity definitions.

\subsection{TOE Security Functional Requirements}

The following table identifies the SFRs that are satisfied by 3e-525/523 TOE.

\begin{tabular}{|c|c|}
\hline Requirement Class & Requirement Component \\
\hline \multirow[t]{3}{*}{ FAU: Security audit } & NDcPP20E:FAU GEN.1: Audit Data Generation \\
\hline & NDcPP20E:FAU GEN.2: User identity association \\
\hline & NDcPP20E:FAU_STG_EXT.1: Protected Audit Event Storage \\
\hline \multirow[t]{15}{*}{ FCS: Cryptographic support } & NDcPP20E:FCS_CKM.1: Cryptographic Key Generation \\
\hline & $\begin{array}{l}\text { WLANAScEP10:FCS CKM.1(2): Cryptographic Key Generation } \\
\text { (Symmetric Keys for WPA2 Connections) }\end{array}$ \\
\hline & NDcPP20E.FCS_CKM.2: Cryptographic Key Establishment \\
\hline & $\begin{array}{l}\text { WLANAScEP10:FCS_CKM.2(2): Cryptographic Key Distribution } \\
\text { (PMK) }\end{array}$ \\
\hline & $\begin{array}{l}\text { WLANAScEP10:FCS_CKM.2(3): Cryptographic Key Distribution } \\
\text { (GTK) }\end{array}$ \\
\hline & NDcPP20E.FCS CKM.4: Cryptographic Key Destruction \\
\hline & $\begin{array}{l}\text { NDcPP20E:FCS_COP.1/DataEncryption: Cryptographic Operation } \\
\text { (AES Data Encryption/Decryption) }\end{array}$ \\
\hline & $\begin{array}{l}\text { WLANAScEP10:FCS_COP.1(1): Cryptographic Operation (AES } \\
\text { Data Encryption/Decryption) }\end{array}$ \\
\hline & $\begin{array}{l}\text { NDcPP20E:FCS_COP.1/SigGen: Cryptographic Operation (Signature } \\
\text { Generation and Verification) }\end{array}$ \\
\hline & $\begin{array}{l}\text { NDcPP20E:FCS_COP.1/Hash: Cryptographic Operation (Hash } \\
\text { Algorithm) }\end{array}$ \\
\hline & $\begin{array}{l}\text { NDcPP20E:FCS_COP.1/KeyedHash: Cryptographic Operation } \\
\text { (Keyed Hash Algorithm) }\end{array}$ \\
\hline & NDCPY2UE:FCS_HI"I'PS EXI:1: H'l'PS'Protocol \\
\hline & NDcPP20E/WLANAScEP10:FCS_IPSEC EXT.1: IPsec Protocol \\
\hline & NDcPP20E:FCS RBG EXT.1: Random Bit Generation \\
\hline & NDCPP20E:FCS_TLSS_EXT.1: TLS Server Protocol \\
\hline \multirow[t]{4}{*}{$\begin{array}{l}\text { FIA: Identification and } \\
\text { authentication }\end{array}$} & $\begin{array}{l}\text { WLANAScEP10:FIA_8021X_EXT.1: Extended: } 802.1 \mathrm{X} \text { Port Access } \\
\text { Entity (Authenticator) Authentication }\end{array}$ \\
\hline & NDcPP20E:FIA AFL.1: Authentication Failure Management \\
\hline & WLANAScEP10:FIA_AFL.1: Authentication Failure Handling \\
\hline & NDcPP20E:FIA PMG EXT.1: Password Management \\
\hline
\end{tabular}




\begin{tabular}{|c|c|}
\hline & $\begin{array}{l}\text { WLANAScEP10:FIA_PSK_EXT.1: Extended: Pre-Shared Key } \\
\text { Composition }\end{array}$ \\
\hline & WLANAScEP10:FIA UAU 6 : Re-authenticating \\
\hline & NDcPP20E:FIA UAU.7: Protected Authentication Feedback \\
\hline & $\begin{array}{l}\text { NDcPP20E:FIA_UAU_EXT.2: Password-based Authentication } \\
\text { Mechanism }\end{array}$ \\
\hline & NDcPP20E:FIA UIA_EXT.1: User Identification and Authentication \\
\hline & $\begin{array}{l}\text { NDcPP20E:FIA_X509_EXT.1/Rev: X.509 Certificate Validation } \\
\text { (Rev) }\end{array}$ \\
\hline & NT):PP20F:FTA X509 FXT 2: X 509 Certificate Authentication \\
\hline & NDcPP20E:FIA_ X509_EXT.3: X.509 Certificate Requests \\
\hline \multirow[t]{5}{*}{ FMT: Security management } & $\begin{array}{l}\text { NDcPP20E:FMT_MOF.1/ManualUpdate: Management of security } \\
\text { functions behaviour (Manual Update) }\end{array}$ \\
\hline & $\begin{array}{l}\text { NDcPP20E:FMT_MTD.1/CoreData: Management of TSF Data } \\
\text { (CoreData) }\end{array}$ \\
\hline & NDcPP20E:FMT_SMF.1: Specification of Management Functions \\
\hline & WLANAScEP10:FMT SMR.1: Security Management Roles \\
\hline & NDcPP20E:FMT SMR 2: Restrictions on Security Roles \\
\hline \multirow[t]{7}{*}{ FPT: Protection of the TSF } & $\begin{array}{l}\text { NDcPP20E:FPT_APW_EXT.1: Protection of Administrator } \\
\text { Passwords }\end{array}$ \\
\hline & WLANAScEP10:FPT_FLS.1: Failure with preservation of secure state \\
\hline & $\begin{array}{l}\text { NDcPP20E:FPT_SKP_EXT.1: Protection of TSF Data (for reading of } \\
\text { all pre-shared, symmetric and private keys) }\end{array}$ \\
\hline & NDcPP20E:FPT_STM_EXT.1:Reliable Time Stamps \\
\hline & NDcPP20E:FPT_TST_EXT.1:TSF testing \\
\hline & WLANAScEP10:FPT TST EXT.1: Extended: TSF Testing \\
\hline & NDePP20E.FPT_TUD_EXT.1. Ti usted updiate \\
\hline \multirow[t]{5}{*}{ FTA: TOE access } & NDcPP20E:FTA_SSL.3: TSF-initiated Termination \\
\hline & NDcPP20E:FTA SSL.4: User-initiated Termination \\
\hline & NDePP20E FTA SSL EXT.1: TSF-initiated Session Locking \\
\hline & NDcPP20E:FTA TAB.1: Default TOE Access Banners \\
\hline & WLANAScEP10:FTA TSE.1: TOE Session Establishment \\
\hline \multirow[t]{3}{*}{ FTP: Trusted path/channels } & NDcPP20E FTP ITC.1: Inter-TSF trusted channel \\
\hline & WLANAScEP10:FTP ITC.1: Inter-TSF Trusted Channel \\
\hline & NDcPP20E:FTP TRP.1/Admin Trusted Path: Trusted Path (Admin) \\
\hline
\end{tabular}

Table 1 TOE Security Functional Components

5.1.1 Security audit (FAU)

5.1.1.1 Audit Data Generation (NDrPP20E:FAU_GFN.1)

NDcPP20E:FAU_GEN.1.1

The TSF shall be able to generate an audit record of the following auditable events:

a) Start-up and shut-down of the audit functions;

b) All auditable events for the not specified level of audit; and

c) All administrative actions comprising:

- Administrative login and logout (name of user account shall be logged if individual user accounts are required for administrators)

- Changes to TSF data related to configuration changes (in addition to the information that a change occurred it shall be logged what has been changed) 
- Generating/import of, changing, or deleting of cryptographic keys (in addition to

the action itself a unique key name or key reference shall be logged).

- Resetting passwords (name of related user account shall be logged).

[no other actions];

d) Specifically defined auditable events listed in Table 2

\begin{tabular}{|c|c|c|}
\hline Requirement & Auditable Events & Additional Content \\
\hline NDCPP20E:FAU_GEN.1 & None & None \\
\hline NDCPP20E:FAU GEN.2 & None & None \\
\hline NDcPP20E:FAU STG EXT.1 & None & None \\
\hline NDcPP20E:FCS CKM.1 & None & None \\
\hline WLANAScEP10:FCS_CKM.1(2) & $\begin{array}{l}\text { Failure of the key generation } \\
\text { activity. }\end{array}$ & None \\
\hline NDcPP20E:FCS CKM.2 & None & None \\
\hline WLANAScEP10:FCS_CKM.2(2) & $\begin{array}{l}\text { Failure of the key distribution } \\
\text { activity. }\end{array}$ & None \\
\hline WLANAScEP10:FCS_CKM.2(3) & $\begin{array}{l}\text { Failure of the key distribution } \\
\text { activity, including failures relate } \\
\text { to wrapping the GTK. }\end{array}$ & $\begin{array}{l}\text { Tdentifier(s) for intended } \\
\text { recipients of wrapped key. }\end{array}$ \\
\hline NDcPP20E:FCS_CKM.4 & None & None \\
\hline NDcPP20E:FCS COP.1/DataEncryption & None & None \\
\hline WLANAScEP10:FCS_COP.1(1) & $\begin{array}{l}\text { Failure of the WPA2 encryption } \\
\text { or decryption. }\end{array}$ & $\begin{array}{l}\text { Cryptographic mode of } \\
\text { operation, name/identifier of } \\
\text { object being } \\
\text { encrypted/decrypted, non- } \\
\text { TOF endpoint of connection } \\
\text { (IP address). }\end{array}$ \\
\hline NDcPP20E:FCS_COP.1/SigGen & None & None \\
\hline NDcPP20E:FCS_COP.1/Hash & None & None \\
\hline NDcPP20E:FCS COP.1/KeyedHash & None & None \\
\hline NDcPP20E:FCS_HTTPS_EXT.1 & $\begin{array}{l}\text { Failure to establish a HTTPS } \\
\text { Session. }\end{array}$ & Reason for failure. \\
\hline NDcPP20E:FCS IPSEC EXT.1 & Failure to establish an IPsec SA. & Reason for failure. \\
\hline WLANAScEP10:FCS_IPSEC_EXT.1 & $\begin{array}{l}\text { Protocol failures. } \\
\text { Establishment/Termination of an } \\
\text { IPsec SA. Negotiation "down } \\
\text { from an IKEv2 to IKEv 1 } \\
\text { exchange. }\end{array}$ & $\begin{array}{l}\text { Reason for failure. Non-TOE } \\
\text { endpoint of connection (IP } \\
\text { address) for both successes } \\
\text { and failures. }\end{array}$ \\
\hline NDcPP20E: FCS_RBG_EXT.1 & None & None \\
\hline NDcPP20E:FCS_TLSS_EXT.1 & $\begin{array}{l}\text { Failure to establish a TLS } \\
\text { Session. }\end{array}$ & Reason for failure. \\
\hline WLANAScEP10:FIA_8021X_EXT.1 & $\begin{array}{l}\text { Attempts to access the } 802.1 \mathrm{X} \\
\text { controlled port prior to successful } \\
\text { completion of the authentication } \\
\text { exchange. }\end{array}$ & $\begin{array}{l}\text { Provided client identity (MAC } \\
\text { address). }\end{array}$ \\
\hline NDcPP20E:FIA_AFL.1 & $\begin{array}{l}\text { Unsuccessful login attempt limit } \\
\text { is met or exceeded. }\end{array}$ & $\begin{array}{l}\text { Origin of the attempt (e.g., IP } \\
\text { address). }\end{array}$ \\
\hline WLANAScEP10:FIA_AFL.1 & $\begin{array}{l}\text { The reaching of the threshold for } \\
\text { the unsuccessful authentication } \\
\text { attempts and the actions taken } \\
\text { (e.g., disabling of an account) } \\
\text { and the subsequent, if } \\
\text { appropriate, restoration to the }\end{array}$ & None \\
\hline
\end{tabular}




\begin{tabular}{|c|c|c|}
\hline & $\begin{array}{l}\text { normal state (e.g., re-enabling of } \\
\text { a terminal). }\end{array}$ & \\
\hline NDcPP20E:FIA_PMG_EXT.1 & None & None \\
\hline WLANAScEP10:FIA PSK EXT.1 & None & None \\
\hline WLANAScEP10:FIA_UAU.6 & Attempts to re-authenticate. & $\begin{array}{l}\text { Origin of the attempt (e.g., IP } \\
\text { address). }\end{array}$ \\
\hline NDcPP20E:FIA_UAU.7 & None & None \\
\hline NDcPP20E:FIA_UAU_EXT.2 & $\begin{array}{l}\text { All use of identification and } \\
\text { authentication mechanism. }\end{array}$ & $\begin{array}{l}\text { Origin of the attempt (e.g., IP } \\
\text { address). }\end{array}$ \\
\hline NDcPP20E:FLA_ITA_EXT.1 & $\begin{array}{l}\text { A11 use of identification and } \\
\text { authentication mechanism. }\end{array}$ & $\begin{array}{l}\text { Origin of the attempt (e g, IP } \\
\text { address). }\end{array}$ \\
\hline NDcPP20E:FIA_X509_EXT.1/Rev & $\begin{array}{l}\text { Unsuccessful attempt to validate } \\
\text { a certificate. }\end{array}$ & Reason for failure. \\
\hline NDcPP20E:FIA_X509_EXT.2 & None & None \\
\hline NDcPP20E: FIA X509 EXT.3 & None & None \\
\hline NDcPP20E:FMT_MOF.1/ManualUpdate & $\begin{array}{l}\text { Any attempt to initiate a manual } \\
\text { update. }\end{array}$ & \\
\hline NDcPP20E:FMT_MTD.1/CoreData & $\begin{array}{l}\text { All management activities of TSF } \\
\text { data. }\end{array}$ & \\
\hline NDcPP20E:FMT SMF.1 & None & None \\
\hline WLANAScEP10:FMT SMR.1 & None & None \\
\hline NDcPP20E:FMT SMR.2 & None & None \\
\hline NDcPP20E:FPT APW EXT.1 & None & None \\
\hline WLANAScEP10:FPT_FLS.1 & Failure of the TSF. & $\begin{array}{l}\text { Indication that the TSF has } \\
\text { failed with the type of failure } \\
\text { that occurred. }\end{array}$ \\
\hline NDcPP20E:FTT_SKT_EXT.1 & None & None \\
\hline NDcPP20E:FPT_STM_EXT.1 & $\begin{array}{l}\text { Discontinuous changes to time - } \\
\text { either Administrator actuated or } \\
\text { changed via an automated } \\
\text { process. (Note that no continuous } \\
\text { changes to time need to be } \\
\text { logged. See also application note } \\
\text { on FPT STM EXT.1) }\end{array}$ & $\begin{array}{l}\text { For discontinuous changes to } \\
\text { time: The old and new values } \\
\text { for the time. Origin of the } \\
\text { attempt to change time for } \\
\text { success and failure (e.g., IP } \\
\text { address). }\end{array}$ \\
\hline NDcPP20E:FPT TST_EXT.1 & None & None \\
\hline WLANAScEP10:FPT_TST_EXT.1 & $\begin{array}{l}\text { Execution of this set of TSF self- } \\
\text { tests. Detected integrity } \\
\text { violations. }\end{array}$ & $\begin{array}{l}\text { For integrity violations, the } \\
\text { TSF code file that caused the } \\
\text { integrity violation. }\end{array}$ \\
\hline NDcPP20E:FPT_TUD_EXT.1 & $\begin{array}{l}\text { Initiation of update; result of the } \\
\text { update attempt (success or } \\
\text { failure). }\end{array}$ & None \\
\hline NDcPP20E:FTA_SSL.3 & $\begin{array}{l}\text { The termination of a remote } \\
\text { session by the session locking } \\
\text { mechanism. }\end{array}$ & None \\
\hline NDcPP20E:FTA_SSL.4 & $\begin{array}{l}\text { The termination of an interactive } \\
\text { session. }\end{array}$ & None \\
\hline NDcPP20E:FTA_SSL_EXT.1 & $\begin{array}{l}\text { (if 'lock the session' is selected) } \\
\text { Any attempts at unlocking of an } \\
\text { interactive session. (if 'terminate } \\
\text { the session' is selected) The } \\
\text { termination of a local session by } \\
\text { the session locking mechanism. }\end{array}$ & None \\
\hline NDcPP20E:FTA_TAB.1 & None & None \\
\hline
\end{tabular}




\begin{tabular}{|l|l|l|}
\hline WLANAScEP10:FTA_TSE.1 & $\begin{array}{l}\text { Denial of a session establishment } \\
\text { due to the session establishment } \\
\text { mechanism. }\end{array}$ & $\begin{array}{l}\text { Reason for denial, origin of } \\
\text { establishment attempt. }\end{array}$ \\
\hline NDcPP20E:FTP_ITC.1 & $\begin{array}{l}\text { Initiation of the trusted channel. } \\
\text { Termination of the trusted } \\
\text { channel. Failure of the trusted } \\
\text { channel functions. }\end{array}$ & $\begin{array}{l}\text { Identification of the initiator } \\
\text { and target of failed trusted } \\
\text { channels establishment } \\
\text { attempt. }\end{array}$ \\
\hline WLANAScEP10:FTP_ITC.1 & $\begin{array}{l}\text { Failed attempts to establish a } \\
\text { trusted channel (including IEEE } \\
802.11) . \text { Detection of } \\
\text { modification of channel data. }\end{array}$ & $\begin{array}{l}\text { Identification of the initiator } \\
\text { and target of channel. }\end{array}$ \\
\hline NDcPP20E:FTP_TRP.1/Admin & $\begin{array}{l}\text { Initiation of the trusted path. } \\
\text { Termination of the trusted path. } \\
\text { Failure of the trusted path } \\
\text { functions. }\end{array}$ & \\
\hline
\end{tabular}

Table 2 Auditable Events

NDcPP20E:FAU_GEN.1.2

The TSF shall record within each audit record at least the following information:

a) Date and time of the event, type of event, subject identity, and the outcome (success or failure)

of the event; and

b) For each audit event type, based on the auditable event definitions of the functional components included in the cPP/ST, information specified in column three of Table 2.

\subsubsection{User identity association (NDcPP20E:FAU_GEN.2)}

NDcPP20E:FAU GEN.2.1

For audit events resulting from actions of identified users, the TSF shall be able to associate each auditable event with the identity of the user that caused the event.

\subsubsection{Protected Audit Event Storage (NDcPP20E:FAU_STG_EXT.1)}

NDcPP20E:FAU STG EXT.1.1

$\bar{T}$ The TSF shall be able to transmit the generated audit data to an external IT entity using a trusted channel according to FTP_ITC.1.

NDcPP20E:FAU_STG_EXT.1.2

The TSF shall be able to store generated audit data on the TOE itself

NDcPP20E:FAU STG_EXT.1.3

$\bar{T}$ The TSF shall [overwrite previous audit records according to the following rule: / when allotted space has reached its threshold]] when the local storage space for audit data is full.

5.1 .2 Cryptographic support (FCS)

5.1.2.1 Cryptographic Key Generation (NDcPP20E:FCS_CKM.1)

NDcPP20E:FCS_CKM.1.1

The TSF shall generate asymmetric cryptographic keys in accordance with a specified cryptographic key generation algorithm: [

- RSA schemes using cryptographic key sizes of 2048-bit or greater that meet the following FIPS PUB 186-4, 'Digital Signature Standard (DSS)', Appen dix B.3,

- ECC schemes using 'NIST curves' $[P-256, P-384, P-521]$ that meet the following: FIPS PUB 186-4, 'Digital Signature Stan dard (DSS)', Appendix B.4, 


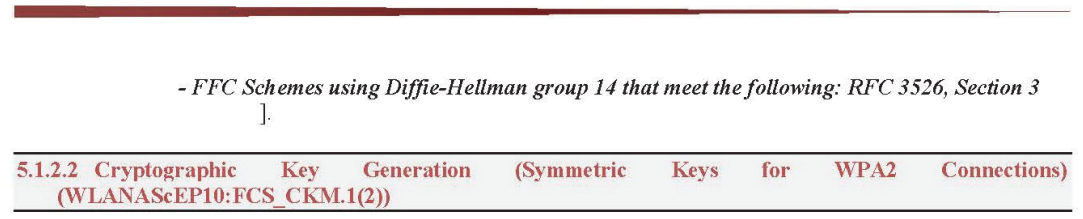

\section{WLANASCEP10:FCS CKM 1(2)}

Refinement: The TSF shall generate symmetric cryptographic keys in accordance with a specified cryptographic key generation algorithm PRF-384 and [no other] and specified cryptographic key sizes 128 bits and [no other key sizes] using a Random Bit Generator as specified in

FCS_RBG_EXT.1 that meet the following: IEEE 802.11-2012 and [no other standards].

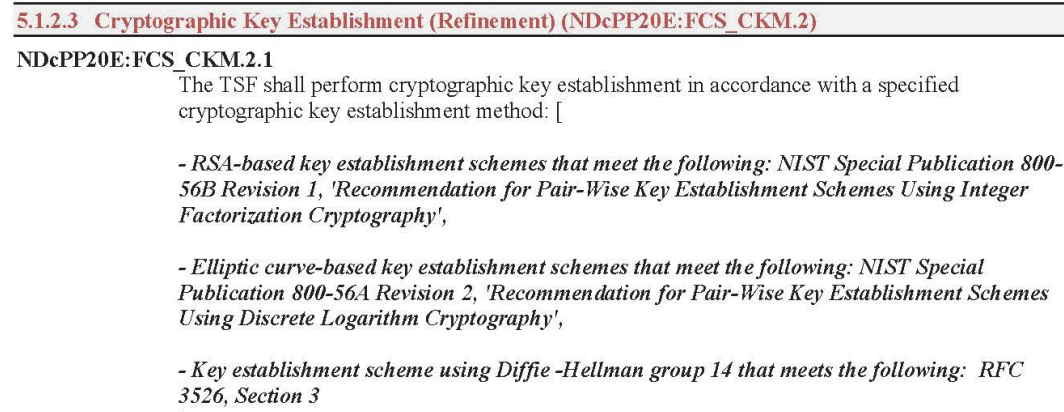

The TSF shall perform cryptographic key establishment in accordance with a specified cryptographic key establishment method: [

- RSA-based key establishment schemes that meet the following: NIST Special Publication 800$56 B$ Revision 1, Recommendation for Pair-Wise Key Establishment Schemes Using Integer Factorization Cryptography',

- Elliptic curve-based key establishment schemes that meet the following: NIST Special Publication 800-564 Revision 2, 'Recommendation for Pair-Wise Key Establishment Schemes Using Discrete Logarithm Cryptography',

- Key establishment scheme using Diffie-Hellman group 14 that meets the following: $R F C$ 3526 , Section 3

\subsubsection{Cryptographic Key Distribution (PMK) (WLANAScEP10:FCS_CKM.2(2))}

WLANAScEP10:FCS_CKM.2(2).1

Refinement: The TSF shall receive the 802.11 Pairwise Master Key (PMK) in accordance with a specified cryptographic key distribution method: from $802.1 \mathrm{X}$ Authorization Server that meets the following: IEEE 802.11-2012 and does not expose the cryptographic keys.

\subsubsection{Cryptographic Key Distribution (GTK) (WLANAScEP10:FCS_CKM.2(3))}

WLANAScEP10:FCS_CKM.2(3).1

Refinement: The TSF shall distribute Group Temporal Key (GTK) in accordance with a specified cryptographic key distribution method: AES Key Wrap in an EAPOL-Key frame that meets the following: NIST SP 800-38F, IEEE 802.11-2012 for the packet format and timing considerations and does not expose the cryptographic keys.

5.1.2.6 Cryptographic Key Destruction (NDcPP20E:FCS_CKM.4)

\section{NDcPP20E:FCS_CKM.4.1}

The TSF shall destroy cryptographic keys in accordance with a specified cryptographic key destruction method:

- For plaintext keys in volatile storage, the destruction shall be executed by a [single overwrite consisting of [zeroes]; 
- For plaintext keys in non-volatile storage, the destruction shall be executed by the invocation of an interface provided by a part of the TSF that I

o logically addresses the storage location of the key and performs a [single-pass] overwrite consisting of [a new value of the key]]

that meets the following: No Standard.

\begin{tabular}{l}
\hline 5.1.2.7 Cryptographic $\begin{array}{c}\text { Operation } \\
\text { (NDcPP20E:FCS_COP.1/DataEncryption)) }\end{array}$ \\
NDcPP20E:FCS_COP.1.1/DataEncryption \\
The TSF shall perform encryption/decryption in accordance with a specified cryptographic \\
algorithm AES used in $[C B C, G C M]$ mode and cryptographic key sizes $[128$ bits, 256 bits $]$ that \\
meet the following: AES as specified in ISO 18033-3, [CBC as specified in ISO 10116, GCM as \\
specified in ISO 19772].
\end{tabular}

5.1.2.8 Cryptographic Operation (AES Data Encryption/Decryption) (WLANAScEP10:FCS_COP.1(1))

WLANAScEP10:FCS COP.1(1).1

Refinement: The TSF shall perform encryption/decryption in accordance with a specified

cryptographic algorithm AES used in CBC, CCMP [GCM] mode and cryptographic key sizes 128

bits [256 bits] that meet the following: AES as specified in ISO 18033-3, CCMP as defined in

NIST SP 800-38C and IEEE 802.11-2012, [CBC as specified in ISO 10116, GCM as specified in

ISO 19772, CCMP as specified in NIST SP800-38D].

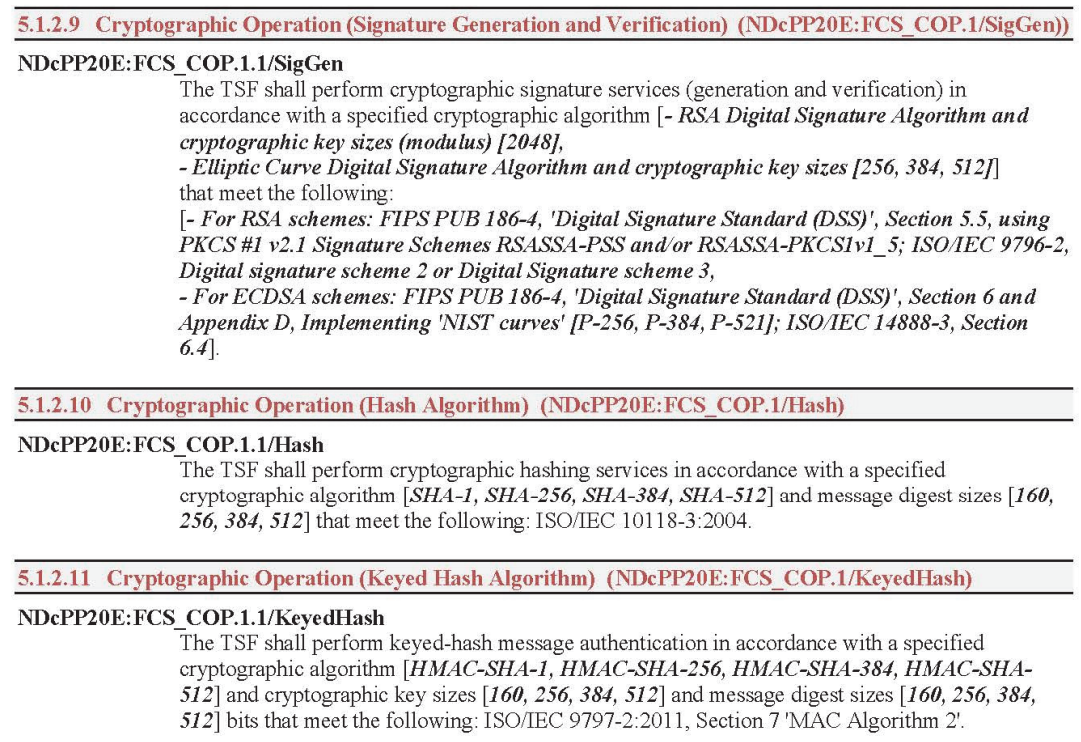




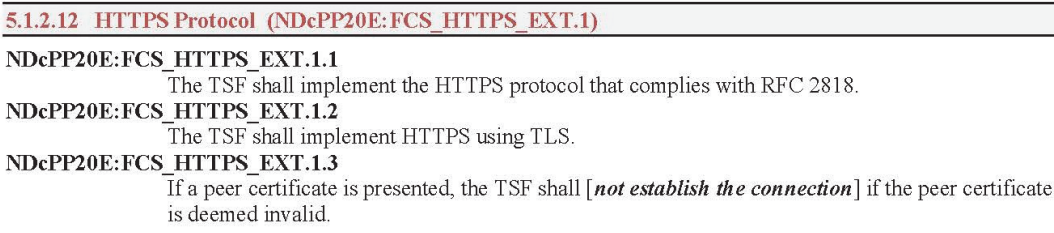

NDcPP20E/ WLANAScEP10:FCS IPSEC EXT.1.5

The TSF shall implement the protocol: [-IKEv2 as defined in RFC 5996 and [with mandatory support for NAT traversal as specified in RFC 5996, section 2.23], and [no other RFCs for hash functions]].

NDcPP20E/WLANAScEP10:FCS_IPSEC_EXT.1.6

The TSF shall ensure the encrypted payload in the $[I K E v 2]$ protocol uses the cryptographic algorithms [AES-CBC-128, AES-CBC-256 (specified in RFC 3602)].

NDcPP20E/WLANAScEP10:FCS_IPSEC_EXT.1.7

The TSF shall ensure that [-IKEv2 SA lifetimes can be configured by a Security Administrator based on [length of time, where the time values can be configured within [1-24] hours]].

NDcPP20E/WLANAScEP10:FCS_IPSEC_EXT.1.8

The TSF shall ensure that [-IKEv2 Child SA lifetimes can be configured by a Security Administrator based on [number of bytes, length of time, where the time values can be configured within [1-8] hours]].

NDcPP20E/WLANAScEP10:FCS_IPSEC_EXT.1.9

The TSF shall generate the secret value $\mathrm{x}$ used in the IKE Diffie-Hellman key exchange (' $\mathrm{x}$ ' in $\mathrm{g}^{\wedge} \mathrm{x}$ mod p) using the random bit generator specified in FCS RBG EXT.1, and having a length of at least $[112,128,192]$ bits.

NDcPP20E/ WLANAScEP10:FCS_IPSEC_EXT.1.10

The TSF shall generate nonces used in [IKEv2] exchanges of length [according to the security strength associated with the negotiated Diffie-Hellman group].

NDcPP20E/WLANAScEP10:FCS IPSEC EXT.1.11

The TSF shall ensure that all IKE protocols implement DH Group(s) [14 (2048-bit MODP), 19 (256-bit Random ECP), 20 (384-bit Random ECP)].

NDcPP20E/ WLANAScEP10:FCS_IPSEC_EXT.1.12

The TSF shall be able to ensure by default that the strength of the symmetric algorithm (in terms of the number of bits in the key) negotiated to protect the $[I K E v 2 I K E S A]$ connection is greater than or equal to the strength of the symmetric algorithm (in terms of the number of bits in the key) negotiated to protect the [IKEv2 CHILD_SA] connection.

NDcPP20E/ WLANAScEP10:FCS_IPSEC_EXT.1.13

The TSF shall ensure that all IKE protocols perform peer authentication using [RSA, ECDSA] that use X.509v3 certificates that conform to RFC 4945 and [Pre-shared Keys]. 
NDcPP20E/WLANAScEP10:FCS IPSEC EXT.1.14

The TSF shall only establish a trusted channel if the presented identifier in the received certificate matches the configured reference identifier, where the presented and reference identifiers are of the following types: [Distinguished Name (DN), IP Address, Fully Qualified Domain Name $(F Q D N)]$ and [ $n o$ other reference identifier type].

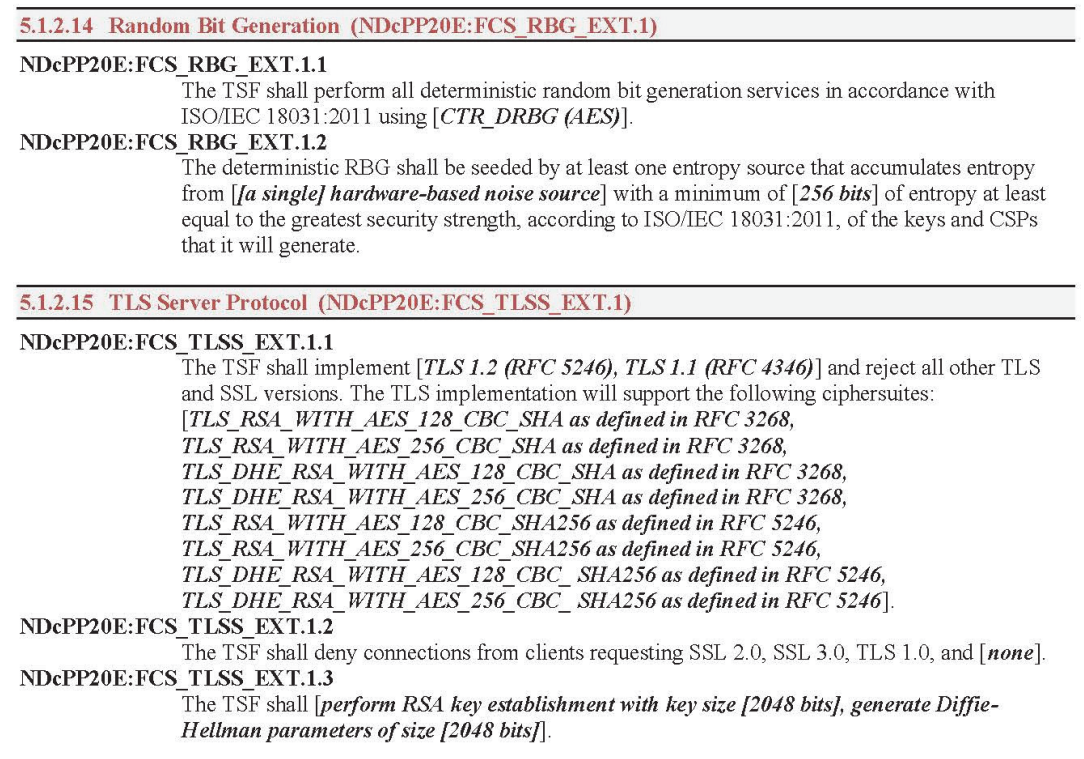

\begin{tabular}{l}
\hline 5.1.3 Identification and authentication (FIA) \\
$\begin{array}{l}\text { 5.1.3.1 Extended: 802.1X Port Access Entity (Authenticator) Authentication } \\
\text { (WLANAScEP10:FIA_8021X_EXT.1) }\end{array}$
\end{tabular}

WLANAScEP10:FIA 8021X_EXT.1.1

The TSF shall conform to IEEE Standard $802.1 \mathrm{X}$ for a Port Access Entity (PAE) in the 'Authenticator' role.

WLANAScEP10:FLA_8021X_EXT.1.2

The TSF shall support communications to a RADIUS authentication server conforming to RFCs 2865 and 3579.

WLANAScEP10:FIA_8021X_EXT.1.3

The TSF shall ensure that no access to its $802.1 \mathrm{X}$ controlled port is given to the wireless client prior to successful completion of this authentication exchange.

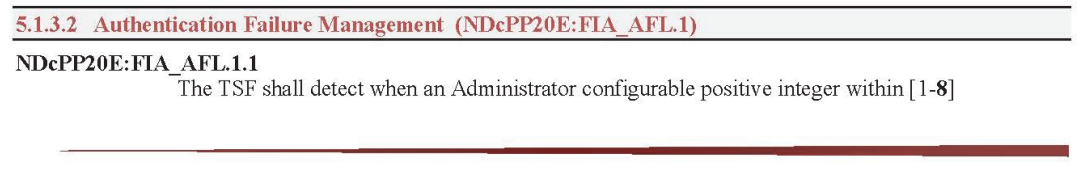


unsuccessful authentication attempts occur related to Administrators attempting to authenticate remotely.

NDcPP20E:FIA_AFL.1.2

When the defined number of unsuccessful authentication attempts has been met, the TSF shall [prevent the offending remote Administrator from successfully authenticating until an

Administrator defined time period has elapsed].

5.1.3.3 Authentication Failure Handling (WLANAScEP10:FIA_AFL.1)

WLANAScEP10:FIA_AFL.1.1

Refinement: The TSF shall detect when an Administrator configurable positive integer of successive unsuccessful authentication attempts occur related to administrators attempting to

WLANAScEP10: FIA AFL.1.2 authenticate remotely.

When the defined number of unsuccessful authentication attempts has been met, the TSF shall [prevent the offending remote administrator from successfully authenticating until an Administrator defined time period has elapsed].

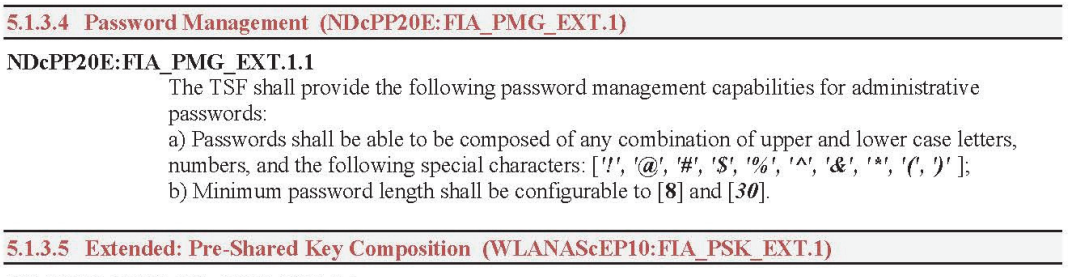

WLANAScEP10: FLA_PSK_EXT.1.1

The TSF shall be able to use pre-shared keys for IPsec and [IEEE 802.11 WPA2-PSK]

(TD0277 applied) 0:FTA PSK EXT.1.2

The TSF shall be able to accept text-based pre-shared keys that:

- are 22 characters and [lengths from 8 to 63 characters for WPA2; and lengths from 16 to 32 characters for IPsec];

- are composed of any combination of upper and lower case letters, numbers, and special characters (that include: '!', '@', '"', '\$', '\%', 'N', '\&', '*', '(', and ')').

WLANASCEP10:FLA PSK EXT.1.3

The TSF shall be able to [accept $]$ bit-based pre-shared keys
:FIA_PSK_EXT.1.3

\subsubsection{Re-authenticating (WLANAScEP10:FIA_UAU.6)}

WLANAScEP10:FTA UAU.6.1

The TSF shall re-authenticate the administrative user under the conditions: when the user changes their password, [following a TSF initiated session locking].

\footnotetext{
5.1.3.7 Protected Authentication Feedback (NDcPP20E:FIA_UAU.7)

NDcPP20E:FIA UAU.7.1

The TSF shall provide only obscured feedback to the administrative user while the authentication is in progress at the local console.
} 
5.1.3.8 Password-based Authentication Mechanism (NDcPP20E:HIA_UAU_EXT.2) NDePP20E:FIA UAU EXT.2.1

The TSF shall provide a local password-based authentication mechanism, and [no other authentication mechanism] to perform local administrative user authentication.

5.1.3.9 User Identification and Authentication (NDcPP20E: FTA UTA EXT.1)

NDcPP20E:FIA_UIA_EXT.1.1

The TSF shall allow the following actions prior to requiring the non-TOE entity to initiate the identification and authentication process:

- Display the warning banner in accordance with FTA_TAB.1;

- [network packets configured by an authorized administrator may flow through the TOE].

NDcPP20E:FIA_UIA_EXT.1.2

The TSF shall require each administrative user to be successfully identified and authenticated before allowing any other TSF-mediated actions on behalf of that administrative user.

5.1.3.10 X.509 Certificate Validation (Rev) (NDePP20E:FIA X509 EXT.1/Rev)

NDcPP20E:FIA_X509_EXT.1.1/Rev

The TS F shall validate certificates in accordance with the following rules:

- RFC 5280 certificate validation and certificate path validation supporting a minimum path length of three certificates.

- The certificate path must terminate with a trusted CA certificate.

- The TSF shall validate a certificate path by ensuring the presence of the basic Constraints extension and that the CA flag is set to TRUE for all CA certificates.

- The TSF shall validate the revocation status of the certificate using [a Certificate Revocation List (CRL) as specified in RFC 5280 Section 6.3]

- The TSF shall validate the extendedKeyUsage field according to the following rules:

o Certificates used for trusted updates and executable code integrity verification shall have the Code Signing purpose (id-kp 3 with OID $1 \cdot 3.6 .1 .5 .5 .7 .3 .3)$ in the

extendedKeyUsage field.

o Server certificates presented for TLS shall have the Server Authentication purpose

(id-kp 1 with OID 1.3.6.1.5.5.7.3.1) in the extendedKeyUsage field.

o Client certificates presented for TLS shall have the Client Authentication purpose (id-kp 2 with OID 1.3.6.1.5.5.7.3.2) in the extendedKeyUsage field

o OCSP certificates presented for OCSP responses shall have the OCSP Signing purpose NDcPP20E:FIA X509 EXT.1.2/REV

(id-kp 9 with OID 1.3.6.1.5.5.7.3.9) in the extendedKeyUsage field.

The TSFF shall only treat a certificate as a CA certificate if the basicConstraints extension is present and the CA flag is set to TRUE.

5.1.3.11 X.509 Certificate Authentication (NDcPP20E:FIA_X509_EXT.2)

NDcPP20E:FIA X509 EXT.2.1

The TSF shall use X.509v3 certificates as defined by RFC 5280 to support authentication for [HTTPS, IPsec, TLS], and [no additional uses]

NDcPP20E:FTA_X509_EXT.2.2

When the TSF cannot establish a connection to determine the validity of a certificate, the TSF shall [accept the certificate].

5.1.3.12 X.509 Certificate Requests (NDcPP20E:FIA_X509_EXT.3)

NDcPP20E:FIA_X509_EXT.3.1

The TSF shall generate a Certificate Request Message as specified by RFC 2986 and be able to 
provide the following information in the request: public key and [Common Name, Organization, Country].

NDcPP20E:FIA_X509_EXT.3.2

The TSF shall validate the chain of certificates from the Root CA upon receiving the CA Certificate Response.

5.1.4 Security management (FMT)

5.1.4.1 Management of security functions behaviour (Manual Update) (NDcPP20E:FMT_MOF.1/Manual Update)

NDcPP20E:FMT_MOF.1.1/ManualUpdate

The TSF shall restrict the ability to enable the functions to perform manual update to Security Administrators.

5.1.4.2 Management of TSF Data (CoreData) (NDcPP20E:FMT_MTD.1/CoreData))

NDcPP20E:FMT MTD.1.1/CoreData

The TSF shall restrict the ability to manage the TSF data to Security Administrators.

5.1.4.3 Specification of Management Functions (NDcPP20E:FMT SMF.1)

NDcPP20E:FMT_SMF.1.1

The TSF shall be capable of performing the following management functions:

- Ability to administer the TOE locally and remotely;

- Ability to configure the access banner;

- Ability to configure the session inactivity time before session termination or locking;

- Ability to update the TOE, and to verify the updates using [digital signature] capability prior to installing those updates;

- Ability to configure the authentication failure parameters for FIA AFL.1;

- Ability to configure audit behavior.

Ability to configure lifetime for IPsec SAs

Ability to configure the cryptographic functionality,

Ability to set the time which is used for time-stamps;

Ability to configure the reference identifier for the peer;].

5.1.4.4 Security Management Roles (WLANAScEP10:FMT_SMR.1)

WLANASCEP10:FMT SMR.1.1

The TS $\bar{S}$ shall ensure that the ability to remotely administer the TOE from a wireless client shall be disabled by default.

\subsubsection{Restrictions on Security Roles (NDcPP20E:FMT_SMR.2)}

NDCPP20E:FMT SMR.2.1

The TSF shall maintain the roles: - Security Administrator.

NDcPP20E:FMT_SMR.2.2

The TSF shall be able to associate users with roles

NDcPP20E:FMT SMR.2.3

The TSF shall ensure that the conditions

- The Security Administrator role shall be able to administer the TOE locally;

- The Security Administrator role shall be able to administer the TOE remotely

are satisfied. 
5.1.5 Protection of the TSF (FPT)

5.1.5.1 Protection of Administrator Passwords (NDcPP20E:FPT_APW EXT.1)

NDCPP20E:FPT_APW_EXT.1.1

The TSF shall store passwords in non-plaintext form.

NDcPP20E:FPT_APW_EXT.1.2

The TSF shall prevent the reading of plaintext passwords

5.1.5.2 Failure with preservation of secure state (WLANAScEP10:FPT_FLS.1)

WLANAScEP10:FPT_FLS.1.1

The TSF shall preserve a secure state when the following types of failures occur: failure of the power-on self-tests.

5.1.5.3 Protection of TSF Data (for reading of all pre-shared, symmetric and private keys) (NDcPP20E:FPT_SKP_EXT.1)

NDcPP20E:FPT_SKP_EXT.1.1

The TSF shall prevent reading of all pre-shared keys, symmetric keys, and private keys.

\begin{tabular}{l}
\hline 5.1.5.4 Reliable Time Stamps (NDcPP20E:FPT_STM_EXT.1) \\
\hline NDcPP20E:FPT_STM_EXT.1.1 \\
The TSF shall be able to provide reliable time stamps for its own use. \\
NDcPP20E:FPT_STM_EXT.1.2 \\
The TSEF shall [allow the Security Administrator to set the time, synchronise time with external \\
time sources].
\end{tabular}

5.1.5.5 TSF testing (NDcPP20E:FPT_TST_EXT.1)

NDcPP20E:FPT_TST_EXT.1.1

The TSF shall run a suite of the following self-tests [during initial start-up (on power on), at the request of the authorized user] to demonstrate the correct operation of the TSF: [Software integrity test and AES, SHA, HMAC, DRBG, RSA, and ECDSA Known Answer Tests].

5.1.5.6 Extended: TSF Testing (WLANAScEP10:FPT TST EXT.1)

WLANAScEP10:FPT_TST_EXT.1.1

The TSF shall run a suite of the following self-tests during initial start-up (on power on) and [at the request of the authorized user] to demonstrate the correct operation of the TSF: [Software integrity test and AES, SHA, HMAC, DRBG, RSA, and ECDSA Known Answer Tests]

WLANAScEP10:FPT TST EXT.1.2

The TSF shall provide the capability to verify the integrity of stored TSF executable code when it is loaded for execution through the use of the TSF-provided cryptographic service specified in FCS COP.1(2).

5.1.5.7 Trusted update (NDcPP20E:HPT_TUD_EXT.1)

NDcPP20E:FPT_TUD_EXT.1.1

The $\mathrm{T} \overline{\mathrm{S}} \mathrm{F}$ shall provide Security Administrators the ability to query the currently executing version of the TOE firmware/software and [no other TOE firmware/software version].

NDcPP20E:FPT TUD EXT.1.2

The TSF shall provide Security Administrators the ability to manually initiate updates to TOE firmware/software and [no other update mechanism]. 
NDcPP20E:FPT TUD EXT.1.3

The TSE shall provide means to authenticate firmware/software updates to the TOE using a [digital signature mechanism] prior to installing those updates.

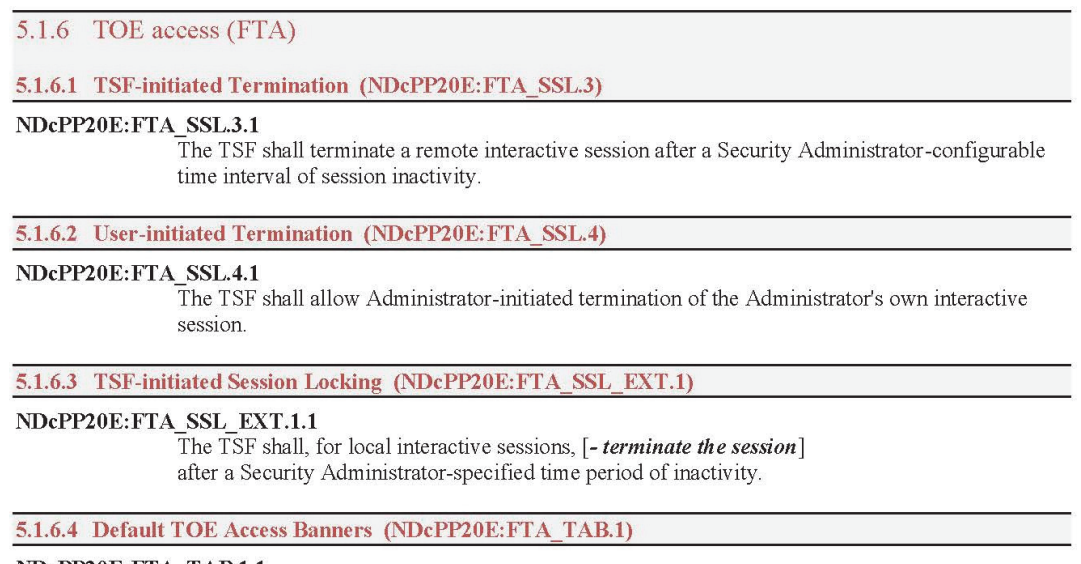

\section{NDcPP20E:FTA TAB.1.1}

Before establishing an administrative user session the TSF shall display a Security Administratorspecified advisory notice and consent warning message regarding use of the TOE.

\subsubsection{TOE Session Establishment (WLANAScEP10:FTA_TSE.1)}

\section{WLANAScEP10:FTA TSE.1.1}

Refinement: The TSF shall be able to deny establishment of a wireless client session based on TOE interface, time, day, [wireless client's MAC address].

5.1.7 Trusted path/channels (FTP)

5.1.7.1 Inter-TSF trusted channel (NDcPP20E:FTP_ITC.1)

NDcPP20E:FTP_ITC.1.1

The TSF shall be capable of using [IPsec] to provide a trusted communication channel between itself and authorized IT entities supporting the following capabilities: audit server, [INTP server]] that is logically distinct from other communication channels and provides assured identification of its end points and protection of the channel data from disclosure and detection of modification of the channel data.

NDcPP20E:FTP_ITC.1.2

The TSF shall permit the TSF or the authorized IT entities to initiate communication via the

NDcPP20E:FTP ITC.1.3 trusted channel.

The TSF shall initiate communication via the trusted channel for [audit logging, accessing an NTP server]. 
5.1.7.2 Inter-TSF Trusted Channel (WLANAScEP10:FTP_ITC.1)

WLANAScEP10:FTP ITC.1.1

Refinement: The TSF shall be capable of using IEEE 802.11-2012 (WPA2), IEEE 802.1X, [IPsec]

and [no other protocol] to provide a trusted communication channel between itself and authorized

IT entities supporting the following capabilities: WLAN clients, audit servers, $802.1 \mathrm{X}$

authentication servers, and [NTP server] that is logically distinct from other communication

channels and provides assured identification of its end points and protection of the channel data

from disclosure and detection of modification of the channel data. (TD0271 applied)

5.1.7.3 Trusted Path (Admin) (NDcPP20E:FTP TRP.1/Admin Trusted Path (Refinement))

NDcPP20E:FTP_TRP.1.1/Admin

The TSF shall be capable of using [TLS, HTTPS] to provide a communication path between itself and authorized remote Administrators that is logically distinct from other communication paths and provides assured identification of its end points and protection of the communicated data from disclosure and provides detection of modification of the channel data.

NDcPP20E:FTP_TRP.1.2/Admin

The TSF shall permit remote Administrators to initiate communication via the trusted path.

NDcPP20E:FTP TRP.1.3/Admin

The TSF shall require the use of the trusted path for initial Administrator authentication and all remote administration actions.

\subsection{TOE Security Assurance Requirements}

The SARs for the TOE are the components as specified in Part 3 of the Common Criteria. Note that the SARs have effectively been refined with the assurance activities explicitly defined in association with both the SFRs and SARs.

\begin{tabular}{|l|l|}
\hline \multicolumn{1}{|c|}{ Requirement Class } & \multicolumn{1}{c|}{ Requirement Component } \\
\hline ADV: Development & ADV FSP.1: Basic Functional Specification \\
\hline AGD: Guidance documents & AGD OPE.1: Operational User Guidance \\
\hline & AGD PRE.1: Preparative Procedures \\
\hline ALC: Life-cycle support & ALC CMC.1: Labelling of the TOE \\
\hline & ALC CMS.1: TOE CM Coverage \\
\hline ASE: Security Target & ASE TSS.1: Security Target \\
\hline ATE: Tests & ATE IND.1: Independent Testing - Conformance \\
\hline AVA: Vulnerability assessment & AVA_VAN.1: Vulnerability Survey \\
\hline
\end{tabular}

Table 3 Assurance Components

5.2.1 Development (ADV)

5.2.1.1 Basic Functional Specification (ADV_FSP.1)

ADV FSP.1.1d

ADV_FSP.1.2d

ADV_FSP.1.1c

The developer shall provide a functional specification

The developer shall provide a tracing from the functional specification to the SFRs.

The functional specification shall describe the purpose and method of use for each SFR-enforcing and SFR-supporting TSFI 
ADV_FSP.1.2c

The functional specification shall identify all parameters associated with each SFR-enforcing and ADV_FSP.1.3c SFR-supporting TSFI.

ADV_FSP.1.4c

The functional specification shall provide rationale for the implicit categorization of interfaces as SFR-non-interfering.

ADV_FSP.1.1e

The tracing shall demonstrate that the SFRs trace to TSFIs in the functional specification.

ADV FSP.1.2e

The evaluator shall confirm that the information provided meets all requirements for content and presentation of evidence.

The evaluator shall determine that the functional specification is an accurate and complete instantiation of the SFRs.

5.2.2 Guidance documents (AGD)

5.2.2.1 Operational User Guidance (AGD OPE.1)

AGD_OPE.1.1d

AGD_OPE.1.1c

The developer shall provide operational user guidance.

The operational user guidance shall describe, for each user role, the user accessible functions and privileges that should be controlled in a secure processing environment, including appropriate

AGD_OPE.1.2c warnings.

$-$

The operational user guidance shall describe, for each user role, how to use the available interfaces provided by the TOE in a secure manner.

The operational user guidance shall describe, for each user role, the available functions and interfaces, in particular all security parameters under the control of the user, indicating secure

AGD_OPE.1.4c values as appropriate.

The operational user guidance shall, for each user role, clearly present each type of securityrelevant event relative to the user-accessible functions that need to be performed, including

AGD_OPE.1.5c changing the security characteristics of entities under the control of the TSF.

The operational user guidance shall identify all possible modes of operation of the TOE (including operation following failure or operational error), their consequences, and implications for

AGD_OPE.1.6c maintaining secure operation.

AGD_OPE.1.7c

The operational user guidance shall, for each user role, describe the security measures to be followed in order to fulfill the security objectives for the operational environment as described in the ST.

AGD_OPE.1.1e

The operational user guidance shall be clear and reasonable

The evaluator shall confirm that the information provided meets all requirements for content and presentation of evidence.

5.2.2.2 Preparative Procedures (AGD PRE.1)

AGD_PRE.1.1d

The developer shall provide the TOE, including its preparative procedures 
AGD_PRE.1.1c

AGD_PRE.1.2c

The preparative procedures shall describe all the steps necessary for secure acceptance of the delivered TOE in accordance with the developer's delivery procedures.

The preparative procedures shall describe all the steps necessary for secure installation of the TOE and for the secure preparation of the operational environment in accordance with the security

AGD_PRE.1.1e objectives for the operational environment as described in the ST.

AGD PRE.1.2e

The evaluator shall confirm that the information provided meets all requirements for content and presentation of evidence.

The evaluator shall apply the preparative procedures to confirm that the TOE can be prepared securely for operation.

5.2.3 Life-cycle support (ALC)

5.2.3.1 Labelling of the TOE (ALC_CMC.1)

ALC_CMC.1.1d

ALC_CMC.1.1c

The developer shall provide the TOE and a reference for the TOE.

ALC_CMC.1.1e

The TOE shall be labelled with its unique reference.

The evaluator shall confirm that the information provided meets all requirements for content and presentation of evidence.

\subsubsection{TOE CM Coverage (ALC_CMS.1)}

ALC_CMS.1.1d

ALC_CMS.1.1c

The developer shall provide a configuration list for the TOE.

ALC_CMS.1.2c

The configuration list shall include the following: the TOE itself; and the evaluation evidence

ALC_CMS.1.1e required by the SARs.

The evaluator shall confirm that the information provided meets all requirements for content and presentation of evidence.

5.2.4 Security Target (ASE)

5.2.4.1 Security Target (ASE_TSS.1)

ASE_TSS.1.1c

For distributed TOEs only the SFRs classified as 'all' have to be fulfilled by all TOE parts. The SFRs classified as 'One' or 'Feature Dependent' only have to be fulfilled by either one or some TOE parts, respectively. To make sure that the distributed TOE as a whole fulfills all the SFRs the following actions for ASE_TSS.1 have to be performed as part of ASE_TSS.1.1E.

The evaluator shall examine the TSS to determine that it is clear which TOE components

contribute to each SFR or how the components combine to meet each SFR.

The evaluator shall verify the sufficiency to fulfil the related SFRs. This includes checking that the

TOE as a whole fully covers all SFRs and that all functionality that is required to be audited is in

fact audited regardless of the component that carries it out. 
AirGuard 3e-525/523 Access Points (NDcPP20ENLANAScEP10) Security Target

Version $0.5,05 / 08 / 18$

5.2 .5 Tests (ATE)

5.2.5.1 Independent Testing Conformance (ATE_IND.1)

ATE_IND.1.1d

The developer shall provide the TOE for testing.

ATE_IND.1.1c

ATE_IND.1.1e

The TOE shall be suitable for testing.

presentation of evidence.

ATE_IND.1.2e

The evaluator shall test a subset of the TSF to confirm that the TSF operates as specified.

5.2.6 Vulnerability assessment (AVA)

5.2.6.1 Vulnerability Survey (AVA_VAN.1)

AVA_VAN.1.1d

AVA_VAN.1.1c

The developer shall provide the TOE for testing.

AVA_VAN.1.1e The evaluator shall confirm that the information provided meets all requirements for content and

The TOE shall be suitable for testing AVA_VAN.1.2e The evaluator shall confirm that the information provided meets all requirements for content and
presentation of evidence. AVA_VAN.1.3e

The evaluator shall perform a search of public domain sources to identify potential vulnerabilities in the TOE.

The evaluator shall conduct penetration testing, based on the identified potential vulnerabilities, to determine that the TOE is resistant to attacks performed by an attacker possessing Basic attack potential. 


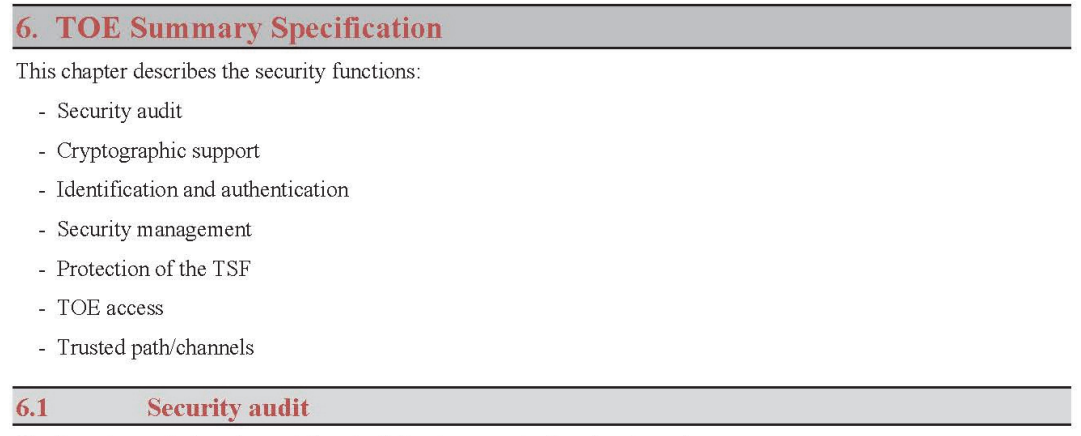

The Security audit function satisfies the following security functional requirements:

NDcPP20E:FAU_GEN.1:

The TOE is capable of generating log records for a wide range of security relevant and other events as they occur. The events that can cause an audit record to be logged include starting and stopping the audit function, Security Administrator's configuration of CSPs and security functions as well as all of the events identified in Error! Reference source not found. The TOE generates records for several separate classes of events:

authentication/access to the system, actions taken directly on the system by network clients, and management of security functions by authorized administrators.

All audit records include the date/time of the event, the identity associated with the event (such as the service, computer or user), the success/failure of the event and a definition of the event (by code or explanation). For example, when the administrator creates, modifies or deletes the cryptographic keys, the key name will be logged in the event to identify the relevant key

\section{NDcPP20E:FAU_GEN.2-Notes:}

All actions performed by the TOE are associated with a unique identifier such as administrator's user name, wireless client's MAC/IP address and NTP/RADIUS/Log server's IP address. This information is maintained in the audit record, allowing the events stored there to be traced directly to the user or system for which they were performed. NDCPP20E:FAU STG_EXT.1:

The TOE stores audit logs locally with up to a fixed size of $256 \mathrm{~K}$ bytes. Local password based authentication and authorization limits the access to the local audit log records. Only the Security Administrator can gain access to the local audit $\log$ records

When the TOE is configured to export audit logs to an external SYSLOG server, it simultaneously sends the message to the server and local store. The TOE requires the external audit server and itself to be connected via an IPsec session. The Admin Guide provides details about the "Export Audit Logs" configuration. 'The l'OE exports audit data over IPsec using AES128/256 bit encryption. Disconnection to external entities such as syslog server will result in $\log$ of communication error and attempt to re-establish secure channel. At no point, will plaintext be transmitted. The TOE does not implement an automatic synchronization mechanism between the local and remote audit storage.

When the audit log storage space is full, the TOE also provides the Authorized Administrator the option of overwriting "old" audit records rather than preventing auditable events. 
There are two cryptographic engines within the TOE models as shown in the figure below. All models share the same hardware MPC8378E cryptographic core and OpenSSL library.

Figure 6-1: TOE Cryptographic Cores

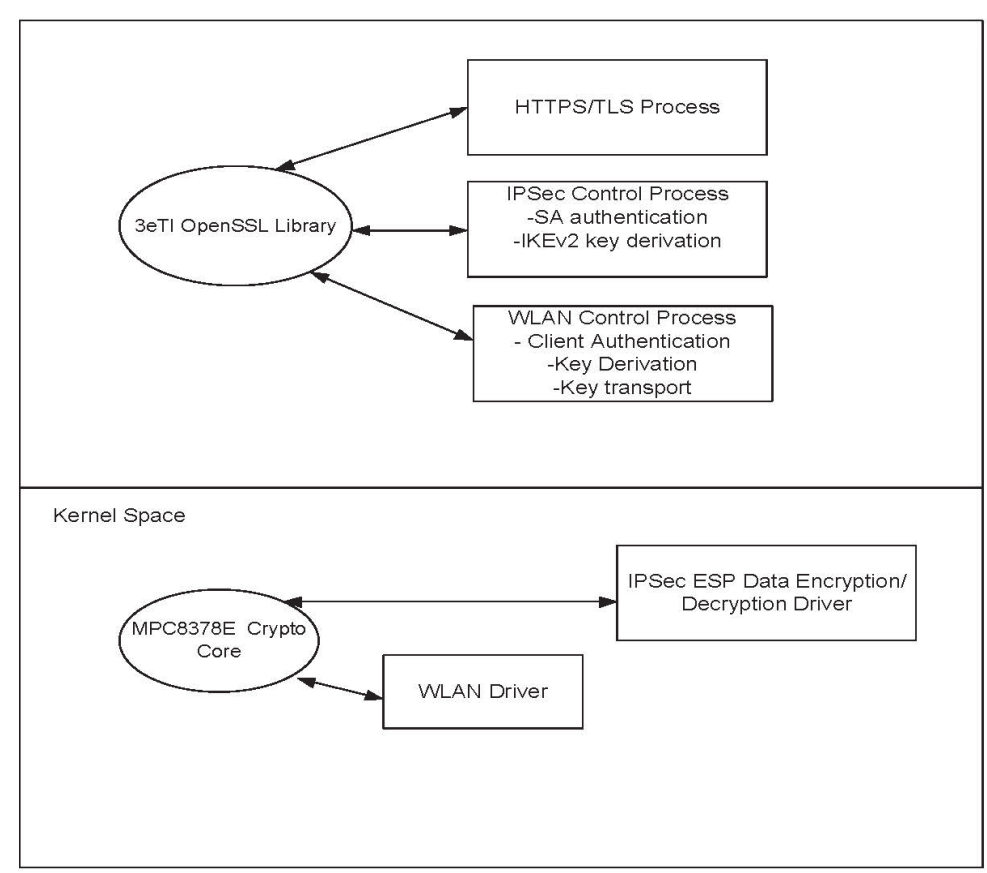

First is the 3eTI's own OpenSSL library. 3eTI's OpenSSL Library serves as the sole user application level cryptographic library. It provides the FCS_COP functions listed below. All user level applications, such as HTTPS/TLS Web U, Wireless LAN Control Application and IPsec SA authentication module use this library. 3eTI's OpenSSL provides the following cryptographic algorithms: AES, RSA, HMAC, SHS, ECDSA and DRBG.

The 3eTI OpenSSL Library represents not the entire OpenSSL Library, but the FIPS Object Module that is compiled into the larger OpenSSL Library. Because 3eTI has already compiled the FIPS Object Module and then links that same, identical Object Module into different versions of the larger OpenSSL library, the version of the larger OpenSSL Library is not relevant. 
There is a FreeScale MPC8378E cryptographic core within the TOE as well. It provides cryptographic functions for the Linux kernel drivers. Wireless client data encryption/decryption functions using AES-CCMP is provided by this engine. IPsec ESP data encryption/decryption using AES-CBC with SHS or AES-GCM is provided by this engine The MPC8378E cryptographic core provides the following cryptographic algorithms in FIPS mode: AES (CBC, GCM, CCM), HMAC, SHS.

The TOE utilizes version 2.0 of its OpenSSL Algorithm Implementation and version 1.0 of the MPC8378E cryptographic core in firmware version 5.1.0 of the TOE.

Table 6-1: TOE CAVP Tested Algorithms

\begin{tabular}{|c|c|c|}
\hline Algorithm & Cert No. & SFR Mapping \\
\hline \multicolumn{3}{|l|}{ 3eTIOpenSSL } \\
\hline AES (CBC, 128,256 bits key) & 2060 & FCS_COP.1/DataEncryption \\
\hline AES-KW & 2060 & FCS CKM.2(3) \\
\hline CVL KAS ECC & 1357 & FCS_CKM.2 \\
\hline $\begin{array}{l}\text { ECDSA, KeyPairGen, sign/verify with P256, P384 } \\
\text { and P521 }\end{array}$ & $\begin{array}{l}415 \\
303\end{array}$ & $\begin{array}{l}\text { FCS_COP.1/SigGen } \\
\text { FCS_CKM.1 }\end{array}$ \\
\hline SHS (SHA-1, SHA-256, SHA-384, SHA-512) & 1801 & FCS COP.1/Hash \\
\hline $\begin{array}{l}\text { HMAC (HMAC-SHA-1, HMAC-SHA-256, HMAC- } \\
\text { SHA-384, HMAC-SHA-512) }\end{array}$ & 1253 & FCS_COP.1/KeyedHash \\
\hline RSA key generation (2048 bits) & 2568 & FCS_CKM.1 \\
\hline RSA sign/verify (2048 bits) & 1491 & FCS COP.1/SigGen \\
\hline $\begin{array}{l}\text { DRBG NIST SP800-90 with one independent } \\
\text { hardware based noise source of } 256 \text { bits of non- } \\
\text { deterministic (CTR_DRBG (AES)) }\end{array}$ & 822 & FCS_RBG_EXT.1 \\
\hline \multicolumn{3}{|l|}{ MPC8378E Cryptographic Core } \\
\hline AES (CBC 128, 256 bits; CCM 128, 256 bits) & 2078 & FCS_COP.1(1) \\
\hline AES (GCM 128, 256 bits) & 2105 & FCS COP.1(1) \\
\hline $\begin{array}{l}\text { HMAC (HMAC-SHA-1, HMAC-SHA-256, HMAC- } \\
\text { SHA-384, HMAC-SHA-512) }\end{array}$ & 1259 & FCS_COP.1/KeyedHash \\
\hline SHS (SHA-1, SHA-256, SHA-384, SHA-512) & 1807 & FCS COP.1/Hash \\
\hline
\end{tabular}

As shown in Figure 6.1, the 3eTI OpenSSL library provides cryptographic services to the TOE's application modules. The IPsec module uses the OpenSSL library during the IKEv2 SA authentication and key establishment. The Wireless Access Point module uses the library's cryptographic service during the client's authentication and the TEEE $802.11 \mathrm{i}$ 4-way handshake key establishment. The MPC 8378E cryptographic core provides services for fast path data encryption. For IPsec, this module provides the IPsec ESP encryption with AES-GCM or AES-CBC-HMAC-SHS. For WLAN data communication between the TOE and the wireless client, this core provides AES-CCM encryption/decryption service

\section{NDcPP20E:FCS_CKM.1:}

The TOE support both RSA and ECDSA key generation. The key generation is used by the TOE when it creates a Certificate Signing Request (CSR) to apply for a certificate from the Certificate Authority (CA). The TOE enforces the key size of 2048 or greater for RSA key pairs and supports NIST curves P256, P384 and P521 for ECDSA key pairs. The TOE implements FFC schemes using Diffie-Hellman group 14. The TOE implementation of DiffieHellman group 14 (2048 MODP) meets RFC 3526, Section 3. The scheme is used by both IPsec IKEv2 and TLS module. 
The symmetric key of size 128 bits for communications between the TOE and the wireless client is generated during the $802.11 \mathrm{i}$ defined 4-way handshake process using random numbers generated by ISO/IEC 18031:2011DRBG random bits generator. 802.11-2012 specified cryptographic key derivation algorithm [PRF-384] is strictly followed by the TOE. The TOE is Wi-Fi Alliance certified (Certification ID: WFA56890) to prove the correctness of key derivation and interoperability between the TOE and other commercial Wi-Fi products.

End-to-end wireless encryption between the TOE and the wireless client is implemented using WPA2. The PMK is generated by the RADIUS Server in coordination with the wireless client, encrypted by the IPsec tunnel, and passed to the AP in the RADIUS ACCESS_ACCEPT message. The AP uses the PMK and the $802.11 \mathrm{i}$ four-way handshake to generate the Pairwise Transient Key (PTK) and the GTK (Group Temporal Key)

The PTK is generated by concatenating the following attributes: PMK, AP nonce (ANonce), the client (station) nonce (SNonce), AP MAC address, and client MAC address. The product is then put through a cryptographic hash function. The four steps are as follows:

1. The AP sends a nonce-value to the client (ANonce). The client now has all the attributes to construct the PTK.

2. The client sends its own nonce-value (SNonce) to the AP together with a MIC, including authentication, which is really a Message Authentication and Integrity Code: (MAIC).

3. The AP sends the GTK and a sequence number together with another MIC. This sequence number will be used in the next multicast or broadcast frame, so that the receiving STA can perform basic replay detection.

4. The client sends an acknowledgement to the $\mathrm{AP}$.

The messages exchanged during the handshake are depicted in Figure 6-2 below.

Figure 6-2: 802.11i Four Way Handshake

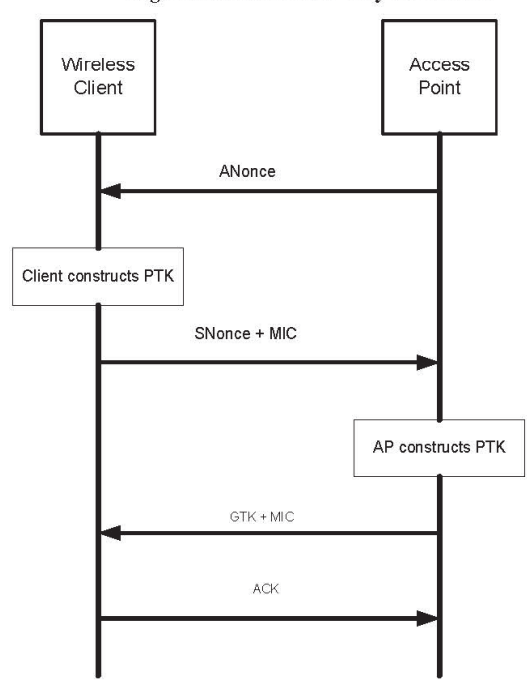

The PTK is divided into the individual session keys including the Key Encryption Key (KEK), the Key Confirmation Key (KCK) and the temporal key (TK)) for encrypting the wireless traffic with each wireless client that has been authenticated. The KEK is used by the EAPOL-Key frames to provide confidentiality. The KCK is 
used by IEEE $802.11 \mathrm{i}$ to provide data origin authenticity. The TK, also known as the CCMP key, is the $802.11 \mathrm{i}$ session key for unicast communications.

The TSF distributes the Group Temporal Key (GTK) by using AES Key Wrap in an EAPOL-Key frame that meets RFC 3394 for AES Key Wrap and 802.11-2012 standard for the packet format and timing considerations.

The TOE allows for the detection of modification of user data while carrying out network communications on the wireless network through the use of AES operating in CCM (CCMP). This is done through the integrity protection capabilities of the algorithm. The Cipher Block Chaining Message Authentication Code (CBC-MAC) component of CCMP provides data integrity. The CBC-MAC allows for the detection of a modified packet. If a CBC-MAC indicates a packet has been modified the packet is dropped

\section{NDcPP20E:FCS_CKM.2:}

The TOE acts as both receiver and sender for RSA-based key establishment and ECDSA based key establishment in cryptographic operations. The TOE implementation of Diffie-Hellman-group-14 meets RFC 3526, Section 3 by virtue of using a 2048-bit MODP group for key establishment.

\section{WLANAScEP10:FCS_CKM.2(2):}

The TSF's key material, such as the Pair-wise Master Key (PMK) for Wireless Protected Access (WPA2) is distributed by the RADIUS server to the TOE's $802.1 \mathrm{X}$ authenticator components via the ACCESS_ACCEPT message after the wireless client's successful authentication with the RADIUS server. The MSK/PMK is included in the message with attribute: MS MPPE SEND KEY (16) Vendor Specific Attribute (VSA) as defined by RFC 2548. The IPsec tunnel between the TOE and RADIUS server protects the PMK from exposure.

The TSF distribute Group Temporal Key (GTK) by using AES Key Wrap in an EAPOL-Key frame that meets NIST SP 800-38F and IEEE 802.11-2012 standard for the packet format and timing considerations. The GTK is first distributed to the client after the client's successful authentication with the RADIUS server, followed by the process of $802.11 \mathrm{i}$ 4-way handshakes which is detailed in Figure 6-2 illustrates the GTK distribution

The TOE has a configurable GTK timeout value. At the configured time expiration, the TOE will update each client with the GTK using the AES key wrap mechanism just described.

\section{NDcPP20E:FCS CKM.4:}

Table 6- below lists all the keys and CSPs used and managed by the TOE.

\begin{tabular}{|l|l|l|l|l|l|l|}
\multicolumn{7}{|c|}{ Table 6-2: TOE CSPs Use and Management } \\
\hline \multicolumn{7}{|c|}{ Non-Protocol Keys/CSPs } \\
\hline Key/CSP & Type & $\begin{array}{l}\text { Generation/ } \\
\text { Input }\end{array}$ & Output & Storage & Zeroization & Use \\
\hline $\begin{array}{l}\text { Operator } \\
\text { passwords }\end{array}$ & ASCII string & $\begin{array}{l}\text { Input } \\
\text { encrypted } \\
\text { (using TLS } \\
\text { session key) }\end{array}$ & Not output & $\begin{array}{l}\text { PKCS5 hash } \\
\text { in flash }\end{array}$ & $\begin{array}{l}\text { Zeroized } \\
\text { when reset to } \\
\text { factory } \\
\text { settings. }\end{array}$ & $\begin{array}{l}\text { Used to } \\
\text { authenticate } \\
\text { Security } \\
\text { Admin and } \\
\text { Admin role } \\
\text { operators }\end{array}$ \\
\hline $\begin{array}{l}\text { Firmware } \\
\text { verification } \\
\text { key }\end{array}$ & $\begin{array}{l}\text { ECDSA } \\
\text { public key }\end{array}$ & $\begin{array}{l}\text { Embedded in } \\
\text { firmware at } \\
\text { compile time. } \\
\text { Firmware } \\
\text { upgrade is } \\
\text { through } \\
\text { encrypted } \\
\text { (using TLS } \\
\text { session key) }\end{array}$ & Not output & $\begin{array}{l}\text { Plaintext in } \\
\text { flash }\end{array}$ & $\begin{array}{l}\text { Zeroized } \\
\text { when } \\
\text { firmware is } \\
\text { upgraded. }\end{array}$ & $\begin{array}{l}\text { Used for } \\
\text { firmware } \\
\text { digital } \\
\text { signature } \\
\text { verification }\end{array}$ \\
\hline
\end{tabular}


AirGuard 3e-525/523 Access Points (NDcPP20ENLANAScEP10) Security Target

Version $0.5,05 / 08 / 18$

\begin{tabular}{|c|c|c|c|c|c|c|}
\hline $\begin{array}{l}802.1 \mathrm{X} \\
\text { RADIUS } \\
\text { Server } \\
\text { Password }\end{array}$ & ASCII string & $\begin{array}{l}\text { Input } \\
\text { encrypted } \\
\text { (using TLS } \\
\text { session key) }\end{array}$ & Not output & $\begin{array}{l}\text { Plaintext in } \\
\text { flash }\end{array}$ & $\begin{array}{l}\text { Zeroized } \\
\text { when } \\
\text { password is } \\
\text { upgraded. }\end{array}$ & $\begin{array}{l}\text { Used to create } \\
\text { authentication } \\
\text { hash value } \\
\text { with RADIUS } \\
\text { server }\end{array}$ \\
\hline \multicolumn{7}{|c|}{ RBG Keys/CSPs } \\
\hline $\mathrm{Key} / \mathrm{CSP}$ & Type & $\begin{array}{l}\text { Generation/ } \\
\text { Input }\end{array}$ & Output & Storage & Zeroization & Use \\
\hline $\begin{array}{l}\text { DRBGCTR } \\
\text { V }\end{array}$ & 32-byte value & $\begin{array}{l}32 \text { bytes from } \\
\text { /dev/random } \\
\text { file, } \\
\text { /dev/random } \\
\text { is populated } \\
\text { by hardware } \\
\text { noise } \\
\text { generator }\end{array}$ & Not output & $\begin{array}{l}\text { Plaintext in } \\
\text { RAM }\end{array}$ & $\begin{array}{l}\text { Zeroized } \\
\text { every time a } \\
\text { new random } \\
\text { number is } \\
\text { generated } \\
\text { using the } \\
\text { FIPS DRBG } \\
\text { after it is } \\
\text { used. } \\
\end{array}$ & $\begin{array}{l}\text { Used as CTR } \\
\text { V value for } \\
\text { FIPS DRBG. }\end{array}$ \\
\hline $\begin{array}{l}\text { DRBG CTR } \\
\text { Key }\end{array}$ & 32 -byte value & $\begin{array}{l}32 \text { bytes from } \\
\text { /dev/random } \\
\text { file, } \\
\text { /dev/random } \\
\text { is populated } \\
\text { by hardware } \\
\text { noise } \\
\text { generator }\end{array}$ & Not output & $\begin{array}{l}\text { Plaintext in } \\
\text { RAM }\end{array}$ & $\begin{array}{l}\text { Zeroized } \\
\text { every time a } \\
\text { new random } \\
\text { number is } \\
\text { generated } \\
\text { using the } \\
\text { FIPS DRBG } \\
\text { after it is } \\
\text { used. }\end{array}$ & $\begin{array}{l}\text { Used as CTR } \\
\text { key for FIPS } \\
\text { DRBG. }\end{array}$ \\
\hline \multicolumn{7}{|c|}{ RFC 2818 HTTPS KeysCSPs } \\
\hline $\mathrm{Key} / \mathrm{CSP}$ & Type & $\begin{array}{l}\text { Generation/ } \\
\text { Input }\end{array}$ & Output & Storage & Zeroization & Use \\
\hline $\begin{array}{l}\text { RSA private } \\
\text { key }\end{array}$ & $\begin{array}{l}\text { RSA (2048) } \\
\text { (key } \\
\text { wrapping; key } \\
\text { establishment } \\
\text { methodology } \\
\text { provides 112- } \\
\text { bits of } \\
\text { encryption } \\
\text { strength) }\end{array}$ & $\begin{array}{l}\text { Not input } \\
\text { (installed at } \\
\text { factory) }\end{array}$ & Not output & $\begin{array}{l}\text { Plaintext in } \\
\text { flash }\end{array}$ & $\begin{array}{l}\text { Zeroized } \\
\text { when new } \\
\text { private key is } \\
\text { uploaded }\end{array}$ & $\begin{array}{l}\text { Used to } \\
\text { support } \\
\text { Security } \\
\text { Admin and } \\
\text { Admin } \\
\text { TLS/HTTPS } \\
\text { interfaces. }\end{array}$ \\
\hline $\begin{array}{l}\text { TLS session } \\
\text { key for } \\
\text { encryption }\end{array}$ & \begin{tabular}{|l} 
AES \\
$(128 / 256)$
\end{tabular} & $\begin{array}{l}\text { Not input, } \\
\text { derived using } \\
\text { TLS protocol }\end{array}$ & Not output & $\begin{array}{l}\text { Plaintext in } \\
\text { RAM }\end{array}$ & $\begin{array}{l}\text { Zeroized } \\
\text { when a page } \\
\text { of the web UI } \\
\text { is served after } \\
\text { it is used. }\end{array}$ & $\begin{array}{l}\text { Used to } \\
\text { protect. } \\
\text { TLS/HTTPS } \\
\text { session. }\end{array}$ \\
\hline \multicolumn{7}{|c|}{ Public Security Parameter } \\
\hline $\begin{array}{l}\text { HTTPS Public } \\
\text { certificate }\end{array}$ & RSA (2048) & $\begin{array}{l}\text { Input } \\
\text { encrypted } \\
\text { (using TLS } \\
\text { session key) }\end{array}$ & $\begin{array}{c}\text { During TLS } \\
\text { session setup }\end{array}$ & & & $\begin{array}{l}\text { Used to setup } \\
\text { TLS session } \\
\text { for } \\
\text { TLS/HTTPS }\end{array}$ \\
\hline $\begin{array}{l}\text { HTTPS root } \\
\text { certificate }\end{array}$ & RSA (2048) & $\begin{array}{l}\text { Input } \\
\text { encrypted } \\
\text { (using TLS } \\
\text { session key) }\end{array}$ & Not output & & & $\begin{array}{l}\text { Used to setup } \\
\text { TLS session } \\
\text { for } \\
\text { TLS/HTTPS }\end{array}$ \\
\hline \multicolumn{7}{|c|}{ IPsec Keys } \\
\hline $\begin{array}{l}\text { DH Private } \\
\text { Key }\end{array}$ & $\begin{array}{l}2048 \text { bits } \\
\text { private key }\end{array}$ & Generated & Not output & $\begin{array}{l}\text { Plaintext in } \\
\text { RAM }\end{array}$ & $\begin{array}{l}\text { Zeroized } \\
\text { when no } \\
\text { longer used }\end{array}$ & $\begin{array}{l}\text { IKE v2 SA } \\
\text { setup }\end{array}$ \\
\hline
\end{tabular}


AirGuard 3e-525/523 Access Points (NDcPP20ENLANAScEP10) Security Target

Version $0.5,05 / 08 / 18$

\begin{tabular}{|c|c|c|c|c|c|c|}
\hline $\begin{array}{l}\text { ECCDH } \\
\text { Private Key }\end{array}$ & $\begin{array}{l}256,384,521 \\
\text { bits }\end{array}$ & Generated & Not output & $\begin{array}{l}\text { Plaintext in } \\
\text { RAM and } \\
\text { encrypted in } \\
\text { FLASH } \\
\end{array}$ & $\begin{array}{l}\text { RAM copy } \\
\text { zeroized when } \\
\text { no longer used }\end{array}$ & $\begin{array}{l}\text { IKE v2 SA } \\
\text { setup }\end{array}$ \\
\hline $\begin{array}{l}\text { IPSec SA } \\
\text { Authenticatio } \\
\text { n certificate } \\
\text { private key } \\
\end{array}$ & $\begin{array}{l}\text { RSA (2048), } \\
\text { ECDSA } \\
(256,384,512)\end{array}$ & $\begin{array}{l}\text { Input } \\
\text { encrypted } \\
\text { using TLS } \\
\text { session key } \\
\end{array}$ & Not output & $\begin{array}{l}\text { Plaintext in } \\
\text { RAM and } \\
\text { encrypted in } \\
\text { FLASH } \\
\end{array}$ & $\begin{array}{l}\text { RAM copy } \\
\text { zeroized when } \\
\text { no longer used }\end{array}$ & $\begin{array}{l}\text { IKE v2 SA } \\
\text { authentication }\end{array}$ \\
\hline $\begin{array}{l}\text { IPSec SA } \\
\text { private key } \\
\text { password }\end{array}$ & Text string & $\begin{array}{l}\text { Input } \\
\text { encrypted } \\
\text { using TLS } \\
\text { session key }\end{array}$ & Not output & $\begin{array}{l}\text { Plaintext in } \\
\text { RAM and } \\
\text { encrypted in } \\
\text { FLASH } \\
\end{array}$ & $\begin{array}{l}\text { Zeroized } \\
\text { when no } \\
\text { longer used }\end{array}$ & $\begin{array}{l}\text { Encrypt the } \\
\text { IPSec SA } \\
\text { certificate } \\
\text { private key }\end{array}$ \\
\hline $\begin{array}{l}\text { IPSec SA } \\
\text { session key }\end{array}$ & $\begin{array}{l}\text { Derived from } \\
\text { DH/ECCDH } \\
\text { key exchange }\end{array}$ & Not input & Not output & $\begin{array}{l}\text { Plaintext in } \\
\text { RAM }\end{array}$ & $\begin{array}{l}\text { Zeroized } \\
\text { when no } \\
\text { longer used }\end{array}$ & $\begin{array}{l}\text { Encrypt and } \\
\text { Authenticate } \\
\text { SA_Auth } \\
\text { messages of } \\
\text { IKE v2 }\end{array}$ \\
\hline $\begin{array}{l}\text { IPSec ESP } \\
\text { symmetric } \\
\text { Data } \\
\text { encryption } \\
\text { key }\end{array}$ & $\begin{array}{l}\text { AES, } \\
\text { AES_GCM } \\
(128,256)\end{array}$ & $\begin{array}{l}\text { Not input } \\
\text { (derived from } \\
\text { SA setup) }\end{array}$ & Not output & $\begin{array}{l}\text { Plaintext in } \\
\text { RAM }\end{array}$ & $\begin{array}{l}\text { Zeroized } \\
\text { when child SA } \\
\text { lifetime } \\
\text { expired }\end{array}$ & $\begin{array}{l}\text { Encrypt IPSec } \\
\text { ESP data }\end{array}$ \\
\hline \multicolumn{7}{|c|}{ Wireless Access Point Keys } \\
\hline PMK & $\begin{array}{l}802.11 \mathrm{i} \\
\text { pairwise } \\
\text { master key }\end{array}$ & $\begin{array}{l}\text { If } 802.11 \mathrm{i} \\
\text { PSK, it's input } \\
\text { directly as a } \\
\text { Hex string. } \\
\text { Input } \\
\text { encrypted } \\
\text { using the TI.S } \\
\text { session key. } \\
\text { If } 802.11 \mathrm{i} \\
\text { EAP-TLS, } \\
\text { then not input, } \\
\text { instead } \\
\text { derived (TLS } \\
\text { master secret } \\
\text { resulting from } \\
\text { successfil } \\
\text { User EAP- } \\
\text { TLS } \\
\text { authentication } \\
\text { ) }\end{array}$ & Not output & $\begin{array}{l}\text { If } 802.11 \mathrm{i} \\
\text { PSK, then } \\
\text { plaintext in } \\
\text { flash } \\
\text { For both } \\
802.11 \mathrm{i} \text { PSK } \\
\text { and EAP-TLS, } \\
\text { plaintext in } \\
\text { RAM }\end{array}$ & $\begin{array}{l}\text { Zeroized } \\
\text { when wireless } \\
\text { user } \\
\text { disconnect or } \\
\text { at PMK } \\
\text { expiration } \\
\text { If } 802.11 \text { i } \\
\text { PSK, zeroized } \\
\text { when reset to } \\
\text { factory } \\
\text { settings. }\end{array}$ & 802.1 li PMK \\
\hline $\mathrm{KCK}$ & $\begin{array}{l}\text { HMAC key } \\
\text { (128 bits from } \\
\text { PTK) }\end{array}$ & $\begin{array}{l}\text { Not input } \\
\text { (derived from } \\
\text { PTK) }\end{array}$ & Not output & $\begin{array}{l}\text { Plaintext in } \\
\text { RAM }\end{array}$ & $\begin{array}{l}\text { When } 802.11 \mathrm{i} \\
\text { session ends. }\end{array}$ & $802.11 \mathrm{i} \mathrm{KCK}$ \\
\hline KEK & $\begin{array}{l}\operatorname{AES} \operatorname{ECB}(\mathrm{e} / \mathrm{d} \text {; } \\
128)\end{array}$ & $\begin{array}{l}\text { Not input } \\
\text { (derived from } \\
\text { PTK) }\end{array}$ & Not output & $\begin{array}{l}\text { Plaintext in } \\
\text { RAM }\end{array}$ & $\begin{array}{l}\text { When } 802.11 \mathrm{i} \\
\text { session ends. }\end{array}$ & 802.11 KEK \\
\hline PTK & $\begin{array}{l}\text { AES CCM } \\
(\mathrm{e} / \mathrm{d} ; 128)\end{array}$ & $\begin{array}{l}\text { Not input } \\
\text { (derived from } \\
\text { PTK) }\end{array}$ & Not output & $\begin{array}{l}\text { Plaintext in } \\
\text { RAM }\end{array}$ & $\begin{array}{l}\text { When } 802.11 \mathrm{i} \\
\text { session ends. }\end{array}$ & $802.1 \mathrm{li} \mathrm{TK}$ \\
\hline $\begin{array}{l}\text { PTK (copy in } \\
\text { driver) }\end{array}$ & $\begin{array}{l}\text { AES CCM } \\
(\mathrm{e} / \mathrm{d} ; 128)\end{array}$ & $\begin{array}{l}\text { Not input } \\
\text { (derived from } \\
\text { PTK) }\end{array}$ & Not output & $\begin{array}{l}\text { Plaintext in } \\
\text { RAM }\end{array}$ & $\begin{array}{l}\text { When } 802.11 \mathrm{i} \\
\text { session ends. }\end{array}$ & $802.11 \mathrm{i}$ TK \\
\hline GTK & $\begin{array}{l}\text { AES CCM } \\
(\mathrm{e} / \mathrm{d} ; 128)\end{array}$ & $\begin{array}{l}\text { Not input } \\
\text { (derived from } \\
\text { GMK) }\end{array}$ & $\begin{array}{l}\text { Output } \\
\text { encrypted } \\
\text { (using KEK) }\end{array}$ & $\begin{array}{l}\text { Plaintext in } \\
\text { RAM }\end{array}$ & $\begin{array}{l}\text { Zeroized } \\
\text { when local } \\
\text { antennae }\end{array}$ & $802.11 \mathrm{i}$ GTK \\
\hline
\end{tabular}




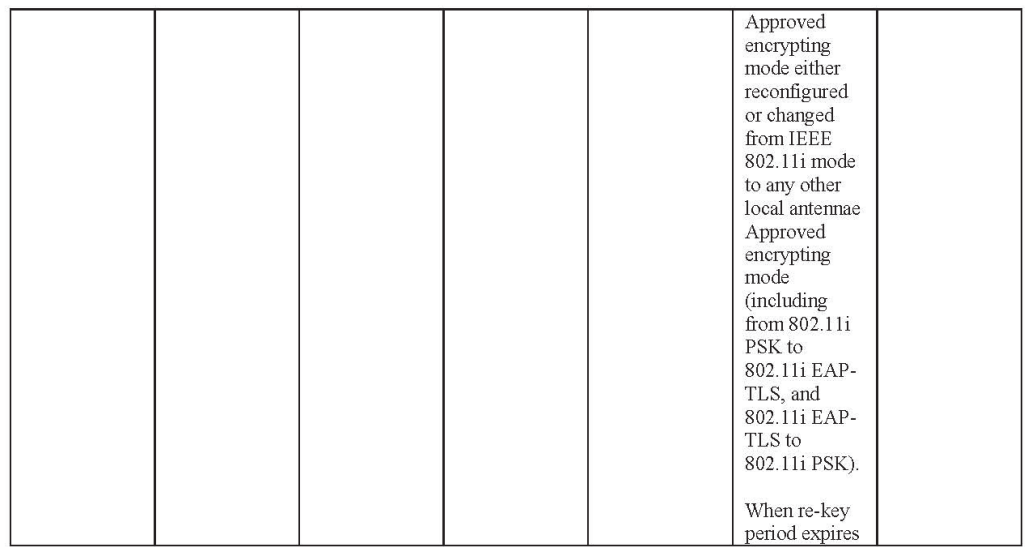

The zeroization technique is to write all $0 x a 5$, then $0 \times 5 a, 0 x f f$ and finally all zeros to the memory location where the key is stored in non-volatile storage. A read-verify is performed after the zeroization. The technique is implemented in the firmware that uses Linux file system APIs. The TOE does not store keys in EEPROM. For keys stored in RAM, a single overwrite of all zeroes is performed. IPsec authentication certificate's private keys are encrypted with the corresponding private key passwords. The passwords are stored as plain text in flash and they are zeroized the same way as described above.

NDcPP20E:FCS_COP.1/DataEncryption

WLANAScEP10:FCS_COP.1(1):

AES is implemented with key sizes of 128 , and 256 bits in Cipher Block Chaining (CBC) mode and Galois Counter Mode (GCM) and 128 bits in CCMP mode. The 3eTI's OpenSSL Library provides AES services for application level data encryption and decryption. The management interface uses this library to provide Transport Layer Security (TLS/HTTPS). For the TOE's TLS interface, AES_CBC with 128 or 256 bits key is used. 3eTI's

MPC8378E Cryptographic Core provides AES_GCM and AES_CBC services for IPsec data encryption. 128 and 256 bits keys are supported. AES_CCM with 128 bits is provided by the MPC8378E core for WLAN data packets encryption/decryption.

Table 6-1 lists the AES mode and key sizes; all AES algorithm implementations are NIST CAVP validated.

\section{NDcPP20E:FCS_COP.1/SigGen:}

The 3eTI OpenSSL Library provides the RSA Digital Signature Algorithm (rDSA) to the TLS/HTTPS Daemon for the TLS session. The TLS/HTTPS Daemon enforces a 2048 or larger bits RSA key length for use with the RSA.

TOE Firmware's digital signature is using ECDSA with P256. The 3eTI OpenSSL library provides ECDSA

sign/verify operation support. IPsec tunnels can be configured to use RSA (2048 bits) or ECDSA with key size 256 384 , and 521 bits using NIST curve P256, P384 and P521 certificate for IPsec SA authentication. Table 6-1 lists

RSA and ECDSA CAVP validation certificate numbers. 
NDcPP20E:FCS_COP.1/KeyedHash:

The TOE's OpenSSL Library and the MPC8378E cryptographic core both implement HMAC-SHA-1, HMACSHA-256, HMAC-SHA-384, and HMAC-SHA-512, keyed-hash message authentication supporting digest sizes: 160, 256,384, and 512 bits and key size of 160 bits, 256 bits, 384 and 512 bits implemented to meet ISO/IEC 9797 2:2011, Section 7 "MAC Algorithm 2. The block size is 512 bits for HMAC-SHA-1, HMAC-SHA-256 and 1024 bits for HMAC-SHA-384, and HMAC-SHA-512 and the output size is the same as the underlying hash function.

\section{NDCPP20E:TCS_IITTPS EXT.1.1:}

The Web UI with remote administration station is always TLS/HTTPS. The HTTPS implementation is fully compliant with RFC 2818. The TOE acts as an HTTPS server and waits for client connections on TCP port 443 . The TOE's HTTPS server permits an HTTP client to close the connection at any time, and the HTTPS server will recover gracefully. In particular, the HTTPS server is prepared to receive an incomplete close from the client, and is willing to resume TLS sessions closed in this fashion.

NDcPP20E/WLANAScEP10:FCS_IPSEC_EXT.1:

The TOE implements IPsec protocol in full compliance with IETF RFCs as specified by NDcPP20E. Within the TOE, the NTP client uses an IPsec tunnel for communications with an NTP server. The TOE uses IPsec to protect communications with the remote log server as well.

The TOE supports IKEv2 only as defined by RFCs 5996 and always attempts NAT traversal, hence an administrator need not configure either. During the Security Association (SA) setup phase, the TOE supports the following DH groups:

- $\operatorname{ecp} 384$

- ecp256

- $\operatorname{modp} 2048$

If the administrator selects "auto negotiation" from IPsec "Cipher Suites" configuration Web UI, then the groups listed above will be send to the IPsec peer during the IKEv2 negotiation. If "Suite B GCM128" is selected, then the TOE will use ecp 256 group. If "Suite B GCM256" is selected, then the TOE will use ecp384 group.

The TOE chooses and enforces the group and AES cipher to make sure that the SA confidentiality strength is equivalent or greater than the configured ESP confidentiality strength. For example, the TOE (when configured for "Auto Negotiation") will reject any ESP proposal with an AES key length greater than the negotiated IKE AES key length. Similarly, administrator selection of "Suite B GCM128" or similar ciphers ensure the TOE will enforce a single, prescribed set of modes to make sure that the parents IPsec SA confidentiality strength is equal or greater than the child SA's strength.

\begin{tabular}{|l|l|l|l|l|l|l|}
\hline \multirow{2}{*}{ Mode } & \multicolumn{3}{|l|}{ IKE (openssl library) } & ESP (Hardware encryption) \\
\cline { 2 - 7 } & Encryption & Integrity & $\begin{array}{l}\text { Pseudo Random } \\
\text { Function }\end{array}$ & DH Group & Encryption & $\begin{array}{l}\text { Integrity } \\
\text { (where } \\
\text { applicable) }\end{array}$ \\
\hline Suite B GCM 128 & aes128cbc & sha256 & sha256 & ecp256 & aes128gcm128 & - \\
\hline
\end{tabular}

The TOE uses ISO/IEC 18031:2011 DRBG to generate the nonce and " $\mathrm{x}$ " in each DH group with lengths of 112 (for DH group 14), 128 (for DH group 19 and 192 (for DH group 20). After the Diffie Hellman exchanges that setup the session keys, the IKEv2 payload is protected by the following encryption algorithms:

- AES-CBC-256

- $\mathrm{AES}-\mathrm{CBC}-128$ 
SHA-512, SHA-384, SHA-256 and SHA1 are used to provide payload data integrity. X 509 certificates with $r$ DSA 2048 bits or larger key or ECDSA 256, 384, and 521 bits key with NIST P256, P384 and P521 are used for IPsec tunnel authentication with its peer.

The TOE supports IPsec tunnel mode and transport mode which allows the payload of packets to be encrypted. The TOE requires no administrative configuration and negotiates either depending on the peer. The TOE uses IPsec standard encapsulating Security Payload (ESP) protocol to provide authentication, encryption and anti-replay services. It uses the following ciphers to encrypt the IPsec data payload:

1. GCM mode with Nonce length of 128,96 and 64 bits

- AES-GCM-128

- AES-GCM-256

2. CBC mode with HMAC-SHA-512, HMAC-SHA-384, HMAC-SHA-256 and HMAC-SHAl as integrity

- AES-CBC-128

- AES-CBC-256

When the administrator chooses AES-GCM-128 for ESP encryption, the TOE will automatically choose SHA-256 for the IKE's integrity and pseudo random function. If the choice is AES-GCM-256, then SHA-256 will be used in IKE integrity and SHA-384 will be used for the pseudo random function. If the administrator chooses "Auto Negotiation" for IPsec, the TOE will send cipher list ranked with the highest security first to its peer. For example, the IKE integrity list will be sent as: SHA-512, SHA-384, SHA-256 and SHA1. It's expected that the peer will pick the strongest one it could support. There is no need to explicitly configure security hashing functions in the IPsec configuration.

The IPsec daemon module implements implicit policies such that only expected data packages are allowed. Any data packages that violate the policy will be discarded

The TOE allows the Security Administrator to configure the IKEv2 SA and child SA lifetime by minutes (20-1440, default 180) and the TOE additionally allows the Administrator to configure child SA lifetime by number of bytes ( 90 to $2047 \mathrm{~Kb}, 0$ for unlimited) or by number of data packets (192 to $2097151 \mathrm{~K}, 0$ for unlimited)

The TOE supports X.509 certificate for IPsec mutual authentication. RSA certificate with 2048 bits, ECDSA certificates with 256,384 , and 521 bits key is supported implementing NIST curves P256, P384 and P521. When certificates are used for authentication, the Distinguished Name (DN), IP Address and Fully Qualified Domain Name (FQDN) are verified to ensure the certificate is valid and is from a valid entity. These reference identifiers in the certificate are compared with the expected reference identifiers (as specified by an administrator) and deemed valid if the attribute types are the same and the values are the same and as expected.

The TOE can be configured to use pre-shared key for IPsec authentication as well. The security administrator first selects "Pre-Shared Key" under "Authentication" when configuring the IPsec tunnel via the Web UT, then enters the pre-shared key manually

The TOE's IPsec Security Policy Database (SPD) is dynamically configured based upon the trusted paths IPsec is used to protect. For example, the TOE uses IPsec to protect a remote syslog trusted path. In this instance, records are written into the SPD to protect packets passing between the TOE and the remote syslog server based on source address, destination address, protocol and port number. When protecting remote syslog trusted path, the SPD will have record matching ingress UDP traffic with source address and port corresponding to the remote syslog server. Additionally, the SPD will have record matching egress traffic with destination address and port corresponding to the remote syslog server. Traffic passing through the security boundary and matching either of these two records will be classified as "PROTECTED" using IPsec transport mode. Traffic that does not match any records in the SPD but does match the local firewall access list will be allows to "BYPASS" the security boundary unperturbed. Traffic that does not match any records in the SPD will be "DISCARDED". Additional records are written into the SPD when additional trusted paths are configured for IPsec protection (i.e. remote audit $\log$, NTP and RADIUS server). 
NDcPP2OE:FCS_RBG_EXT.1:

The TOE implements RBG as defined ISO/EC 18031:2011 using CTR_DRBG (AES). The entropy source is a hardware based noise generator. Entropy is obtained from a zener diode operated in avalanche mode. The noise from the diode is passed through a cascaded pair of operational amplifiers, then applied to the input of a Microchip MCP3221. MCP3221 is a successive approximation analog to digital converter (ADC) with a 12 bit resolution. The TOE communicates with the MCP3221 hardware over the 2 -wire I2C and reads in the raw entropy. The raw entropy is further conditioned by the Linux kernel to produce 8 bits of entropy per byte. Then the random bytes are read by the DRBG implementation of 256 bits of DRBG key and DRBG seed.

\section{NDcPP20E: FCS TLSS EXT.1:}

The TOE's HTTPS server supports TLS version 1.1/1.2 only and will deny connection requests from TLS clients with a lower version. It supports the following ciphers:

- TLS_RSA_WITH_AES_128_CBC_SHA

- TLS_RSA_WITH_AES_256 CBC SHA

- TLS_DHE_RSA_WITH_AES_128_CBC_SHA

- tLS DHE RSA WTTH AES 256 CBC SHA

- TLS_RSA_WITH_AES_128_CBC_SHA256

- TLS_RSA_WITH_AES_256_CBC_SHA256

- TLS_DHE RSA WTTH AES 128 CBC SHA256

- TLS_DHE_RSA_WITH_AES_256_CBC_SHA256

The TOE's TLS/HTTPS server uses RSA 2048 bits certificate for TLS authentication. After the TLS session's successful setup, the security administrator logs into the TOE via user name and passwords. If the failure count reaches the configured threshold, the TLS/HTTPS session will be terminated by the TLS/HTTPS server. The DiffieHellman group 14 with parameters of size 2048 bits is used for the key agreement.

\subsection{Identification and authentication}

The Identification and authentication function satisfies the following security functional requirements

\section{WLANAScEP10:FIA 8021X EXT.1:}

When a wireless user attempts to associate to a given network, they must first associate with an Access Point (AP). The TOE maintains the userID and MAC address for the user (and their client) throughout the user's session. During the security policy discovery phase of 802.11 , the wireless client determines the security methods enforced by the TOE that are advertised by the AP. The Extensible Authentication Protocol (EAP) over LAN (EAPOL) protocol is used for communication between the wireless client and the TOE. The TOE functions as Authenticator as defined by $802.1 \mathrm{X}-2010$. The TOE strictly follows the port-based network control model as defined in section 7.1 of $802.1 \mathrm{X}-2010$

Once the wireless client and the TOE have negotiated the required security methods, the authentication phase of the process is initiated. The TOE acts as $802.1 \mathrm{X}$ authenticator and provides remote EAP-TLS authentication pass through to the RADIUS server. The TOE implements the Authenticator's state machine and counters as defined in section $8.9 \& 8.10$ of $802.1 \mathrm{X}-2010$. The TOE's authenticator module strictly enforces EAPOL PDU format as defined in section 11 of $802.1 \mathrm{X}-2010$. The TOE's authenticator implementation uses all $8021 . \mathrm{X}-2010$ mandatory definitions. No optional or non-conformance definitions of 802.1X are implemented. The TOE is Wi-Fi Alliance certified for WPA2 interoperability. The interoperability tests include many EAP methods, among which EAP-TLS is included. 
The TOE establishes IPsec tunnel with the RADIUS server to protect the EAPOL data packets between itself and the RADIUS server. The IPsec tunnel is also used to protect the PMK sent by the RADIUS server as part of RADIUS Access-Accept message. During this $802.1 \mathrm{x}$ authentication state, the TOE denies all packets sent by the client that are not 802.1x EAP packets.

After successful authentication of a wireless client, an IP address is also associated with the client. The IP address may be obtained from a DHCP server on the wired network, or if the client is not using DHCP, then the IP address already configured into the client will be used as an additional identifier for the client along with the MAC address.

NDCPP20E:FIA_AFL.1:

WLANAScEP10:FIA_AFL.1:

If the administrator's authentication fails with the TOE, the failure counter will be incremented. Once the failure counter reaches the configured threshold, the TOE's HTTPS server will refuse connection from this end point for an administrator. The authentication failure threshold is configurable by an authorized security administrator with a maximum value of 8 , the default value is 3 . Once a connection is refused, the administrator would have to re-login after the configurable lockout time period has elapsed, the login failure counter would be reset to 0 by then. The default lockout period is 10 minutes and is configurable between ranges of 0-90 minutes. In order to ensure that remote administrator authentication failures do not lead to a situation where no administrator access is available, an administrator account with local access can be configured with a lockout period of " 0 " minutes. When the value is set at 0 , the administrator won't be locked out even after the login threshold is reached

NDcPP20E:FIA_PMG_EXT.1:

The TOE supports the local definition of users with corresponding passwords. The passwords can be composed of any combination of upper and lower case letters, numbers, and the following special characters: ' $"$ ', ' $@$ ', ' $\#$ ', ' $\$$ ', "\%', ' $'$ ' ' $\&$ ', '*', ' $($ ', ' ')'. Minimum password length is settable by the Authorized Administrator and can be configured for minimum password lengths of 8 to 30 characters. Additionally, the TOE supports password lengths up to 32

characters long. NOTE: The TOE will truncate passwords that are longer than 32 characters when creating a user or changing passwords for an existing user.

\section{WLANASCEP10:FIA PSK EXT.1}

The TOE uses pre-shared keys for IPsec. The TOE accepts IPsec PSK keys between 16 and 32 characters in length. The TOE also supports PSK for wireless client authentication. The pre-shared key for wireless client is between 8 and 63 characters. The pre-shared keys must be composed of any combination of upper and lower case letters,

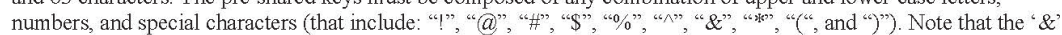
character is not supported for WPA2 pre-shared keys, but it is supported for IPsec pre-shared keys.

For IPsec, the TOE conditions the text-based pre-shared keys using the SHA-1, SHA-256, and SHA-384 hash algorithm and can accept bit based pre-shared keys. For WPA2, the TOE only accepts text based PSKs that are transformed into bit-based PSKs. Text-based keys are conditioned using PBKDF2, as specified in 802.11i.

WLANAScEP10:FIA_UAU.6:

An administrative user is required to re-authenticate when he/she changes password, and following a TSF-initiated locking as described in any of the FTA_SSL requirements in this ST

NDCPP20E:FIA_UAU.7:

When a user is entering their password information, the password is obscured such that no observer could read the password off the screen.

NDcPP20E:FIA_UAU_EXT.2: 
NDcPP20E:FIA_UIA_EXT.1:

The TOE requires all users to be successfully identified and authenticated before allowing any TSF mediated actions to be performed except for the login banner that is displayed prior to user authentication and any network packets as configured by the authorized administrator may flow through the TOE

The administrator logs on the TOE through either dedicated local Ethernet port or over WAN Ethernet port to access the Web UI. The Web UI is accessible over HTTPS only and the TOE supports TLS 1.1/1.2. There is no local access such as a serial console port. Therefore, the local and remote management is considered the same for this evaluation.

A successful authentication is determined by a successful username and password combination after the HTTPS connection. An incorrect password will result in a failed authentication attempt. The TOE does not provide a reason for failure in the cases of a login failure. The TOE supports local authentication using a local user database.

NDcPP20E:FIA_X509_EXT.1/Rev:

NDcPP20E:FIA_X509_EXT.2:

NDcPP20E:FIA X509_EXT.3:

The TOE uses X.509 certificates for IPsec authentications. The TOE can be configured with the certificates and their corresponding private key by security administrator or by creating CSRs and importing the CA signed CSRs to the TOE. The security administrator can load and delete certificates for usage of IPsec authentication, load and delete CAs, intermediated CAs and CRLs. The TOE checks that the basicConstraints extension and CA flag are set to TRUE for all CA certificates before their acceptance. During the IPsec authentication using X509 certificate, the TOE develops a certificate path from a trust anchor configured by security administrator which is fully compliant with RFC 5280. When a certificate chain is received from a peer, the TOE processes the certificate chain path until the first trusted certificate, or trust point, is reached. The TOE uses CRL that have been preloaded by the administrator to validate the peer certificates, and the TOE will accept as valid, a certificate for which the administrator has not loaded a CRL. Failure by the TOE to establish the certification path to a trust anchor will lead to the failure of establishment of this IPsec trusted channel.

The TOE allows the security administrator to view the certificates and CAs. The TOE's Web UT will display the certificates' name, subject name, issuer name, valid-start date, expiration date

\subsection{Security management}

The Security management function satisfies the following security functional requirements:

WLANAScEP10:FMT SMR.1:

By default, the TOE disallows wireless client devices to access the TOE's Web UT interface. For each wireless client that successfully authenticates with the TOE and obtains an IP address, the TOE will use its MAC address and IP address to build an entry in the TOE's access table to disallow the client's access to the TOE's Web UT.

NDcPP20E:FMT_MOF.1/ManualUpdate

NDcPP20E:FMT MTD. 1/CoreData:

INDCPP2OE:FMT_SMF.1:

NDCPP20E:FMT SMR.2:

The Web UI over HTTP/TLS provides capabilities for the authorized administrator to manage the TOE security functions and data. The TOE provides the role of Security Administrator (encompassing both the Crypto Officer and Administrator role as described in the guidance). The Security Administrator accesses the TOE through the Web UI over TLS/HTTPS. Upon successful authentication with the TOE, the Security Administrator role can perform the following operations: 
- Configure cryptographic functionality - generate/import a key, change a key, delete/zeroize a key, set a key lifetime, set the cryptographic algorithms, set the TOE to encrypt or not to encrypt wireless transmissions, execute self tests of the cryptographic functions.

- Configure lifetime for IPsec SAs

- Configure authentication failure parameters by setting the number of authentication failures that may occur before the TOE takes action to prevent future logins for an administrator defined time period.

- Configure session inactivity by setting the length of time a session may remain inactive before it is terminated.

- Configure the TOE Access Banner

- Configure the time used for timestamps on the TOE - set the time locally on the TOE, set the time using an NTP server

- Configure the reference identifier for the peer

- Configure the audit behavior - remote audit logging, audit storage exhaustion settings

- Perform TOE updates and verification of those updates using digital signatures.

- Configure Users - create and manage user accounts

- Reset Passwords - administrators can modify their own passwords

No Web UI interfaces are accessible to the user prior to authentication. The TOE enforces authentication then enables the TSF data configuration interfaces. The Non-security administrators have no access to those TSF data configuration interfaces.

\subsection{Protection of the TSF}

The Protection of the TSF function satisfies the following security functional requirements:

NDCPP20E:FPT_APW_EXT.1:

NDCPP20E:FPT_SKP_EXT.1:

The authentication passwords are stored in PKCS5 format in the TOE. All other CSPs are stored in encrypted format in the TOE on non-volatile memory. The file system that holds the hashed password and encrypted CSPs are made read-only during runtime to avoid data corruption. None of the files or CSPs is available through any external interfaces to users/administrators. The Web UI allows the security administrator to input keys/passwords to the TOE with no output capabilities.

\section{NDcPP20E:FPT STM EXT.1:}

The TOE has a running NTP daemon to synchronize the local time with an external NTP server. IPsec tunnel is setup between the TOE and NTP server to protect the integrity and privacy of the time source. In the absence of an NTP server in the Operational Environment, the authorized administrator has the capability to set the time locally The local time is used for the following security functions identified in this ST:

- Time stamping each audit record

- Verifying the validity of the Web Server X509v3 Certificate.

- Verifying the validity of the IPsec tunnel peer's Certificate.

- Verifying the validity of the Firmware X509v3 Certificate during the firmware upload process.

- Enforcing user lockout periods for "Bad Password" login attempts

- Timing out login sessions due to inactivity 
NDcPP20E:FPT_TST_EXT.1:

WLANAScEP10:FPT_TST_EXT.1

WLANAScEP10:FPT_FLS.1:

The TSF performs a firmware integrity check and a configuration file integrity check on system start up. Algorithm Known Answer Tests are run at startup time or at security administrator's request as shown below:

Power-on self-tests:

Software Integrity Test

- Bootloader Integrity Test

- Firmware Integrity Test

FreeScale PowerQUTCC Crypto Engine Power-on self-tests:

- AES_GCM

- AES CCM

- SHA-1, SHA256, SHA384, SHA512

- HMAC SHA-1, SHA256, SHA384, SHA512

encrypt/decrypt KAT

encrypt/decrypt KAT

KAT

KAT

3eTI OpenSSL library Power-on self-tests:

- HMAC SHA-1, SHA256, SHA384, SHA512

- SHA-1, SHA256, SHA384, SHA512

- FIPS SP800-90 DRBG

- RSA sign/verify

- ECDSA sign/verify

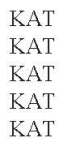

Vectors for each known answer test (KAT) are compiled into the Firmware. The known inputs are provided to the cryptographic function and the output of that function is compared to the known output. The firmware is halted if any of the known answer tests fail

After the device is powered on, the first thing done by the bootloader is to check its own integrity. If the integrity is broken, the firmware won't boot. Firmware integrity is performed at firmware boot up. Both firmware and bootloader are digitally signed with ECDSA.

NDcPP20E:FPT_TUD_EXT.1:

Security Administrators can query the currently active TOE version via the Web UI by going to the System Administration $>$ Help screen and clicking on the 'About' button. When a new version of firmware is released, the customers are notified by $3 \mathrm{eTI}$ 's customer service department, normally via e-mail. If a customer desires to get a copy of the new firmware, the customer will be provided with an URL link to the secured download site together with a onetime valid user name and password

The Security Administrator can update the TOE's firmware. The firmware is digitally signed with ECDSA. The TOE uses the public key to verify the digital signature. Upon successful verification, the TOE will load the new update upon reboot. The update will be rejected if the verification fails.

\subsection{TOE access}

The TOE access function satisfies the following security functional requirements:

NDcPP20E:FTA_SSL.3 
NDCPP20E:FTA_SSL 4 :

NDcPP20E:FTA_SSL_EXT.1:

The Web UI terminates the remote or local session if it detects inactivity longer than the configured time period. The default time period is 10 minutes. The remote session will be closed by the Web UI together with the HTTPS session. The Security Administrator is required to re-authenticate with the TOE and setup a new session. The time intervals are configurable by the security administrator.

\section{NDcPP20E:FTA_TAB.1:}

The Web UI displays a customizable TOE access banner to the remote administrative user before the user can log into the system.

\section{WLANAScEP10:FTA TSE.1:}

The TOE implements wireless client MAC address filtering functions. The security administrator can specify white list (allow) or black list (deny) or both. The list's attributes are IP address, client WiFi MAC address, time (hour and minute) and day.

An authorized security administrator can configure the TSF to deny establishment of a wireless client based on that client's location, time or day. The location is based on client MAC address as the client will usually move within one wireless Basic Service Set (BSS).

\subsection{Trusted path/channels}

The Trusted path/channels function satisfies the following security functional requirements

NDCPP20E:FTP_ITC. 1 :

WLANAScEP10:FTP_ITC.1:

The TOE provide a trusted communication channel between itself and all authorized IT entities that is logically distinct from other communication channels. IPsec is setup between the TOE and the audit log server, RADIUS and NTP server. The trusted channel can be initiated either by the TOE or by the remote IT entities. The TOE establishes WPA2 with 802.1x EAP-TLS connections with wireless clients and uses IPsec for communications with authentication servers.

NDcPP20E:FTP_TRP.1(1):

All remote administrative communications take place over a secure encrypted TLS session. The HTTPS/TLS implementation allows web browser clients to connect to the TOE's HTTPS server. The remote users are able to initiate TLS communications with the TOE 


\section{Appendix S: Tactical Microgrid Standard Data Distribution Service Implementation Guide}


MIL-STD-TMS 21 July 2017

\title{
TMS OMG DDS Implementation Guide
}

Release 1.5

For TMSC Draft Dated 1 February 2017

\author{
This draft, dated 29 March 2017, prepared by TMSC, has not been \\ approved for use and is subject to modification. Questions, \\ suggestions, or comments about the document or its maturity should be \\ addressed to Tom Bozada, the TMSC Project Manager, \\ Thomas.a.bozada@usace. army.mil.
}




\section{Contents}

1

1.1

2

2.1

2.2

2.3

3

3.1

3.2

3.3

3.4

4

4.1

4.2

1.3

4.4

4.5

4.6

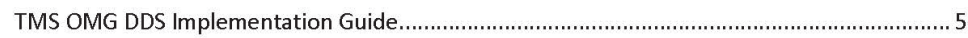

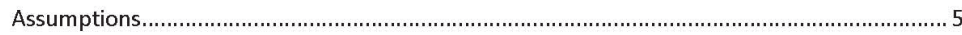

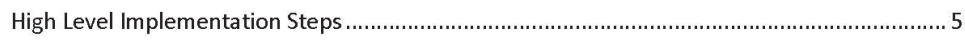

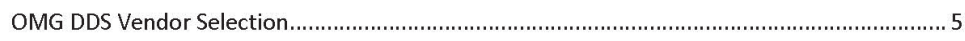

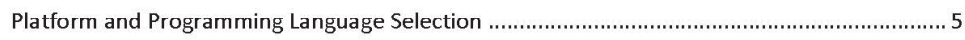

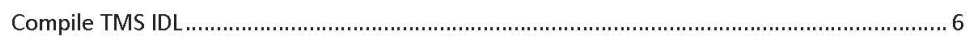

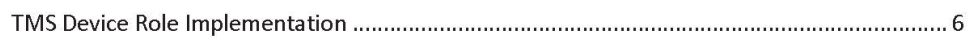

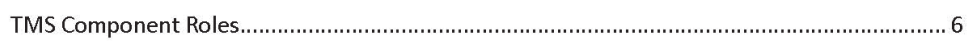

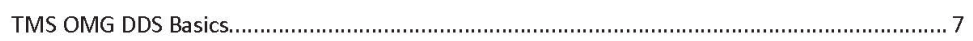

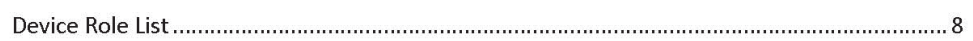

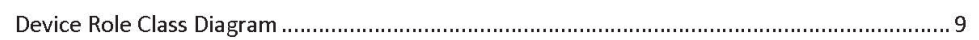

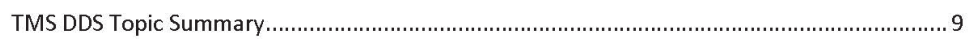

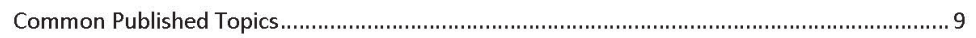

Power Device Published Topics (SRC, STOR, DIST, and LOAD) ......................................... 10

Source (SRC) and Storage (STOR) Published Topics..................................................... 10

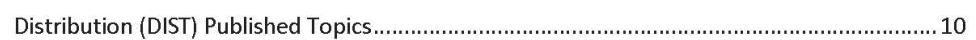

Load (LOAD) Published Topics (FUTURE) ..................................................................... 10

Microgrid System Manager (MSM) Published Topics ...................................................... 10

Microgrid Controller (MC) Published Topics.................................................................. 10

TMS DDS Topic Publishers and Subscribers ......................................................................11

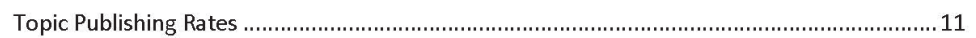

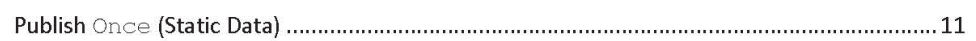

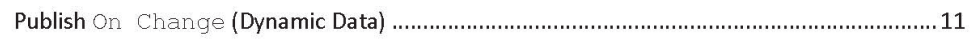

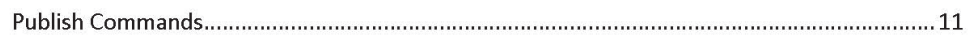

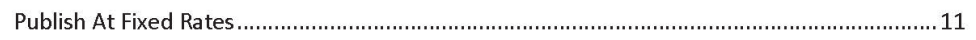

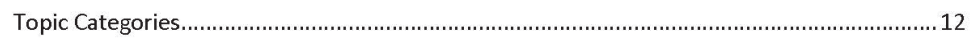

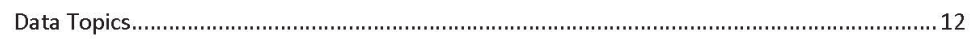

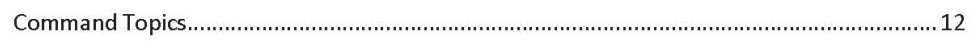

DDS Publisher and Subscriber Implementation ..............................................................12

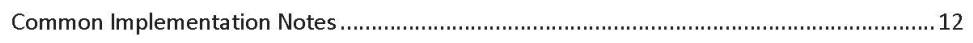

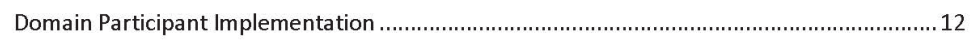




\begin{tabular}{|c|c|}
\hline 6.3 & 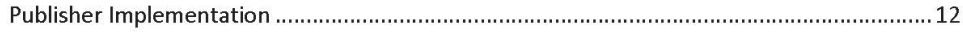 \\
\hline 6.4 & Subscriber Implementation .................................. \\
\hline 6.5 & DDS QoS Profiles \\
\hline 6.5 .1 & Publish Last Profile \\
\hline 6.5 .2 & Rate Based Profiles \\
\hline 6.5 .3 & Command Profile \\
\hline 6.5 .4 & QoS Settings \\
\hline 6.6 & Topic to Device Role Mapping \\
\hline 6.7 & Topic to IDL Data Type \\
\hline 6.8 & 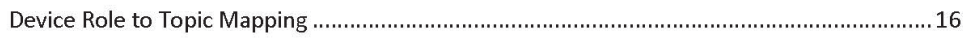 \\
\hline 7 & 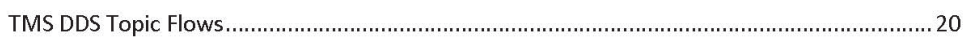 \\
\hline 7.1 & Device Discovery \\
\hline 7.2 & Source Startup \\
\hline 7.3 & Topology Discovery \\
\hline 7.4 & 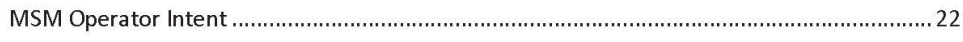 \\
\hline 7.5 & 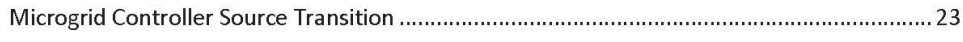 \\
\hline 7.6 & 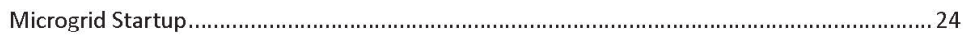 \\
\hline 7.7 & 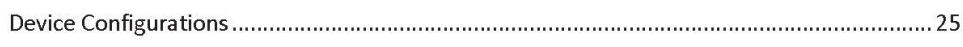 \\
\hline 7.7 .1 & 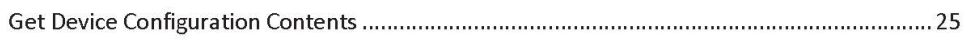 \\
\hline 7.7 .2 & Reserve, Copy and Release \\
\hline 7.8 & 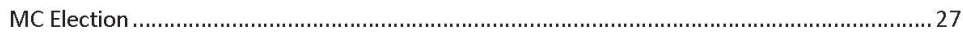 \\
\hline 8 & 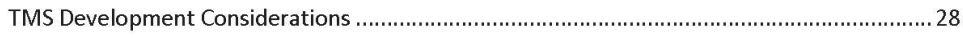 \\
\hline 8.1 & OMG DDS Vendor Interoperability \\
\hline 8.2 & 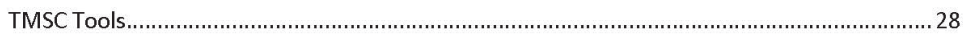 \\
\hline 8.2 .1 & 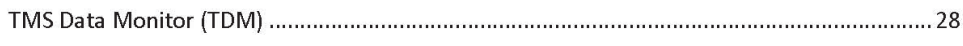 \\
\hline 8.2 .2 & EIO MSM (Future) \\
\hline 8.3 & Additional Tools \\
\hline 8.3 .1 & 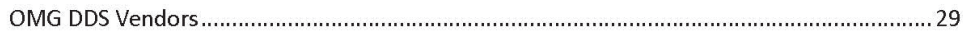 \\
\hline 8.3 .2 & Wireshark \\
\hline
\end{tabular}


MIL-STD-TMS 21 July 2017

\section{Table of Figures}

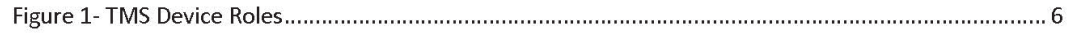

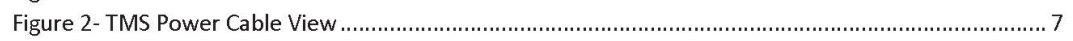

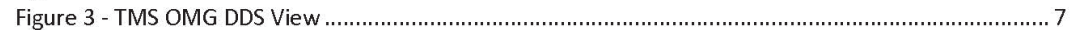

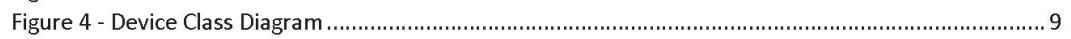

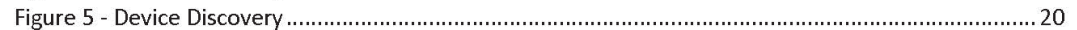

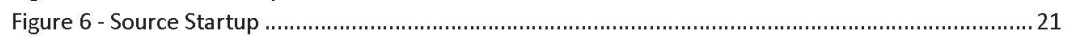

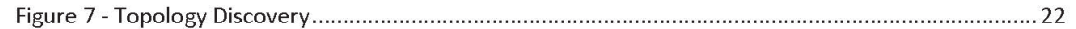

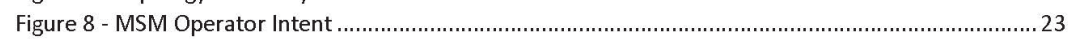

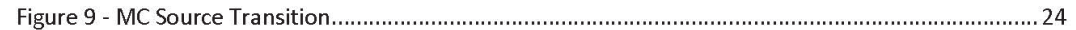

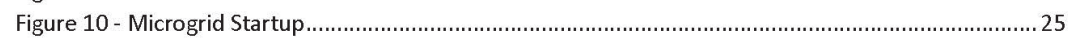

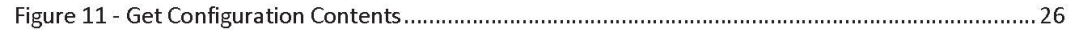

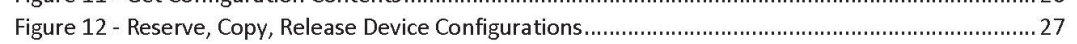


MIL-STD-TMS 21 July 2017

\section{Change Summary}

\begin{tabular}{|c|c|}
\hline Release & Changes \\
\hline 1.2 & $\begin{array}{l}\text { Added "GetConfig<Device Command>Response" topics for the response to the } \\
\text { GetConfigContentsRequest topic. Clarified USER_DATA QoS settings. Simplified } \\
\text { the tables defining the IDL data types and rates. }\end{array}$ \\
\hline 1.3 & Added new SecondaryControl Capabilities topic name. \\
\hline 1.4 & Added section on determining Device liveliness. \\
\hline 1.5 & $\begin{array}{l}\text { - Clarified Liveliness kind and lease duration for "Publish Last" QOS Profile. Only the } \\
\text { DeviceAnnoucement topic has a lease duration. } \\
\text { - Added Resource Limits to QOS Profiles } \\
\text { - Change "Continuous" QOS Profile deadline from } 1 \text { to } 2 \text { seconds. } \\
\text { - Added "Response" to QOS Profile to indicate which topics or published in response to } \\
\text { a command. The new "Response" profile has the same QOS settings as the } \\
\text { "Command" profile. } \\
\text { - Corrected topic "ReserveConfigReply" as a command "Response" QOS profile. } \\
\text { - Updated the tms.idl to reflect changes during the TMSC DDS IO Loop Check. }\end{array}$ \\
\hline & \\
\hline & \\
\hline
\end{tabular}


1 TMS OMG DDS Implementation Guide

This document provides additional details needed to implement the DEPARTMENT OF DEFENSE INTERFACE STANDARD TACTICAL MICROGRID STANDARD (TMS).

The following list are important references to sections in the TMS standards.

- Appendix B - Data Model Object Definitions

Defines the data types named in this document.

1.1 Assumptions

- The reader is familiar with "Publish / Subscribe" based architectures such as OMG DDS.

- The reader is familiar with UML.

- Data Model Provisional Sections are subject to change which may result in changes to this implementation guide.

- Assignment of TMS devices to a Microgrid not covered. Assume all devices are part of a single microgrid.

- Vendor specific OMG DDS implementation steps are not provided. An example is compilation of the tms.idl file. Refer to the selected OMG DDS Vendor Documentation for details.

2 High Level Implementation Steps

The following sections outline the high level steps required to implement a TMS compliant device.

2.1 OMG DDS Vendor Selection

The OMG DDS Standard is designed to be vendor-neutral. TMS IDL is tested to compile with products from the following vendors.

- $\underline{\text { RTI }}$

- Twin Oaks Computing INC.

- $\underline{\text { PrismTech }}$

The following OMG DDS Specifications are required for TMS.

- http://WWw.omg.org/spec/DDS/1.2

- http://Www.omg.org/spec/DDS|-RTPS/2.1

Future releases of TMS may require support for the following OMG DDS Extensions

- DDS-XTypes v1.1

- DDS-Security 1.0 Beta 1

- DDS-RPC V1.0 Beta 1

2.2 Platform and Programming Language Selection

As part of the OMG DDS vendor selection, ensure the vendor supports the target platform (hardware / operating system) and programming language. 
MIL-STD-TMS 21 July 2017

2.3 Compile TMS IDL

After acquiring the selected OMG DDS vendor software and setting up a target development environment, the next step is to compile the TMS IDL and generate code in the target programming language.

3 TMS Device Role Implementation

Implement the required TMS device roles based on the following sections.

3.1 TMS Component Roles

Figure 1-TMS Device Roles below illustrates the TMS device roles that are software-only or that include power ports. TMS currently plans to deploy the Microgrid Controller (MC) software as part of the Distribution (DIST) device but the 'TMS Software Only' device roles can be deployed on any computer networked with the other TMS devices.

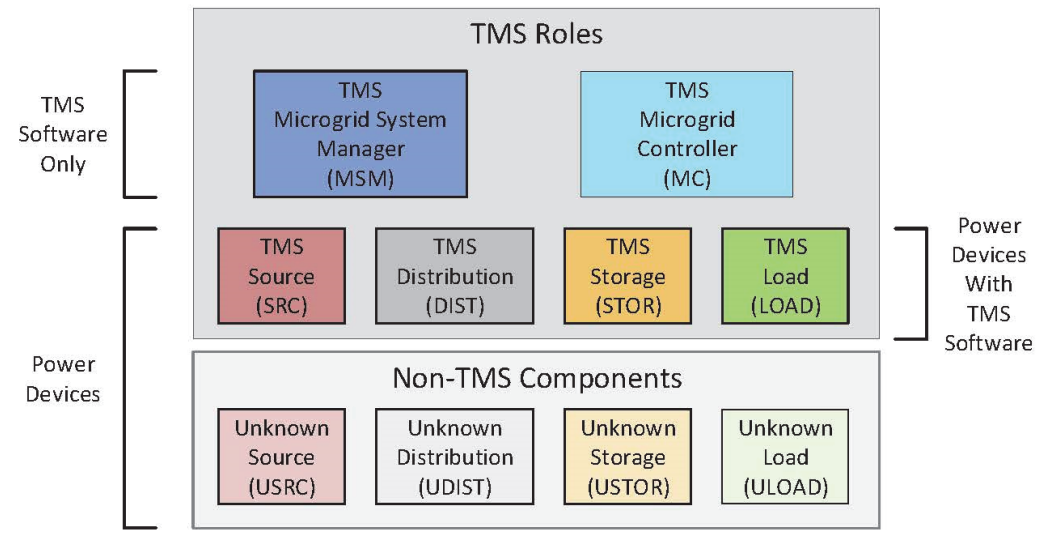

Figure 1-TMS Device Roles 
Figure 2-TMS Power Cable View below provides an example of the power connections between various TMS device roles.

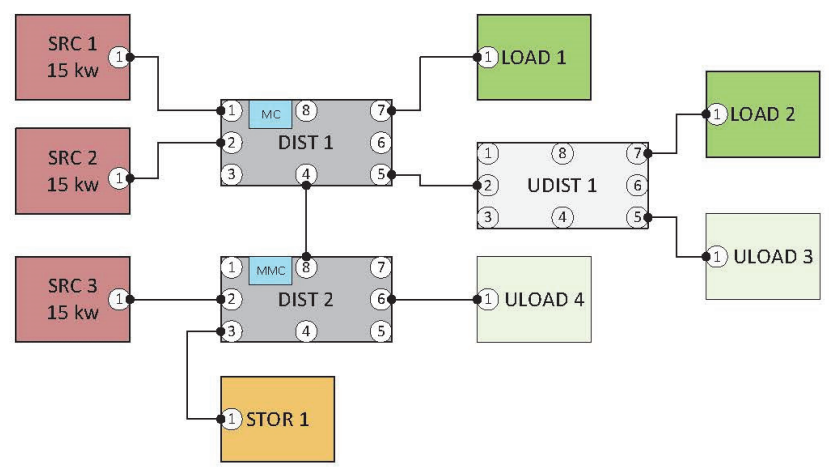

Figure 2- TMS Power Cable View

3.2 TMS OMG DDS Basics

A TMS compliant device uses OMG DDS standard for communications with other TMS compliant devices. Refer to an OMG DDS compliant vendor software product for additional details.

Figure 3 below provides the OMG DDS view of TMS devices roles all participating in the DDS global data exchange. Each link between the TMS device and the DDS cloud represent data being published or subscribed to by each device. This section focuses on the steps required to implement a TMS compliant device that interfaces/communicates with other TMS compliant devices using the DDS Global Data.

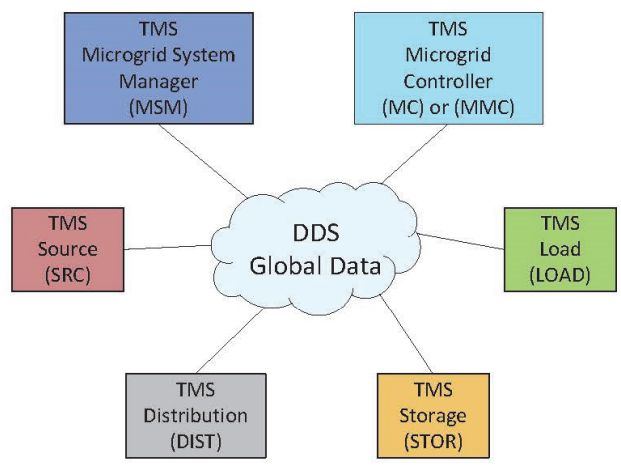

Figure 3 - TMS OMG DDS View 
3.3 Device Role List

The following table summarizes the TMS device roles used defined in the Data Model.

\begin{tabular}{|c|c|c|c|}
\hline Role & Description & $\begin{array}{l}\text { TMS } \\
\text { Compliant }\end{array}$ & $\begin{array}{l}\text { Required } \\
\text { Instances }\end{array}$ \\
\hline MSM & $\begin{array}{l}\text { Microgrid System Manager } \\
\text { Software component that serves as the } \\
\text { operator's primary Human Machine Interface } \\
\text { (HMI) used to monitor and configure the } \\
\text { Microgrid operations. }\end{array}$ & Yes & $\begin{array}{l}0 \text { - many } \\
\text { (typically } 1 \text { ) }\end{array}$ \\
\hline MC & $\begin{array}{l}\text { Microgrid Controller } \\
\text { Software component authorized to send } \\
\text { configuration settings and commands to other } \\
\text { TMS compliant devices in the Microgrid. }\end{array}$ & Yes & $\begin{array}{l}0 \text { - many } \\
\text { (typically } \\
\text { many) }\end{array}$ \\
\hline MGR & $\begin{array}{l}\text { Manager } \\
\text { An alias for the MSM and MC. MGR is used } \\
\text { later in this document to simplify the sequence } \\
\text { diagrams when either the MSM or MC can be } \\
\text { included. }\end{array}$ & Yes & $\begin{array}{l}0 \text { - many } \\
\text { (typically } \\
\text { many) }\end{array}$ \\
\hline MMC & $\begin{array}{l}\text { Master Microgrid Controller } \\
\text { The elected master MC. Only the MMC will } \\
\text { send configuration settings and commands to } \\
\text { the other TMS compliant Microgrid devices. }\end{array}$ & Yes & $\begin{array}{l}0-1 . \text { Exactly } \\
1 \text { if MC } \\
\text { included }\end{array}$ \\
\hline SRC & $\begin{array}{l}\text { Source Power Device } \\
\text { Hardware component that provides energy to } \\
\text { the Microgrid. Note: A microgrid will typically } \\
\text { include at least one SRC or STOR device. }\end{array}$ & Yes & $\begin{array}{l}0-\text { many } \\
\text { (typically } \\
\text { many) }\end{array}$ \\
\hline USRC & Unknown Source & No & 0 - many \\
\hline STOR & $\begin{array}{l}\text { Storage Power Device } \\
\text { Hardware component that consumes energy } \\
\text { with the intent to provide it later. }\end{array}$ & Yes & 0 - many \\
\hline DIST & $\begin{array}{l}\text { Distribution Power Device } \\
\text { Hardware component with cabling and } \\
\text { switching that transports energy }\end{array}$ & Yes & $\begin{array}{l}0-\text { many } \\
\text { (typically } \\
\text { many) }\end{array}$ \\
\hline LOAD & $\begin{array}{l}\text { Load Power Device } \\
\text { Hardware component that consumes energy } \\
\text { from the Microgrid. }\end{array}$ & Yes & 0 - many \\
\hline ULOAD & $\begin{array}{l}\text { Unknown Load } \\
\text { Hardware component that consumes energy } \\
\text { from the Microgrid. It is classified as } \\
\text { "unknown" because it is a non-TMS compliant } \\
\text { device or communications is currently } \\
\text { unavailable. }\end{array}$ & Yes or No & 0 - many \\
\hline DEV & $\begin{array}{l}\text { Device } \\
\text { Denotes a generic device that is participating in } \\
\text { the microgrid communications. Includes MSM, } \\
\text { MC, SRC, STOR, DIST, and LOAD }\end{array}$ & Yes & - \\
\hline
\end{tabular}




\begin{tabular}{|l|l|l|l|}
\hline PDEV & $\begin{array}{l}\text { Power Device } \\
\text { Denotes a generic device that provides, } \\
\text { consumes, or transports energy. Includes SRC, } \\
\text { STOR, DIST, and LOAD. PDEV is used later in } \\
\text { this document to simplify the sequence } \\
\text { diagrams when any of the power device roles } \\
\text { are allowed. }\end{array}$ & Yes & - many \\
\hline
\end{tabular}

3.4 Device Role Class Diagram

The following class diagram illustrates the TMS Device hierarchy.

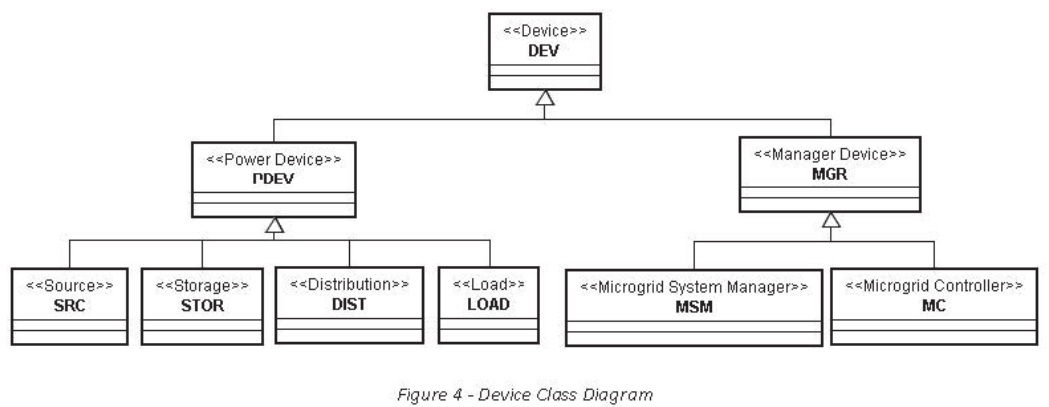

4 TMS DDS Topic Summary

This section summarizes the individual topics that are required to implement the TMS Device Roles. A topic is defined by a unique "Topic Name". Each topic has an associated data type defined in the

tms.idl. The topic names give additional meaning to the data type as multiple topics can share the same IDL data type. Every topic has one or more publishers and one or more subscribers. The publishers and subscribers are allocated to the different TMS device roles. See the Topics Spreadsheet for the publisher subscriber allocations by TMS device role.

The topics are summarized from the publisher's perspective. See the Topic Spreadsheet for the corresponding subscribers.

4.1 Common Published Topics

This section describes the topics that are published by all TMS device roles.

- Device Announcement: Used to discover TMS compliance devices.

- Device Clock Status: Reports changes to a device dock(s). A device must support relative time and can optional support absolute time.

- Active Diagnostics: Reports a devices diagnostics state including information and alarm states.

- Request Response: Used to implement a generic request (command) and response (reply) pattern. 
4.2 Power Device Published Topics (SRC, STOR, DIST, and LOAD)

This section describes the topics that all TMS Power Devices publish. A power device is any device that provides, consumes, or transports energy.

- Device Power Port List: Reports static data for a devices power ports.

- Device Power Status List: Reports dynamic state changes for a devices power ports.

- Discovered Connection List: Reports device discovered power connections to a devices power ports.

- Device Power Measurement List: Reports dynamic power measurements for a devices power ports.

4.3 Source (SRC) and Storage (STOR) Published Topics

This sections describes the topics all TMS SRC and STOR device roles publish.

- Engine State: Reports dynamic state changes of a SRC/STOR device if an engine is present.

- Source Transition State: Reports the operational state (off, on, running, sourcing) of the SRC/STOR device

4.4 Distribution (DIST) Published Topics

The DIST devices publish the topics already described in section 4.1 Common Published Topics and section 4.2 Power Device Published Topics (SRC, STOR, DIST, and LOAD)

4.5 Load (LOAD) Published Topics (FUTURE)

This section describes the topics that only the LOAD device role publishes.

- Energy Ration: Reports the LOAD devices energy consumption limits (power budget).

4.6 Microgrid System Manager (MSM) Published Topics

This section describes the topics that are only published by the MSM device role.

- Operator Connection List: Part of topology discovery to report operator device power connections when a device does not support connection discovery.

- Operator Intent Request: Used to set a microgrid control directives that influence how the microgrid behaves.

4.7 Microgrid Controller (MC) Published Topics

This section describes the topics that are only published by the MC device role.

- Microgrid Connection List: Part of topology discovery used to report the effective microgrid topology.

- Load Sharing: Used to configure and select microgrid load sharing algorithms.

- Consensus Protocol (FUTURE): Used to implement distributed control across the microgrid.

- Source Transition Request: User to request a change in a SRC/STOR device state.

- Device Configurations: Used to define a set of commands for execution by a power device. 
5 TMS DDS Topic Publishers and Subscribers

This section describes key concepts required to implement the publishers and subscribers. The concepts discussed include the publishing rates, topic categories, DDS implementation, and DDS QoS profiles.

5.1 Topic Publishing Rates

The rate a topic is published is specified by the "QoS Profile", "Publisher Rate" columns in the Topics Spreadsheet. The following table summarizes the supported rates of a TMS device.

\begin{tabular}{|l|l|l|}
\hline $\begin{array}{l}\text { Publisher Rate } \\
\text { Names }\end{array}$ & Data Model Rate & Publisher Rate Summary \\
\hline Once & Rate-0 & Only published once after device joins a DDS domain. \\
\hline On Change & Rate-0 & $\begin{array}{l}\text { Published after device joins a DDS domain and again } \\
\text { when a previously published value has changed. }\end{array}$ \\
\hline Command & Command & $\begin{array}{l}\text { Published when a device state change is being } \\
\text { requested. See section } 5.2 .2 .\end{array}$ \\
\hline Fixed Maximum Rates & Rate-1ks & Published every 1000 seconds. \\
\hline Rare & Rate-10s & Published every 10 seconds. \\
\hline Slow & Rate-01s & Published every 100 milliseconds. \\
\hline Continuous & Rate-10s-bur & Published in bursts 10 seconds. \\
\hline Burst & \multicolumn{2}{|l|}{ Table 2 - Publisher Rates }
\end{tabular}

5.1.1 Publish Once (Static Data)

The publisher must publish the topic once. The data is static and is not expected to change for the time the device is part of the current microgrid. An example of a Once topic is the rated power of a generator.

5.1.2 Publish On Change (Dynamic Data)

The publisher is expected to publish the topics when the device data defined for the topic changes from the previously published value. These topics are expected to change over time but at no pre-

determined rate. An example of an On Change topic is the position of a power switch / contactor on a distribution box.

5.1.3 Publish Commands

The topic represents a command/request from the publisher to the subscribing device to perform a state change. See Command Topics below.

5.1.4 Publish At Fixed Rates

The publisher must publish the topic at the specified rate, even if the values have not changed from the previous published data. Topics assigned to fixed rate published are expected to change frequently and the liveliness of the published data determines the overall state of the microgrid. An example of a fixed rate topic is the active power measured at a power port of a device. 
MIL-STD-TMS 21 July 2017

5.2 Topic Categories

Topics are divided into two categories, Data Topics or Command Topics.

5.2.1 Data Topics

Data Topics are published asynchronously by a TMS device to share static configuration data and

dynamic state changes. An example Data Topic is EngineStatus or DeviceAnnouncement.

5.2.2 Command Topics

Command Topics are published by a TMS device when some action or device state change is required on another TMS device. A TMS device receives a Command Topic by subscribing to specific topic names.

Then, the receiving TMS device publishes a generic CommandResponse topic to report on the results of the specific Command Topic. In addition to the generic CommandResponse the device will publish one or more additional Data Topics that report dependent device state changes. Example Command topics are included in later sections.

6 DDS Publisher and Subscriber Implementation

This section outlines the basic steps a device role must follow to implement to publish or subscribe to a DDS topic.

6.1 Common Implementation Notes

IMPORTANT: Since DDS is inherently asynchronous, no assumptions can be made about the order different topics are received by a subscriber.

6.2 Domain Participant Implementation

A single DDS Domain Participant is required per TMS device.

Create a DDS Domain Participant with a configurable domain ID. Recommendation is to make the domain ID runtime configurable to allow for flexibility during testing and deployment.

6.3 Publisher Implementation

A DDS publisher publishes to a topic with the following steps. In general the first four steps occur during device startup. Only the last step is required to publish additional data for a specific topic. These are the logical steps to publish a message, the actual required steps depend on the selected OMG DDS vendor.

a) Creates a DDS Publisher

b) Registers the IDL Data Type specified for the topic.

c) Creates a DDS Topic with the specified Topic Name and registered IDL Data Type.

d) Creates a DDS DataWriter for the specified IDL Data Type and QoS profile. See Table 3 - QoS Settings Part 1 for details.

e) Invokes the write method on the DDS DataWriter with an instance of the IDL Data Type specified for the topic. 
6.4 Subscriber Implementation

A DDS subscriber subscribes to a topic with the following steps. These steps are repeated for each subscriber topic a device role implements. These are the logical steps to subscribe to a topic, the actual required steps depend on the selected OMG DDS vendor.

a) Creates a DDS Subscriber

b) Registers the IDL Data Type specified for the topic.

c) Creates a DDS Topic with the specified Topic Name and registered IDL Data Type.

d) Creates a DDS DataReader for the specified IDL Data Type and QoS profile.

e) Either

a. Invoke the read method on the DDS DataReader to poll for the subscribed data or

b. Registers a DataReader listener to receive the subscribed data asynchronously.

6.5 DDS QoS Profiles

OMG DDS publishers and subscribers use Quality of Service (QoS) settings to control the behavior of individual topics. This section describes the QoS profiles used by TMS. Refer to the Topics Spreadsheet "QoS Profiles" tab for detailed settings.

\subsubsection{Publish Last Profile}

The Publish Last QoS profile is used by publisher rates "Once" and "On Change". This profile ensures that the order publishers and subscribes join the DDS domain does not impact the availability of the topic data. The last published data is cached inside the DDS middleware for re-publishing as needed. There are two possible cases;

1) Subscriber is running before the Publisher. In this case, when the publisher writes topic data, the subscriber is already running and receives the published data.

2) Publisher is running before the Subscriber. In this case, the subscriber starts after a publisher has written topic data. Using the Publish Last profile, the DDS middleware recognizes the new subscriber matches the published data and re-sends the cached last published topic data to the new subscriber. This behavior is transparent to the publishers reducing the complexity of application.

6.5.2 Rate Based Profiles

The next set of QoS profiles is used by all fixed rate publishers. That includes Rare, Sl ow, Continuous, and Burst. Since fixed rate publishers are expected to publish at a predetermined interval, the rate based profiles is used to trigger "liveliness" changes in the subscriber when the publishers fails to meet the predetermined interval. This liveliness trigger allows the subscriber to detect the missing time sensitive topic data and take appropriate actions.

6.5.3 Command Profile

The final QoS profile is the Command profile. This profile is used for all Conmand Topics described in section 5.2.2 Command Topics.

\subsubsection{QoS Settings}

The following two tables define the QoS settings by QoS Profile and Publisher Rate. These QoS settings must be specified for each DDS DataWriter and Data Reader using the vendor specific technique. The QoS settings are specified using two tables for formatting purposes only. 
MIL-STD-TMS 21 July 2017

\begin{tabular}{|l|l|l|l|r|r|r|}
\hline & & User Data & \multicolumn{2}{|c|}{ History } & \multicolumn{2}{|c|}{ kineliness } \\
\hline $\begin{array}{l}\text { QoS Profile } \\
\text { (Topic Name) }\end{array}$ & $\begin{array}{l}\text { Publisher } \\
\text { Rate }\end{array}$ & value & kind & depth & $\begin{array}{r}\text { lease } \\
\text { duration } \\
\text { (seconds) }\end{array}$ \\
\hline $\begin{array}{l}\text { Publish Last } \\
\text { (DeviceAnnoucement) }\end{array}$ & $\begin{array}{l}\text { On } \\
\text { change }\end{array}$ & Fingerprint & KEEP_LAST & 1 & MANUAL BY \\
\hline $\begin{array}{l}\text { Publish Last } \\
\text { (All Other Topics) }\end{array}$ & $\begin{array}{l}\text { On } \\
\text { change }\end{array}$ & Fingerprint & KEEP_LAST & 1 & AUTOMATIC & INFINITE $^{2}$ \\
\hline Rare & rate-1ks & Fingerprint & KEEP_LAST & 1 & AUTOMATIC & INFINITE \\
\hline Slow & rate-10s & Fingerprint & KEEP_LAST & 1 & AUTOMATIC & INFINITE \\
\hline Continuous & rate-01s & Fingerprint & KEEP_LAST & 1 & AUTOMATIC & INFINITE \\
\hline Burst & $\begin{array}{l}\text { rate-10s- } \\
\text { burst }\end{array}$ & Fingerprint & KEEP_LAST & 1 & AUTOMATIC & INFINITE \\
\hline Command & Command & Fingerprint & KEEP_LAST & 1 & AUTOMATIC & INFINITE \\
\hline Response & Response & Fingerprint & KEEP_LAST & 1 & AUTOMATIC & INFINITE \\
\hline
\end{tabular}

\begin{tabular}{|c|c|c|c|c|c|c|}
\hline & & Reliability & Durability & Deadline & Ownership & $\begin{array}{l}\text { Resource } \\
\text { Limits }\end{array}$ \\
\hline QoS Profile & $\begin{array}{l}\text { Publisher } \\
\text { Rate }\end{array}$ & kind & kind & $\begin{array}{l}\text { period } \\
\text { (seconds) }\end{array}$ & kind & \\
\hline Publish Last & On change & RELIABLE & TRANSIENT_LOCAL & INFINITE & EXCLUSIVE & None $^{3}$ \\
\hline Rare & rate-1ks & BEST_EFFORT & VOLATILE & 2000 & EXCLUSIVE & None \\
\hline Slow & rate-10s & BEST_EFFORT & VOLATILE & 20 & EXCLUSIVE & None \\
\hline Continuous & rate-01s & BEST_EFFORT & VOLATILE & 2 & EXCLUSIVE & None \\
\hline Burst & $\begin{array}{l}\text { rate-10s- } \\
\text { burst }\end{array}$ & BEST_EFFORT & VOLATILE & INFINITE & EXCLUSIVE & None \\
\hline Command & Command & RELIABLE & VOLATILE & INFINITE & EXCLUSIVE & None \\
\hline Response & Response & RELIABLE & VOLATILE & INFINITE & EXCLUSIVE & None \\
\hline
\end{tabular}

6.5.5 Device Liveliness

6.6 Topic to Device Role Mapping

The following table provides a mapping from a TMS topic name to the device roles that publish or subscribe to each role.

NOTE: The topic names are included in the TMS IDL as constant strings named TOPIC_<topic name>.

\begin{tabular}{|l|l|l|l|l|l|l|}
\hline Topic Name & MSM & MC & SRC & STOR & DIST & LOAD \\
\hline ActiveDiagnostics & sub & pub/sub & pub & pub & pub & pub \\
\hline ConfigReservationState & - & sub & pub & pub & pub & pub \\
\hline
\end{tabular}

${ }^{1}$ The 32 byte unique device ID.

${ }^{2}$ OMG DDS Vendor specific value for infinite number of seconds.

${ }^{3}$ Based on future performance testing, this is likely to change. 
MIL-STD-TMS 21 July 2017

\begin{tabular}{|l|l|l|l|l|l|l|}
\hline CopyConfigRequest & - & pub & sub & sub & sub & sub \\
\hline DeviceAnnouncement & pub/sub & pub/sub & pub/sub & pub/sub & pub/sub & pub/sub \\
\hline DeviceClockStatus & pub/sub & pub/sub & pub & pub & pub & pub \\
\hline DevicePowerMeasurementList & sub & sub & pub & pub & pub & pub \\
\hline DevicePowerPortList & sub & sub & pub & pub & pub & pub \\
\hline DevicePowerStatusList & sub & sub & pub & pub & pub & pub \\
\hline DiscoveredConnectionList & - & sub & pub & pub & pub & pub \\
\hline EngineState & sub & sub & pub & - & - & - \\
\hline GetConfigContentsRequest & - & pub & sub & sub & sub & sub \\
\hline GetConfigPowerSwitchResponse & - & sub & pub & pub & pub & pub \\
\hline GetConfigPrimaryControIResponse & - & sub & pub & pub & - & - \\
\hline GetConfigSecondaryControlResponse & - & sub & pub & pub & - & - \\
\hline GetConfigSourceTransitionResponse & - & sub & pub & pub & - & - \\
\hline MicrogridConnectionList & sub & pub & - & - & - & - \\
\hline OperatorConnectionList & pub & sub & - & - & - & - \\
\hline OperatorIntentRequest & pub & sub & - & - & - & - \\
\hline OperatorIntentState & sub & pub & - & - & - & - \\
\hline PowerSwitchRequest & - & pub & - & - & sub & - \\
\hline PrimaryControIRequest & - & pub & sub & sub & - & - \\
\hline ReleaseConfigRequest & - & pub & sub & sub & sub & sub \\
\hline RequestResponse & sub & pub/sub & pub & pub & pub & pub \\
\hline ReserveConfigReply & - & sub & pub & pub & pub & pub \\
\hline ReserveConfigRequest & - & pub & sub & sub & sub & sub \\
\hline SecondaryControICapabilities & - & sub & pub & pub & - & - \\
\hline SecondaryControIRequest & - & pub & sub & sub & - & - \\
\hline SourceTransitionRequest & sub & sub & sub & sub & - & - \\
\hline SourceTransitionState & - & pub & pub & - & - \\
\hline StandardConfigMaster & pub & pub & pub & pub \\
\hline
\end{tabular}

6.7 Topic to IDL Data Type

The following table defined the IDL data type and publisher rate for each of the TMS topic names.

\begin{tabular}{|l|l|l|}
\hline Topic Name & IDL Data Type & $\begin{array}{l}\text { Publisher } \\
\text { Rate }\end{array}$ \\
\hline ActiveDiagnostics & tms.ActiveDiagnosticMessages & On Change \\
\hline ConfigReservationState & tms.ConfigReservationList & On Change \\
\hline CopyConfigRequest & tms.CopyConfigRequest & Command \\
\hline DeviceAnnouncement & tms.Productlnfo & Once \\
\hline DeviceClockStatus & tms.ClockStatus & On Change \\
\hline DevicePowerMeasurementList & tms.DevicePowerMeasurements & Continuous \\
\hline DevicePowerPortList & tms.DevicePowerPortTypes & Once \\
\hline DevicePowerStatusList & tms.DevicePowerPortStatuses & On Change \\
\hline DiscoveredConnectionList & tms.PowerConnectionList & On Change \\
\hline
\end{tabular}


MIL-STD-TMS 21 July 2017

\begin{tabular}{|l|l|l|}
\hline EngineState & tms. EngineState & Slow \\
\hline GetConfigContentsRequest & tms.GetConfigContentsRequest & Command \\
\hline GetConfigPowerSwitchResponse & tms.PowerSwitchCommand & Response \\
\hline GetConfigPrimaryControIResponse & tms.PrimaryControlRequest & Response \\
\hline GetConfigSecondaryControIResponse & tms.SecondaryControlRequest & Response \\
\hline GetConfigSourceTransitionResponse & tms.SourceTransitionRequest & Response \\
\hline MicrogridConnectionList & tms.PowerConnectionList & On Change \\
\hline OperatorConnectionList & tms.PowerConnectionList & On Change \\
\hline OperatorIntentRequest & tms. OperatorlntentRequest & Command \\
\hline OperatorIntentState & tms.OperatorIntentState & On Change \\
\hline PowerSwitchRequest & tms.PowerSwitchCommand & Command \\
\hline PrimaryControIRequest & tms.PrimaryControlRequest & Command \\
\hline ReleaseConfigRequest & tms. ReleaseConfigRequest & Command \\
\hline RequestResponse & tms.RequestResponse & Response \\
\hline ReserveConfigReply & tms. ReserveConfigReply & Response \\
\hline ReserveConfigRequest & tms. ReserveConfigRequest & Command \\
\hline SecondaryControlCapabilities & tms.SecondaryControlCapabilities & Once \\
\hline SecondaryControIRequest & tms.SecondaryControlRequest & Command \\
\hline SourceTransitionRequest & tms.SourceTransitionRequest & Command \\
\hline SourceTransitionState & tms.SourceTransitionState & On Change \\
\hline StandardConfigMaster & tms.StandardConfigMaster & Slow \\
\hline & Table 6 TopicIDL Data Types & \\
\hline
\end{tabular}

6.8 Device Role to Topic Mapping

The following table provides a mapping from the TMS Device Roles to the topics publish or subscribed to. The table contains similar information to

\begin{tabular}{|l|l|l|l|l|l|l|}
\hline ActiveDiagnostics & sub & pub/sub & pub & pub & pub & pub \\
\hline ConfigReservationState & - & sub & pub & pub & pub & pub \\
\hline CopyConfigRequest & - & pub & sub & sub & sub & sub \\
\hline DeviceAnnouncement & pub/sub & pub/sub & pub/sub & pub/sub & pub/sub & pub/sub \\
\hline DeviceClockStatus & pub/sub & pub/sub & pub & pub & pub & pub \\
\hline DevicePowerMeasurementList & sub & sub & pub & pub & pub & pub \\
\hline DevicePowerPortList & sub & sub & pub & pub & pub & pub \\
\hline DevicePowerStatusList & sub & sub & pub & pub & pub & pub \\
\hline DiscoveredConnectionList & - & sub & pub & pub & pub & pub \\
\hline EngineState & sub & sub & pub & - & - & - \\
\hline GetConfigContentsRequest & - & pub & sub & sub & sub & sub \\
\hline GetConfigPowerSwitchResponse & - & sub & pub & pub & pub & pub \\
\hline GetConfigPrimaryControlResponse & - & sub & pub & pub & - & - \\
\hline GetConfigSecondaryControlResponse & - & sub & pub & pub & - & - \\
\hline GetConfigSourceTransitionResponse & - & sub & pub & pub & - & - \\
\hline MicrogridConnectionList & sub & pub & - & - & - & - \\
\hline OperatorConnectionList & pub & sub & - & - & - & - \\
\hline
\end{tabular}


MIL-STD-TMS 21 July 2017

\begin{tabular}{|l|l|l|l|l|l|l|}
\hline OperatorIntentRequest & pub & sub & - & - & - & - \\
\hline OperatorIntentState & sub & pub & - & - & - & - \\
\hline PowerSwitchRequest & - & pub & - & - & sub & - \\
\hline PrimaryControlRequest & - & pub & sub & sub & - & - \\
\hline ReleaseConfigRequest & - & pub & sub & sub & sub & sub \\
\hline RequestResponse & sub & pub/sub & pub & pub & pub & pub \\
\hline ReserveConfigReply & - & sub & pub & pub & pub & pub \\
\hline ReserveConfigRequest & - & pub & sub & sub & sub & sub \\
\hline SecondaryControlCapabilities & - & sub & pub & pub & - & - \\
\hline SecondaryControlRequest & - & pub & sub & sub & - & - \\
\hline SourceTransitionRequest & - & pub & sub & sub & - & - \\
\hline SourceTransitionState & sub & sub & pub & pub & - & - \\
\hline StandardConfigMaster & sub & sub & pub & pub & pub & pub \\
\hline
\end{tabular}

Table 5 - Topic Name to Device Roles but is organized by device role.

\begin{tabular}{|l|l|l|}
\hline Device Role & Pub/Sub & Topic Name \\
\hline Distribution / DIST & pub & ActiveDiagnostics \\
\hline & pub & ConfigReservationState \\
\hline & pub & DeviceAnnouncement \\
\hline & pub & DeviceClockStatus \\
\hline & pub & DevicePowerMeasurementList \\
\hline & pub & DevicePowerPortList \\
\hline & pub & DevicePowerStatusList \\
\hline & pub & DiscoveredConnectionList \\
\hline & pub & GetConfigPowerSwitchResponse \\
\hline & pub & RequestResponse \\
\hline & pub & ReserveConfigReply \\
\hline & pub & StandardConfigMaster \\
\hline & sub & CopyConfigRequest \\
\hline & sub & DeviceAnnouncement \\
\hline & sub & GetConfigContentsRequest \\
\hline & sub & PowerSwitchRequest \\
\hline & sub & ReleaseConfigRequest \\
\hline & sub & ReserveConfigRequest \\
\hline & pub & ActiveDiagnostics \\
\hline & pub & ConfigReservationState \\
\hline & pub & DeviceAnnouncement \\
\hline & pub & DeviceClockStatus \\
\hline Load/LOAD & pub & DevicePowerMeasurementList \\
\hline & pub & DevicePowerPortList \\
\hline & pub & DevicePowerStatusList \\
\hline & pub & DiscoveredConnectionList \\
\hline & pub & GetConfigPowerSwitchResponse \\
\hline & & \\
\hline & & \\
\hline & &
\end{tabular}


MIL-STD-TMS 21 July 2017

\begin{tabular}{|c|c|c|}
\hline Device Role & Pub/Sub & Topic Name \\
\hline & pub & RequestResponse \\
\hline & pub & ReserveConfigReply \\
\hline & pub & StandardConfigMaster \\
\hline & sub & CopyConfigRequest \\
\hline & sub & DeviceAnnouncement \\
\hline & sub & GetConfigContentsRequest \\
\hline & sub & ReleaseConfigRequest \\
\hline & sub & ReserveConfigRequest \\
\hline \multirow[t]{34}{*}{ MicrogridController / MC } & pub & ActiveDiagnostics \\
\hline & pub & CopyConfigRequest \\
\hline & pub & DeviceAnnouncement \\
\hline & pub & DeviceClockStatus \\
\hline & pub & GetConfigContentsRequest \\
\hline & pub & MicrogridConnectionList \\
\hline & pub & OperatorIntentState \\
\hline & pub & PowerSwitchRequest \\
\hline & pub & PrimaryControlRequest \\
\hline & pub & ReleaseConfigRequest \\
\hline & pub & RequestResponse \\
\hline & pub & ReserveConfigRequest \\
\hline & pub & SecondaryControlRequest \\
\hline & pub & SourceTransitionRequest \\
\hline & sub & ActiveDiagnostics \\
\hline & sub & ConfigReservationState \\
\hline & sub & DeviceAnnouncement \\
\hline & sub & DeviceClockStatus \\
\hline & sub & DevicePowerMeasurementList \\
\hline & sub & DevicePowerPortList \\
\hline & sub & DevicePowerStatusList \\
\hline & sub & DiscoveredConnectionList \\
\hline & sub & EngineState \\
\hline & sub & GetConfigPowerSwitchResponse \\
\hline & sub & GetConfigPrimaryControlResponse \\
\hline & sub & GetConfigSecondaryControlResponse \\
\hline & sub & GetConfigSourceTransitionResponse \\
\hline & sub & OperatorConnectionList \\
\hline & sub & OperatorIntentRequest \\
\hline & sub & RequestResponse \\
\hline & sub & ReserveConfigReply \\
\hline & sub & SecondaryControlCapabilities \\
\hline & sub & SourceTransitionState \\
\hline & sub & StandardConfigMaster \\
\hline
\end{tabular}


MIL-STD-TMS 21 July 2017

\begin{tabular}{|c|c|c|}
\hline Device Role & Pub/Sub & Topic Name \\
\hline \multirow{16}{*}{$\begin{array}{l}\text { MicrogridSystemManager / } \\
\text { MSM }\end{array}$} & pub & DeviceAnnouncement \\
\hline & pub & DeviceClockStatus \\
\hline & pub & OperatorConnectionList \\
\hline & pub & OperatorIntentRequest \\
\hline & sub & ActiveDiagnostics \\
\hline & sub & DeviceAnnouncement \\
\hline & sub & DeviceClockStatus \\
\hline & sub & DevicePowerMeasurementList \\
\hline & sub & DevicePowerPortList \\
\hline & sub & DevicePowerStatusList \\
\hline & sub & EngineState \\
\hline & sub & MicrogridConnectionList \\
\hline & sub & OperatorIntentState \\
\hline & sub & RequestResponse \\
\hline & sub & SourceTransitionState \\
\hline & sub & StandardConfigMaster \\
\hline \multirow[t]{25}{*}{ Source / SRC } & pub & ActiveDiagnostics \\
\hline & pub & ConfigReservationState \\
\hline & pub & DeviceAnnouncement \\
\hline & pub & DeviceClockStatus \\
\hline & pub & DevicePowerMeasurementList \\
\hline & pub & DevicePowerPortlist \\
\hline & pub & DevicePowerSta tusList \\
\hline & pub & DiscoveredConnectionList \\
\hline & pub & EngineState \\
\hline & pub & GetConfigPowerSwitchResponse \\
\hline & pub & GetConfigPrimaryControlResponse \\
\hline & pub & GetConfigSecondaryControlResponse \\
\hline & pub & GetConfigSourceTransition Response \\
\hline & pub & RequestResponse \\
\hline & pub & ReserveConfigReply \\
\hline & pub & SecondaryControlCapabilities \\
\hline & pub & SourceTransitionState \\
\hline & pub & StandardConfigMaster \\
\hline & sub & CopyConfigRequest \\
\hline & sub & DeviceAnnouncement \\
\hline & sub & GetConfigContentsRequest \\
\hline & sub & PrimaryControlRequest \\
\hline & sub & ReleaseConfigRequest \\
\hline & sub & ReserveConfigRequest \\
\hline & sub & SecondaryControlRequest \\
\hline
\end{tabular}


MIL-STD-TMS 21 July 2017

\begin{tabular}{|l|l|l|}
\hline Device Role & Pub/Sub & Topic Name \\
\hline & sub & SourceTransitionRequest \\
\hline Storage / STOR & pub & ActiveDiagnostics \\
\hline & pub & ConfigReservationState \\
\hline & pub & DeviceAnnouncement \\
\hline & pub & DeviceClockStatus \\
\hline & pub & DevicePowerMeasurementList \\
\hline & pub & DevicePowerPortList \\
\hline & pub & DevicePowerStatusList \\
\hline & pub & DiscoveredConnectionList \\
\hline & pub & GetConfigPowerSwitchResponse \\
\hline & pub & GetConfigPrimaryControlResponse \\
\hline & pub & GetConfigSecondaryControlResponse \\
\hline & pub & GetConfigSourceTransitionResponse \\
\hline & pub & RequestResponse \\
\hline & pub & ReserveConfigReply \\
\hline & pub & SecondaryControlCapabilities \\
\hline & pub & SourceTransitionState \\
\hline & pub & StandardConfigMaster \\
\hline & sub & CopyConfigRequest \\
\hline & sub & DeviceAnnouncement \\
\hline & sub & GetConfigContentsRequest \\
\hline & sub & PrimaryControlRequest \\
\hline & sub & ReleaseConfigRequest \\
\hline & sub & ReserveConfigRequest \\
\hline & sub & SecondaryControlRequest \\
\hline & sub & SourceTransitionRequest \\
\hline & Table 7- Device Role to Topics \\
\hline & & \\
\hline & &
\end{tabular}

\section{TMS DDS Topic Flows}

This section expands on the individual topics described in the previous section by outlining the sequence of steps involved in compound interactions between the various TMS device roles. It describes the relationships between when device roles are expected to publish or receive subscribed data for various scenarios supported by TMS.

\section{Assumptions}

1. Read is familiar with UML sequence diagrams. In the following illustration, the message name matches the TMS topic names. The class name matches a TMS device role.

2. All TMS devices are connected to the same communications network.

7.1 Device Discovery

The following sequence diagram illustrates the topics used during device discovery. 
MIL-STD-TMS 21 July 2017

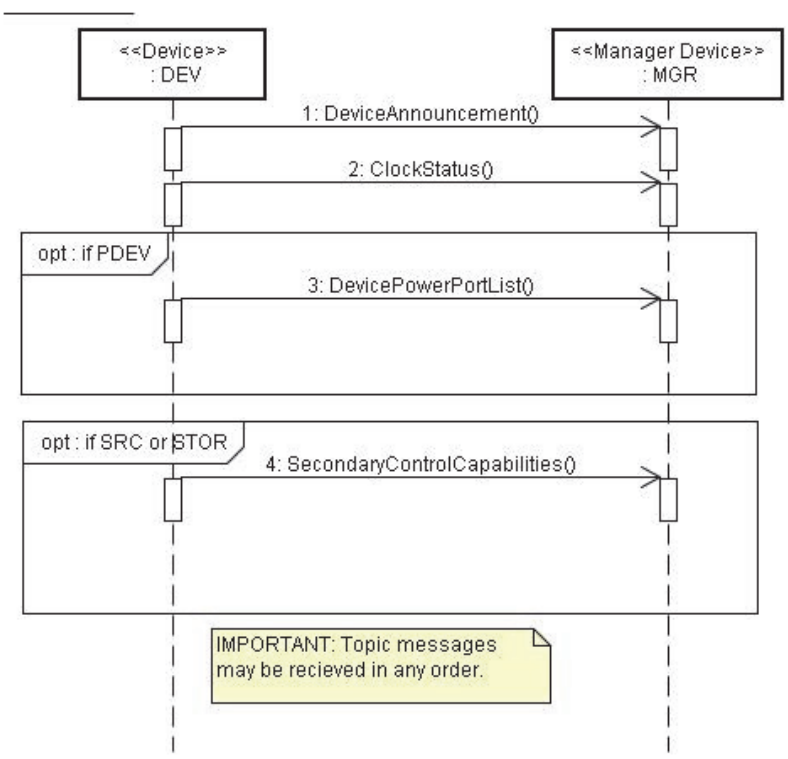

Figure 5 - Device Discovery

7.2 Source Startup

The following sequence diagram illustrates the topics used by a SRC device during startup. 
MIL-STD-TMS 21 July 2017

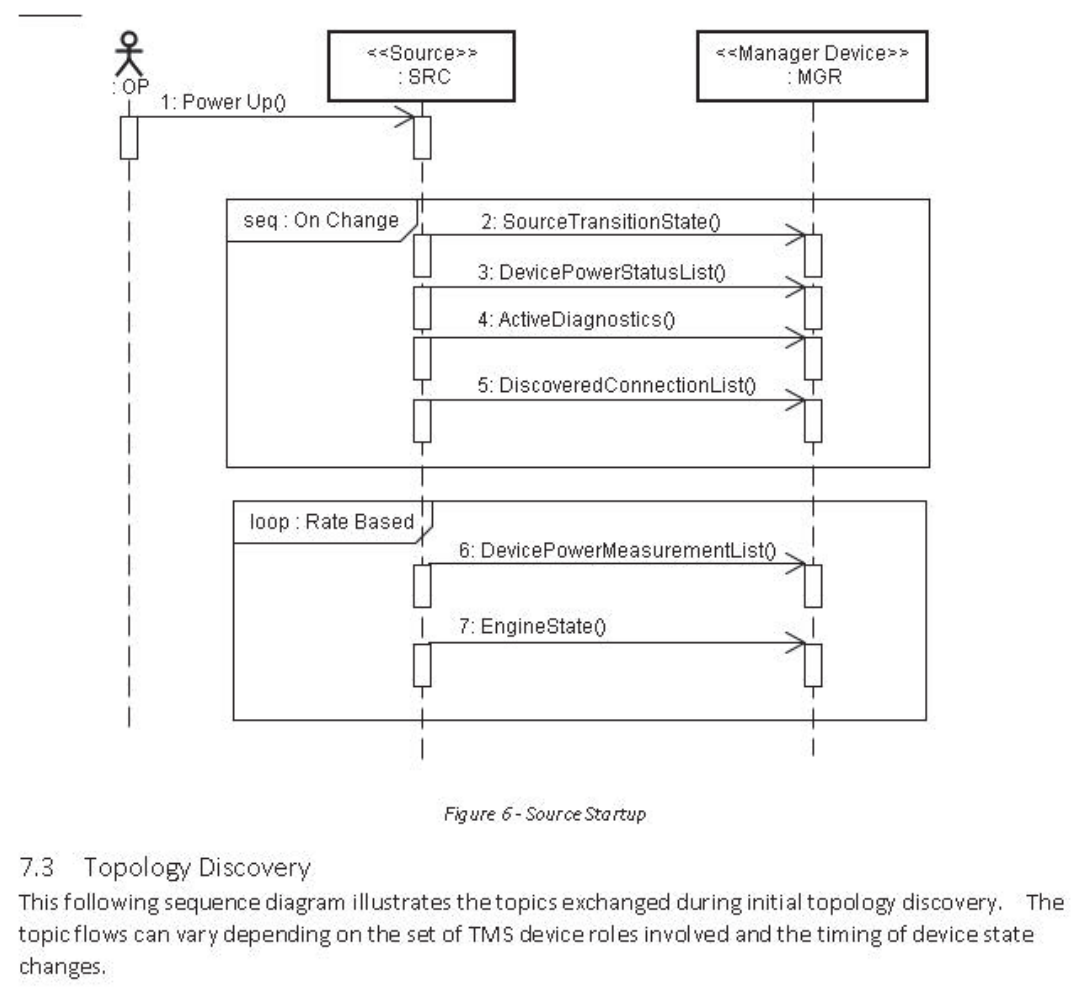


MIL-STD-TMS 21 July 2017

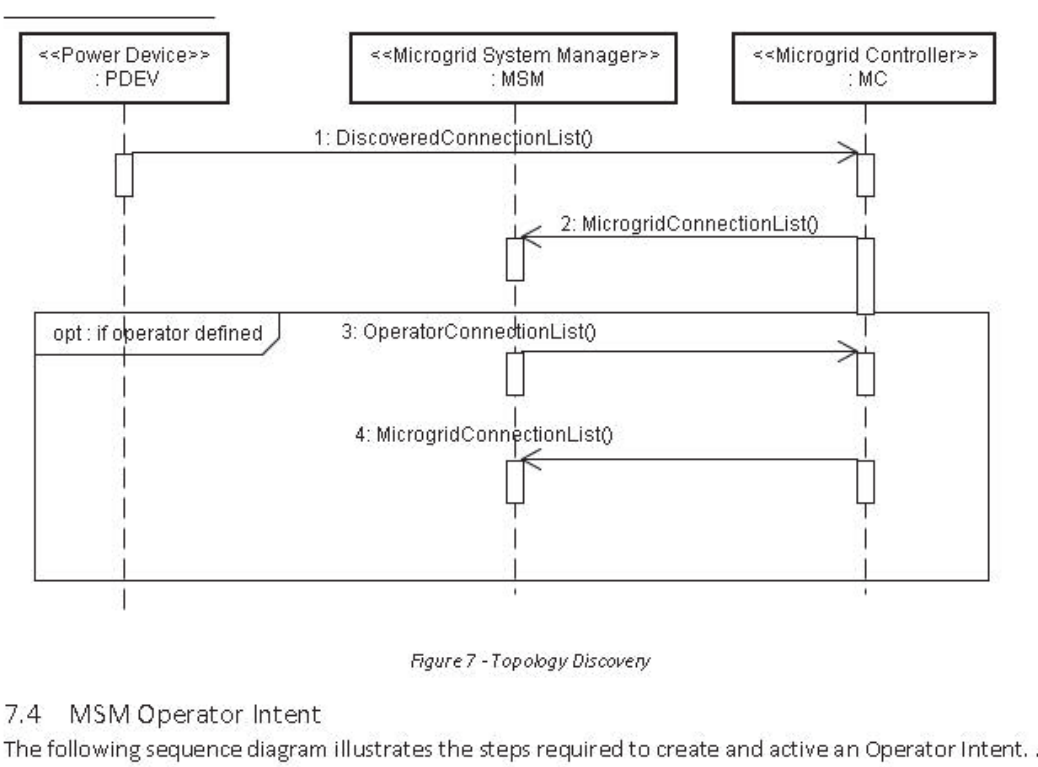


MIL-STD-TMS 21 July 2017

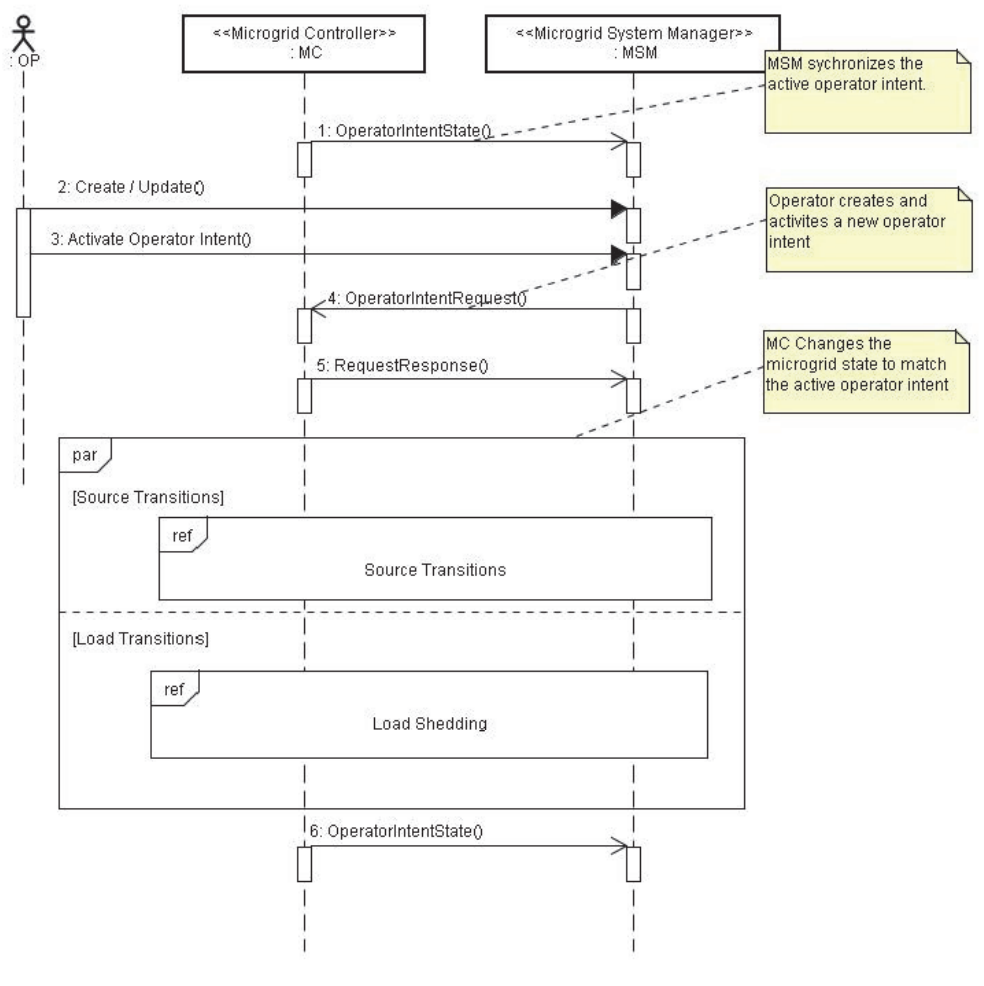

Figure 8 -MSM Operator intent

7.5 Microgrid Controller Source Transition

The following sequence diagram illustrates the topics exchanged when the MC decides to add or remove a SRC device from the current power sharing set of devices.

The following sequence assumes that device configuration ID (configld) is set to CONFIG_ACTIVE. 
MIL-STD-TMS 21 July 2017

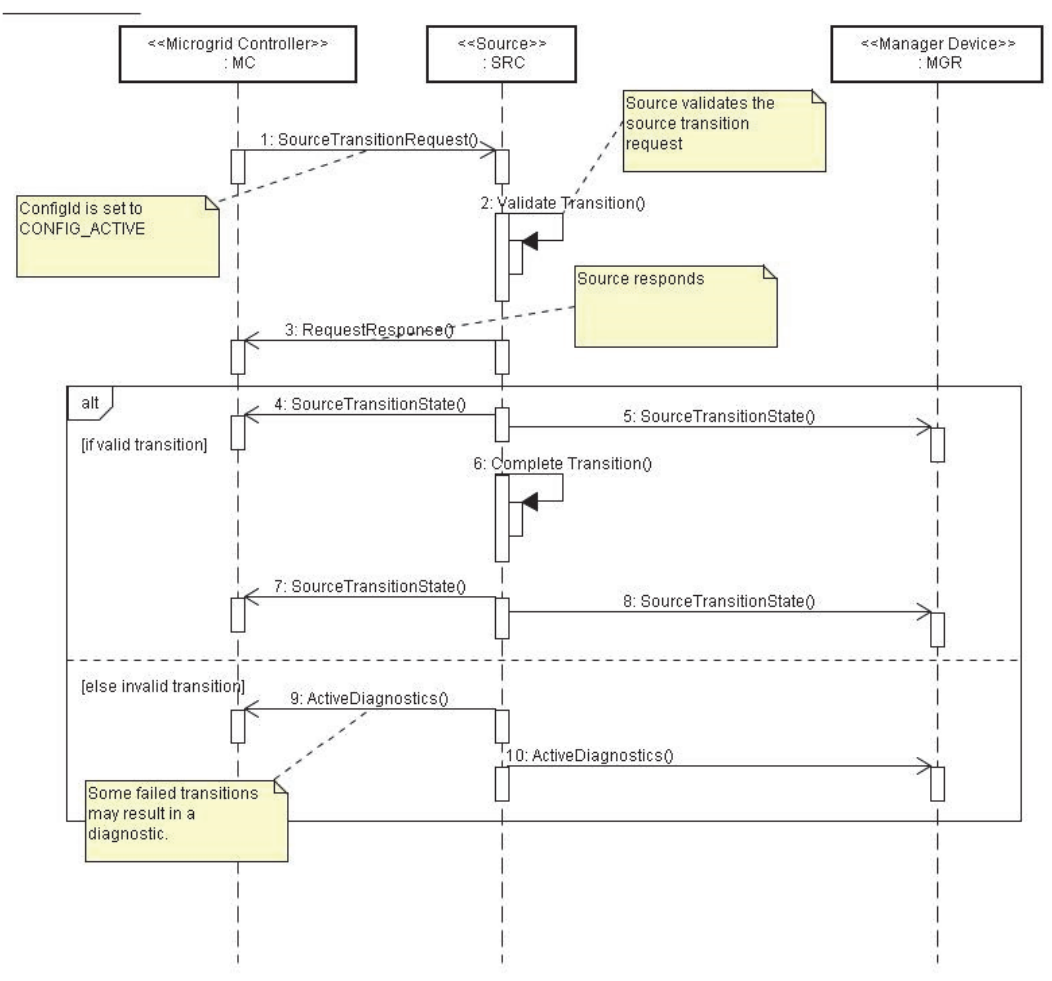

Figure 9 -MC Source Transition

7.6 Microgrid Startup

The foll owing sequence diagram illustrates initial microg rid star tup. 
MIL-STD-TMS 21 July 2017

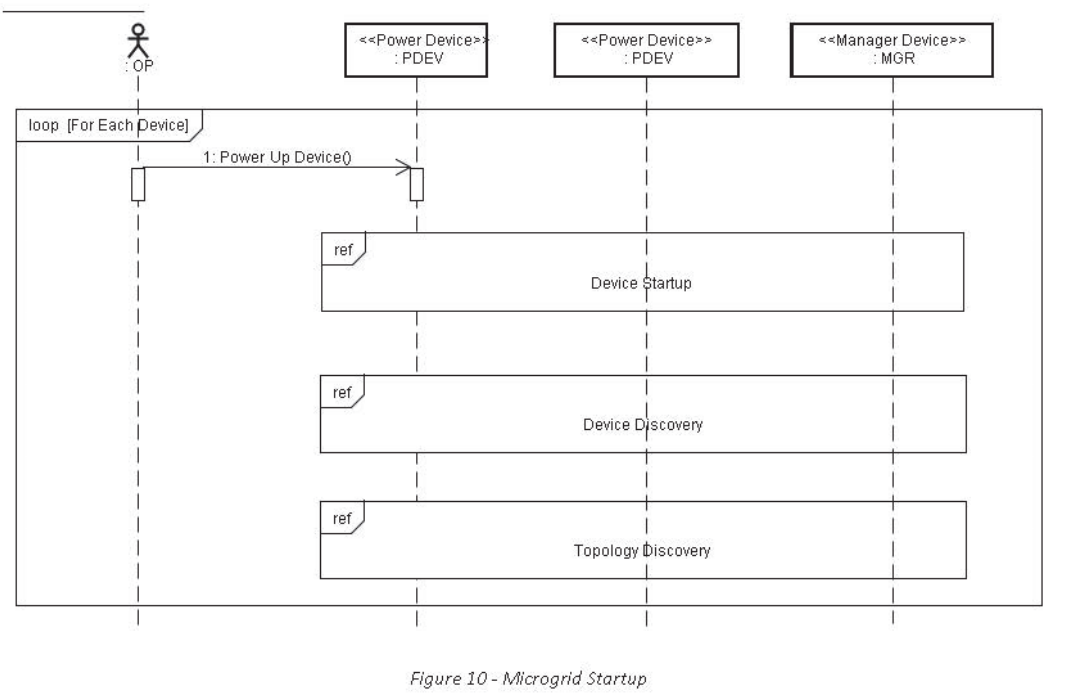

7.7 Device Configurations

This section illustrates sequence diagrams related to device configurations.

7.7.1 Get Device Configuration Contents

The following sequence diagram illustrates the topics used by the $M C$ to get the contents of a device configuration. 
MIL-STD-TMS21 July 2017

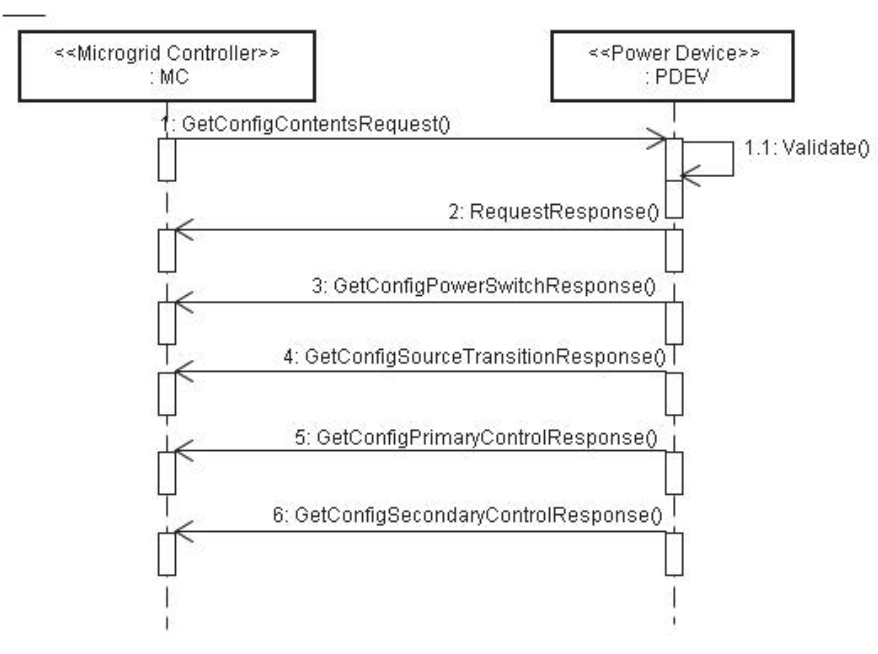

Figure 11 - Get Configuration Contents

7.7.2 Reserve, Copy and Release

The following sequence diagram illustrates the topics used to reserve, copy, and release a device configuration. 
MIL-STD-TMS 21 July 2017

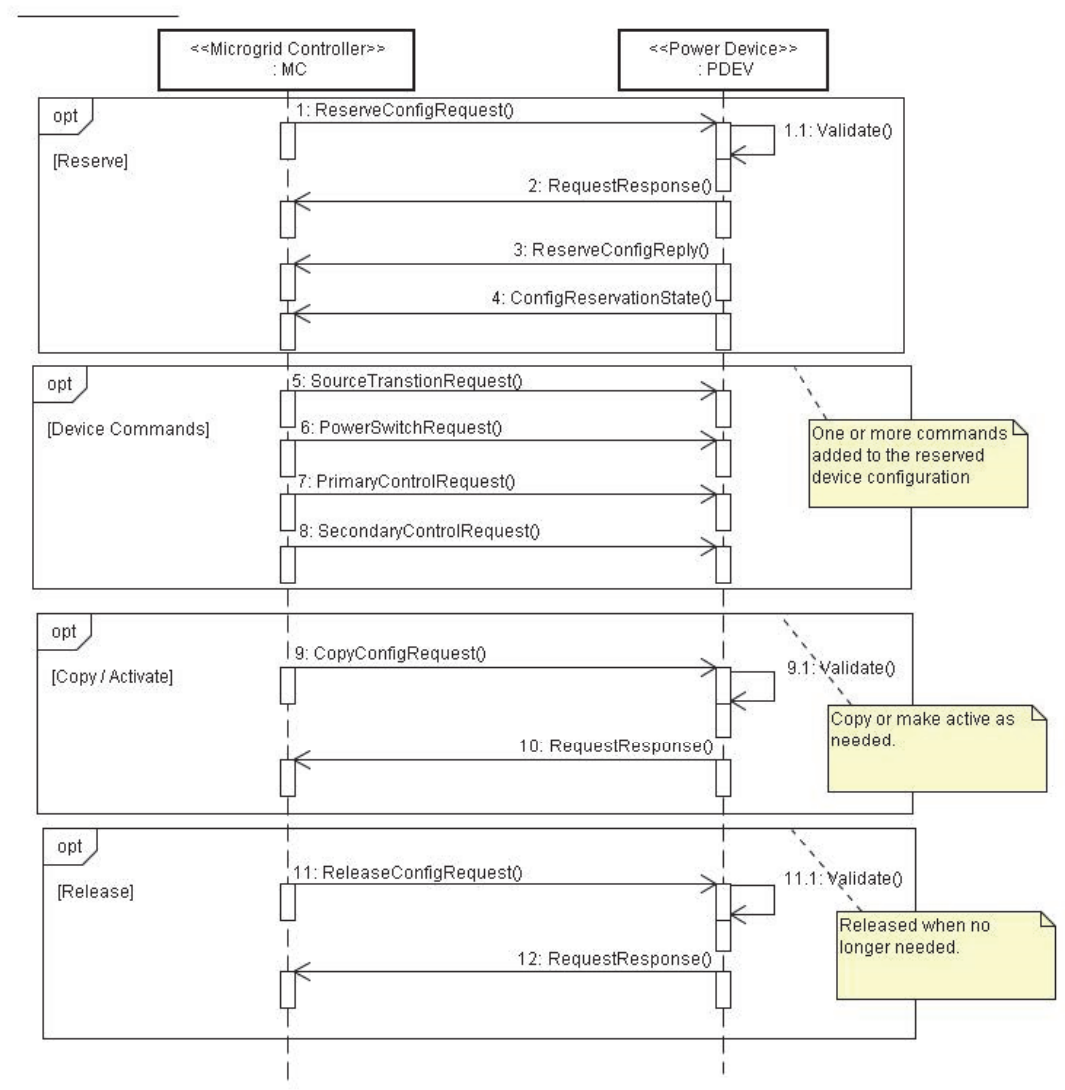

Figure 12 - Reserve, Copy, Release Device Configurations

7.8 MC Election

The TMS allows for one or more MC's (Microgrid Controllers) to participate in a single microgrid. The MC Election process is used to determine which MC is the elected "master" or Master Microgrid Controller (MMC). See the Consensus Protocol section of the Data Model appendix for details on how an MMC is de termined. 


\section{TMS Development Considerations}

\subsection{OMG DDS Vendor Interoperability}

This section covers OMG DDS vendor interoperability lessons learned by the TMSC team. Refer to vendor specific documentation for additional details.

a) The tms.idl only defines bounded sequences and strings because RTI bindings do not currently support unbounded strings.

b) The RTI proprietary shared memory transport must be disabled on hosts that are running DDS domain participants from other vendors. This is because RTI assumes all domain participants on the same host are RTI participants.

c) If using PrismTech, the following configuration must be enabled to interoperate with Twin Oaks. $<$ ExplicitlyPublishQosSetToDefault $>$ true $</$ ExplicitlyPublishQosSetTo Default $>$

d) Twin Oaks IDL compiler generated API includes a short and full data type name. The full data type name must be used to interoperate with other DDS vendors. An example of the full type name if "tms. Enginestate" where the IDL module name is prepended to the nested data type name. The short data type name is just "EngineState". The type name is used when creating a topic.

e) The QOS partition name defaults to different values across DDS vendors. If the partition names do not match across two domain participants, the publishers and subscribers will not match resulting in no data being exchanged. The partition name should always be specified using the vendor specific method to avoid the default values not matching.

8.2 TMSC Tools

TMSC is planning to make the following tools available.

8.2.1 TMS Data Monitor (TDM)

The TMS Data Monitor subscribes to all TMS topics and displays a list of TMS devices (publishers) along with the published data.

The OMG DDS uses a binary data format so the TDM logs and displays the data as ISON. The JSON representation follows the hierarchy defined in the IDL and includes the attributes names as they appear in the IDL. Primitive attributes values (numeric, character, string, and boolean) are displayed in their natural format. IDL enumeration values are represented as integer by DDS but the TDM includes the string name of the enumerator as it appears in the IDL.

8.2.2 EIO MSM (Future)

A TMS compliant implementation of the Microgrid System Manager (MSM) will be provided in the future by ElO to support development and testing of TMS devices. Energy Informed Operations (EIO) is a R\&D project of the CERDEC Command, Power, and Integration (CP\&I) Power Division.

8.3 Additional Tools

In addition to the TMSC provide tools, may other tools exist. This section includes a sample used during TMS development. 
MIL-STD-TMS 21 July 2017

8.3.1 OMG DDS Vendors

Some OMG DDS vendors include tools to monitor DDS domain participants and topic data.

8.3.2 Wireshark

Wireshark includes plugins that decode the OMG DDS wire protocol. 


\section{Appendix T: Coritech Fast Charger Product Brochure}

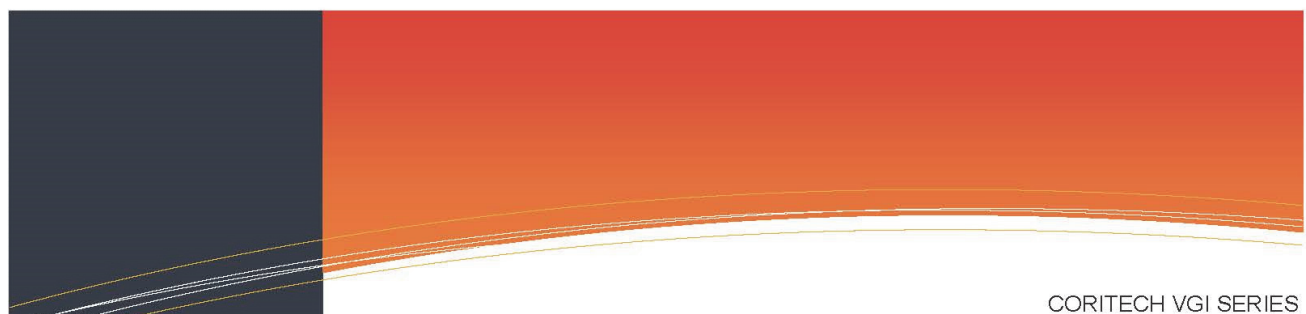

Electric Vehicle DC V2G Fast Charger

BI-DIRECTIONAL

Provides $\mathrm{DC}$ fast charging and

reverse power flow (V2G)

capability for grid-tie applications

to allow vehicles to act as power

sources.

DC LEVEL 1 \& 2

CHARGING

Coritech VGI Series chargers

provide up to $30 \mathrm{~kW}$ (Level 2)

charging with SAE J1772-CCS

and CHAdeMO connectors.

Maximum charging capacity of

$60 \mathrm{~kW}$ is avalable with dual

inverter and rated cable (See

VGI-60 model).

V2G SUPPORT

VGI Chargers provide full

communications capability for

connectivity to Utillty ISO's

Aggregators and Microgrid

Controllers using high speed and

secure Ethernet-Based

protocols.

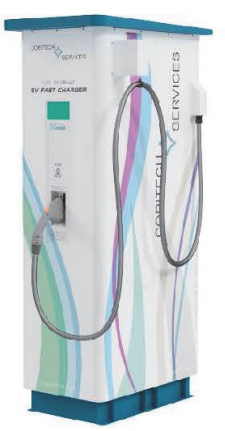

CORITECH VGH 30

OVERVIEW

Plug-in electric vehicles are commercially available today

with most vehicles having Level 1 and Level 2 AC

charging capability only. $A C$ charging requires on-board

chargers within the vehicle to convert $A C$ power to $D C$

power, which the vehicle battery requires. The use of on-

board chargers adds burden to the vehicles in the form of

slze, welght, cost and heat. In addition, AC charging at

240 Volts $A C$ has limitations to the amount of power or

Kilowatts that can be delivered to the vehicle. This

limitation results in lengthy, undesirable charging times to

"fill up" the vehicle's battery. DC Fast Charging is a

method in which the charger is placed off-board and the

AC power conversion to $\mathrm{DC}$ takes place within the

external stationary charger thus reducing burden on the

vehicle.

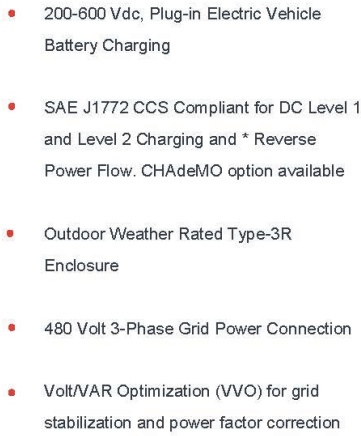

stabilization and power factor correction

Coritech VGI Series DC Fast Chargers contain stateof-the-art power electronics (bi-directional inverters) which efficiently convert $A C$-to-DC power for charging and $\mathrm{DC}-\mathrm{to}-\mathrm{AC}$ for sending vehicle battery power to the utility grid for Vehicle-to-Grid (V2G) operation. The inverters are $U L-A p p r o v e d$ and meet $U L-1741$ requirements for utllity connected power Inverters. Coritech chargers provide many safety features including over-current protection, ground fault detection and emergency shutdown capability to assure safe and reliable fast charging of electric vehicles. An easy to use color touchscreen operator interface guides the user through a simple procedure to initiate each charging session. 


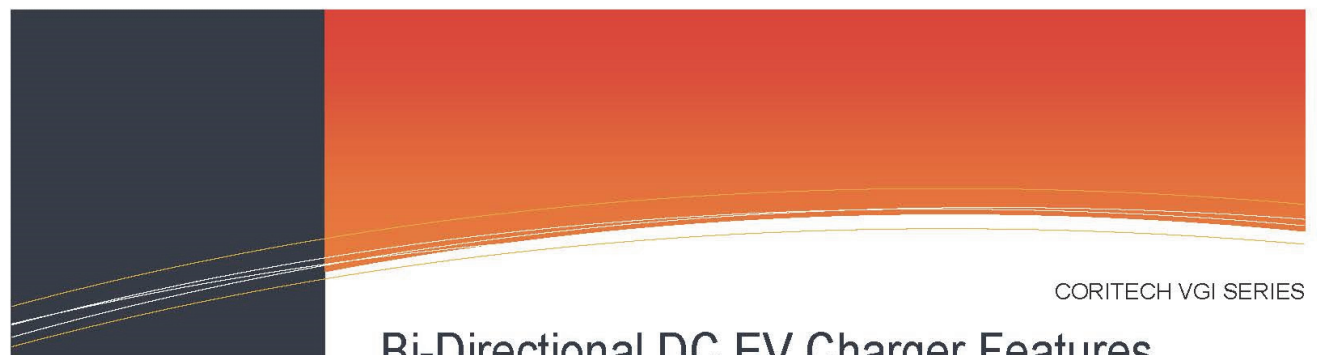

\section{Bi-Directional DC EV Charger Features}

TECHNICAL SUPPORT

Coritech provides full technical

support for all hardware and

software. Chargers are all

equipped with remote access

support to allow dial-in

diagnostics and troubleshooting

support.

VEHICLE INTEGRATION

Coritech provides integration and

engineering services and support

to develop harciware and

communications integration

between the vehicle and charger

Coritech has developed a

communications interface which

conforms to SAE standards for

electric vehicle charging

including SAE J1772 and J2847

and ISO-15118.

TURNKEY SOLUTIONS

Coritech can provide a turnkey

solution through their network of

partners to supply infrastructure,

vehicles, chargers and

aggregator software and

systems.

For more information on any of

our products or senvices please

visit us on the Web at:

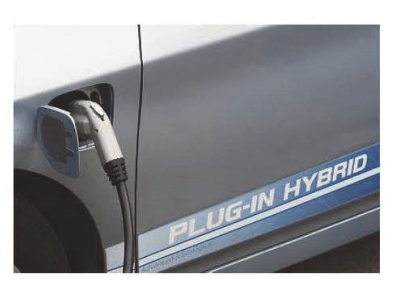

AGGREGATOR CONTROL

Coritech chargers provide the ability for each

vehicle's charging activity to be controlled from

a remote grid "Aggregator" system which

manages overall grid performance, health and

stability. Aggregators also can communicate

with local utility ISO's for participation in the

energy markets.

\section{Technical Specifications}

- Utility Grid Connection: 480 Vac, 3 Phase, 44A Max.

- Vehicle Connection: SAE J1772-Combo CCS or CHAdeMO

- Aggregator Connection: Ethernet, Modbus TCPIP, SEP 2.0

- Dimensions: $36 \times 24 \times 81$ inches

- Weight: 400 lbs Approx.

- Voltvar Optimization:-18 to +18 kVAR

- Battery Voltage Range: 200-600 Vdc

- Battery Charging Power*: $30 \mathrm{~kW} 60 \mathrm{~A}$ Max

- Reverse Power Flow V2G*: $30 \mathrm{~kW} 60 \mathrm{~A}$ Max

- Operator Interface: $7^{\prime \prime}$ Color Touchscreen

- Environmental:

Enclosure: Outdoor Rated Type 3R

Operating Temp: -20 to +120 Degrees Fahrenheit Humidity: 0-95\%

- Safety: Over-Current Circuit Breaker, GFCl, DC Isolation

- Emergency Shutdown Pushbutton

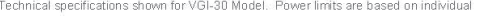

vehicle battery voltage range; to utit is full $30 \mathrm{~kW}$ capacity of the charger the battery yoltage

must be greatter than spprax 500 Vdd. Digtal cormumuications hetween vehicle and ch hrger

assures that power tran ser is a tways wothin safe op erating limits of both charger and venicle
Coritech VGI Series chargers provide the fastest power transfer ailable today for DC charging, limited only by the maximum urrent capacity of the industry standard SAE J1772-CCS or

many benefits to owning electric vehicles is to have the

chargers provide the ability to influence the stability of the y providing WO to contribute or absorb reactive power (KVAR) the grid power factor.

\section{SERVICES AVAILABLE \\ Technical Support \\ Installation and Setup \\ Maintenance \\ Application Support \\ Hardware Support \\ Guaranteed Warranty}

Certifications and Standards

- UL1741, IEEE1547a, IEC62109-1,2

- UL2202, UL2231-1, UL2231-2

CORITECH

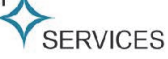

4716 Delemere Blvd Royal Oak, MI 48073 Phone 248.549.3300 Fax 248.549 .3302 


\section{Appendix U: Coritech Fast Charger User Manual}

SORITECH

EV FAST CHARGER

VGI-30-DC-CHA User Manual

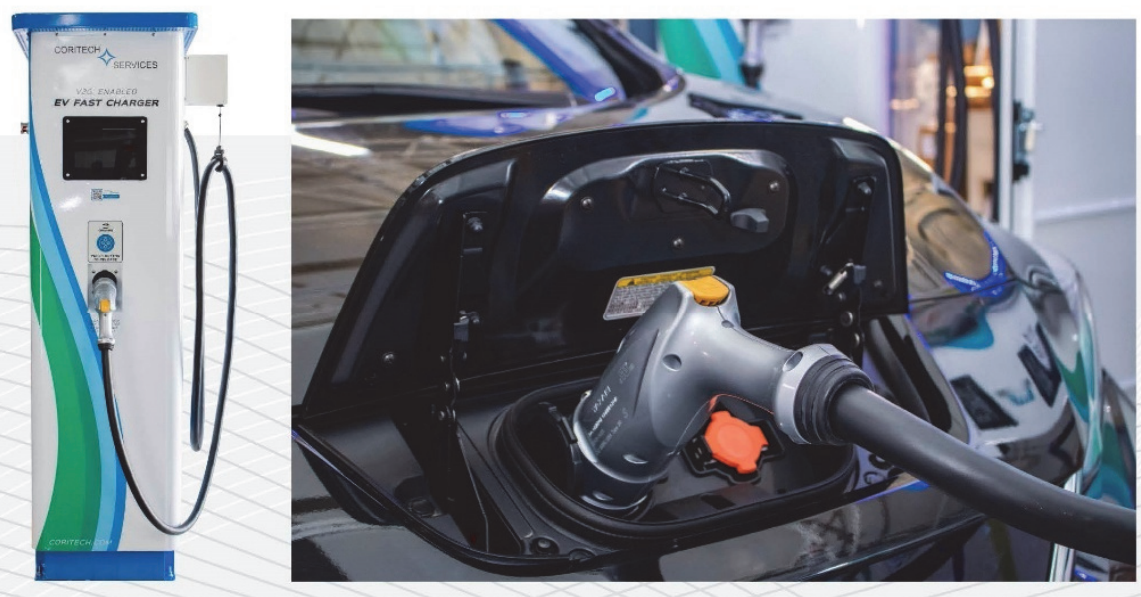

Discover the future, today. 


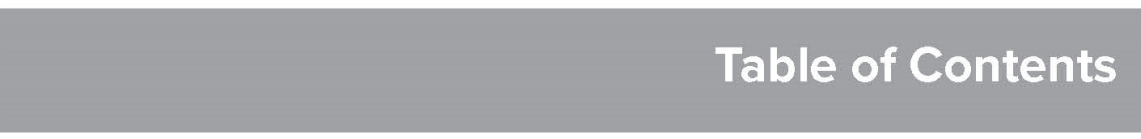

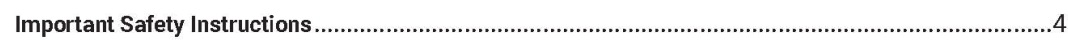

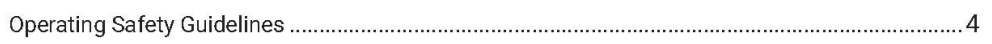

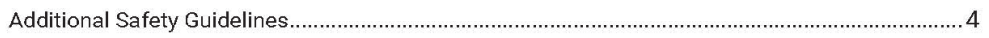

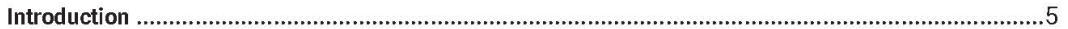

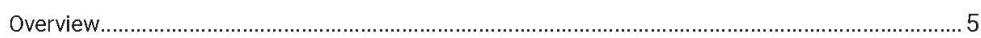

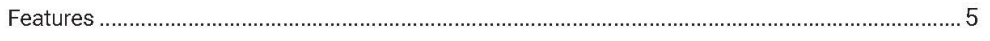

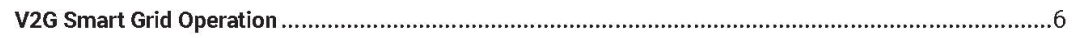

V2G Technology

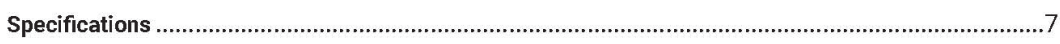

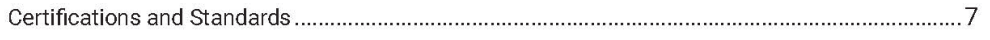

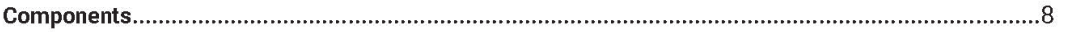

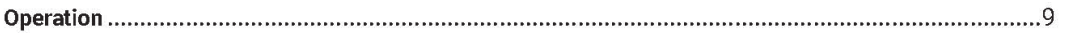

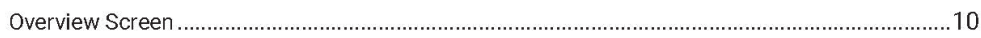

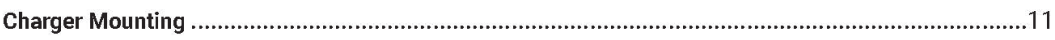

Installation Considerations ......................................................................................................... 11

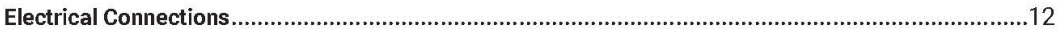

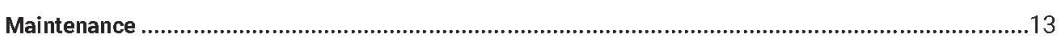

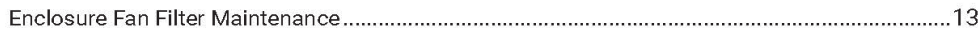

$\gamma_{\text {SERVICES }}$ CORIECH Cortech VGI-30-DC-CHA Charger User Guide (revision 1.0) 3 


\section{Important Safety Instructions}

Carefully read the following instructions along with charging instructions in your vehicle owner's manual prior to operating this charger with your electric vehicle.

\section{OPERATING SAFETy GUIDELINES}

A Warning-Use this charger to charge electric vehicles equipped with a CHAdeMO charge port only.

A Warning-Make certain that the charging cable is positioned so it will not be stepped on, tripped over or otherwise subjected to damage or stress.

\section{ADDITIONAL SAFETY GUIDELINES}

A Danger-To avoid an electric shock during servicing, verify that the charger's external $\mathrm{AC}$ and DC Disconnects are open (off). A minimum wait time of five (5) minutes is required after both $\mathrm{AC}$ and $\mathrm{DC}$ Disconnects remain in the off position during all service periods.

A Danger-The enclosure contains exposed high voltage conductors. The enclosure access doors must remain closed, except during installation, commissioning, or maintenance by trained service personnel. Do not open enclosure doors if extreme moisture is present (rain, snow, or heavy dew).

A Danger-Ensure that the equipment is adequately installed and grounded per this manual and all applicable codes.

A Danger-Do not leave foreign objects in the charger enclosure. Keep the area around the enclosure clear of trash, debris, and other combustible materials.
Warning-Do not operate the charger with
any visible damage to the cable or enclosure/pedestal. Contact Coritech Services immediately for service.

A Warning-This device should be supervised when used around children.

1. Warning-Do not put fingers into the electric vehicle connector.

A Warning-Personnel Qualification: Inspections and operations requiring access to lethal AC or DC voltages should only be performed by qualified personnel.

A Warning-All field wiring must conform to the codes set forth in the National Electric Code ANSI/NFPA 70, and/or applicable codes per Authority Having Jurisdiction (AHJ).

1. Warning-The charger contains ESDsensitive equipment. Failure to use ESD control measures while servicing the equipment may result in component damage and void the warranty.

A Warning-The VGI-30-DC-CHA charging station weighs approximately 400 pounds. Follow all safety rules when transporting or installing. Failure to do so could result in personal injury or equipment damage. 


\section{Introduction}

The Coritech VGI-30-DC-CHA Charger provides enhanced fast V2G charging capability to electric vehicles equipped for DC Fast Charging, in compliance with CHAdeMO version 1.2 and $\mathrm{V} 2 \mathrm{H}$ Guideline 2.1 standards.

\section{OVERVIEW}

Most commercially available plug-in electric vehicle chargers require on-board chargers within the vehicle to convert $\mathrm{AC}$ power to $\mathrm{DC}$ power. The use of on-board chargers adds a burden to the vehicles in the form of size, weight, cost and heat. In addition, AC-charging at $120 / 208 / 240$ Volts AC limits the amount of power that can be delivered to the vehicle. This limitation results in lengthy, undesirable charging times to "fill up" the vehicle's battery. DC Fast Charging is a method in which the charger is placed off-board (external to vehicle), and the AC power conversion to DC takes place within the external charger, thus reducing burden on the vehicle.

Coritech VGI Series DC Fast Chargers contain state-of-the-art power electronics (galvanically isolated bi-directional inverters) which efficiently convert AC-to-DC power for charging, and DC-to-AC for sending vehicle battery power to the utility grid for Vehicle-to-Grid (V2G) operation. The inverters

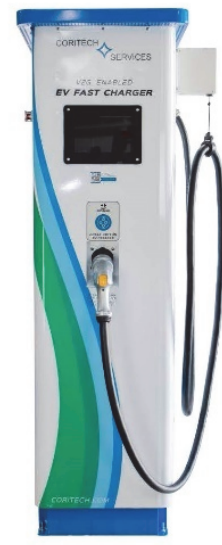

are UL-Approved, meeting UL-1741 requirements for utility-connected power inverters. Coritech chargers provide many safety features including over-current protection, ground fault detection and emergency shutdown capability to assure safe and reliable fast charging of electric vehicles. An easy-to-use color touchscreen operator interface guides the user through a simple procedure to initiate each charging session.

\section{FEATURES}

- $100 \mathrm{Vdc}-500 \mathrm{Vdc}$, Plug-in Electric Vehicle Battery Charging

- Outdoor Weather-Rated Type-3R Enclosure

- 480 Volt 3 Phase AC Grid Power Connection

- Volt/VAR Optimization (VVO) for grid stabilization and power factor correction

OfF-BOARD AC TO DC CONVERSION Externalizes power electronics for vastly more efficient charging.

\section{Bi-DiRectional}

Provides DC fast charging and reverse power flow (V2G) capability for grid-tie applications to allow vehicles to act as power sources.

\section{V2G SUPPORT}

Provides full communications capability for connectivity to Energy Management Servers (EMS), and Utility ISOs. 


\section{V2G Smart Grid Operation}

\section{V2G TECHNOLOGY}

This charger has the ability to provide automated V2G Smart Grid operation with an external Energy Management Server (EMS). The EMS can be used for:

- Microgrid Management: Manage overall grid performance, health and stability. Vehicle charge rates can be optimized for maximum charge efficiency with multiple vehicle types, and adapt to situations in a complex environment including vehicle charging clients, a solar photovoltaic array, battery storage system, and communications with local utility Independent System Operators (ISOs).

- V2G (Vehicle to Grid): An EMS can communicate with local utility ISOs for participation in the energy markets. One of many benefits of owning an electric vehicle is the ability to use the vehicle as an additional energy source when not in use. Analogous to distributed computing, the energy stored in an electric vehicle can be used to provide power back to the utility AC power grid for many benefits such as offsetting power demand, reducing peak demand charges, and stabilizing the grid during power outages and high demand periods.

- Vvo (Volt Var Optimization): Coritech chargers provide the ability to influence the stability of the grid by providing $\mathrm{VVO}$, which changes grid power factor by contributing or absorbing reactive power (kVAR).

If no EMS is integrated with the charger, then the station will charge at the rate requested by the vehicle. If an EMS is present, vehicles that do not support V2G will still charge at EMS-controlled rates, but be unable to discharge. Please contact Coritech Services to determine if a particular vehicle is capable of V2G Smart Grid operation with this charger.

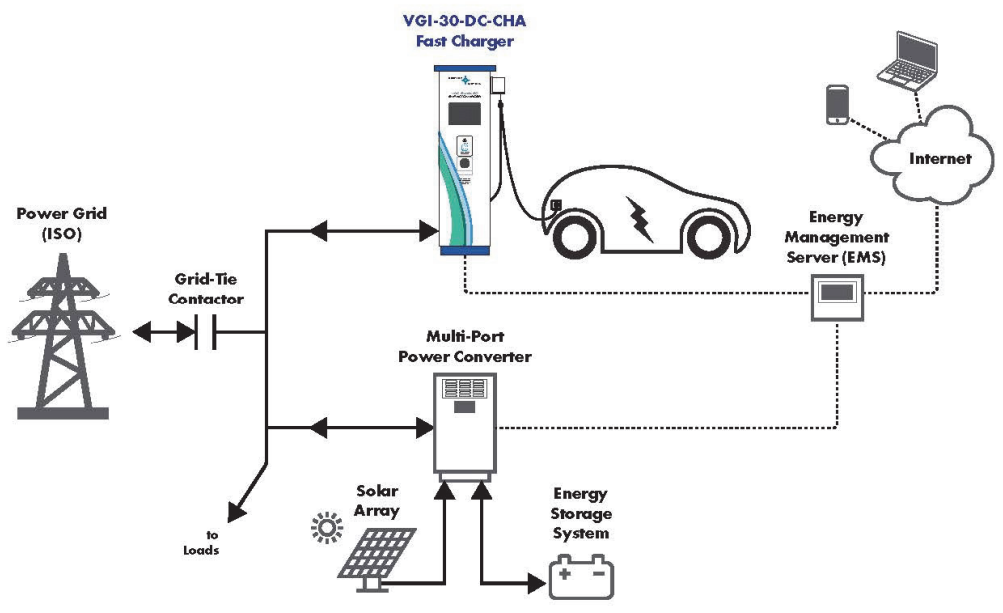




\section{Specifications}

- Util. Grid Connection: 480Vac,3Ph, 47A Max

- Real Power: $30 \mathrm{~kW}^{+}$

- Volt/VAR Optimization: -18kVAR-18kVAR

- Vehicle Voltage Range: $100 \mathrm{Vdc}-500 \mathrm{Vdc}$

- Vehicle Charging Power ${ }^{+}: 30$ kW 60Adc Max

- Reverse Power Flow V2G : 30 kW 60Adc Max

- Enclosure: Outdoor-Rated, Type 3R

- Operating Temp: $-20^{\circ} \mathrm{F}-120^{\circ} \mathrm{F}$

- Dimensions: $41^{\prime \prime} \times 26^{\prime \prime}$ x 81.75"

- Base Dimensions: $33^{\prime \prime} \times 21^{\prime \prime}$
- Weight: 400 lbs Approx.

- Humidity: 0-95\%

- Operator Interface: 10" Color Touchscreen

- Vehicle Connection: DC Charging Connector conforming to CHAdeMO 0.9, 1.0 specifications

- Energy Management Server (ESM) Connection: Ethernet, Modbus TCP/IP, SEP 2.0, MQTT

- Safety: Over-Current Circuit Breaker, GFCI, DC Isolation, Emergency Shutdown Pushbutton

* The VGH-30-DC-CHA is a 30kW model, but can be configured for lower power levels (for example: CHA-10K for $10 \mathrm{~kW})$, or other custom real and reactive power combinations.

† Technical specifications shown for VGI-30 Model. Power limits are based on individual vehicle battery voltage range; to utilize full $30 \mathrm{~kW}$ capacity of the charger the battery voltage must be approx. $500 \mathrm{Vdc}$. Digital communications between vehicle and charger assures that power transfer is always within safe operating limits of both charger and vehicle.
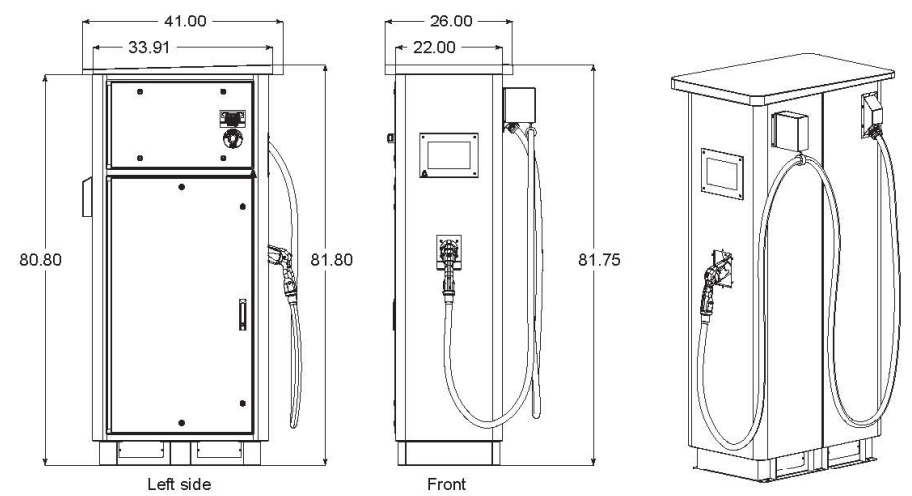

\section{CERTIFICATIONS AND STANDARDS}
- $\quad$ CHAdeMO 1.2, V2H Guideline 2.1
- UL1741, IEEE1547a, IEC62109-1,2
- $\quad$ CSA SPE-1000-13,
- UL2202, UL2231-1, UL2231-2
CSA C22.2 N.107.1-16
CSA C22.2 N.281.1-12 CSA C22.2 N.281.2-12 


\section{Components}

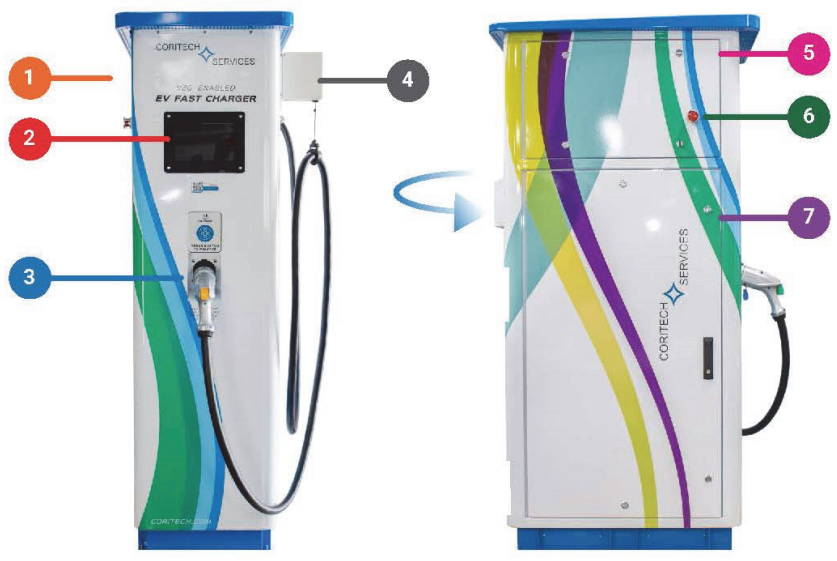

1. OUtdoor-Rated, Type 3R Enclosure

Rugged indoor/outdoor enclosure provides protec-

tion to personnel against access to hazardous parts; also provides protection against rain/sleet/snow.

\section{Operating Screen}

10 " touchscreen for display of charge session data.

\section{Charging Connector}

(CHAdeMO Charge Connector) Charge connector with cable that extends up to 18 feet.

\section{Cable Retractor}

Spring-loaded cable reel retracts charge cable back up for next use.

5. Top Access PANel

Contains control components for the unit.
A Warning-only qualified, trained personnel should open this panel. This panel contains elements which could present electrically dangerous hazards.

6. EMERGENCY STOP BUTtON

Pressing will interrupt current charging session and all high power will be terminated.

7. LOWER ACCESS PANEL

Contains a galvanically isolated bi-directional inverter and installation connections (480Vac and high-voltage DC).

Warning-only qualified, trained personnel should open this panel. This panel contains elements which could present electrically dangerous hazards. 


\section{Operation}

Beginning a Charging Session

1. Press the release button on the top of the charge connector handle to release.

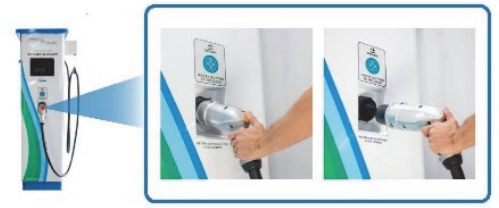

2. Insert the charge connector into the vehicle charging port. The connector will lock into place. On the screen, press the Start button.

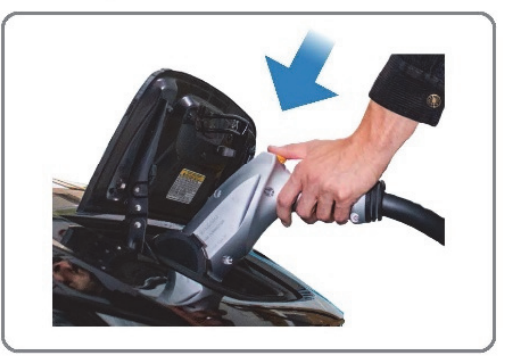

3. The Overview screen on the charger will change to reflect that a new charging session has been initiated. Parameters will be exchanged with the vehicle and a cable check will be performed. After safety checks, contactors will close and vehicle charging will be underway.

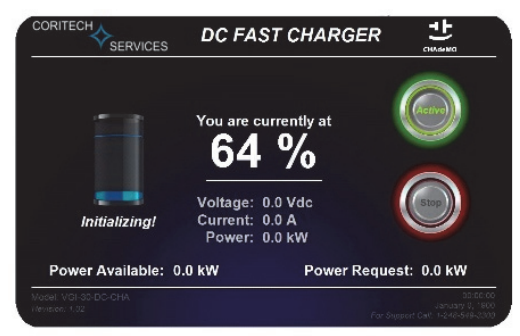

During a charge, the charge connector will not unlock by pressing the release button. It can only be removed after the session has been ended either manually or automatically.

manually Ending a Charging Session

To manually end a charging session before charging has completed, press the Stop Charge Button on the display screen. In an emergency, press the red Emergency Stop Button on the left side of the charger.
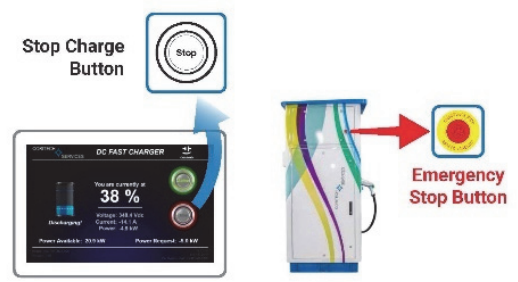

Automatically Ending a ChaRging Session 1. The charging session will complete automatically once SOC (State of Charge) has reached $100 \%$, which is shown on the Overview screen. At this moment, the charger will cease charging the vehicle, the connector will unlock, and the screen's status message will go blank.

2. Return the charge connector to the holster socket on the charger.

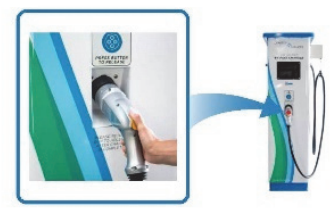




\section{Using the Touchscreen}

\section{OVERVIEW SCREEN}

The Overview Screen automatically opens when a charging session is underway. This screen displays all relevant values to the charging session: progress of vehicle State of Charge, voltage, current, power, and EMS Power Request. The operator can manually end the session at any time or view faults.

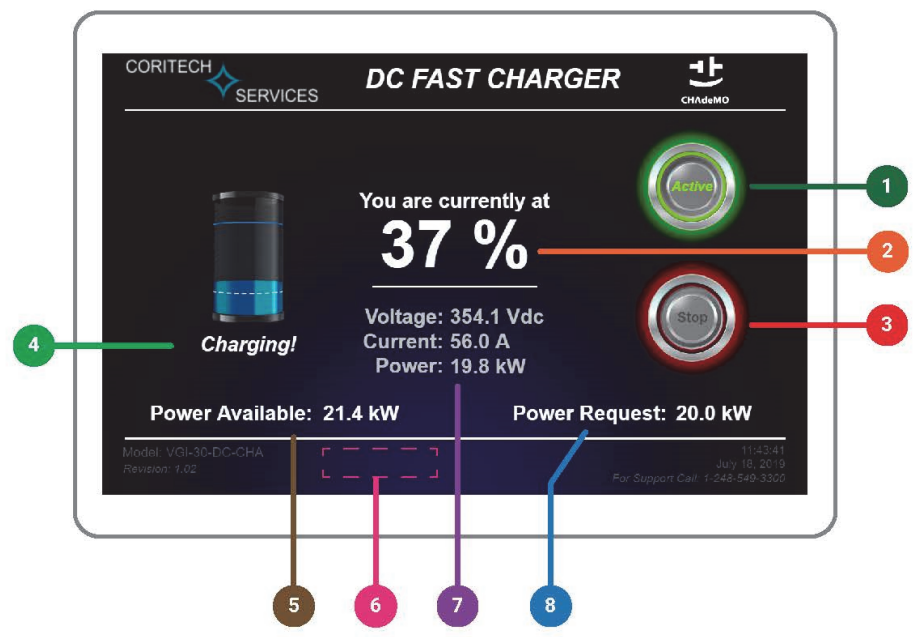

1. StaRt Charge

Pressing this button will initiate a new charging session.

\section{STATE OF CHARGE INDICATOR}

Vehicle battery State of Charge (SOC). When the SOC reaches $100 \%$, the charge session will end.

\section{STOP CHARGE}

Pressing this button will immediately end the active charging session.

\section{Status Message}

Displays one of the four active modes: Initializing, Charging, Discharging and Idle. When no car is connected, the status message area is blank.

\section{Power AVailable}

Displays the vehicle-determined amount of power that can be drawn from the vehicle.

6. Alarms and Statuses Display ARea Any alarms or status reports are displayed at the bottom of the screen; multiple items will form a list that cycles through its members.

\section{Charger Voltage, Current, Power} Voltage, current and power for the charging session.

\section{Charger Power Reouest}

This is determined by the Energy Management Server (EMS), which coordinates its determination with the vehicle. 


\section{Charger Mounting}

\section{INSTALLATION CONSIDERATIONS}

The VGI-30-DC-CHA Charger is a stand-alone pedestal design intended to be mounted to a concrete pad with conduit connection for wiring through the bottom of the enclosure. See drawings for base mounting-hole dimensions and fastener instructions. The base has two pockets for lifting by forklift, $2.88^{\prime} \times 7.75^{\prime \prime}$ each.

Approximate weight: $400 \mathrm{lbs}$.

Required area to be kept clear around unit: 30 " minimum

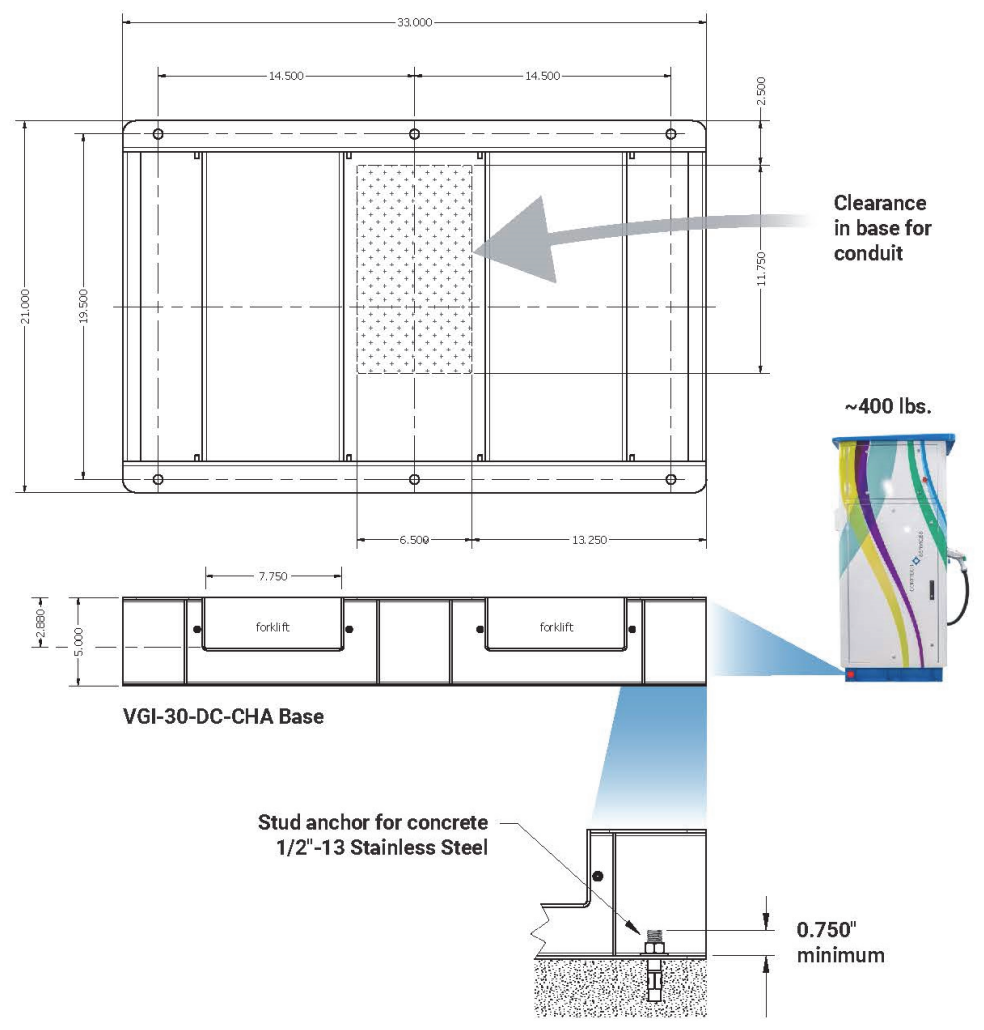




\section{Electrical Connections}

The VGI-30-DC-CHA Charger has electrical connections for the electrical service, neutral deriving transformer circuit breaker status, and connections to a Grid Interface Connection Box (GICB). All electrical connections to the charger are located in the lower enclosure.

The VGI-30-DC-CHA Charger is a three-phase 480 Vac power device. It requires a 3-wire electrical service (no neutral connection), as all power is made between phase legs. The VGI-30-DC-CHA must be installed with external 3-wire AC circuit protection rated at $480 \mathrm{Vac}$, sized in accordance with local electrical codes. Likewise, the gauge of the wire must be sized in accordance with NEC ANSI/NFPA 70, and/or applicable codes per AHJ.

This product must be connected to a grounded, metal, permanent wiring system, or an equipment- grounding conductor must be run with the circuit conductors and connected to either the equipment-grounding terminal or lead on the product.

WARNING: Do not connect to a three-phase 480Vac floating delta electrical service. If service is floating delta, a neutral deriving transformer ("zigzag") must be used to anchor each phase in reference to ground. Contact Coritech Services support if your electrical service is floating.

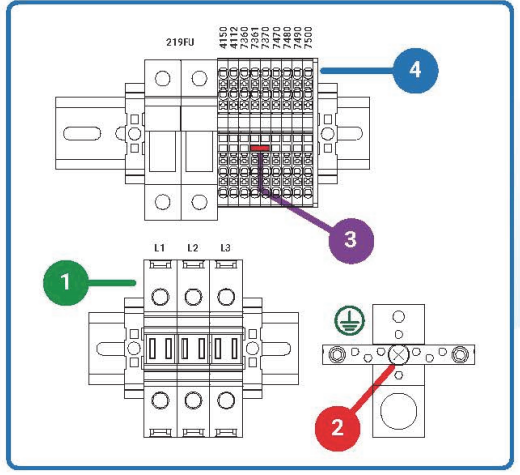

1. AC POWER CONNECTIONS (ELECTRICAL SERVICE)

L1, L2, L3: 480 Vac

\section{Main Ground Lug (Electrical Service)}

Equipment ground connection.

Caution: proper ground connection must be installed per National Electric Code ANSI/NFPA 70, and/or applicable codes per AHJ.

\section{JUMPER}

BETWEEN 7361 AND 7370

If neutral deriving transformer is being used,

this jumper must be removed.

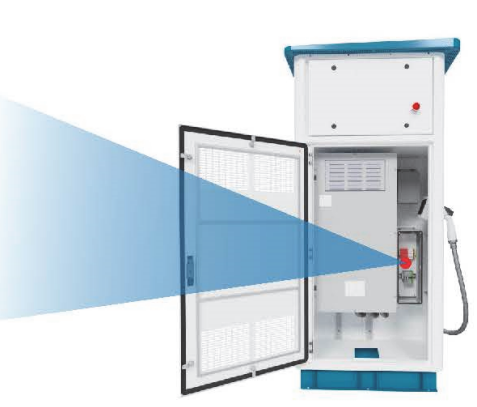

Left view 


\section{Maintenance}

\section{EnClosure FAn Filter MAINTENANCE}

There are three fan filters inside the unit that protect components from particulate buildup. Once per year, these filters should be removed and cleaned.

Warning: only qualified, trained personnel should open this panel. This panel contains elements which could present electrically dangerous hazards.

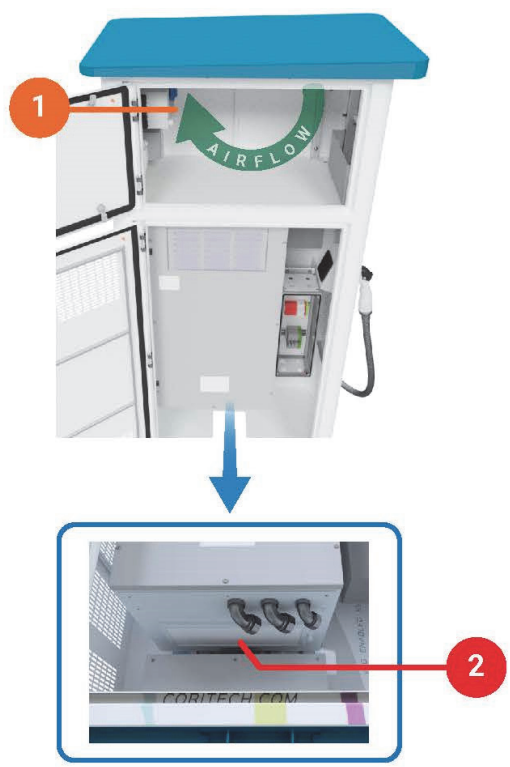

\section{INVERTER FAN FILTER}

This filter is located beneath the inverter in the bottom cabinet. It is accessed by unscrewing one knob.

A Danger-To avoid an electric shock during servicing, verify that the charger's external $A C$ and DC Disconnects are open (off). A minimum wait time of five (5) minutes is required after both $A C$ and $D C$ Disconnects remain in the off position during all service periods.

A Danger-The enclosure contains exposed high voltage conductors. The enclosure access doors must remain closed, except during installation, commissioning, or maintenance by trained service personnel. Do not open enclosure doors if extreme moisture is present (rain, snow, or heavy dew).

1. Warning-Personnel Qualification: Inspections and operations requiring access to lethal $A C$ or $D C$ voltages should only be performed by qualified personnel.

1. Upper Cabinet Circulation Exhaust AND FAN INLET Filters

These filters clean inlet air and exhaust air from the upper cabinet; both are located in the upper cabinet's ceiling. They are accessed by opening the upper cabinet and sliding the filters in or out. 


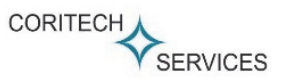

4716 Delemere Blvd Royal Oak MI 48073 Tel.: + 1/(248)549/3300 • Fax: +1(248)549/3302 info@coritech.com •www.coritechservices.com 


\section{Appendix V: Coritech Fast Charger Memory Map}

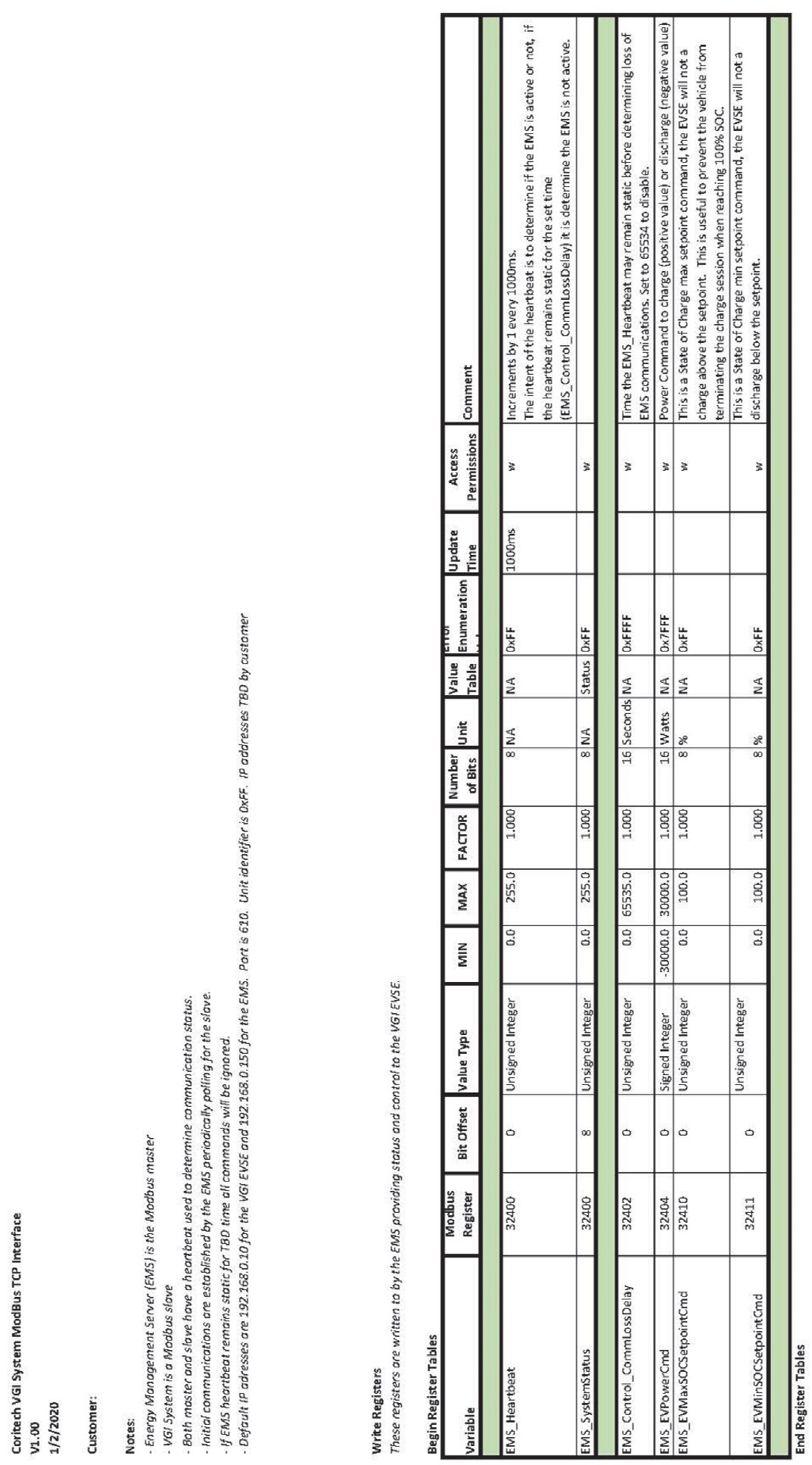




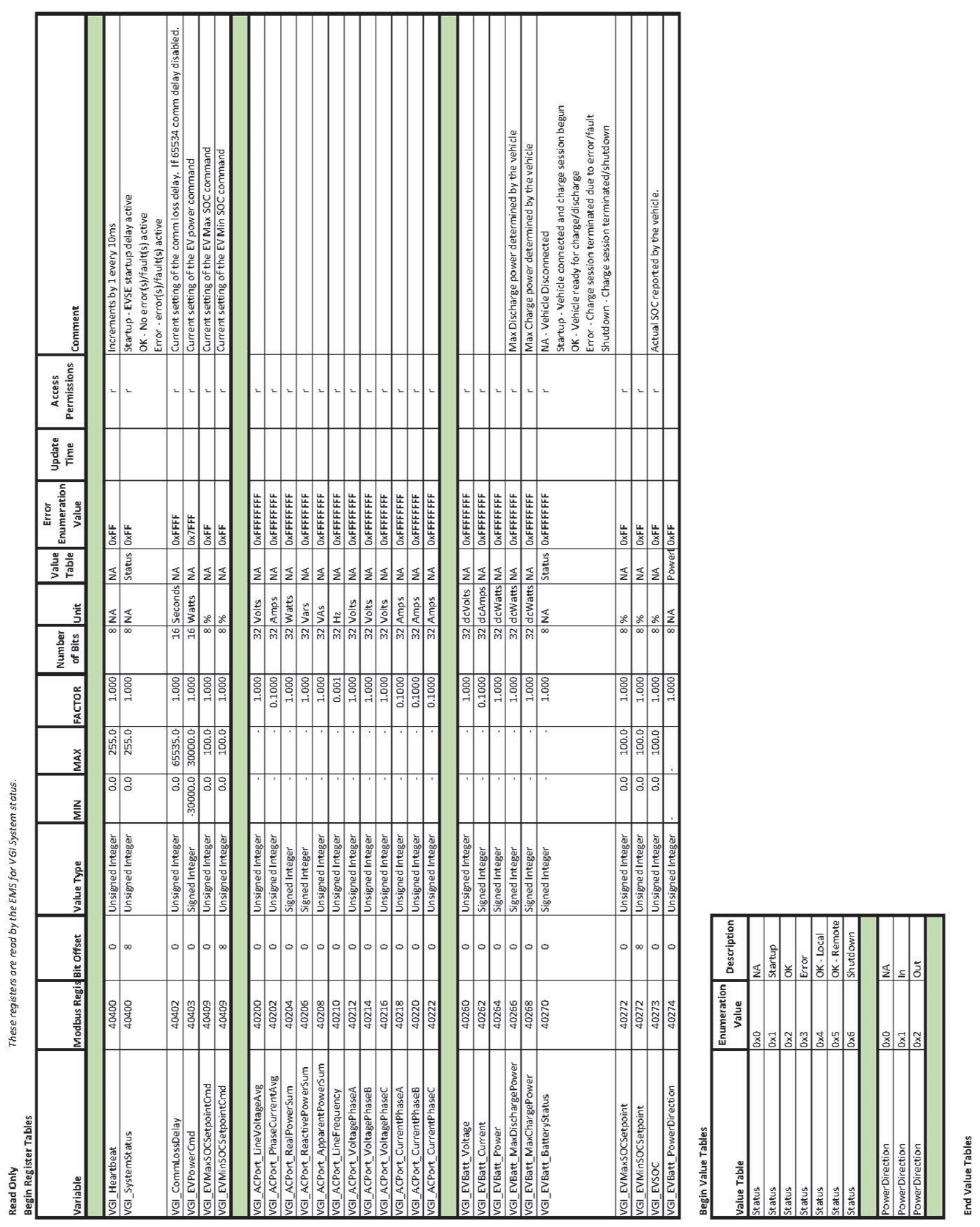




\section{REPORT DOCUMENTATION PAGE}

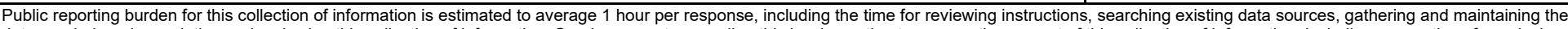

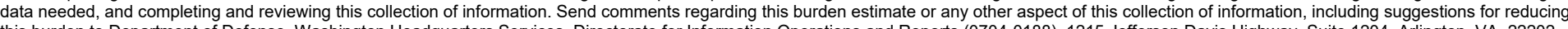

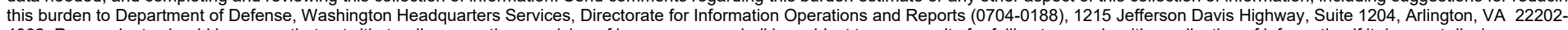

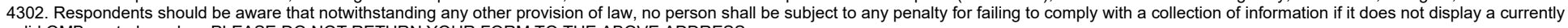
valid OMB control number. PLEASE DO NOT RETURN YOUR FORM TO THE ABOVE ADDRESS.

\section{REPORT DATE (DD-MM-YYYY) \\ September 2021 \\ 2. REPORT TYPE \\ Final Technical Report (TR)}

\section{TITLE AND SUBTITLE}

Integration of Autonomous Electric Transport Vehicles into a Tactical Microgrid: Final Report

6. AUTHOR(S)

Angela Rolufs, Amelia Trout, Kevin Palmer, Clark Boriack, Bryan Brilhart and Annette L. Stumpf

3. DATES COVERED (From - To)

5a. CONTRACT NUMBER

W9132T19CoOO3

5b. GRANT NUMBER

5c. PROGRAM ELEMENT

5d. PROJECT NUMBER

6LKD1G

5e. TASK NUMBER

\section{5f. WORK UNIT NUMBER}

6LKD1G

8. PERFORMING ORGANIZATION REPORT NUMBER

ERDC/CERL TR-21-19

\section{SPONSORING / MONITORING AGENCY NAME(S) AND ADDRESS(ES)}

Headquarters, U.S. Army Corps of Engineers (HQUSACE)

441 G St., NW

Washington, DC 20314-1000

\section{SPONSOR/MONITOR'S ACRONYM(S)}

11. SPONSOR/MONITOR'S REPORT NUMBER(S)

\section{DISTRIBUTION / AVAILABILITY STATEMENT}

Approved for public release; distribution is unlimited.

\section{SUPPLEMENTARY NOTES}

\section{ABSTRACT}

The objective of the Autonomous Transport Innovation (ATI) technical research program is to investigate current gaps and challenges and develop solutions to integrate emerging electric transport vehicles, vehicle autonomy, vehicle-to-grid (V2G) charging and microgrid technologies with military legacy equipment. The ATI research area objectives are to: identify unique military requirements for autonomous transportation technologies; identify currently available technologies that can be adopted for military applications and validate the suitability of these technologies to close need gaps; identify research and operational tests for autonomous transport vehicles; investigate requirements for testing and demonstrating of bidirectional-vehicle charging within a tactical environment; develop requirements for a sensored, living laboratory that will be used to assess the performance of autonomous innovations; and integrate open standards to promote interoperability and broad-platform compatibility. This final report summarizes the team's research, which resulted in an approach to develop a sensored, living laboratory with operational testing capability to assess the safety, utility, interoperability, and resiliency of autonomous electric transport and $\mathrm{V}_{2} \mathrm{G}$ technologies in a tactical microgrid. The living laboratory will support research and assessment of emerging technologies and determine the prospect for implementation in defense transport operations and contingency base energy resilience.

\section{SUBJECT TERMS}

Automated vehicles; Electric vehicles; Microgrids (Smart power grids); Battery charging stations (Electric vehicles); Transportation, Military

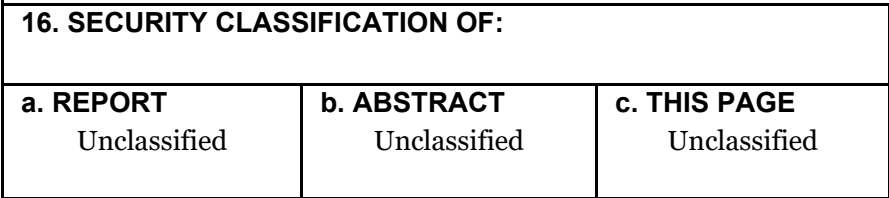

\section{LIMITATION} OF ABSTRACT

SAR
18. NUMBER OF PAGES

462 19a. NAME OF RESPONSIBLE PERSON

19b. TELEPHONE NUMBER (include area code) 
U.S. Army Engineer Research and Development Center (ERDC)

Construction Engineering Research Laboratory (CERL)

2902 Newmark Dr.

Champaign, IL 61824

Paragon Business Solutions, Inc.

610 N. Olive Street

Rolla, MO 65401

CS2 (Center for Sustainable Solutions)

2700 Vinton Ct.

Charlottesville, VA 22901

TechFlow

9889 Willow Creek Road, Suite 100

San Diego, CA 92131

Robotic Research

555 Quince Orchard Road, Suite 300

Gaithersburg, MD 20878 